\title{
Die Demographie des Alten Ägypten
}

Eine Phänomenologie anhand altägyptischer Quellen

\author{
Dissertation \\ zur \\ Erlangung des akademischen Grades \\ >Doktor der Philosophie< \\ der Philosophischen Fakultät \\ der Georg-August-Universität Göttingen
}

\author{
vorgelegt von \\ JÜRGEN KRAUS \\ aus Kassel
}

Göttingen 2004 
(c) Copyright 2004 Jürgen Kraus

Seminar für Ägyptologie \& Koptologie

Georg-August-Universität Göttingen

Diese Dissertation wurde mit den Textsatzsystem LTEX erstellt.

Hieroglyphensatz: HieroTEX von Serge Rosmorduc, Université de Paris 8

Zusätzliche Hieroglyphenzeichen: Extended Library, (c) 1993-1996 CCER Utrecht

Zeichensatz für ägyptologische Umschrift von Friedrich Junge, Universität Göttingen

Zeichensatz für Koptisch: Coptic Regular von Dirk Van Damme und Gregor Wurst

Zeichensatz für Altgriechisch: Ibycus4 von Pierre A. MacKay, University of Washington

Bibelzitate nach der Lutherübersetzung in der Revision von 1964. 


\section{Vorwort}

$\mathrm{D}$ AS Thema der vorliegenden Arbeit hat seinen Ursprung in einem Vorschlag meines Doktorvaters Friedrich Junge, der mir bereits 1994 nach meinem Wechsel an die Universität Göttingen vorschlug, das Thema >Ägyptische Demographie< auf eine breitere statistische Basis zu stellen. Ein Thema, das ich seinerzeit jedoch verwarf. Als ich 1996 die Ägyptische Landeskunde von Fouad Ibrahim in den Händen hielt, griff ich den Gedanken wieder auf, und es entstand daraus die Idee, eine Altägyptische Landeskunde nach dem Muster Ibrahims zu schreiben, jedoch moderner als die bereits von Hermann Kees Mitte der 50er Jahre vorgelegte. Beim Lesen der Sekundärliteratur zeigte sich, daß allein das Kapitel Demographie sich als weitaus umfangreicher herausstellte, als es zu Beginn der Arbeit hätte vorausgesehen werden können. So entstand aus dem ursprünglich auf ein einzelnes Kapitel beschränkten Teilaspekt eine eigenständige Arbeit.

Es ist mir eine angenehme Pflicht, an dieser Stelle allen zu danken, die mir in den vergangenen Jahren auf die verschiedenste Weise bei der Entstehung dieser Arbeit beigestanden haben. An erster Stelle möchte ich meinem Doktorvater Friedrich Junge danken, der trotz der verschlungenen Pfade, die meine Themensuche genommen hat, als Initiator der vorliegenden Arbeit gelten muß und deren Entstehungsphase mit ständigem Interesse und wissenschaftlicher Neugier begleitet hat.

Emel Çoban-Kraus, Helga Kraus, Elke Mählitz, Matthias Müller und Franziska Vahle haben die Mühsal des Korrekturlesens auf sich genommen und so manche Kante im Text geglättet. Ihnen gilt mein besonderer Dank.

Ein herzlicher Dank sei auch den Angehörigen des Göttinger Seminars ausgesprochen, die auf vielfältige Weise zum Gelingen der Arbeit beigetragen haben: Heike Behlmer, Albrecht Endruweit, Ralf Ernst, Khaled Hamza Awad, Rainer Hannig, Marion Hesse, Jürgen Horn, Frank Kammerzell, Clemens Liedtke, Matthias Müller, Hubertus Münch, Christian Opitz, Malte Rosenau und Daniel Werning. Daß viele an mich herangetragene Anregungen nicht den Weg in diese Arbeit gefunden haben, liegt allein in meiner Verantwortung.

Widmen möchte ich diese Arbeit meinen Eltern, die mit Geduld und umfassender Unterstützung dazu beigetragen haben, daß alles ein glückliches Ende gefunden hat. 


\section{Inhaltsverzeichnis}

1 Einleitung 1

1.1 Der Gegenstand demographischer Forschung . . . . . . . . . . . . . 1

1.2 Fragestellung und Zielsetzung . . . . . . . . . . . . . . . 2

1.3 Demographie als Forschungszweig . . . . . . . . . . . . . . . . 5

1.3.1 Die Wegbereiter: David Hume und Robert Malthus . . . . . . . . . . 5

1.3.2 Exkurs: Malthus, Bevölkerungsdruck und die carrying capacity . . . 10

1.3.3 Anfänge ägyptologischer Demographie . . . . . . . . . . . . . . 12

1.3.3.1 Die $>$ Déscription de l'Égypte $<\ldots \ldots . \ldots . . \ldots 12$

1.3.3.2 Zwei Reisende des 19. Jahrhunderts . . . . . . . . . . 13

1.4 Ägyptologie und Demographie im 20. Jahrhundert . . . . . . . . . . . . . 16

1.4.1 Moderne Ansätze und Berechnungen . . . . . . . . . . . . . . . . 16

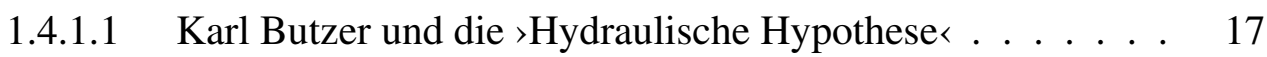

1.4.1.2 Die Untersuchungen von Fekri Hassan . . . . . . . . . . 21

1.4.2 Neue Methoden halten Einzug . . . . . . . . . . . . . . . 23

1.5 Ausblick: Lineares oder nichtlineares Verhalten? . . . . . . . . . . . . 26

2 Die griechische Überlieferung 30

2.1 Der Umgang mit griechischen Quellen . . . . . . . . . . . . . . 30

2.1.1 Exkurs: Über die Herkunft von Zahlenangaben . . . . . . . . . . . . 31

2.1.2 Verschiedene Lesarten für unterschiedliche Intentionen . . . . . . . . 32

2.2 Extreme Schätzungen der Gesamtbevölkerung auf Basis antiker Autoren . . . 33

2.3 Das Ägyptenbild in der klassischen Antike . . . . . . . . . . . . . . . . 36

2.4 Zensusverfahren in griechisch-römischer Zeit . . . . . . . . . . . . . 39

2.5 Die historische Überlieferung von Diodor und Josephus . . . . . . . . . . . . 45 
2.5.1 Das Werk Diodors in der Kritik . . . . . . . . . . . . . . 45

2.5.2 Der Bericht des Josephus . . . . . . . . . . . . . . . . . . . 53

2.6 Eine neue Hypothese zur Bevölkerungsentwicklung . . . . . . . . . . . . . . 57

2.6.1 Prämissen . . . . . . . . . . . . . . . . 57

2.6.2 Frühdatierung und Spätdatierung der Quelle . . . . . . . . . . . . . 58

2.6.3 Bevölkerungsentwicklung und technischer Fortschritt . . . . . . . . . 64

2.7 Zusammenfassung . . . . . . . . . . . . . . . . 67

3 Zensus-Papyri aus pharaonischer Zeit 69

3.1 Zur Situation . . . . . . . . . . . . . . . . . . . . 69

3.1.1 Umfang der Quellen . . . . . . . . . . . . . . . . . . . . . . 69

3.1 .2 Zeitstellung ...................... 70

3.2 Zensustexte aus den Gebelein-Papyri . . . . . . . . . . . . . . . 71

3.2 .1 Herkunft . . . . . . . . . . . . . . . . . 71

3.2 .2 Aufbau .......................... 72

3.2.3 Inhaltliche Auswertung . . . . . . . . . . . . . . . . . 72

3.3 Die Kahun-Papyri . . . . . . . . . . . . . . . . . . . . . . . . . . . 75

3.3.1 Herkunft ....................... 75

3.3 .2 Aufbau \& Struktur . . . . . . . . . . . . . . . . . 77

3.3.3 Zur Interpretation der Aktenvermerke . . . . . . . . . . . . . . . 85

3.3.4 Die Auflistung der Dienerschaft . . . . . . . . . . . . . . . . . . 88

3.4 Eine Häuserliste aus Theben-West . . . . . . . . . . . . . . . . . . . . 91

3.4 .1 Aufbau ........................... 91

3.4 .2 Zweckbestimmung .......................... 92

3.5 Zusammenfassung . . . . . . . . . . . . . . . . . . 97

4 Die Bevölkerung in Siedlungen und Regionen 99

4.1 Über die Verwertbarkeit der Daten aus den Papyri . . . . . . . . . . . . . . . 99

4.2 Statistiken über die Wohnflächen als Gegenprobe . . . . . . . . . . . . . . . 101

4.3 Die Arbeitersiedlung (>Eastern Village $<$ ) in Amarna . . . . . . . . . . . . . . 102

4.3.1 Aufbau ....................... 102

4.3.2 Die $>$ Main City< Amarnas als Vergleichsobjekt . . . . . . . . . . . . 104

4.3.3 Statistische Auswertung . . . . . . . . . . . . . . . . . . . 104

4.3.4 Die Grenzen der Berechnungsmöglichkeiten . . . . . . . . . . . 106 
4.4 Die Arbeitersiedlung Deir el-Medineh . . . . . . . . . . . . . . . . . 108

4.4.1 Aufbau . . . . . . . . . . . . . . . . . 108

4.4.2 Statistische Auswertung . . . . . . . . . . . . . . . . 108

4.5 Die Siedlungsanlage von Kahun - Versuch einer Näherung . . . . . . . . . . 110

4.5 .1 Aufbau . . . . . . . . . . . . . . . . . 110

4.5.2 Gebäudetypen und Verteilung . . . . . . . . . . . . . . 111

4.5.3 Modellrechnungen . . . . . . . . . . . . . . . . . 113

4.6 Hochrechnungen auf regionaler Ebene . . . . . . . . . . . . . . 115

4.6.1 Papyrus Harris I . . . . . . . . . . . . . . . . . 116

4.6 .2 Die Lehre für Merikare . . . . . . . . . . . . . . . . . . 117

4.7 Zusammenfassung . . . . . . . . . . . . . . . . 119

5 Pharaonische Quellen: Zahlen und Verfahren $\quad 121$

5.1 Topos kontra Realität . . . . . . . . . . . . . . . . . . . . . 121

5.1.1 Vom Umgang mit überlieferten Zahlen . . . . . . . . . . . . . . . 121

5.1.2 Untersuchungsmethode . . . . . . . . . . . . . . . . . . . . . . 124

5.2 Zählungen im pharaonischen Ägypten . . . . . . . . . . . . . . 125

5.2.1 Terminologie . . . . . . . . . . . . . . 125

5.2.2 Zur Zweckbestimmung von Zählungen . . . . . . . . . . . . . . . . 126

5.2.2.1 Erfassung wehrfähiger Männer . . . . . . . . . . . . 128

5.2.2.2 Fallbeispiel: Eine einzelne Zahl und ihr Informationsgehalt 133

5.2.2.3 Steuer- und Abgabenerfassung . . . . . . . . . . . . . 139

5.2.2.4 Subjekt und Objekt, oder: Was ist ein wpw.t ? . . . . . . . 145

5.2.2.5 Arbeitsverpflichtung und Frondienst . . . . . . . . . . 154

5.2.3 Der demographische Wert der ältesten Belege . . . . . . . . . . . . . 157

5.2.3.1 Der Keulenkopf des Narmer . . . . . . . . . . . . . . . 157

5.2.3.2 Die Inschrift des Dewen auf dem Annalenstein . . . . . . . 165

5.2.3.3 Die toten Feinde des Chasechemui . . . . . . . . . . . . . 169

5.2.3.4 Die Kriegsgefangenen des Snofru . . . . . . . . . . . . 171

5.3 Zusammenfassung . . . . . . . . . . . . . . . . 177

6 Exogene demographische Faktoren $\quad 178$

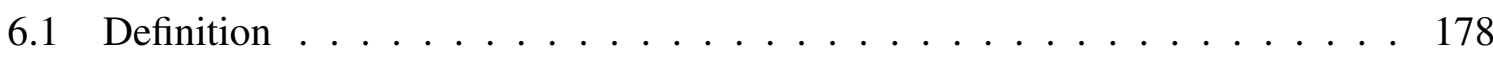

6.2 Naturkatastrophen: Überschwemmungen und Dürre . . . . . . . . . . . . . . 178 
6.3 Krankheiten . . . . . . . . . . . . . . . . . . . . . 181

6.3 .1 Die $>$ Pest $<\ldots \ldots \ldots \ldots \ldots$. . . . . . . . . . . . 181

6.3.1.1 Definition .................. 181

6.3.1.2 Ratten und Flöhe als Überträger . . . . . . . . . . . . . . . 183

6.3.1.3 Historische Evidenz . . . . . . . . . . . . . . . . . . . . . 184

6.3.1.4 Saisonale Sterblichkeitsraten . . . . . . . . . . . . . . 191

6.3 .2 Andere Infektionskrankheiten . . . . . . . . . . . . . . . . . 196

6.4 Hungersnot . . . . . . . . . . . . . . . . . . . . . . . . . . . 198

6.4.1 Nahrungsmangel als demographischer Faktor? . . . . . . . . . . . . 198

6.4.2 Entkoppelung von Hungersnot und Populationsrückgang . . . . . . . 201

6.5 Geburtsraten und Kindersterblichkeit . . . . . . . . . . . . . . . . . . . 203

6.5.1 Konzeption und Kontrazeption . . . . . . . . . . . . . . . . . . . . . 204

6.5.2 Sterbeziffern, Konstitution und Fertilität . . . . . . . . . . . . . . . 206

6.6 Soziale Auswirkungen demographischer Einbrüche . . . . . . . . . . . . . 208

6.7 Zusammenfassung . . . . . . . . . . . . . . . . . . 212

7 Die ägyptische Population im 19. Jahrhundert 214

7.1 Moderne Bevölkerungsstatistiken . . . . . . . . . . . . . . . . . . . . . 214

7.1.1 Volkszählungen und Modellrechnungen als Datenbasis . . . . . . . . 215

7.1.2 Beobachtungen und Ergebnisse . . . . . . . . . . . . . . . . 220

7.1.3 Lineare Wachstumsberechnungen . . . . . . . . . . . . . . 221

7.2 Grenzen der Vergleichbarkeit . . . . . . . . . . . . . . . . . . . 223

7.3 Faktoren der Bevölkerungsentwicklung Deutschlands . . . . . . . . . . . . . 227

7.4 Zusammenfassung . . . . . . . . . . . . . . . 231

8 Zusammenfassung der Ergebnisse 233

$\begin{array}{ll}\text { Siglen und Abkürzungen } & 238\end{array}$

$\begin{array}{ll}\text { Literaturverzeichnis } & 239\end{array}$ 


\section{Abbildungsverzeichnis}

1.1 Zwei Interpretationen zur Auswirkung der carrying capacity

$($ nach HASSAN 1981: 165) $\ldots \ldots \ldots \ldots$. . . . . . . . . . . 8

2.1 Interpolierte Populationskurven nach den Angaben griechischer Autoren

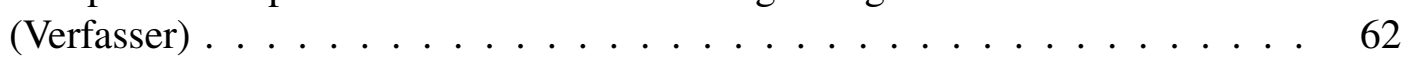

4.1 Das sogenannte $>$ Eastern Village $<$ von Amarna (nach BADAWY 1968: 111) . . . . . . . . . . . . . . . . . 103

4.2 Die dritte Bauphase der Arbeitersiedlung Deir el-Medineh (nach BRUYÈrE 1939: Taf. 7) . . . . . . . . . . . . . . . . . . . 106

4.3 Die Siedlungsanlage von Kahun (nach KEMP 1991: 150) . . . . . . . . . . . . . . . . . . . . . . . . . 112

5.1 Rang-Größen-Verteilung der Agrarflächen im Papyrus Wilbour (ADAMs 1997: 100) . . . . . . . . . . . . . . . . . . . 142

5.2 Der Keulenkopf des Narmer (Millet 1990: 54) . . . . . . . . . . . . . . . . . . . . . . . . . 159

5.3 Das Jahr $\mathrm{x}+4$ des Königs Dewen auf dem Annalenstein (SCHÄFER 1902: 19) . . . . . . . . . . . . . . . . . . . . . . . 165

5.4 Abbildungen auf den Sockeln der Chasechemui-Statuetten (ADAMS 1990: 162) . . . . . . . . . . . . . . . . . . . 170

5.5 Die Jahre 14 und 15 des Königs Snofru auf dem Annalenstein (SCHÄFER 1902: 30) . . . . . . . . . . . . . . . . . . . . . . 172

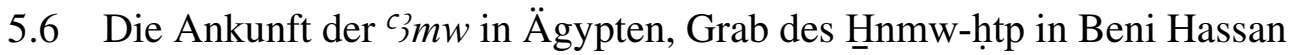
(NEWBERRY 1893: Taf. 30) . . . . . . . . . . . . . . . . 176

6.1 Vergleichende Verlaufskurve zur Mortalität (nach ScHEIDEL 2001a: Tab. 1.1, 1.3, Abb. 1.42) . . . . . . . . . . . . . . . 194

7.1 Bevölkerungsentwicklung in Ägypten seit 1800 (Verfasser) . . . . . . . . . . . . . . . . . . . . . . . . . . 219

7.2 Bevölkerungsentwicklung in Deutschland zwischen 1816 und 1915 (nach MARSCHALCK 1984: 145 f., Tab. 1.1-1.3) . . . . . . . . . . . . . 228 


\section{Tabellenverzeichnis}

1.1 ButZERs Eigenschaftskatalog zur Klassifizierung von Siedlungen

(BUTZER 1976: 59) . . . . . . . . . . . . . . . . . . . 19

1.2 Hypothetische Bevölkerungsentwicklung Ägyptens nach BUTZER

(ButZer 1976: 83 Tab. 4) . . . . . . . . . . . . . . . 21

2.1 Eckdaten einer Früh- und Spätdatierung von Diodors Angaben

(Verfasser) . . . . . . . . . . . . . . . . . . . . . 61

4.1 Hausgrößen und Haushaltsgrößen in Tell el-Amarna

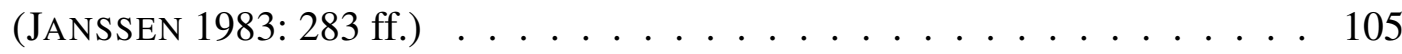

4.2 Berechnung der Bewohnerzahl Amarnas

(Daten nach JANSSEN 1983 und Tietze 1985) . . . . . . . . . . . . . . . . 107

4.3 Haustypen in Kahun nach Einteilung von RoIK

(ROIK 1988: 8 ff.) . . . . . . . . . . . . . . . . . . 111

5.1 Zusammenstellung der Expeditionsgrößen des Mittleren Reiches

(SEYFRIED 1981:219f.) . . . . . . . . . . . . . . . . . 136

5.2 Mannschaft einer Expedition in das Wadi el-Hudi (nach SADEK 1980: 16 ff.) . . . . . . . . . . . . . . . . . . . . . . 137

5.3 Vergleich der Schreibweisen für wpw.t/wp.t

(Verfasser) . . . . . . . . . . . . . . . . . . . . . . . . . . . . . . 149

5.4 Vergleich von Listen mit Angaben zu Rindern und Kleinvieh

(Verfasser) . . . . . . . . . . . . . . . . . . . . . . . 160

5.5 Osteoarchäologische Befunde aus dem Nildelta

(Verfasser) . . . . . . . . . . . . . . . . . . . . 162

6.1 Die saisonale Verbreitung der Pest im modernen Ägypten

(KuHnke 1992: 73 Anm. 18) . . . . . . . . . . . . . . . . . . . . . 192

7.1 Schätzungen und Zensusdaten aus dem Ägypten des 19. Jahrhunderts (nach ScHEIDEL 2001a: 205 f., Tab. 3.1) . . . . . . . . . . . . . . . . . . 217

7.2 Bevölkerungsentwicklung im Ägypten des 19. Jhs.

(nach SCHEIDEL 2001a: 211 f.) 


\section{Einleitung}

Die phänomenologische Methode ist [...] eine spezifische Schau der Dinge, die darauf abzielt, an ihnen Aspekte aufzudecken, welche der üblichen Sicht durch Gewohnheit verdeckt sind.

Vilém Flusser, Lob der Oberflächlichkeit

\subsection{Der Gegenstand demographischer Forschung}

"NCIENT demography has finally arrived. « Mit diesen Worten leitete Walter SCHEIDEL
im Jahr 2001 einen Sammelband mit Beiträgen zur römischen Demographie ein. ${ }^{1}$ Dieses Buch stellt zusammen mit SCHEIDELs im gleichen Jahr erschienener Monographie Death on the Nile ${ }^{2}$ einen vorläufigen Höhepunkt in der Forschung zur (antiken) historischen Demographie dar. Mit der Veröffentlichung beider Werke wurde zugleich deutlich, daß sich in den vergangenen Jahrzehnten in der demographischen Forschung ein massiver Wandel vollzogen hat. Obwohl der Gegenstand der Forschung weiterhin die »Erklärung von Strukturen und Entwicklungen von (antiken) Bevölkerungen in ihrem Verhältnis zum Lebensraum « bleibt, ${ }^{3}$ haben sich bei der Wahl von geeigneten Verfahren tiefgreifende Veränderungen vollzogen, wie die aktuelle Definition für Demographie beweist: ${ }^{4}$

»Die Demographie $[\ldots$. $]$ befaßt sich überwiegend quantitativ mit der wissenschaftlichen Untersuchung menschlicher Bevölkerungen hinsichtlich ihrer Größe, Struktur, Entwicklung und ihrer allgemeinen Merkmale.«

Auch in der historischen Demographie stehen die überlieferten Textzeugnisse nicht mehr als primäre Quelle im Zentrum des Interesses, sondern müssen sich in ihrer Aussagequalität an Rechenmodellen zur Bevölkerungsentwicklung messen lassen. Es sind nicht nur durch neue

1 SCHEIDEL (2001b).

2 SCHEIDEL (2001a). Diese Untersuchung befaßt sich abermals ausschließlich mit dem Ägypten der römischen Epoche.

3 WIESEHÖFER und RENGER (1997).

4 HöHN u.a. (1987: $§ 101.1)$; im weiteren Verlauf dieser Arbeit orientiert sich die verwendete Terminologie ausschließlich an den Definitionen des Demographischen Wörterbuchs. 
historische Erkenntnisse auch neue Fragen aufgeworfen worden, sondern aufgrund der immer stärkeren Standardisierung der (modernen) demographischen Forschung weltweit hat sich der Zweig der historischen Demographie diesem Trend angeschlossen und den Schwerpunkt auf die numerischen Aspekte und Verfahren verlagert. Vorrangiges Ziel der Analyse scheint hier die Vergleichbarkeit des ausgewerteten Zahlenmaterials zu sein. So operieren selbst gestandene Althistoriker plötzlich mit Sterbetafeln und streiten darüber, ob für die untersuchte Gesellschaft die Tabelle West Level 3 oder West Level 2 sinnvoller anzuwenden ist. ${ }^{5}$ Gleichzeitig wird den historischen Wissenschaften eine »complete ignorance of the growing body of modern research on determinants of fertility and fertility transition theory « vorgeworfen. ${ }^{6}$ Damit scheint sich zu bestätigen, daß sich die Demographie - als historische und textkritische Disziplin - der >quantitativen $<$ Demographie schleichend untergeordnet hat.

Selbst wenn die reine Abschätzung einer Gesamtbevölkerungsgröße als »Demography at its crudest level « bezeichnet wird, ${ }^{7}$ so wäre die Ägyptologie schon dankbar, wenn in diesen Bereich etwas mehr Licht gebracht und das Zahlenmaterial zumindest auf einer soliden Basis von Erkenntnissen stehen würde. ${ }^{8}$ Doch erst wenn der Autor den Weg zur Ermittlung dieser Zahl darlegt, besteht die Möglichkeit einer Überprüfung seiner Kriterien. Diese einfache Regel wird allerdings in den meisten Publikationen nicht befolgt. Gleichwohl verlangt die Forschung nach verläßlichen Angaben zur altägyptischen Population. ${ }^{9}$ Dies ist in besonderem Maße einem Trend zu verdanken, der in den letzten Jahrzehnten das Interesse der Forschung auf die Siedlungsarchäologie gelenkt hat. ${ }^{10}$ Die neuen Fragestellungen der Siedlungsgeographie verlangen nach fundiertem Datenmaterial zu Haushaltsgrößen, Familienstrukturen und Bevölkerungsdichte. ${ }^{11}$

\subsection{Fragestellung und Zielsetzung}

Die vorliegende Arbeit weicht in vielerlei Hinsicht von den typischen demographischen Fragestellungen ab. Die Menge an verwertbaren Dokumenten ist gering und allein diese unbestreitbare Tatsache hat gerade in jüngerer Zeit zu einer generellen Ablehnung jeglicher For-

5 SCHEIDEL (2001b: $19 \mathrm{ff}$.).

6 SCHEIDEL (2001b: 37).

7 Hollingsworth (1969: 37); zum geringen Wert einer einzelnen Gesamtzahl im Sinne der modernen demographischen Forschung siehe auch SCHEIDEL (2001b: 49 f. Anm. 196).

8 SCHEIDEL (2001b: 50): »Nevertheless, population size matters, and is of more than antiquarian interest.«

9 Vgl. etwa die Untersuchung zur altägyptischen Literarizität von BAINES und EYRE (1983).

10 Siehe z. B. BIETAK (1975: 55 Anm. 167).

$11 \mathrm{Zu}$ einzelnen Aspekten der Siedlungsgeographie vgl. BUTZER (1984). 
schung zur Demographie des Alten Ägypten geführt. Daher soll an dieser Stelle die Definition von >Demographie< etwas erweitert werden. Im Zentrum des Interesses stehen zum einen die Textquellen und die Möglichkeiten ihrer Auswertung, zum anderen werden Indizien für Faktoren gesammelt, die einen positiven oder negativen Einfluß auf die Bevölkerungsentwicklung haben. Ziel ist es, ein Gerüst zu erstellen, in das künftige Beobachtungen integriert werden können und sich nach und nach wie ein Mosaik zu einem Gesamtbild der altägyptischen Bevölkerung zusammenfügen.

Faktisch bleibt damit eine Gesellschaft wie die des pharaonischen Ägypten für die moderne Demographie eine terra incognita. Diese freiwillige Selbstbeschränkung ist jedoch alles andere als befriedigend. Die auf die Untersuchungen zum pharaonischen Ägypten folgende Kritik wurde stets recht grundsätzlich formuliert - den Unterschied zwischen >Demographie< einerseits und >historischer Demographie< andererseits verkennend. Die von Peter BILlER vorgeschlagene Differenzierung scheint dagegen dem zu untersuchenden Material Rechnung zu tragen: ${ }^{12}$

»Historical demography is this way of thinking and studying, but applied to past peoples by modern historians, historical demographers. The initial definition of demography needs to be adapted when applied to historical demography, with the phrase 'by statistical methods' replaced by 'according to quantity'.«

BILLER hat in seiner Definition der historischen Demographie exakt herausgearbeitet, wo die Kernkompetenzen dieses Forschungszweiges liegen können und müssen: eine verifizierbare Grundlage demographischer Phänomene außerhalb eines statistischen Rahmens zu erarbeiten. Der Mangel an Daten aus dem Alten Ägypten für halbwegs verläßliche Erhebungen zur Geburtenrate oder der Geschlechtsgliederung ist nicht zu leugnen. An deren Stelle müssen Einzelbeobachtungen aus den Texten treten, die mittels Vergleich mit besser bekanntem Material auf ihre Korrektheit und Reichweite überprüft werden müssen. So kann ein einzelner Hinweis aus einem Papyrus auf eine Kleinfamilie mit vier Personen nicht sofort als Paradigma für eine ganze Epoche herangezogen werden. Vielmehr gilt es, diese Information mit anderen Einzelbeobachtungen abzugleichen.

Die folgenden Kapitel sind dabei thematisch strikt voneinander abgegrenzt. Im zweiten Kapitel werden die Textquellen der griechisch-römischen Antike unter die Lupe genommen. Dies geschieht aus zweierlei Gründen: Erstens wird für die Angaben von Diodor und Josephus eine neue Interpretation angeboten, die sich sowohl in die historischen Gegebenheiten, als auch in die auf anderem Weg erarbeiteten Bevölkerungsschätzungen für die römische Kaiserzeit in Ägypten gut einpaßt und Probleme mit den Größenordungen der Überlieferung vermei-

12 BiLler (2000: 6). 
det, zweitens dient die Beschreibung der griechisch-römischen Zensusverfahren als Erbe der pharaonischen Administration der Stärkung des Arguments einer nahtlosen Entwicklung der Zensusverfahren. Die altägyptischen Zählungen müssen weitaus zuverlässiger und umfangreicher gewesen sein, als die wenigen erhalten gebliebenen Zeugnisse heute preisgeben. Eben jene wenigen Fragmente pharaonischer Zählung von Personengruppen sind Thema des dritten Kapitels. Aus allen drei Hauptepochen der altägyptischen Geschichte sind einzelne Aktenstücke in unterschiedlichem Erhaltungsgrad überliefert, von denen die Haushaltsaufstellungen des Mittleren Reiches aus Kahun die detailliertesten und interessantesten Einblicke in einen gehobenen ägyptischen Haushalt und die Lebensumstände seiner Bewohner bieten. Für eine Vergleichbarkeit der Familienstrukturen mit anderen Kulturkreisen und Epochen wurde auf das graphische Modell von Eugene HAMMEL und Peter LASLETT zurückgegriffen, das eine standardisierte Wiedergabe der Strukturen ermöglicht. Aufbauend auf die aus den Zensuspapyri erarbeiteten Familiengrößen erfolgt eine Betrachtung von Siedlungen und deren Bevölkerungsgröße in Kapitel vier. Neben dem Versuch einer Hochrechnung anhand der durchschnittlichen Haushaltsgrößen für ausgewählte Siedlungsanlagen Ägyptens zu verschiedenen Zeiten, kamen hier auch aus der Ethnologie entlehnte Formeln für die Bevölkerungsdichte von Siedlungen als Gegenprobe zur Anwendung, die das zuvor gewonnene Ergebnis stützen oder widerlegen sollten. Kapitel fünf kehrt thematisch nochmals zu den schriftlichen Quellen des Alten Ägypten zurück. Neben den Zensuspapyri haben sich in den verschiedensten Dokumenten zahllose Angaben zur Größe bestimmter Personenkreise erhalten, die in der Vergangenheit häufig als Beleg für demographische Angaben über das Alte Ägypten herangezogen, oder aber aufgrund mangelnder Erklärbarkeit als Übertreibung zurückgewiesen wurden. In dem Kapitel werden sowohl verschiedene zweckgebundene Erhebungen von Personen diskutiert, als auch die ältesten Belege für Zahlenangaben von Menschen auf ihre Plausibilität überprüft. Kapitel sechs widmet sich einem recht problematischen Teil demographischer Forschung, nämlich der Überprüfung von externen Faktoren, die für demographische Schwankungen verantwortlich sein können. Dazu gehören Naturkatastrophen, Epidemien sowie grundsätzliche Aspekte der Gesundheit ägyptischer Frauen, die sich auf deren Fertilität auswirken. Das siebte Kapitel schließlich beschäftigt sich mit dem modernen Ägypten des 19. Jahrhunderts, das immer wieder gerne für Vergleiche mit dem Alten Ägypten herangezogen wurde, um bestimmte Parallelen in der Bevölkerungsentwicklung zu bestätigen. Anhand neuer Forschungsergebnisse und eines Vergleichs mit der Bevölkerungsentwicklung Deutschlands im gleichen Zeitraum kann gezeigt werden, daß sich eine solche grundsätzliche - und damit vergleichbare - Übereinstimmung nicht $a$ priori festlegen läßt. 
Es sei nochmals deutlich hervorgehoben, daß im Fokus der Arbeit nicht die Materialschau, respektive eine möglichst Vollständigkeit anstrebende Katalogisierung aller Zeugnisse stand, die zum Thema Zensus oder Zählung von Menschen aus dem Alten Ägypten bekannt sind. Stattdessen sind die Fragestellungen problembezogen, und die Wahl und Auswertung der Quellen wird allein durch die zuvor gestellte Frage bestimmt. Diese Form der Herangehensweise hat gewisse Vorteile, da so auch Quellen als Zeugen dienen können, die nicht primär mit dem Thema >Demographie< in Zusammenhang zu bringen sind. Es liegt daher in der Natur des Gegenstandes dieser Arbeit, daß mancher Aspekt unberücksichtigt bleiben mußte. Dazu gehören etwa die Auswertungen von Nekropolen, deren statistische Analyse möglicherweise noch manche Überraschung bereit hält. Aber auch auf eine Untersuchung der Ostraka aus Deir el-Medineh unter dem Gesichtspunkt genealogischer Gruppierungen wurde zugunsten anderer Schwerpunkte am altägyptischen Material verzichtet. Solche Detailarbeiten, die leicht ein eigenes Buch füllen könnten, bleiben künftigen Forschungen vorbehalten.

Mehr als einhundert Jahre sind seit der Untersuchung von Julius BELOCH zur Bevölkerung der antiken Welt vergangen; genug Zeit, damit folgendes Zitat seiner damaligen Einleitung wieder einen Grund darstellt, die folgenden Kapitel zu schreiben. ${ }^{13}$

»Es giebt kein grösseres Werk über alte Geschichte, das nicht statistische Angaben in Menge enthielte, und wären es auch nur Angaben über die Stärke der Heere; und Niemand wird diese Zahlen aus der Geschichte verbannen wollen. Bei ihrer Verwerthung aber herrscht noch immer der roheste Empirismus, und grobe Irrthümer finden sich selbst in unseren besten Arbeiten. Ist es da nicht wünschenswerth, dass das gesammte überlieferte Zahlenmaterial einmal in kritischer Weise verarbeitet wird?«

\subsection{Demographie als Forschungszweig}

\subsubsection{Die Wegbereiter: David Hume und Robert Malthus}

Als einflußreichste Arbeit in den frühen Studien zur antiken Demographie darf das 1752 vom britischen Philosophen und Historiker David HumE veröffentlichte Essay Of the Populousness of Ancient Nations gelten. ${ }^{14}$ Ausgangspunkt war seinerzeit die allgemein vertretene These, daß die Bevölkerungzahl in der Antike größer gewesen sein muß als im modernen Europa. ${ }^{15}$

13 BELOCH (1886: vii f.).

14 Die weiteren Ausführungen basieren auf einem Reprint der Ausgabe von 1882, im folgenden als HUME (1964) zitiert.

15 Allgemeiner formuliert: Populationen haben sich im Laufe der Zeit in der Tendenz immer verringert. Dem Vorwurf, wenigstens ansatzweise diese Sichtweise vertreten zu haben, muß sich teilweise auch die ältere 
HuME versucht eine Widerlegung, indem er die allgemeinen Verhältnisse beleuchtet, die zu Populationsveränderungen führen können. Ausführlich beschäftigt er sich mit Status und Anzahl der Sklaven in der Antike. Dazu finden sich in seiner Abhandlung auch Erörterungen über ökonomische Notwendigkeiten, die einen Mann erst in die Lage versetzten, zu heiraten und für Nachkommen zu sorgen.

Als > Geißel der Menschheit< bezeichnet er drei Faktoren: Kriege, Pest und Hunger. ${ }^{16}$ Vor allem in den kriegerischen Auseinandersetzungen der Antike sieht HUME einen entscheidenden Faktor für verheerende Verluste unter den Soldaten, da Waffentechnik und Form der Kriegsführung seiner Einschätzung nach zwangsläufig blutigere Kämpfe zur Folge hatten als dies in der Mitte des 18. Jahrhunderts der Fall war. ${ }^{17}$ Dagegen können Epidemien zwar einen tiefen Einschnitt in die Population bedeuten, den demographischen Trend jedoch nicht umkehren, da sie innerhalb von zwei Generationen kompensierbar sind. ${ }^{18}$

Darüber hinaus analysiert HUME die aus den Texten der klassischen Antike überlieferten Zahlenangaben, wie zum Beispiel die Anzahl der Bewohner einer Stadt, die Größe eines Heeres oder die Sklaven, die ein römischer Patrizier besaß. Dabei verweist er jedoch ausdrücklich auf die geringe Verläßlichkeit, die er den Aussagen antiker Autoren zumißt. ${ }^{19}$

»The facts, delivered by ancient authors, are either so uncertain or so imperfect as to afford us nothing positive in this matter. How indeed could it be otherwise? [.. ] It is probable, that there was formerly a good foundation for the number of citizens assigned to any free city; because they entered for a share in the government, and there were exact registers kept of them. But as the number of slaves is seldom mentioned, this leaves us in a great uncertainty as ever, with regard to the populousness even of single cities.«

Wiederholt versuchte er Hochrechnungen mit den aus antiken Texten extrahierten Daten und gelangte schließlich zu der Erkenntnis, daß eine oder mehrere Angaben zu Ereignissen und Orten nicht stimmen könnten, da sie zueinander im Widerspruch ständen oder aufgrund fehlender Differenzierung ihrer Herkunft als Quelle wertlos seien. Als Beispiel für das scharfsinnige Problembewußtsein Humes seien hier etwa die Angaben zu den römischen Getreidespenden genannt, von denen überliefert ist, daß es in Rom zur Zeit des Kaisers Augustus über 200000 Empfänger der öffentlichen Getreideverteilung (annonae) gegeben haben soll.

ägyptologische Forschung stellen. So vermuteten ERMAN (1903: 16 f.) und SCHAEDEL (1936: 52 ff.) bei ihren Untersuchungen zum Papyrus Harris I allein aufgrund des Diodor-Zitats von 7 Millionen Menschen zur Zeit der Ptolemäer, daß die Population der Ramessidenzeit größer gewesen sein müsse und setzten dafür eine Zahl von 8-9 Millionen Menschen an.

16 HuME (1964: 383).

17 HuME (1964: 400 f.).

18 HUME (1964: 384).

19 Hume (1964: 413 f.). 
HumE sah sich jedoch außerstande, diese Personenzahl nach Status, Alter oder Geschlecht zu differenzieren, so daß sich die vermeintlich aussagekräftige Angabe für eine Beurteilung der römischen Gesamtbevölkerung als ungenügend herausstellt. ${ }^{20}$ An anderer Stelle dagegen errechnet HUME anhand der Angaben Appians und Diodors die Zahl der Gesamtbevölkerung Galliens auf 200 Millionen Menschen - eine Zahl, die ihm selbst kaum glaubwürdig erscheint. $^{21}$

Auch die Überlieferung Diodors zum Alten Ägypten findet an zwei Stellen Erwähnung. Zum einen die Zahl von drei Millionen Einwohnern Ägyptens (Diodor I 31,8), die HUME für sehr niedrig und mit Diodors Angabe von 18000 Städten in Ägypten (I 31,7) für unvereinbar hält. ${ }^{22}$ Diodors Hinweis auf die sieben Millionen Ägypter in >früheren Zeiten< sind für ihn dagegen ein Indiz für die allgemeine Bewunderung und Wertschätzung, die man vergangenen Epochen entgegenbrachte. Die andere Textstelle Diodors bezieht sich auf die 300000 Bewohner Alexandrias, eine Zahl, die nach Meinung HumEs zwar Frauen und Kinder mit einschließt, jedoch aller Wahrscheinlichkeit nach die Sklaven nicht mit berücksichtigt. ${ }^{23}$ Die Argumente HuMEs gegen die Ausgangsthese lassen sich wie folgt zusammenfassen: Die Situation der Sklaven und deren Beitrag zur Gesamtbevölkerung waren unklar, Gewalt und Kriege drückten auf das Bevölkerungswachstum, die überlieferten Dimensionen antiker Städte waren unvereinbar mit deren angeblicher Bewohnerzahl und die antiken Ökonomien besaßen nicht die Leistungsfähigkeit, große Populationen angemessen zu versorgen. Daraus folgernd mußten die häufig gerundeten Zahlen - antiker Beschreibungen reine Spekulationen oder gar Verklärung ihrer Autoren bleiben und stellten keine verläßliche Quelle für die historische Forschung dar.

Die Thesen HumEs blieben unter seinen Zeitgenossen nicht undiskutiert. So veröffentlichte der Hauptgegner HuMEs, Robert WALlACE, nur ein Jahr später eine Erwiderung, die auch ausdrücklich gegen die Thesen HuMEs Stellung bezog und der traditionellen Meinung verpflichtet blieb. ${ }^{24}$ Doch sollte es danach noch etliche Jahre dauern, bis abermals ein umfassendes Werk zur Population erschien. Im Jahr 1798 veröffentlichte der spätere Professor für >politische Ökonomie< Thomas Robert MALTHUS seine Überlegungen zur Bevölkerungsentwicklung in der Abhandlung An Essay on the Principle of Population. ${ }^{25}$ Darin vertrat er die These, daß eine Bevölkerung immer bis an die Grenzen der Ressourcen ihrer Umwelt und

20 HUME (1964: 427 f.).

21 HuME (1964: 436 f.).

22 HUME (1964: 415).

23 HuME (1964: 429).

24 Wallace (1753). Aufgrund seiner Herangehensweise hat SCHEIDEL (2001b: 4) die Arbeit von WALLACE als »methodologically reactionary« gescholten. Eine ausführlichere Präsentation seiner Thesen, insbesondere zur Bevölkerung des Alten Ägypten, erfolgt im Kap. 2.2.

25 Zugrunde gelegt wurde die von Philip APPLEMAN (1976) herausgegebene kritische Ausgabe. 

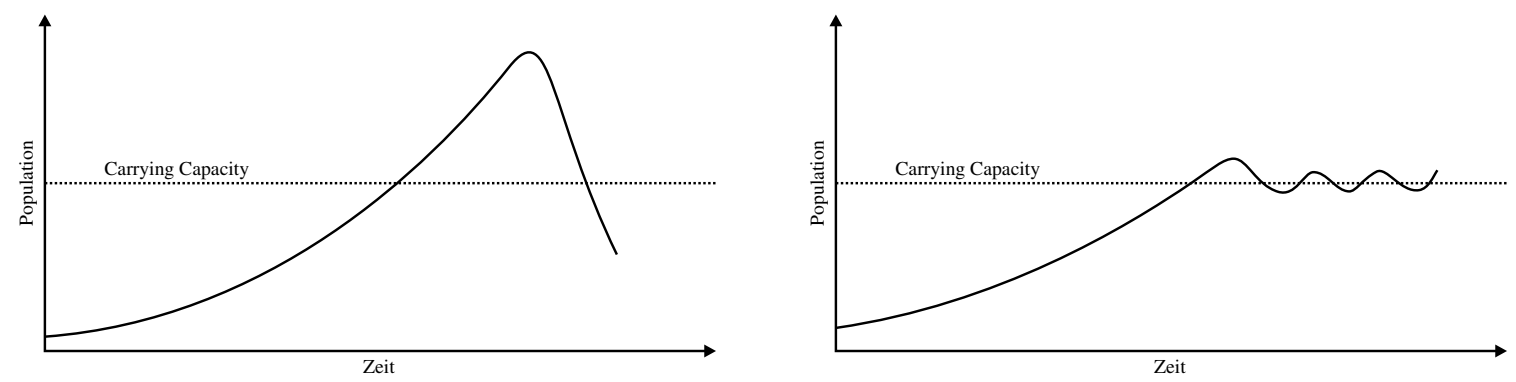

Abbildung 1.1: Zwei Interpretationen zur Auswirkung der carrying capacity

auch darüber hinaus wächst, und dabei Population und Ressourcen gänzlich unterschiedliche Wachstumsraten aufweisen. ${ }^{26}$ Während die Bevölkerung geometrisch wächst, vergrößern sich im gleichen Zeitraum die Ressourcen der Versorgungsgrundlage nur linear. Aufgrund dieses Umstandes ist nach MALTHus' Meinung eine natürliche >Verrechnung ${ }^{27}$ die zwangsläufige Folge, die in Form von Epidemien und Hungersnöten die Sterblichkeitsrate schnell ansteigen läßt, so daß der demographische Trend umgekehrt und die Bevölkerung wieder unter den Schwellenwert gedrückt wird. Mit dieser Umkehrung von Ursache und Wirkung hinsichtlich der Faktoren, die für Populationsrückgänge verantwortlich sind, stand MALTHUS im Widerspruch zu den Ausführungen HuMEs.

Aus der von ihm entworfenen These des Wachstums zieht MALTHus die Konsequenzen, indem er zwei Mechanismen formuliert, die das ungehemmte Wachstum kontrollieren sollen: eine verzögerte Heirat und damit einhergehend eine geringere Anzahl Kinder (vorbeugende Hemmnisse, preventive checks) oder Kriege, Seuchen und Hungersnöte (nachwirkende Hemmnisse, positive checks). Während das erste Hemmnis auf die Verringerung der Geburtenrate abzielt, bewirkt das zweite Hemmnis eine Erhöhung der Sterberate.

MALThus arbeitete kontinuierlich an seinem Buch weiter, so daß er 1803 eine wesentlich überarbeitete und stark erweiterte Auflage herausgeben konnte, die er um viele Belege für seine Thesen ergänzt hatte. ${ }^{28}$ Gleichzeitig relativierte er seine ursprünglich pessimistischen Einschätzungen und entschärfte etliche seiner Formulierungen. Auch stammt sein Vorschlag, das Bevölkerungswachstum über späte Heirat zu regulieren, aus der späteren Edition. Zusammengefaßt lassen sich MALTHUS' Thesen wie folgt formulieren: ${ }^{29}$

26 APPLEMAN (1976: 20, 23).

27 HeRlihy (2000: 28).

28 APPLEMAN (1976: xviii).

29 LiVI-BACCI (1992: 77). 
1. Nahrung ist die wichtigste Ressource. Mangel führt zu erhöhter Sterblichkeit, die sogar zur Umkehr des demographischen Trends führen kann, damit wieder ein Gleichgewicht hergestellt wird.

2. Eine Population kämpft immer gegen das Gesetz sinkender Erträge an. Kultivierung neuen Bodens und erhöhter Arbeitseinsatz als Reaktion auf Bevölkerungswachstum bedingen zunehmend kleinere Erträge pro Einheit.

3. Produktivitätssteigerung durch Innovation entlastet den Bevölkerungsdruck nur kurzfristig, da sie durch weiteres Wachstum wieder kompensiert wird.

4. Eine Population kann die Spirale von Wachstum und als Folge daraus resultierenden $>$ Hemmnissen< (siehe 1) durchbrechen, indem sie Eheschließungen beschränkt und damit die Geburtenrate kontrolliert.

\section{Kritik an Malthus' Thesen}

Die statische Einschätzung der Agrarwirtschaft und die zu jener Zeit noch nicht absehbare industrielle Revolution des 19. Jahrhunderts waren nicht die einzigen Schwachpunkte in MALThUS' Argumentation, die daher vielfach als überholt galt. ${ }^{30}$ Bereits zu Lebzeiten konnten Malthus gravierende methodische Mängel im Umgang mit seinen Quellen nachgewiesen werden, doch unterlagen bis weit in das 20. Jahrhundert hinein die Stimmen der Kritiker der Mehrheit an Befürwortern. ${ }^{31}$ Mit einer gewissen Hartnäckigkeit konnten sich die Thesen besonders in den historischen Disziplinen halten und rückten erst in den vergangenen Jahren wieder in das Zentrum der Kritik. Eine recht ausführliche Beurteilung hat erst kürzlich der Bevölkerungswissenschaftler Herwig BIRG formuliert und dabei die grundsätzlichen Probleme von MAlThus' Prämissen herausgearbeitet. ${ }^{32}$ BIRG unterstellt MALThus' Arbeit, sie erfülle nicht die Ansprüche an eine wissenschaftlich fundierte Theorie. Weder enthalte sie eine klare, nichttriviale Hypothese, noch definiere sie Bedingungen, unter denen die These bestätigt oder widerlegt werden könne. Auch eine Anwendung der These in der Realität fehle völlig.

Tatsächlich läßt sich zeigen, daß die Voraussetzung eines linearen Wachstums der Nahrungsmittelmenge falsch ist. Vielmehr bedingten Verbesserungen in den Anbaumethoden, neue Tier- und Pflanzenzüchtungen sowie die Einführung des Kunstdüngers in der Mitte des 19. Jahrhunderts ebenfalls ein geometrisches Wachstum. ${ }^{33}$ Schließlich zeigt insbesondere die

30 Zur Diskussion bis in die jüngste Zeit vgl. Herlihy (2000: 32 f.); Hassan (1981: 5 f.); Appleman (1976: $167 \mathrm{ff}$.$) .$

31 BIRG (1996: 33).

32 BIRG (1996: $30 \mathrm{ff}$ ).

33 BIRG (1996: 32 f.). 
Einführung der sexuellen Enthaltsamkeit (moral restraint) als drittes Hemmnis in der überarbeiteten Fassung von 1803 recht deutlich die Motivation, aus der heraus MALTHUS sein Werk schrieb. Er verquickte die Höhe der Geburtenrate mit der moralischen Qualität der unteren sozialen Schichten. Damit stand jedoch endgültig nicht mehr die demographische Entwicklung im Zentrum seiner Betrachtungen, sondern MALTHUS schuf, wie BIRG es formuliert, »eine sozialphilosophische, demographisch begründete Theorie der Moral «. ${ }^{34}$

Letztlich dürfen sowohl HUME als auch MALTHUS als Initiatoren der demographischen Forschung gelten, die mit ihren Thesen zur Bevölkerungsentwicklung zwar in verschiedenen Punkten irrten, andererseits aber den Grundstein dafür legten, wie über Populationen und deren Dynamik nachgedacht werden muß. Nicht zuletzt deshalb hat sich MALTHUS' Vorstellung von der Abhängigkeit zwischen Ressourcen und Populationswachstum - bei aller Kritik - bis in die moderne Forschung halten können. ${ }^{35}$

\subsubsection{Exkurs: Malthus, Bevölkerungsdruck und die carrying capacity}

Galten für HumE Kriege, Hunger und Krankheit noch als >Geißel der Menschheit<, so beschreibt MALTHUS sie als >Hemmnisse $<$, die als Regularien für die Bevölkerungsentwicklung ihren natürlichen Zweck erfüllen. ${ }^{36}$ Im einzelnen mag die Beschreibung der Hemmnisse noch eine Verkennung von Ursache und Wirkung widerspiegeln, wie etwa bei der Gleichstellung aller drei Faktoren. Kriege und Krankheit sind eher eine Folge von Hunger, zum einen, um unter Anwendung von Gewalt an neue Ressourcen zu gelangen, zum anderen, weil der von Hunger geschwächte Körper anfälliger für Krankheiten ist. Im übrigen bezweifelt die neuere Forschung einen Zusammenhang zwischen demographischen Einbrüchen und Hungersnot. ${ }^{37}$ Doch greift MALTHUS in seiner Situationsbeschreibung bereits Konzepten vor, die in der modernen Demographie als >Bevölkerungsdruck< und die sogenannte carrying capacity bekannt sind. Der Bevölkerungsdruck ist dabei definiert als der Druck, der von der Bevölkerungszahl eines Gebietes auf die Ressourcen, besonders in Bezug auf Ernährung, ausgeübt wird. Hoher Bevölkerungsdruck spielt also für die Bestimmung der ökonomischen Tragfähigkeit einer Region eine wesentliche Rolle. Die carrying capacity ist ein Teilaspekt moderner Ökosystem-

34 BIRG (1996: 69).

35 SCHÄFERS (2001: 38) bezeichnet sie als »populärste und einflußreichste Bevölkerungs-Theorie«; auch für die mathematische Biologie bildet MALThus' These eines exponentiellen Bevölkerungswachstums die Ausgangsbasis (BAZYKIN 1998: 1).

36 Appleman (1976: 44).

37 Siehe dazu auch ausführlich Kap. 6.4. 
Analysen, die als Werkzeug zur Ermittlung von Bevölkerungsgrößen immer noch Anwendung findet, jedoch in der Qualität ihrer Ergebnisse nicht unumstritten ist. ${ }^{38}$ Im wesentlichen basiert das Konzept der carrying capacity darauf, daß es für das Wachstum der Population eine Obergrenze an Ressourcen gibt. Die Population wächst unter Ausbeutung ihrer Umwelt über das Maß ihrer Möglichkeiten hinaus, das heißt der Umwelt können nicht mehr ausreichend Ressourcen für die Versorgung der Bewohner entzogen werden. Die Folge ist ein plötzlicher oder kontinuierlicher Rückgang der Population, der in seltenen Fällen sogar bis hin zu ihrem Aussterben führen kann. Letzteres gilt insbesondere für kleine Populationen, deren Anzahl unter die Mindestgröße für die Lebensfähigkeit solcher Gruppen sinkt. ${ }^{39}$ Auswirkungen dieser Art lassen sich im übrigen bereits bei Aristoteles nachlesen, der hinsichtlich Bevölkerungsmigration beschreibt, daß ein Teil der Gruppe abwandert, während der andere Teil bleibt, bis die Region ihre Bewohner nicht mehr ernähren kann. ${ }^{40}$

Die Modelle beschreiben das Anwachsen der Population entweder als extreme Überbeanspruchung der Ressourcen und einem jähen Abfall der Bevölkerungsgröße oder als ständiges Oszillieren um die Obergrenze, so daß sich die Population auf einen stabilen Level einpendelt (Abb. 1.1). Die dagegen stehenden Kritikpunkte beziehen sich hauptsächlich auf die Problematik, daß Nahrungserträge pro Fläche kaum exakt zu ermitteln sind, was insbesondere für Gesellschaften von Jägern und Sammlern gilt. Auch lassen sich keine genauen Abschätzungen über die Auswirkungen unterschiedlicher Technologien auf die Ertragshöhe des Bodens treffen. Ein weiterer immer wieder anzutreffender Problemfall ist auch die Beschränkung der carrying capacity allein auf Getreiderationen pro Kopf ${ }^{41}$ sowie die (unausgesprochene) Prämisse, daß die zu erwartende Bevölkerungsgröße immer am Limit der carrying capacity liegt, wie es auch die in Abb. 1.1 gezeigten Diagramme suggerieren. ${ }^{42}$

Immerhin läßt sich festhalten, daß Fragen der Versorgungsmöglichkeit zumindest eine Berücksichtigung finden müssen, wenn man mikroskalierend die Bevölkerungsgrößen von Siedlungen untersucht. Eine durch andere Methoden ermittelte Population benötigt auch eine adäquate Versorgungsfläche.

38 Beispiele für neuere Versuche findet man u. a. bei HASSAN $(1993,1994)$ [Ägypten], ZoRN (1994) [Israel] oder SANDERS (1984) [Griechenland]; zu Konzept und Kritik vgl. auch SCHEIDEL (2001b: 50), HASSAN (1981: 5 f., 164 ff.), MitCHELL (1979: 176 ff.).

39 HASSAN (1981: ebenda).

40 BILLER (2000: 219).

41 Vgl. dazu SHAW (1981).

42 Gegen diese weit verbreitete Meinung haben sich bereits ButZer (1976: 77 Anm. 2) und DuRAND (1972: $370 \mathrm{f}$.) ausgesprochen. BUTZER schätzt die tatsächliche Auslastung der carrying capacity auf maximal $60 \%$, so auch weitestgehend HASSAN (1973: 536) und (1975: 32). 


\subsubsection{Anfänge ägyptologischer Demographie}

\subsubsection{Die >Déscription de l’Égypte‘}

Seit sich die Ägyptologie als eigenständige Forschungsdisziplin mit allen Aspekten des altägyptischen Lebens beschäftigt, hat sich - abseits der großen Forschungsschwerpunkte - bereits in der ersten Stunde der Wissenschaft die demographische Forschung ihren Platz gesucht. Es ist daher mit Sicherheit kein Zufall, daß bereits in der >Description de l'Égypte< im Jahr 1818 eine umfangreiche Abhandlung erschien, die unter dem Titel >Mémoire sur la population comparée de l'Égypte ancienne et moderne< eine Zusammenstellung von Material zu dieser Fragestellung anbot und - obwohl später in den Schlußfolgerungen korrekturbedürftig ${ }^{43}-$ als Pionierarbeit gelten darf. Dies ist das Verdienst des französischen Gelehrten Edmé JOMARD (1777-1862), der als Mitglied der wissenschaftlichen Delegation an der ägyptischen Expedition Napoleons in den Jahren 1798/99 teilnahm. 151 Wissenschaftler begleiteten damals den Zug des französischen Heeres nach Ägypten und sorgten dafür, daß trotz der militärischen Niederlage, die Napoleon gegen die Engländer erlitt, das Unternehmen wissenschaftlich gesehen ein grandioser Erfolg wurde. Die gesammelten Erkenntnisse aller Disziplinen wurden in einem 20 Bände umfassenden Werk zusammengefaßt und illustriert. Neben dem Ägypten der Antike fand auch das moderne Ägypten des ausgehenden 18. Jahrhunderts eine gebührende Beachtung. Einen weiteren Schwerpunkt bildete die Beschreibung von Fauna und Flora Ägyptens. Die so entstandene >Déscription de l'Égypte< gilt vielen als die Geburtsstunde der Ägyptologie und ermöglichte dem Okzident erstmals einen umfassenden Blick auf den Reichtum an Hinterlassenschaften, den Napoleons Truppen in Ägypten vorgefunden hatten. Neben dem umfangreichen Tafelteil bestand die Déscription auch zu einem wesentlichen Teil aus Abhandlungen über bestimmte Themenkomplexe, zu denen Edmé JOMARD seinen ausführlichen Artikel über die antike und moderne Population Ägyptens beisteuerte. Obwohl eine solche Pioniertat noch mit Irrtümern und Mängeln behaftet war, nahm sie dennoch in weiten Teilen Verfahren und Konzepte zur demographischen Informationsgewinnung voraus. Die Abhandlung wurde im Rahmen einer Neuauflage der Déscription erneut veröffentlicht (1818, 1829) und scheint dabei auch redaktionellen Überarbeitungen unterworfen gewesen zu sein. ${ }^{44}$

43 Kritische Auseinandersetzung mit dem Material bei El-DARWISH (1929) und MCCARTHY (1976); zu den fehlerhaften Prämissen und Irrtümern vgl. auch unten Kap. 7.1.1.

44 Russell (1966: 70 Anm. 10) beruft sich bei den Angaben zum Zensus von 1821 auf JomARDs Ausgabe von 1829. Dieser Zensus kann in der ersten Auflage natürlich noch nicht berücksichtigt worden sein. 
Alle Faktoren, die auch in späteren Untersuchungen Grundlage der Kalkulationen (und Spekulationen) waren, sind in JOMARDs Arbeit bereits genannt worden. Er untersuchte die Fläche des Landes, die Anzahl der Siedlungen (Lieux habités) und die Verteilung der Bevölkerung auf selbige, das proportionale Verhältnis zwischen Männern und Frauen wie auch die Fruchtbarkeit der Frauen, Nahrungsmittelproduktion und -verbrauch und unternahm abschließend eine Auswertung der griechischen Quellen und einen Vergleich zwischen dem alten und dem >modernen< Ägypten, wie JOMARD es um 1798 im Geleit des Napoleonfeldzuges kennenlernte.

Die Déscription de l'Égypte hatte ein kleines Vorspiel, das hier zur Vervollständigung der Ereignisse jener Zeit Erwähnung finden sollte. Anfang der 80er Jahre des 18. Jahrhunderts unternahm der französische Diplomat Constantin François Chassebceuf DE Volney Reisen durch Ägypten und Syrien, deren Erlebnisse er später in Form eines Reiseberichtes veröffentlichte, der zur Pflichtlektüre nachfolgender Reisender wurde und sogar Napoleon als Vorbereitung für seine Expedition nach Ägypten diente. ${ }^{45}$ Unter anderem schätzte er darin die Bevölkerung des pharaonischen Ägypten - gegen die ihm bekannten Behauptungen Diodors - auf etwa 2,3 Millionen Menschen. Worauf CHASSEBEUf seine Einschätzung begründete, erfährt man bei BowRING leider nicht, so daß die Angabe lediglich eine historische Fußnote als Vorläufer der ausführlichen Bearbeitung JOMARDs bleibt. Die Nähe der genannten Größenordnung zur >zeitgenössischen< Population um 1800, wie sie später JOMARD errechnete, mag dabei ein reiner Zufall sein.

\subsubsection{Zwei Reisende des 19. Jahrhunderts}

\section{John Bowring (1840)}

Eine weitere bedeutende Beschreibung der Situation Ägyptens im 19. Jahrhundert, besonders unter sozialen und ökonomischen Aspekten, lieferte der Engländer John BOWRING (17921872) basierend auf seiner Reise in den Jahren 1837 bis 1838. Zentrales Thema seines Reports war eine Beschreibung der industriellen Entwicklung Ägyptens in der Zeit Mohammed Alis, die für die Wirtschaftsgrößen Großbritanniens von besonderem Interesse war: ${ }^{46}$

\footnotetext{
»Nothing very defined is known as to the number of its inhabitants. In the early periods of its history Herodotus speaks of 20,000 well-peopled towns ; but this is a vague manner of writing. It has, however, been supposed

45 Comte C.F. Chassebceuf de Volney, Voyage en Syrie et en Égypte, pendant les années 1783, 1784 et 1785, avec deux cartes géographiques et duex planches gravées, Paris 1787; das Buch war nicht zugänglich, so daß im folgenden auf ein Zitat von John BowRING (s. u.) Bezug genommen werden muß. Zu ChAsseberfs Reisebeschreibungen siehe auch Hollingsworth (1969: 248 Anm. 2).

46 Report on Egypt 1823-1838; zitiert wird der kommentierte Nachdruck: BowRING (1998: 9 f.).
} 
that the population was not less than 8,000,000 in very remote times; they were only 3,000,000 in the time of Diodorus Siculus and when Volney wrote, he estimated them at 2,300,000 souls, which is, perhaps, not very far from the present amount of population. The government estimates it at 3,200,000; but I have found it impossible to come to anything like a certain calculation. The opinion of the best informed is that the number of inhabitants is from $2,000,000$ to $2,500,000$ «

Die von BOWRING angestellten Überlegungen führen einmal mehr zurück zu den antiken Quellen. Neben den 20000 Siedlungen nach Auskunft Herodots findet auch die Zahl von 3000000 Menschen aus der Quelle Diodors Erwähnung. Auch taucht an dieser Stelle die geschätzte Zahl von 8 Millionen Menschen auf, jedoch ohne Nachweis der Herkunft dieser Überlegung. Unklar ist auch die Herkunft der zeitgenössischen >Regierungsschätzung< über 3,2 Millionen Menschen. Diese Angabe kann nicht in Einklang mit den bekannten Erhebungen und Schätzungen des 19. Jahrhunderts in Ägypten gebracht werden. Keine der in Tabelle 7.1 zusammengefaßten Volkszählungen ergeben die von BowRING zitierte Zahlenangabe. Es könnte sich also um einen weiteren halboffiziellen Zensus vor dem Jahr 1840, in dem BOWRING seine Reisebeschreibung veröffentlichte, handeln. Über die Qualität der Erhebung läßt sich keine zuverlässige Aussage treffen, vermutlich geht es - nach der Wortwahl BOWRINGs zu urteilen - eher um Schätzungen auf der Grundlage von vorhandenen Daten wie zum Beispiel Steuerlisten, als um einen echten Zensus. Es handelt sich demnach um eine ähnliche Maßnahme wie sie bereits zuvor im Jahr 1821 durchgeführt wurde.

Insgesamt ist die Meinung BowRINGs zur Population Ägyptens von einer starken Skepsis geprägt. Ähnlich wie schon HUME mißtraut er den Quellen wie auch den Versuchen der Behörden und Statistiker seiner Zeit, eine verbindliche und korrekte Zahl aus dem Datenmaterial erarbeiten zu können. ${ }^{47}$ BOWRING ist damit auch ein Kronzeuge für die zeitgenössische Beurteilung der Volkszählungen des 19. Jahrhunderts, die oft genug unkritisch als Fakten ausgewertet wurden und erst in jüngerer Zeit einer gründlichen Prüfung auf ihren Aussagewert hin unterzogen wurden. ${ }^{48}$ BowRINGs Beschreibung des Populationsverlaufes von 8 Millionen über 3 Millionen hin zu 2,3 Millionen steht im Widerspruch zu den neueren Erkenntnissen und veranlaßte die Herausgeber seines Berichtes zu einem kurzen Kommentar über die Bevölkerungsentwicklung Ägyptens. ${ }^{49}$ In der Tat scheinen die Ausführungen BowRINGs eher von der bereits durch HuME kritisierten These einer stärkeren Population in der Antike getragen zu werden. Ob BOWRING jedoch tatsächlich von den Vertretern dieser Meinung beeinflußt war,

47 Bowring (1998: 6 f.).

48 Siehe dazu auch ausführlich Kap. 7.1.

49 Bowring (1998: 10 Anm. 6). 
läßt sich kaum mit Sicherheit sagen, obwohl die Nachdrücklichkeit seiner Formulierungen diesen Verdacht nahelegt.

\section{Edward William Lane (1852)}

Eine kurze, doch in ihrer Art recht bemerkenswerte Aussage zur Bevölkerung des Alten Ägypten traf der Arabist Edward LANE (1801-1876), der mehrfach einige Jahre in Ägypten verbrachte, seine Erlebnisse und Beobachtungen über ägyptische Lebensgewohnheiten sammelte und schließlich bereits 1836 veröffentlichte. ${ }^{50}$ Obgleich seine eigentlichen Leistungen unbestritten im Bereich der arabischen Sprache lagen, für die er durch Lexika und Übersetzungen der Disziplin eine reichhaltige Grundlage schuf, bieten seine Eindrücke als Augenzeuge der ägyptischen Gesellschaft des 19. Jahrhunderts bis heute unschätzbare Einblicke in das Alltagsleben und die Gepflogenheiten, wie sie in dieser Form längst nicht mehr existieren.

Im Kontrast zu seinen Ausführungen über die Bevölkerung des modernen Ägypten wußte er über das Alte Ägypten folgendes zu berichten: ${ }^{51}$

\footnotetext{
»Zur Zeit der Pharaonen hatte Egypten aller Wahrscheinlichkeit nach eine Bevölkerung von sechs bis sieben Millionen. Der Ertrag des Bodens in jetziger Zeit könnte, wenn nichts ausgeführt würde, zum Unterhalt einer Bevölkerung von mehr als vier Millionen hinreichen; und wenn alles culturfähige Land besät würde, für acht Millionen. Dies aber wäre die höchste Anzahl welche Egypten in den Jahren fruchtbarer Ueberschwemmung ernähren könnte; ich rechne daher, dass die alte Bevölkerung zu der Zeit, als der Ackerbau in voller Blüthe stand, sich bis zu der angegebenen Höhe belaufen habe, muss aber vermuthen, dass sie zur Zeit der Ptolemäer und später, als jährlich eine grosse Quantität Getreide ausgeführt wurde, kaum mehr als die Hälfte kann betragen haben. Diese Berechnung stimmt mit der Angabe Diodor's (I, 31) überein, welcher sagt, dass Egypten zur Zeit der alten Könige sieben Millionen Einwohner hatte, zu seiner Zeit aber nicht unter drei Millionen.«
}

Bemerkenswert ist hierbei, wie LANE bereits Überlegungen zu den Parametern anstellte, die eine Abschätzung der Bevölkerungsgröße ermöglichen könnten. Er bezog seine Darlegungen auf den Ertrag des Ackerbodens - eine Idee, die erst über 80 Jahre später im Modell der sogenannten carrying capacity konsequent ausformuliert wurde. Und in der Tat stellt diese Form der Berechnung ein bis heute verwendetes, wenn auch immer wieder kritisiertes Konzept dar. ${ }^{52}$ Die Angaben der antiken Autoren hielt er dagegen für wenig aussagekräftig, weshalb sie in seinen Überlegungen keine Berücksichtigung fanden. ${ }^{53}$

50 Zitiert wird im folgenden nach der dreibändigen deutschen Ausgabe: LANE (1852).

51 LANE (1852: Bd. 1, 19).

52 Vgl. dazu auch oben Kap. 1.3.2.

53 LANE (1852: Bd. 1, 19 Anm. 2): »Ich gebe wenig auf die Erzählungen der alten Schriftsteller über diesen Gegenstand.« 
Gleichermaßen verfuhr LANE im übrigen mit seinen Ausführungen zum modernen Ägypten. Zum Zeitpunkt seiner ersten Aufenthalte stand Ägypten die erste Volkszählung erst noch bevor, so daß LANE auf eigene Beobachtungen angewiesen war. Richtig erkannte er bereits die Notwendigkeit der in Ägypten fehlenden Geburten- und Sterberegister, die für ihn die zwingende Grundlage für eine statistisch verläßliche Aussage bildeten. Ähnlich wie bereits JOMARD verlegte er sich daher auf Überschlagsrechnungen anhand der Anzahl von Hausbewohnern, die er für Kairo auf acht Personen, auf dem Lande jedoch nur auf vier bezifferte. Allerdings mißtraute er seinem eigenen Pauschalurteil und rechnete für verschiedene Orte wie Alexandria oder Damietta doch mit einer dichteren Population. Die Summe seiner Schätzungen betrug danach etwa $21 / 2$ Millionen Menschen, von denen weniger als die Hälfte (1,2 Millionen) männlich waren. ${ }^{54}$

\section{4 Ägyptologie und Demographie im 20. Jahrhundert}

\subsubsection{Moderne Ansätze und Berechnungen}

Gegen Ende der 50er Jahre geriet die demographische Forschung erneut in Bewegung, als der amerikanische Ägyptologe Klaus BAER mit Vorarbeiten für eine Parametrisierung der Bevölkerungsentwicklung begann und einzelne Schätzungen zur Populationsgröße in seine Aufsätze mit einarbeitete. Kern seiner Überlegungen stellten die Ertragsfähigkeit des Ackerbodens und die für die Versorgung eines Haushalts nötige Agrarfläche dar. ${ }^{55}$ BAER vermutete für das Neue Reich eine Gesamtzahl von 4 1/2 Millionen Menschen unter Berufung auf den Nährgehalt ägyptischen Bodens, gab aber gleichzeitig eine hohe Fehlerrate zu. ${ }^{56}$ Bedingt durch schwere Krankheit und seinen frühen Tod war es BAER jedoch nicht mehr vergönnt, seine Ideen auszuformulieren. ${ }^{57}$

Weitere theoretische Vorüberlegungen stammten zum Beispiel von Jac JANSSEN, der sich vorwiegend auf anthropologische Untersuchungen und Nekropolenstatistiken stützte, ${ }^{58}$ dar-

54 LANE (1852: Bd. 1, 17).

55 BAER (1962) auf Grundlage der Untersuchungen zum Stickstoffgehalt des Bodens von Hans JENNY (1962); BAER (1963: 14).

56 BAER (1962: 43 f.); später hat BAER diese Zahl offenbar auf 2,4 Millionen bis 3,6 Millionen Menschen eingeschränkt (mündlicher Hinweis, zitiert bei BUTZER 1976: 76).

57 Für eine thematische Zusammenstellung der im Oriental Institute verwahrten Notizen BAERs zum Stichwort >Population of Ancient Egypt< siehe WILFONG (1994: 302).

58 JANSSEN (1975b: 135 ff.). 
über hinaus jedoch lediglich ältere Arbeiten heranzog. ${ }^{59}$ Mit seinem abschließenden Vorschlag rückte er die Arbeitersiedlung Deir el-Medineh wieder ins Zentrum des Interesses, die er als archäologisch und epigraphisch gut dokumentierte Siedlung der künftigen Forschung zur Auswertung nahelegte. In Ermangelung einer genügend großen Anzahl antiker Textüberlieferungen mußten also verschiedene methodische Neuansätze in der Soziologie und Ethnologie entwickelt werden, die allein auf Basis archäologischer Erkenntnisse eine Abschätzung der Bevölkerungszahlen liefern sollten. Einen eigenen Ansatz verfolgte Karl BUTZER, der seine Analysen in zwei Phasen aufteilte. ${ }^{60}$ Zunächst sammelte er die Daten zu allen archäologisch oder anderweitig bekannten Siedlungen in Ägypten unabhängig von deren Größe. Diese wurden im zweiten Schritt jeweils in eine Größenkategorie eingeteilt, wobei er jeder Kategorie einen Mittelwert für die Anzahl der Bewohner zuwies. Die Gesamtzahl der Population wurde zum Schluß durch einfaches Aufsummieren der Siedlungsgrößen extrapoliert. Neueste Zahlen stammen von Fekri HASSAN, der die von BUTZER errechneten Zahlen nochmals nach unten korrigiert, allerdings sein dazu herangezogenes Material nicht nachvollziehbar offenlegt. ${ }^{61}$ Die Ansätze beider Autoren werden in den folgenden Abschnitten noch ausführlicher vorgestellt.

Häufig genug fand jedoch nur ein unkritisches Kopieren älterer Literatur, gleichsam eine >Genealogie des Abschreibens < statt, die auf die Verläßlichkeit der auf solche Weise tradierten Daten vertraute. ${ }^{62}$ Es ist mit einem gewissen Bedauern festzustellen, daß seit der Monographie BUTZERs keine intensivere Forschung zur Demographie des Alten Ägypten mehr stattgefunden hat. Den einzigen Neuansatz wagte Dominique VAlbelle in der Mitte der 80er Jahre, die ihre Untersuchungen aber nicht über die Vorarbeiten einer kommentierten Katalogisierung von Textquellen zum Thema Zensus hinaus weiterführte. ${ }^{63}$

\subsubsection{Karl Butzer und die `Hydraulische Hypothese،}

Einen neuen Schub erhielt die demographische Forschung, als der Geograph Karl BUTZER 1976 sein Buch über die Early Hydraulic Civilization herausbrachte. ${ }^{64}$ BuTZER griff darin

59 BAER (1962); O'CONNOR (1972b).

60 BUTZER (1976).

61 HASSAN (1994).

62 David AyAlon (1985: 1) hat dazu treffend bemerkt: »Figures look always as a very tangible thing, and, therefore, once there is an estimate expressed by numbers, it tends to be copied and spread quickly, with its dubious origin not always remembered.«

63 VAlbelle (1985a, 1987).

64 BUTZER (1976). 
die sogenannte >hydraulische Hypothese < auf, die gegen Ende der 50er Jahre von Karl WITTFOGEL in ihrer extremsten Ausprägung formuliert wurde. ${ }^{65}$ Um die Argumentationen BUTZERs zur Bevölkerungsentwicklung im Alten Ägypten nachvollziehen zu können, soll daher zunächst eine knappe Beschreibung der hydraulischen Hypothese den weiteren Ausführugen vorangestellt werden. Kern der Hypothese ist der Umstand, daß alle frühen Hochkulturen abhängig von künstlicher Bewässerung waren, um die Ernährung sicherzustellen. Aus der gesellschaftlichen Notwendigkeit der Bewässerung entwickelte sich eine zentrale Organisation, die sämtliche Maßnahmen koordinierte, sich jedoch auch gleichzeitig als absolutistisches Machtzentrum zur Despotie entwickelte, wie WiTTFOGEL es bezeichnet. Diese Hypothese wurde besonders von amerikanischen Ethnologen aufgegriffen und - um ihre politische Komponente entschärft - weitergeführt. ${ }^{66}$ Der Bewässerung wurde zwar ihr Stellenwert für die gesellschaftliche Entwicklung zuerkannt, jedoch der kausale Zusammenhang von Ursache und Wirkung aufgebrochen, so daß das Entstehen früher Hochkulturen mit der Bewässerungstechnologie einherging, jedoch nicht zwingend aus dieser resultierte. Und obgleich BUTZER die ideologische Färbung WITTFOGELs kritisch zurückweist, ${ }^{67}$ genauso wie eine simplifizierende Kausalverkettung Krise/Belastung/Druck $\rightarrow$ Bewässerung $\rightarrow$ Verwaltungsbürokratie $\rightarrow$ despotische Kontrolle, ${ }^{68}$ so betont er dennoch die Bedeutung dreier Faktoren, die entscheidenden Einfluß auf die soziale Organisation haben, nämlich Umwelt, Technologie und Population. ${ }^{69}$ In jüngerer Zeit wird gerade für den Kulturraum Ägypten die >Hydraulische Hypothese< wieder relativiert. So bemerkt etwa Christopher EYRE, daß es keines besonderen Organisationsgrades für die Aufrechterhaltung der Bewässerung bedurfte, der als ein bürokratisches Verwaltungsmodell für die Rekrutierung und Kontrolle von Arbeitermassen fungiert haben könnte. ${ }^{70}$

Obwohl die demographischen Einschätzungen nur einen Teilaspekt seiner Arbeit ausmachten, bleibt das Buch bis heute das am häufigsten zitierte, wenn es darum geht, Zahlen zur Bevölkerungsgröße des Alten Ägypten heranzuziehen. Auch methodisch schlug BuTZER neue Wege ein. Statt einmal mehr über Hochrechungen aus der carrying capacity die antike Populationsgröße zu ermitteln, wählte er ein statistisches Verfahren aus der Siedlungsgeographie. Ausgangsmaterial seiner demographischen Untersuchung waren bei ihm die ägyptischen Siedlungen, die anhand archäologischer und inschriftlicher Befunde bekannt waren. ${ }^{71}$ Die Sich-

65 WitTFogel (1962).

66 Zur Diskussion vgl. SCHENKEL (1978: 12).

67 BUTZER (1976: xiii).

68 BUTZER (1976: 111).

69 BUTZER (1976: xiv).

70 EYRE (1987: 18).

71 ButZer (1976: 59). 
tung des Materials ergab eine Liste von 217 Siedlungen, von denen etwa $57 \%$ exakt lokalisierbar waren. Im nächsten Schritt wurde auf alle Siedlungen ein Katalog von Eigenschaften (Tab. 1.1) angewendet, für deren Existenz jeweils Punkte vergeben wurden. Je mehr Eigenschaften eine Stadt besaß, desto mehr Punkte fielen auf sie und desto zentraler mußte ihre Bedeutung gegenüber den Siedlungen im Umland sein. Dieses Modell ist auch als >ZentralortTheorie< (engl. Central Place Theory, kurz CPT) bekannt. ${ }^{72}$

\begin{tabular}{cllr}
\hline Rubrik & Eigenschaft & Punkte \\
\cline { 3 - 3 } A & Hauptstadt des Nomos & 3 \\
& Königliche Residenz & 6 \\
& Alternative Hauptstadt des Nomos & 1 \\
B & Gräber der Oberschicht & 3 \\
& Königsgräber & 3 \\
& Beides & 4 \\
C & Es gibt einen Ortsvorsteher $\left(h^{3}{ }^{3} j{ }^{-}{ }^{-}\right)$ & 2 \\
D & Ein einzelner Tempel & 2 \\
& Mehrere Tempel & 3 \\
E & Wohnsiedlung & 1 \\
F & Festung oder Wehrbau & 1 \\
G & Villen, Domänen oder >Vororteく & 1 \\
H & Steinbruch mit Ortsbezug & 1 \\
\hline
\end{tabular}

Tabelle 1.1: BUTZERs Eigenschaftskatalog zur Klassifizierung von Siedlungen

Da bekannt war, daß man längst nicht alle Informationen für jede Siedlung zusammentragen konnte, mußten gewisse Abstriche in der statistischen Verteilung in Kauf genommen werden. Die gesammelten Daten schienen jedoch ausreichend, um die analysierten Siedlungen in vier Kategorien einteilen zu können: große Dörfer (1-3 Pkt.), kleine Zentren (4-6 Pkt.), große Zentren (7-10 Pkt.) und >Städte< $>10$ Pkt.), wobei letztere durch die beiden Metropolen Luxor/Karnak und Memphis repräsentiert wurden. ${ }^{73}$ Wie bereits David O'CONNOR nahm BUTZER als zusätzlichen Faktor die Schätzungen über die regionale Verteilung kultivierbaren Bodens mit in seine Auswertungen auf. ${ }^{74}$ Die Problematik der Datenauswertung zeigt sich jedoch an den entgegengesetzten Ergebnissen, die beide für die Populationsdichte vorlegten. O'CONNOR postulierte, daß in den mittleren Regionen des Niltals zwischen Theben und

72 Zur Definition vgl. auch BERNBECK (1997: 169 ff.).

73 Am Rande sei vermerkt, daß hier der Begriff der >Stadt< als reine Konvention übernommen wurde, obwohl er durch andere Kulturepochen, besonders in Europa, stark geprägt wurde. Der wissenschaftliche Diskurs über Konzepte und Gestaltung einer Stadt im Alten Ägypten (MÜLLER-WolLERMANn 1991; KoLB 1984) soll an dieser Stelle nicht weiter aufgegriffen werden.

74 O'CONNOR (1972a); BUTZER (1976: 76 ff.). 
Assiut, um Nechen und Elkab sowie nahe Memphis, wo eine dichte und zahlenmäßig hohe Population herrschte, die Zentralorte näher zueinander lagen und regelmäßiger verteilt waren, während sie im Süden mit seiner ungleichmäßig verteilten Population weiter auseinander angelegt wurden. ${ }^{75}$ Dagegen befand BUTZER, daß die Populationsdichte - im Gegensatz zum Süden - eher im mittleren Niltal niedriger war. ${ }^{76}$

Als Neuerung im Verfahren versuchte BUTZER eine Hochrechnung der Anzahl an Siedlungen, die bei der Zusammenstellung des Ausgangsmaterials durch das Raster gefallen waren und zu einer Unterrepräsentation der Siedlungskategorie innerhalb ihrer Region führten. Dazu bediente er sich der Hypothese aus der Zentralort-Theorie, daß das Verhältnis der aufeinander folgenden Kategorien zumindest $2: 1$, im Idealfall aber $3: 1$ betragen sollte. ${ }^{77}$ Da die beiden höchsten Kategorien zumindest aus den Textquellen als erschlossen gelten dürfen, können sie als Grundlage dafür dienen, die entsprechende Mindestzahl an kleinen Zentren (Kategorie 2) zu errechnen. Die Ergebnisse waren jedoch ernüchternd, da aufgrund der starken Unterschiede zwischen der Besiedlung einzelner Nomoi keine Konsistenz erzielt werden konnte. Das Ergebnis verleitete BUTZER zu der Einschätzung, daß die Zentralort-Theorie wegen der linearen Anordung der Besiedlung im Niltal für Ägypten nicht anwendbar sei. ${ }^{78}$ Diese pessimistische Beurteilung fand in der Folgezeit jedoch keine Bestätigung. Beispielsweise konnten ägyptischen Katasterpapyri mit Siedlungsgeographischen Methoden durchaus interessante neue Erkenntnisse abgerungen werden. ${ }^{79}$

Auch das von BUTZER gewählte Verfahren war noch keineswegs perfekt. BUTZER selbst nannte bereits drei Kernprobleme seiner Datenerhebung, die zu Verzerrungen in den Schlußfolgerungen führen könnten: ${ }^{80}$

$\triangleright$ In der Aufstellung fehlen zwangsläufig kleine Dörfer, Gehöfte, etc., wie sie zum Beispiel im Papyrus Wilbour verzeichnet sind.

$\triangleright$ Die Verteilung der untersuchten Siedlungen über das gesamte ägyptische Siedlungsgebiet ist nicht ausgewogen, was zu statistischen Über- oder Unterrepräsentationen führen kann.

$\triangleright$ Die im Verzeichnis erfaßten Siedlungshinweise stammen aus einem Zeitraum von 2000 Jahren.

75 O'CONNOR (1972a: 688).

76 ButZer (1976: 79, Abb. 12). Zusammenfassend bei KAUfFMAn (1981: 45 ff.).

77 Dies wird als $>$ Bifurkationsrate $<$ bezeichnet; BUTZER (1976: 72).

78 Dieser Vorwurf wurde von KAUFFMAN (1981: 49) allerdings mit Hinweis darauf zurückgewiesen, daß gerade Flußtäler als ideale Umgebung für eine Zentralort-Analyse gelten, da sie dem geographischen Ideal einer einheitlichen Fläche am nächsten kommen.

79 Vgl. dazu ausführlich Kap. 5.2.2.3.

80 BUTZER (1976: 71 ff.). 
Um diese Mängel zu kompensieren, griff BUTZER manuell in seine Tabellen ein und korrigierte einzelne Zwischenergebnisse nach oben oder unten. Zudem verwies er auf den glücklichen Umstand einer überwiegenden Datenherkunft aus dem Neuen Reich. ${ }^{81}$ Ein weiterer Schwachpunkt in BUTZERs Arbeit bleibt die unzureichende Erklärung seiner Relation zwischen den einzelnen Siedlungstypen und der daraus resultierenden Bevölkerungszahl. Dies betrifft nicht die von ihm gewählte Bifurkationsrate, sondern die implizit getroffene Prämisse einer Korrelation von Größe und regionaler Wichtigkeit einerseits und der Bevölkerungsgröße andererseits. Dieser Zusammenhang ist zwar generell zutreffend, läßt sich aber nur in den seltensten Fällen in absolute Zahlen zur Bevölkerungsgröße von Siedlungen übertragen, so daß hier mit einer unscharfen Größe von Durchschnittswerten operiert werden muß, deren Fehlerrate sich bei der Gesamtsumme der Bevölkerung Ägyptens kaum noch nachvollziehen läßt. ${ }^{82}$

In der abschließenden Betrachtung setzte BUTZER die zuvor gewonnenen Daten einmal mehr in Relation zur Gesamtfläche an nutzbarem Ackerboden. Durch eine Verrechnung von Kulturland und Bevölkerungsdichte kam er abschließend auf die Bevölkerungsgrößen in den verschiedenen Epochen der ägyptischen Geschichte (Tab. 1.2), die lange Zeit der Maßstab für die Population des Alten Ägypten geblieben sind und bis heute als gültige Lehrmeinung zitiert werden.

\begin{tabular}{|c|c|c|c|c|c|}
\hline \multirow[b]{2}{*}{ Zeit } & \multirow[b]{2}{*}{ Bevölkerung } & \multirow{2}{*}{$\begin{array}{r}\text { Kultivierbarer } \\
\text { Boden (qkm) }\end{array}$} & \multicolumn{3}{|c|}{ Bevölkerungsdichte (Personen/qkm) } \\
\hline & & & Niltal & Fajjum & Delta \\
\hline 4000 v. Chr. & 350000 & 16100 & 30 & 30 & 10 \\
\hline 3000 v. Chr. & 870000 & 15100 & 75 & 60 & 30 \\
\hline 2500 v. Chr. & 1600000 & 17100 & 130 & 90 & 60 \\
\hline 1800 v. Chr. & 2000000 & 18450 & 140 & 135 & 75 \\
\hline 1250 v. Chr. & 2900000 & 22400 & 180 & 180 & 90 \\
\hline 150 v. Chr. & 4900000 & 27300 & 240 & 240 & 135 \\
\hline
\end{tabular}

Tabelle 1.2: Hypothetische Bevölkerungsentwicklung Ägyptens nach BUTZER

\subsubsection{Die Untersuchungen von Fekri Hassan}

Der ägyptische Geologe und Ethnologe Fekri HASSAN hat sich seit den 70er Jahren auf sozioökonomische Untersuchungen früher Gesellschaften spezialisiert. Insbesondere seine Beiträ-

81 BUTZER (1976: 76).

82 Vgl. dazu Kap. 4.2. 
ge zu den Grundlagen der Populationsentwicklungen im Übergang von Jäger und SammlerKulturen zu seßhaften Farmern ${ }^{83}$ waren auch für die ägyptologische Diskussion nutzbringend. ${ }^{84}$ Dabei ging es im wesentlichen um die Frage, welche Auswirkungen veränderte Lebensbedingungen auf die Fortpflanzung haben und welche Faktoren dabei zu gewichten sind. So stand etwa die Korrelation von Mobilität und Bevölkerungsgröße zur Diskussion, die nach Meinung HASSANs darauf hinauslief, daß bessere Versorgung durch Ressourcen und deren Verarbeitung die Seßhaftigkeit fördern, während Mangel und geringe Verteilung von Ressourcen zwangsläufig zur stärkeren Mobilität und - ebenfalls aus dem Mangel resultierend - zu geringerem Wachstum führen. ${ }^{85}$ Wesentliches Argument war dabei die carrying capacity, die dem Wachstum natürliche Grenzen setzte und damit als wichtiger Faktor für Bevölkerungswachstum galt. Eine Erhöhung des Ertrags pro Fläche aus den Ressourcen durch technische Innovation erlaubte der Bevölkerung weiteres Wachstum bis zu dem neuen Limit. Sollte dieses überschritten werden, so fällt die Bevölkerungsgröße auf einen Stand zurück, der unterhalb der carrying capacity liegt, und damit von den Ressourcen der Umwelt noch getragen werden kann. ${ }^{86}$ Weitere Untersuchungen HASSANs befaßten sich speziell mit den prähistorischen Siedlungen in Ägypten und Mesopotamien.

Es ist das Verdienst HASSANs, daß er die gewonnenen Erfahrungen gerade mit Methoden zur Demographie für vorhistorische und frühe Hochkulturen in seinem maßgeblichen Buch Demographic Archaeology zusammenfaßte. ${ }^{87}$ Zum einen wurden mathematische Verfahren anhand konkreter Befunde durchgespielt und erläutert, zum anderen bot das Buch erstmals eine handliche Zusammenstellung gängiger demographischer Methoden für die Archäologie, da andere Kompendien stark auf die Bedürfnisse neuzeitlicher Forschung zugeschnitten waren, für die eine entsprechend gute Datengrundlage vorliegen muß. ${ }^{88}$

Leider erlag auch HASSAN in seinen jüngeren Publikationen der Versuchung, durch allzu kurz greifende Argumentationsketten seine Hypothesen vermeintlich zu untermauern. ${ }^{89}$ Dazu gehören insbesondere Informationen aus antiken Quellen, die positivistisch zur Grundlage darauf aufbauender Berechnungen erklärt werden, ohne sie genügend mit anderen historischen Daten abzusichern. Darüber hinaus arbeitet HASSAN mit Vergleichen zum (vor-)modernen

83 HASSAN $(1973,1975)$.

84 BUTZER (1976).

85 HASSAN (1973: 536).

86 Siehe dazu bereits ausführlich oben Kap. 1.3.2.

87 HASSAN (1981).

88 Stellvertretend für diesen Zweig demographischer Forschung seien die beiden vielzitierten Standardwerke von REHER und SCHOFIELD (1993) und NEWELL (1988) genannt.

89 HASSAN (1994: 168 ff.). 
Ägypten und setzt ohne nähere Erläuterung Prämissen hinsichtlich der durchschnittlichen Familiengröße in den verschiedenen Epochen Ägyptens fest. Schließlich entlarvt er sich mit folgender Aussage als Anhänger der Thesen von MALThus: ${ }^{90}$

»A system of deliberate population controls (e. g. minimum age for marriage, enforced abstinence and abortion) must have regulated the population in order to meet the demands of subsistence and taxation without incurring excessive misery.«

HASSAN liefert mit seinen Arbeiten zwar eine umfassende Quellensammlung zur Demographie des Alten Ägypten, verfängt sich aber allzu häufig in seinem Bestreben nach raschen Ergebnissen. Daher sind insbesondere seine Arbeiten speziell zum pharaonischen Ägypten mit einer gewissen Vorsicht zu behandeln.

\subsubsection{Neue Methoden halten Einzug}

Seit Anfang der 90er Jahre ist der Einfluß moderner demographischer Verfahren in den historischen Wissenschaften deutlich spürbar geworden. Basierend auf der umfassenden Materialsammlung von Marcel HOMBERT und Claire PRÉAUX sowie gestärkt durch einen seit den 60er Jahren neu etablierten Forschungszweig der >historischen Demographie< versucht man, die für die moderne demographische Forschung neu entwickelten statistischen Verfahren auch auf die Antike zu übertragen. ${ }^{91}$ Dabei geht es nicht mehr allein um absolute Bevölkerungszahlen zu bestimmten Zeitpunkten der Geschichte, sondern der Fokus liegt neuerdings auf Fragestellungen zu Kerndaten demographischer Entwicklung in diachroner Betrachtung: Altersgruppierung einer Bevölkerung, Fertilität, Geburts- und Sterberaten. Das zu diesem Zweck notwendige Material, nämlich Zensusdaten in ausreichender Zahl und Qualität, fand sich jedoch nur für die griechisch-römische Zeit. Dies führte zum einen zu einer Flut von Arbeiten über das zur Verfügung stehende Material, zum anderen distanzierten sich die Historiker zunehmend vom pharaonischen Ägypten, das - gemessen an den Anforderungen an die Quellen - als nicht mehr wissenschaftlich erfaßbar galt. Jüngere Publikationen verschweigen die vorgriechischen Epochen entweder völlig oder verurteilen weniger materialorientierte Versuche als zu oberflächlich oder gar spekulativ.

Auslöser dieses Wandels waren die häufigen Vertreter von Arbeiten zur antiken Demographie, die einen unkontrollierten und positivistischen Umgang mit ihren Quellen pflegten,

90 HASSAN (1994: 176).

91 Eckpfeiler dieser neuen Entwicklung waren BAGNALL und FRIER (1994) und SCHEIDEL (2001a). 
ohne eine qualifizierende Kontrollinstanz in ihre Auswertungen einzubauen, die ein Mindestmaß an Plausibilität garantiert. ${ }^{92}$ Dieser recht freimütige Umgang mit literarischen, epigraphischen oder osteologischen Befunden führte zu recht exzentrischen und nicht selten offenkundig falschen Ergebnissen. Die Folge war daher eine radikale Abkehr von den historischen Quellen, denen man keine repräsentativen oder zumindest glaubwürdigen Ergebnisse abringen konnte. Verfechter dieser extremen Gegenposition war der Historiker Keith HoPKINS, der seit Beginn der 60er Jahre die ausschließliche Verwendung moderner demographischer Rechenmodelle und Tabellen propagierte - eine Meinung, die sich in der Folge weitere Historiker zu eigen machten. Zwar waren die verwendeten Modelle für neuzeitliche Bevölkerungen entwickelt worden, doch schienen die so gewonnenen Ergebnisse den Befürwortern immer noch glaubwürdiger, als die statistischen Resultate aus historischen Quellen, deren Umstände ihrer Entstehung im Dunkel lagen. ${ }^{93}$ Die Konsequenz war jedoch eine >Entfremdung<von der Antike als Forschungsgegenstand. Eine Rückbesinnung auf die Quellen fand erst in den frühen 80er Jahren statt, als Bruce FRIER den Versuch einer Synthese aus den beiden gegensätzlichen Forschungsansätzen wagte. Die modernen Sterbetafeln und demographischen Modelle dienten ihm dabei zur Überprüfung, welche Aussagekraft den aus den antiken Quellen gezogenen prima facie-Schlüssen zugemessen werden konnten. ${ }^{94}$ Damit versuchte FRIER auch erstmals eine Brücke zwischen moderner und historischer Demographie zu schlagen.

Diese Entwicklung war allerdings auch die Ursache dafür, daß weiterführende Überlegungen zur Demographie des pharaonischen Ägypten fast gänzlich zum Erliegen gekommen sind. Mangels ausführlicher Zensusdaten oder anderer Quellen, die vom neuen Zweig der historischen Demographie akzeptiert werden, kann für das Alte Ägypten eine im strengen Sinne vergleichbare Leistung wie für die griechisch-römische Epoche nicht erbracht werden. Vielmehr stellt sich pharaonische Demographie nach wie vor als Konstrukt aus Rückschlüssen und Quervergleichen aus den spärlich überlieferten Daten dar, an dessen Ende selten eine gesicherte Zahl, sondern allenfalls eine Tendenz steht, in der sich die Größenordnungen bewegen. Dies jedoch würde gegenüber dem bisherigen Umgang mit dem Material bereits einen gewaltigen Fortschritt bedeuten.

Es liegt also in der Natur der Sache, daß es gelingen muß, Aussagen jenseits rein statistischer Interpretationen zu finden. Dazu sei an dieser Stelle der Aufruf SCHEIDELs an die

92 SCHEIDEL (2001b: 9 Anm. 35, 11).

93 SCHEIDEL (2001b: 11).

94 Vgl. zuletzt FRIER (2001); siehe auch SCHEIDEL (2001b: 12 f.). 
historischen Disziplinen aufgegriffen, die schmale Datenbasis antiker Autoren zu erweitern und nach Vergleichsmöglichkeiten zu suchen. ${ }^{95}$

Die Erfahrung hat gezeigt, daß das europäische Mittelalter als Vergleichsobjekt und Kontrastfolie einen geeigneten Hintergrund liefert, um sich über Ursachen und Wirkungen bestimmter Faktoren ein Bild zu machen. ${ }^{96}$ Auch kann das gut bekannte Material griechischrömischer Zeit den einen oder anderen Hinweis darauf geben, wie bestimmte Phänomene des pharaonischen Ägypten einzuschätzen sind. Zusätzlich können Vergleiche mit dem vorindustriellen Ägypten des 19. Jahrhunderts als Grundlage dienen, um zu besseren Einschätzungen über das Alte Ägypten zu gelangen. ${ }^{97}$ Zum Schluß sollte die Erkenntnis stehen, daß man von der reinen, ungesteuerten Spekulation Abstand nehmen muß, um mehr Klarheit darüber zu schaffen, welche Informationen über das Alte Ägypten möglich sind - und welche nicht.

Der traditionelle Weg, der seit dem Beginn demographischer Forschung in allen Bereichen historischer Wissenschaften eingeschlagen worden war, bestand in der Auswertung von dokumentarisch oder osteologisch bezeugten zeitgenössischen oder zumindest zeitnahen Quellen zu bestimmten Aspekten der Bevölkerung. SCHEIDEL führt dazu eine ganze Reihe von Gegenargumenten ins Feld, die eine Qualitätsminderung des Informationsgehaltes bedingen können. Intention und Kenntnisgrad der antiken Autoren, ein besonderes Faible für symbolische oder gerundete Zahlen, Überlieferungsverfälschungen oder schlicht Unvollständigkeit sind nur einige der von ihm genannten Gründe, die gegen eine positivistische Auswertung der historischen Quellen sprechen. Ähnliche Vorbehalte gelten für die Auswertung von Nekropolen hinsichtlich Lebenserwartung, Sterblichkeitsraten oder Geschlechtsverteilung, die nicht als repräsentativ gelten dürfen. ${ }^{98}$ Eine kritische Kontrolle ihrer Quellen wird den Historikern daher von SCHEIDEL nachdrücklich ins Stammbuch geschrieben, verkennt aber teilweise die Situation in den historischen Wissenschaften, wogegen sich bereits Wolfgang SCHENKEL gewehrt hat: ${ }^{99}$

»Die grundsätzlich negative Beurteilung >spekulativer< Elemente basiert nämlich auf der ungesagten Voraussetzung, daß historische Wissenschaften ihren Schlußfolgerungen einzig und allein >objektive< empirische Befunde zugrunde legen, mit anderen Worten: induktiv vorgehen. Dies ist aber weder theoretisch begründet, noch entspricht es der Forschungspraxis.«

95 SCHEIDEL (2001b: ix).

96 So z. B. in den Arbeiten von Behlmer (1998) oder Alston (2001).

97 Bereits als Option von RATHBONE (1990) erwähnt; vgl. zu Möglichkeiten und Gefahren ausführlich unten Kap. 7 sowie SCHEIDEL (2001a: $181 \mathrm{ff}$.), SCHEIDEL (2001b: 58 f.).

98 SCHEIDEL (2001b: 19).

99 SCHENKEL (1978: 15 f.). 
Wenn man sich die Vorwürfe von SCHEIDEL gegenüber der Quellenmethode genauer betrachtet, so fällt zweierlei auf: Zum einen gelten die von ihm vorgebrachten Beobachtungen hinsichtlich der Spaltung in zwei komplementäre Verfahren im wesentlichen für die griechischrömische Epoche Ägyptens, die den Schwerpunkt seiner Arbeit bildet. Zum anderen - und als direkte Ableitung aus dem vorangehenden Punkt - ist die Quellenlage in der von SCHEIDEL bearbeiteten Epoche deutlich günstiger. Die bei vielen Historikern angebrachte Kritik an ihren Methoden kann dadurch untermauert werden, daß es für die griechisch-römische Zeit tatsächlich eine methodische Alternative in Form einer statistischen Auswertung gibt, die trotz ihrer unausgewogenen Überlieferungssituation eine prinzipiell tragfähige Grundlage für die Anwendung numerischer Verfahren moderner Demographie bietet.

Diese günstige Situation kann für viele andere Archäologien antiker Gesellschaften nicht in Anspruch genommen werden, was auch für die Ägyptologie gilt. Der Mangel an numerischen Fakten hätte nach der strikten Herangehensweise von SCHEIDEL und anderen zur Folge, daß sich eine rein auf historischen Zeugnissen aufbauende >Demographie< solcher Gesellschaften grundsätzlich verbietet, oder zumindest in ihren Ergebnissen fragwürdig, wenn nicht gar obskur ist. ${ }^{100}$ Richtig ist dagegen die Behauptung, daß über die ausschließliche Quellenforschung nur schwer oder gar nicht Untersuchungen zu Geburts- und Sterblichkeitsraten sowie der generellen Lebenserwartung geleistet werden können. Dieses Manko muß die Ägyptologie akzeptieren, wenn sie sich nicht völlig aus der Disziplin demographischer Forschung zurückziehen will. Gleichzeitig sollte im kritischen Umgang mit den historischen Zeugnissen deren Reichweite für darüber hinausgehende Hypothesen niemals außer acht gelassen werden. Aus einzelnen Siedlungsgrößen auf eine Gesamtbevölkerung eines Landes zu schließen muß zwangsläufig in einer statistischen Streuung resultieren, die den Aussagewert des Ergebnisses erheblich in Frage stellt. Sofern also Mittel und Wege gefunden werden, die eine Überprüfung von überlieferten isolierten Aussagen ermöglichen, hat auch die Ägyptologie wieder die Möglichkeit der Untersuchung demographischer Einzelaspekte.

\subsection{Ausblick: Lineares oder nichtlineares Verhalten?}

Die neuesten Entwicklungen in der mathematischen Forschung halten indes noch einige Herausforderungen für die archäologischen Disziplinen bereit. In der vorliegenden Arbeit werden

100 Vgl. bei SCHEIDEL (2001b: 6) etwa die despektierliche Bemerkung über »conjectural numbers games (another term for parametric modelling)«. 
für die Berechnungen von Wachstumsraten ausschließlich lineare Modelle herangezogen. ${ }^{101}$ Es hat sich jedoch gezeigt, daß dies ein zu grobes Abbild von Populationsverhalten darstellt. Eine Lösung zur Entwicklung neuer und genauerer Modelle versprach erst der Durchbruch der sogenannten >Chaos-Theorie<, auch Theorie Nichtlinearer Dynamischer Systeme genannt. Die Chaos-Theorie ist jedoch nicht gleichzusetzen mit dem Prinzip des >Zufalls $<$, wie dies in alltagshermeneutischer Verwendung immer wieder passiert. Vielmehr ist es das Wesen des Chaos, daß der Verlauf vorherbestimmt ist und deshalb in gewisser Weise berechenbar bleibt. Die Schwierigkeit liegt in den Differenzen der Ausgangskonfiguration, die äußerst gering ausfallen und zudem nicht beliebig präzisiert werden können. Dies führt dazu, daß bei zeitabhängiger Betrachtung nach kurzer Zeit keine verläßlichen Prognosen mehr möglich sind. Dies bedeutet - vereinfacht gesprochen - folgendes: Während in linearen Modellen das Fehlen von Daten in den Anfangswerten dadurch kompensiert werden kann, daß die daraus resultierende >Lücke< in den Ausgangswerten anhand von benachbarten Ergebniswerten interpoliert wird, ist dies bei nichtlinearen Modellen nicht möglich. Diese fehlende Vorhersagbarkeit zwingt zu einer hohen Informationsdichte der Anfangsdaten, wenn man solche Ergebnislücken vermeiden will. Für einen Umgang mit historischen Daten, deren Informationsdichte und Genauigkeit tatsächlich nicht beliebig vergrößerbar sind, offenbart sich für die statistisch-quantitativen Verfahren der Demographie damit eine ähnlich gravierende Schwäche, die von deren Verfechtern den historischen Quellen angelastet wird.

Die Bevölkerungsentwicklung, insbesondere ihre Vorhersagbarkeit anhand definierter Parameter, ist zwar deterministisch, aber nicht linear. ${ }^{102}$ Für die Entwicklung mathematischer Modelle gibt es verschiedene mögliche Szenarien. Die stochastische Bewegung, die mit dem Prinzip des Zufalls gleichzusetzen ist und daher nicht deterministisch ist, kann für die weiteren Betrachtungen außer acht gelassen werden. Es stehen dann noch zwei Szenarien zur Auswahl: eine komplexe, aber trotzdem deterministische und vorhersagbare Bewegung, und eine chaotische Bewegung, die zwar deterministisch, aber nicht langfristig vorhersagbar ist. Die Frage, ob Populationen nun komplexen oder chaotischen Gesetzmäßigkeiten gehorchen, ist auch in der Mathematik nicht abschließend geklärt. ${ }^{103}$ Dennoch wird in der mathematischen Biologie

101 Vgl. ausführlich unten Kap. 7.1.3.

102 Die Definition für Determiniertheit lautet: Beschreibung eines Algorithmus, der jeden möglichen Anfangswert nur jeweils auf exakt einem Ausgangswert abbildet. Deterministische Abläufe basieren also auf dem Kausalprinzip von Ursache und Wirkung. Nichtdeterministische (stochastische) Abläufe können bei gleichen Anfangswerten unterschiedliche Ausgangswerte einnehmen.

103 Es gibt Thesen, die beispielsweise statt chaotischem Oszillieren von Populationsgrößen eher mathematische Artefakte hinter den Ergebnissen vermuten; vgl. z. B. WEHR (2002: 179). 
seit einigen Jahren wieder mit verstärkter Intensität die Forschung auf dem Gebiet nichtlinearer Dynamik von Populationen vorangetrieben. ${ }^{104}$

Um nochmals zu verdeutlichen, wie die Probleme einer mathematischen Beschreibung von Prozessen gelagert sind, sei im folgenden das bisherige Naturverständnis dem neuen, durch die Chaostheorie geprägten Verständnis gegenübergestellt:

\begin{tabular}{lll}
\hline $\begin{array}{l}>\text { Klassisches }<\text { Modell } \\
\text { (basiert auf linearen Gleichungen) }\end{array}$ & \begin{tabular}{l}
$\begin{array}{l}\text { Neues Modell } \\
\text { (basiert auf nichtlinearen Gleichungen) }\end{array}$ \\
\cline { 1 - 2 } $\begin{array}{l}\text { Gleiche Ursachen haben gleiche Wirkung. } \\
\text { Ähnliche Ursachen haben ähnliche Wirkung. }\end{array}$
\end{tabular} & $\begin{array}{l}\text { Gleiche Ursachen haben gleiche Wirkung. } \\
\text { Ähnliche Ursachen können ähnliche Wirkung haben, } \\
\text { es sind jedoch auch völlig andere Ergebnisse möglich. }\end{array}$ \\
\hline
\end{tabular}

Es sei abschließend mit einem Beispiel beschrieben, wie mathematische Modelle einer Bevölkerungsdynamik beschrieben werden, welche Ziele die mathematische Biologie verfolgt und welche Prämissen gesetzt sein müssen, um Umweltvariablen überhaupt parametrisierbar zu gestalten. Grundsätzlich wird zunächst unterschieden, ob eine Population isoliert betrachtet wird, oder mehrere Populationen, die innerhalb des gleichen Raumes interagieren. ${ }^{105}$ Letzteres kann wiederum in zwei verschiedene Szenarien aufgeteilt werden: Entweder verhalten sie sich in Form von Wettbewerb und Symbiose, was eine Gleichrangigkeit der daran beteiligten Populationen voraussetzt, oder sie funktionieren als System von Jäger und Beute. Die Dynamik eines solchen ökologischen Systems basiert auf den bereits bekannten Parametern wie zum Beispiel Geburt, Tod, Wettbewerb, Verbrauch, die als regulierende Mechanismen die Stabilität eines solchen Systems bestimmen. ${ }^{106}$

Die parametrisierte Modellierung eines solchen Ökosystems ist jedoch - wie bei jeder Modellbildung - gezwungen, bestimmte vereinfachende Prämissen zu setzen, um die Abhängigkeiten zwischen den einzelnen Parametern überschaubar zu halten. Folgende Vereinfachungen und Idealisierungen werden zuvor festgelegt: ${ }^{107}$

1. Die externen Bedingungen bleiben unverändert. Obgleich das Wissen um den Einfluß exogener Faktoren auf die Dynamik wünschenswert wäre, wird mit dieser Prämisse das zentrale Augenmerk auf die Population selbst als autonomes System gelegt.

104 ISLAM (2002); BAZYKIN (1998).

105 Es sei an dieser Stelle darauf hingewiesen, daß >Population $<$ im biologischen Sinne weiter gefaßt ist und dabei auch die Tierwelt mit einschließt. Dennoch sind die Modelle ausdrücklich so formuliert, daß humanoide Populationen gleichermaßen damit erschlossen werden können.

106 BAZYKIN (1998: ix).

107 BAZYKIN (1998: 4 f.). 
2. Die Bevölkerungsentwicklung wird als kontinuierlicher Wert beschrieben. Zufällige Fluktuationen sind vernachlässigbar, so daß nur die Dynamik der durchschnittlichen Populationsgröße betrachtet wird.

3. Es werden Vorausannahmen hinsichtlich der Altersgliederung einer Population getroffen, beispielsweise etwa eine synchrone Fortpflanzung zu einem festgelegten Alter. Generationen überlappen zwar einander, doch die Variation in ihrer Größe wird in Abhängigkeit zur Gesamtpopulation an einem zurückliegenden Zeitpunkt bestimmt.

4. Es muß differenziert werden zwischen lokalen Systemen, die einen eng begrenzten, für alle Individuen zugänglichen Lebensraum beschreiben und Systemen, die einen übergroßen Lebensraum beschreiben, der eine weite räumliche Verteilung der Population(en) erlaubt.

Trotz - oder gerade wegen - dieser strengen Limitierung der Parameter können zentrale Fragen zum Verhalten ökologischer Gruppen gestellt werden. ${ }^{108}$ Dazu gehört zunächst die Frage nach der Stabilität einer solchen Population, oder ob sie oszillierend oder chaotisch schwankt. Ein weiterer Aspekt ist die Bedeutung des Anfangswertes für das Verhalten einer Gruppe und die Frage, ob der Anfangswert überhaupt für die Entwicklung einer Population ausschlaggebend ist. Einen weiteren Ansatz stellt der Einfluß der Umwelt auf die Gruppe dar. Hier kann die Rolle exogener Faktoren als kurzfristige oder dauerhafte Einwirkung genauer betrachtet werden. Der letzte Gesichtspunkt betrifft die Ausbreitung der Individuen in ihrem Lebensraum und die möglichen Konsequenzen, die aus einer ungleichen Verteilung für die Entwicklung des Ökosystems zu ziehen sind.

Es liegt auf der Hand, daß die hier vorgestellten mathematischen Modelle recht hohe Voraussetzungen an die Daten der zu untersuchenden Population stellen. Doch scheinen diese Verfahren das Potential zu haben, eines Tages die auf >herkömmlichem< Weg gewonnenen Bevölkerungsdaten des Alten Ägypten, wie sie zum Beispiel in Tabelle 1.2 vorliegen, zu bestätigen oder zu widerlegen. Dies wäre für die ägyptologische Problemstellung bereits ein großer Fortschritt. Der aktuelle Forschungsstand in der Ägyptologie läßt dies allerdings vorerst nicht zu.

108 BAZYKIN (1998: 6). 


\section{Die griechische Überlieferung}

Leider ist es unmöglich, jeden einzelnen aus der Menge beiseite zu nehmen [... ] oder alle der Reihe nach zu fragen.

Dion von Prusa, Reden 7,100

\subsection{Der Umgang mit griechischen Quellen}

$\mathrm{Z}$ U Beginn der Betrachtungen über die Demographie des Alten Ägypten soll eine Bestandsaufnahme der Überlieferungen aus der klassischen Antike stehen. Die griechischen und römischen Autoren stellten lange Zeit die wichtigste Quelle über die Ägypter und ihr Alltagsleben dar. Als Hauptvertreter für Informationen aus >erster Hand dürfen dabei sicherlich Herodot und Diodor gelten, deren Reputation im Laufe der Forschung jedoch starken Schwankungen unterworfen war. Galten ihre Beschreibungen anfangs häufig noch als unumstößliche Tatsachenberichte, so wurden sie später als Sammlungen freier Erfindungen oder gar Unmöglichkeiten verurteilt, die für die historische Forschung von minderem Wert seien. Erst in jüngerer Zeit entsteht wieder ein etwas anderes Bild, da mittlerweile ein differenzierteres Verständnis für das Werk und die Intention antiker Autoren entwickelt wurde. Erst dadurch gelang es, die alten Texte neu zu lesen und zu interpretieren.

Der Quellenkritik in der ägyptologischen Forschung dienten die Zitate aus der Feder griechischer wie römischer Autoren - den eigenen Überlegungen vorangestellt - eher der Illustration. Ein dabei immer wieder zu beobachtendes Phänomen wissenschaftlicher Auseinandersetzung mit diesen Quellen ist eine allgemeine Tendenz zu allzu vordergründiger Lesart der darin enthaltenen Informationen. In der traditionellen Herangehensweise werden den Texten einzelne Aussagen entnommen, die sich anschließend einem kritischen Vergleich mit Erkenntnissen anderer Quellen - zumeist archäologischer Herkunft - stellen müssen. Da sich mit diesem Verfahren ein Abgleich zwischen den Quellengattungen mitunter recht schwierig gestaltet und im Zweifelsfall die archäologische Evidenz als Realie nicht wegzudiskutieren ist, suchte 
man den Fehler bei den antiken Autoren. Die unmittelbare und wortgetreue Exegese der Texte dürfte als Hauptursache dafür gelten, weshalb in den vergangenen Jahren das einst allzu blinde Vertrauen in die klassischen Quellen einem Hyperskeptizismus gewichen ist, der einen von den antiken Überlieferungen unabhängigen Neuansatz versucht. Die Ursache dafür lag in einem Wandel der Einstellung, die den historischen Zahlen eine zu große Leistungsfähigkeit abverlangte, die sie im Rahmen ihres Kontextes weder erfüllen konnten noch wollten. Die Forderung an die antiken Autoren war die zuverlässige Dokumentation historischer Daten, und zwar in einer Exaktheit, wie sie sonst nur in Form einer Zählung oder auf mathematischem Wege erreicht werden kann. Einerseits bedient man sich also immer noch der Informationen aus den griechischen Quellen, um die eigenen Ausführungen hinsichtlich antiker Populationen zu kontrastieren. Auf der anderen Seite steht die statistische Forschung, die - unbelastet von der griechischen Textlektüre - anhand mathematischer Methoden die Population jener Zeit anders beurteilt und die historischen Quellen vermeintlich Lügen straft.

\subsubsection{Exkurs: Über die Herkunft von Zahlenangaben}

Wenn man im Zweifel für die historischen Quellen zunächst einmal davon ausgeht, daß die antiken Autoren von der Richtigkeit ihrer Angaben überzeugt waren, dann gilt es in einem zweiten Schritt, die Motivation für das Niederschreiben dieser Informationen zu ergründen. Es gibt verschiedene Möglichkeiten, numerische Informationen in der Qualität ihrer Aussage zu definieren. Stark vereinfacht lassen sich - in absteigender Güte - drei Varianten unterscheiden:

Berechnete oder gezählte Zahlen: Methodische Sauberkeit vorausgesetzt, ist diese Form die präziseste. Durch ein geeignetes mathematisches Verfahren oder schlichtes Auszählen wird ein Ergebnis erzeugt, das der Realität am nächsten steht.

Geschätzte Zahlen: Die Methode der Schätzung birgt bereits die Gefahr von Ungenauigkeiten. Anhand von bekannten Faktoren und Beobachtungen wird versucht, aus Stichproben eine Hochrechnung des Ergebnisses zu ermöglichen (>maximum likelihood<). Je nach Vollständigkeit der zugrundeliegenden Informationen muß eine gewisse Fehlerrate dabei einkalkuliert werden, doch ist eine Stichprobe in der Regel nicht durch das anschließende exakte Auszählen falsifizierbar.

-Gewußte Zahlen: Die unpräziseste Form einer Dateninformation besteht aus einem groben Wissen über die Dinge, verbunden mit individuellen Vorstellungen und unvollständiger Kenntnis über ihre Zusammenhänge. Aus diesem persönlichen Bild heraus wird ein Ergebnis formuliert. 


\subsubsection{Verschiedene Lesarten für unterschiedliche Intentionen}

Wenn ein antiker Autor zu einem bestimmten Sachverhalt eine Größenordnung oder Zahlenangabe beifügt, dann gibt es zwei Möglichkeiten des Umgangs mit dieser Information. Die erste Lesart wäre eine rein numerische, nämlich die Zahl in ihrer absoluten mathematischen Größe aufzufassen und auszuwerten. Diese Form der Interpretation ist allerdings nur dann zulässig, wenn dies ausdrücklich als Intention des Verfassers zu verstehen ist. Die zweite Möglichkeit besteht in der Vernachlässigung der numerischen Exaktheit einer Information zugunsten der Gesamtaussage des Satzes. In diesem Fall wäre das numerische Prädikat dem intensionalen Kontext untergeordnet und hat trotz seiner fehlenden Exaktheit keinen Einfluß auf die Gültigkeit der damit getroffenen Aussage. Es geht bei der Distinktion dieser beiden Herangehensweisen darum, ob die Analyse einer Aussage hinsichtlich dessen getroffen wird, was sie tatsächlich ist oder aber für was wir sie halten. Wir haben hier eine Differenzierung von Dimensionsangabe versus gezählter Zahl. Um das Gebiet der Ägyptologie nicht allzu weit zu verlassen, sei hier auf Alan GARDINER verwiesen, der bereits in seiner Arbeit zur Theory of Speech and Language darauf hinwies, daß der Aussagewert eines Satzes von dessen Intentionstiefe abhänge. Über diesen angestrebten Punkt der Intentionstiefe hinaus erübrige sich eine Analyse des Wahrheitsgehaltes. ${ }^{109}$

Ziel der folgenden Ausführungen soll es sein, die Qualität antiker Zitate zur ägyptischen Demographie auf eine neue Grundlage zu stellen, die von Josephus und Diodor überlieferten Informationen zu rehabilitieren und deren Zahlenmaterial für das Alte Ägypten ernstzunehmen. Die von den beiden Autoren getroffenen Aussagen können - gemessen an ihrer Intentionstiefe - durchaus als >wahr $<$ bezeichnet werden, ohne daß die Teilaussagen im Einzelfall exakt genug sein müssen. Letztlich sind es die einzigen Autoren, die für die Population Ägyptens eine konkrete Zahl nennen und als Ausgangsbasis für weitergehende Betrachtungen genutzt werden können. Schließlich sollen die für diesen Zweck benötigten Kontexte und Lesarten der gebotenen Information einer neuen Betrachtungsweise unterzogen werden, die es ermöglicht, anhand des historischen Kontextes im 3. bis 1. Jahrhundert v. Chr. die Einzelinformationen plausibel zu gestalten. Eine Verwertung der darüber hinaus bei Herodot und Diodor zitierten Anzahl von Städten (oder Siedlungen) scheint dagegen kaum möglich, da die Kenntnisse der modernen Forschung über Siedlungsgrößen eher als gering einzustufen sind und somit eine Schätzung der Gesamtbevölkerung auf einer weiteren Schätzung, nämlich der Größe der Siedlungen, aufbauen würde.

109 GARDINER (1932: 52 f., § 17). 


\subsection{Extreme Schätzungen der Gesamtbevölkerung auf Basis antiker Autoren}

Zum Schluß dieses Kapitels soll noch einmal kurz darauf eingegangen werden, wie anhand der griechischen Quellen auch extreme Interpretationen ihren Weg in die Forschung fanden und dort zumindest zeitweilig auch Aufmerksamkeit erfuhren. Besonders die Gelehrten des 18. Jahrhunderts zogen mit fundiertem Wissen über die antiken Autoren, vor allem aber auch blindem Vertrauen in den Wahrheitsgehalt der Überlieferungen ihre Schlußfolgerungen, lange bevor die Napoleonische Expedition im Jahr 1798 an den Nil aufbrach. ${ }^{110}$

Neben dem bereits vorgestellten David HuME hatte sich auch dessen Widersacher Robert WALLACE Mitte des 18. Jahrhunderts mit dem Problem antiker Populationen beschäftigt. Sehr ausführlich analysierte er die Quellen griechischer und römischer Historiker zu den Ländern des Altertums, um daraus die Bevölkerungsgrößen zu errechnen. ${ }^{111}$ Es sei vorab nochmals darauf hingewiesen, daß WALLACE ein Vertreter jener Forschergruppe war, die sich - verglichen mit der Situation zu seiner Zeit - für eine deutlich höhere Bevölkerungszahl in der Antike aussprach und dafür mit Nachdruck nach Indizien suchte. Berechnungsgrundlage waren für WALLACE wiederholt die ihm bekannten Länder seiner eigenen Epoche, hauptsächlich England und Frankreich. So leitete er etwa einen ersten Berechnungsversuch für das alte Ägypten damit ein, daß er die Fläche Englands mit der Ägyptens verglich und daraus ein GröBenverhältnis von $1: 2,84$ ermittelte. Bei der Schätzung der damaligen Bevölkerung Englands auf etwa 8 Millionen Menschen, kam WALLACE - unter der Voraussetzung einer identischen Bevölkerungsdichte - auf eine Zahl von 22,7 Millionen Menschen für Ägypten.

Mit dieser >Arbeitsgrundlage < wagte sich WALLACE danach an die antiken Autoren, deren Daten nach seiner Meinung Beleg für noch weitaus höhere Populationsgrößen Ägyptens lieferten. Zunächst widmete er sich einer Aussage Diodors, der zu berichten wußte, daß am Tage der Geburt des Königs Sesostris weitere 1700 männliche Kinder in ganz Ägypten das Licht der Welt erblickten. ${ }^{112}$ Für WALLACE sprach nichts dagegen, daß eine solche Zahl an jedem Tag des Jahres geboren wurde, was ihn zu einer jährlichen Zahl von 620500 männlichen Nachkommen brachte, aus der sich durch Kalkulation die Gesamtzahl von 17 Millionen Männern ergab. ${ }^{113}$ Bei einem Verhältnis von 1:1 zwischen Männern und Frauen lautet das Ergebnis nach dieser Rechnung also auf 34 Millionen Menschen.

110 Vgl. oben Kap. 1.3.3.1.

111 WALLACE (1753: $41 \mathrm{ff}$.).

112 Diodor I,53-54; WALLACE (1753: 41 f.).

113 WALLACE zitierte für diese Kalkulation einen Dr. HALLEY, das Verfahren der Berechnung wird leider nicht offengelegt. 
Als nächste Datengrundlage wurden die von Herodot zitierten 20000 Städte verwendet. Auch hier operierte WALLACE mit Durchschnittsgrößen und rechnete mit einem mittleren Wert von 2000 Menschen pro Stadt, was für ihn in Anbetracht der beiden überproportional großen Metropolen Theben und Memphis als moderate Größe erschien. ${ }^{114}$ Im Ergebnis kommt er damit auf eine Gesamtzahl von 40 Millionen Menschen.

Auch die bereits einschlägig untersuchte Passage bei Diodor wurde von WALLACE herangezogen, die die Bevölkerung Ägyptens auf >damals< 7 Millionen, doch später nur noch 3 Millionen Menschen bezifferte. ${ }^{115}$ Aufgrund seiner generellen Vorstellungen über die Antike war es für WALLACE allerdings unmöglich, die Angaben Diodors als Gesamtpopulationen aufzufassen. Daher deutete er sie um und bezog die Zahlen auf Familienoberhäupter oder waffenfähige Männer. ${ }^{116}$ Aus bereits zuvor getätigten Kalkulationen verwendete WALLACE den Faktor vier, um von den waffenfähigen Männern auf die Gesamtbevölkerung hochzurechnen, was ihn abermals in eine Dimension von 12 bis 28 Millionen Menschen brachte. ${ }^{117}$

Als letztes Beispiel für den Umgang mit den Quellen soll das Zitat Herodots dienen, der die Größe der ägyptischen Kriegerkaste mit 410000 Mann bezifferte. ${ }^{118}$ Für einen Vergleich zog WALLACE das > wehrhafte< Frankreich heran, dessen Bevölkerung er auf 16 bis 20 Millionen, das französische Heer auf ungefähr 200000 Mann schätzte. Trotz der Überlegung, daß antike Gesellschaften eine geringere Truppengröße hatten als moderne Nationen, setzte WALLACE eine identische Relation an und kam zu dem Ergebnis, daß Ägypten zwischen 32 und 40 Millionen Bewohner gehabt habe. ${ }^{119}$

114 WALLACE (1753: 43).

115 Diodor I,31.6; bemerkenswert in Hinblick auf WALLACEs Ausführungen sind zum einen das Gefälle der Bevölkerungszahl, das gut in sein eigenes Geschichtsbild paßt, und zum anderen, daß er bereits 1753 ganz selbstverständlich mit zwei verschiedenen Zahlen operierte, obwohl diese Stelle später von verschiedenen Forschern emendiert wurde. Vgl. dazu auch unten Kap. 2.5.1.

116 WALLACE (1753: 45); Ähnlich äußerte sich noch rund 150 Jahre später Julius BELOCH, der in den 3 Millionen nur die männliche, kopfsteuerpflichtige Bevölkerung sah, während die Gesamtbevölkerung 10 Millionen betragen habe; siehe auch RosTOVTZEFF (1984: 907 Anm. 73).

117 Zur Methode und Bewertung derartiger Faktoren-basierter Berechnungen vgl. HoLLINGSWORTH (1969: 49).

118 Herodot II,164-168.

119 WALLACE (1753: 46). Einen vergleichbaren Umgang mit historischen Zahlen hat noch in jüngster Zeit Fekri HASSAN (1994: 168 ff.) vorgeführt, jedoch mit weitaus weniger extremen Ergebnissen. Werner Huss (2001: 30 f.) dagegen bleibt ganz in der Tradition von WALLACE, wenn er die Zahl der Kriegerkaste mit vier (plus Ehefrau und zwei Kinder) multipliziert, um eine Gesamtzahl der Bevölkerung zu erhalten. An ihm scheinen 250 Jahre Forschungsgeschichte spurlos vorübergegangen zu sein. Ein ähnliches und im Ergebnis kaum haltbares Verfahren zur ägyptischen Bevölkerung des Deltas in der Zeit des Königs Narmer auch bei MiLLET (1990: 57 f.). 
Alle seine Ergebnisse zusammengenommen befand WALLACE, daß das antike Ägypten von rund 34 Millionen Menschen bewohnt gewesen sein muß und sah sich aufgrund der ersten Nachrichten, die Mitte des 18. Jahrhundert aus Ägypten nach Europa drangen, in seiner Hypothese über die Bevölkerungsentwicklung der Menschheit bestätigt. Aus moderner Sichtweise und mit dem in der Zwischenzeit erlangten Wissen um demographische Entwicklungen erscheinen die von WALLACE vorgetragenen Beobachtungen in gewisser Weise naiv. Andererseits muß ihm zugute gehalten werden, daß er seine Überlegungen stets begründet und versucht ist, seine Thesen durch Argumente zu stützen. Aufschlußreich ist auch die Erkenntnis, daß WALLACEs Arbeitsweise wiederholt moderne Nachahmer gefunden hat, die einfache Kombinatorik an die Stelle kritischer Schätzungen setzten.

Erst 1937 griff ein polnischer Historiker namens Tadeusz WAŁEK-CZERNECKI die alten Hypothesen erneut auf und beschäftigte sich mit der Demographie Ägyptens auf Basis der Quellen der klassischen Antike. ${ }^{120}$ Insbesondere die frühen Überlegungen BELOCHs, daß Diodor nur die Zahl der waffenfähigen Männer gemeint haben konnte, fanden seine Unterstützung. ${ }^{121}$ Dagegen sprach sich WAŁEK-CZERNECKI vehement gegen die >Frühdatierung< aus, da sie nach seiner Meinung philologisch nicht begründbar sei. ${ }^{122}$ Da das Ergebnis der Kalkulation mit 25 Millionen Menschen bis zum Ende der Ptolemäerzeit für WAŁEK-CZERNECKI doch zu unwahrscheinlich klang, präferierte er für diese Zeit eine Obergrenze von maximal 12 bis 13 Millionen Menschen. ${ }^{123}$ Die Berechnungen für die pharaonische Zeit verwarf er dagegen völlig, da sie nur auf die Attestation durch Hekataios von Abdera beruhen, der nach Einschätzung WAŁEK-CZERNECKIs kein ausgewiesener Kenner der pharaonischen Epoche war.

Im weiteren Verlauf seiner Analyse beschäftigte sich WAŁEK-CZERNECKI näher mit der Frage nach dem genauen Inhalt der Zahlenangaben. Aufgrund Diodors Wortwahl, der die Bevölkerung statt mit $\delta \tilde{\eta} \mu o \varsigma$ mit dem universelleren Begriff $\lambda \alpha o ́ \varsigma$ bezeichnet, ist nach WAŁEKCZERNECKI davon auszugehen, daß die Gesamtzahl aller Menschen inklusive Frauen und Kinder gemeint sein muß. Eine letzte Evidenz dafür, daß die Vermutung Julius BELOCHs zu den Zahlen zurückgewiesen werden muß, findet WAŁEK-CZERNECKI bei der Textstelle

120 WAŁEK-CZERNECKI (1937).

121 Siehe oben Anm. 116.

122 WAŁEK-CZERnECKI (1937: 10). Zur Hypothese einer >Frühdatierung<von Diodors Angaben vgl. ausführlich unten Kap. 2.6.2.

123 WAŁEK-CZERNECKI (1937: ebenda). 
des Josephus. ${ }^{124}$ Trotz der Zweifel an der Herkunft der Daten aus Steuerlisten, wie Josephus behauptet, ist es doch ein Indiz für eine Gesamtzahl der Bevölkerung und nicht nur Teilen davon. ${ }^{125}$ Aufgrund der Vermutung, daß Josephus durch seine guten Beziehungen zur Obrigkeit Zugang zu den römischen Verwaltungsakten hatte, stuft WAŁEK-CZERNECKI ihn letztlich doch als vertrauenswürdig ein.

In seiner abschließenden Textkritik setzt WAŁEK-CZERNECKI zwei Eckpfeiler an Daten: Da die Schere zwischen den vermuteten 12 bis 13 Millionen Menschen und den 7 1/2 Millionen Menschen zur Zeit des Josephus zu groß ist, muß es sich bei den Zahlen Diodors um Gesamtangaben handeln. Das schließt für WAŁEK-CZERNECKI aber auch aus, daß die Behauptung Diodors, zu seiner Zeit habe es 3 Millionen Menschen gegeben, richtig ist. Die Emendation

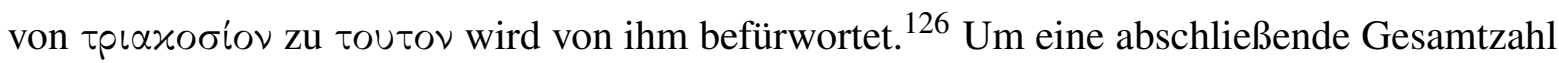
zu erhalten, muß allerdings noch eine offene Frage bei Josephus geklärt werden, der in seiner Kalkulation die Bewohner Alexandrias ausdrücklich nicht mit einbezogen hatte. Nach Auskunft Diodors bewohnten Alexandria zu seiner Zeit 300000 freie Bürger. Auch hier besteht Uneinigkeit über Art und Entstehung der Zahl. WAŁEK-CZERNECKI präferiert in diesem Fall die Interpretation als >erwachsene Männer<. Da Diodor auch betont, daß Alexandria die damals größte Stadt der alten Welt gewesen sei, kann eine Gesamtbevölkerung Alexandrias von nur $1 / 2$ Million kaum möglich sein, da die Bevölkerung Roms zur gleichen Zeit auf über eine Million Menschen geschätzt wird. So bleibt als Schlußfolgerung nur die Hochrechnung aus den 300000 Männern auf eine Gesamtbevölkerung von 1,2 bis 1,5 Millionen Menschen.

\subsection{Das Ägyptenbild in der klassischen Antike}

Für den Zweig der Demographie gibt es nur wenig Evidenz. Neben den bekannten Aussagen von Diodor und Josephus, auf die unten ausführlich eingegangen wird, gibt es nur wenige Autoren, die sich zur Bevölkerung Ägyptens geäußert haben. Keine dieser Textstellen weist konkrete Zahlen auf, so daß eine Verwertung zunächst unmöglich scheint. Doch verraten die Zitate viel von der seinerzeit herrschenden Einschätzung über Ägypten, also genau das, was Peter BILLER als >demographic thought $<$ bezeichnet hat. ${ }^{127}$ Es geht dabei nicht so sehr um die

124 Siehe unten Kap. 2.5.2.

125 WAEEK-CZERNECKI (1937: 11).

126 WAŁEK-CZERneCKI (1937: 12). Zur Kritik an dieser Emendation vgl. unten Kap. 2.5.1.

127 BiLLER (2000: 6 f.). 
demographische Realität jener Zeit, sondern vielmehr um das >demographische Bewußsein< der Zeitgenossen, deren Vorstellungen von Populationsentwicklungen und die Ursachen, die sie dafür verantwortlich machten. Wichtig war hierbei nicht die konkrete Aussage, sondern das Gespür oder die - begründete - Vermutung über die Zustände in Ägypten, sei es durch eigene Beobachtung oder aber über Berichte von Gewährsleuten aus zweiter Hand.

So wurde zum Beispiel dem Nilwasser zugesprochen, daß es die Fruchtbarkeit der Frauen fördere. ${ }^{128}$ Diese Erkenntnis dürfte allerdings nicht neu gewesen sein, sondern lag in der alten Erfahrung begründet, daß der Nil während seiner jährlichen Überschwemmung dem Ackerboden die Fruchtbarkeit bringt. Beobachtungen wie das Entstehen der Frösche im feuchten Nilboden ${ }^{129}$ haben sicher ihren Teil dazu beigetragen, auch wenn die Quellen über die Hintergründe dieser Vorstellungen schweigen. Die Funktion des Nilwassers als >Lebensspender findet sich dabei bereits im rituellen Gebrauch während der Zeit des pharaonischen Ägypten wieder, da sein Genuß für die Verstorbenen als Mittel der Regeneration galt. ${ }^{130}$ Ein Zusammenhang zwischen Regeneration und Fruchtbarkeit läßt sich indes für die pharaonische Zeit nur schwer herstellen. ${ }^{131}$ Dennoch haben sich bis zu Beginn des 20. Jahrhunderts in der ägyptischen Bevölkerung gewisse Traditionen halten können, die einen Zusammenhang zwischen Fruchtbarkeit der Frau und dem Nil herstellt, wie etwa die Beschreibung eines Mittels, um erneut schwanger zu werden: Die Frau soll an den Nil gehen und mit einem Fährboot einmal zum gegenüberliegenden Ufer und zurück übersetzen. ${ }^{132}$ Doch könnten derartige Rezepte bloße Reflexe längst in Vergessenheit geratener Vorstellungen sein.

Auch andere Autoren hatten eine ähnliche Meinung über Ägypten. Ungefähr einhundert Jahre nach Herodot spottete Timon von Phleius über die Philosophen seiner Zeit: ${ }^{133}$

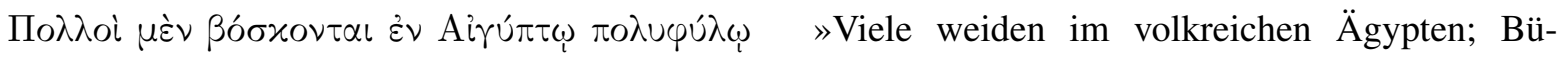
cherkundige, Eingepferchte, endlos Streitende im

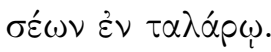
Hühnerstall der Musen.«

128 Plinius, Naturalis historiae VII § 33; Seneca, Naturales Quaestiones III 25,11; Strabon, Geographica XV 1,22; etwas allgemeiner formuliert es Theophrast, in: Athenaios, Deipnosophistae II $41 \mathrm{f}$.

129 Beschrieben bei Horapollo I 25.

130 PAMMINGER (1991).

131 BRUNNER (1977: 337) verneint einen Zusammenhang zwischen Fruchtbarkeit des Bodens und der Menschen; PAMminger (1991: 74 Anm. 17) dagegen zitiert Sargtext Spruch 405 (DE BUCK 1954: V 209.m-n), in dem Überschwemmungswasser und männliche Potenz in einen Kontext zueinander gebracht werden: $\underline{d} j$ $z w j=f m w h r b 3 b 3 . t j m j r j=f n \underline{d} m m . t » \mathrm{Laß}$ ihn Überschwemmungswasser trinken, damit er seine sexuelle Lust befriedigt.«

132 WinKLER (1934: 114).

133 Das Zitat ist nur noch als Fragment im Werk Deipnosophistae des Athenaios erhalten geblieben. 
Bevor er mit konkreten Zahlen und Daten aufwartete, beschrieb Diodor einleitend seinen allgemeinen Eindruck über Ägypten und dessen Bewohner:

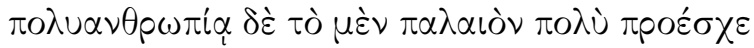

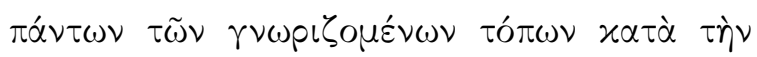

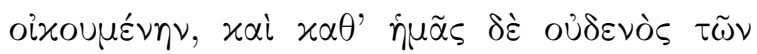

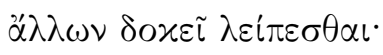

»Hinsichtlich seiner Bevölkerungsdichte überragte es [scil. Ägypten] damals alle bekannten Gebiete der bewohnten Welt, und sogar heutzutage scheint es mit keinem anderen vergleichbar.«

Diodor zitierte in seinem Werk auch Scipio den Jüngeren, der während der Regierung Ptolemaios' VIII. zwischen 141 und 139 v. Chr. Ägypten bereiste und in seinem Reisebericht unter anderem auf die Bevölkerungssituation Ägyptens einging.

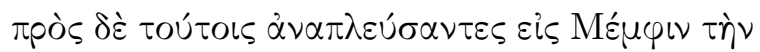

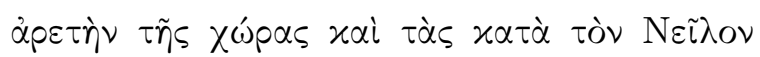

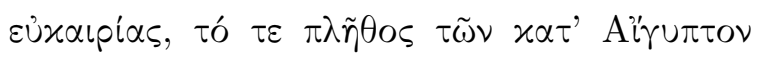

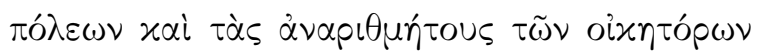
$\mu \cup \rho\llcorner\alpha ́ \delta \alpha \varsigma . .$.
»Weiter nilaufwärts nach Memphis fahrend [sahen sie] die Qualität des Bodens und die Segnungen, die ihm durch den Nil zuteil werden, die große Zahl ägyptischer Städte und die unzähligen Myriaden ihrer Bewohner, ...«

Und auch der Geschichtsschreiber Prokop vermerkte im 6. Jahrhundert n. Chr. am Rande die besondere Bevölkerungssituation in Ägypten, die er anscheinend zu seiner eigenen Zeit noch ähnlich einschätzte.

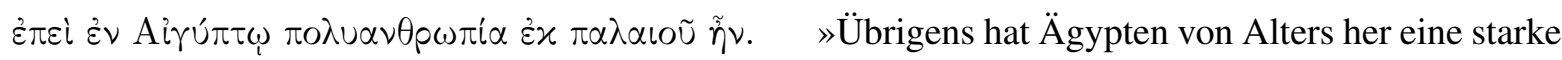
Bevölkerung.«

Diodor 131,6

Die einzelnen Zitate zeigen deutlich, daß die klassische Antike Ägypten für ein - in jeder Hinsicht - fruchtbares Land hielt, das seine Prosperität auch in einer großen Bevölkerungsgröße zeigte. Sogar im Mittelalter zeigte diese antike Einschätzung über das Volk Ägyptens Wirkung in den frühen Reiseberichten. Noch gegen Ende des 13. Jahrhunderts schrieb der Dominikanermönch Burkard de Monte Sion in seinem Bericht über seine Reisen nach Ägypten, Syrien und Palästina: ${ }^{134}$

»Ägypten hat ein zahlreiches Volk, das von den Sarazenen regiert wird.«

Burkard traf damit eine Aussage, die sicher aus den antiken Quellen genährt wurde und verdeutlicht, wie tief dieser seit alters verwurzelte Eindruck über das ägyptische Volk auch in spätere Generationen hineinwirkte.

134 КнатТАВ (1982: 82). 


\subsection{Zensusverfahren in griechisch-römischer Zeit}

Auch wenn das >demographische Bewußtsein< der klassischen Autoren häufig allein auf allgemeinen Einschätzungen zur Bevölkerungssituation ihrer Zeit beruhte, so muß dieses recht einhellige Urteil doch einen gewissen abgesicherten Hintergrund aufweisen. Dies bedeutet allerdings, daß das Zensussystem der griechisch-römischen Zeit ausgeprägt genug war, um aus den regelmäßigen Erhebungen wenigstens eine grobe Vorstellung über die Gesamtbevölkerung jener Zeit ermitteln zu können. Als Kernargument stand bisher die zweifelhafte Quellenlage dagegen, die sowohl Diodor als auch Josephus nach eigener Auskunft für ihre Angaben herangezogen haben. Diodor spricht einerseits von den >heiligen Schriften $<$ und Zensuslisten, andererseits vertraut er auf die Überlieferung, wobei nicht näher erläutert wird, ob es sich dabei um eine mündliche oder schriftliche Tradition handelt. Josephus spricht zweifelsfrei von den Steuerlisten, aus denen er seine Angaben ermittelt haben will.

Beide Autoren verweisen also auf - in Form und Inhalt nicht näher bestimmbare - Akten der öffentlichen Verwaltung, die geeignete Informationen zum Inhalt haben. Um einen Eindruck davon zu erhalten, welche Formen eines Zensus seit der Zeit der ersten Ptolemäer für Ägypten angenommen werden darf, sollen im folgenden einzelne Verfahren, ihr Zweck und ihr formaler Aufbau kurz skizziert werden.

Grundsätzlich lassen sich zwei verschiedene Methoden der Zensuserfassung unterscheiden: die persönliche Deklaration durch den Steuerpflichtigen selbst und die Erfassung durch Beamte der königlichen Administration. ${ }^{135}$ Die einzelnen Kategorien von Steuern und deren Bezeichnung waren dagegen vielfältig und gelegentlich finden sich in Hinblick auf Zweck und Zielgruppe auch Überschneidungen. Spezielle Steuern auf Güter oder bestimmte Personenund Berufsgruppen finden in dieser Aufstellung keine Berücksichtigung.

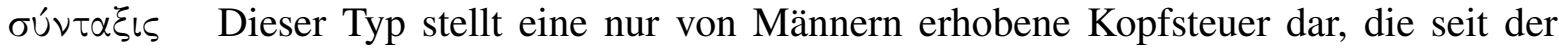
Ptolemäerzeit nachgewiesen ist. ${ }^{136}$

$\lambda \alpha O \gamma p \alpha \varphi i \alpha$ Dies ist die römische Entsprechung der ptolemäischen $\sigma u ́ v \tau \alpha \xi\llcorner\varsigma$, die Augustus im wesentlichen beibehalten hat. ${ }^{137}$ Gelegentlich wird diese Kopfsteuer in den römi-

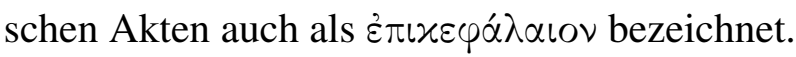

135 THOMPSON (1997: 244).

136 WILCKEN (1912: $171 \mathrm{f}$.).

137 HOMBert und PrÉAUX (1952: 47); WiLCKEN (1912: 186). 


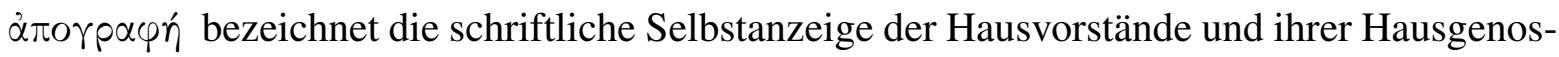
sen, was mit hoher Wahrscheinlichkeit auf jährlicher Basis stattfand. ${ }^{138}$ Besonders interessant ist die $\dot{\alpha} \pi$ r $p \alpha \varphi \hat{n}$ auch deshalb, weil neben den Hausbewohnern auch Objekte (Immobilien und Mobilien) Grundlage der Deklaration waren. Während die Immobilien nicht einer jährlichen Deklarationspflicht unterlagen, sondern durch besondere königliche Verfügung erhoben wurden, bestand insbesondere für die landwirtschaftlichen Güter wie Getreide und Vieh ein Zwang zur jährlichen Auskunft. ${ }^{139}$ Die Angaben waren dabei knapp gehalten und auf das Notwendigste reduziert.

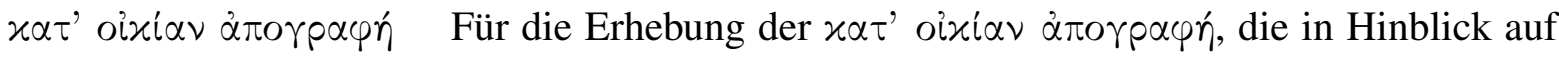
die Steuerpflicht ab dem 14. Lebensjahr in einem Zyklus von 14 Jahren erfolgte, gibt es keine Hinweise vor der römischen Zeit. ${ }^{140}$ Die Datenaufnahme war äußerst detailreich. Erfaßt wurden Hausbesitzer, Hausstand, Beruf, Personalstand, Alter und Informationen über den Wohnraum, was gleichzeitig als Bestandaufnahme der Wohnhäuser diente. ${ }^{141}$ Die Ausführlichkeit der Aufstellung hat Ulrich WILCKEN dazu verleitet,

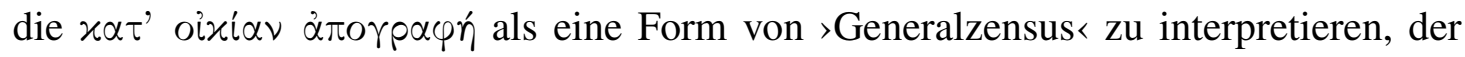
die Feststellung der Gesamtbevölkerung zum Ziel hatte; ein jeder nach seiner Heimat $(i \delta i \alpha) .{ }^{142}$ In der jüngeren Forschung ist dieses Urteil wieder relativiert worden und es hat sich die Interpretation als >Haushaltsbasierter Zensus< etabliert. ${ }^{143}$ Dieser Erklärungsversuch läßt allerdings eine ausreichende Differenzierung zum etablierten Verfahren

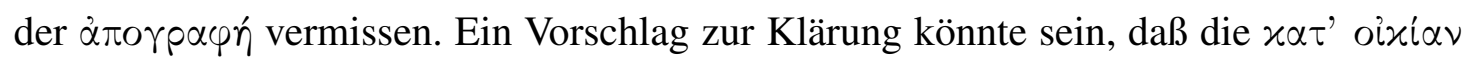
$\dot{\alpha} \pi 0 \curlyvee p \alpha \varphi \hat{n}$ eine ausführliche Ergänzung in längeren Zyklen darstellte, da die $\alpha \dot{\pi} 0 \curlyvee p \alpha \varphi \eta^{\prime}$ zwar aktuellere, dafür aber weniger Daten umfaßte.

Die Befreiung von Kopfsteuern bedeutet dabei nicht notwendigerweise auch die Abwesenheit des betroffenen Personenkreises in den Registern, wie auch Roger BAGNALL und Bruce FRIER betonen. ${ }^{144}$ Wie die römischen Zensusdeklarationen zeigen, wurden Frauen, Kinder und Greise sehr wohl in den amtlichen Registern erfaßt, obwohl sie von Steuern befreit wa-

138 WILCKEN (1912: $173 \mathrm{f}$.).

139 WILCKEN (1912: 175).

140 Hombert und PréAuX (1952: 47 ff.); Wilcken (1912: 193 f.); die von WALlaCe (1938) konstruierte

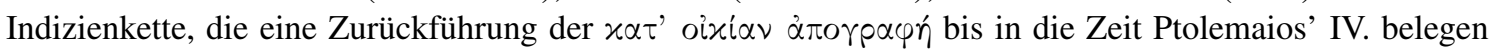
soll, hat sich nicht bewährt.

141 WILCKEN (1912: 194).

142 WILCKEN (1912: 193).

143 Hombert und PrÉaux (1952: 65 f.); Bagnall und Frier (1994: 57).

144 BAGNALL und FRIER (1994: 12). 
ren. Eine weitere Bestätigung findet sich in einem Beispiel für eine demotische Haushaltsliste aus der Zeit nach 251/0 v. Chr., die recht ausführlich die einzelnen Haushalte, ihre Vorstände sowie weitere Familienangehörige nebst Alter dokumentiert. ${ }^{145}$ Dorothy THOMPSON weist ausdrücklich auf die Einzigartigkeit dieses Dokuments hin. ${ }^{146}$ Es ist sowohl eines der ausführlichsten erhalten gebliebenen Register, als auch das einzige Exemplar, das für die Haushaltsangehörigen das Alter vermerkt. Im folgenden ist der von THOMPSON publizierte Textauszug unverändert wiedergegeben: ${ }^{147}$

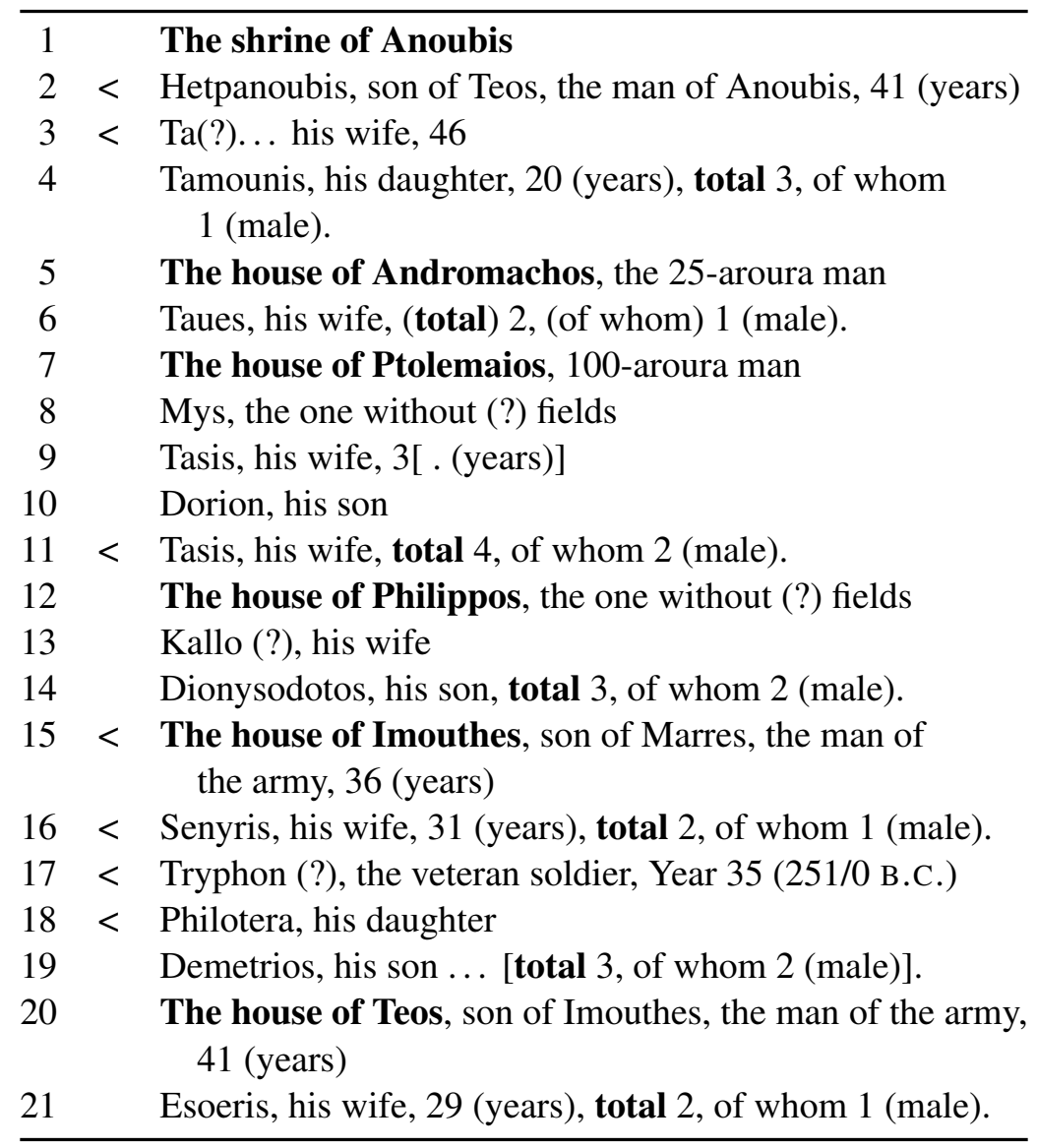

Dieses Beispiel beweist die Existenz detaillierter Bestandsaufnahmen bereits zur Zeit von Ptolemaios II. Dabei steht die Erfassung aller Angehörigen eines Haushalts im Vordergrund,

145 pAsh.dem. 1984.93; THOMPSON (1997: 249 f., Tab. 2); der Papyrus ist unveröffentlicht und wird von ThOMPSOn zusammen mit Willy ClarysSE in der im Druck befindlichen Monographie Counting the People publiziert. Alle folgenden Angaben beziehen sich auf die auszugsweise Übersetzung THOMPSONs.

146 ThOMPSON (1997: 251).

147 Thompson (1997: 249 f. Tab. 2). 
wobei zwischen Männern, Frauen und Kindern nicht unterschieden wird, der Aspekt der Steuerpflicht bestenfalls der expliziten Nennung des Hausherrn zu entnehmen ist und die Angabe einer Gesamtsumme an Einwohnern pro Haushalt eine einfache Aufsummierung der Einwohnerzahl eines Dorfes ermöglicht. Mit diesem Papyrus liegt ein eindeutiger Beleg dafür vor, daß die griechisch-römischen Zensusakten nicht ausschließlich die männliche kopfsteuerpflichtige Bevölkerung erfaßten, sondern Dokumente existierten, die eine Kalkulation der Bevölkerungssituation ermöglichten. Dies ist ein wichtiges Argument gegen die Kritik an den Angaben von Diodor und Josephus.

Fraglich bleibt, ob für die griechisch-römische Zeit tatsächlich sauber zwischen Steuerdeklarationen einerseits und echten Volkszählungen andererseits differenziert werden kann. ${ }^{148}$

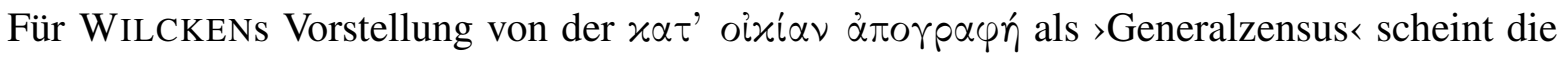
Beweislage aus den antiken Papyri zu dünn, um eine Volkszählung zu konstatieren. Selbst der gängige Vergleich mit dem Zensus in Judäa im biblischen Lukasevangelium (Lk 2,1-5) er-

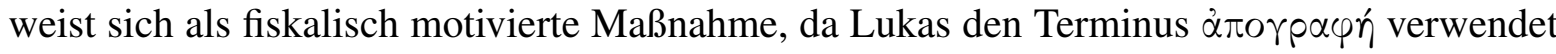
hat. ${ }^{149}$ Doch gerade diese Steuerakten präsentieren sich als detailreiche und ergiebige Quellen, die - bei flächendeckender Erhebung - durchaus das Potential besaßen, Hochrechnungen auf die Gesamtbevölkerung zu ermöglichen.

\section{Zu den pharaonischen Ursprüngen der Verfahren}

Die Überlegungen, daß das griechisch-römische Zensussystem auf pharaonischen Wurzeln basiert, sind nicht neu. Spätestens seit der Publikation der Kahun-Papyri durch Francis Llewellyn GRIFFITH, ${ }^{150}$ die auch Fragmente von Deklarationen der Familienverhältnisse in Wohnhäusern des Mittleren Reiches enthielten, wird auf diese Hinweise Bezug genommen und eine gewisse Tradition bereits bewährter pharaonischer Verfahren in der griechisch-römischen Epoche vermutet. ${ }^{151}$ In der gleichen Weise, wie die römischen Kaiser die bereits von den ersten Ptolemäern etablierten Verfahren zur Erfassung der ägyptischen Bevölkerung für sich nutzten, so wird den Ptolemäern ebenfalls nachgesagt, daß sie sich der langen altägyptischen Erfahrung in der Verwaltungsorganisation bedienten. Eine vollständiger Verfahrenswechsel konnte nicht im Interesse der neuen Herrscher liegen, da Ägypten ein modernes Staatssystem mit einen voll funktionsfähigen Verwaltungsapparat aufweisen konnte. Eine Änderung des sta-

148 Trennung zwischen Steuerdeklarationen und Zensus bereits bei WILCKEN (1912: 174) sowie zuletzt HUSS (2001: 221 Anm. 1).

149 Zum biblischen Zensus vgl. auch UNRUH (2001) sowie PALME (1993).

150 GRIFFITH (1898), siehe auch unten Kap. 3.3.

151 BORCHARDT (1904: 392); kritisch bis ablehnend beurteilt von WILCKEN (1912: 173) und WALLACE (1938: 418 Anm. 4); Zustimmung von HOMBERT und PRÉAUX (1952: 44) sowie BelOCH (1886: 5). 
tus quo war weder sinnvoll noch notwendig. ${ }^{152}$ Bestätigung findet diese Hypothese in einem Hinweis Herodots, nach dessen Worten die jährliche Selbstdeklaration der Bevölkerung keine genuin hellenistische Erfindung ist. Seinen Recherchen zufolge existierte dieses Gesetz bereits lange bevor die Griechen die Herrschaft am Nil übernahmen. Und wenn seinen Worten Glauben geschenkt werden darf, so hatte sich dieses Gesetz in der Praxis derart bewährt, daß die Ptolemäer mit hoher Wahrscheinlichkeit nach der Regierungsübernahme in Ägypten ein dort bereits etabliertes System weitergeführt haben. ${ }^{153}$

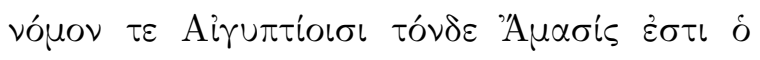

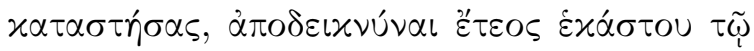

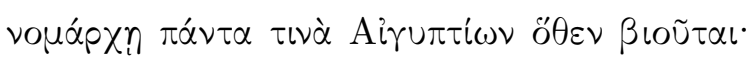

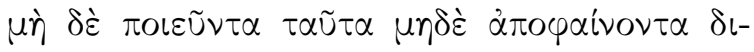

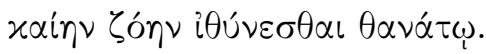

»Amasis gab den Ägyptern auch folgendes Gesetz: Jeder Ägypter mußte jährlich dem Verwalter des Gaues sein Einkommen angeben. Wer das nicht tat und keine rechtmäßigen Einkünfte nachweisen konnte, wurde mit dem Tode bestraft.«

Interessant wird Herodots Bericht für die Frage nach dem Alter eines derartigen Gesetzes oder dessen Vorläufern. Obwohl Herodot nur von der Deklaration der Einkünfte der Personen spricht, gibt es in den griechischen Steuerpapyri Beispiele dafür, daß die Angabe des Einkommens mit anderen Auskunftsverpflichtungen, darunter auch der Personenliste des Haushalts, verbunden war. ${ }^{154}$ Damit dürfte Herodots Hinweis auf die Zeit des Amasis als sehr wahrscheinliches Datierungskriterium für dieses Gesetz zugrundegelegt werden. Zumindest zeigen die Belege, daß die Ptolemäer im Prinzip die von Herodot beschriebene Methode der Steuerdeklaration anwendeten, also auf etwas zurückgegriffen hatten, das vor der Zeit ihrer Herrschaft in Ägypten bereits als gängiges Verfahren bekannt war. Es läßt sich zwar nicht ausschließen, daß diese Information in Herodots Aufzeichnungen zu den inhaltlich schwächer abgesicherten zählt, doch da zwischen der Zeit des Amasis und Herodots Besuch in Ägypten kaum mehr als 120 Jahre vergangen waren, konnte das Wissen um dieses Athener Gesetz und seine Herkunft noch immer im Bewußtsein gewesen sein. Nach Aussage Herodots hat Solon dieses Gesetz von einem Besuch in Ägypten nach Athen mitgebracht. ${ }^{155}$

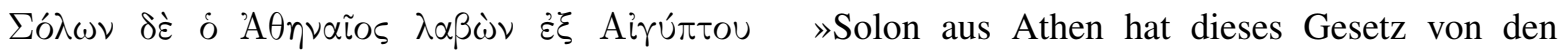

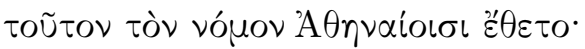

Ägyptern übernommen und in Athen eingeführt.«

Übereinstimmend äußert sich auch Diodor, der vielleicht aber nur - ohne Nennung seiner

152 Zur Verzahnung von altägyptischer Tradition und deren hellenistischer Fortsetzung vgl. allgemein HöLBL (1994: 4, 27 Anm. 72) sowie BRAUnERT (1964: 100).

153 Text und Übersetzung nach FEIX (1963: 356 f.).

154 WilcKen (1912: 173 Anm. 5).

155 Text und Übersetzung nach FEIX (1963: 356 f.). 
Quelle - die Ausführungen Herodots zitiert. ${ }^{156}$ Lediglich Plutarch schreibt die Einführung eines ähnlichen Gesetzes - aufgrund eines schriftlichen Hinweises von Theophrast - nicht Solon, sondern Peisistrates zu. ${ }^{157}$ Der Bericht, daß Amasis nach dem Bürgerkrieg und dem Sieg gegen Apries als Gesetzgeber in Erscheinung trat, wird zwar von Diodor bestätigt, ${ }^{158}$ ansonsten bleibt die Quellenlage aus jener Zeit jedoch fragmentarisch. Es bestehen allenfalls Mutmaßungen darüber, ob und in welchem Maße der ägyptische Verwaltungsapparat Änderungen unterzogen wurde. Alan Lloyd führt dazu zwei Indizien an: ${ }^{159}$ Zum einen weisen einige hieroglyphische Texte aus der Regierungszeit des Amasis darauf hin, daß eine Reorganisation sowohl im Verwaltungs- als auch im religiösen Bereich stattfand. Zum anderen verdrängte die von Amasis als Verwaltungsschrift eingeführte demotische Schrift nach einigen Jahren das sogenannte >Abnormhieratische< als Amtsschrift. LLOYD selbst räumt jedoch ein, daß diese Änderungen im Verwaltungsbereich über ganz Ägypten verstreut gewesen sind und stets nur in regionaler Begrenzung auftraten. Aus den beiden Indizien läßt sich kaum entnehmen, daß Amasis auch landesweit Umbaumaßnahmen in der Verwaltung durch die Einführung neuer Gesetze auf den Weg gebracht hat. Deshalb geht LLOYD in seiner Untersuchung davon aus, daß die Beschreibung des Amasis als Gesetzgeber eher darauf beruht, daß dieser nach dem Bürgerkrieg die Zustände, die vor dem Krieg herrschten, wieder etablierte, also eine Form von Restauration betrieb. ${ }^{160}$ Diese Annahme läßt sich mit weiteren Argumenten stützen. Folgt man einem Hinweis von Girgis MатTHA, so korrespondiert das demotische Wort $h p$ mit dem griechischen vóuos und bedeutet in beiden Fällen ursprünglich nicht >(kodifiziertes) Gesetz<, sondern >Sitte, Gewohnheit, Konvention $<.{ }^{161}$ Man könnte Herodots Aussage zur Gesetzgebung des Amasis also auch dahingehend auslegen, daß Amasis nach den Wirren des Bürgerkriegs die alten Gewohnheiten wieder etablierte, was auch für die Gesetze gilt, die vor dem Ausbruch des Bürgerkrieges in Kraft waren. Für die Überlegungen zum Steuersystem im pharaonischen Ägypten würde dieser Sachverhalt darauf hinweisen, daß die von Herodot geschilderten Verfahren bereits vor dem Bürgerkrieg in Gebrauch gewesen sein könnten. Damit wäre der Regierungsantritt des Amasis im Jahr 570 v. Chr. ein terminus ante quem für die Existenz eines Gesetzes zur Erhebung von Abgaben und damit auch zur Erfassung der Bevölkerung.

156 Diodor I 77,5.

157 Plutarch, Solon 31,2; zur Diskussion der Textstelle bei Herodot vgl. auch LLOYD (1988: Bd. 3, 220 f.).

158 Diodor I 95,1.

159 LLOYD (1988: Bd. 3, 220 f.).

160 LLOYD (1988: ebenda).

161 MATTHA (1975: xii); zu $h p$ vgl. zuletzt auch BonTTy (1997). 


\subsection{Die historische Überlieferung von Diodor und Josephus}

\subsubsection{Das Werk Diodors in der Kritik}

Über seine Zeit in Ägypten gibt Diodor in seinen Büchern ausführlich Auskunft. Laut eigener Aussage hat er selbst Ägypten bereist ${ }^{162}$ und vielleicht sogar einige Zeit dort gelebt. ${ }^{163}$ Man darf ihm daher unterstellen, daß er wenigstens ein Minimum an eigener Anschauung in seine Ausführungen zum Alten Ägypten einbringen konnte. Der Aufenthalt fand nach Diodors Aussage während der 180. Olympiade statt, also zwischen 60 und 56 v. Chr., der Zeit des Königs Ptolemaios XII. ${ }^{164}$ Welche Orte Diodor in Ägypten aufsuchte, ist nicht mehr zweifelsfrei feststellbar. Man kann sicher davon ausgehen, daß er in Alexandria ägyptischen Boden betrat und dort auch seinen zeitweiligen Wohnsitz nahm, nachdem er sich von Sizilien aus nach Ägypten eingeschifft hatte. Anne BURTON geht aufgrund von Diodors Beschreibung eines Lynchmordes an einem Römer, der versehentlich eine Katze getötet hatte, davon aus, daß Diodor sich auch in Bubastis als der Heimatstadt der Katzengöttin Bastet aufhielt. ${ }^{165}$ Doch aufgrund der allgemeineren Ausführungen Herodots, ${ }^{166}$ der in Ägypten eine Todesstrafe für alle Personen bestätigt, die - versehentlich oder nicht - ein >heiliges< Tier töten, und der Anmerkung Günther HöLBLs, der in dem von Diodor berichteten Vorfall einen willkommenen Anlaß für die Ägypter sieht, einen verhaßten Römer zu töten, ${ }^{167}$ ist die Evidenz für einen Aufenthalt Diodors speziell in Bubastis wieder relativiert. Obwohl auch vorsichtiger Zweifel angebracht wäre, ob der beschriebene Vorfall eines Lynchmordes auf >offener Straße < in Alexandria stattfinden konnte, muß davon ausgegangen werden, daß Alexandria als Metropole und >Schmelztiegel verschiedenster Kulturkreise auch entsprechende Vorstädte mit ethnischen Ghettos aufwies, in denen sich die Szene abgespielt haben könnte.

\section{Zur Bevölkerungsfluktuation}

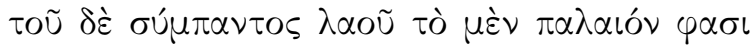

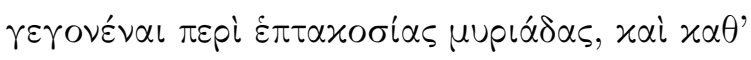

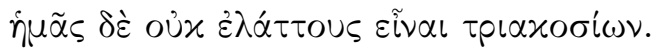

»Die Bevölkerung, so sagt man, war früher über sieben Millionen und ihre Zahl ist in unserer Zeit nicht weniger als drei Millionen.«

162 Diodor I 44,1.

163 Diodor I 83,9.

164 SACKS (1990: 165) und RAthbone (1990: 104) legen sich auf 59 v. Chr. fest.

165 Diodor I 83,8; BURTON (1972: 39).

166 Herodot II 65,5

167 HÖLBL (1994: 199). 
Besonders diese Textstelle Diodors ist seit dem Beginn ägyptologischer demographischer Untersuchungen unter den Forschern heftig umstritten. Grund für die Auseinandersetzung ist eine Abweichung einer einzelnen Handschrift aus dem Überlieferungskorpus. Diese eine

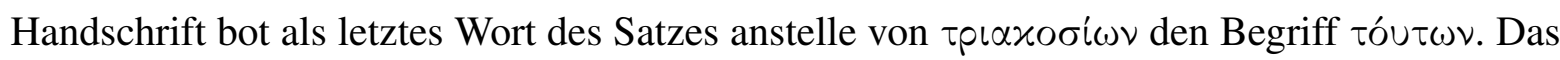
Ergebnis war eine nachhaltige Sinnveränderung der Textstelle, denn die Aussage lautete nun nicht mehr, daß die Population in früheren Zeiten höher war als zum Zeitpunkt der Feststellung, sondern daß sich an der (hohen) Bevölkerungszahl in der Vergangenheit nichts Wesentliches mehr geändert habe. Neben dem Althistoriker WILCKEN ${ }^{168}$ plädierten im 19. Jahrhundert auch Ludwig DINDORF und Immanuel BEKKER ${ }^{169}$ für die Emendation und finden bis in die heutige Zeit Anhänger. ${ }^{170}$ Die Gründe dafür sind kaum nachvollziehbar, gilt doch sonst in der Textkritik eher das Prinzip, daß übereinstimmende Quellen mit hoher Wahrscheinlichkeit die besseren Lesungen boten, als eine singuläre Alternative. Möglicherweise stand diese jedoch der damals vorherrschenden Meinung über die Bevölkerung Ägptens näher, so daß man die Version dieses einzelnen Manuskripts favorisierte, wenngleich sie nicht unumstritten war.

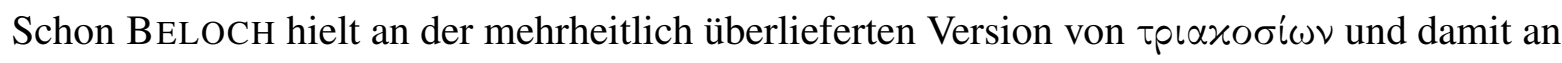
der Angabe von 3 Millionen Menschen fest. ${ }^{171}$ Und auch in jüngerer Zeit ist der Widerstand gegen diese Emendation wieder gewachsen. ${ }^{172}$

Eine weitere Zahlenangabe zur Bevölkerung Ägyptens übermittelt uns Diodor in seiner Beschreibung der Stadt Alexandria im 17. Buch. Dort heißt es zur Situation Alexandrias:

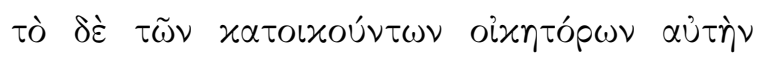

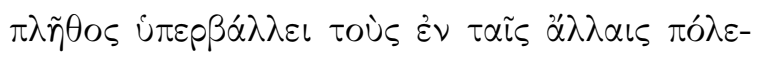

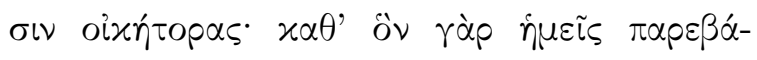

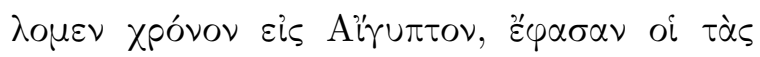

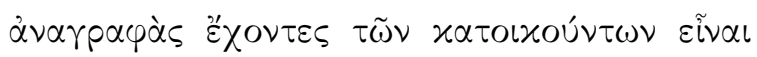

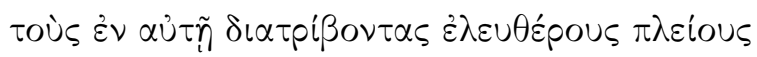
$\tau \tilde{\omega} \nu \tau \rho\llcorner\alpha ́ x o \nu \tau \alpha \mu u \rho ı \alpha ́ \alpha \omega \nu, \ldots$
»Die Zahl ihrer [scil. Alexandria] Bewohner überragt alle anderen Städte. Zum Zeitpunkt, als wir in Ägypten waren, sagten diejenigen, die für die Zensuslisten zuständig waren, daß die Zahl ihrer freien Einwohner über 300000 war.«

Bereits Hume wies darauf hin, daß der Text von $\varepsilon \dot{\lambda} \varepsilon \dot{u} \theta \varepsilon \rho \circ$ anstelle von $\pi 0 \lambda \tilde{\imath} \tau \alpha \iota$ spricht und nur letztere allein die erwachsenen Männer mit Bürgerrecht meint. Falls diese Interpreta-

168 WiLCKEN (1899: 489 f.).

169 BELOCH (1886: 255 f.).

170 Wachsmuth (1903); OldFAther (1968: 104 f.); RostovtzeFF (1984: 907 Anm. 73); Nothers (1992: 59).

171 BELOCH (1886: ebenda).

172 BAgnall und Frier (1994: 53); besonders von RATHBone (1990: 104) stammt harsche Kritik an der vermeintlich korrigierten Lesung. 
tion richtig sein sollte, hat Diodor vielleicht alle freien Einwohner Alexandrias einschließlich der Frauen in die Gesamtzahl miteinbezogen. ${ }^{173}$ Damit blieben aber immer noch die Sklaven und Unfreien in der Kalkulation Diodors unberücksichtigt. Obgleich દ่ $\lambda \varepsilon u ́ \theta \varepsilon \rho \circ \iota$ keine offizielle Bezeichnung für eine bestimmte Bevölkerungsgruppe darstellt, besteht mittlerweile eine gewisse Einigkeit darüber, daß Diodor damit nicht auf die gesamte Bevölkerung Alexandrias Bezug genommen hat. Welcher Teil der Einwohner damit letztlich gemeint war, ist jedoch bis heute nicht schlüssig zu beantworten. ${ }^{174}$ Die Arbeiten der jüngeren Forschung favorisieren allerdings eine Größenordung von rund 500000 bis 600000 Menschen, die ab der zweiten Hälfte des 1. Jahrhunderts v. Chr. in Alexandria lebten.

\section{Diodors Quellen}

Eine weitere Problematik stellt sich bei der Frage, ob die Auskunft Diodors über die Gesamtbevölkerung Ägyptens aus älteren Quellen entnommen ist, oder aus eigenen Recherchen stammt. Aufgrund der Art des Materials, das von Diodor aus verschiedenen anderen Quellen kompiliert wurde, ist für seine Daten ein etwas früheres Datum anzunehmen. Dies gilt besonders für das erste Buch Diodors, das in weiten Teilen auf dem Werk des Hekataios von Abdera, einem Zeitgenossen von Ptolemaios I., basieren soll. Eine Aufschlüsselung von Diodors Überlieferung nach Fremdübernahmen und >Eigenleistung < gestaltet sich indes schwierig und die Diskussion darüber ist in der philologischen Forschung anscheinend noch nicht abgeschlossen. ${ }^{175}$

Bezüglich der Angaben Diodors zur Gesamtbevölkerung Ägyptens scheint nach Meinung von BURTON die Beweislage besonders zweifelhaft. ${ }^{176}$ BURTON befaßte sich erneut mit den Textfragmenten, die als Begründung für Hekataios als Urheber der Daten herangezogen wurden. Dabei handelt es sich zum einen um eine Stelle aus Porphyrs Quaestiones Homericae, zum anderen eine fast identische Aussage im Werk des Stephanus Byzantinus unter dem Stich-

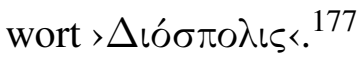

173 Hume (1964: 429 Anm. 6).

174 Vgl. dazu BagNaLL und FrIER (1994: 54); siehe auch unten S. 55 Anm. 202.

175 So schrieb noch in jüngerer Zeit NOTHERS (1992: 289): »Die Zahlen stammen von Hekataios, Diodor hat sie nur oberflächlich aktualisiert.«, ohne jedoch näher auf die unterstellten Aktualisierungen einzugehen. Auch MÜLLER (1997: 31) geht von einer geschlossenen Übernahme aus dem Werk des Hekataios aus.

176 BURTON (1972: 8 f.).

177 >Fragmente Griechischer Historiker 264, F19a/b (JACOBY 1957); auch RATHBONE (1990: 104 Anm. 3).

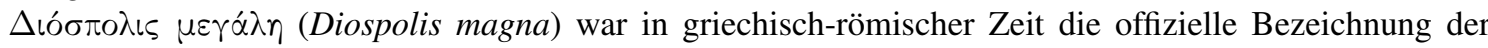
oberägyptischen Stadt Theben. 


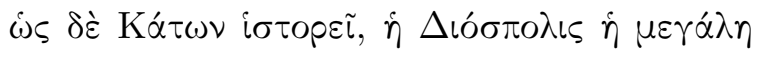

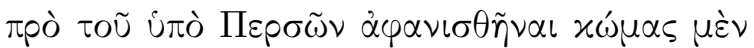

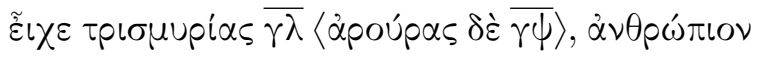

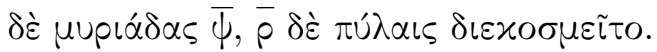

»Wie Katon sagt, hatte Diospolis vor seiner Zerstörung durch die Perser 33030 Siedlungen, 3700 (?) Aruren und 7 Millionen Menschen, unterteilt durch 100 Tore.«

Die Verbindung dieser beiden Textfragmente zur Urheberschaft des Hekataios stellte Felix JACOB Y, der Herausgeber der >Fragmente griechischer Historiker< (F Gr Hist), her. Er identi-

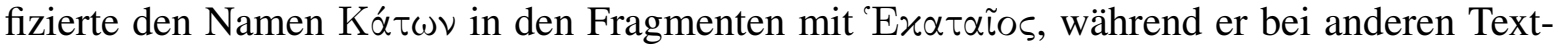
fragmenten dagegen eine Gleichsetzung von Kó $\tau \omega \nu$ mit Ká $\sigma \tau \omega \rho$ präferierte. ${ }^{178}$ Die Tatsache einer von zwei verschiedenen Personen nahezu identisch überlieferten Textfassung ließ BURTON die Vermutung aussprechen, daß es sozusagen eine unbekannte gemeinsame Quelle $>Q$ für die Informationen gab, aus der sich alle Autoren unabhängig voneinander bedient hatten. Die Überlegungen BURTONs konnten sich jedoch nicht durchsetzen. Trotz Zweifeln an der eindeutigen Zuweisung zu Hekataios scheint auch die neue Interpretation des Namens kaum eindeutiger zu sein. Die jüngere Forschung zeigt sich nach wie vor hinsichtlich der These von Hekataios als Quelle für Diodor gespalten. ${ }^{179}$ Die Diskussion über die Quelle, aus der sich Diodor bediente, hat einerseits chronologische Konsequenzen für den Gültigkeitszeitraum der gebotenen Informationen, andererseits stellt sich die Frage nach der Qualität und Vertrauenswürdigkeit von Diodors Zusammenstellung. Zur Problematik der Chronologie könnte auch die explizite Erwähnung des >Ptolemaios, Sohn des Lagos< (Ptolemaios I.) ein Indiz für eine ältere Quelle darstellen, die allerdings keine zwangsläufige Urheberschaft des Hekataios bedeuten muß, sondern möglicherweise von einem anderen Zeitgenossen Ptolemaios' I. stammt. Damit wäre der Vorschlag BURTONs für eine gemeinsame ältere Quelle wieder wahrscheinlicher geworden.

Anders sieht die Situation bei Diodors Angaben zur Stadt Alexandria aus. Hier erwähnte der Verfasser ausdrücklich, woher seine Erkenntnisse stammen: Er erhielt die Auskünfte von Beamten der städtischen Verwaltung, die für den Zensus zuständig waren. Eine öffentliche Einsichtnahme in die Steuerdokumente durch einen Bürger muß daraus nicht notwendigerweise geschlossen werden. ${ }^{180}$ Diodor könnte sich seine Informationen über Gespräche mit den Beamten geholt haben. Die knappen Angaben in seinen Texten deuten darauf hin, daß er

178 BURTON (1972: 8 f.).

179 SACKS (1990: 91) zweifelt den Bezug nicht an, RATHBONE (1990: 104 f.) dagegen lehnt die HekataiosHypothese ab und folgt im wesentlichen den von BURTON vorgebrachten Einwänden.

180 Siehe zur ähnlich gelagerten Diskussion um die Quellen von Josephus auch unten Kap. 2.5.2. 
nicht selbst Einsicht in die Zensusdokumente genommen hat, sondern lediglich von offizieller Seite einige Auskünfte erringen konnte, die er dann in seinen Beschreibungen verarbeitet hat.

\section{Das Städteproblem}

Neben der Problematik einer korrekten chronologischen Einbettung von Diodors Überlieferung zur Bevölkerungsgröße des Alten Ägypten ist auch seine Angabe zur Anzahl der ägyptischen Siedlungen ein bis heute nicht zufriedenstellend gelöster Streitpunkt. Die häufigste Lesung der Ausführungen Diodors lautet:

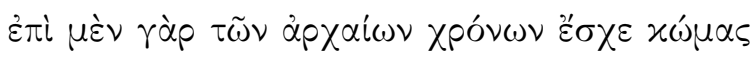

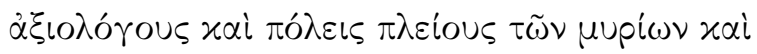

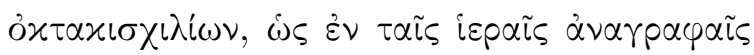

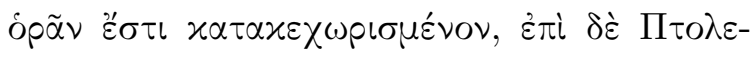

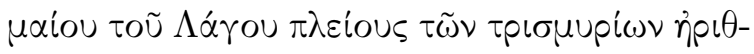

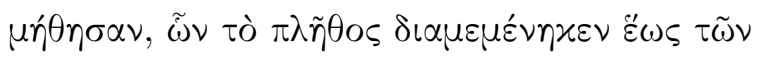

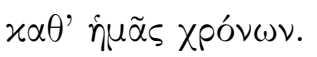

»In alter Zeit hatte es [scil. Ägypten] über 18000 wichtige Dörfer und Städte, wie man aus den heiligen Schriften ersehen kann, während unter Ptolemaios, Sohn des Lagos, diese auf über 30000 anwuchsen und diese hohe Zahl bis zur heutigen Zeit fortwährte.«

Nach dieser Beschreibung hat es in der Zeit des ersten Ptolemäers einen sprunghaften Anstieg der Siedlungszahl gegeben. Eine Expansion um 150\% auf ungefähr 30000 Siedlungsplätze läßt sich indes auch mit den optimistischen ökonomischen Entwicklungen während der frühen Ptolemäerzeit nicht in Einklang bringen. Daher wies bereits BELOCH auf die besseren Lesungen anderer Codices hin, die statt der üblichen 30000 nur 3000 Städte verzeichneten. ${ }^{181}$ Auch von Dominic RATHBONE wird die hohe Zahl bestritten, der darin ein > Textmanöver < der neueren Forschung sieht, die Diodor nicht unterstellen will, er habe in Zeiten wirtschaftlichen Wachstums unter den frühen Ptolemäern keinen Bevölkerungsrückgang für Ägypten konstatieren können oder wollen. ${ }^{182}$ Vielmehr deutet RATHBONE die Aussage Diodors dahingehend, daß dieser auf den Mangel an Arbeitskraft hinweisen wollte, der die Ptolemäer nicht in die Lage versetzte, mit den Bauleistungen der Pharaonenzeit zu konkurrieren.

Die Befürworter der höheren Zahl von 30000 Siedlungen zogen als Argument gerne eine Parallele aus dem fragmentarisch überlieferten Werk von Theokrit heran. ${ }^{183}$ In dem stark zerstörten Textfragment, einer Eulogie auf den König Ptolemaios, findet sich auch eine Angabe zu der Zahl von Städten in Ägypten. ${ }^{184}$

181 BELOCH (1886: 255 f.).

182 RATHBONE (1990: 104 f.).

183 Zum Beispiel OldFather (1968: 103 Anm. 2) und Nothers (1992: 289).

184 Text und Übersetzung nach FRITZ (1970: 122 ff.). 


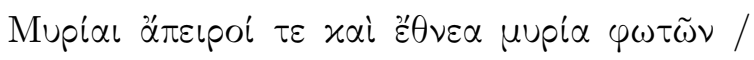

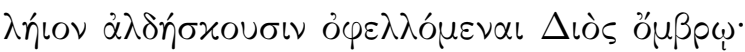

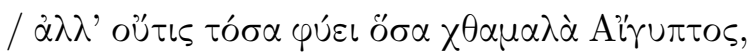

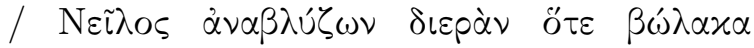

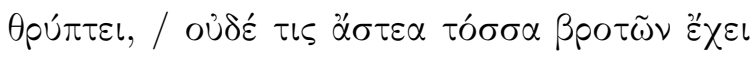

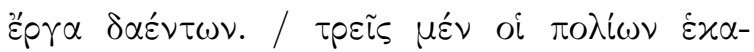

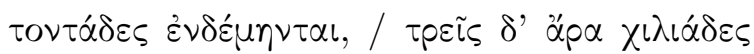

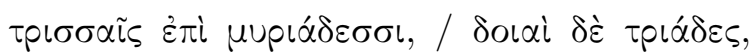

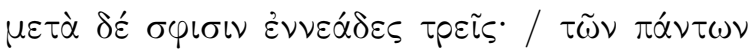

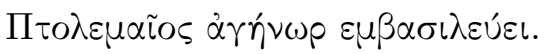

»Zahllose Länder der Erde und zahllose Menschengeschlechter / Bringen, vom Regen des Himmels begünstigt, die Saat zum Gedeihen. / Keines jedoch bringt so viel hervor wie die Niedrung Ägyptens, / Wenn der schwellende Nil den Boden durchfeuchtet und lockert, / Keines auch hat so viele Städte mit Menschen, die Arbeit erlernten. / Denn es sind drei mal einhundert Städte erbaut in dem Lande, / Drei mal tausend und drei mal zehntausend kommen dazu noch, / Außerdem zwei mal drei sowie drei mal neun noch als letztes / Über sie alle ist König der tapfere Held Ptolemaios.«

Theokrit, ein Zeitgenosse und Günstling Ptolemaios' II, war allerdings eher ein Literat als ein Geschichtsschreiber. Die in diesem Zitat enthaltene spielerische Zahl wurde bisher mehrheitlich als dichterische Gestaltung bewertet und damit ihre Verwertbarkeit in Zweifel gezogen. ${ }^{185}$ Auch vermag der Vergleich zum Textfragment F Gr Hist 19a nicht zu überzeugen, das - wie bereits oben gezeigt - von 33030 Siedlungen vor der Perserzeit berichtet. Da die hier $\mathrm{zu}$ vergleichenden Textstellen jeweils eindeutig den Zeitpunkt, auf den sie Bezug nehmen, datieren, ist ein Zusammenhang zwischen Diodor und Porphyrs Ausführungen zu $\Delta$ เó $\sigma \pi \mathrm{\lambda} \iota \varsigma$ unwahrscheinlich.

Für die niedrigere Zahl an Siedlungen gibt es dagegen einige gute Gründe, die jedoch letzlich auch keine zweifelsfreie Erklärung bieten können. Zum einen wäre das der Hinweis Diodors, daß die Zahl an Siedlungen bis zum Zeitpunkt seines Ägyptenbesuches gleich blieb. Diese Beobachtung kann mit dem Trend einer verstärkten Urbanisierung erklärt werden, die eine Landflucht der Bevölkerung und ein Wachstum der regionalen Metropolen bedeutete und im ausgehenden 1. Jahrhundert v. Chr. bereits deutliche Auswirkungen auf die ägyptische Siedlungsstruktur gehabt haben dürfte. ${ }^{186}$ Diodors Aussage verrät nichts über die Schwankungen in der Entwicklung, die Ägypten seit den ersten Ptolemäern genommen hatte. Ein möglicher Anstieg der Siedlungsgründungen unter dem Eindruck der allgemeinen politischen und ökonomischen Verbesserungen während der Regierungszeit Ptolemaios' I. und II., der erst durch den Beginn der Urbanisierung wieder umgekehrt wurde, lag außerhalb von Diodors Be-

185 FRITZ (1970: 301 f.) kommentiert die Zahl 33333 als »Heilige Dreizahl« und »Zahlenmystik«.

$186 \mathrm{Zu}$ den Anzeichen für eine zunehmende Verschiebung zugunsten der Metropolen ab er zweiten Hälfte der Ptolemäerzeit vgl. auch BRAUnERT (1964: 86 ff.). 
obachtungsmöglichkeiten, so daß er seine Kenntnisse um die alten Verhältnisse in direkten Zusammenhang zur Situation in seiner eigenen Zeit setzte. Diese Interpretation von Diodors Ausführungen bietet damit zugleich auch Widerspruchsfreiheit zu der Entwicklung der ägyptischen Population, da nicht gleichzeitig die Bevölkerungszahl um etwa die Hälfte gesunken sein kann, die Anzahl der Siedlungen sich aber im gleichen Zeitraum mehr als verdoppelt hat.

Diodors Angabe von 18000 Dörfern und Städten in >alter Zeit< findet eine annähernde Bestätigung bei Herodot, der die Regierungszeit des Königs Amasis gegen Ende der 26. Dynastie beschreibt. ${ }^{187}$

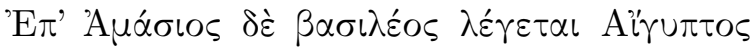

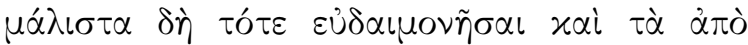

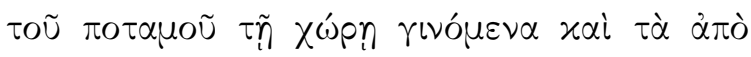

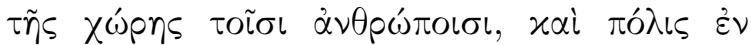

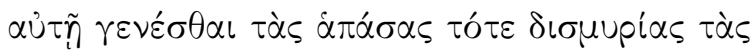
oixeouévas.
»Zur Zeit des Königs Amasis soll sich Ägypten in einer besonders glücklichen Lage befunden haben: Der Strom beschenkte das Land und das Land die Menschen. Damals soll es insgesamt 20000 Städte gegeben haben, die dicht bewohnt waren.«

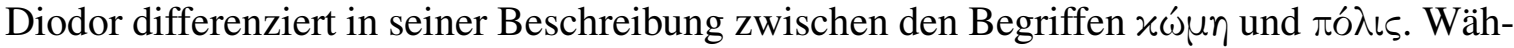
rend ersterer im wesentlichen als >unbefestigte Siedlung< aufgefaßt wird und in der Übersetzung meist mit >Dorf< wiedergegeben wird, bedeutet letzterer als Gegensatzpaar nicht nur im engeren Sinne - die klassisch griechische Polis mit ihren politischen und sozialen Implikationen, sondern auch einfach nur eine befestigte Stadt. ${ }^{188}$ Dagegen bezeichnet Herodot die Siedlungen einheitlich als $\pi o ́ \lambda \varepsilon\llcorner\varsigma$, was allerdings mit einer Unterspezifizierung erklärt werden kann, die Herodot die Dörfer und Städte unter einem einzigen Begriff zusammenfassen ließ. Die numerische Differenz zu den 18000 Siedlungen Diodors macht gerade einmal $11 \%$ aus, was für eine allgemeine Dimensionsangabe völlig ausreicht. Die Überschneidung der beiden Angaben erlaubt es auch, durch Herodots exakte Zeitangabe darauf zu schließen, daß Diodor

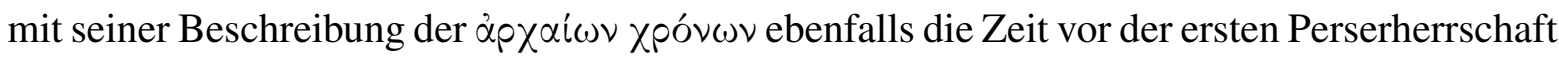
gemeint hat.

\section{Methodenkritik und der Umgang mit historischen Quellen}

Die Art und Weise, wie in den vorangegangenen Abschnitten mit dem antiken Textmaterial umgegangen wurde, bietet durchaus eine breite Angriffsfläche für Kritik. Die Probleme, die mit einem faktenbezogenen Lesen klassischer Quellen einhergehen, sind durchaus bekannt und werden durch die bisher getätigte Form des Umgangs auch kaum gemindert. Die Mo-

187 Text und Übersetzung nach FEIX (1963: 356 f.); vgl. auch LLOYD (1988: 219 f.).

188 So auch Huss (2001: 221 Anm. 2). 
tivation für einen erneuten Versuch rührt aus zwei Überlegungen. Zum einen ist es relativ gesichert, daß die frühen Historiker bei der Kompilation ihrer Werke durchaus kritisch an die ihnen zur Verfügung stehenden Quellen herangingen. Die Aufnahme eines Informationsfragments in das Manuskript erfolgte dann, wenn der Autor dessen Inhalt innerhalb seines Weltbildes für >glaubwürdig< hielt. Diese Beurteilung entzieht sich zwar völlig einer objektiven Klassifizierung nach > wahr $<$ oder >falsch $<$, bietet aber immerhin die Gewissheit, daß die zu beurteilende Situation in ihrem historischen Kontext möglich war. Als weiteres Argument für ihre kritische Sichtweise können die häufig anzutreffenden Kommentare gelten, in denen die Autoren ihre Zweifel oder ihre Ungläubigkeit hinsichtlich bestimmter Sachverhalte zum Ausdruck bringen, die ihnen von dritter Seite berichtet wurden. Dabei dürfte diese Form von $>$ Gewährsleuten<, auf die bereits Herodot vielfach zurückgriff, die häufigste Quelle für diejenigen Auskünfte sein, die Diodor nicht offenkundig von anderen Autoren abschrieb oder als Augenzeuge miterlebte. Die zweite Überlegung bezieht sich auf die Art der Auswertung antiker Quellen. Die gebotenen Informationen sind nicht per se die gewünschte historische Aussage, die eine zweifelsfreie und eindeutige Beschreibung eines historischen Sachverhalts beschreibt. Stattdessen müssen sich diese Informationen im Kontext mit anderen Quellen als plausibel erweisen. Zu diesem Zweck sollen in Kap. 2.6 die Optionen ausgetestet werden, in denen die Aussagen Diodors zur ägyptischen Bevölkerung zum historischen Gesamtbild passen.

Ein anderer und nicht minder schwerer Einwand bringt der Verweis auf die >alten Zeiten< mit sich, die in der vorliegenden Arbeit auf die Regierungszeit des Königs Amasis datiert werden. Ein solcher Rückgriff, der insbesondere auf eine beinahe legendäre politische Figur Bezug nimmt, wird in der Regel hinsichtlich der tatsächlich zu jener Zeit erbrachten Leistungen von der Forschung mit Argwohn betrachtet. Zu groß ist das Risiko, daß Herodot wie auch Diodor in ihren Aussagen einen politischen Mythos widerspiegeln, in dem die Ereignisse historisch verklärt worden sind. Dem stehen jedoch die Beobachtungen LLOYDs entgegen, der für die Zeit der 26. Dynastie deutliche Hinweise auf Veränderungen in der ägyptischen Administration feststellen konnte, die sich mit den Aussagen Herodots und Diodors in Einklang bringen lassen. Die Blütezeit der 26. Dynastie ist mehr als nur ein Mythos - sie ist aus den schriftlichen Quellen gut belegt und basiert im wesentlichen auf der Friedens- und Handelspolitk jener Zeit, die für einen kurzen Zeitraum nochmals Sicherheit und Wohlstand für die Bewohner Ägyptens bedeutete. Daß dies in der Erinnerung späterer Generationen einen positiven Nachklang fand, dürfte dabei kaum überraschen. 


\subsubsection{Der Bericht des Josephus}

Die Bücher des Josephus sind ungefähr einhundert Jahre nach Diodor entstanden. Josephus war jüdischer Abstammung und seine Eltern gehörten der Jerusalemer Oberschicht an. Über diverse Umwege war sein Schicksal mit dem römischen Kaiser Vespasian verbunden, den er auch auf einer Ägyptenreise begleitete. Aus dieser Verbindung zu den Römern rührt auch sein Namenszusatz >Flavius< - in Anlehnung an das flavische Herrscherhaus - her. ${ }^{189}$ Sein Wechsel in das römische Leben und seine durch den römischen Kaiser ermöglichten Privilegien als Bürger in Rom haben die stark pro-römisch gefärbten Aussagen in seinen Büchern immer wieder in das Zentrum der Kritik rücken lassen. ${ }^{190}$ Übertreibungen, Verherrlichungen und teilweise gar patriotisch gefärbte Stellungnahmen haben den Günstling des Kaisers in ein schlechtes Licht gerückt und seine Qualität als zuverlässiger Berichterstatter in Frage gestellt, obgleich er den unbestreitbaren Vorteil eines Augenzeugen für sich in Anspruch nehmen konnte. Die für die demographische Forschung Ägyptens wichtige Textstelle findet sich in seinem Werk über den Jüdischen Krieg (De Bello Judaico), das aus dem Zeitraum um das Jahr 75 n. Chr. stammt: ${ }^{191}$

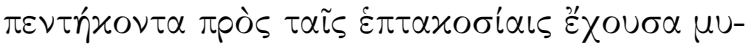

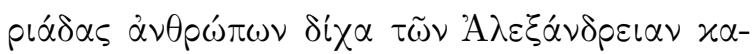

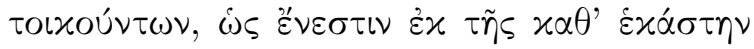

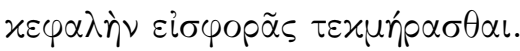

»... [Ägypten] hat eine Einwohnerschaft von 7 1/2 Millionen Menschen ohne die Bevölkerung Alexandriens, wie man aus der Kopfsteuer berechnen kann.«

In der Diskussion dieser Textstelle attestierte man Josephus, er sei »notorisch unzuverlässig in statistischen Dingen ${ }^{192}$, und daher sei seine Überlieferung »völlig wertlos ${ }^{193}$. Und noch in jüngerer Zeit war man davon überzeugt »the figure of 7.5 million is fiction ${ }^{194}$ oder zumindest $»$ of doubtful provenance ${ }^{195}$. Demgegenüber standen vereinzelt Feststellungen, Josephus' Angaben seien »zur Zeit noch nicht verwertbar « ${ }^{196}$ oder »von der Wahrheit nicht allzuweit [... ] entfernt « ${ }^{197}$, die immerhin die Möglichkeit anderer Herangehensweisen offenhielten. Auf solche Weise dem Säurebad des Skeptizismus ausgesetzt, blieb von Josephus' Überlieferung als demographisch verwertbare Quelle nicht viel übrig.

189 MICHEL und BAUERNFEIND (1959: xiii).

190 MiCHEL und BAUERNFEIND (1959: xx f.).

191 Text und Übersetzung nach MiCHEL und BAUERNFEIND (1959: $258 \mathrm{f}$.).

192 BELOCH (1886: 258).

193 WiLCKEN (1899: 239).

194 RATHBONE (1990: 106).

195 BAGNALL und FRIER (1994: 53).

196 WACHSMUTH (1903: 273).

197 WiLCKEN (1899: 491) - im scharfen Kontrast zu seiner mehrfach geäußerten Kritik. 


\section{Quellenbeschaffung und Quellenverwertung}

Zentraler Kritikpunkt an Josephus' Überlieferung ist dessen Quellenangabe, aus der er seine Erkenntnisse gewonnen haben will. Schon WILCKEN bemängelte an dem Verfahren, daß mittels Division der Steuersumme durch den Kopfbetrag die Gesamtzahl der Bevölkerung nicht gefunden werden könne und führte rein pragmatische Einwände dagegen an. ${ }^{198}$ Das am schwersten wiegende Gegenargument ist die in den einzelnen Kommunen erhobene Kopfsteuer, die von Ort zu Ort eine unterschiedliche Höhe aufweisen konnte, in den meisten Dörfern jedoch bei etwa 10 Drachmen pro Jahr lag. Dieser Betrag wurde von allen Steuerpflichtigen der jeweiligen Kommune einheitlich erhoben und war nicht abhängig vom Vermögen.

Ein anderer Einwand betraf die Ausnahmen von der Steuerpflicht für Kinder, Greise und anderen Gruppen, die nicht zu den Bürgern Alexandrias ('A $\lambda \varepsilon \xi \alpha \nu \delta \rho \varepsilon \tilde{\varsigma} \varsigma$ ) gehörten. In jedem Fall kann aufgrund der Dimensionierung von $7 \frac{1}{2}$ Millionen Menschen nur die Gesamtheit der Bevölkerung gemeint sein, wie es bereits für die Angaben Diodors bestimmt werden konnte. Wenn also diese Zahl tatsächlich die Gesamtheit der ägyptischen Bevölkerung beziffern soll, dann konnte sie kaum mittels Division aus der Kopfsteuer ermittelt worden sein, da zu viele Bevölkerungsteile fehlen. Zwei relevante Informationen müssen also nach Meinung der älteren Forschung Josephus zugänglich gewesen sein: Der Steuersatz $w$, den jeder Steuerpflichtige zu zahlen hatte sowie die Gesamtsumme $\Sigma$ der Steuereinnahme eines Jahres. Josephus hat dann die Kopfsteuer für jeden Einwohner Ägyptens einheitlich veranschlagt und stellte zwangsläufig die folgende Rechnung auf:

$$
\frac{\Sigma}{w}=7500000
$$

Gleich mehrere Fehler müssen in diesem Fall Josephus unterstellt werden: die Unkenntnis über unterschiedliche Steuersätze an verschiedenen Orten Ägyptens und die Steuerbefreiung für bestimmte Gruppen oder aber geringe Kenntnisse der Mathematik. Im letzteren Fall müßte man (hypothetisch) noch Frauen, Kinder und Greise zu den 7 1/2 Millionen >Steuerpflichtigen $<$ hinzurechnen. Das allerdings würde eine Gesamtzahl der Bevölkerung weit jenseits der 10 Millionen zur Folge haben. Wahrscheinlicher ist, daß Josephus selbst bereits eine $>$ Schätzung < aufgrund der ihm vorliegenden Akten vorgenommen hat. Als Grundlage könnten ihm

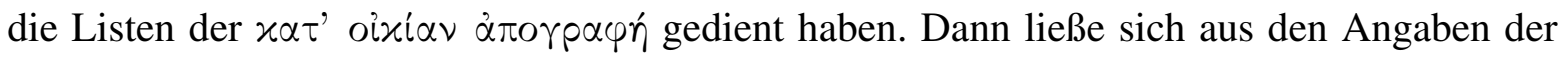
steuerpflichtigen Haushaltsvorstände und ihrer Mitbewohner auf eine ungefähre Gesamtzahl der Bevölkerung schließen. Der Status Alexandrias muß dabei allerdings gesondert betrachtet

198 WiLCKEN (1899: 238 f.). 
werden. Diese Erklärung für den Zugang zu den Daten bleibt unbewiesen, ist aber grundsätzlich denkbar, weil die Zensusdokumente für Bürger zur Einsichtnahme öffentlich waren und für zivilrechtliche Streitfälle als Beweismittel jederzeit konsultiert werden konnten. ${ }^{199}$ Als Beleg für diesen recht freizügigen Umgang mit offiziellen Akten können mehrere Papyri angeführt werden. In einem Fall handelt es sich beispielsweise um einen Streit um die Besitzrechte an einem Sklaven, der mit Hilfe einer $x \alpha \tau^{\prime}$ oixi $\alpha \nu \dot{\alpha} \pi 0 \gamma p \alpha \varphi \hat{n}$-Deklaration entschieden werden konnte. ${ }^{200}$ Ein anderer Fall betraf die Klärung von Verwandtschaftsverhältnissen zwi-

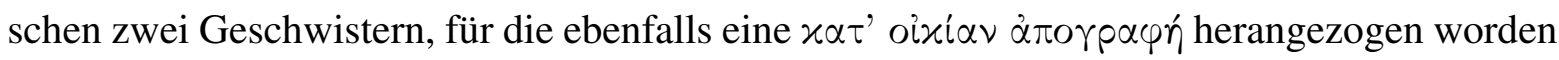
war. $^{201}$

Überhaupt ist es fraglich, was genau Josephus mit seiner Bezeichnung der Kopfsteuer gemeint hat. Der von ihm verwendete Terminus $\varepsilon x \alpha ́ \sigma \tau \eta \nu ~ x \varepsilon \varphi \alpha \lambda \eta े \nu \varepsilon i \sigma \varphi \circ \rho \tilde{\alpha} \varsigma$ ist keineswegs eine offizielle Bezeichnung eines römischen Verwaltungsvorgangs, sondern die Umschreibung einer Pro-Kopf-Abgabe. Es ist zwar durchaus möglich, daß Josephus die römische $\lambda \alpha o \gamma p \alpha \varphi i \alpha$ oder $\varepsilon \pi \iota \varkappa \varepsilon \varphi \alpha ́ \lambda \alpha \iota \circ v$ gemeint hat, doch werden diese im vorliegenden Text nicht explizit erwähnt. Weder das dahinterstehende Verfahren, noch die Form der Steuerakten kann aus Josephus' Angaben eindeutig identifiziert werden. Es ist noch nicht einmal gesichert, daß hier ein mathematischer Rechenvorgang zum Ergebnis geführt hat, da griech. $\tau \varepsilon x \mu \alpha i \rho \omega$ auch allgemeiner für >folgern $<$ oder >schließen aus< stehen kann. Wenn man alle diese Einwände zusammenführt, dann kann Josephus’ Aussage auch so gelesen werden: »Ägypten hat eine Einwohnerschaft von 7 1/2 Millionen Menschen ohne die Bevölkerung Alexandriens, wie aus den (Kopf-)Steuerlisten erschlossen werden kann.«Für Alexandria kann als Arbeitsgrundlage mit etwa 500000 Menschen gerechnet werden, so daß eine Gesamtzahl von ca. 8 Millionen Menschen $\mathrm{zu}$ veranschlagen ist. ${ }^{202}$

Erst kürzlich hat sich RATHBONE nochmals den Überlieferungen aus der klassischen Antike angenommen und einer erneuten Überprüfung unterzogen. ${ }^{203}$ Auch nach seinem Urteil besitzt die Überlieferung des Josephus keinen inhaltlichen Wert. ${ }^{204}$ Stattdessen rekonstruiert RATHBONE deren Ursprung wie folgt: Ein römischer >Lobredner < (sic!) hat die bekannte Zahl von 7 Millionen Ägyptern übernommen und um eine halbe Million Menschen erweitert, um

199 BELOCH (1886: 6); WILCKEN (1899: 487).

200 BGU 388 II 19; WILCKEN (1899: 486); vgl. dazu auch unten S. 151 für eine identische Situation aus der pharaonischen Zeit, die in den >Admonitions $<$ beschrieben wird.

201 pLond. 324; HomberT und PRÉAUX (1952: 147); WiLCKEN (1899: 487).

202 Die aus der Summe aller Faktoren in der älteren Forschungsmeinung gewonnene >Schätzung < von 500000 Menschen für Alexandria wird auch von BAGNALL und FRIER (1994: 54) akzeptiert.

203 RATHBONE (1990).

204 RATHBone (1990: 105 f.). 
die Prosperität des Landes unter römischer Herrschaft zu verdeutlichen. Er oder ein anderer Redakteur ergänzten den Text um den dokumentarischen Hinweis auf eine Steuererhebung und erklärten Alexandria - in vager Kenntnis der speziellen Regelungen für die Bürger dieser Stadt - zum Sonderfall. ${ }^{205}$

Gerade diesen besonderen Status Alexandrias hat RATHBONE zum Anlaß für seine Ablehnung genommen, da eine Person, die die Bürgerrechte der Stadt Alexandria oder aber das römische Bürgerrecht innehatte, überall in Ägypten von Steuern befreit war. ${ }^{206}$ Es bestand keine zwingende Notwendigkeit, auch seinen Wohnsitz in Alexandria nehmen zu müssen. Daher macht die Berufung Josephus' auf die Kopfsteuerlisten keinen Sinn, da sie für die Berechnung der Bevölkerungszahl unvollständig sind. Auch die Uminterpretation von Josephus' Quellen als Zensuslisten weist RATHBONE zurück, obgleich er zugeben muß, daß die Kopfsteuerlisten jener Zeit aus den Zensuslisten abgeleitet waren. ${ }^{207}$ Dennoch hält er an dem Vorwurf fest, Josephus habe sich an dieser Stelle geirrt und eine profunde Unkenntnis über die ägyptische Administration gezeigt.

RATHBONEs negativ gefärbter Gegenentwurf scheint allerdings ebenso wenig tragfähig. Zuvor bot RATHBONE noch gute Argumente dafür an, die Überlieferung Diodors mit seinem >Einbruch< der Bevölkerungszahlen von 7 Millionen auf 3 Millionen Menschen zu akzeptieren und die zweite Zahlenangabe nicht zu korrigieren. ${ }^{208}$ Mit seiner These zur Josephus-Stelle behauptet RATHBONE, daß einerseits Josephus die Bücher Diodors nicht bekannt waren, was außerhalb unserer Beweismöglichkeiten liegt, andererseits aber die bei Porphyr und Stephanus nachzulesende Zahl zur Bevölkerung >Thebens< von 7 Millionen Menschen zum seinerzeit bekannten Gemeingut gehört haben mußten. Darüber hinaus ist RATHBONEs Lesart der antiken Texte stark traditionell gefärbt und versucht erst gar nicht, diese Quellen auf Plausibilität zu testen. Der Josephus unterstellte Hinweis auf Prosperität in Form einer Steigerung von einer halben Million Menschen mutet in Anbetracht der sonst beklagten >Übertreibungen $<$ geradezu realistisch an.

Gegen Josephus spricht nicht unbedingt seine Nähe zum römischen Kaiserhaus und seine möglicherweise daraus resultierende Voreingenommenheit bezüglich der Darstellung historischer Sachverhalte. Vielmehr mahnt seine nicht-ägyptische Abstammung und sein verhältnismäßig kurzer Aufenthalt in Ägypten zur Vorsicht, wenngleich er prinzipiell als hoher >rö-

205 RATHBONE (1990: 106).

206 RAThbone (1990: ebenda); das besondere Steuerrecht für Alexandria hat bereits WILCKEN (1899: 239) angedeutet.

207 RATHBONE (1990: 106 Anm. 10).

208 RATHBONE (1990: 104). 
mischer Vertreter einfachen Zugang zu den Verwaltungsarchiven finden konnte. Es läßt sich allerdings nicht völlig ausschließen, daß Josephus auf ältere Literatur zurückgriff, um sich über die Größe Ägyptens zu informieren.

\subsection{Eine neue Hypothese zur Bevölkerungsentwicklung}

Im folgenden geht es um die Frage, ob die Angaben Diodors und Josephus' prinzipiell mit der historischen Situation jener Zeit vereinbar und in Einklang zueinander zu bringen sind. Die Kritik an den absoluten Zahlenangaben soll dafür außer Acht gelassen werden, sondern es gilt, die grundsätzliche Bevölkerungsentwicklung nachzuvollziehen. Konkret bedeutet das sowohl die Betrachtung des historischen Kontextes, in dem die Informationen verortet sind, als auch die rein rechnerische Überprüfung, ob ein lineares Bevölkerungswachstum, beziehungsweise dessen Rückgang innerhalb eines Wertebereichs liegt, der von der demographischen Forschung als praktisch möglich zugelassen wird. Die hier vorgestellte Hypothese entfernt sich dabei durchaus stärker von den sicheren Pfaden der Belege. Dies erschien notwendig, um die Herausstellung einiger Zusammenhänge zu ermöglichen, denen in der bisherigen Forschung keine Beachtung geschenkt wurde, die sich aber sowohl historisch als auch statistisch gut in Einklang zueinander bringen lassen.

\subsubsection{Prämissen}

Für die Konstruktion der Hypothese werden einige Prämissen vorausgesetzt, die sich aus der Betrachtung von Diodors und Josephus' Aussagen ergeben. Zunächst werden den Texten der beiden Autoren drei voneinander verschiedene Zahlenangaben entnommen. Diodor spricht von 7 Millionen Menschen »damals« und mindestens 3 Millionen Menschen »zu unserer Zeit«. Mit dieser Festlegung von Diodors Aussagen wird gleichzeitig die mehrfach geforderte Emendation der Textstelle verworfen. ${ }^{209}$ Die Angabe des Josephus von $71 / 2$ Millionen Bewohnern Ägyptens (ohne Alexandria) zu seiner Zeit um das Jahr 75 n. Chr. bleibt unverändert bestehen. Rechnet man auf Josephus' Zahl noch die heute allgemein akzeptierte Schätzung für Alexandria in Höhe von 500000 Menschen hinzu, dann kann der späteste Fixpunkt in der Reihe der demographischen Angaben auf 8 Millionen Menschen festgelegt werden.

Für Diodor gibt es dagegen drei verschiedene Möglichkeiten einer Datierung der von ihm niedergeschriebenen Zahlenangaben:

209 Zur Emendation vgl. auch ausführlich oben Kap. 2.5.1. 
1. Beide Angaben Diodors sind Übernahmen aus älteren Quellen.

2. Die Angabe Diodors, die sich auf die ägyptische Vergangenheit bezieht, stammt aus einer älteren Quelle, während die zweite Zahl von Diodor selbst und aus seiner Zeit stammt.

3. Beide Angaben basieren auf Recherchen Diodors in zeitgenössischen Quellen.

Die dritte Erklärung muß als recht unwahrscheinlich gelten, da bei einer Differenzierung der zwei Zahlen in >damals < und >heute < zumindest die ältere Zahl aus einer archivalischen Quelle stammen muß und daher von Diodor ohne Überprüfungsmöglichkeit übernommen wurde. Dagegen werden die beiden anderen Möglichkeiten im folgenden ausführlich auf ihre Tragfähigkeit hin überprüft. Hinsichtlich ihrer unterschiedlichen Zeitstellung, die die Daten anhand ihrer Herkunft einnehmen, soll die 1. Möglichkeit im folgenden als Frühdatierung und die 2. Möglichkeit als Spätdatierung bezeichnet werden.

Eine letzte Vorentscheidung betrifft die Voraussetzung, wer in den überlieferten Zahlen mitgezählt wurde. Auch in diesem Punkt ist die Forschungsmeinung gespalten, ob die Angaben sich lediglich auf die (kopf-)steuerpflichtige männliche Bevölkerung oder alle Bewohner Ägyptens beziehen. ${ }^{210}$ Zwei Gründe sprechen dafür, daß für die weiteren Überlegungen von einer Gesamtbevölkerung ausgegangen werden darf. Erstens hat Diodor zwar bei seiner Beschreibung der Stadt Alexandria offenbar nicht die Gesamtzahl der Einwohner gemeint, dies allerdings recht deutlich durch die Wortwahl seiner Beschreibung, in der er sie als é̀ bezeichnet, kenntlich gemacht. Zweitens sei auf eine gewisse Tradition verwiesen, die schon in Kap. 2.3 aus den Berichten anderer Autoren über Ägypten ersichtlich wurde. Hinsichtlich der Angabe einer zahlreichen Bevölkerung Ägyptens fand keine Differenzierung zwischen bestimmten sozialen Gruppen statt. Dies sei daher auch für die Gesamtzahlen Diodors und Josephus' angenommen.

\subsubsection{Frühdatierung und Spätdatierung der Quelle}

Wie bereits in Kap. 2.5.1 ausführlich besprochen wurde, wird die sogenannte >HekataiosHypothese< in der althistorischen Forschung immer noch kontrovers diskutiert und von manchem Vertreter altphilologischer Disziplinen auch favorisiert. Im Kern geht es dabei um die Frage, ob ganze Passagen in Diodors Büchern aus älteren Werken übernommen wurden und die darin enthaltenen Aussagen somit auch nicht für die Zeit Diodors in der zweiten Hälfte des 1. Jahrhunderts v. Chr. gültig sind, sondern die Situation eines bereits länger zurückliegenden 210 U.a. Hombert und PrÉaux (1952: 45 Anm. 1); HuSs (2001: 221 f. Anm. 3). 
Zeitraums beschreiben. Eine dieser Quellen, auf die man sich bei der Analyse von Diodors Texten wiederholt beruft, ist das verlorengegangene Werk des Hekataios von Abdera, der zur Zeit des Königs Ptolemaios' I. und damit über 200 Jahre vor Diodor lebte. ${ }^{211}$ Falls es sich bei dem Textauszug aus Diodor I 31,8 tatsächlich um eine Übernahme aus den Niederschriften des Hekataios oder eines Zeitgenossen von ihm handelt, dann stellt sich das chronologische Gerüst Diodors Angaben wie in Tabelle 2.1 unter der Spalte Frühdatierung zusammengefaßt dar. $^{212}$ Beide dem Text Diodors entnommenen Zahlenangaben müssen in diesem Fall aus der Zeit Ptolemaios I. oder davor stammen, dessen Lebensdaten als Hekataios' Zeitgenosse einen terminus ante quem bilden. Ein literarischer Verweis in die ägyptische Vergangenheit übersprang die Zeiten der Fremdherrschaft durch die Perser, so daß die frühen Ptolemäer an die 26. Dynastie anknüpften, die als letzte große pharaonische Glanzzeit in Erinnerung blieb, ${ }^{213}$ bevor sie im Jahr 525 v. Chr. mit der Eroberung Ägyptens durch den persischen König Kambyses endete. Dieses Datum ist daher der erste Eckpunkt einer chronologischen Neubewertung der Bevölkerungszahlen.

Als zweiter Eckpunkt kann die niedrigere der zwei Zahlenangaben Diodors in den Zeitraum der ersten beiden Ptolemäer gesetzt werden. Dies hat seinen Grund in der Betrachtung der geschichtlichen Ereignisse. Während für die Zeit der Perserherrschaft ab 525 v. Chr. kein kontinuierliches Bevölkerungswachstum erwartet werden darf und sich auch zu Beginn der Ptolemäerzeit Ägypten nur mühsam von den persischen Repressionen erholte, ${ }^{214}$ gibt es spätestens ab der Zeit Ptolemaios' II. mehrere Indizien für einen neuen Aufschwung. Zum einen spricht ein verstärktes staatliches Bauprogramm für ökonomischen Reichtum und fehlende außenpolitische Probleme. ${ }^{215}$ Zum anderen zeichnet sich bereits ab dem Neuen Reich ein spürbarer technischer Fortschritt in der Landwirtschaft ab, wie etwa die vereinfachte Bewässerung durch Einführung des shadufs, die bessere Getreideerträge ermöglichte und ein steigendes Bevölkerungswachstum garantierte. ${ }^{216}$ Die Zunahme der Bevölkerung läßt sich unter anderem an den Siedlungsprogrammen von Ptolemaios II. ablesen, der - wie auch sein Nachfolger -

$211 \mathrm{Zu}$ Hekataios und seinem vermutlichen Aufenthalt in Alexandria vgl. Huss (2001: 233 Anm. 34).

212 Den Vorschlag, daß beide Zahlenangaben Diodors auf Hekataios beruhen könnten, unterbreiteten - bisher unbeachtet - bereits BELOCH (1886: 255 f.) und WACHSMUTH (1903: 279).

213 So auch BIETAK (1975: 55 Anm. 167). Zum Rückgriff auf die »Staatsidee der Saïten« durch Ptolemaios I. vgl. auch HöLBL (1994: 30). Die Fragmente F Gr Hist 264, F19a/b (vgl. oben S. 48) beziehen sich ebenfalls ausdrücklich auf die vorpersische Zeit.

214 Sternberg-El Hotabi (2000).

215 Zum Bauprogramm der frühen Ptolemäer vgl. HöLBL (1994: 78 ff.).

216 Der enge Zusammenhang zwischen Agrarinnovation und dem Bevölkerungswachstum wurde bereits von O'CONNOR (1972b: 78) betont und eine gründliche Bearbeitung dieses Themenkomplexes für die pharaonischen Epochen als Desiderat angemahnt. 
besonders im Gebiet des Fajjum neugeschaffenen Nomos Arsinoitēs eine rege Ansiedlungspolitik für griechische Söldner, aber auch für Ägypter betrieb, nachdem weite Teile des Gebietes trockengelegt waren. ${ }^{217}$

So dürften während der Zeit der ersten Perserherrschaft weniger die vermeintlichen Massentötungen und gewaltsamen Unterdrückungen der fortwährend auflodernden Aufstände zur Trendwende in der Population beigetragen haben, als vielmehr der permanente wirtschaftliche Druck - insbesondere auf die Landbevölkerung - und die daraus resultierende Anachorese oder aber auch die Zwangsverschleppung der ägyptischen Bevölkerung zur Sklavenarbeit. ${ }^{218}$ Eine schwierige Versorgungslage und allgemein schlechte Lebensbedingungen waren geeignete Voraussetzungen für erhöhte Kindersterblichkeit, die als Auslöser für einen kontinuierlichen Rückgang der Bevölkerungszahlen verantwortlich gemacht werden kann. Dagegen sind während der nur 125 Jahre dauernden Perserzeit keine massiven Einbrüche in der Population zu erwarten. In diesen kurzen Zeitraum dürften kurzfristige extreme Schwankungen nach oben oder unten lediglich im Bereich von etwa hunderttausend Menschen liegen, so daß für eine Gesamtbetrachtung der Population jener Zeit dieser Aspekt vernachlässigbar ist. ${ }^{219}$

Sofern man Diodors Angaben - wie schon in der Einleitung dieses Kapitels diskutiert nicht wörtlich als absoluten Zahlenwert auffaßt, sondern lediglich als Bestimmung einer ungefähren Größenordnung versteht, kann der Verlauf der Bevölkerungsfluktuation recht gut nachvollzogen werden. Die zuvor diskutierten Faktoren haben zwar zur negativen Entwicklung der Bevölkerung beigetragen, jedoch sicher nicht in dem extremen Umfang, wie man ihn aus den Angaben Diodors vordergründig herauslesen will. Daher tangiert die Kurve der ägyptischen Population (Abb. 2.1) nicht die von Diodor festgelegten Extremwerte, sondern bildet einen interpolierten Verlauf, der die Untergrenze von 3 Millionen nicht durchschreitet. Konkret bedeutet dies für die Bevölkerung der Perser- und Ptolemäerzeit, daß sie eher zwischen 6 und 4 Millionen Menschen lag, als zwischen 7 und 3 Millionen.

Um den für die Frühdatierung unterstellten Kurvenverlauf einer weiteren Prüfung zu unterziehen, wurde zusätzlich zu den Angaben aus den antiken Quellen eine weitere Bevölkerungszahl hinzugezogen. Diese stellt zwar lediglich eine moderne Schätzung dar, hat aber den Vorteil, daß sie allgemein akzeptiert ist und allein auf statistischen Daten der römischen Zeit Ägyptens beruht. Mit dieser >Hilfskonstruktion< besteht die Möglichkeit, die Gesamtent-

217 BRAUNERT (1964: 91); CRAWFORD (1971: 39 ff.); HÖLBL (1994: 62 f.); HuSS (2001: 313).

218 STERNBERG-EL HotABi (2000: 166).

219 Anders dagegen STERnBERG-EL HotABI (2000: 154 Anm. 11).

220 Siehe Text. 
2 Die griechische Überlieferung

\begin{tabular}{|c|c|c|c|c|c|}
\hline \multirow[b]{2}{*}{ Datum } & \multirow[b]{2}{*}{ Beschreibung } & \multicolumn{2}{|c|}{ Frühdatierung } & \multicolumn{2}{|c|}{ Spätdatierung } \\
\hline & & Bevölkerung & $\varnothing$ Wachstum & Bevölkerung & $\varnothing$ Wachstum \\
\hline 525 v. Chr. & Beginn Perserzeit & 7 Mio. & & 7 Mio. & \\
\hline 282 v. Chr. & Tod Ptolemaios I. & 3 Mio. & $-0,30 \%$ & & \\
\hline 59 v. Chr. & Diodor in Ägypten & 5 Mio. $^{220}$ & $0,23 \%$ & 3 Mio. & $-0,35 \%$ \\
\hline 75 n. Chr. & Flavius Josephus & 8 Mio. & $0,27 \%$ & 8 Mio. & $0,73 \%$ \\
\hline
\end{tabular}

Tabelle 2.1: Eckdaten einer Früh- und Spätdatierung von Diodors Angaben

wicklung der Populationskurve indirekt zu stützen, ohne diese zugleich als Argumentationsgrundlage zu verwenden. Für die Zeit Diodors - also die Mitte des 1. Jahrhunderts v. Chr. vermuten Karl BUTZER wie auch RATHBONE übereinstimmend eine Population von maximal 5 Millionen Menschen, möglicherweise auch etwas niedriger, ${ }^{221}$ so daß sich die Eckdaten einer Frühdatierung wie in Tab. 2.1 aufgelistet darstellen. Gestützt wird diese Schätzung durch die Arbeiten Walter ScHEIDELs, der zwar eine generell kritisch bis ablehnende Haltung gegenüber den rein quellenbasierten Forschungen einnimmt, doch auf Basis seiner eigenen statistischen Auswertungen eine Bevölkerungsgröße des frühen kaiserzeitlichen Ägypten von fünf bis sieben Millionen Menschen für wahrscheinlich hält. ${ }^{222}$ Ein entscheidendes Kriterium für die Plausibilität der Populationskurve ist die Vermeidung allzu starker Wachstumsraten, auch wenn diese nur einen kurzen Zeitraum andauern. Wachstumsraten, die bei $1 \%$ oder sogar darüber liegen, sind zwar nicht ausgeschlossen, kollidieren jedoch mit den Erfahrungswerten, die bisher in der demographischen Forschung gewonnen wurden. BAGNALL und FRIER setzen aufgrund ihrer eigenen Berechnungen für die römische Zeit eine durchschnittliche jährliche Wachstumsrate von nur 0,2\% an. ${ }^{223}$ Die Wachstumsraten der hier vorgestellten Frühdatierung liegen nur knapp darüber und decken sich deshalb hervorragend mit den statistischen Eckdaten für das Ägypten der griechisch-römischen Zeit.

Die Frühdatierung hat jedoch auch ihre Schwächen. Diodor wäre die einzige Überlieferung von zwei Zahlen, während Porphyr und Stephanus jeweils nur auf eine einzelne Angabe von 7 Millionen Menschen Bezug nehmen. Andererseits liegt uns die Originalquelle nicht vor, und es ist daher kaum möglich zu entscheiden, welche Informationen sie tatsächlich zur Verfügung stellte, die von späteren Autoren übernommen wurden. Ob Hekataios der Verfasser dieser Quelle war, basiert ohnehin auf wackeligen Argumenten. Ebenso liegt es im Bereich

221 BUTZER (1976: 92); RATHBONE (1990: 123).

222 SCHEIDEL (2001b: 58 f.); eine ähnliche Obergrenze von sechs Millionen Menschen hat HASSAN (1994: 167) auf Basis von Ernteerträgen berechnet.

223 BAGNALL und FRIER (1994: 139). 


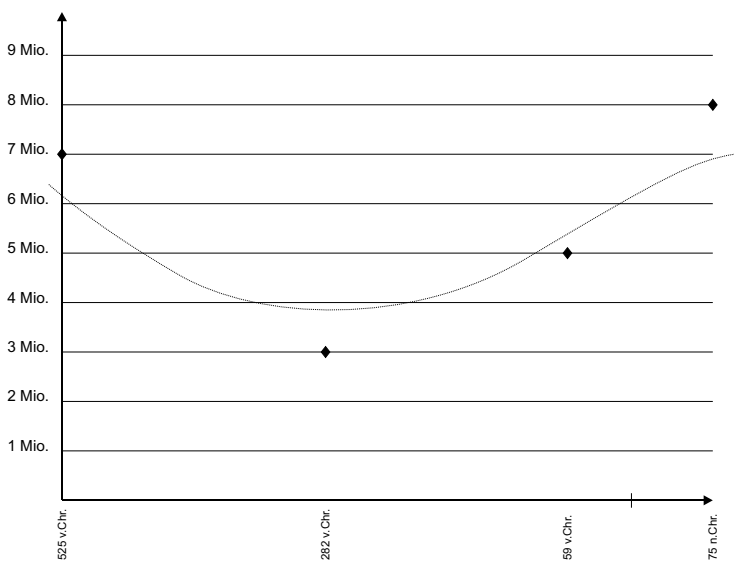

(a) Frühdatierung

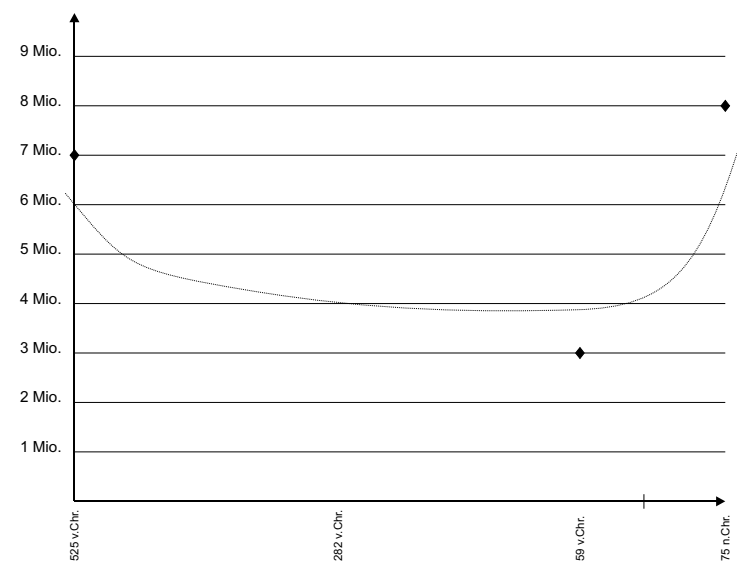

(b) Spätdatierung

Abbildung 2.1: Interpolierte Populationskurven nach den Angaben griechischer Autoren

des Möglichen, daß sowohl die Angabe der 7 Millionen als auch der 3 Millionen Menschen aus der Feder irgendeines Zeitgenossen Ptolemaios' I. stammen.

\section{Die Spätdatierung und ihre Implikationen}

Eine Spätdatierung der Diodor-Daten bezieht sich auf eine Aufspaltung der Datierung beider Zahlenangaben. Die ältere Zahl von 7 Millionen wird dabei als Übernahme Diodors aus älteren Quellen der Zeit Ptolemaios' I. aufgefaßt, die jüngere Zahl von 3 Millionen dagegen interpretiert man als eigene Ergänzung Diodors für die Zeit seines Ägyptenaufenthalts (Tab. 2.1, Spalte Spätdatierung). ${ }^{24}$ Wenn Diodor also die zweite Zahlenangabe tatsächlich auf seine eigene Zeit bezogen hat, dann gilt für das Jahr 59 v. Chr. eine Untergrenze von etwa 3 Millionen Menschen in Ägypten. Die Zahlenangabe des Josephus bleibt für das Jahr 75 n. Chr. unverändert die Obergrenze von etwa 8 Millionen Menschen. Zwischen diesen beiden Angaben liegt eine Zeitspanne von 116 Jahren. In dieser Zeit müßte - wollte man beiden Autoren Glauben schenken - ein Bevölkerungswachstum um maximal 5 Millionen Menschen nachweisbar oder zumindest vorstellbar sein.

Bereits Alan BOWMAN hat gezeigt, daß bei einer jährlichen Wachstumsrate von $2 \%$ diese Bevölkerungszunahme in etwa 50 Jahren zu erreichen ist. ${ }^{225}$ Gegenüber konservativen Schät-

224 SACKS (1990: 91); auch RATHBONE (1990: 107) geht trotz seiner ablehnenden Haltung gegen die Hekataios-Hypothese von einer älteren Überlieferung aus.

225 BowmAN (1986: 17). Er betont darüber hinaus, daß in dieser Rechnung Aspekte der Bevölkerungswande- 
zungen zu langfristigen Wachstumsraten von Populationen erscheint dieser Wert allerdings zu hoch - selbst bei Betrachtung der durchschnittlichen Raten im Ägypten des 19. Jahrhunderts. ${ }^{226}$ Für das moderne Ägypten wird von einem schwankenden Wachstum unter 1,5\% ausgegangen. ${ }^{227}$ Da die Angaben der antiken Autoren ohnehin nur als grobe Richtlinie und nicht als präzise Aktenauskunft aufzufassen sind, kann man davon ausgehen, daß ein Anstieg der Bevölkerung von 3 auf 8 Millionen in 116 Jahren eine durchschnittliche Wachstumsrate zwischen 0,8 und $0,9 \%$ voraussetzte, was im Sinne demographischer Forschung zwar bereits als >rapides Wachstum $<$ gilt, ${ }^{228}$ andererseits aber niedrig genug liegt, um über einen begrenzten Zeitraum hinweg möglich zu sein.

Um allerdings den Anschluß an die Vorgabe für die Zeit vor den Persern halten zu können, müßte dann postuliert werden, daß die Zahl der ägyptischen Bevölkerung seit der Perserzeit bis in die erste Hälfte des 1. Jahrhunderts kontinuierlich zurückgegangen ist. Dies widerspricht den Thesen ButZERs, der im Gegenteil auf die bis zum Zeitpunkt Diodors expandierende Bevölkerung hingewiesen hat. ${ }^{229}$ Einerseits wird die Möglichkeit einer niedrigen Population im 1. Jahrhundert v. Chr. durch die dramatischen ökonomischen Verhältnisse unter den letzten Ptolemäern gestützt, andererseits steht der Verlauf im Widerspruch zu den zahlreichen belegten Neugründungen von Siedlungen durch die ersten Ptolemäer sowie den erheblichen Verbesserungen der Landwirtschaftstechnik, die höhere Erträge und zwei Ernten pro Jahr ermöglichte und damit eine grundsätzliche Entlastung der Ernährungssituation bedeutete. ${ }^{230}$ Diese Indizien weisen auf einen erneuten Anstieg seit dem 3. Jahrhundert hin. Der für das Ende der ptolemäischen Zeit häufig vermutete Bevölkerungsrückgang hat besonders im ländlichen Raum trotz der nachweislich schlechten wirtschaftlichen Lage nicht so sprunghaft und massiv eingesetzt, wie aus den älteren Forschungsergebnissen immer wieder interpretiert wurde, sondern muß eher als anhaltender Schwund aufgefaßt werden. ${ }^{231}$ Dazu trug die Landflucht in großem Ausmaß zur Verödung ganzer Landstriche bei. ${ }^{232}$ Inwieweit beide Faktoren für eine echte Verringerung der Bevölkerungszahl verantwortlich waren oder hauptsächlich ein sicht-

rung (Immigration) noch gar nicht berücksichtigt sind.

226 Vgl. z. B. die extrem niedrig angesetzten Wachstumsraten für die pharaonischen Epochen bei HASSAN (1993: 170 ff.) oder die Schätzung von BUTZER (1976: 86) von 0,08 \% für das Alte Reich.

227 Siehe dazu auch ausführlich Kap. 7.

228 HASSAN (1981: 234).

229 ButZer (1976: 92 Anm. 8).

230 Die Auswirkung dieser Verbesserung der Lebenssituation zeigt sich dabei nicht so sehr in einer Steigerung der Geburtenrate, sondern vermutlich in der Verringerung der Kindersterblichkeit. Siehe dazu auch Kap. 6.4.2.

231 BRAUNERT (1964: 94 ff.).

232 HöLBL (1994: 193). 
barer Beleg für eine Konzentration der Bevölkerung in Siedlungszentren ist, läßt sich nicht abschließend beurteilen.

\subsubsection{Bevölkerungsentwicklung und technischer Fortschritt}

Die zuvor dargelegten Überlegungen zu einer hypothetischen Früh- oder Spätdatierung der durch Diodor auf uns gekommenen Bevölkerungsgrößen können nur im Kontext zur allgemeinen Entwicklung Ägyptens während der Zeit der Ptolemäerherrschaft betrachtet werden. Im folgenden soll es nun darum gehen, inwieweit sich Parameter dafür finden lassen, daß die Bevölkerungsentwicklung der Ptolemäerzeit tatsächlich den in Abb. 2.1 a vorgeschlagenen Verlauf genommen haben könnte. Ob die Prosperität Ägyptens tatsächlich seit der Zeit Ptolemaios I. zutraf, entzieht sich leider unserer Kenntnis, da während der Regierung Ptolemaios' I. die Praxis der Wiederverwendung von Papyri für Mumienkartonagen nur geringe Verbreitung erfuhr und auf diesem Weg nur eine kleine Anzahl von Papyri erhalten blieb. ${ }^{233}$ Solche Papyri, deren Inhalte aus Briefen, Aktenstücken und ähnlichen Schriftstücken bestehen, sind für die Vervollständigung eines historischen Gesamtbildes unabdingbar. Erst ab der Zeit Ptolemaios' II. gibt es eine beachtliche Zahl von Papyrusfragmenten, die Aufschluß über das Alltagsund Wirtschaftsleben geben. Wenn man jedoch die Maßnahmen der nachfolgenden Ptolemäer nur als Fortsetzung dessen versteht, was von Ptolemaios' I. bereits auf den Weg gebracht wurde, dann darf man vielleicht - mit THOMPSON - davon ausgehen, daß im Fajjum bereits seit Beginn der Ptolemäerzeit mit Aktivitäten zur Bodenkultivierung, Bewässerung, Deichbau, Schweinezucht sowie der Erweiterung des Ackerbaus durch Immigranten zu rechnen ist. ${ }^{234}$

Intensivierte Landwirtschaft und höhere Erträge stellen ein wesentliches Merkmal für die Ökonomie der frühen Ptolemäer dar. ${ }^{235}$ Die Bevölkerung profitierte davon, daß die Organisation darauf ausgerichtet war, möglichst eine autarke Versorgung zu ermöglichen. Bei den Anbauprodukten dominierten deshalb die Grundnahrungsmittel wie Weizen, daneben auch

233 THOMPSON (1999b: 125).

234 ThOMPSON (1999b: ebenda); Welche Personengruppe unter THOMPSONs >Immigranten< fällt, ist unklar. Die Situation des Fajjum bleibt unter Ptolemaios I. mangels Quellen im Dunkeln. Lediglich über indirekte Indizien können frühe Aktivitäten in dieser Region vermutet werden, etwa durch den Ortsnamen Berenikis, der sich vom Namen der Gemahlin Ptolemaios' I. ableitet, oder die archäologische Evidenz für eine

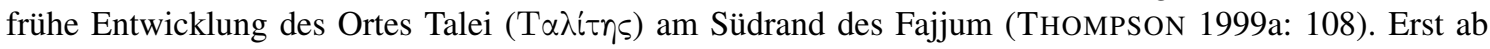
Ptolemaios II. und verstärkt unter Ptolemaios III. können ein breiter Immigrantenzustrom aus dem Norden (BRAUNERT 1964: 48) sowie eine zunehmende Niederlassung griechischer Militärsiedler, der sogenannten Kleruchen (HÖLBL 1994: 61), nachgewiesen werden. Siehe dazu auch oben S. 60 Anm. 217.

235 SCHÜRMANN (1991: 116 ff.), obwohl von SCHENKEL (1978: 68) im Vergleich zu den Bewässerungsrevolutionen pharaonischer Zeit als »unvergleichlich weniger dramatisch« charakterisert. 
Flachs, Hopfen, Papyrus sowie Sesam, Kroton und Lein. Auch die Haltung von Kleinvieh wie Schaf und Ziege wurde gefördert. ${ }^{236}$ Ein Großteil der Erträge blieb für den Export bestimmt. Bereits unter den Ptolemäern konnte sich Ägypten als >Kornkammer < des Mittelmeeres etablieren und hatte Abnehmer in Griechenland und Kleinasien. ${ }^{237}$ Daneben gab es Bestrebungen, für die eingewanderten Griechen Produkte verfügbar zu machen, die sie aus ihrer Heimat kannten, die aber bisher nur über teure Importe nach Ägypten gelangten, wie zum Beispiel Wein, Oliven, Obst und Salat. Dafür erschloß man neuen Boden und trieb den Ausbau an Agrarfläche voran. Es lag also im doppelten Interesse der ptolemäischen Herrscher, die landwirtschaftliche Nutzung zu verbessern und daraus persönlichen Nutzen zu ziehen. ${ }^{238} \mathrm{Um}$ diesen Umstand mit gängigen Hypothesen zur Population in Einklang zu bringen, seien im folgenden nochmals kurz zwei konträre Modelle angerissen. Die Beziehung zwischen dem Pro-Kopf-Ertrag zur Versorgung, technischer Innovation und Bevölkerungswachstum wird hinsichtlich ihrer Verhältnisse von Ursache und Wirkung recht uneinheitlich beurteilt.

Die >klassische< Theorie, wie sie zum Beispiel Thomas MALTHUS formulierte, besagt, daß Nahrungsmangel zu erhöhter Sterblichkeit, verlangsamten Bevölkerungswachstum oder sogar zu Bevölkerungsrückgang führt. ${ }^{239}$ Auch geht die traditionelle Sichtweise davon aus, daß Innovation und Erfindung als Ursache für Bevölkerungswachstum anzusehen sind. Die neuen Technologien in der Landwirtschaft, der Tierzucht und der Ernte verbesserten und stabilisierten den gesamten Produktionsproze $\beta$ und förderten somit das Wachstum. ${ }^{240}$ Andere Theorien verkehren diesen Prozeß in das genaue Gegenteil: Das rapide Anwachsen der Bevölkerung und die Ausdehnung ihrer Ansiedlung auf die gesamte nutzbare Fläche zwang zu Innovation und Intensivierung, um das Überleben zu sichern. ${ }^{241}$ Zentraler Aspekt war dabei die einfache und schnelle Reproduktion von Nahrung, was mithin der Hauptgrund für das Umschwenken der Jäger und Sammler-Gesellschaften zum Ackerbau war. ${ }^{242}$

Nach dem Zeugnis der griechischen Autoren ist für die Ptolemäerzeit faktisch mit einem Bevölkerungswachstum zu rechnen, das als Prämisse für die weiteren Überlegungen gelten

236 SCHÜRMANN (1991: 118).

237 SCHÜRMANN (1991: ebenda).

238 SCHÜRMANN (1991: 117) mit Schätzungen für die jährlichen Einkünfte des ptolemäischen Herrscherhauses aus der Landwirtschaft.

239 Siehe zu MALTHUS ausführlich Kap. 1.3.1.

240 LIVI-BACCI (1992: 90).

241 Bereits 1901 formulierte Julius WOLF die bevölkerungstheoretische These: »Die Bevölkerung hat nicht die Tendenz, über die Unterhaltsmittel hinauszuwachsen, vielmehr haben die Unterhaltsmittel die Tendenz, über die Bevölkerung hinauszuwachsen.« (zitiert bei BIRG 1996: 53).

242 Vgl. Boserup $(1970,1981)$ als führende Vertreterin dieser Theorie. In ihrem Modell ist Population keine Variable, die von der Entwicklung abhängt, sondern diese bestimmt. 
soll. In diesen Zeitraum fällt auch eine technische Entwicklung hinsichtlich der Bewässerung und der Verbesserung der landwirtschaftlichen Produktion, die archäologisch nachvollziehbar ist. ${ }^{243}$ Wenn man für die Ptolemäerzeit davon ausgeht, daß kein Bevölkerungsdruck die Herrscher zu den beschriebenen Maßnahmen zwang, sondern die Erhöhung der Erträge und in Folge davon eine Vergrößerung ihres Exportgewinns ihr Handeln leitete, dann dürfte das Modell Ester BOSERUPs nicht zutreffend sein. ${ }^{244}$ Vielmehr ergibt sich aus den Verbesserungen der Agrarwirtschaft und der daraus resultierenden ökonomischen Entlastung der Landbevölkerung quasi als positiver Nebeneffekt ein Stimulans für das Bevölkerungswachstum. Diese Tendenz hielt während der gesamten Ptolemäerzeit an und konnte auch nicht durch das Mißmanagement der letzten ptolemäischen Könige gestoppt oder gar umgekehrt werden, da die positiven Effekte der Reformen tiefgreifend genug waren, um für die vergleichsweise kurze Zeit des Mangels keine demographischen Auswirkungen zu haben.

Schließlich erreichte mit den Römern ab 30 v. Chr. die exportorientierte Organisation der Provinz Aegyptus ihren Höhepunkt, da die neuen Herrscher für die Befriedigung ihrer eigenen Bedürfnisse das ägyptische Agrarsystem optimiert hatten, was jedoch nicht unbedingt zum individuellen Wohlergehen der ägyptischen Bevölkerung führte. ${ }^{245}$ In diesen Zusammenhang paßt auch eine Beobachtung WILCKENs, der darauf hinwies, daß die Getreidebesteuerung

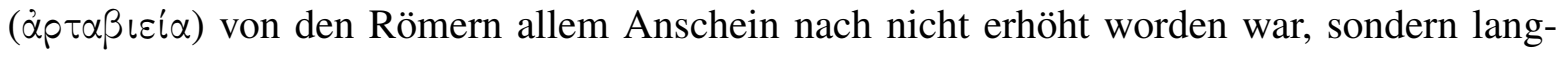
fristig bei 1-2 Artaben pro Arure blieb. Den Überschuß für den Export nach Rom erzielte man offenbar durch Neugewinnung von Agrarflächen. ${ }^{246}$ Die Fruchtbarkeit des Bodens und die Leistungsfähigkeit der Landwirtschaft blieben unverändert hoch, wie auch der Historiker Strabon zu berichten weiß, der um 25 v. Chr. Ägypten bereiste und unter anderem auch das Fajjum aufsuchte. ${ }^{247}$ Die Lebenssituation blieb für breite Bevölkerungsschichten erträglich, so daß davon auszugehen ist, daß die Maßnahmen der neuen römischen Herren keinen negativen Einfluß auf die Population Ägyptens hatten. Dazu sei auch auf eine Beobachtung BosERUPs hingewiesen, die der Ansicht war, daß die Getreidelieferungen nach Rom nur aus Gebieten mit einer hohen Bevölkerungsdichte kamen. Das Niltal wies eine frühe Urbanisierung auf und

243 Vgl. dazu SchürmanN (1991: 93 ff.)

244 Dies ist jedoch kein Argument für grundsätzliche Zweifel an BosERUPs Modell. Für die modernen westlichen Gesellschaften hat sich das Modell bestätigt. Wachsende Bevölkerungszahlen zwingen zu einer Intensivierung aller Bereiche der Nahrungsmittelproduktion, um die Menschen ernähren zu können. Hier zeigt sich der Zusammenhang zwischen Bevölkerungsdruck und Ernährungsproblemen in seiner ausgeprägten Form.

245 BUTZER (1976: $91 \mathrm{f}$.).

246 WiLCKEN (1912: 187 f.).

247 Strabon, Geographica XVII 1,35. 
hatte nach dem Ranking BosERUPs den höchsten Grad an Bevölkerungsdichte. ${ }^{248}$ Dies paßt ausgezeichnet in das Bild, daß die ägyptische Bevölkerungsdichte ihren vorläufigen Höhepunkt im 1. Jahrhundert n. Chr. erreichte. ${ }^{249}$

\subsection{Zusammenfassung}

Gleich aus mehreren Gründen präsentiert sich die hier vorgeführte >Frühdatierung< als die besser begründbare Hypothese. Zunächst einmal passen die historischen Fakten gut in das Gesamtbild: Eine prosperierende Gesellschaft, deren Zahl während der Perserherrschaft durch Anachorese und der Belastung einer allgemein schlechten wirtschaftlichen Situation spürbar, doch nicht dramatisch sank, um dann durch die Maßnahmen unter den ersten Ptolemäern wieder zu neuem Wachstum zu gelangen. Das Mißmanagement der späten Ptolemäer und die Ausbeutung der Provinz Aegyptus durch die Römer verschlechterte zwar die Lebensbedingungen, bewirkte jedoch aufgrund des eingetretenen Innovationsschubs keine Umkehrung des Trends mehr, so daß bis in das erste nachchristliche Jahrhundert hinein die Bevölkerung lediglich aufgrund einer durch die Römer optimal organisierten Kolonie anstieg. Auch weisen die Faktoren für die durchschnittlichen jährlichen Wachstumsraten moderate Werte auf. Weder im Niedergang noch im Anstieg wird eine jährliche Rate von 0,3\% überschritten, was auf eine gemäßigte Entwicklung hinweist, die deutlich unter dem Limit für rapides Wachstum bleibt und demographisch gut vertretbar ist. Als letztes stützendes Argument helfen die von BUTZER und RATHBONE vorgeschlagenen Bevölkerungsgrößen für die Mitte des ersten vorchristlichen Jahrhunderts. Auf gänzlich anderem Datenmaterial basierend fügt sich ihre Schätzung nahtlos in den hypothetischen Populationsverlauf ein und ermöglicht eine Absicherung der Kurve. Sollte sich die Frühdatierung der Diodor-Angaben anhand anderer Indizien bestätigen, so wirft dies auch ein neues Licht auf die Perserzeit Ägyptens. Die politische, ökonomische und soziale Situation der ägyptischen Bevölkerung jener Zeit würde sich dann durch die Evidenz eines Bevölkerungsrückgangs deutlich schlechter darstellen, als bisher vermutet wurde und durch die Textquellen jener Zeit zuvor nicht beweisbar war.

Auch wenn Diodor im Urteil der historischen Forschung hinsichtlich seines Werks besser beurteilt wurde als etwa Josephus, so stand auch Diodor häufig genug in der Kritik. ${ }^{250}$ Dieses

248 BOSERUP (1981: 74); diese Einschätzung wird auch von RATHBONE (1990: 120 f.) geteilt.

249 BUtZer (1976: ebenda).

$250 \mathrm{Zu}$ den Schmähungen gegen Diodor in der älteren Forschung vgl. SPOERRI (1991: 310 f. Anm. 4); LLOYD (1988: Bd. I, ix ff.). 
Urteil erfährt erst in jüngerer Zeit eine Revision, und man würdigt Diodor als wichtigste und teilweise sogar einzige Quelle für die historische Überlieferung, ${ }^{251}$ relativiert die Vorwürfe des Plagiarismus und unkritischen Zitierens ${ }^{252}$ und attestiert ihm im Falle von Vergleichsmöglichkeiten sogar seine Zuverlässigkeit als Kopist. ${ }^{253}$ Als Fazit bleibt daher die Feststellung, daß sowohl Diodor als auch Josephus mit ihren Beurteilungen der ägyptischen Population Recht behielten, zwar nicht in absoluten Zahlen gemessen, wohl aber im prinzipiellen Verlauf der Entwicklung, den die Bevölkerung in der griechisch-römischen Zeit genommen hat. Als Trendmesser für den demographischen Prozeß jener Zeit haben sich beide Autoren - aller modernen Kritik zum Trotz - bewährt, so daß ihnen zumindest dieses Verdienst zuerkannt werden darf. ${ }^{254}$

251 WIRTH (1993: 3)

252 WiRTH (1993: 6 Anm. 13) befindet »Nach ihren Zitiermethoden zu fragen, scheint für antike Autoren freilich so sinnlos, wie für die meisten modernen.«; in ähnlicher Weise äußert sich auch SACKS (1990: 9).

253 SACKS (1990: 99).

254 SCHEIDEL (2001b: 49). 


\section{Zensus-Papyri aus pharaonischer Zeit}

Denn was man schwarz auf weiß besitzt, kann man getrost nach Hause tragen.

Goethe, Faust I

\subsection{Zur Situation}

\subsubsection{Umfang der Quellen}

$\mathrm{D}$ IE Menge der ägyptischen Papyri, die - im weitesten Sinne - mit dem Begriff des >Zensus < zu tun haben, scheint auf den ersten Blick überschaubar: Nur eine Handvoll Quellen hat die Zeiten überdauert und bietet einen begrenzten Einblick in diesen speziellen Aspekt pharaonischer Administration. Überdies harrt etliches, was erhalten geblieben ist, noch der Publikation oder ist bereits seit längerer Zeit in der Bearbeitung, jedoch ohne Aussicht auf baldige Zugänglichkeit. Dies gilt gleichermaßen für die Veröffentlichung der Papyri aus Gebelein, wie auch für die Fragmente des Stato Civile. ${ }^{255}$ Einen detaillierteren Einblick in Form und Inhalt der Papyri zu gewinnen, gleicht dem Zusammenfügen eines Puzzles, dessen Teile weit über die verfügbaren Publikationen verstreut sind. ${ }^{256}$

Der Ägyptologie bleibt so nur der neidvolle Blick in die Nachbardisziplinen der klassischen Antike, die mit einer Fülle von Material aufwarten können, das neben den rein historischen Analysen auch in beschränktem Umfang statistische Auswertungen ermöglicht. ${ }^{257}$ Diese Form einer Auswertung bleibt der Ägyptologie vorerst noch verschlossen, und es ist

255 Über die Arbeiten an den Gebelein-Papyri gibt es keine aktuellen Informationen. Die Bearbeitung des Stato Civile liegt derzeit in den Händen von Robert DemaréE. Zum letzten Stand der Arbeit siehe DemaréE (1993: 105).

256 Für einen Katalog aller Quellen des pharaonischen Ägypten vom Alten bis zum Neuen Reich, die im weitesten Sinne etwas mit >Zensus < zu tun haben, vgl. VALBELlE (1987). VALBELLEs Zusammenstellung hat 40 Einträge, darunter jedoch auch Texte, die sich ausschließlich auf Viehzählungen beziehen.

257 BAgNALl und Frier (1994); HoMbERT und PrÉAUX (1952). 
zweifelhaft, ob Menge und Qualität der Daten jemals ein Niveau erreichen, das den Minimalanforderungen für derartige Untersuchungsmethoden entspricht. Was bleibt, ist der Versuch einer Einschätzung von Größenordnungen, die in Kenntnis der wenigen konkreten Daten das Unwahrscheinliche ausschließt. Dieses Verfahren, im Angloamerikanischen prägnant als >informed guesses $<$ bezeichnet, hinterläßt als Ergebnis jedoch immer noch eine weit gefaßte Zahl von Möglichkeiten und sollte daher nur als Orientierung verstanden werden, anhand dessen sich künftige Aussagen zu dieser Problematik besser einordnen lassen.

In diesem wie auch im folgenden Kapitel soll anhand ausgewählter Beispiele die Grundlage für einen besseren Zugang zur demographischen Situation im Alten Ägypten gelegt werden. Während in diesem Kapitel ausschließlich der Blick auf die Textquellen gerichtet ist, wird im Kapitel 4 ein Versuch unternommen, die am Text gewonnenen Ergebnisse direkt an verschiedenen Siedlungen zu überprüfen. Ein höherer Grad an Genauigkeit soll dabei durch eine strikte Selektion der Quellen erreicht werden, für die zuvor einheitliche Kriterien definiert worden sind. Darüber hinaus werden den Ergebnissen Alternativen gegenübergestellt, die auf anderem Wege errechnet wurden. Anhand dieses Vergleichsmaterials lassen sich Differenzen und Übereinstimmungen gut beobachten.

\subsubsection{Zeitstellung}

Im wesentlichen lassen sich drei >Gruppen< von Papyri fassen, jede für sich aus einem geschlossenen Fundkontext. Alle drei Hauptepochen des pharaonischen Ägypten sind dabei abgedeckt. Die frühesten Zeugnisse stammen aus einem Grabfund des Alten Reiches in Gebelein. Sie sind einer eher listenartigen Aufstellung verhaftet und bieten keine exakte Erfassung nach Haushalten oder Sippen. Die nächste große Entdeckung stammt aus der am Taltempel der Pyramide Sesostris' II. gelegenen Arbeitersiedlung Kahun aus dem Mittleren Reich. Eine Handvoll Fragmente erlaubt tiefere Einblicke in die Erfassung einzelner Haushalte, die in einem regelmäßigen Turnus aktualisiert wurden. Die dritte und letzte Gruppe bilden Papyri aus dem Neuen Reich. Hier stehen besonders zwei Fundkomplexe im Vordergrund. Zum einen eine Liste, die auf dem Verso des Papyrus Abbott - eigentlich der Gruppe der sogenannten $>$ Grabräuber-Papyri< zugehörig - erhalten geblieben ist und in geographischer Reihenfolge eine beträchtliche Zahl von Häusern und deren jeweiligen Vorstand benennt. Zum anderen existiert im Museum von Turin eine Anzahl Fragmente eines oder mehrerer Papyri aus Deir el-Medineh, die - ähnlich wie schon die Listen aus Kahun - eine recht präzise Erfassung einzelner Haushalte und deren Bewohner enthält. 
Neben diesen Hauptvertretern der Gattung >Zensustexte< läßt sich noch eine Anzahl von Hinweisen und Einzelinformationen aus anderen Texten benennen, die für sich genommen nicht im engeren Sinne dieser Gattung zugeordnet werden dürfen, deren Informationsgehalt aber die ansonsten düstere Situation der demographischen Erfassung ein wenig aufzuhellen hilft. Diese Quellen werden in Kapitel 5 ausführlicher betrachtet.

\subsection{Zensustexte aus den Gebelein-Papyri}

\subsubsection{Herkunft}

Die Gebelein-Papyri stellen nach heutiger Kenntnis die ältesten Verwaltungsakten dar, die aus Ägypten bekannt sind. Verpackt in einer kleinen Holzkiste, wurden sie 1936 von Giulio FARINA als Teil der Beigaben in einem Grab gefunden. ${ }^{258}$ Die Papyri sind bis heute unpubliziert, es liegen lediglich einige Vorberichte von Paule POSENER-KRIÉGER vor, der es leider nicht mehr vergönnt war, die Arbeiten an der Publikation abzuschließen. ${ }^{259}$ Selbst Fotos der Papyri sind nur teilweise verfügbar, darunter auch Ausschnitte der hier zu besprechenden. ${ }^{260}$ Neben fünf fast vollständig erhaltenen Rollen, die heute im Kairener Museum aufbewahrt werden, ${ }^{261}$ enthielt die Kiste auch eine kleine Anzahl von Papyrusfragmenten, die teilweise in das $\mathrm{Mu}$ seo Egizio in Turin gekommen sind. Von den fünf Rollen enthalten drei Abrechnungen über Getreide, eine Rolle enthält eine Aufstellung über Stoffe und Kleidung und eine stellt eine Liste mit Personennamen dar. Fundort und Datierung lassen nach wie vor viele Fragen offen. PosEnER-KRIÉGER verglich die Gebelein-Papyri mit den Funden aus Abusir und stellte fest, daß weder inhaltlich noch bezüglich des Fundortes in einem Grab die Papyri zueinander >passen ${ }^{262}$ Eher hätte man einen Fund in einem Administrationsgebäude erwartet. So bleibt nur die Vermutung, daß die Papyri nicht mehr benötigt und daher ausgemustert wurden, so daß sie als Palimpsest für andere Zwecke wiederverwendet werden konnten. Da der Grabinhaber offenbar ein Schreiber war, könnte er sich die alten Aktenstücke angeeignet haben, die ihm schließlich als Statussymbol seines Berufes bei der Bestattung mitgegeben wurden. Ebenso schwierig ist die genaue Datierung der Papyri, da keiner eine präzise Datumsangabe enthält. Zum überwiegenden Teil ist der Beginn der Papyri verloren. Nur von einer Rolle ist der Anfang erhalten, doch hatte der Schreiber noch nicht den Namen des regierenden Königs einge-

258 Der Fund wurde nur kommuniziert über CdE 10 (1935), $269 \mathrm{f}$.

259 POSENER-KRIÉGER $(1986,1975)$.

260 POSENER-KriÉGER (1986: Taf. 2-6).

261 Kairo JE 66844.

262 Posener-KriÉGer (1986: 26 f.). 
tragen, sondern lediglich Leerraum dafür reserviert. Aufgund der archaischen Orthographie sowie der Tatsache, daß Stofflisten zu Beginn der 5. Dynastie aus den Inventaren der Gräber wieder verschwinden, datiert Posener-KriÉger die Papyri in das Ende der 4. Dynastie und hält sogar eine noch frühere Entstehungszeit für möglich. ${ }^{263}$

\subsubsection{Aufbau}

Zwei Papyri aus dem Gebelein-Fund sind im Rahmen der Fragestellung dieser Arbeit von Interesse. Der erste Papyrus - von POSENER-KRIÉGER als >Rolle I $<$ bezeichnet - ist 1,23 m lang und 0,20 m hoch. Der Anfang der Rolle ist nicht erhalten. Das Recto enthält vier Personenlisten, von denen drei in Tabellenform verfaßt sind. Dabei ist in einem Fall die Rubrik erhalten geblieben, so daß sich der Zweck der Auflistung erkennen läßt: Die verzeichneten Männer und Frauen waren zu einer Baumaßnahme für einen lokalen Tempel rekrutiert worden, dessen Name auf König Snofru hinweist. Eine andere Liste faßt die Personen unter der Bezeichung $n f r . w$ zusammen. Hier dürfte es sich also um Arbeiter handeln, wie sie bereits aus Expeditionen, aber auch im militärischen Bereich als >Rekruten< für besondere Aufgaben bekannt sind. Die Personennamen sind dabei nach den Ortschaften ihrer Herkunft gruppiert und mit Titeln vermerkt. Auf dem Verso befindet sich eine Liste von 104 Namen ohne Titel, daneben eine Liste mit weiteren 74 Namen. Andere Listen mit nicht personenbezogenen Aufstellungen finden sich auf beiden Seiten des Papyrus. ${ }^{264}$

Auch die $>$ Rolle IV $<$ liefert indirekt interessante Fakten zur demographischen Situation des Alten Reiches. Die Länge des Papyrus beträgt 1,16 m, die Höhe 0,21 m. Das Ende des Papyrus ist beschädigt. Der Inhalt befaßt sich mit einer Aufstellung von Stoffen, die als Abgabeleistung eines $p r-\underline{d} t$ erfaßt wurden. In 99 Spalten wurden die Namen der Bewohner des $p r-\underline{d} t$ vermerkt, dazu ihre Titel oder Tätigkeitsbezeichnungen. Das $p r-\underline{d} t$ besteht aus zwei Dörfern, entsprechend sind die Einträge der Bewohner in zwei Gruppen aufgeteilt. Fragmente von Papyri mit vergleichbarem Inhalt sind bereits aus Abusir bekannt. ${ }^{265}$

\subsubsection{Inhaltliche Auswertung}

Der Inhalt von >Rolle I $<$ stellt sich als typischer Vertreter einer >Arbeitsliste $<$ dar, mit deren Hilfe die für Baumaßnahmen ausgewählten Personen einer Ortschaft verwaltungstechnisch

263 POSENER-KRIÉGER (1986: ebenda).

264 Alle Angaben nach POSENER-KRIÉGER (1975: 212).

265 PoSENER-KriÉGER (1975: 213); von Rolle IV existieren zumindest zwei Abbildungen: PosENERKRIÉGER (1986: Taf. 2, 4). 
erfaßt wurden. Obgleich dieser Aktentypus keinesfalls eine Ausnahme in der altägyptischen Administration darstellt, liegt mit dem Beispiel aus Gebelein der älteste Beleg dafür vor. Christopher EYRE bringt diese Listen in Verbindung mit anderweitigen Aushebungen von Corvée für verschiedenste Arbeitstätigkeiten im großen Stil und und weist darauf hin, daß diese genau wie die im folgenden genannten $n f r . w$ - als Ungelernte mit niedrigem Status zur Gruppe der >Arbeitermasse< gezählt werden müssen, ${ }^{266}$ wobei zu vermuten ist, daß diese Listen keinen Gesamtbestand an verfügbarem Personal wiedergaben, aus denen man sich bei Bedarf bedienen konnte, sondern nur die tatsächlich herangezogenen Personen erfaßte, was durch die explizite Nennung der Arbeitsmaßnahme in der Überschrift bestätigt wird.

Die >Rolle IV < liefert nicht nur interessante Einblicke in die Struktur von Verwaltungsabläufen des Alten Reiches, sondern bietet auch einige Fakten, die bisher nur auf der Grundlage von Schätzungen in die demographische Forschung mit einbezogen wurden. Die Liste trägt die Bezeichnung 20 (t $3 w)$, was von POSENER-KRIÉGER mit »registre u übersetzt wird. ${ }^{267}$ Die Namen der beiden Orte, die dem $p r-\underline{d} t$ angehören, sind ebenfalls aus dem Pa-

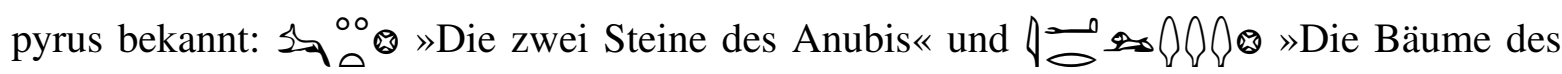

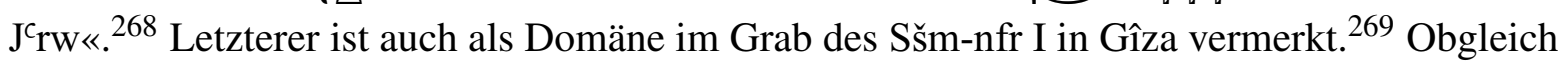
das Grab des Sšm-nfr I in den Beginn der 5. Dynastie datiert wird, spricht nichts grundsätzlich dagegen, daß die Domäne bereits zuvor existiert hat, so daß die Chronologie hinsichtlich der Gebelein-Papyri erhalten bleibt. Eine weitere bemerkenswerte Beobachtung besteht darin, daß eine beträchtliche Anzahl der in Rolle IV verzeichneten Bewohner den Titel hm-nzw, beziehungsweise $h m . t-n z w$ trugen. Der Status dieser Personen, deren Bezeichnung ursprünglich erst ab dem Mittleren Reich bekannt ist, ${ }^{270}$ läßt sich nur schwer feststellen. POSENER-KRIÉGER versuchte durch Heranziehung zweier Belege aus dem Alten Reich eine Klärung des Sachverhalts. Aus zwei Gräbern von hohen Beamten, die gegen Ende der 5. Dynastie als oberste Verwalter des 15. und 16. oberägyptischen Nomos fungierten, ist die Bezeichnung hm-nzw bereits bekannt. ${ }^{271}$ In beiden Fällen arbeitete die Personengruppe bei der Getreideernte. Dennoch ist nicht gesichert, ob die hm.w-nzw ausschließlich in der Landwirtschaft tätig waren und ob sie für die Nomos-Verwalter persönlich arbeiteten. Genausogut wäre vorstellbar, daß die beiden Beamten diese Gruppe im Rahmen ihrer Aufsichtstätigkeiten in ihren Gräbern erwähn-

266 EYRE (1987: 19).

267 Zur Diskussion vgl. Posener-KriéGer (1975: 216 Anm. 17).

268 POSENER-KRIÉGER (1975: 218).

269 JACQUET-GORDON (1962: 240).

270 Vgl. HAYES (1955: 90 ff.) und BAKIR (1952: 30).

271 PosenER-KRIÉGER (1975: 218 f.). 
ten. Andererseits läßt sich für das $p r-\underline{d} t$ der Rolle IV auch nicht klären, ob es sich dabei um eine landwirtschaftliche Domäne gehandelt hat, die dem Königshaus unterstellt war.

Folgt man weiter William HAYES' Ausführungen, dann waren diese $h m . w-n z w$ nicht an den Hofstaat des Königs gekoppelt, sondern erhielten ihren Status als >Diener ${ }^{272}$ zwar durch die Autorität des Königs, waren aber auch nicht-königlichen Personen für Arbeitsdienste zugewiesen. Zumindest muß der betroffene Personenkreis einen recht niedrigen Rang innerhalb der sozialen Hierarchie eingenommen haben, da HAYES für die spätere Zeit Beispiele für $>$ Vorsteher der $h m . w-n z w<$ zitiert, die gleichgestellt waren mit >Vorstehern der Rinder $<$ oder $>$ Vorstehern der Pferde $<.273$

Neben einigen Schreibern und anderen Personen mit speziellen Aufgaben werden in den Papyri zu einem überwiegenden Teil Handwerker verschiedenster Prägung erwähnt. ${ }^{274}$ So zeigt sich die Bevölkerung in einer typischen dörflichen Zusammenstellung, auch wenn in den Akten auf die Verwandtschaftsangaben zu Frauen, Kindern oder Eltern verzichtet wird. Die wenigen Argumente zusammengenommen, läßt sich etwa folgender Entwurf der Situation im Alten Reich skizzieren: Neben der Verpflichtung zu Gemeinschaftsarbeiten in größerem Umfang waren die Bewohner des pr-d $t$ auch zu Abgabenleistungen verpflichtet. ${ }^{275}$ Erwähnt werden in den Aufstellungen anscheinend nur diejenigen Personen, die dazu volle Arbeitskraft erbringen - Männer und Frauen gleichermaßen. Aus der Anzahl von 99 Personen kann geschlossen werden, daß für jedes der beiden Dörfer als Mittelwert 50 Personen dafür herangezogen wurden. Obgleich sich weitere Rechenexempel hinsichtlich der Gesamtzahl inklusive Kindern und Alten aufgrund unserer mangelnden Kenntnis der altägyptischen Alterspyramide verbieten, scheint die Größenordnung einer Ansiedlung, wie sie zum Beispiel von Rolf GundLACH aus den Annalen-Einträgen des Königs Snofru errechnet wurden, plausibler geworden zu sein. ${ }^{276}$ Zumindest passen die Größenordnungen, die aus beiden Quellen gewonnen wurden, gut zueinander.

Eine weitere Textstelle aus dem Alten Reich liefert einen Hinweis darauf, wie in verschiedenen Fällen solche Siedlungen aufgrund des Eingreifens durch die staatliche Administration entstanden. Es handelt sich dabei um die Biographie des Ḥnq.w aus Deir el-Gebrawi im 12. oberägyptischen Nomos, dessen Grab ungefähr in den Übergang zwischen Altem Reich und Erster Zwischenzeit datiert wird. Dort heißt es unter anderem: ${ }^{277}$

272 Der Terminus >Sklave< soll an dieser Stelle vermieden werden, da der soziale Status der $h m . w-n z w$ kaum zweifelsfrei geklärt werden kann.

273 HAYES (1955: 91).

274 PoSENER-KRIÉGER (1975: 219).

$275 \mathrm{Zu}$ den Strukturen und Verpflichtungen eines $p r-\underline{d} t$ vgl. auch GOEDECKEN (1976: $307 \mathrm{ff}$.).

276 Siehe dazu ausführlich Kap. 5.2.3.4.

277 Siehe auch Helck (1958: 119 Anm. 6). 


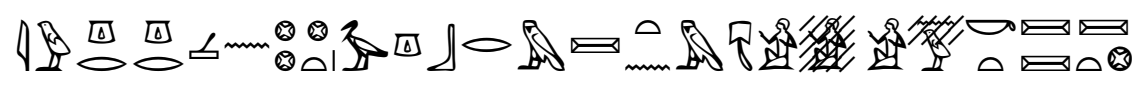

jw-gr grg.n(=j) njw.wt bjgb-r'm sp3.t tn $m^{c}$ w.t rmt.w n.w k.t sp3.wt

$»$ Auch habe ich die verstummten ${ }^{278}$ Städte in diesem Nomos mit Vieh und Leuten aus anderen Nomoi wieder eingerichtet.«

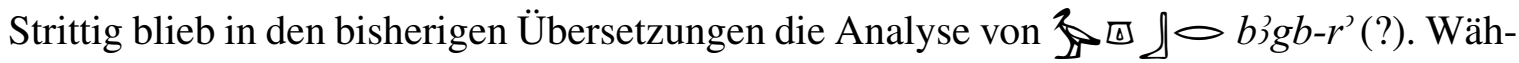
rend Wolfgang SCHENKEL es als Zeichen einer Depopulation auffaßt, der mit staatlichen Maßnahmen begegnet werden muß, läßt Wolfgang HELCK die unklare Stelle völlig offen und interpretiert den gesamten Vorgang als eine reine Umsiedlungsmaßnahme, quasi ein Standortwechsel von Arbeitstruppen. Eindeutiger ist dagegen der Ausdruck $\uparrow$ wohl kein unbekanntes Lexem verbirgt, wie etwa das von SCHENKEL vermutete, gleichwohl mit Fragezeichen versehene >Arbeiter , sondern eine Kombination von Tier und Mensch, wie es hier als ${ }^{c} w . t$ und rmt vorgeschlagen werden soll. Das Mitführen von Kleinvieh stellt für die Selbstversorgung der Leute eine sinnvolle zusätzliche Maßnahme dar. Die gesamte Szenerie paßt zudem in die Vorstellung, daß die Binnenkolonisation ein zentrales Anliegen des ägyptischen Staates im Alten Reich war, auch wenn die vorliegende Textstelle tendenziell an das Ende dieser Epoche datiert wird und zu diesem Zeitpunkt in Oberägypten aufgrund von Ressourcenverknappung ein starker Rückgang der Kolonisationsmaßnahmen zu beobachten ist. ${ }^{279}$ Auch läßt sich die Dreifachsetzung des Ideogramms anstelle des geläufigeren $\frac{\text { p. }}{11}$ problemlos mit der Entstehungszeit des Textes in Einklang bringen.

\subsection{Die Kahun-Papyri}

\subsubsection{Herkunft}

Zwischen 1889 und 1890 grub William Matthew Flinders PETRIE die antike Arbeitersiedlung neben dem modernen Ort el-Lahun aus. ${ }^{280}$ Unter den Funden, die dabei zutage traten, befand sich auch eine beträchtliche Anzahl Papyri, die offenbar einem Tempel-Archiv entstammten. Der genaue Kontext des Fundortes ist unklar, doch dürfte es sich um eine Abraumhalde gehandelt haben, wie sie auch Ludwig BORCHARDT beschrieb, der dort 1899 den zweiten großen Papyrusfund machen konnte. ${ }^{281}$ Die von PETRIE gefundenen Papyri befinden sich heute im

278 Vgl. dazu SchenKel (1965: 43 Anm. h).

279 Müller-WollermanN (1987: 266 f.); Müller-Wollermann (1986: 101 f.); SCHENKEL (1978: 56).

280 PETRIE (1891).

281 BORCHARDT (1899). 
University College in London und wurden von Francis Llewellyn GRIFFITH bereits wenige Jahre nach ihrer Entdeckung in einer Auswahl der wichtigsten Stücke publiziert. ${ }^{282}$

Von den zahlreichen Texten, deren Inhalt die verschiedensten Bereiche öffentlicher Verwaltung abdeckt, interessieren hier besonders die wenigen Beispiele für die Haushaltsdeklarationen, die eine detaillierte Liste der Mitglieder eines Haushalts protokollieren. Für lange Zeit gerieten die Zensusakten aus dem Blickfeld der weiteren Forschung, bis sie in jüngster Zeit erneute Würdigung erfuhren und aktuelle Analysen der Zensusakten vorgelegt wurden. ${ }^{283}$ Die erhaltenen Fragmente der betreffenden Papyri haben im wesentlichen die fortlaufende Erfassung eines einzigen Familienverbands zum Inhalt. Es sind die Familien des Soldaten ( $\left.{ }^{c} h 3 w . t j\right)$ Dḥw.tj und seines Sohnes Ḥrj, deren Geschichte sich anhand der Fragmente grob nachzeichnen läßt. Der Zeitraum ist dabei auf die 13. Dynastie festgelegt, wie aus vereinzelt erhaltenen absoluten Datierungen hervorgeht.

Um trotz der wenig repäsentativen Ergebnisse hinsichtlich der Familienstrukturen eine prinzipiell bessere Vergleichbarkeit zu ermöglichen, wurde im folgenden die Struktur der Familien, wie sie den Zensusakten zu entnehmen ist, in das Schema von Eugene HAMMEL und Peter LASLETT übertragen, das bereits bei den Auswertungen der römischen Zensusakten durch Roger BAGNALL und Bruce FRIER Anwendung fand. ${ }^{284}$ Im Gegensatz zu den in griechischrömischen Zensusdeklarationen oft anzutreffenden Altersangaben beschränken sich die Einträge in den pharaonischen Akten auf die Unterscheidung zwischen Männern (通), Frauen ( schieden wird, sondern lediglich am Namen oder am grammatikalischen Geschlecht der Verwandtschaftsbezeichnung erkennbar ist. Wie alt die Kinder tatsächlich waren und ab welchem Alter sie als Erwachsene erfaßt wurden, entzieht sich so zumeist unserer Kenntnis. Lediglich größere Zeiträume lassen sich aus den Determinativen ziehen, wie etwa der Vermerk 单负 auf dem Fragment I.4, der auf ein jüngst geborenes Kind hinweist, oder gelegentliche explizite Altersangaben hinter dem Kind-Determinativ. ${ }^{285}$ Warum jedoch gerade eine relativ fein abgestufte Determinierung für Kleinstkinder in verschiedenen Stadien des Alters vorzufinden ist, während sich für ältere Kinder und Erwachsene praktisch keine Klassifizierung nachweisen läßt, muß unbeantwortet bleiben.

\footnotetext{
282 GRIFFITH (1898).

283 KeMP (1991: 156 ff.); VAlbelle (1985a); neuerdings Kóthay (2001).

284 Hammel und Laslett (1974), Bagnall und Frier (1994). Auch Kóthay (2001) folgt dem Modell, übernimmt aber nicht dessen graphische Umsetzung.

285 GRIFFITH (1898: 28, Taf. 10,16), dort auch mit weiteren Beispielen.
} 


\subsubsection{Aufbau \& Struktur}

Auf die Bedeutung des Terminus wpw.t, der im Fall der Kahun-Papyri als immer wiederkehrender Begriff für die Deklarationen Verwendung findet, und auf Vergleiche mit Deklarationen anderer Epochen wird später in Kap. 5.2.2.4 genauer eingegangen. Die Papyri sind in der chronologisch wahrscheinlichsten Reihenfolge wiedergegeben. ${ }^{286}$ Nicht berücksichtigt wurden für die Diagramme das Fragment I.6, da es keine verwertbaren Informationen zur Familienstruktur trägt und auch bei GRIFFITH gar nicht erst abgebildet war, sowie das Fragment I.4 recto. Das längste und ausführlichste Fragment IV.1 wird zusätzlich komplett in Hieroglyphen, Transliteration und Übersetzung nach der Edition von GRIFFITH wiedergegeben. ${ }^{287}$

Datierung: Unbekannt

\section{Struktur:}

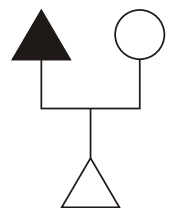

Deklarant: Hrj

\section{Familienmitglieder:}

1. seine Ehefrau Šps.t

2. sein Sohn Snfr.w

freie Nichtangehörige, Sklaven: Keine

Quelle: GRIFFITH (1898: I.4. verso Taf. 9)

Diskussion: Zu Beginn des Fragments ist noch ein beträchtliches Stück erhalten geblieben, das den Ort der Deklaration und den zuständigen Beamten vermerkt. Indiz für die relative Chronologie innerhalb der Fragmente ist das Wort jm.t-pr(w) (>Testament $<$ ) auf dem recto, aus dem geschlossen wurde, daß im Todesjahr von Ḥjj's Vater Dḥw.tj von beiden Haushalten eine Aufstellung gemacht wurde, die auf dem gleichen Papyrus Platz fand. Die Tatsache, daß Hrj’s Sohn Snfr.w gerade erst geboren wurde und es sich somit um

286 Vgl. GRIFFITH (1898: 24); alle späteren Bearbeiter sind seiner Chronologie ohne Modifikationen gefolgt.

287 Unklare Passagen in der Rubrik >Rufnamen< sind in der Übersetzung unverändert und kursiv wiedergegeben. Zum zweiten Fragment, Z. 6 und 8, vgl. Vernus (1986: 77, Nr. 355 und 357); allgemein zu den >Rufnamen< auch VERNUS (1986: 98 f. § 12). 
eine recht junge Familie handelt, die einen eigenen Haushalt gegründet hat, könnte mit Blick auf die Inhalte der anderen Fragmente - die These stützen.

Datierung: Unbekannt

\section{Struktur:}

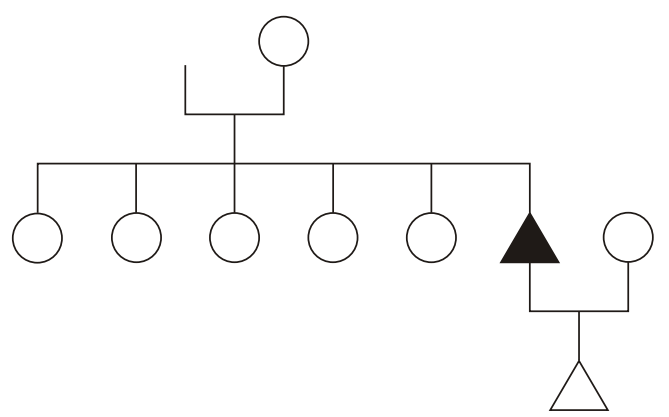

Deklarant: Hirj

\section{Familienmitglieder:}

1. seine Ehefrau Šps.t

2. sein Sohn Snfr.w

3. seine Mutter H’̉-rh-nj

4. ihre Tochter Z3.t-Snfr.w (= Hrj's Schwester)

5. (ihre Tochter) Mktn

6. ihre Tochter 3s.t

7. ihre Tochter Rwd.w.t

8. ihre Tochter Snfr.w

freie Nichtangehörige, Sklaven: Keine

Quelle: GRIFFITH (1898: I.5. Taf. 9)

Diskussion: Die Geschwister des Ḥrj werden als Filiation seiner Mutter angegeben. Seit der Deklaration I.4 ist in der Organisation des Haushalts einiges geschehen. Nach dem Tod seines Vaters nahm Hrj dessen Haushalt in seinen eigenen mit auf. Wann genau dies passierte, kann nicht mit Sicherheit erschlossen werden. Mit Hinweis auf das Alter des Kindes sprach GRIFFITH von »very little later than I.4«, ${ }^{288}$ während Katalin KÓTHAY 288 GRIFFITH (1898: 24). 
aus unbekannten Gründen diese Deklaration genau ein Jahr später als I.4 datiert. ${ }^{289}$ Weil auch unter der Voraussetzung, daß das Kind-Determinativ tatsächlich mit einer Jugendlocke im Papyrus vermerkt ist, eine Datierung ungenau bleiben muß, da das Tragen der Jugendlocke einen weiten Zeitraum abdecken kann, scheinen die bisherigen Datierungsversuche zu optimistisch. Der Zeitraum läßt sich lediglich auf die ersten Jahre nach dem Tod Dḥw.tj’s eingrenzen. Andernfalls müßte eine Zwangszusammenführung vermutet werden, nachdem das Familienoberhaupt Dḥw.tj verstorben ist und H’3-rh-nj keinen eigenen Haushalt führen darf. Dafür gibt es jedoch keine Evidenz.

Datierung: 13. Dynastie; Jahr 3 des Jmn-m-ḥ̉t-(z')-snb=f

\section{Struktur:}

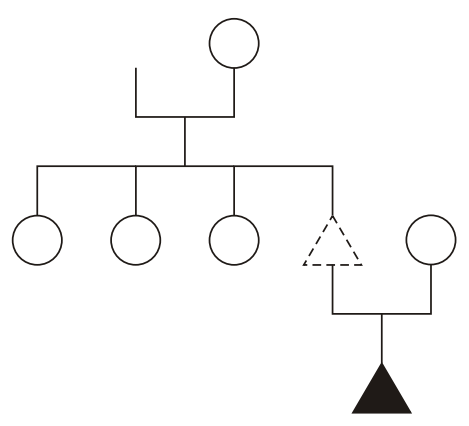

Deklarant: Der Soldat Snfr.w, Sohn des Ḥrj

\section{Familienmitglieder:}

1. seine Mutter Šps.t

2. die Mutter seines Vaters $\mathbf{H}_{3}$-rh-nj

3. die Schwester seines Vaters Kjj.t-sn.wt

4. die Schwester seines Vaters is.t

5. die Schwester seines Vaters Z3.t-Snfr.w

freie Nichtangehörige, Sklaven: Keine

Quelle: GRIFFITH (1898: I.3. Taf. 9)

Diskussion: Mit dieser Deklaration endet die uns bekannte >Familienchronik $<$. Snfr.w, der zum Zeitpunkt der Abfassung von I.4 gerade geboren und in I.5 noch als Kind erwähnt

289 KÓTHAY (2001: 353). 
wurde, ist nun selbst zum Familienvorstand aufgerückt. Zwei seiner Tanten haben den Haushalt verlassen, haben also entweder eigene Familien gegründet oder sind verstorben. Da das Fragment einen Hinweis darauf enthält, daß Snfr.w's Vater noch innerhalb des vorangegangenen Jahres eine eigene Deklaration vorgelegt hatte, kann daraus geschlossen werden, daß Hrj zwischen dem zweiten und dritten Regierungsjahr des Jmnm-hảt-(z) $)$-snb=f starb. Bemerkenswert ist, daß Snfr.w im wesentlichen die Liste, die bereits aus I.5 bekannt ist, bestätigt. Weder von einer Ehefrau Snfr.w's noch von Kindern ist die Rede. Also hat Snfr.w ein Alter erreicht, in dem er zwar bereits >geschäftsfähig war, aber noch keine eigene Familie gegründet hatte. Es ist auch zu vermuten, daß alle Personen immer noch unter dem gleichen Dach wohnten.

Datierung: 13. Dynastie; Jahr 1 des Shm-R $\mathrm{R}^{\mathrm{c}}$ hwj-t\}wj

\section{Struktur:}
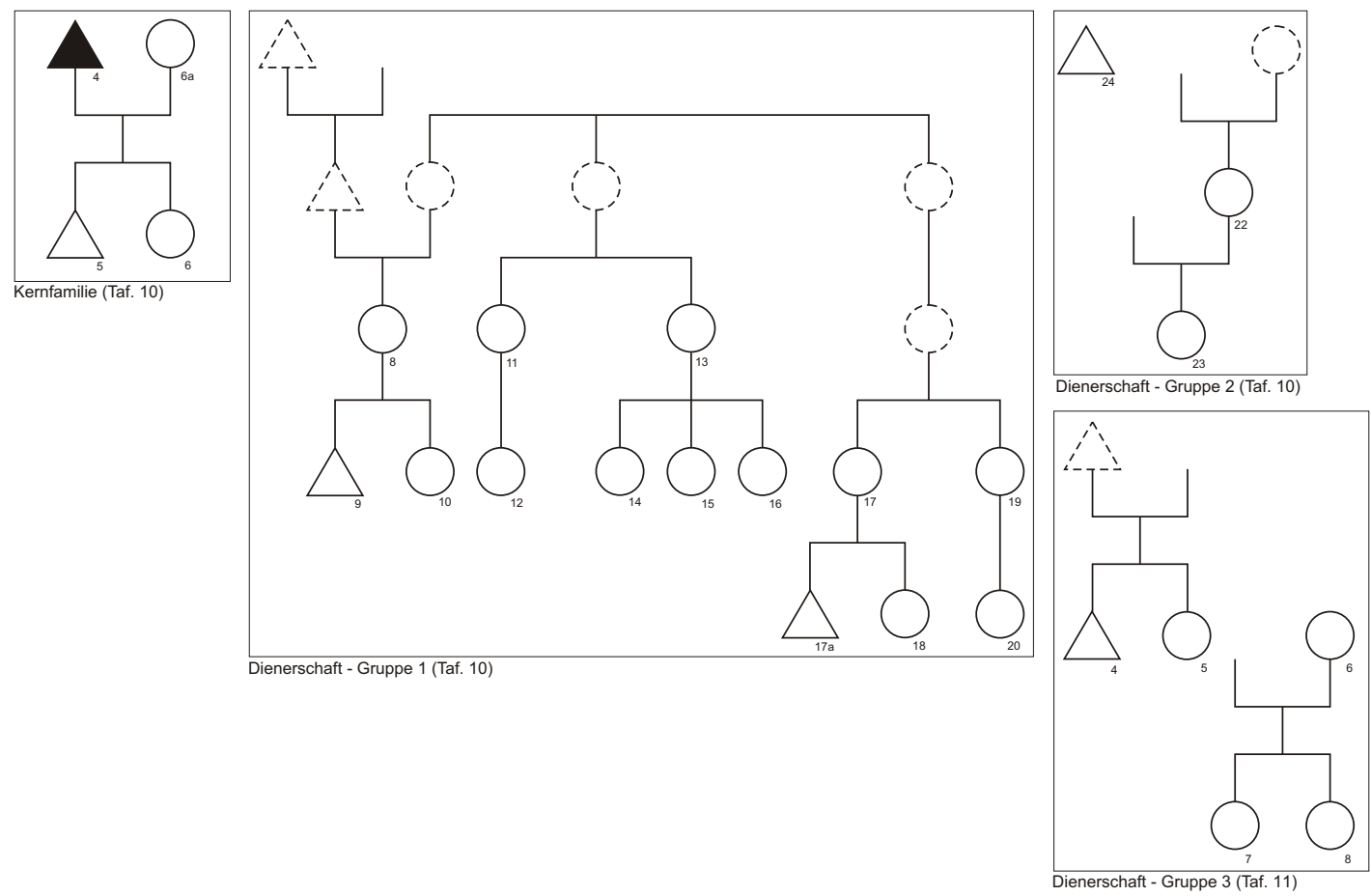

Deklarant: Der Vorlesepriester $\underline{H}^{c} \mathrm{j}-\mathrm{k} 3 . \mathrm{w}-\mathrm{R}^{\mathrm{c}} \cdot \mathrm{w}$

\section{Familienmitglieder:}

1. sein Sohn Zj-nj-wsr.t-snb

2. seine Tochter Zn.t 


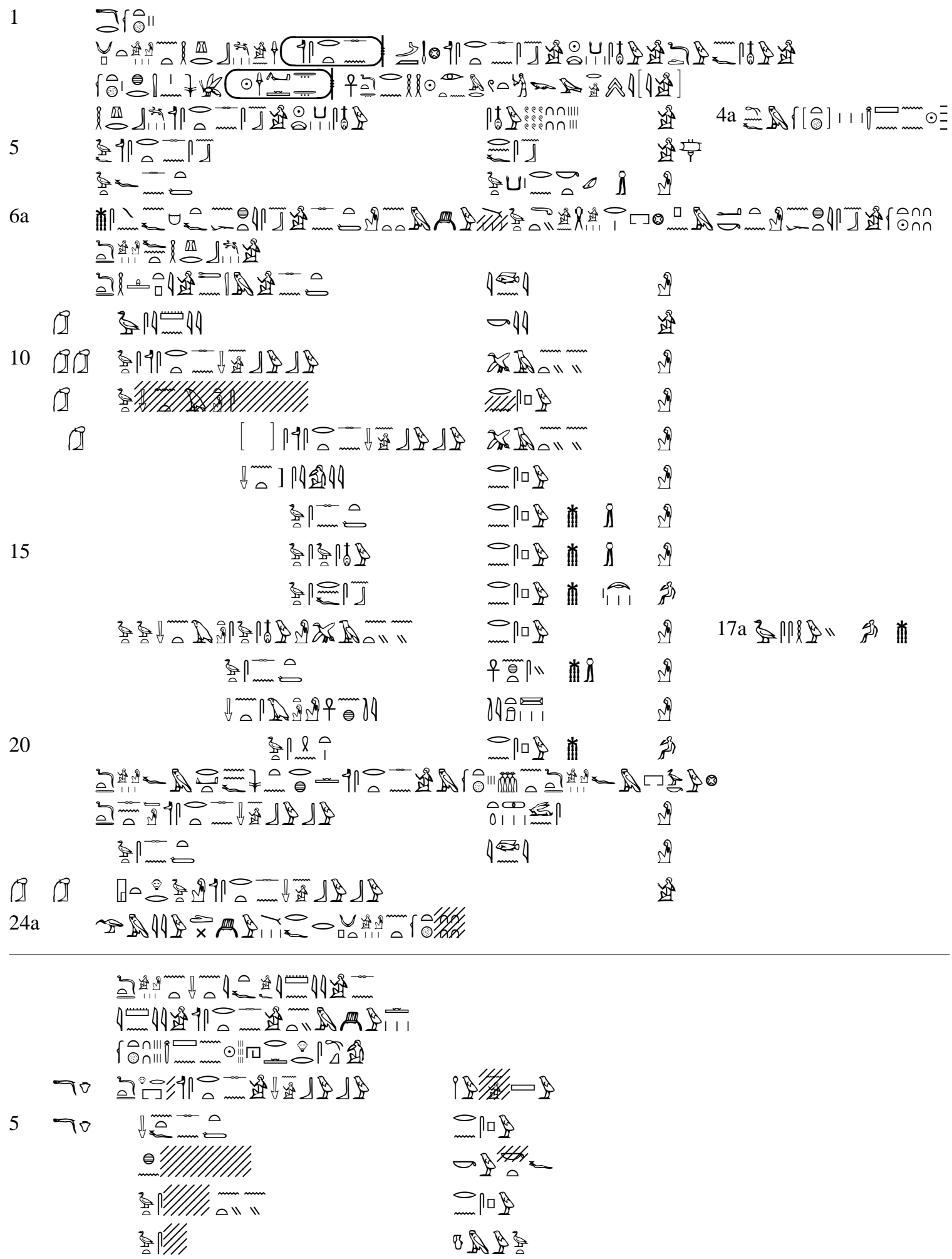

Papyrus Kahun, Fragment IV.1 - Hieroglyphische Umschreibung 
$1 \quad$ mh r r rnp.t 2

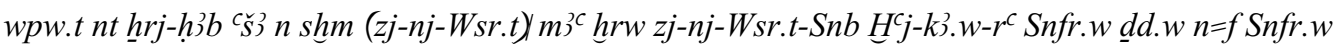

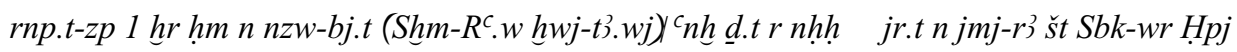

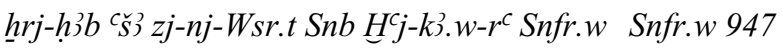

4a ${ }^{c}$ rq. $n=f m$ rnp.t-zp 3 tp-šmw sw 4

$5 \quad z 3=f z j-n j-W$ sr.t $S n b$

$$
r n=f \operatorname{Snb}
$$

$z$ 3.t $=f Z n . t$

z’.t k3 rn ktt

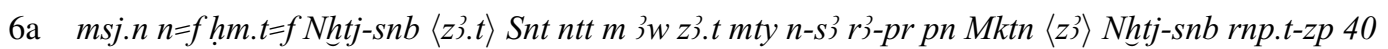

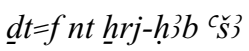

dt Htpj Tnm Zn.t

Jnj

$z 3=s \mathrm{Jmn} \cdot j j$

$K j j$

10

z3.t=s Wsr.t-sn Snbwbw

P)-ntj-nj

z3.t Zn.t mw.t=s...

$r n=s-p w$

$[z 3 . t]=s$ Wsr.t-sn Snbwbw

P3-ntj-nj

$s n . t=s \mathrm{Jjj}$

$r n=s-p w$

$z$ 3.t $=s \mathrm{Zn.t}$

$r n=s-p w$

15

$z 3 . t=s$ Z3.t-Snfr.w $\quad r n=s-p w$

$z 3 . t=s R n=f-s n b \quad r n=s-p w$

z3.t z3.t sn.t mw.t=s Z3.t-snfr.w P3-ntj-nj

$r n=s-p w$

17a $z 3=s S h w j$

$z$ 3.t $t=s$ Zn.t

cnh.t-sj

sn.t=s Mw.t- ${ }^{c}$ h..$t j$

Tjt?

20

$$
z 3 . t=s \check{S} n . t \quad r n=s-p w
$$

$\underline{d} t=f m$ rdj.t $n n=f r h-n z w$ Wsr.t-sn $m$ rnp.t-zp 3 hntj $\underline{d} t=f m$ pr-bjkw

dt Zn.t Wsr.t-sn Snbwbw

$T-w n=s$

z3.t=s Zn.t

Hw.t-hrr z3.t Wsr.t-sn Snbwbw

24a gmj.w wdj $3 w$ rf $r$ wpw.t nt rnp.t-zp 40

$\underline{d}$ t nt sn.t jtj=f Jmn.jj $\mathrm{Zn}\langle. t\rangle$

Jmn.jj zj-nj-Wsr.t ntj $m$ 3w

rnp.t-zp 26 tp-šmw sw 9 hrw hr=s ${ }^{c} r q$

dt hrj-pr Wsr.t-sn Snbwbw

$W \underline{d}-n-\check{S} w$

5

$$
\begin{aligned}
& \text { sn.t }=f Z n . t \\
& \text { hn... } \\
& z 3 . t=s \ldots\langle P\rangle\rangle-n t j-n j \\
& z \text { ). } t=s \ldots
\end{aligned}
$$$$
r n=s-p w
$$$$
k w-k t t=f
$$$$
r n=s-p w
$$

Hnmw z 3.t

Papyrus Kahun, Fragment IV.1 - Transliteration 
Das zweite Jahr

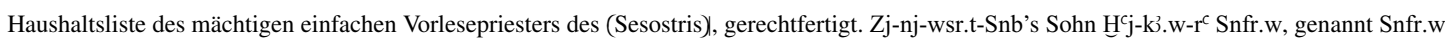
Jahr 1 seiner Majestät des Königs (Shm-R ${ }^{c}$.w ḩwj-t3.wj), er lebe ewig; erstellt durch den Steuerbeamten, Sbk-wr's Sohn Ḥj Der einfache Vorleser, Zj-nj-wsr.t Snb's Sohn $\underline{H}^{c} j-k 3$ j.w-r ${ }^{c}$ Snfr.w （genannt) Snfr.w 947 4a hat im Jahr 3/1. šmw/4. Tag einen Eid abgelegt 5
sein Sohn Zj-nj-wsr.t Snb
genannt Snb
seine Tochter Zn.t
z3.t k3 rn ktt

$6 \mathrm{a}$ Es hat ihm geboren seine Frau Nhtj-snb's Tochter Snt, die verstorben ist, die Tochter des Phylenvorstehers dieses Tempels, Mktn's Sohn Nhtj-snb, (im) Jahr 40.

seine Diener, die zum Priesteramt gehören

die Dienerin, Tochter von H̦tpj’s Sohn Tnm, (namens) Zn.t Jnj

ihr Sohn Jmn.jj $\quad$ Kjj

10

ihre Tochter zj-nj-wsr.t Snj Bwbw P3-ntj-nj

die Tochter ihrer Tante (Schwester ihrer Mutter) ... selber Name

ihre [Tochter?] Zj-nj-wsr.t Snj Bwbw P3-ntj-nj

[ihre Schwester] Jjj selber Name

ihre Tochter Zn.t selber Name

15

ihre Tochter Z3.t-Snfr.w selber Name

ihre Tochter Rn=f-snb selber Name

die Tochter ihrer Cousine Z3.t-snfr.w's Tochter P'-ntj-nj $\quad$ selber Name $\quad 17 \mathrm{a}$ ihr Sohn Shwj

ihre Tochter Zn.t $\quad$ cnh.t-sj

ihre Schwester Mw.t- ${ }^{-}$nh.tj $\quad$ Tjt?

20 ihre Tochter Šn.t selber Name

die Diener, die ihm vom >Königsbekannten $<$ Zj-nj-wsr.t im Jahr 3 aus dessen Dienerschaft des Personalgebäudes übergeben wurden.

die Dienerin, Zn.t’s Tochter Zj-nj-wsr.t Snj Bwbw

$\mathrm{T}-\mathrm{wn}=\mathrm{s}$

ihre Tochter Zn.t

Ḥw.t-ḥr's Sohn Zj-nj-wsr.t Snj Bwbw

24a Es wurde amtlich festgestellt, daß ihm der Todesfall in Bezug auf die Haushaltsliste des Jahres 40 angezeigt wurde.

die Diener seiner Tante, Jmnjj’s Tochter $\mathrm{Zn}\langle. \mathrm{t}\rangle$

Jmnjj's Sohn Zj-nj-wsr.t, der verstorben ist

Jahr 26/1. šmw/9. Tag von ihr; Schwur

der Diener und Hausverwalter Zj-nj-wsr.t's Sohn Snj Bwbw Wd-n-Šw

5

$\begin{array}{ll}\text { seine Schwester Zn.t } & \text { selber Name } \\ \ldots & k w \text { - } k t t=f \\ \text { ihre Tochter ... }\langle\mathrm{P} 3\rangle \text {-ntj-nj } & \text { selber Name } \\ \text { ihre Tochter ... } & \underline{\text { Hnmw z3.t }}\end{array}$

Papyrus Kahun, Fragment IV.1 - Übersetzung 


\section{freie Nichtangehörige, Sklaven:}

1. Die zum Priesteramt gehörenden Diener:

a) die Tochter von Ḥtpj's Sohn Tnm, (namens) Zn.t

b) ihr Sohn Jmn.jj

c) ihre Tochter Zj-nj-wsr.t Snj Bwbw

d) die Tochter ihrer Tante (Schwester ihrer Mutter) [Name fehlt]

e) (ihre Tochter?) Zj-nj-wsr.t šnj Bwbw

f) (ihre Schwester?) Jjj

g) ihre Tochter Zn.t

h) ihre Tochter Z3.t-Snfr.w

i) ihre Tochter $\mathrm{Rn}=\mathrm{f}$-snb

j) die Tochter ihrer Cousine, Z3.t-Snfr.w's Tochter P3-ntj-nj

k) ihr Sohn Shww

1) ihre Tochter $Z n . t$

m) ihre Schwester Mw.t- ${ }^{c}$ hh.tj

n) ihre Tochter Šn.t (?)

2. Die vom $r h$ - nzw Zj-nj-wsr.t übernommenen Diener

a) Zn.t's Tochter Zj-nj-wsr.t Snj Bwbw

b) ihre Tochter Zn.t

c) Z3.t-Ḥthr's Sohn Zj-nj-wsr.t Snj Bwbw

3. Die von seiner Tante Zn.t übernommenen Diener

a) der Hausverwalter, Zj-nj-wsr.t's Sohn, Snj Bwbw

b) seine Schwester Zn.t

c) Hnm.t

d) ihre Tochter P'-ntj-nj

e) ihre Tochter [Name fehlt]

f) [hier bricht der Text ab]

Quelle: GRIFFITH (1898: IV.1. Taf. 10-11)

Diskussion: Die Aufzeichnung dieser Deklaration birgt einige Widersprüche, die sich auf den ersten Blick nicht auflösen lassen. Um die komplexe Struktur der Angaben besser abbilden zu können, stellen die Zahlen im Diagramm - entgegen den Vorgaben von HAMMEL und LASLETT - keine Altersangaben dar, sondern geben die Zeilen im Papyrus an, auf die sich das Symbol bezieht. 
Die Kernfamilie ist nicht (mehr) vollständig. Neben dem Vater stehen lediglich die leiblichen Kinder - Sohn und Tochter - auf der Liste, während seine Ehefrau nur durch einen Aktenvermerk hinter dem Namen der Kinder Erwähnung findet. Besonders auffällig jedoch ist die Liste der Dienerschaft, zweifellos eine beträchtliche Anzahl, die auf einen reichen Besitzer hinweist. Bereits GRIFFITH bemerkte, daß bis auf zwei erwachsene Söhne ausschließlich Frauen als Diener aufgelistet wurden. ${ }^{290}$ GRIFFITH versuchte eine Erklärung, indem er die Namen der Männer auf anderen Zensuslisten vermutete, ${ }^{291}$ doch scheint dies aus praktischer Sicht kaum wahrscheinlich. Es würde bedeuten, daß Diener zwar ein gemeinsames Leben einschließlich Kindern führen, jedoch fast sämtlich in verschiedenen Haushalten leben und arbeiten. Oder aber GRIFFITH hat mit seiner (angedeuteten) zweiten Vermutung recht, daß die Dienerinnen keine Lebensgemeinschaft mit den Vätern ihrer Kinder führen. ${ }^{292}$ GRIFFITH hielt es nicht einmal für gesichert, daß Familie und Diener unter dem gleichen Dach leben. Das würde allerdings der Intention dieser Listen, die einen Haushalt und dessen Bewohner erfassen, elementar widersprechen.

\subsubsection{Zur Interpretation der Aktenvermerke}

Auch in anderen Details bereitet das Fragment IV.1 Schwierigkeiten. Am linken Rand befinden sich hinter der Zeile des Deklaranten und seines Sohnes zwei >Aktenvermerke <, deren Inhalt kontrovers diskutiert wurde. Der erste Vermerk bezieht sich auf einen Schwur, den der Deklarant zu einem bestimmten Datum abgelegt hatte. Der zweite Vermerk stellt eine Ergänzung zur offiziellen Personenliste der Deklaration dar: ${ }^{293}$

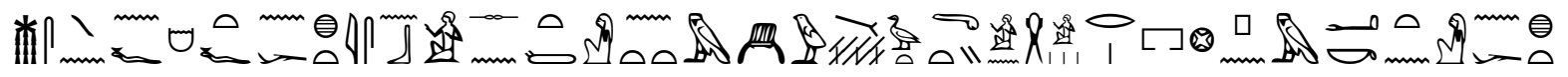
ำ 造 $\left\{\begin{array}{l}\odot \cap \cap \\ \odot \cap \cap\end{array}\right.$

$m s j . n n=f h m . t=f$ Nhtj-snb $\langle z 3 . t\rangle$ Snt ntt $m$ 3w z3.t mtj-n-s3 rij-pr pn Mktn $\left\langle z^{3}\right\rangle$ Nhtj-snb rnp.t-zp 40

»Es hat ihm geboren seine Frau, Nhtj-snb's Tochter Snt, die verstorben ist, die Tochter des Phylenvorstehers dieses Tempels, Mktn's Sohn Nhtj-snb, (im) Jahr 40.«

290 Überraschend an der Ausschließlichkeit von Frauen ist, daß aus anderen Quellen Männer oder gemischte Gruppen von $\underline{d} t$ bekannt sind, z. B. Stele Kairo Cat. Gén. 20161 oder auch die Textstelle Bauer B1 20 (R 6.6 [alt: R 40]), die von einem Mann und seinem Sohn als $\underline{d} t p w n t ~ j m j-r 3$ pr-wr spricht. Eine Klärung der Geschlechterverteilung ist nur schwer möglich, da der Tätigkeitsbereich der $\underline{d} t$ nicht eindeutig umrissen ist.

291 GRIFFITH (1898: 28).

292 Eine dritte Überlegung sei hier nur am Rande erwähnt, da sie reine Spekulation ist: der Hausherr selbst könnte in einigen Fällen Vater der Kinder seiner Dienerinnen gewesen sein.

293 GRIFFITH (1898: Taf. 10,6a). 
GRIFFITH selbst war in seinen Lesungen noch unsicher und übersetzte $n t t m 3 w$ als $>$ reich sein<, doch hat sich mittlerweile als Übersetzung eher >tot, verstorben< durchgesetzt, zumal es dafür eine Anzahl weiterer Belege mit gesichertem Kontext gibt. ${ }^{294}$ Eine neue Interpretation der fraglichen Stelle hat jetzt KÓTHAY vorgelegt. ${ }^{295}$ Sie deutet den Vermerk als Erweiterung der bestehenden Liste und will $n t t m 3 w$ als »as an extension « verstehen. Obwohl dieser Vorschlag gut in das von KÓTHAY beschriebene Szenario des Ursprungs dieser Ergänzung paßt, stehen dem jedoch schwerwiegende Argumente entgegen. Zwei Wortfelder lassen sich für $3 w$ definieren: zum einen >Schaden, Übel, Todesfall<, zum anderen >Länge, Ausdehnung, Weite<, was sowohl räumlich als auch zeitlich aufgefaßt werden kann. Keinen Beleg gibt es jedoch in der Verwendung des Begriffs im übertragenen Sinne als >ausdehnen< oder >erweitern <, obwohl eine grundsätzliche Möglichkeit dafür nicht von der Hand zu weisen ist. Auch inhaltlich ist die Vorstellung einer >Ergänzung « nicht unproblematisch. Wie aus den erhaltenen Fragmenten hervorgeht, fand eine Aktualisierung der Listen entweder regelmäßig oder nach Bedarf statt. Ein fester Turnus der Erhebung läßt sich aus den wenigen Belegen nicht erschließen, was allerdings nicht bedeutet, daß es keinen gegeben hat.

Um die Hintergründe für die Ergänzung der Zeile 6a näher zu beleuchten, soll im folgenden die Deklaration nochmals ausführlicher analysiert werden. Die Deklaration wurde im Jahr 1 des Königs Shm-R ${ }^{c}$ hwwj-ţwj verfaßt. Die Identifikation und Datierung dieses Königs ist immer noch nicht zweifelsfrei geleistet, obgleich es sich durchgesetzt zu haben scheint, daß es sich auch hier um den ersten Herrscher der 13. Dynastie, der unter dem Namen Sobekhotep I. geführt wird, gehandelt haben dürfte. ${ }^{296}$ Da der Beginn der 13. Dynastie durch die Festlegung des Endes der 12. Dynastie auf das Jahr $1794 / 93$ v. Chr. chronologisch festgelegt ist, ${ }^{297}$ hängt die endgültige Fixierung des Deklarationsdatums einzig von der Identifizierung des Königs ab. КÓTHAY hat bereits zu Recht darauf hingewiesen, daß das in der Ergänzung vermerkte Jahr 40 sich nur auf die lange Regierungszeit Amenemhets III. beziehen kann. ${ }^{298}$ Je nach Einordnung des gesuchten Königs liegen zwischen dem 40. Regierungsjahr Amenemhets III. und dem Deklarationsdatum etwa 20 bis 30 Jahre. Nach der neueren Übersetzung starb die Mutter also einige Jahrzehnte zuvor unter der Herrschaft Amenemhets III. Daß die Mutter zum Zeitpunkt der Erstellung des Dokuments entweder tot oder zumindest keine Angehörige

294 Vgl. zum Ausdruck auch ERMAN (1917: 34).

295 KÓTHAY (2001: 355).

296 RyHolt (1997: 336) und VALBelle (1985a: 77) gegen VOn BeCKERATH (1999: 92 f.), der ihn als 16. Herrscher Sobekhotep II. führt.

297 VON BECKERATH (1997: 136).

298 KÓTHAY (2001: 356). Nach vON BECKERATH (1997: 135) wäre dies das Jahr 1813 v. Chr. 
des Haushalts mehr war, kann auch daraus geschlossen werden, daß sie in der regulären Liste nicht verzeichnet ist, sondern ausschließlich in der Anmerkung. KóTHAYs Kernargument gegen diese These ist das Zeichen $\bigwedge$, das hinter der Tochter in Zeile 6 vermerkt ist und nach bisheriger Interpretation für ein Kleinstkind steht, das noch getragen werden muß. ${ }^{299}$ Wenn diese Überlegung richtig ist, dann wäre die Existenz einer Tocher im Kleinkindalter von einer Mutter, die bereits lange verstorben ist, in der Tat nicht plausibel. Die gesamte Konstruktion dieser Argumentation gleicht allerdings einem Kartenhaus, da sie einzig von der Interpretation der Hieroglyphe $\bigwedge$ abhängt. ${ }^{300}$ Während ansonsten die Kinder - selbst die >älteren< mit Altersangabe - noch mit 乌̧ $^{3}$ determiniert werden, steht hinter den angeblich jüngsten das Zeichen in. Zum Vergleich sind die Kombinationen nachfolgend tabellarisch aufgestellt:

\begin{tabular}{|c|c|c|c|}
\hline Zeile(n) & Ver & merk & \\
\hline $14,15,18$ & 尚 & $\AA$ & तो \\
\hline 16 & & 111 & 㖞 \\
\hline $20,(17 a)$ & 䛔 & & 䑸 \\
\hline 6 & & $\AA$ & in \\
\hline
\end{tabular}

Neben der weit auseinandergezogenen Schreibung, die darauf hindeutet, daß die einzelnen Determinative nicht zwangsläufig eine zusammengehörige Funktion haben müssen, ist die Situation speziell für $₫$ alles andere als eindeutig. Zumindest läßt sich aus dem vorliegenden Material keinesfalls herauslesen, daß es sich um Kleinstkinder handelt. Dies wird auch durch das fehlende in in Zeile 6 unterstützt, was nicht als Schreibfehler ignoriert werden sollte. Wenn aber die Interpretation als >Kleinkind < hinfällig ist, kippt auch das Argument gegen den lange zurückliegenden Tod der Mutter. Die Kinder sind dann beide im Erwachsenenalter, wie es die Determinative am Ende der jeweiligen Zeilen auch ausdrücken. ${ }^{301}$

Damit bliebe immer noch die Frage, welche Bedeutung den Vermerken in der Akte zukommt. Wenn man sich für $₫ j n j$ die Grundbedeutung wie >herbeibringen< oder verwandte Ausdrücke ins Gedächtnis ruft, dann könnte vielleicht vermutet werden, daß die so in der Liste bezeichneten Angehörigen bei der Deklaration persönlich anwesend waren, zumal der

299 Ко́тHAY (2001: ebenda); diese Interpretation geht bereits auf GRIFFITH (1898: 28) zurück.

300 Der von Kóthay (2001: 356 Anm. 34) herangezogene Kronzeuge HelCK (1974: 61) bezieht sich in seiner Aussage einzig auf das Fragment des Kahun-Papyrus, so daß hier ein Hapax vorliegt.

301 Weitere Evidenz für Altersangaben in der hier anzutreffenden Form sind auf einem anderen Fragment aus Kahun zu finden, vgl. GRIFFITH (1898: Taf. 13,9 ff.). 
Ausdruck in Rechtsurkunden häufig für das Herbeibringen von Zeugen oder anderen Personen verwendet wird. Doch selbst dies muß im vorliegenden Fall reine Spekulation bleiben.

\subsubsection{Die Auflistung der Dienerschaft}

Neben der recht kurzen Liste der Kernfamilie des Priesters $\underline{H}^{c} \mathrm{j}-\mathrm{k} 3 . \mathrm{w}-\mathrm{R}^{\mathrm{c}}$.w ist außerdem noch eine beträchtliche Anzahl an ihm unterstellten Dienern auf der Liste verzeichnet. Sie sind in drei Gruppen unterteilt und werden auf der Deklaration getrennt vermerkt. Die größte Gruppe mit 14 Personen scheint nach Auskunft der Akte direkt an das Priesteramt des Deklaranten gebunden zu sein. Drei weitere Diener stammen aus dem Personal eines anderen Beamten, die dieser dem Deklaranten überlassen hatte, sowie mindestens fünf Personen, die eine Übernahme innerhalb der Familie von einer Schwester seines Vaters darstellen.

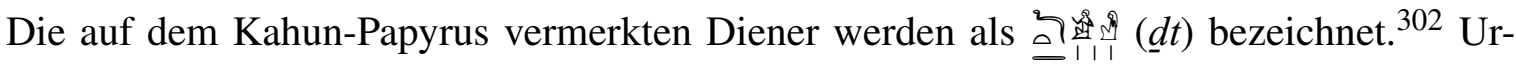
sprünglich noch als $>$ servants $<^{303}$ oder $>$ serfs $<^{304}$ übersetzt, wurde diese strikte Interpretation sehr bald in Zweifel gezogen. In seiner grundlegenden Arbeit über Sklaverei im Alten Ägypten untersuchte Abd el-Mohsen BAKIR auch den Terminus $\underline{d} t$ und kam nach Analyse der Belege zu recht zwiespältigen Resultaten. Einerseits zog er den Schluß, daß die auf diese Weise klassifizierten Personen keinesfalls zweifelsfrei als »assigned property « einer anderen Person gelten können, ${ }^{305}$ stellte aber im weiteren Verlauf fest, daß in der Erzählung des Sinuhe die Textstelle B $238 \mathrm{ff}$. durchaus darauf hinweist, daß $\underline{d} t$ als Eigentum aufgefaßt werden kann, was ihn zu einer Übersetzung als »human chattels« verleitet und von der Ausgangsposition seiner Überlegungen inhaltlich nicht weit entfernt liegt. ${ }^{306}$

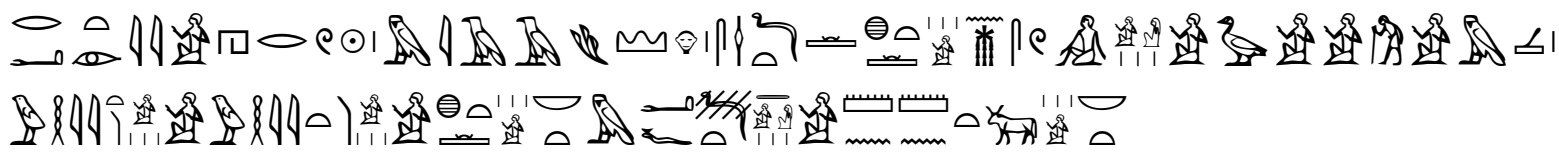

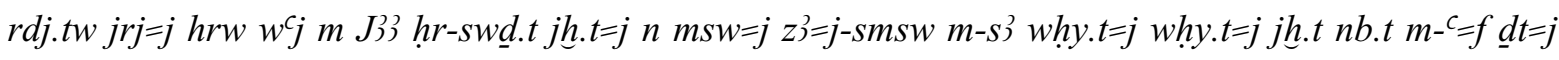
mnmn.t $=j n b . t$

»Man ließ mich einen Tag in Araru verbringen, und ich übertrug meinen Besitz auf meine Kinder. Mein ältester Sohn übernahm die Verantwortung für die Sippe; meine Sippe und mein gesamter Besitz lagen in seiner Hand: meine $\underline{d} t$, meine gesamte Herde, ... «

$302 \mathrm{Zu}$ verschiedenen hieroglyphischen Schreibvarianten vgl. BAKIR (1952: 40).

303 GRIFFITH (1898: 27), aber auch zuletzt noch PARKINSON (1997: 39).

304 GRIFFITH (1898: 28); GARDINER (1909: 48).

305 BAKIR (1952: 36 f.), bes. S. 36 a).

306 Schließlich läßt BAKIR (1952: 100) sämtliche Differenzierungen fallen und spricht im Zusammenhang mit den Kahun-Papyri wieder von »slaves«. 
Es war jedoch bereits aus dem Kahun-Papyrus ersichtlich, daß die $\underline{d} t$ in gewisser Weise von einer Person auf eine andere übertragbar sind. Anders wären die entsprechenden Passagen Kahun 10,21 und 11,1 nicht zu erklären, in denen Übernahmen von einer anderen Person einem anderen Familienmitglied - vermerkt sind. Über den juristischen Status der erwähnten Personen sagt dieses Faktum allein allerdings recht wenig aus. Auch ein - wie auch immer geartetes - Bedienstetenverhältnis kann weitergegeben oder die Verpflichtungen daraus auf die Kinder übertragen werden. HAYES mißtraute ebenfalls den bisherigen Interpretationen und deutete die $\underline{d} t$ als Mittelding zwischen einem Pächter und einem Diener. ${ }^{307}$ Er bezweifelte auch grundsätzlich, daß die $\underline{d} t$ jemals Rechtlose waren. ${ }^{308}$ Aus der Ersten Zwischenzeit gibt es Hinweise in Autobiographien, daß Diener mit niedrigstem Status, die von ihren Herren auch regelrecht gekauft werden konnten, mit dem Begriff $b 3 k$ bezeichnet wurden. ${ }^{309}$ Auch dafür liefern die Kahun-Papyri bezüglich der $\underline{d} t$ keine Evidenz, obwohl es sich auch nicht ausdrücklich ausschließen läßt, daß der Transfer dieser Personen mit einem finanziellen Ausgleich geschah.

Der neuerdings von KóTHAY dazu gemachte Vorschlag, daß es sich bei den $\underline{d} t$ eher um eine Kategorie von Arbeitern als um Hörige oder Abhängige gehandelt haben dürfte, verdient weitere Beachtung. ${ }^{310}$ Nach ihren Ausführungen verrichteten die $\underline{d} t$ ähnlich wie die $h s b w$ bestimmte Tätigkeiten, die ursprünglich ihren >Herren< auferlegt waren. Daraus folgert KóTHAY weiter, daß die wpw.t-Listen keine Zensus-Listen im engeren Sinne waren, sondern eher ein Verzeichnis der Zugehörigkeit der $\underline{d} t$-Personen zu den Staatsdienern darstellten. ${ }^{311}$ Sie übersieht allerdings, daß die zum Vergleich herangezogenen $h s b w$ anderen Verwaltungsstrukturen unterworfen waren und die $\underline{d} t$ nie in für die Corvée vorgesehenen Listen registriert wurden, sondern zumeist direkt bestimmten Personen, auch dem König, unterstellt sein konnten. ${ }^{312}$ Darüber hinaus zeigen die anderen aus Kahun erhaltenen wpw.t, daß der Familienverband des Hauses durchaus von Interesse war und eine regelmäßige Erfassung etwaiger Änderungen akribisch aufgezeichnet wurde. Das bestätigt auch eine Parallele einer Stele, die von BAKIR angeführt wird, und eine Personenliste von Familienmitgliedern eines Haushalts und einer Anzahl von mindestens vierzehn $\underline{d} t$ beinhaltet. ${ }^{313}$ Für den Fall einer >Besitzliste $<$ der $\underline{d} t$ hätte die Nennung des Herren genügt. Der Rest der Familie und des Haushalts wäre in einem solchen Fall redundant gewesen. Auch reicht der von KÓTHAY aufgeführte Beleg aus den Admoniti-

307 HAYES (1955: 30 Anm. 92).

308 HAYES (1955: 130 Anm. 463).

309 Moreno Garcia (2000).

310 KÓTHAY (2001: 360 f.).

311 KÓTHAY (2001: ebenda).

312 BAKIR (1952: 36); vgl. auch HELCK (1975b: 59 f.).

313 Stele Kairo Cat. Gén. 20161; BAKIR (1952: ebenda). Die Inschrift ist unvollständig, so daß nach BAKIR die genaue Zahl der $\underline{d} t$ nicht feststeht. 
ons 6,7 als Kronzeuge nicht aus, da die Intention der Aussage allein auf die $\underline{d} t$ polarisiert ist und die anderen Aspekte vernachlässigt wurden. ${ }^{314}$

$\mathrm{Ob}$ die in diesen Listen erfaßten $\underline{d} t$ auch tatsächlich Angehörige des Haushalts waren, läßt sich aus den Texten nicht erschließen. Bereits GRIFFITH hielt Zweifel daran für angebracht, zumal das völlige Fehlen erwachsener Männer ihn irritierte. ${ }^{315}$ Auch BAKIR und zuletzt KÓTHAY sehen keine Gründe dafür, daß alle Personen gemeinsam den Haushalt bewohnten. ${ }^{316}$ Wie viele Personen insgesamt den $\underline{d} t$ zugerechnet werden müssen, entzieht sich unserer Kenntnis, da der Schluß des Papyrus verloren ist. Für eine ungefähre Einschätzung der Situation weist Barry KEMP auf eine Parallele aus Theben hin, die aus dem Mittleren Reich stammt und im Museum von Brooklyn aufbewahrt wird. ${ }^{317}$ Dort ist für einen Beamten die stattliche Anzahl von 95 Dienern aufgelistet, von denen über die Hälfte Asiaten sind und das Verhältnis von Frauen zu Männern etwa 2:1 beträgt. ${ }^{318}$ Jedoch übersieht KEMP in seinem Vergleich die unterschiedlichen Klassifizierungen der Arbeitskräfte. In dem Beispiel aus Brooklyn wird die Dienerschaft als $\underline{h} n m w$ bezeichnet und trägt damit einen ganz anderen Status als die Personengruppe aus dem Kahun-Papyrus. ${ }^{319}$ Aufschlußreicher ist der Vergleich zum Brooklyn-Papyrus hinsichtlich des recht ähnlich gestalteten Aufbaus des Formulars. Im Brooklyn-Papyrus werden die Daten der Personen in vier Spalten unterteilt:

Name und >Titel< - Rufname - Tätigkeitsbezeichnung - Geschlecht und Alter

Sämtliche dieser Daten werden auch im Kahun-Papyrus vermerkt. Lediglich kleinere Unterschiede lassen sich dabei feststellen. Eine besondere Erwähnung von Titeln gibt es im KahunPapyrus nicht und die Altersangabe ist im Papyrus Brooklyn nur grob vermerkt. Kinder tragen

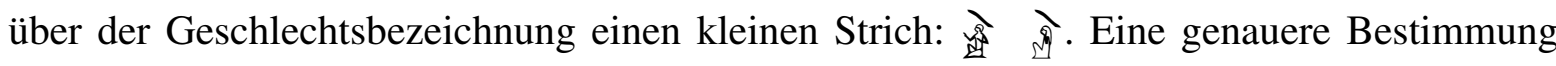
des Alters der Personen ist auch in diesem Fall nicht möglich.

Es scheint im Fall der $\underline{d} t$ eindeutig, daß sie zwar in einem engen Dienstverhältnis zu ihren Herren standen und diese ihre Dienerschaft auch an andere Personen weiterreichen konnten. Eine eindeutige Identifikation der $\underline{d} t$ als rechtlose >Diener $<$ läßt sich aus den vorliegenden Quellen nicht herauslesen. Allerdings gibt es ein kleines, bislang unbeachtetes Indiz im

314 Siehe dazu auch unten S. 151.

315 GRIFFITH (1898: 28).

316 BAKIR (1952: 36); KóTHAY (2001: 361), dort auch Gegenmeinung von Oleg BERLEV zitiert, der die $\underline{d} t$ für ständige Mitglieder eines Haushalts des Mittleren Reiches hielt.

317 pBrooklyn 35.1446; HAYES (1955); KEMP (1991: 156). Dagegen stammen von QUIRKE (1990: 149) Überlegungen, daß es sich bei der Liste weder um ein offizielles Dokument, noch um einen >typischen< Haushalt der Oberklasse handelt, sondern um die Dienerschaft der thebanischen Anwesen des Wesirs Resseneb.

318 HAYES (1955: $87 \mathrm{ff}$.).

319 HAYES (1955: ebenda). 
Kahun-Fragment IV.1, das eine engere Zugehörigkeit der $\underline{d} t$ zum Haushalt wahrscheinlicher macht. Taf. 11, Zeile 4 beginnt mit als Personenname aufgefaßt und mit >Her·per< übersetzt wurde. ${ }^{320}$ Tatsächlich verbirgt sich dahinter jedoch der Titel $h r j-p r$, der eine Art Hausverwalter bezeichnet. ${ }^{321}$ Damit ist der $\underline{d} t$ zugleich auch für Haustätigkeiten abgestellt und dürfte vermutlich direkt zum Haushalt gehören, der im vorliegenden wpw.t erfaßt wurde.

\subsection{Eine Häuserliste aus Theben-West}

Ein gänzlich anderer Typus einer Auflistung stammt aus Theben-West und ist als Verso eines Textes, der unter die Gattung der sogenannten >Grabräuber-Papyri< fällt, erhalten geblieben. Der Papyrus befindet sich heute unter der Nummer 10068 im Besitz des Britischen Museums in London, das ihn Mitte des 19. Jahrhunderts angekauft hatte. Eine erste ausführliche Besprechung fand die Liste im Rahmen der Publikation Thomas PEETs über die GrabräuberPapyri. ${ }^{322}$ Darüber hinaus hat dieser Papyrus nur wenig Aufmerksamkeit erfahren und fand eher zusammenfassend Erwähnung. ${ }^{323}$ Erst mit der erneuten Bearbeitung durch JANSSEN erfuhr der Text wieder eine Würdigung, die über die bereits von PEET erzielten Ergebnisse hinausging. ${ }^{324}$

\subsubsection{Aufbau}

Der Papyrus ist mit seinen Ausmaßen von $156 \times 44 \mathrm{~cm}$ recht groß und gut erhalten. ${ }^{325}$ Neben den Grabräuber-Akten auf dem recto aus dem Jahr 17 Ramses' IX, auf die hier nicht näher eingegangen wird, befinden sich auf dem verso zwei verschiedene Listen. Die erste Liste ist ein Verzeichnis über den Empfang der šrmt. Die zweite Liste besteht aus einer Aufstellung von Häusern und - vermutlich - deren Haushaltsvorstehern mit insgesamt 182 Einträgen, wovon allerdings drei Einträge einen Tempel bezeichnen. ${ }^{326}$ Die Listen sind in mehreren Spalten

320 GRIFFITH (1898: gegenüber 26).

321 So auch WARD (1982: Nr. 977); der gleiche Titel taucht bereits auf Fragment I.4 (Taf. 9,26) auf und wurde dort von GRIFFITH (1898: 23) unsicher, jedoch korrekt als »chief (?) of a house« erkannt.

322 KRI VI 747-755; PEET (1930: 79 ff.).

323 VALBELLE (1985a, 1987).

324 JANSSEN (1992).

325 PeEt (1930: 79).

326 Kemp (1991: 306 f.) beschreibt, daß von den 179 aufgelisteten Häusern 155 zur gleichen Siedlung namens Maiunehes gehörten. Mit Hilfe des >Mittelwertes` von 6 Personen pro Haushalt errechnet er eine ungefähre Größe der Siedlung von rund 1000 Einwohnern. 
angeordnet. Da die letzte Spalte aus nur noch 15 Zeilen besteht, kann daraus geschlossen werden, daß die Häuserliste vollständig ist. ${ }^{327}$ Jeder Eintrag fängt stereotyp mit den Zeichen $\square$ ( pr $n$, >Haus des $\ldots<$ an, gefolgt von einer Berufsbezeichnung und einem männlichen Personennamen. Von diesem Aufbau gibt es drei Ausnahmen, die sich direkt auf Tempel und einen jeweils dafür zuständigen Priester beziehen, was PEET zu der Vermutung veranlaßte, daß sich auch die Einleitung $p r n$ der anderen Zeilen auf einen Tempel bezieht. ${ }^{328}$ Von JANSSEN stammt dagegen ein plausiblerer Vorschlag, der davon ausgeht, daß die Einleitung $\square$ I der Zeilen schon vorbereitet war und der Schreiber beim Erfassen der Daten es schlicht vergessen hat, bei den Tempelnamen die Einleitungs-Floskel zu tilgen. ${ }^{329}$ Die dritte und in der chronologischen Abfolge vermutlich letzte Liste vermerkt eine Reihe von Abgaben, die mit dem Begriff $\check{s} r m t$ bezeichnet werden. Die Reihenfolge der Aufzeichnung ergibt sich aus der Beobachtung, daß manche Zeilen der šrmt-Liste in die Leerräume der sauber niedergeschriebenen Häuserliste >hineinragen $<$. Obwohl in der Datumsangabe über der šrmt-Liste die Jahresangabe aus unbekannten Gründen fehlt, läßt sich durch die eindeutig datierte Häuserliste der Zeitraum recht gut eingrenzen. Die Häuserliste datiert auf den III šmw 13 des Jahres 12, was sich nach Jaroslav ČERNÝ ausschließlich auf die Regierungszeit Ramses' III. beziehen kann. ${ }^{330}$ Alle drei Inhalte sind von verschiedenen Schreibern verfaßt worden, wie die epigraphische Analyse zweifelsfrei bestätigt. ${ }^{331}$

\subsubsection{Zweckbestimmung}

Welche Funktion die Aufstellung dieser Listen hatte, ist bis heute nicht eindeutig geklärt. Der erste Vorschlag von PEET bezog sich auf die ebenfalls auf dem Verso enthaltene šrmt-Liste. Die Personennamen sowohl der $\check{s} r m t$-Liste, als auch der Häuserliste zeigen weitestgehend eine Übereinstimmung, so daß PEET davon ausging, daß die Häuserliste eigens dafür aufgestellt wurde, von diesen Haushalten die šrmt-Abgaben einzufordern. ${ }^{332}$ Dies steht auch nicht im Widerspruch zur Reihenfolge der Niederschrift, da eine Gesamtliste der Siedlung durchaus vor der Maßnahme der Einsammlung von Abgaben stehen konnte und nur ein Teil der Personen aus der Häuserliste auch in der šrmt-Liste auftaucht. Diese Hypothese wurde von JANS-

327 JANSSEN (1992: 8).

328 PEET (1930: 83).

329 JANSSEN (1992: 9 Anm. 11, 10 f.).

330 JANSSEN (1992: 9).

331 PEET (1930: 79), bestätigt von JANSSEN (1992: 8).

332 PEET (1930: 86). 
SEN zurückgewiesen. Seiner Meinung nach gibt es keinen unmittelbaren Bezug zwischen den Listen, ${ }^{333}$ die Übereinstimmungen der in den Listen vermerkten Namen rühren vom gemeinsamen Bezug auf die gleiche Siedlung und der zeitlichen Nähe her, in der beide Listen verfaßt wurden und müssen als purer Zufall gelten. ${ }^{334}$ In eine ähnliche Richtung weisen auch Vorbehalte Dominique VAlbelles, die sich entschieden dagegen wendet, die Häuserliste als polizeiliche Maßnahme im Zusammenhang mit den spektakulären Raubfällen im Tal der Könige zu sehen, da die Liste auf dem Verso einer Grabraub-Akte aufgeschrieben ist. ${ }^{335}$

Die Liste ist in ihrer Form nicht einzigartig, sondern es gibt vom gleichen Typus auch ein weiteres Fragment mit einer ähnlichen Zeitstellung. Es handelt sich dabei um ein Ostrakon aus Deir el-Medineh, das eine - sicher unvollständige - Aufstellung über 26 Häuser enthält, die ebenfalls stereotyp mit $\square$ । eingeleitet wird, gefolgt von den Namen des darin wohnenden Nekropolenarbeiters (ohne Familie!) sowie gelegentlich eine Nennung des Vaters. ${ }^{336}$ Der Zweck dieser Liste ist eindeutig zu bestimmen, da mit Bezug auf die genannten Häuser Wasserrationen von $1 / 2$ bzw. $1 / 4 \underline{h} 3 r(1 \underline{h} 3 r=76,881)$ erwähnt werden. ${ }^{337}$

Daß es sich bei der auf Papyrus BM 10068 verzeichneten Thebanischen Häuserliste mit hoher Wahrscheinlichkeit nicht um eine normale und regelmäßig erfolgende Maßnahme handelt, die im weitesten Sinne dem Bereich des Zensus zugerechnet werden kann, beweist ein weiterer unpublizierter Fund, der aus der Arbeitersiedlung Deir el-Medineh stammt, in die Zeit der 20. Dynastie datiert wird und unter der Bezeichnung Stato Civile bekannt geworden ist. ${ }^{338} \mathrm{Im}$ Museum von Turin existieren etwa 80 Fragmente, die mindestens zwei verschiedenen Papyri zuzuordnen sind. ${ }^{339}$

Die von diesem Papyrus erhalten gebliebenen Fragmente weisen zum Papyrus BM 10068 dahingehend Übereinstimmung auf, daß auch hier eine Reihe von Haushalten erfaßt und aktenkundig gemacht wird. Auch die Einleitung eines Eintrags ist durch die stereotype Formel $\square$ /mm identisch. Danach enthalten die Turiner Fragmente jedoch keine Berufsbezeichnung des Hausinhabers, sondern geben eine - mehr oder weniger - ausführliche Familienliste wieder. Die typische Struktur eines Eintrags sieht wie folgt aus: ${ }^{340}$

333 JANSSEN (1992: 8).

334 JANSSEN (1992: 23).

335 VAlbelle (1985b: 60 Anm. 7) weist zu Recht auf die weit auseinanderliegende Datierung der Einträge auf Recto und Verso hin.

336 oStockholm MM 14126 ro.; alle Angaben nach VALbeLLE (1985a: 81 Anm. 45).

337 JANSSEN (1979).

338 MCDowell (1999: 51 f.); VAlbelle (1985b: 56 ff.); BоTti (1922).

339 Tlw. numeriert pTurin 1963, vgl. Demarée (1993: 103); VALbelle (1985b: ebenda).

340 Beispiel entnommen aus BOTTI (1922: 392). 


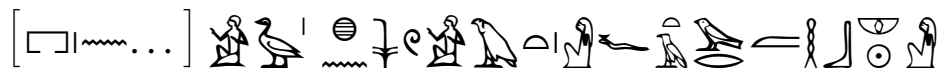

[pr $n$...] z 3 H Hnsw mw.t=f T3-wr-m- hb

»[Haus des ... ], Sohn des Hnsw, seine Mutter ist T3-wr-m-ḥb.«

Neben der patronymen Filiationsangabe sind auch die Namen aller weiteren Hausbewohner aus der Familie und deren Familienstatus in der Liste vermerkt. ${ }^{341}$ Es ist hier also ein echter Zensus greifbar, der die genaue Personenzahl ermittelt, die in den jeweiligen Häusern lebt. Der Aufbau derartiger Personenlisten ist auch aus anderen Zusammenhängen bekannt, etwa aus dem Papyrus Bologna 1086, einem Brief, in dem über einen Leibeigenen Auskunft erteilt wird. ${ }^{342}$

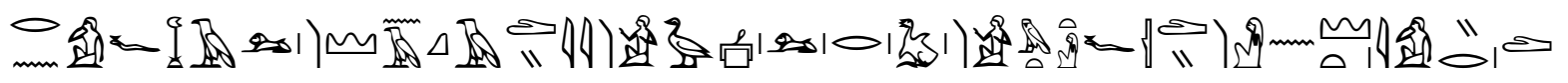
e)凹

$r n=f \underline{h} 3 r w N q d j$ z $3 r r \underline{t} m w t=f Q d j n$ h్ $3 s t$ Jrt

»Sein Name ist Nqdj der Syrer, Sohn des Srrț, seine Mutter ist Qdj, aus dem Land Arzawa.«

Aus der Form des Zitats schließt Walther WOLF auf das Schema, das in einer Akte vorliegt: Name, Filiationsangabe, Abstammungsort. Daß eine solche Liste nicht ausschließlich auf unfreie Personen beschränkt bleibt, zeigt unter anderem eine Parallele aus Papyrus Abbott, der die Identifikation eines Handwerkers im Rahmen der Grabräuberprozesse in ähnlicher Weise bezeugt: ${ }^{343}$

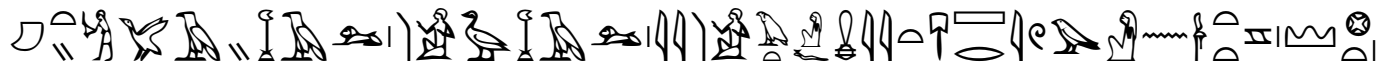
hmtj PЗj-hr z’ Hrj $m w . t=f M j . t-s ̌ r j j m n t . t n ~ n j w . t$

»Der Kupferschmied P3j-ḩr, Sohn des Hrj, seine Mutter ist Mj.t-šrj, aus dem Westen der Stadt.«

Entgegen WOLFs Annahme, daß es sich in allen Fällen um Sklaven oder Leibeigene handelte, scheint zumindest der in Papyrus Abbott erwähnte Arbeiter eher ein Angehöriger einer Hilfsarbeiter-Truppe gewesen zu sein, die mit smdt bezeichnet wird. Angehörige solcher Arbeitseinheiten waren zwar ungelernt und wurden deshalb von den Spezialisten unterschieden, standen aber dennoch in keinem Abhängigkeitsverhältnis zu anderen. ${ }^{344}$

In diesem Zusammenhang soll auch kurz auf den Informationsumfang in Bezug auf die Filiationsangaben eingegangen werden. Im überwiegenden Fall der hier vorgestellten Textstellen

341 Zur Struktur und Varietät der genealogischen Angaben vgl. VALBELLE (1985b: 57).

342 Wolf (1930: 92 f.).

343 Peet (1930: 39, Taf. 2); VAlbelle (1985b: 57) mit weiteren Beispielen.

344 ČERNÝ (1973: 183 ff.). 
dient die patronyme Filiationsangabe als - möglichst - eindeutiges distinktives Merkmal, um Personen mit identischen Namen voneinander unterscheiden zu können. Auch wird in einzelnen Fällen neben dem offiziellen Namen der Rufname in einer eigenen Spalte vermerkt, falls die betreffende Person einen solchen besitzt. ${ }^{345}$ Patronyme sind also durchaus ein gängiges Mittel, um in den vorliegenden Fällen die Identifikation Einzelner zu erleichtern. Auch in anderen Arten von Akten ist dieses Verfahren zu finden und so erstaunt es, daß Sven VLEEMING in seiner Publikation des Papyrus Reinhardt, einer Landliste des 10. Jahrhunderts v. Chr., ausdrücklich den Ausnahmecharakter einer solchen Patronymisierung betont. ${ }^{346}$

Ein paar Überlegungen sollen zum Schluß noch hinsichtlich des Stato Civile angestellt werden. Zunächst einmal fällt auf, daß es in Aufbau und Inhalt deutliche Abweichungen von den aus Kahun bekannten wpw.t-Listen gibt. Die Erfassung der Personen erfolgte dort nicht >per Haus<, sondern nach Haushalt. Darüber hinaus zeigen die Einleitungen der Kahun-Texte, daß sich der Deklarant zu einer eigens dafür zuständigen Behörde bemühen mußte, um seine Familie zu registrieren. Entsprechend ausführlich waren die Einträge in den Listen, zum Teil sogar mit Rufnamen und Altersangabe der Familienmitglieder. Nichts von alledem scheint in den Fragmenten aus Deir el-Medineh vorhanden zu sein. Hier steht die Personenerfassung in direktem Bezug zum Kataster der Häuser, darüber hinausgehende Angaben sucht man vergeblich. Es liegt auf der Hand, daß es sich hier um zwei grundsätzlich verschiedene Typen von Zensus handeln muß, die zwar im Zweck möglicherweise übereinstimmten, aber in der Detailfülle differierten. Es stellt sich daher die Frage, ob sich die Methode der Erhebung im Laufe der Zeit geändert hat, so daß hier eine neue Form der Deklaration vor uns liegt, oder ob die Erfassung in Form eines wpw.t auch im Neuen Reich immer noch existierte und Beispiele dafür noch ausstehen. Dieses argumentum ex silentio läßt sich schwer einschätzen, doch dürfte aufgrund der ähnlich strukturierten Zensusdeklarationen in der griechisch-römischen Zeit das Verfahren des wpw.t nicht gänzlich in Vergessenheit geraten sein. Darüber hinaus ist die Quellenlage für Zensusakten der pharaonischen Zeit generell nicht so breit gefächert, um daraus verbindliche Rückschlüsse über Verfahrenswechsel zu ziehen. Wenn jedoch ein Verfahrenswechsel stattgefunden hat, dann bleibt das Fehlen von Dienstpersonal oder Dienern in den Dokumenten unerklärbar. Daß es faktisch auch >Privatbesitzく an Dienern und Sklaven un-

345 Siehe oben pKahun, Taf. 10 und pBrooklyn 35.1446.

346 VleEMING (1993: 59 f. Anm. 182) mit Verweis auf JANSSEN (1975a: 16). Als Gegenbeispiel zitiert VLEEMING Papyrus Wilbour, in dem gänzlich auf Filiationsangaben verzichtet wird. Der Hinweis auf JANSSENs Arbeit zu den >Commodity Prices < verkennt die unterschiedlichen Ausrichtungen der Quellen: hier staatliche Akten, dort (zumeist) private Rechtsurkunden. 
ter den Handwerkern von Deir el-Medineh gegeben hat, ist aus diversen Ostraka bekannt. ${ }^{347}$ Es bliebe nur die Vermutung, daß dieser Besitzstand separat aufgezeichnet wurde, wofür es allerdings (noch) keine Evidenz gibt.

Einen anderen Aspekt betrifft die Beobachtung von VALBELLE, die darauf hinwies, daß im Stato Civile - im Gegensatz zu den Beispielen der >Aktenauszüge< aus anderen Papyri - kein Herkunftsort vermerkt ist. ${ }^{348}$ Sie folgerte daraus, daß diese Information fortgelassen wurde, da ohnehin alle Bewohner aus Deir el-Medineh stammten. Sollte dies tatsächlich der Grund für diese Variante sein, so hätte dies zwei gravierende Folgen für die Beurteilung eines solchen Zensus. Zum einen bedeutet dies, daß das >Formular< einer solchen Akte nicht statisch ist, sondern von den Schreibern nach Bedarf abgewandelt werden kann. ${ }^{349}$ Zum anderen - und das ist bedeutend wichtiger - muß daraus geschlossen werden, daß ein derartiger Zensus immer ein regionaler Verwaltungsakt war, dessen Inhalte zumindest nicht in der Urform ihrer Erfassung die Verwaltungszentren erreichte. Ansonsten wäre eine regionale oder gar landesweite Institution damit überfordert, einen solchen Papyrus korrekt zuzuordnen.

Tatsächlich ist die Beleglage für die Frage nach der Aufbewahrung der Verwaltungsakten unsicher. Dies liegt insbesondere daran, daß die Strukturen und Verknüpfungen zwischen zentraler Verwaltung einerseits und regionaler Verwaltung andererseits auch für das Neue Reich nur in groben Zügen bekannt sind. Eine Skizzierung der Situation versuchte zuletzt David O’CONNOR. ${ }^{350}$

Üblicherweise ging man davon aus, daß etwa Steuerlisten und ähnliche Akten in den Schatzhäusern vor Ort aufbewahrt wurden. Ein Hinweis von Alan GARDINER zeigt, daß dies nicht zwangsläufig der Fall sein mußte. In seiner Publikation der Grabinschrift des Mes kann er schlüssig vorführen, daß personenbezogene Katasterlisten während der Regierungszeit Ramses' II. auch in einer Kopie im zentralen Archiv der königlichen Kornkammern vorhanden waren, die sich in der Residenz von Piramesse befanden. ${ }^{351}$ Ausgangspunkt der Inschrift ist ein juristischer Streitfall um ein Landstück, das ursprünglich als Geschenk des Pharao an eine Familie übertragen wurde und deren Besitzanspruch von einem hohen Beamten streitig gemacht wird. Da dieses Land nicht von Steuern befreit war, sondern allem Anschein nach Getreide in einem bestimmten Umfang an die staatliche Getreideverwaltung geliefert werden

347 JANSSEN (1997: 23 ff.).

348 VALBELLE (1985b: 57).

349 Für die griechisch-römische Zeit ist die Differenzierung regionaler Unterschiede einfacher, vgl. WILCKEN (1899: 440).

350 TRIGGER u. a. (1983: 204 ff.).

351 GARDINER (1964: 39); vgl. auch HELCK (1958: 155 f.). 
mußte, lagen entsprechende >Grundbucheinträge <, genannt $d n j t$, vor und konnten als Nachweis der Rechtmäßigkeit des Besitzes vorgebracht werden.

Gleichwohl bleibt die Inschrift des Mes für den Versuch einer Generalisierung dieses Verfahrens problematisch. Aufgrund der direkten Unterstellung der Abgabeverpflichtung unter die königlichen Kornspeicher hatte die staatliche Administration ein ausdrückliches Interesse daran, Steuerpflichtige in eigenen Listen zu erfassen, um nicht von den lokalen Verwaltungsträgern abhängig zu sein. Daß dies gleichfalls für Zensus-Daten gilt, kann unterstellt werden, bleibt aber mangels Fakten reine Mutmaßung. Auch steht die Grundstücksaufteilung, die Bodenqualität und die Höhe der Steuer oder Pacht im Kern des Interesses. ${ }^{352}$ Die dazugehörigen Personen interessieren nur insoweit, als sie zum einen fortlaufend und erblich als Besitzer dokumentiert sind und zum anderen die jeweils lebenden Besitzer gegenüber dem König steuerpflichtig sind. Erst in zweiter Linie lassen sich über die Listen genealogische Zuordnungen treffen, was schließlich auch gar nicht deren eigentlicher Zweck ist. In diesem Zusammenhang trägt auch der von GARDINER verwendete Terminus >census-lists< kaum zur Klärung der Sachverhalte bei. ${ }^{353}$ Es muß vielmehr - mit Erwin SEIDL - davon ausgegangen werden, daß es sich bei den Akten, die in der Inschrift des Mes erwähnt werden, um Katasterbücher oder Auszüge davon handelt, die für die Klärung des Rechtsstreits herangezogen worden waren. Gleichwohl wirft der vorliegende Fall ein bezeichnendes Licht darauf, was GARDINER unter dem von ihm gewählten Begriff >census-lists< subsumiert, nämlich jegliche Form einer geordneten und regelmäßigen Erfassung von Subjekten wie Objekten durch die altägyptische Administration.

\subsection{Zusammenfassung}

Die ausführliche Untersuchung der altägyptischen Zensus-Papyri hat gezeigt, daß nur in den wenigsten Fällen die Datenbasis gut genug ist, um davon weitergehende Aussagen deduzieren zu können. Während die Angaben in den Gebelein-Papyri zumindest eine grobe Vorstellung davon geben, welche Größe bei einer typischen Domäne des Alten Reiches erwartet werden darf, ist der Namensliste des Papyrus BM 10068 kaum eine demographisch verwertbare Information zu entnehmen. Lediglich die Fragmente aus Kahun erlauben es aufgrund ihrer fortlaufenden Auszüge aus der Familiengeschichte des Soldaten Ḥrj, eine gesicherte Aussage über Familiengrößen und Angehörige eines Haushalts zu treffen. Die Singularität dieses Belegs

352 SEIDL (1982: 179 f.).

353 GaRdiner (1964: 39). 
verhindert jedoch dessen Überprüfbarkeit. Ob die Familienverhältnisse Hirj's typisch waren oder ein außergewöhnliches, durch Zufall erhaltenes Beispiel darstellen, kann für die Zeit des Mittleren Reiches nicht abschließend beantwortet werden, obgleich die Wahrscheinlichkeit der Überlieferung eher für den Normalfall einer Kategorie, als für die Ausnahme spricht. Das Hinzuziehen der Fragmente des Stato Civile aus dem Neuen Reich ist aufgrund der zeitlichen und räumlichen Unterschiede nur bedingt möglich. Zumindest zeigt der Vergleich beider Papyri, daß der überlieferte Kahun-Haushalt zeitweise größer als die größten Haushalte des Stato Civile war. Die starken Schwankungen in der Personenzahl innerhalb weniger Jahre weisen allerdings auch auf die dynamischen Veränderungen hin, die im Stato Civile nicht greifbar sind.

Lange Zeit galten allein diese Angaben aus den Papyri als verläßliche Datengrundlage für demographische Rechnungen, obwohl häufig weder die Gesamtfläche einer Siedlung, noch die Fläche eines Hauses als wichtige zusätzliche Informationen für die demographische Situation bekannt sind. Auch deshalb sollte das von KEMP für den Papyrus BM 10068 demonstrierte Rechenexempel mit dem >Mittelwert< von 6 Personen mit Vorsicht betrachtet werden. Im folgenden Kapitel wird deshalb ein statistisches Verfahren zum Überprüfen der auf Basis der bisherigen Methode gewonnenen Ergebnisse vorgestellt. 


\title{
4 Die Bevölkerung in Siedlungen und Regionen
}

Yet Egypt the nation had nothing which can be recognized as a city in modern terms [...]

John A. Wilson, Egypt through the New Kingdom. Civilization without Cities

\section{1 Über die Verwertbarkeit der Daten aus den Papyri}

\begin{abstract}
$\Lambda^{\mathrm{Us}}$ US den wenigen bekannten Angaben, die sich aus den zuvor vorgestellten Papyri ziehen lassen, ein tragfähiges Gerüst für die Schätzung einer Haushaltsgröße zu geben, scheint ein schwieriges, wenn nicht gar hoffnungsloses Unterfangen. Zu viele Unsicherheiten begleiten die Daten, und häufig fehlt die Möglichkeit einer Einschätzung, ob das vorliegende Material ein singulärer Ausnahmefall ist, oder als repräsentativ für vergleichbare andere Siedlungen gelten darf. Anhand der existierenden Daten soll im folgenden dennoch der Versuch gewagt werden, zunächst die ungefähre Haushaltsgröße und darauf basierend die Bevölkerungszahl für Siedlungen näher einzugrenzen.

Die frühesten Informationen können den Kahun-Papyri der Zweiten Zwischenzeit entnommen werden. Sie umfassen mehrere Erhebungen desselben Haushalts, so daß sich aus den Zugängen und Fluktuationen ein Mittelwert errechnen läßt. Aus den Fragmenten I.3, I.4 und I.5 ergibt sich somit die durchschnittliche Größe von 6 Personen im Haushalt. In Anbetracht der Tatsache, daß in diesem speziellen Fall keine Diener und fremde Personen dazugehören, liegt die Zahl bereits am oberen Limit, wie die folgenden Überlegungen zu den Belegen des Neuen Reiches zeigen werden. Eine Sonderstellung nimmt sicher auch das Fragment IV.1 ein, das neben einer Kernfamilie von 4 Personen einen recht großen Stamm an Bediensteten auflistet. Wie bereits im Kap. 3.3.4 dargelegt wurde, ist nicht zwingend darauf zu schließen, daß
\end{abstract}


die genannten Personen notwendigerweise auf dem selben Anwesen wohnten, wenngleich die Stellung des Deklaranten das Vorhandensein eines repräsentativen Wohnsitzes nahelegt.

Für das Neue Reich sieht die Überlieferungssituation ähnlich eingeschränkt aus. Aus der Häuserliste des Papyrus BM 10068 kann lediglich eine Untergrenze der Besiedlung ermittelt werden, sofern man die aus anderen Quellen gewonnenen Haushaltsgrößen mit der Zahl von 179 Häusern multipliziert. Dabei ist nicht einmal sicher, welche Haushaltsgröße hier zu erwarten ist, da mangels Angaben über die Größe der Häuser keine Aussagen hinsichtlich eines Durchschnitts getroffen werden können. Dabei muß berücksichtigt werden, daß Form und Kontext der Aufzeichnung den Schluß zulassen, daß nicht alle existierenden Häuser in der Liste Erwähnung fanden. ${ }^{354}$ Wieviele Häuser tatsächlich zwischen den Totentempeln in Theben-West standen, oder welche Gesamtgröße der Ort Maiunehes aufwies, entzieht sich unserer Kenntnis. 355

Wenn die Daten, die zuvor aus den Kahun-Papyri abgeleitet wurden, die Fragmente des Stato Civile sowie Vergleichsdaten aus dem Nahen Osten ${ }^{356}$ betrachtet werden, kristallisiert sich eine Kerngröße von etwa 5-6 Personen pro Haushalt heraus. Im Einzelfall ist die Zahl der Hausbewohner nach Auskunft der Dokumente allerdings Schwankungen unterworfen. ${ }^{357}$ Dominique VALBELLE bestätigt die relative Häufigkeit von Ehepaaren mit zwei bis drei Kindern, zitiert aber auch Belege für >Single<-Haushalte, von denen sie - bei allem Vorbehalt vermutet, daß die Gebäude neu angeworbenen jungen Handwerkern zugewiesen worden waren. ${ }^{358}$ Dabei ist allerdings zu berücksichtigen, daß die Durchschnittsgrößen eher auf Konsens basieren als auf exakt abgesicherten Fakten.

Was im Fall des Stato Civile besonders auffällt, ist die gegenüber dem errechneten Durchschnitt geringere Größe der Familien. Selten sind mehr als vier oder fünf Personen erwähnt. Die dabei vorgefundene Struktur der Familienmitglieder entspricht ungefähr derjenigen, die bereits aus den Kahun-Papyri des Mittleren Reiches bekannt ist. Neben der eigenen Familie, bestehend aus Ehefrau und einem oder mehreren Kindern, wohnen häufig auch Geschwister und Eltern oder Schwiegereltern unter einem Dach. Letztere besonders dann, wenn ein Eltern-

354 Dazu ausführlich oben Kap. 3.4.

$355 \mathrm{Zu}$ den Einzelangaben PeEt (1930: 83 f.).

356 ZORN (1994: 33) gibt für Tell en-Nasbeh/Israel eine durchschnittliche Haushaltsgröße von etwa 4 bis 5,5 Personen an. Auf eine ähnliche Größenordnung kommt GARR (1987) bei seiner Auswertung der Daten aus Ugarit im späten 2. Jahrtausend. Nach Auskunft der ugaritischen Verwaltungsakten lag die durchschnittliche Familiengröße bei 5,25 Personen ohne Diener. Die Auswertung der Wohnfläche nach statistischen Gesichtspunkten festigte diesen Wert mit einem Mittelwert von 7,635 Bewohnern - allerdings mit extremen Schwankungen bei unterschiedlichen Berechnungsverfahren. Alle Angaben stehen unter dem Vorbehalt der nicht ohne weiteres gesicherten 1:1 Übertragbarkeit von Daten aus Nachbarkulturen.

357 VALBELle (1985b: 57).

358 VAlbelle (1985b: 122); vgl. dazu auch MCDowell (1999: 51). 
teil verstorben ist, und der andere zwecks Versorgung in den eigenen Haushalt aufgenommen wird. ${ }^{359} \mathrm{Da}$ keine Aufstellungen über Dienstpersonal in den Listen existieren, dürfte mit der Klientel des Zensus zusammenhängen, die als Mitglieder der Handwerkerschicht - von Ausnahmen abgesehen - in der Regel über keine eigenen Diener verfügten.

Vorläufig läßt sich feststellen, daß die aus den Papyri gewonnenen Angaben zur Haushaltsgröße als grobe Orientierung genügen, im folgenden jedoch durch die Einbeziehung der Wohnfläche präzisiert werden müssen.

\subsection{Statistiken über die Wohnflächen als Gegenprobe}

Um eine verläßlichere Aussage über die Bevölkerungsverhältnisse der hier betrachteten Siedlungen zu treffen, genügt es nicht, lediglich eine Aufsummierung der Haushalte aufgrund der aus den Papyri gewonnenen Familiengrößen vorzunehmen. Die auf diesem Wege gewonnenen Zahlen müssen anhand anderer Verfahren überprüft werden, um die Aussage stützen zu können. Zum Beispiel könnten statistische Berechnungen als Grundlage dienen, die auf Basis von Siedlungsfläche und Größe der Wohneinheiten eine Gesamtzahl liefern. Um für beide Berechnungsverfahren eine einheitliche Datenbasis liefern zu können, sollten die untersuchten Siedlungsplätze folgende Merkmale aufweisen:

$\triangleright$ Siedlungsgebiet mit fest umrissener, bekannter Fläche

$\triangleright$ (relativ) gleichförmige Bebauung

$\triangleright$ eng gesteckter Besiedlungszeitraum

Geeignet für diesen Vergleich scheinen die sogenannten Arbeitersiedlungen von Amarna, Deir el-Medineh und - mit gewissen Einschränkungen - auch Kahun zu sein. Dagegen können die erst kürzlich von Ernst CZERNY vorgelegten Untersuchungen zur Siedlung des frühen Mittleren Reiches in Tell el-Dab ${ }^{c}$ a für demographische Zwecke leider (noch) nicht ausgewertet werden. ${ }^{360}$ Obwohl auf einer Fläche von fast 10000 qm 189 Häuser nachgewiesen und - aufgrund der äußerst regelmäßigen Anlage der Gebäude - weitere 153 Häuser erschlossen werden konnten, ${ }^{361}$ ist die Fläche nur durch den Befund einer Nordmauer eingegrenzt, in alle anderen Himmelsrichtungen dagegen offen, so daß keine weiteren Schlußfolgerungen gezogen werden können. Immerhin überrascht die im Vergleich zu den anderen in diesem Kapitel vorgeführten Siedlungen recht kleine Bauweise der einzelnen Häuser, die jeweils ungefähr eine Größe von 5,20 × 5,40 m (27 qm) aufweisen, was etwa $10 \times 10$ Ellen entspricht. ${ }^{362}$

359 Vgl. dazu die Textbeispiele bei MCDowell (1999: ebenda).

360 CZERNY (1999).

361 CZERNY (1999: $17 \mathrm{f}$.).

362 CZERNY (1999: 20). 
Alle drei Beispiele sollen im folgenden als Musterfälle für eine Berechnung dienen. Ausgangspunkt dafür ist die Untersuchung von Raoul NAROLL, der an 18 unterschiedlichen Kulturen weltweit eine universelle Formel für die Relation zwischen Siedlungsfläche und Populationsgröße aufstellen konnte. ${ }^{363}$

$$
A=21,7 \times P^{0,84195}
$$

$A$ steht stellvertretend für die Siedlungsfläche und $P$ für die Anzahl der Personen, die diese Fläche bewohnt. Löst man diese Formel nach $P$ auf, so erhält man

$$
P=\left(\frac{A}{21,7}\right)^{1,187719}
$$

NAROLL hingegen vereinfachte seine Formel ohne nähere Angabe von Gründen auf

$$
P=\frac{A}{10 \mathrm{~m}^{2}}
$$

was vereinzelt auf Widerspruch stieß. ${ }^{364}$ Die Kernaussage der vereinfachten Formel lautet, daß jede Person durschnittlich 10 qm Raum beansprucht, was deutlich unter den Schätzungen Alexander BADAWYs liegt. NAROLLs Formel fand in archäologischen Arbeiten jüngerer Zeit wiederholt Anwendung und scheint sich dahingehend bewährt zu haben, daß mit ihrer Hilfe zumindest eine grobe statistische Einschätzung über die Population eines abgeschlossenen Siedlungsbereichs zu erhalten ist. ${ }^{365}$

\subsection{Die Arbeitersiedlung (๖Eastern Village`) in Amarna}

\subsubsection{Aufbau}

Die Arbeitersiedlung Amarnas liegt ungefähr 1,2 km südöstlich der Zentralsiedlung in einer Felsschlucht, was ihr den Beinamen >Eastern Village< gegeben hat. ${ }^{366}$ Sie setzt sich zusammen aus einer quadratisch angelegten Kernsiedlung (Abb. 4.1), die von einer aus Schlammziegeln gemauerten Umwallung von etwa $70 \times 70 \mathrm{~m}$ eingefaßt ist. Außerhalb dieser Anlage grup-

363 NAROLL (1962).

364 Zur Diskussion der NAROLL-Formel vgl. HASSAN (1981: 73). Hervorzuheben ist u. a., daß die Ergebnisse der Formel anscheinend bei Siedlungsflächen unter $1000 \mathrm{qm}$ zu einer Unterschätzung der Population tendieren.

365 Vgl. z. B. KRAMER (1980) für prähistorische Siedlungen des Irak, GARR (1987) für Ugarit im 2. Jt. v. Chr. $366 \operatorname{KEMP~(1984:~1).~}$ 
pieren sich in loser Anordnung weitere Gebäude sowie Kapellen, die für die Auswertungen unberücksichtigt bleiben. Fünf Straßen durchziehen in regelmäßigen Abständen in Nord-SüdRichtung die Kernsiedlung und teilen das Areal in sechs Häuserreihen. ${ }^{367}$ Die zwei westlichen Häuserreihen sind von den anderen nochmals durch eine stärkere Mauer abgeteilt. Da die Grundrisse der meisten Gebäude archäologisch nicht mehr nachweisbar sind, hat man aufgrund der recht schematischen Einteilung in einzelne Wohneinheiten deren Gesamtzahl erschlossen. So ergibt sich die in der älteren Literatur noch häufig anzutreffende Gesamtmenge von 75 Häusern, ${ }^{368}$ basierend auf der Aussage der ersten Ausgräber Thomas PEET und Leonard WoOLLEY, die angaben, daß sie mit 36 vollständig ausgegrabenen Häusern gut die Hälfte der Anlage freigelegt hätten. ${ }^{369}$ Dazu kamen einige Anschnitte von weiteren Gebäudekomplexen.

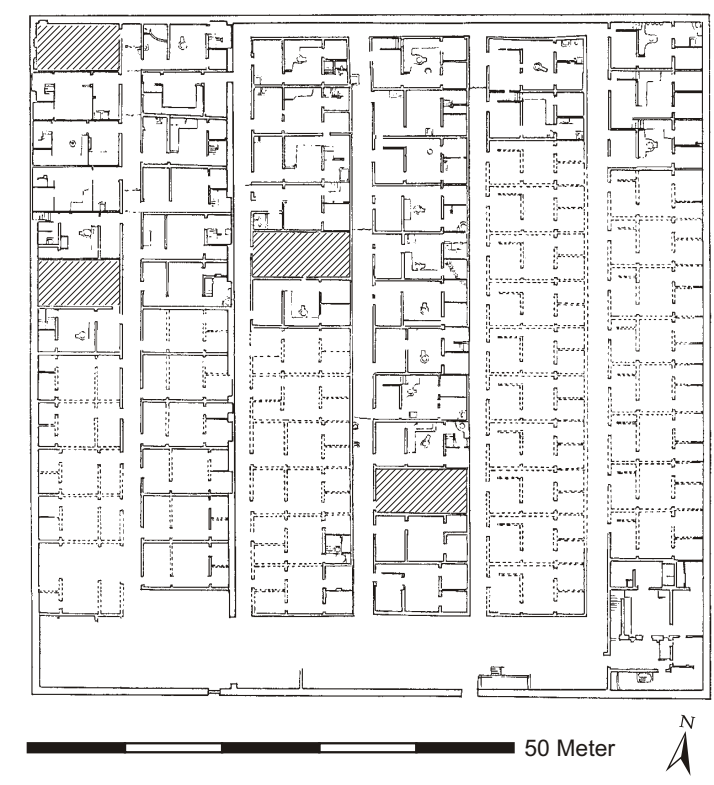

Abbildung 4.1: Das sogenannte >Eastern Village< von Amarna

Die neueren Untersuchungen der Egypt Exploration Society haben hinsichtlich der Datenbasis für die Arbeitersiedlung Amarnas zu einigen Korrekturen geführt. So hat sich etwa die Anzahl der Grundrisse, die einem Standard-Wohnhaus zuzurechnen sind, auf 69 verringert. ${ }^{370}$

367 KEMP (1987b: 22).

368 Zum Beispiel BADAWY (1968: 61), aber auch noch RoIK (1988: 18).

369 Zusammen mit den ersten Grabungsergebnissen der Egypt Exploration Society waren es 38 StandardHäuser; KEMP (1987a: 41 Anm. 7).

370 Vgl. dazu auch KEMP (1987a: 40 ff.); KEMP (1987b: 29). Die Flächen, die nach KEMP vermutlich nicht als Wohnraum dienten, sind in Abb. 4.1 schraffiert. 
Dazu kommen noch zwei Anlagen, die nach Aufbau und Dimensionierung den Wohnraum für Personen mit höherem Status vermuten lassen. Barry KEMP betont darüber hinaus, daß aufgrund der neu gewonnenen Erkenntnisse nicht mehr von einem Standardtypus eines Hauses gesprochen werden kann, da durch wiederholte Umbaumaßnahmen die Häuser in ihrer Grundform differierten. ${ }^{371} \mathrm{Ob}$ diese Tendenz zu einer individuelleren Gestaltung einzelner Wohneinheiten Auswirkungen auf die Bewohnerzahl hat, läßt sich nur schwer einschätzen. Daher bleiben diese baulichen Veränderungen in den Berechnungen unberücksichtigt.

\subsubsection{Die >Main City` Amarnas als Vergleichsobjekt}

Ergänzt werden die Informationen durch einige Schätzungen von Jac JANSSEN, der für die Siedlung Amarna anhand von Gebäudebefunden Überlegungen zur Haushaltsgröße angestellt hat. ${ }^{372}$ JANSSEN hat dazu die erhaltenen Gebäude der Grabungsflächen Q46 und Q 47 am Nordende der >Main City< in fünf Kategorien eingeteilt (Tab. 4.1), die eine bestimmte Hausgröße repräsentieren. ${ }^{373}$ Nur insgesamt sechs Gebäude konnten aufgrund des stark zerstörten Befundes keiner der fünf Kategorien zugewiesen werden. Andere Gebäude wiederum konnten zwar als Typ A klassifiziert werden, ihre genaue Zahl ließ sich jedoch nicht klären. Beide Unsicherheiten finden bei JANSSEN keine mathematische Berücksichtigung, lassen ihn aber im Ergebnis dazu tendieren, die so errechneten Zahlen eher aufzurunden. Die von JANSSEN vorgeschlagenen Haushaltsgrößen ${ }^{374}$ stellen Mindestzahlen dar, die Zahlen in Klammern sind höhere Alternativvorschläge. Die Angaben sind allerdings keine harten Fakten, sondern stellen eine Arbeitsgrundlage dar, die - bei sorgfältiger Anwendung - die Ergebnisse etwas näher an die tatsächlichen Gegebenheiten rücken kann. ${ }^{375}$

\subsubsection{Statistische Auswertung}

Innerhalb des Rahmens bisheriger Schätzungen von Haushaltsgrößen im Alten Ägypten lag bereits die Familiengröße, die BADAWY für die Analyse der >Eastern Village< genannten Ar-

371 KEMP (1987a: ebenda).

372 JANSSEN (1983).

373 JANSSEN (1983: 283 ff.).

374 Zur Unterscheidung zwischen >Haushalt< einerseits und >Familie< andererseits vgl. das Zitat bei JANSSEN (1983: 284 Anm. 86): »>A household consists of all the persons who occupy a housing unit<, whereas $>$ family< is defined as >the entire group of (two or more) persons in a household who are related by blood, marriage, or adoption « «.

375 JANSSEN (1983: 283) selbst bemerkt dazu, daß »anything near an exact answer can not now, and will probably never be given.« 


\begin{tabular}{|c|c|c|c|c|}
\hline Тур & Bezeichnung & Größe & Räume & Personen \\
\hline A & sehr kleines Wohnhaus & $\leq 50 \mathrm{qm}$ & $\leq 6$ & $5(6)$ \\
\hline B & kleines Wohnhaus & $50-80 \mathrm{qm}$ & $>6$ & $5(6)$ \\
\hline $\mathrm{C}$ & Mittelklassehaus & 80-160 qm & $\geq 8$ & $71 / 2(8)$ \\
\hline $\mathrm{D}$ & große Residenz & $160-200 \mathrm{qm}$ & $>15$ & $15(20)$ \\
\hline $\mathrm{E}$ & sehr große Residenz & ab 200 qm & $>15$ & $15(20)$ \\
\hline
\end{tabular}

Tabelle 4.1: Hausgrößen und Haushaltsgrößen in Tell el-Amarna

beitersiedlung Tell el-Amarnas wählte. Als Vergleichsbasis nahm er die durchschnittliche GröBe einer Familie in ländlichen Regionen des modernen Ägypten von 4,18 Personen. ${ }^{376}$ Bei einer Zahl von 75 Häusern kam er so auf eine Summe von 313 Personen, denen jeweils 15,63 qm Raum zur Verfügung stand. Hinsichtlich des Sonderfalls von staatlich gefördertem Wohnraum für eine privilegierte Handwerkerschicht überrascht auch nicht die relative Größe an Raum pro Person. Aufgrund des fest umrissenen Wohnbereichs greifen die bisher in der Ethnologie vorgeschlagenen Schätzungen zu Personenzahl und Wohnfläche nicht, da keine freie Ansiedlung auf einer offenen Fläche gegeben ist. ${ }^{377}$

Das Ergebnis BADAWYs verschiebt sich noch ein wenig, wenn zusätzlich diejenigen Personen, die nicht zur Familie gehören, mit einkalkuliert werden müssen. Bei einer durchschnittlichen Haushaltsgröße von 5 Personen beläuft sich die Gesamtzahl der Bewohner auf 375. Eine höhere Personenzahl pro Haushalt deckt sich dabei durchaus mit den Daten, die JANSSEN für den Südbezirk Amarnas vorgeschlagen hat. Eine Wohneinheit in der Arbeitersiedlung Amarnas läßt sich mit einer Größe von ungefähr 50 qm veranschlagen, ${ }^{378}$ und fällt damit unter JANSSENs Kategorie B. Darauf aufbauend kann noch ein weiterer Test an den vorliegenden Daten vorgenommen werden.

Wendet man nun die Formeln auf die Angaben für die Arbeitersiedlung Amarnas an, kommt man auf recht unterschiedliche Ergebnisse. Basierend auf NAROLLs ursprünglicher Formel (2) kommt man auf rund 625 Bewohner, also 8,33 Bewohner pro Haus. Verglichen mit den Schät-

376 BADAWY (1968: 60 f.). Die Herkunft der Vergleichszahl aus dem Jahr 1968 ließ sich nicht ermitteln. Bei ihrem Gebrauch sollte man sich jedoch der Tatsache bewußt sein, daß solche statistisch ermittelten Angaben alles andere als statisch sind. Ein Vergleich mit den ausführlicheren Untersuchungen von KELLEY u.a. (1982: 8 ff.) zeigt, daß allein die tatsächliche Zahl der Kinder einer ländlichen Familie zum Zeitpunkt der neuen Erhebungen bereits auf 5,2 angewachsen ist.

377 Bei freier Ansiedlung wurde basierend auf Daten aus der Altamerikanistik ein Raumverhältnis von 13,92 qm gemeinsam für die ersten sechs Personen einer Wohneinheit vorgeschlagen. Ob solche Datenauswertungen für einzelne Kulturkreise eine universelle Gültigkeit beanspruchen dürfen, ist fraglich. Vgl. zur Problematik auch HASSAN (1981: 72 ff.).

378 Durchschnittliche Größe $5 \times 10$ m nach RoIK (1988: 18). 


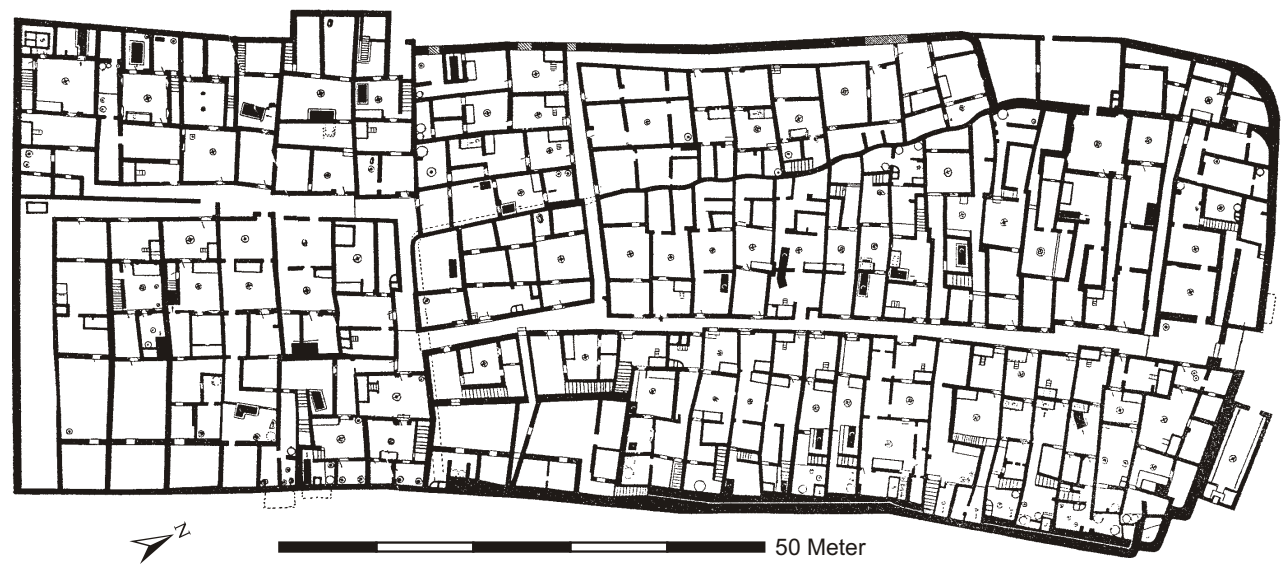

Abbildung 4.2: Die dritte Bauphase der Arbeitersiedlung Deir el-Medineh

zungen JANSSENs ist dieser Wert recht hoch, doch nicht völlig unwahrscheinlich. Verwendet man dagegen die vereinfachte Formel (3), so ergeben sich daraus 490 Personen, durchschnittlich 6,53 Bewohner pro Haus. Dies liegt nur geringfügig über den von JANSSEN vorgeschlagenen Werten. Die Berechnungen stehen unter der Prämisse, daß alle Gebäude einstöckig sind. Eine mehrstöckige Bebauung, die zudem noch unregelmäßig erfolgen kann, läßt sich mit diesen statischen Methoden nicht erfassen. Zumindest in Einzelfällen konnten bisher Aufbauten auf Häusern rekonstruiert werden, jedoch mit dem ausdrücklichen Vermerk, daß es sich dabei nicht zwangsläufig um einen regelhaften Bautypus handeln muß. ${ }^{379}$

\subsubsection{Die Grenzen der Berechnungsmöglichkeiten}

Wie groß die Differenzen zwischen errechneten und geschätzten Größenangaben tatsächlich liegen, läßt sich ebenfalls am Beispiel Amarnas aufzeigen. Ausgangspunkt sei dabei die Schätzung JANSSENs, der aufgrund seiner Einzelbetrachtungen der Siedlungsanlage zu dem Urteil gelangte, daß in Amarna gegen Ende der Regierungszeit Echnatons etwa 50000 bis 100000 Menschen wohnten. ${ }^{380}$ Dieser Schätzung waren die Ergebnisse der Untersuchungen in den Flächen Q 46 und Q 47 zugrundegelegt. Die Besiedlungsdichte einer Fläche, die jeweils $200 \times 200 \mathrm{~m}$ mißt, ${ }^{381}$ gab JANSSEN mit rund 700 Menschen an. ${ }^{382}$ In einem nächsten Schritt

379 KEMP (1987b: 27).

380 JANSSEN (1983: 287); dagegen setzt KEMP (1991: 269) die Bevölkerung Amarnas niedriger auf 20000 bis 50000 Menschen an und begründet dies mit der zur Verfügung stehenden Agrarfläche, deren Erträge maximal etwa 45000 Menschen versorgen konnte.

381 JANSSEN (1983: 283 Anm. 81).

382 JANSSEN (1983: 285). 
mußte die Anzahl der Flächen ermittelt werden, die nach Lage und Beschaffenheit der architektonischen Reste als Wohngebiet bezeichnet werden konnten. Die Summe dieser Flächen erlaubte dann die approximierte Berechnung der Bewohner Amarnas, die mangels Kenntnis über die tatsächliche Siedlungsfläche zusätzlich noch aufgerundet wurde. Es läßt sich bereits hier feststellen, daß die Ergebnisse JANSSENS eine Kette von Schätzungen darstellen, deren einzelne Fehlerraten sich kaum beurteilen lassen. Daraus erklärt sich auch die recht große Spannweite des Endergebnisses, dessen Nutzen damit fraglich erscheint.

Für den Versuch einer Gegenrechnung dieser Schätzung wurden die Daten über die ab 1911 von der Deutschen Orientgesellschft unter der Leitung von Ludwig BORCHARDT ausgegrabenen Wohnhäuser Amarnas herangezogen, die Christian TIETZE für seine eigenen Untersuchungen statistisch aufbereitet hat. ${ }^{383}$ Legt man wiederum die Vorschläge JANSSENs hinsichtlich der Anzahl der Bewohner einer >Wohneinheit $<$ zugrunde (Tab. 4.1) und rechnet diese Zahlen mit den Tabellen TIETZEs durch, zeigt sich rasch ein ernüchterndes Bild (Tab. 4.2). Die durch Ausgrabungen erschlossene Wohnfläche würde gerade einmal Raum für 3,5-7\% der von JANSSEN vermuteten Bewohnerzahl Amarnas zur Verfügung stellen.

\begin{tabular}{|c|c|c|c|c|c|c|}
\hline Haustyp & $\varnothing$ Räume & $\varnothing$ Fläche (qm) & Anzahl & Bewohner & $\Sigma(\min )$. & $\Sigma(\max )$. \\
\hline $1 \mathrm{a}$ & 3,07 & 22,52 & 27 & $5(6)$ & 135 & 162 \\
\hline $1 b$ & 4,14 & 33,14 & 70 & $5(6)$ & 350 & 420 \\
\hline $1 \mathrm{c}$ & 5,45 & 46,52 & 105 & $5(6)$ & 525 & 630 \\
\hline $1 d$ & 8,05 & 68,50 & 60 & $5(6)$ & 300 & 360 \\
\hline $2 c$ & 5,91 & 61,73 & 33 & $5(6)$ & 165 & 198 \\
\hline $2 d$ & 9,46 & 102,47 & 89 & $7,5(8)$ & 667,5 & 712 \\
\hline $2 \mathrm{e}$ & 12,89 & 147,26 & 47 & $7,5(8)$ & 352,5 & 376 \\
\hline \multirow[t]{2}{*}{$3 e$} & 18,03 & 330,58 & 36 & $15(20)$ & 540 & 720 \\
\hline & & & & & 3035 & 3500 \\
\hline
\end{tabular}

Tabelle 4.2: Berechnung der Bewohnerzahl Amarnas

Da auch die Datenbasis der ausgegrabenen Häuser lückenhaft und alles andere als klar definiert ist, scheint ein Vergleich zur Flächenberechnung JANSSENs nicht statthaft. Entscheidender Mangel ist zumindest die Unkenntnis über den Anteil, den die Häuser in der Gesamtfläche Amarnas einnehmen. Der Zweck dieser Demonstration kann es auch nur sein, die Unsicherheiten, mit denen sämtliche Verfahren behaftet sind, klarzustellen und die Problematik mangelnder Übereinstimmung der auf verschiedenen Wegen gewonnenen Ergebnisse aufzuzeigen.

383 TIETZE (1985). 


\subsection{Die Arbeitersiedlung Deir el-Medineh}

\subsubsection{Aufbau}

Die Arbeitersiedlung von Deir el-Medineh wurde unter Thutmosis I. angelegt. In ihr siedelte man alle Handwerker und Künstler an, die zu den Arbeiten an den Gräbern im Tal der Könige herangezogen worden waren. Dies hatte einerseits den Vorteil der räumlichen Nähe zum >Arbeitsplatz<, bot aber andererseits der Administration auch eine gewisse Kontrolle über das Personal. Da bei größeren Bauvorhaben die Anzahl der Arbeiter wiederholt aufgestockt wurde, gab es zwei Phasen einer Erweiterung der Anlage, die unter Thutmosis III. ${ }^{384}$ und Sethos I. stattfanden. ${ }^{385}$ In der letzten Ausbaustufe umfaßte die Siedlung ein Areal von ungefähr $50 \times 132 \mathrm{~m}$ in einem regelmäßigen Grundriß, jedoch weitaus weniger schematisch als die Amarna-Siedlung, da unter anderem durch neue Unterteilungen und den Häusern hinzugefügte Zwischenmauern die ursprünglich strikte Konzeption der Gesamtanlage aufgegeben wurde.

Die ummauerte Anlage der dritten und letzten Bauphase (Abb. 4.2) umfaßt etwa 70 Häuser, die eine mittlere Fläche von 72 qm aufweisen. Die Detailangaben zur Größe der Häuser schwanken, VALBELlE gibt eine Breite von 3-7 m sowie eine Länge von 10-24,5 m an, so daß die Hausgrundrisse im Einzelfall zwischen 40 und 120 qm liegen. ${ }^{386}$

Weitere 50 Häuser liegen außerhalb der Ummauerung, finden im folgenden jedoch keine Berücksichtigung, da sie nicht den Prämissen für die hier zu untersuchenden Siedlungen entsprechen.

\subsubsection{Statistische Auswertung}

Eine vielbeachtete Schätzung zur Bevölkerungsgröße der Arbeitersiedlung Deir el-Medineh stammt bereits aus dem Jahr 1972 und wurde von Mario TOSI und Alessandro ROCCATI im Rahmen ihres Katalogs zu den Stelen und epigraphischen Funden aus Deir el-Medineh formuliert. ${ }^{387}$ Insgesamt soll es - nach Auskunft einer Zählung aus der Zeit Ramses’ III. - 120

384 Identifiziert anhand von Ziegelstempeln; DONADONI (2000: 193).

385 Die genaue Datierung der Bauphasen ist umstritten. Es gibt auch Vorschläge, daß erst unter Ramses' II. oder seinen Nachfolgern die 3. Bauphase Deir el-Medinehs fertiggestellt wurde. Da die Fertigstellung in jedem Fall in der 19. Dynastie erfolgt sein dürfte, spielt eine exakte Datierung für die weiteren Betrachtungen der Bevölkerungsgröße nur eine untergeordnete Rolle.

386 Valbelle (1985b: 117); RoiK (1988: 14 Anm. 30) dagegen geht anscheinend von zu kleinen Dimensionierungen aus, da sie nur einen Teil des Geländes auswertet.

387 Tosi und RocCATI (1972: 11). 
Herdstellen (= Haushalte) gegeben haben, die von 1200 Menschen bewohnt wurden, so die vagen Formulierungen. Diese Angaben bergen gleich mehrere Probleme in sich. Zunächst stellt sich die Frage nach der Quelle. Der einzige Papyrus, der hinsichtlich Datierung und der Zahlenangabe von 120 Personen mit dem Zitat bei TOSI und ROCCATI in Einklang gebracht werden kann, ist der Papyrus Turin 1891, auf dessen Recto allerdings keine Zählung verzeichnet ist, sondern die Gründung einer Kommission im 2. Regierungsjahr Ramses' IV., die die Anzahl der Arbeiter auf 120 Männer aufstocken soll. ${ }^{388}$ Darüber hinausgehende demographische Daten lassen sich dem Text nicht entnehmen. Selbst die Erwähnung der 120 Männer ist zu vage, um weitergehende Schlußfolgerungen für die Bevölkerung zu ziehen. Die Gesamtzahl wird schließlich aus einer einfachen 1:10 Relation ermittelt, der eine Argumentationsbasis 1 Mann = 1 Haushalt zugrunde gelegt ist. ${ }^{389}$ Die Schätzung, daß in jedem Haushalt durchschnittlich 10 Personen leben, widerspricht einerseits unserer Kenntnis über Haushaltsgrößen, die aus den Papyri gewonnen wurde, ${ }^{390}$ reicht aber recht nahe an NAROLLs mathematische Approximationen heran, wie im folgenden gezeigt werden kann.

Bei einer Berechnungsgrundlage von 7 1/2 Personen für eine mittlere Hausgröße von 72 qm nach JANSSEN betrug die Bevölkerung Deir el-Medinehs 525 Personen. BADAWY geht sogar von einer möglichen höheren Population aus, da aufgrund des fehlenden Rasters und der geringeren Anzahl von Straßen eine höhere Bevölkerungsdichte möglich war. ${ }^{391}$ Die Gegenprobe mit NAROLLs Formel (3) fällt wiederum mit 660 Personen etwas höher aus und resultiert in durchschnittlich 9,4 Personen pro Haus. Berechnungsgrundlage dafür war - wie bereits im Fall der Amarna-Siedlung - die Gesamtfläche Deir el-Medinehs von rund 6600 qm und nicht nur die Summe der Wohnraumfläche. Sollte BADAWY mit seiner Einschätzung zur Bevölkerungsdichte Deir el-Medinehs Recht haben, dann findet seine Vermutung in der Berechnung nach NAROLL Bestätigung, obgleich die Zahl den Kenntnissen aus den Zensus-Papyri widerspricht. Es muß dann davon ausgegangen werden, daß die Formel >ein Haus = ein Haushalt möglicherweise nicht konsequent für die gesamte Siedlung galt und die von TosI und RocCATI geäußerte Vermutung sogar im Rahmen des Möglichen liegt.

388 KRI VI 76 f.; VAlbelle (1987: 42); Gutgesell (1983: 126 f.); ČERnÝ (1973: 103 f.).

389 Ausgelassen werden dabei die Möglichkeiten, ob sich zuweilen mehrere Familien ein Haus geteilt haben oder ob Vater und Sohn gleichermaßen als Handwerker beschäftigt waren; vgl. dazu auch den Hinweis bei ROIK (1988: 16).

390 Vgl. etwa VAlbelle (1985a: 84), die sogar nur eine Durchschnittsgröße von 3 bis 5 Personen, davon 2 Erwachsene, für eine einfache Familie annimmt und den Bereich von 2 bis 6 Personen als Minimum/Maximum betrachtet, wobei Diener oder Sklaven nicht berücksichtigt sind.

391 BADAWY (1968: 63). 


\subsection{Die Siedlungsanlage von Kahun - Versuch einer Näherung}

\subsubsection{Aufbau}

Schwieriger gestaltet sich eine statistische Herangehensweise bei der Siedlungsanlage von Kahun. Obwohl ihr genauso wie den beiden anderen zuvor besprochenen Siedlungen ein festes Planungskonzept zugrunde zu liegen scheint, weist der bisher archäologisch nachgewiesene Grundriß noch zu viele ungeklärte Fragen auf. Ausgegraben wurde die Siedlung, die in der Nähe des modernen Ortes el-Lahun liegt, in den Jahren zwischen 1888 und 1890 von William Matthew Flinders PetRIE, der zusammen mit Guy BRUnTON 1914 nochmals für kurze Zeit die Arbeiten fortsetzte. ${ }^{392}$ Von PETRIE stammt auch der bis heute geläufige Name $>$ Kahun $<$. Erbaut wurde die Siedlung in der 12. Dynastie während der Regierungszeit Sesostris’ II., der die Anlage als überdimensioniertes Beispiel einer $>$ Pyramidenstadt $<$ in Auftrag gab. Ihre Funktion als Unterkunft für die Priesterschaft des Totenkults ergibt sich aus der unmittelbaren Nachbarschaft zum Taltempel, dessen Achse parallel zur Südmauer Kahuns verläuft sowie die relative Nähe zur Pyramide Sesostris' II., die etwas mehr als 1 km westlich der Siedlung liegt. Auch aus den in Kahun gefundenen Papyrus-Archiven geht hervor, daß die Bewohner in den Totenkult für den verstorbenen Sesostris II. involviert waren. ${ }^{393}$ Die bekannte Fläche hat sich seit den Grabungen PETRIEs nicht wesentlich geändert und ist praktisch im gesamten Südwestteil der Siedlung durch Winderosion und teilweise auch durch die Ausdehnung des Fruchtlandes für die archäologische Auswertung verloren, so daß die Siedlung heute im Übergang zwischen Fruchtland und Wüste liegt. ${ }^{394}$ Als Arbeitsgrundlage für die Ermittlung der Grundfläche wird daher häufig auf die Außenmauern an der Nord- und Westseite verwiesen, deren Längen bekannt sind. Nach KEMP umfaßt die Fläche demnach grob ein Quadrat von ca. $384 \times 335 \mathrm{~m}$ (Abb. 4.3). ${ }^{395}$

KEMP selbst hat an gleicher Stelle auch eigene Schätzungen der Bevölkerungszahl Kahuns vorgenommen. Neben einer Kalkulation anhand der Versorgungssituation auf Basis der in Kahun gefundenen Getreidesilos, ${ }^{396}$ benutzt er auch die aus den Kahun-Papyri I.3-I.5 ermittelte Durchschnittsgröße für einen Haushalt von sechs Personen. ${ }^{397}$

392 KEMP (1991: 149 ff.); DAVID (1986: 101 ff.).

393 KEMP (1991: ebenda).

394 PETRIE (1891: 8).

395 KEMP (1991: 151).

$396 \operatorname{KEMP~(1991:~} 153$ f.).

397 KEMP (1991: 155). 


\subsubsection{Gebäudetypen und Verteilung}

Nach Elke RoIK lassen sich in Kahun fünf Typen von Häusern (Tab. 4.3) unterscheiden. Der kleinste vertretene Typus weist eine Grundfläche von etwa $8,5 \times 5,25 \mathrm{~m}$ auf und ist in zwei Räume, eine kleine Kammer und einen Eingangshof unterteilt. Der nächstgrößere Typus von RoIK in zwei unterschiedliche Subtypen (1 und 2) klassifiziert - hat mit seinen Maßen von 7,5 $\times 8 \mathrm{~m}$ bereits eine beachtliche Dimensionierung. Die dritte und größte Bauform ebenfalls von ROIK in zwei Subtypen (4 und 5) getrennt - dehnt sich auf einer Fläche von ca. 168 qm bei unterschiedlicher Geometrie aus, wobei ROIK nur rund 150 qm als reine Wohnfläche kalkuliert. Die Subtypen bezeichnen dabei lediglich Unterschiede in der Raumaufteilung bei identischer Grundfläche. Zusätzlich zu diesen Häusertypen existieren noch die im Norden Kahuns gefundenen Strukturen, die jedoch nicht mehr als >Haus< bezeichnet werden können, sondern mächtige, repräsentative Wohnanlagen mit Nebengebäuden, Höfen usw. darstellen, die sich über etwa $42 \times 60$ m erstrecken. ${ }^{398}$

\begin{tabular}{|c|c|c|}
\hline Тур & Grundfläche & Anzahl Räume \\
\hline$>$ Herrenhaus & $2700 \mathrm{qm}$ & \\
\hline $4 / 5$ & $168 \mathrm{qm}$ & $5-6$ \\
\hline $1 / 2$ & $60 \mathrm{qm}$ & $3-4$ \\
\hline 3 & $44 \mathrm{qm}$ & $2-3$ \\
\hline
\end{tabular}

Tabelle 4.3: Haustypen in Kahun nach Einteilung von RoIK

Bereits bei der ersten Sichtung des Plans fällt die recht schematische Verteilung der Häusertypen auf. Der häufigste Haustypus von $60 \mathrm{qm}$ läßt sich im wesentlichen im Westen der Siedlung nachweisen, wo er fast durchgehend von Norden bis Süden etwa ein Viertel der Gesamtfläche einnimmt. Vom Rest der Siedlung ist dieser Bezirk nochmals durch eine NordSüd-Mauer abgeteilt, deren Zweck unbekannt ist. ${ }^{399}$ Die auf dem Plan vermerkten Gebäudereste der Südwestecke oberhalb des Tempels geben zu der Vermutung Anlaß, daß weitere Häuserzeilen auf dem Gelände zu erwarten sind.

Die großen >Herrenhäuser`sind alle im Norden der Siedlung zu finden. Ein Teil von ihnen grenzt direkt an die Nordmauer, andere hingegen liegen diesen gegenüber an der selben

398 Vgl. zuletzt ausführlich O'CONNOR (1997). Dieser Typus wird im folgenden nach Vorschlag von RoIK (1988: 8 Anm. 17) als >Herrenhaus< angesprochen, ohne dabei näher auf dessen Funktion einzugehen. Die Angaben zur Größe differieren bei den Autoren: RICKE (1932: 52) und RoIK (1988: 8): $45 \times 60$ m; PETRIE (1891: 7) und KeMP (1991: 151): $42 \times 60 \mathrm{~m}$; BADAWY (1966: 22): $40 \times 60 \mathrm{~m}$.

$399 \operatorname{KeMP}(1991: 151)$. 


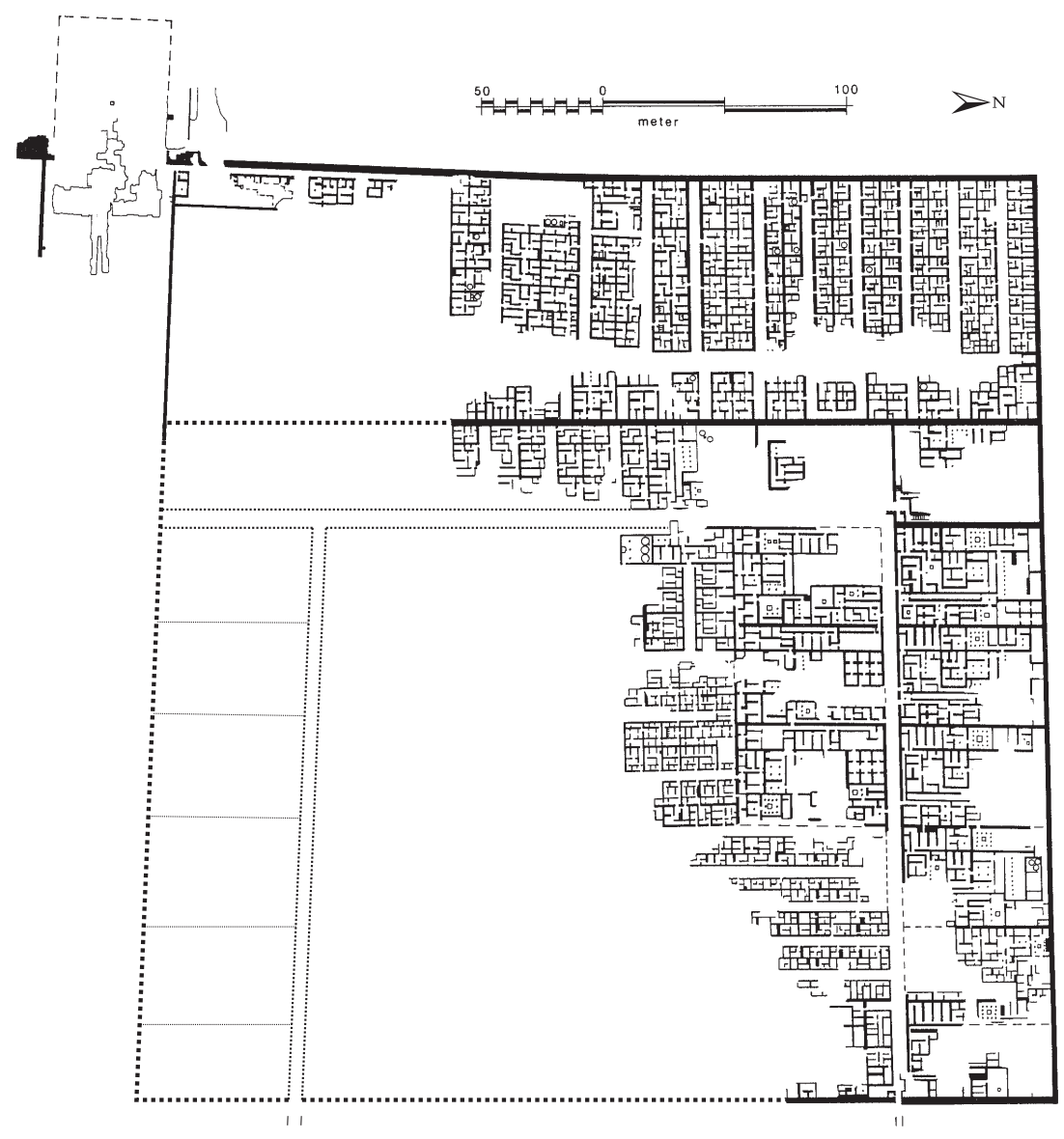

Abbildung 4.3: Die Siedlungsanlage von Kahun

$>$ Hauptstraße <. In der Ecke zwischen Nordmauer und der Trennmauer zur >Weststadt $<$ liegt ein Komplex, der als Königsresidenz identifiziert wird und als höchster Punkt der Siedlung den Namen >Akropolis< erhalten hat. ${ }^{400}$ Insgesamt können von den >Herrenhäusern< acht oder neun nachgewiesen werden. Der größere Haustypus liegt teils ebenfalls direkt an der Hauptstraße im Norden, teils südlich an die >Herrenhäuser< angrenzend und bildet damit eine mittlere Ost-West-Achse in der Siedlung.

Die entscheidende Frage, die auch auf eine näherungsweise Kalkulation der Bevölkerung Einfluß hat, lautet nun, wie sich die Struktur der Siedlung im Süden fortsetzt. Dafür können aus der vorliegenden Anordung einige Anhaltspunkte gewonnen werden, die in dem Plan als gestrichelte Linien eingezeichnet sind (Abb. 4.3). Ausgangspunkt der Überlegungen ist die Suche nach der Zugangsseite der Siedlung. Lediglich in der Nordost-Ecke ist ein Tor nachge400 Petrie (1891: 5 f.); BADAWY (1966: 22); KEMP (1991: 151). 
wiesen, das zudem den Weg auf die Hauptstraße entlang der >Herrenhäuser Route auch den Hauptweg zur Königsresidenz darstellt, steht der repräsentative Charakter der Strecke außer Zweifel. Neben fünf kleineren Straßen, die auf diese Hauptstraße treffen, biegt an der Ecke der Königsresidenz eine weitere breite Straße nach Süden ab. Obwohl unklar ist, wie der tatsächliche Straßenverlauf zu rekonstruieren ist, läßt sich als Hypothese vorschlagen, daß diese Straße nicht notwendigerweise bis zur südlichen Umfassungsmauer verläuft, sondern nochmals nach Osten abbiegt und auf ein zweites Tor im Südosten der Umfassungsmauer trifft. Damit könnte der Ostflanke der Umfassungsmauer als Hauptseite Rechnung getragen werden. ${ }^{401}$ Eine Öffnung nach Westen ist auszuschließen, da sich dahinter das Gelände der Nekropolen und der Pyramide aufspannt und der Wohnbereich der Arbeiter schwerlich als Frontseite der Siedlung begriffen werden kann. Auf der Südseite hingegen würde der Zugang zur Siedlung die Achse des Taltempels kreuzen, die parallel zur Südmauer der Siedlung verläuft. Diese Gründe zusammengenommen sprechen dafür, daß die Ostseite die Zugangsseite zur Siedlung darstellte. ${ }^{402}$ Darauf basierend darf man entlang der Südmauer ebenfalls Wohnbereiche rekonstruieren, die zu beiden Seiten der südlichen $>$ Hauptstraße< liegen und zusammen mit den anderen Wohnvierteln den im Zentrum vermuteten >öffentlichen< Bereich einfassen.

\subsubsection{Modellrechnungen}

Für einen ersten Zugang zu der Bevölkerungszahl Kahuns soll eine Hochrechnung anhand eines Ausschnitts der Westsiedlung vorgenommen werden, weil dieser die homogenste Bebauung aufweist. Aus der Nordwest-Ecke wird ein Rechteck als repräsentative Größe herausgenommen. Die Breite beträgt die volle Ausdehnung zwischen östlicher und westlicher Außenmauer dieses >Stadtteilsく, also ungefähr 100 m. Die Höhe des Rechtecks wurde auf $50 \mathrm{~m}$ festgelegt, so daß die obersten drei Reihen an Gebäudegrundrissen vollständig erfaßt sind. ${ }^{403}$

401 Diesen Vorschlag hat im Prinzip bereits O’Connor (1997: 390, Abb. 1) gemacht. O’ConNors weiteren Vorschlägen zur Unterteilung des durch die U-förmige Umfassung gewonnenen >Stadtviertels< durch eine mittig verlaufende Ost-West-Straße sowie einer weiteren Nord-Süd-Achse, die etwa $50 \mathrm{~m}$ hinter der Ostgrenze der Siedlung liegt, vermag hier nicht gefolgt zu werden.

402 Zur topographischen Situation vgl. PETRIE u. a. (1923: Taf. II).

403 Aufgrund der Verkleinerung der publizierten Pläne und der Ungenauigkeiten in der Skalierung können die gemessenen Abweichungen signifikant sein. Eine Überprüfung der hier am >grünen Tisch< entwickelten Berechnungen durch eine exakte Aufmessung in Kahun hat bereits RoIK (1988: 8 Anm. 17) als Desiderat angemahnt. RICKE (1932: 51) hat gar die Untersuchungen der kleinen Häuser anhand der Pläne aufgrund der mangelhaften Publikation PETRIEs gänzlich in Frage gestellt. KEMP (1991: 155) weist jedoch zu Recht darauf hin, daß die statistische Auswertung der Wohnhäuser den sichersten Zugang zu den einstigen Bevölkerungszahlen bietet. 
Folgende Aspekte fanden darüber hinaus Berücksichtigung: Durch das gesamte Viertel führt in Nord-Süd-Achse eine breite Straße. Die Gebäude östlich davon, die zwischen Straße und der Trennmauer zum Rest der Siedlung liegen, können nicht durchgehend als Wohngebäude nach den Vorgaben von RoIK identifiziert werden. Daher wurden nur die Häuser westlich der Straße in die Zählung mit einbezogen.

Auf der Fläche von 5000 qm befanden sich 45 Häuser des Typs 1 und 2 nach RoIK. ${ }^{404}$ An reinem Wohnraum sind also etwa 2700 qm oder $54 \%$ der Gesamtfläche genutzt. Nimmt man 6 Personen pro Haus - basierend auf der in Tabelle 4.1 vorgeschlagenen Gebäudegröße - als Berechnungsgrundlage, so leben insgesamt 270 Personen auf der $>$ Testfläche $<$. Hochgerechnet auf die gesamte Westsiedlung ergibt sich folgendes Bild: Die Fläche wird entgegen ihrer tatsächlichen Geometrie als echtes Rechteck aufgefaßt. Die Ausdehnung von Norden nach Süden wurde an der östlichen Trennmauer bis zum Auftreffen auf die Reste der Südmauer abgetragen. Die Länge dieser Mauer beträgt ca. 336,5 m, die Gesamtfläche der Westsiedlung demnach $100 \times 336,5 \mathrm{~m}=33650$ qm. Auf $54 \%$ dieser Fläche leben hochgerechnet mindestens 1817 Menschen. Bei dieser Berechnung fand keine Berücksichtigung, daß RoIK im Süden des Stadtteils die größeren Gebäude vom Typ 4 und 5 lokalisiert hat. Da nach JANSSENs Tabelle die Anzahl der Personen pro Haushalt ungefähr proportional steigen, sollte dies keine gravierenden Auswirkungen auf das Endergebnis haben.

Eine Ausdehung dieser Teilberechnung auf die gesamte Fläche Kahuns gestaltet sich als nahezu unmögliches Unterfangen. Wie auf Abb. 4.3 ersichtlich, fehlen erhebliche Informationen zur Bebauungsstruktur besonders in der Südhälfte der Siedlung. Nur wenige Hinweise lassen sich für Rekonstruktionsversuche auswerten. So kann etwa mit einiger Wahrscheinlichkeit ausgeschlossen werden, daß an der Südmauer der Siedlung - spiegelbildlich - weitere große Wohnanlagen standen, wie sie entlang der Nordmauer identifiziert wurden. Der Grund liegt darin, daß die Gebäudekomplexe im Norden das Privileg besaßen, direkt dem kühlen Nordwind ausgesetzt zu sein und damit günstigere klimatische Wohnbedingungen boten. ${ }^{405}$ Auch andere Areale der Siedlungsanlage sind in ihrer Funktion noch nicht gesichert. So liefern zum Beispiel die Strukturen im Zentrum der Siedlung Hinweise darauf, daß es sich dabei um Lagerräume oder ähnliches gehandelt haben könnte. ${ }^{406}$

404 Zur Typeneinteilung siehe oben S. 111, Tab. 4.3.

405 EndRUweit (1994: 70 ff., 168 ff.); die Unterteilung entlang der Südwand in Abb. 4.3 stellt nur eine Markierung der Achsen dar.

406 DAVID (1986: 108). 
Selbst wenn man das Ergebnis der Weststadt mit $1 / 3$ der Gesamtfläche Kahuns als Durchschnitt nimmt und auf die Gesamtfläche hochrechnet, so kommt man im Ergebnis doch nur auf eine Bevölkerungszahl von 5400 Menschen, die zudem noch als absolute Obergrenze gelten muß. Abstriche müssen hinsichtlich der großen Wohnanlagen und der tatsächlichen Besiedlungsdichte einzelner Häuser gemacht werden. Dies entspricht ungefähr der Zahl, die von KEMP auf der Basis optimaler Versorgung geschätzt wurde. ${ }^{407}$ Die höheren Angaben zwischen 8500 und 10000 Einwohnern sind nach Meinung KEMPs nur erreichbar, wenn die kalkulierten Nahrungsrationen pro Person drastisch reduziert werden. ${ }^{408}$ KEMP geht sogar noch weiter, indem er nach PETRIEs Plan die Häuser einzeln abzählt. Doch auch KEMP kämpft mit der unvollständigen Datenbasis Kahuns. Die rund 220 identifizierten Häuser ergeben zu den großen Wohnanlagen ein Verhältnis von mindestens $20: 1$. Diese Relation ist vielleicht sogar noch höher, weil laut KEMP davon ausgegangen werden muß, daß auf der restlichen Fläche Kahuns eher kleine als große Häuser angelegt worden waren. ${ }^{409}$ Bei einer groben Hochrechnung unter der Prämisse, daß PETRIEs Plan nur ungefähr die Hälfte der Wohnhäuser erfaßt hat und jeder Haushalt sechs Personen beherbergte, kommt KEMP auf eine Summe von knapp 3000 Menschen.

Betrachtet man die Summe der Näherungsversuche, so bleiben am Schluß mehr Vermutungen als Erkenntnisse übrig. Die Betrachtungen KEMPs hinsichtlich der Versorgungslage Kahuns und seine Hochrechnung der Häuser, verbunden mit den flächenbasierten Alternativrechnungen, lassen - bei aller Vorsicht - die These zu, daß für Kahun eine Größenordnung zwischen 5000-6000 Bewohnern als Obergrenze eine vertretbare Aussage darstellt.

\subsection{Hochrechnungen auf regionaler Ebene}

Sofern die Kalkulationen für Bevölkerungszahlen auf Siedlungsebene geglückt erscheinen, rückt als nächste Größenordnung eine Siedlungsregion ins Zentrum der Betrachtung. Doch spätestens hier zeigen sich überdeutlich die Grenzen, die einer auf >historischen< Daten basierenden Berechnung gesetzt sind. Zum einen fehlen verläßliche Daten zu Verteilung und Größe der Siedlungsplätze, die über das von Karl BUTZER zusammengestellte Material hinausgehen. ${ }^{410}$ Zum anderen sind in den ägyptischen Quellen nur wenige, kaum verwertbare Hinweise zur regionalen Bevölkerung zu finden.

407 KEMP (1991: 153 f.).

408 Vgl. etwa die Kalkulation von 8747 Einwohnern durch BADAWY (1968: 61) und den eigenwilligen Vergleich mit den 10000 Männern in der Lehre für Merikare.

409 KeMP (1991: 155).

410 BUTZER (1976). 


\subsubsection{Papyrus Harris I}

Der von Ramses IV. in Auftrag gegebene Papyrus Harris I liefert eine Aufstellung über Schenkungen, die Ramses III. an diverse Tempel vergab. Diese Schenkungen bestanden aus arbeitsfähigen >Untertanen < und agrarfähigem Boden. Im einzelnen sind für bestimmte Tempelbezirke folgende Personenzahlen vermerkt worden:

\begin{tabular}{lr}
\hline Theben: & 86486 \\
Heliopolis: & 12364 \\
Memphis: & 3079 \\
Sonstige: & 5686 \\
\hline
\end{tabular}

Dabei hat der Schreiber sich in der Addition der Einzelangaben mehrfach vertan und die Gesamtsumme der Personen um 5818 zu hoch angegeben. ${ }^{411}$ Maßgeblich für die Auswertung der im Papyrus verzeichneten Zahlen ist die Untersuchung von Herbert SCHAEDEL, der bereits 1936 vorschlug, daß es sich bei den Angaben lediglich um die arbeitsfähigen Männer handelt. ${ }^{412}$ Begründet wurde dies damit, daß der Informationsgehalt des Papyrus sich primär um die Größe des königlichen Besitzes dreht und die Familiengröße ein starken Schwankungen unterworfener Faktor ist. Im Zentrum des Interesses stand die auf dem Ackerland verfügbare Arbeitskraft. Aus dieser Grundannahme zog SCHAEDEL weitere Schlüsse. Zum einen errechnete er anhand der Ergebnisse der Volkszählung aus dem Jahr 1882 ein Verhältnis von 1:1 zwischen Männern und Frauen, womit sich die Gesamtzahl bereits auf 215230 Menschen verdoppelt. In einem letzten Schritt addierte er pro Familie mindestens zwei bis drei Kinder dazu, die mit Hinblick auf den Verfall Ägyptens seit der 19. Dynastie als niedrige Schätzung angesetzt wurden. SCHAEDEL kam so zu einer Zahl von rund 500000 Menschen, die von Ramses III. in die Obhut der Tempel überantwortet wurde. Basierend auf seiner Vermutung, daß in der Ramessidenzeit Ägypten eine recht starke Bevölkerung von 8-9 Millionen Menschen besaß, kommt er auf einen Anteil von $6 \%$ der Gesamtbevölkerung.

Die Überlegungen SCHAEDELs sind seitdem ohne nennenswerte Beanstandung von anderen Bearbeitern übernommen worden. ${ }^{413}$ Dabei können schwere Vorbehalte gegen die von SCHAEDEL aufgemachte Rechnung angeführt werden. Bereits die Prämisse, daß es sich bei

411 GRANDET (1994: 89, Tab. 22).

412 SCHAEDEL (1936: $56 \mathrm{f}$.).

413 Von BAER (1962: 42) als »reasonable figure« akzeptiert, da sich für ihn die Umrechnungen von 2 Aruren Land pro Person als realistisch darstellt; zuletzt auch von GRANDET (1994: 88 f.) ohne Einwände übernommen. 
den Angaben des Papyrus Harris nur um die arbeitsfähigen Männer gehandelt hat, ist alles andere als gesichert. Der im Papyrus verwendete Terminus $t p . w$ ist zunächst neutral als $>$ Köpfe $<$ zu verstehen, ganz im Sinne wie der Begriff auch heute noch bei Zahlenangaben von Personen Verwendung findet. ${ }^{414}$ Auch die Methode einer Hochrechnung der Familiengröße muß kritisch hinterfragt werden. Während SCHAEDEL zunächst noch betont, daß die Anzahl der Familienangehörigen gewissen Schwankungen unterliegt, basiert seine darauf folgende Berechnung auf einer schematischen Vorstellung über altägyptische Familien. Wenn nur die Hälfte der von ihm angesetzten Männer verheiratet ist und von diesen möglicherweise nur die Hälfte Kinder besitzt, liegt das Ergebnis mit knapp 270000 Menschen fast $50 \%$ unter der Berechnung ScHAEDELs. Eine pauschale Verheiratung aller männlichen Personen birgt selbst als krude Schätzung zu viele Risiken, zumal die Kalkulationen ScHAEDELs zum Teil auf rezenten Volkszählungen basieren. ${ }^{415}$

Darüber hinaus läßt sich eine regionale Zuordnung der Menschen nicht sicher treffen, da es für die Aussage des Papyrus auch eine andere Interpretationsmöglichkeit gibt. Die Personenangaben sind dort untrennbar mit den Flächenangaben des dazugehörigen Landes verbunden. Oder anders gesagt: Arbeitskraft und Ackerboden stellen eine Einheit dar. Wenn dies der Fall sein sollte, ist eine räumliche Nähe zu dem zugewiesenen Tempel nicht mehr zwingend erforderlich. Man könnte sich vielmehr vorstellen, daß die Zuweisungen an den Tempel in Form einer Domäne an anderen Orten Ägyptens lagen und lediglich deren Ertrag auf das >Konto< des jeweils vermerkten Tempels gutgeschrieben wurde. ${ }^{416}$

Zugleich stellt der Additionsfehler, der dem Verfasser des Papyrus unterlief, ein illustres Beispiel für die Irrtümer und Ungenauigkeiten dar, die auch in den vermeintlich fehlerfreien antiken Quellen auftreten können. Die Textstelle mahnt daher vor allzu blindem Vertrauen, das oftmals in die Belege hinsichtlich ihrer Genauigkeit gesetzt wird.

\subsubsection{Die Lehre für Merikare}

Ein weiteres Beispiel einer Größenangabe, für die jedoch noch ein korrekter Kontext erarbeitet werden muß, findet sich in der Lehre für Merikare. Dort heißt es, daß der Bezirk Djed-sut $(\underline{D} d$-sw.t) 10000 Mann an >Kleinen< zählt, die freigestellt und ohne Verpflichtungen sind. Wolfgang HELCK weist darauf hin, daß die Pyramidenanlage des Teti Djed-sut der Kern des

$414 \mathrm{~Wb} \mathrm{V,268.13-15.}$

415 Zur Problematik der modernen Volkszählungen Ägyptens vgl. auch unten Kap. 7.

416 Ansatzweise wurde diese These bereits von STRUDWICK (1995: 100 Anm. 17) formuliert. 
memphitischen Siedlungsgebietes war. ${ }^{417}$ Deshalb sind die $n \underline{d} s . w$ die Angehörigen dieser Pyramidenstadt zusammen mit ihren Priestern. ${ }^{418}$ Die Ansicht HeLCKs, daß in dieser Textstelle unter $n \underline{d} s . w$ die Bewohner der Pyramidenstadt subsumiert sind, ${ }^{419}$ fand nicht ungeteilte $\mathrm{Zu}$ stimmung. Insbesondere über den Status der $n \underline{d} s . w$ ist in der Vergangenheit heftig gestritten worden, da in der älteren Forschung die definitorische Trennung von >Freien< und >Hörigen nicht recht gelingen wollte. Auch über die Bedeutung der Bezeichnung Djed-sut herrschte keine Einigkeit, doch scheint sich vermehrt die Ansicht durchzusetzen, daß nicht nur die Pyramidenstadt selbst, sondern die gesamte dazugehörige Region unter dieses Toponym fällt. ${ }^{420}$

Erst kürzlich hat sich Detlef FRANKE erneut mit dem Terminus $n \underline{d} s$ auseinandergesetzt und die bisherigen Interpretationen als zu pauschal zurückgewiesen. Er bezieht sich in seinem Aufsatz auf eine russische Arbeit von Oleg BERLEV und weist nach, daß die Verwendung von $n \underline{d} s$ sehr kontextabhängig ist. Für die Textstelle bei Merikare scheint eine militärische Konnotation am besten geeignet, so daß die 10000 Personen durchaus als >Miliz< ansprechbar sind, ${ }^{421}$ was auch in anderen Texten, etwa in dem Ausdruck $n \underline{d} s \underline{n} \underline{d} 3 m w$, Bestätigung findet. ${ }^{422}$ Damit wären $10000>$ Kleine< der militärische Kern in der memphitischen Provinz. ${ }^{423}$

Die Ergebnisse von FRANKE, verbunden mit der Tatsache, daß ohnehin die Textstelle ausdrücklich von Männern ( $z j$ ) spricht, lassen die früheren Überlegungen HELCKs nicht mehr zu, daß die Pyramidenstadt des Teti 10000 Einwohner hatte. Damit verbieten sich jedoch auch Vergleiche mit anderen Siedlungen, wie sie etwa BADAWY für seine Kahun-Berechnungen als stützendes Argument herangezogen hatte. ${ }^{424}$ Der Beleg aus der Lehre für Merikare kann in seiner bisherigen Form nicht länger als Quelle für lokale oder regionale Populationen dienen. Dagegen sei eine - zugegebenermaßen spekulative - These zu den Angaben in der Lehre für Merikare an den Schluß gestellt. Sie bildet eine Synthese aus den Beobachtungen HELCKs und FrankEs. Sollte HelcKs Vermutung zutreffen, daß Djed-sut das Zentrum der Region Memphis darstellte, könnten Überlegungen dahingehend angestellt werden, daß Djed-sut in diesem Fall als pars pro toto für den gesamten 1. unterägyptischen Nomos stand. Zusammen

417 HELCK (1977a: 63).

418 Die explizite Erwähnung der Priester in der älteren Forschung scheint auf einer irrtümlichen Interpretation von $w^{c} b$ zu beruhen. Zu diesem Terminus im Zusammenhang mit der Freistellung von gewissen Verpflichtungen vgl. ausführlich FRANKE (1998: 35 ff.).

419 HELCK (1958: 121).

420 KEES (1962: 127); FRANKE (1998: 34).

421 FRANKE (1998: 46).

422 Franke (1998: 45 Anm. 36).

423 Siehe oben Anm. 420.

424 Siehe auch oben S. 115 Anm. 408. 
mit den Ergebnissen FRANKEs stellen die 10000 nd $d s . w$ dann die potentielle Streitmacht dieses Nomos dar; ein Pool, aus dem sich die staatliche Administration bei Bedarf bedienen konnte. In Verbindung mit den Angaben auf der Stele Kairo CG $20732^{425}$ wäre dann eine Aussage getroffen, daß 1/100 = 100 Mann rekrutiert würden - eine Größenordnung für eine Truppeneinheit, die aus anderen Quellen vertraut und daher absolut plausibel ist.

\subsection{Zusammenfassung}

Da aus den ägyptischen Quellen wenigstens ansatzweise Angaben zur Zahl der Personen eines Haushalts ermittelt werden können, befindet man sich in einer glücklichen Lage, nicht allein auf universelle Formeln für Populationsgrößen angewiesen zu sein. Im Fall der `Eastern Village < konnte die Statistik dazu beitragen, die Grundannahmen zur >typischen< altägyptischen Haushaltsgröße weiter zu untermauern, so daß man auf Basis zukünftiger Siedlungsgrabungen besser gesicherte Erkenntnisse gewinnen kann. Berücksichtigt werden sollte dabei immer, daß die so gewonnenen Ergebnisse als Maximalwerte für das analysierte Areal gelten müssen. Leerstehende Gebäude, Teilbesiedlung und Fluktuation können als dynamische Prozesse von statischen Berechnungen nicht berücksichtigt werden und bleiben auch künftig die Achillesferse der präsentierten Ergebnisse. Eine halbwegs verläßliche Extrapolation der so gewonnenen Daten auf andere Siedlungen oder gar auf eine Gesamtbevölkerung Ägyptens kann mit dem vorliegenden Material nicht geleistet werden. In diesem Zusammenhang muß auch die Schätzung JANSSENs zu Amarna kritisch gesehen werden. Aufgrund der Lückenhaftigkeit der Kenntnisse über den Grundriß der Siedlungsfläche müssen seine Modellrechnungen als zweifelhaft gelten. Trotz der mittlerweile relativ gesicherten Faktenlage für einzelne Haushalte bleibt deren Leistungsfähigkeit für eine universelle Anwendung eingeschränkt.

Insbesondere bleibt die Erkenntnis, daß im Verlauf der Geschichte Schwankungen in der Familiengröße ein wahrscheinlicher Faktor sind und daher die aus den Zensus-Akten Kahuns gewonnene mittlere Größe eines Haushalts des Mittleren Reiches zwar eine willkommene, aber nicht notwendigerweise in andere Epochen übertragbare Einzelinformation darstellt. Die deutlich niedriger angesetzten Größen von VALBELLE für Deir el-Medineh weisen in diese Richtung und könnten ihren Grund in der speziellen sozialen Stratifizierung der Einwohnerschaft in den Arbeitersiedlungen haben. Andererseits weisen moderne Beispiele aus Staaten des Nahen Ostens darauf hin, daß theoriebasierte Betrachtungen zu Wohnfläche und Bevölke-

425 Vgl. dazu ausführlich unten Kap. 5.2.2.2. 
rungsdichte in der Realität ungünstigere Ergebnisse zeigen und die Fläche, die einer einzelnen Person zur Verfügung steht, deutlich geringer ausfällt, was in einer prinzipiell höheren Zahl an Bewohnern pro Wohneinheit resultiert. ${ }^{426}$

Für alle vorgeführten Beispiele gilt gleichermaßen, daß sie Sonderfälle einer Siedlungsanlage darstellen. Sie liegen auf einem fest umrissenen Areal, sind in der Regel von Mauern umfriedet und weisen eine Konstruktion > vom Reißbrett< auf. In den Arbeitersiedlungen von Amarna und Deir el-Medineh gehören die Bewohner einer bestimmten Bevölkerungsschicht an, deren interne Hierarchie zumindest flacher ausfällt als für die Sozialstrukturen anderer Orte Ägyptens anzunehmen ist. ${ }^{427}$ Insofern stellen sie einerseits den Idealfall für demographische Betrachtungen dar, bieten quasi >Laborbedingungen < für die Berechnung von Haushalten und Personen pro Fläche, müssen aber andererseits als Ausnahmeerscheinung gelten, von deren Ergebnissen keine universell gültigen Aussagen deduziert werden können. Diese Einschränkung ist der Forschung zur antiken Stadt wohl bewußt. ${ }^{428}$ Doch liegt gerade darin die Chance, über eine Kontrastierung zu anderen Siedlungsplätzen Faktoren herauszuarbeiten, die grundlegend für die Beschreibung einer ägyptischen Siedlung sind. Dies dürfte ein Feld künftiger Forschungen und Fragestellungen sein.

426 Vgl. zur Siedlungsdichte auch BUTZER (1984: 928).

427 Die Ausnahmesituation von Deir el-Medineh als untypische Siedlung hat bereits JANSSEN (1975b: 137) betont.

428 Vgl. Kolb (1984: 39). 


\section{Pharaonische Quellen: Zahlen und Verfahren}

You can be wholly precise in presenting a vagueness.

Ezra Pound, The Serious Artist

\subsection{Topos kontra Realität}

\subsubsection{Vom Umgang mit überlieferten Zahlen}

$\mathrm{W}$

ENN man der Frage nachgeht, welche konkreten Zahlen über die Bevölkerungen aus dem pharaonischen Ägypten überliefert worden sind, dann stellt man zunächst fest, daß die Informationen breit gestreut sind und es - im Sinne moderner Demographie - keinen Ansatzpunkt für statistische Auswertungen gibt. Detaillierte Geburtenregister oder Steuerlisten fehlen gänzlich. Die Angaben zu Zahl und Größe einzelner Bevölkerungsgruppen sind zeitlich und regional verstreut und nicht auf den ersten Blick in ihrer Qualität einschätzbar. ${ }^{429}$

Dennoch war für die Historiker in der Vergangenheit der Gedanke verführerisch, aus diesen wenigen Einzelinformationen ein mehr oder weniger vollständiges demographisches Gerüst des Alten Ägypten zu errichten, ohne im Einzelfall die Plausibilität ihrer Daten zu hinterfragen. Dieses Problem stellt sich in allen Disziplinen historischer Forschung und hat mitunter bis in die jüngste Zeit recht seltsame Blüten getrieben. David HENIGE nahm diese Situation zum Anlaß und formulierte für den Umgang mit den Quellen in seiner eigenen Disziplin - Mittelamerika zur Zeit der spanischen Eroberung - eine kritische Stellungnahme. ${ }^{430}$ Die von HENIGE den historischen Quellen entgegengebrachte Skepsis bezog sich besonders auf

$429 \mathrm{Zu}$ den vereinzelt erhaltenen Papyri mit Zensusdaten vgl. ausführlich oben Kap. 3.

430 HeNige (1998). 
die Verläßlichkeit ihrer Angaben und die Umstände ihrer Entstehung. Um als verwertbare Quellen im Sinne einer statistischen Auswertung gelten zu können, müssen sie vier Kriterien erfüllen: ${ }^{431}$

1. Fähigkeit zu präzisen Schätzungen oder Zählungen

2. Der Wille oder die Notwendigkeit zu solchen Zählungen

3. Historisch berichtete Zählungen haben tatsächlich stattgefunden

4. Die Ergebnisse sind fehlerfrei überliefert

Der auf diese Weise an die Quellen angelegte Maßstab ist hoch und besonders in der Ägyptologie nicht in allen Punkten an historischen Dokumenten demonstrierbar. ${ }^{432}$ Während über die ersten beiden Punkte in der Forschung noch ein gewisser Konsens herrscht und gerne die aus der Thinitenzeit bekannten frühesten Zählungen zitiert werden, ist der Nachweis, daß einzelne Zahlen auf einer realen Zählung basieren, häufig unmöglich. Ob Tausende Kriegsgefangene der Frühzeit oder die riesigen Expeditionen des Mittleren Reiches in das Wadi Hammamat Realität oder Propaganda sind, muß im Einzelfall durch Plausibilisierung der Angaben überprüft werden. Auch die Fehlerfreiheit kann nicht in jedem Fall garantiert werden, wie die Rechenfehler des Verfassers von Papyrus Harris I beweisen. ${ }^{433}$ Zumindest lassen die ausführlichen Papyri wie in diesem Fall eine Kontrolle der Ergebnisse zu und die fehlende Positionsschreibweise der Zahlen verhindert - anders als in anderen Schriftsystemen - die irrtümliche Veränderung um eine ganze Zehnerpotenz. ${ }^{434}$

Im allgemeinen hat sich heute die Meinung durchgesetzt, daß die aus der ägyptischen Überlieferung bekannten >großen< Zahlenangaben ausschließlich Übertreibungen darstellen. Insbesondere die im folgenden noch zu besprechenden Zahlen der Inschriften aus der Frühzeit sind fast einhellig abgelehnt worden, und es fanden sich in der Vergangenheit nur vereinzelt

431 Henige (1998: 6).

432 Die Ausgangssituation ist jedoch für Ägypten und Mittelamerika nicht identisch. Während die Aufzeichnungen zur Situation Mittelamerikas hauptsächlich aus der Feder spanischer Geistlicher stammen, die von Land und Bevölkerung nur eine vage Vorstellung besaßen, basieren die altägyptischen Daten im wesentlichen auf den Akten, die zeitgenössische Schreiber mit allen Möglichkeiten der eigenen Anschauung verfaßt hatten.

433 Siehe oben Kap. 4.6.1.

434 Die sogenannte Positionsschreibweise, wie sie etwa in unserem heutigen Zahlensystem vorliegt, ermöglicht die Veränderung um eine ganze Zehnerpotenz durch Fortlassen oder Hinzufügen einer einzigen Null, die ohne eigenen Wert als >Platzhalter < fungiert. Da die Zehnerpotenzen im ägyptischen Zahlensystem durch je ein eigenes Zeichen repräsentiert sind und darüber hinaus eine >additive< Form der Schreibung - vergleichbar mit den römischen Ziffern - Anwendung findet, sind irrtümliche Verschiebungen ganzer Größenordnungen nahezu ausgeschlossen. Vgl. auch IMHAUSEN (1996). 
Fürsprecher. ${ }^{435}$ Gleiches gilt auch für einige Überlieferungen zu den Expeditionsgrößen des Mittleren Reiches in das Wadi Hammamat.

Das Argument ist nicht neu und betrifft in ähnlichem Umfang auch andere Epochen historischer Wissenschaften. ${ }^{436}$ Immer dann, wenn Zahlen in bisherige Wissensgerüste schlecht einzupassen sind oder mit anderweitigen Vorstellungen über Zeit und Gesellschaft kollidieren, muß notwendigerweise eine Übertreibung vorliegen. Dagegen finden sich nur selten in gleicher Häufigkeit die dazu passenden Argumente, die diesen Vorwurf stützen könnten. Die Bewertung als >Übertreibung < basiert demnach in vielen Fällen eher auf subjektiven Kriterien als auf gesicherten Fakten. ${ }^{437}$

Dieses pauschale Urteil kollidiert auch mit etablierten Meinungen über die Entstehung altägyptischer Mathematik und des Zahlensystems, zu denen weitgehend eine opinio communis herrscht. Die Herausbildung eines graphischen Systems bis 1000000 war bereits in der Frühzeit abgeschlossen und fand ausgeprägte Anwendung. ${ }^{438}$ Im weiteren Verlauf der Geschichte änderte sich die Verwendung der Zeichen dahingehend, daß die größten Zahlenwerte zunehmend ihre ursprüngliche Bedeutung verloren und zu unbestimmten Ausdrücken für > viel<verkamen. ${ }^{439}$ Will man nun diesen Umstand auf die Zahlenangaben der Frühzeit anwenden, so müßte eine Aufweichung der Stringenz bereits während der Entstehungsphase der Zahlzeichen vorausgesetzt werden. Darüber hinaus bleibt die Frage offen, warum gerade die frühesten und größten Zahlenangaben eine relative Präzision bis auf 1000 aufweisen, wenn es nur ein unbestimmter Ausdruck für eine große Anzahl sein soll. ${ }^{440}$ Es bleibt also zu zeigen, ob die Zahlen tatsächlich jeglicher Realität entbehren, oder ob sie unter bestimmten Umständen doch als $>$ wahr $<$ bezeichnet werden dürfen. Die Entscheidung darüber muß an dieser Stelle zunächst

435 GUNDLACH (1994: 38 Anm. 151) warnt vor einer zu häufigen Unterstellung als >Übertreibung<; auch FECHT (1956: 58) bekennt ausdrücklich: »Jedoch scheint es vorsichtiger und dem Stand unseres Wissens angemessener, diese Zahlen so lange ernst zu nehmen, wie das Gegenteil nicht erwiesen ist.«

436 Vgl. Sonntag (1987: 4) zum weitverbreiteten Vorwurf der Übertreibung in der Mittelalterlichen Geschichtsschreibung. Sie konnte jedoch nachweisen, daß die von ihr untersuchte Quelle (die Libri Historiarum Gregors von Tours) weitaus zuverlässiger ist als ihr die Forschung bis dahin unterstellt hatte. Zwar räumt SONNTAG ein, daß Übertreibungen sich nie wirklich nachweisen lassen, doch scheinen die Zahlenangaben bestenfalls eine Aufrundung erfahren zu haben (SonNTAG, S. 144).

437 In der Wahl der Methoden unterscheiden sich die Kritiker der historischen Quellen nur allzu selten von deren Befürwortern. Für beide gilt gleichermaßen die von Henige (1998: 8) geübte Kritik: »The aim is to convince without being able to demonstrate.«

438 REINEKE (1978: 70).

439 Sethe (1916: 11 ff.). Entgegen der Aussagen von LOPRIENO (1986), der die Verwendung als unbestimmten Terminus bereits in der Frühzeit sehen will, spricht SETHE ausdrücklich von einem Wandel ab dem Neuen Reich.

440 Siehe auch unten Kap. 5.2.3.1. 
offen bleiben. Sowohl für das eine wie für das andere Resultat können bei genauer Quellenanalyse Argumente auftreten, die eine bestimmte Beurteilung zwingend erscheinen lassen.

\subsubsection{Untersuchungsmethode}

Der Umfang an aus dem Alten Ägypten überliefertem Zahlenmaterial scheint fast unerschöpflich. Dies gilt nicht zuletzt auch für Angaben zur Bevölkerung oder speziellen Personengruppen, wobei hier nicht der Grad an Menge und Qualität erwartet werden darf, den moderne Demographen für ihre Arbeit beanspruchen. Sämtliche Zahlen aus ägyptischen Texten einzeln besprechen zu wollen, ist also im Rahmen dieser Arbeit weder möglich noch sinnvoll. Daher sollen im folgenden aus der Fülle an überlieferten Zahlenangaben zu Personen nur diejenigen Belege exemplarisch herausgestellt werden, deren Herkunft und Auswertung weiterführende Erkenntnisse über demographische Sachverhalte des pharaonischen Ägypten versprechen. Eine strikte Trennschärfe hinsichtlich Zeitstellung und Kontext muß dabei eine geringere Rolle spielen als eigentlich erforderlich wäre. Dies ist ein Tribut an die kleine Anzahl von tatsächlich verwertbaren Belegen, die den geforderten Kriterien entsprechen. Verwendung findet stattdessen das epistemologische Mittel der Analogie, um isolierte Aussagen auf ihre Qualität zu prüfen. Auch diejenigen Belege, die bereits in der Vergangenheit von der Forschung für das Thema >Ägyptische Demographie $<$ herangezogen wurden, müssen sich einer erneuten Kontrolle unterziehen.

Bei der Betrachtung der Einzelbelege werden Art der Quelle und Berichtszeitraum geklärt, etwa ob die Angaben >zeitnah< aufgezeichnet worden sind, oder Überlieferungen aus älterer Zeit aufgegriffen und - womöglich ungeprüft - wiedergegeben wurden. Schließlich stellt sich für alle Quellen die Frage nach der Zielsetzung oder sogar Zielgruppe, für die eine solche Information schriftlich festgehalten wurde. Die drei Hauptgruppen stellen hier Königsinschriften, Grabstelen sowie alle Formen administrativer Aufzeichnungen dar, wie zum Beispiel Verwaltungsakten oder Expeditionsinschriften. Letztere können jedoch auch in Intention und Propaganda den Königsinschriften nahestehen und sind daher mit der gleichen Vorsicht zu behandeln. Zusätzlich wird der Versuch unternommen, anhand weiterer Quellen Indizien für oder gegen die Zahlenangaben zu finden und damit einer Plausibilitätsprüfung zu unterziehen. Das Vergleichsmaterial kann dabei sowohl archäologischer wie auch schriftlicher Herkunft sein. Zusätzlich wird zum Zweck einer besseren Differenzierung der zu besprechenden Ereignisse ein Vergleich zu thematisch verwandten Befunden anderer Epochen, wie zum Beispiel dem europäischen Mittelalter, gezogen. Über indirekte Indizien und Belege wird es möglich sein, aus den vagen Zahlenangaben darauf aufbauende Vorschläge für Berechnungen 
oder Schätzungen zu formulieren und neues Licht auf bestimmte Aspekte der altägyptischen Wirtschafts- und Sozialgeschichte zu werfen.

\subsection{Zählungen im pharaonischen Ägypten}

\subsubsection{Terminologie}

Neben der Vielfalt von Begriffen, die mit dem Konzept des >Zählens< in Verbindung gebracht werden können, verschiebt sich auch die hinter dem jeweiligen Ausdruck liegende Vorstellung von der damit verbundenen Tätigkeit. ${ }^{441}$ In der Frühzeit und im Alten Reich ist es noch das Zählen selbst, das im Mittelpunkt steht. Neben dem Begriff $\widetilde{\sigma_{0}} \underline{\underline{t} n w . t} \gg$ Zählung, Zahl $\ll$, der nach der Belegsituation zu urteilen nur verwendet wurde, um Objekte zu zählen, ist $j p$ ein zentraler Terminus in unterschiedlicher Verwendung. Außer der Grundbedeutung »zählen« kommt ihm auch eine Deutung als »Einkünfte (Steuern) berechnen/auferlegen « zu. ${ }^{442}$ In enger Verbindung zu diesen beiden Begriffen steht zu Beginn der ägyptischen Geschichte die Entwicklung der Jahreszählung. Spätestens seit der 2. Dynastie hat sich - mit wiederkehrenden Abweichungen - die Jahreszählung an der im zwei-Jahres-Rhythmus vermutlich zu Steuerzwecken abgehaltenen Vermögenszählung orientiert: rnp.t zp X tnw.t. ${ }^{443}$ Nach Meinung Wolfgang HELCKs erbrachte die Zählung von Rind und Kleinvieh die wesentlichen Steuererträge, weshalb auch die Akten des Alten Reiches danach datiert wurden. ${ }^{444}$ Für diesen Vorgang fand der Terminus jp Verwendung: jp.t jh.w ${ }^{c}$ w.t nb.t Mh.w $\check{S}^{c}{ }^{c} .{ }^{4} .{ }^{445}$

Im Mittleren Reich kam hasb als ein neuer Begriff auf, der jedoch die bisherigen Wörter nicht ablöste, sondern vielmehr ergänzte, so daß nunmehr alle drei Begriffe gleichberechtigt in einem Text erscheinen konnten. Der etymologische Ursprung von $h s b$ ist nicht ganz eindeutig. Vermutlich handelt es sich um eine Ableitung vom mathematischen Begriff $h s b$, der sich - im ursprünglichen Sinne »brechen« - zu einer Bezeichnung für die Bruchzahl 1/4 wandelte und später generell für »(be)rechnen« oder im weiteren Sinne auch »zählen« verwendet werden konnte. ${ }^{446}$ Dabei spielte es keine Rolle, ob das Gezählte aus Personen ${ }^{447}$ oder Objekten und Tieren ${ }^{448}$ bestand.

441 Vgl. dazu auch Husson und VALBelle (1992: 111).

442 Urk. I 106, Urk. I 212, Stele Kairo Cat. Gén. 20025.

443 Sethe (1905: $75 \mathrm{f}$.).

444 HELCK (1975b: 113).

445 Urk. I 112,15.

446 Sethe (1916: 76 f.).

447 Z. B. Urk. V 105; Tb (NAVILle 1886: Bd. 2, 235): 100,10 (nach Pb); Urk. IV 36.

448 Z. B. Davies (1902: Taf. 13); Statue Kairo Cat. Gén. 583, Z. 15; pAnastasi I 13,7. 
Bereits früh waren die Termini und die damit verbundene Aufgabe in ein System von Titeln und Amtsbezeichnungen eingebunden. Aus der Frühzeit liefert Peter KAPLONY ein Beispiel, das einen hỉ.tj-tnw.t erwähnt. ${ }^{449}$ Ab dem Mittleren Reich lassen sich mehrfach Beispiele für einen >Menschenzähler< (hsb.w rmt $)$ finden. ${ }^{450}$ Die Aufgabe, die diese Beamten innehatten, ist kaum klar einzugrenzen, da die Belege für den Titel häufig aus Titelreihen stammen und nur wenig über das Arbeitsfeld der Träger sowie deren konkrete Aufgaben aussagen. Zumindest läßt sich den Titeln entnehmen, daß die ḩsb.w rmt ein eigenes Büro besaßen, da es

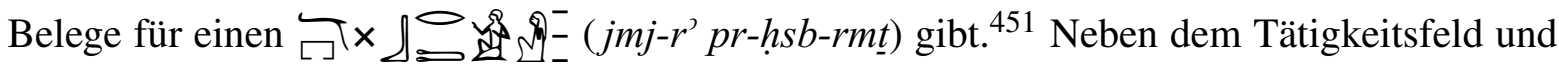
den dazugehörigen Verwaltungsbeamten gibt es ab dem Mittleren Reich auch eine verfeinerte terminologische Ausdifferenzierung zweckgebundener Listen:

$$
\begin{aligned}
& \text { \# } \\
& \text { ӨDl11 rh.t Verzeichnis, Liste } \\
& \text { wpw.t Haushaltsliste } 452
\end{aligned}
$$

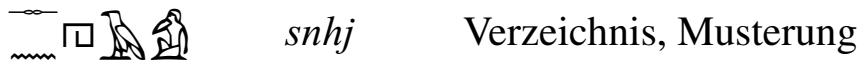

Es sei an dieser Stelle jedoch darauf hingewiesen, daß gerade die verschiedenen Listen nicht unbedingt mit einem Zensus im engeren Sinne zu tun haben. Eine Akte, die eine bestimmte Anzahl von Arbeitern für ein Projekt benennt oder alle Mitglieder einer Phyle aufzählt, erfüllt - rein verwaltungstechnisch - einen völlig anderen Zweck. Ob und inwieweit solche Listen auch sekundär ausgewertet wurden, um zum Beispiel eine Gesamtstärke an Arbeitskraft zu ermitteln, entzieht sich unserer Kenntnis. Auch lassen sich die Listen nur selten einer bestimmten Region oder - als Optimalfall - einem konkreten Ort zuweisen.

\subsubsection{Zur Zweckbestimmung von Zählungen}

Seit frühester Zeit hatten die Ägypter ein Interesse an Zählungen: alle Belange der ökonomischen oder sozialen Komponenten wurden erfaßt. Diese seit Beginn der Existenz von Schriftquellen belegten Ereignisse einer $>$ Bestandsaufnahme $<$ des Landes betrifft natürlich auch die

449 KAPLONY (1963: 808, Nr. 755); basierend auf den anderen bekannten Kontexten für thw.t dürfte es sich auch hier nicht um eine Person handeln, die für das Zählen von Personen zuständig war.

450 Urk. IV 1051,17 und 1055,15 sowie pKahun 14,4, pKahun UC 32168A; QUIRKE (1990: 170 Anm. 52), HELCK (1958: 153).

451 WARD (1982: Nr. 167).

$452 \mathrm{Vgl}$. dazu ausführlich unten Kap. 5.2.2.4. 
Erfassung der Bewohner. Ein derartiges Vorgehen war natürlich keinem Selbstzweck unterworfen, sondern damals wie heute ist die genaue Erfassung einer Bevölkerung von zentraler Bedeutung für die Verwaltung eines Staates.

»Die Ziele einer Volkszählung sind:

1. Feststellung der Einwohnerzahl

2. Feststellung der Zahl der Haushalte und Familien, der Erwerbspersonen, der Gebäude und Wohnungen,

3. Ermittlung von Strukturdaten für die Gesamtheiten der Einwohner, der Haushalte und Familien, der Erwerbspersonen, der Gebäude und Wohnungen, jeweils nach wenigen, aber durchgängig miteinander kombinierbaren Merkmalen,

4. Gewinnung von Basis- und Rahmendaten für Stichproben, Fortschreibung, Adjustierungen und Qualitätsüberprüfungen bei anderen Statistiken, und das alles in kleinräumiger regionaler Gliederung. ${ }^{453}$

Diese Aufstellung ist nicht etwa das Ergebnis der Analyse altägyptischer Zensus-Akten, sondern stellt das Grundkonzept für moderne Zensus-Planungen in Deutschland dar. Die Fragestellungen haben sich also im Prinzip nicht verändert, wohl aber die Verfahren der Erhebung, die Qualitätssicherung sowie die sekundäre Verwendung ihrer Ergebnisse.

Nach heutiger Kenntnis hat der altägyptische Verwaltungsapparat seine Aufgabe nicht in Form eines Generalzensus gelöst, dessen Existenz weiterhin ein Feld für Spekulationen bleibt, sondern anhand spezieller Listen für einen eng umrissenen Zweck. Im wesentlichen lassen sich die zweckgebundenen Erhebungen in drei große Bereiche unterteilen:

$\triangleright$ Militärwesen und Rekrutierung

$\triangleright$ Steuer- und Abgabenverwaltung

$\triangleright$ Organisation von Arbeitskräften

Welchen konkreten Problemen die einzelnen Bereiche gegenüberstanden und in welcher Form die Maßnahmen durchgeführt wurden, soll Gegenstand der folgenden Betrachtungen sein. Auch in Bezug auf eine andere Fragestellung stehen die einzelnen Funktionsbereiche im

453 GROHMANN (1999: 26). 
Zentrum des Interesses. Bereits in Kapitel 2 wurde die Frage aufgeworfen, auf welche Weise Autoren der klassischen Antike wie Diodor oder Flavius Josephus in der Lage gewesen sind, aus den ihnen zugänglichen Akten eine Gesamtzahl für die ägyptische Bevölkerung zu kompilieren. Die Unterschiede zu den Zensusverfahren anderer Regionen der griechisch-römischen Welt wie auch die Ähnlichkeiten zu den Vorläufern aus der altägyptischen Epoche scheinen die These zu bestätigen, daß der Zensus des hellenistischen Ägypten ein Erbe der Pharaonenzeit darstellte. Deshalb ist der Aufbau altägyptischer Akten und Verfahren ein grundlegender Baustein für vergleichende Betrachtungen mit nachpharaonischen Quellen.

\subsubsection{Erfassung wehrfähiger Männer}

Die eigene Kenntnis der Wehrstärke und deren ständige Aktualisierung stellten nicht nur in den Zeiten kriegerischer Auseinandersetzungen eine notwendige Informationsgrundlage für die Handlungsfähigkeit eines Staates dar. Aus diesem Grund treten in den Überlieferungen der Antike besonders Berichte über Zählungen auf, die an diesen speziellen Zweck gebunden waren.

So ist in der Bibel im zweiten Buch Samuel eine Zählung unter König David beschrieben, die als Resultat Hunderttausende von >Schwerttragenden $<$ meldete. ${ }^{454}$ Auch bei Herodot findet sich beispielsweise eine Angabe zur Größe der sogenannten >Kriegerkaste<, die er auf 410000 Mann beziffert. ${ }^{455}$ Schließlich liefert auch Julius Cäsar einen Beleg für eine Art Volkszählung bei den Helvetiern, deren Umstände dafür sprechen, daß es sich nicht um einen Import römischer Verfahren handelt, sondern ein eigenständiger Vorgang in dieser Gemeinschaft war. ${ }^{456}$ Unabhängig vom im Einzelfall nur schwer überprüfbaren Wahrheitsgehalt der Textstellen und deren unterschiedlichen kulturellen und zeitlichen Zuordnung lassen sich doch Gemeinsamkeiten in der Grundaussage feststellen. Zum einen wird der Schwerpunkt der Information auf die Anzahl waffentragender Personen gelegt, zum anderen bieten die Passagen konkrete Zahlen, die zwar nur grobe und sicher gerundete Angaben darstellen, doch eben genaue Größenordnungen umreißen und nicht bloß unbestimmte Ausdrücke für viel sind.

Auch in Ägypten bestand in den Zeiten, da es noch kein Berufsheer aufgestellt hatte, eine gewisse Notwendigkeit, über den Grad an militärischer Stärke Kenntnis zu besitzen. Während HELCK noch die Auffassung vertrat, daß die Bevölkerung des Alten Reiches mangels äußerer

$4542 \operatorname{Sam} 24,2 \mathrm{ff}$.

455 Herodot II, 165-166.

456 Caesar, De Bello Gallico I 29, 1-2; kritische Auseinandersetzung mit den Detailaspekten dieser >Zählung bei HeNIGE (1998: 214 ff.). 
Bedrohung lediglich zu Bauarbeiten und Expeditionen herangezogen wurde, ${ }^{457}$ betont Eckhard EICHLER in seiner Neubetrachtung der Sachverhalte, daß es aus organisatorischen wie militärischen Gründen bereits in der Zeit des Alten Reiches ein stehendes Heer gegeben haben muß. ${ }^{458}$ EICHLER stützt sich dabei auf die Kenntnis von Erstürmungen fremder Festungen durch ägyptische Truppen, die in Gräbern des Alten Reiches abgebildet sind und ein Minimum an Ausbildung für derartige militärische Maßnahmen benötigten. ${ }^{459}$ Weitere Argumente sind das Bestehen einer nubischen Hilfstruppe, die zumindest von einem Kern ägyptischer Einheiten befehligt und kontrolliert werden mußte, sowie die häufige Bildung von Titeln mit dem Begriff $m \check{s}^{c}$.

Wenn man mit EICHLER davon ausgeht, daß die Ägypter des Alten Reiches »einen relativ großen Aufwand mit [...] der Rekrutierung zusätzlicher Zivilisten betrieben «, ${ }^{460}$ dann muß gleichzeitig ein dazugehöriger ausgereifter Verwaltungsapparat existieren. Ein Hinweis dafür liegt zum Beispiel in der Biographie des Wnj vor, der man entnehmen kann, daß der König speziell für den Feldzug Truppen ausheben ließ. Es kann jedoch nicht erschlossen werden, ob diese Truppen zusätzlich zu einem stehenden Heer rekrutiert wurden oder ein Gesamtheer eigens für den Anlaß des Feldzuges aufgestellt wurde.

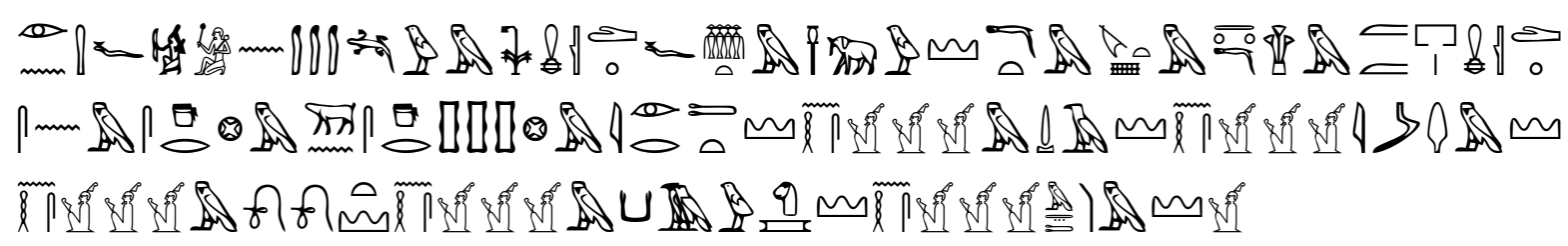

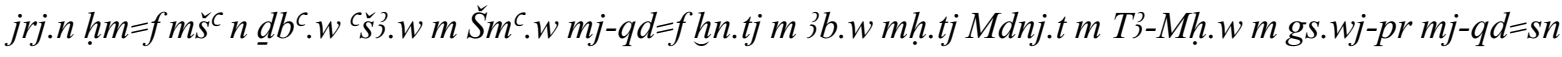

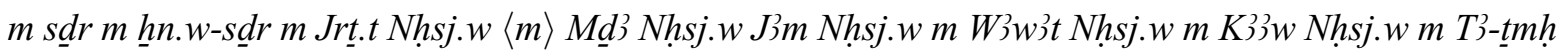
»Aus ganz Oberägypten von Elephantine im Süden bis zum 22. Nomos im Norden; aus den beiden vollständigen Haushälften Unterägyptens; aus der >Festungくaus dem Inneren der Festungen; aus Irtjet, Medja, Yam, Wawat, Kaau, Libyerland Nubiern stellte seine Majestät ein viele Zehntausende zählendes Heer auf.«

Um sich nun über den Ablauf und die Ausführenden einer derartigen Rekrutierung eine bessere Vorstellung zu verschaffen, lassen sich prinzipell zwei mögliche Szenarien dafür beschreiben:

457 HELCK (1982: 128); ähnlich auch GNIRS (1996: 36).

458 EICHLER (1993: 198 ff.) mit Verweis auf FAULKNER (1953).

459 Siehe auch FAULKNER (1953: 35).

460 EICHLER (1993: 204). 
Szenario A: Es existierten Listen mit >wehrfähigen< jungen Männern aus den Ansiedlungen, nach denen bei Bedarf die Truppen rekrutiert werden konnten.

Szenario B: Die Rekrutierer gingen direkt in die Dörfer, um die benötigte Anzahl Personen für den Dienst zu rekrutieren.

Obwohl die in den schriftlichen Quellen getroffenen Aussagen dahingehend interpretiert werden könnten, daß die Administration im Vorfeld von Rekrutenaushebungen Kenntnis über die potenzielle Stärke der zur Verfügung stehenden Gesamtzahl hatte, liegt es aufgrund der Belegsituation näher, daß die Schreiber und Rekrutierungsoffiziere direkt in die Dörfer gingen, um ihren Bedarf an Arbeitskräften zu decken. ${ }^{461}$

Als Kronzeuge für Szenario A gilt HELCK eine Abbildung im Grab des Soldatenschreibers Tnnj aus der Zeit Thutmosis' IV., die eine General-Registratur zeigt, zusammen mit der Beischrift: ${ }^{462}$

荞

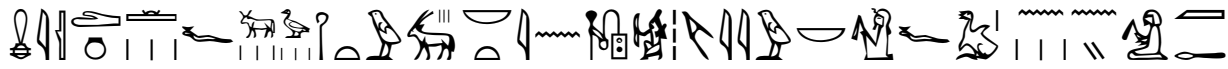

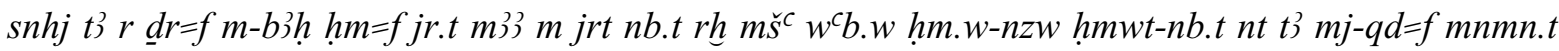
3pd.w ${ }^{c} w . t n b . t j n z \check{s}-m \check{s}^{c} m r j . w n b=f \underline{T} n n j m \jmath^{c}-h r w$

»Registrieren des gesamten Landes vor Seiner Majestät, Beaufsichtigen aller Tätigkeiten, Kenntnisnahme der Soldaten, Priester, Königsdiener, aller Handwerker des ganzen Landes, der Rinder, des Geflügels und des Kleinviehs durch den Soldatenschreiber, geliebt von seinem Herrn, Tnnj, gerechtfertigt.«

Ausdrücklich wird in diesem Text davon berichtet, daß die Soldaten der staatlichen Registratur unterworfen waren. Da es sich hier jedoch um einen Vorgang aus dem Neuen Reich handelt, dürfte sich die Verwaltungsmaßnahme einzig auf die Berufssoldaten beziehen, also das stehende Heer jener Zeit. Die Tatsache, daß die Soldaten zwischen anderen >Berufsgruppen< erwähnt sind, unterstreicht diese Vermutung. Keinesfalls ist hier eine Gesamtaufnahme aller (arbeitsfähigen) Menschen dargestellt, so daß auch weiterhin davon ausgegangen werden kann, daß die Rekruten und wehrfähigen Männer der Administration nicht in Form von Listen bekannt waren. Auch werden die Angehörigen zusätzlich rekrutierter Einheiten - im Gegensatz zur Gesamttruppe - niemals als $m \check{s}^{c}$ bezeichnet, sondern als $\underline{d} 3 m$.w oder $n f r . w$.

461 So auch GNIRS (1996: 17); anders dagegen KADRY (1986: 156): »These lists which recorded all officers and soldiers as well as the whole man power and also the economic resources of the land, were obviously the basis of any general or partial mobilisation of the imperial army.«; bereits HELCK (1939: 19) befürwortete die Existenz ausführlicher >Dienstfähigkeitslisten <. Die direkte und unbürokratische Rekrutierung in den Dörfern fand auch noch im Ägypten des 19. Jahrhunderts statt, wie LANE (1852: Bd. 1, 210) zu berichten wußte.

462 TT 74; BRACK (1977: 34); HelCK (1939: 20). 
Das Szenario B wird gestützt durch zwei Abbildungen in Privatgräbern des Neuen Reiches, die eine lebendige Darstellung solcher Rekrutierungsmaßnahmen in den Dörfern vermitteln. Zum einen ist dies das Grab eines Polizeioffiziers unter der Regierung Thutmosis' IV. namens Nb-Jmn, ${ }^{463}$ zum anderen das Grab des Gouverneurs der Westwüste namens Ddj, ${ }^{464}$ der unter Thutmosis III. und Amenophis II. diente. Dazu ist aus dem Mittleren Reich eine Stele bekannt, auf der ein Beamter namens Mntw-ḥtp davon berichtet, daß er in den abydenischen Nomos reiste, um eine junge Mannschaft zu rekrutieren. ${ }^{465}$ Es läßt sich dabei allerdings nicht zweifelsfrei klären, ob auch in diesem Fall die Rekrutierungen ad hoc oder planvoll nach Listen erfolgten.

Die Zusammenstellung benötigter Truppenkontingente lag während des Mittleren Reiches

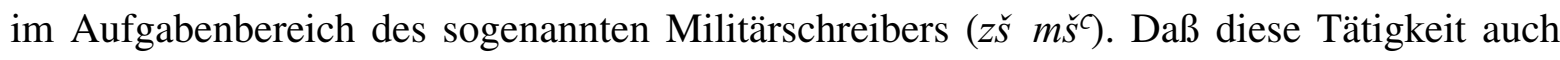
den Militärschreibern des Neuen Reiches oblag, weiß man durch die Aufzeichnungen des Amenophis, Sohn des Hapu, der seine Tätigkeit wie folgt beschreibt: ${ }^{466}$

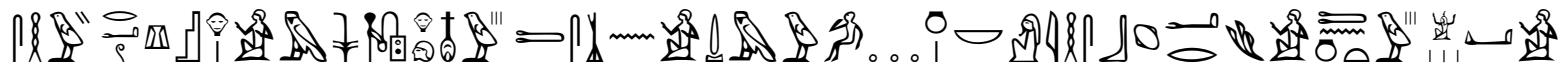

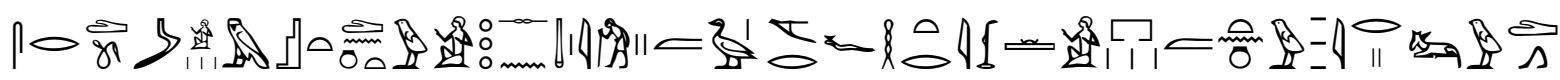

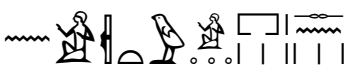

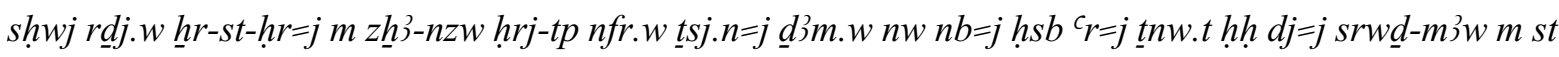
$d n w t=s n \quad m d w-j 3 w j m z 3 \quad m r j=f h t r=j$ pr.w $m$ tn $n$ j jrj jwd.n=j jswt pr.w=sn

»Ein Verzeichnis wurde unter meiner Aufsicht als oberster Königsschreiber angefertigt, nachdem ich eine Truppe Rekruten meinem Herrn ausgehoben habe. Meine Schreibbinse zählte Millionen, wobei ich die jungen Rekruten an den Ort ihrer Familie gab, da der >Stab des Alters $<$ der von ihm geliebte Sohn ist. Ich besteuerte zahlreiche zugehörige Häuser, nachdem ich ich die Truppe und ihre Häuser abgesondert hatte.«

Für HELCK ergeben sich aus dieser Textstelle eine ganze Reihe von Konsequenzen. Kernpunkt seiner Interpretation ist die Stelle $h t r=j$ pr.w $m \underline{t} n w$, die er als »die Orte zinsen nach ihrer Zahl « übersetzt. ${ }^{467}$ Nur mit dieser Übersetzung klingt HELCKs Interpretation schlüssig: Es müssen demnach ausführliche Einwohnerregister für jeden Ort vorgelegen haben, nach denen (»nach ihrer Zahl«) die Militärschreiber die Besteuerung vornahmen. Wollte man den

463 TT 90; DAVIES (1923: Taf. 25).

464 TT 200; SEIPEL (1984: 155).

465 Siehe dazu ausführlich unten Kap. 5.2.2.2.

466 Statue Kairo 583, Z. 13; VARILle (1968: 32 ff., bes. 41); HelcK (1939: 17).

467 HELCK (1939: 20). 
Ausführungen HELCKs folgen, dann hätte man sogar Vermutungen dahingehend anstellen können, daß - ähnlich wie bereits im Alten Reich aus dem Koptos Dekret C bekannt - ein Teil der Abgabenleistung eines Dorfes aus der Arbeitskraft der Männer bestand, die auf Abruf verfügbar sein mußten. ${ }^{468}$ Bei einer kritischen Neubetrachtung der Passage und ihrer Übersetzung können HELCKs Ergebnisse allerdings nicht mehr aufrecht erhalten werden.

Aus dem Alten Reich liegen zum Aufgabenbereich des $z \check{s} m \check{s}^{c}$ überhaupt keine Informationen vor. Lediglich aus der Biographie des Wnj und der Tatsache, daß der Titel $z \check{s}$ (n) $m \check{s}^{c}$ bereits mehrfach im Alten Reich belegt ist, ${ }^{469}$ könnte man - unter Vorbehalt - Überlegungen dahingehend anstellen, daß dies bereits seit frühester Zeit ein Teil des Aufgabenbereiches dieser Personengruppe war. Dies schreibt auch Pierre-Marie CHEvEREAU in seiner Beschreibung des Titels, obwohl die Belege (sieben, wenn man die Variante $z \check{s} n f r . w>$ Rekrutenschreiber $<$ mit einbezieht) diese Information nicht unbedingt hergeben.

Der von Chevereau als > Variante< bezeichnete Titel zš nfr.w birgt im übrigen eine zusätzliche Unsicherheit. Sollte dieser tatsächlich mit $z \check{s} m \check{s}^{c}$ austauschbar sein, wäre der Beleg für Rekrutierungsbeamten des Alten Reiches eindeutig. Es scheint jedoch eher der Fall, daß der Vergleich zwischen beiden Titeln auf der Vermutung über den Aufgabenbereich der $z \check{s} m \check{s}^{c}$ anhand der Belege aus späterer Zeit basiert und über diesen Weg ein Bezug zwischen beiden Titeln hergestellt wurde. Jedoch auch ohne einen gesicherten Zusammenhang beider Titel ist durch den Nachweis einer frühen Existenz des Titels žs nfr.w gesichert, daß es speziell für Rekrutierungsaufgaben zuständiges Personal gab.

Die Inschrift des Tnnj könnte auch als Beleg gegen die Vermutung einer Gleichsetzung von $z \check{s} m \check{s}^{c}$ und $z \check{s} n f r . w$ gelten, da Tnnj sowohl den Titel $z \check{s} m \check{s}^{c}$ als auch (im weiteren Textverlauf) den Titel $z \check{s}$ nfr.w trägt. Für HELCKs Annahme einer Rangfolge vom Soldatenschreiber als niedrigsten Beamtendienst hin zum höheren Dienstgrad des Rekrutenschreibers spricht möglicherweise der Kontext des Rekrutenschreibers, der direkt nach dem Titel >General< (jmj-r) $m \check{s}^{c}$ ) genannt wird. ${ }^{470}$

Die Heranziehung der $n f r . w$ als Arbeitskräfte für Expeditionen ist auf einem Graffito der 6. Dynastie aus Hatnub belegt. ${ }^{471}$ Dort wird von drei Einheiten zu je zweimal 500 und einmal 600 Personen gesprochen. Die betreffende Stelle ist leider sehr schlecht erhalten, doch scheint die Lesung des Zeichens

468 Siehe auch unten S. 138 Anm. 487.

469 Chevereau (1987: 43 f.).

470 Urk. IV 1007,14.

471 Hatnub Gr. 6, Anthes (1964: 21 f.; Taf. 11); EiChler (1993: 44). 


\subsubsection{Fallbeispiel: Eine einzelne Zahl und ihr Informationsgehalt}

Im folgenden soll anhand einer einzelnen überlieferten Angabe exemplarisch vorgeführt werden, welche Möglichkeiten für die Analyse solcher Informationen bestehen, welche Aussagekraft sie besitzen und schließlich der Punkt bestimmt werden, an dem die historische Untersuchung solcher Zahlenangaben an ihre Grenzen stößt.

Im Jahr 1900 publizierte Heinrich ScHÄFER eine Stele des Mittleren Reiches aus Abydos. ${ }^{472}$ Auf dieser Stele berichtet ein Beamter von einer erfolgreichen Rekrutierungsaktion für den König. Den Umfang der Rekrutierung gibt der Stifter mit einem Prozentsatz an: 1 von 100 männlichen Personen, also $1 \%$. Diese Information allein ist noch wenig aussagekräftig, doch scheint allein ihre bloße Erwähnung bemerkenswert. Es gibt keine weiteren Indizien aus dem Alten Ägypten, die darauf schließen lassen, daß nur ein Prozent der - gewiß wehrfähigen - Männer rekrutiert wurden. Die Erwähnung dürfte also darin begründet liegen, daß der Verfasser Sbk-Nht die geringe Anzahl hervorheben will. ${ }^{473}$ Es muß daher davon ausgegangen werden, daß der Faktor an eingezogenen Rekruten in anderen Fällen höher ausfallen kann als in diesem Beispiel überliefert wurde.

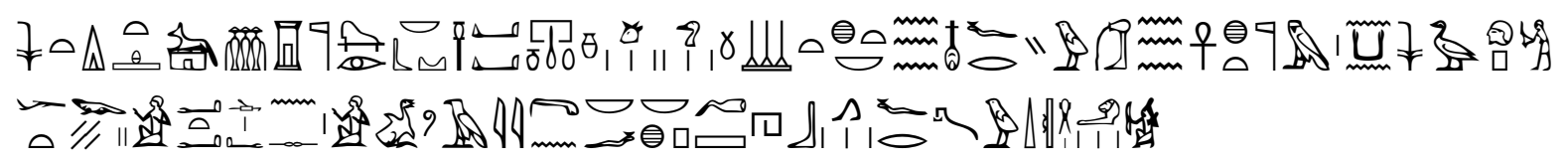

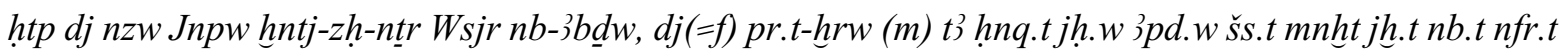

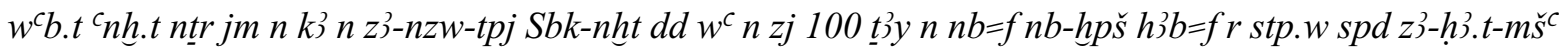
»Ein Gunsterweis des Königs, daß Anubis, der Erste der Gotteshalle und Osiris, der Herr von Abydos ein Invokationsopfer gewähren, bestehend aus Brot, Bier, Rindern, Geflügel, Alabaster, Stoff und alle guten und reinen Dinge, von denen ein Gott lebt, für den Ka des ersten Königssohns Sbk-nht, der einen von hundert erwachsenen Männern seinem Herrn bereitstellte, dem >Herrn der Kraft<, nachdem er geschickt wurde, um eine tüchtige Truppe Kundschafter auszuwählen.«

Interessant an diesem Text ist die Erwähnung der Truppeneinheit $\mathbb{1}_{1}$. Obwohl die genaue Größe dieser Einheit im Mittleren Reich nicht bekannt ist, kann mit einem Beispiel aus der Zeit Ramses' II. zumindest eine Vermutung darüber angestellt werden. Nach Auskunft des Papyrus Turin B war eine so bezeichnete Truppe 200 Mann stark. ${ }^{474}$

472 ERMAN und SCHÄFER (1900); Stele Kairo Cat. Gén. 20732.

473 Bereits SCHÄFER zog den Schluß, daß der hier angegebene Prozentsatz ungewöhnlich gering ist.

474 GARDiner (1937: 126); HelCK (1939: 20 Anm. 2). 


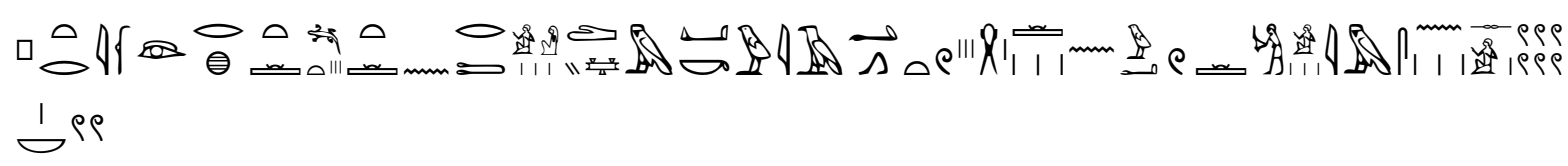

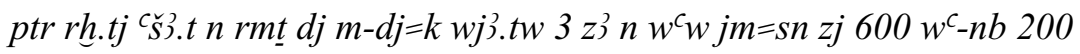

»Siehe, du kennst die Anzahl der Leute, die bei dir sind; abgesehen von drei Abteilungen Soldaten, die aus 600 Männern bestehen, eine jede 200.«

HELCK betont allerdings die Unmöglichkeit, diesen Prozentsatz als allgemeingültig zu nehmen. Seiner Meinung nach ist die Struktur des Militärs im Neuen Reich grundsätzlich verschieden von derjenigen des Mittleren Reiches, so daß die Angabe von $1 \%$ nur eine punktuelle Information darstellen kann. ${ }^{475}$ Mangels anderer Möglichkeiten wird hier dennoch das Risiko eingegangen, die Information des Papyrus Turin B auf das Mittlere Reich zu übertragen. Dafür spricht, daß zumindest kleinste Abteilungen von Rekruten im Mittleren Reich wie auch im Neuen Reich übereinstimmend aus 10 Mann bestanden und sich daher in ihrer Struktur nicht grundsätzlich unterschieden. ${ }^{476}$

Viel gravierender ist allerdings der Mangel an Informationen über die tatsächliche Gesamtstärke des Heeres im Mittleren Reich. Als Grundlage für die Deduktion weiterer Bevölkerungsangaben könnten die Rekrutierungszahlen nur dienen, wenn man weitere vage Zahlen wie etwa eine geschätzte Gesamtbevölkerung und deren Gliederung hinzuzieht. Es muß an dieser Stelle genügen, eine ungefähre Vorstellung über den Anteil der wehrfähigen Männer in einer Ortsgemeinschaft gewonnen zu haben.

Ein weiterer Aspekt ist der geographische Raum, in dem diese Rekrutierungen vorgenommen wurden. Die Kairener Stele gibt dazu keine Auskunft, wohl aber die gleichzeitig von Adolf ERMAN publizierte Berliner Stele aus der Zeit Amenemhets III. ${ }^{477}$ Dort beschreibt der Soldatenschreiber Mntw-ḥtp eine seiner Missionen, die ihn in den 8. oberägyptischen Nomos führte.

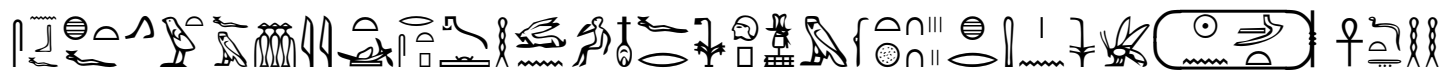

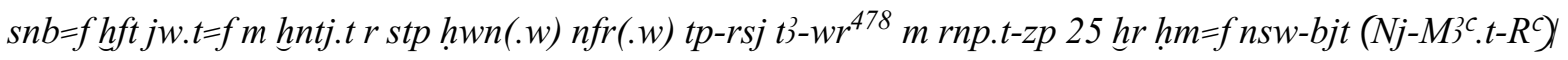

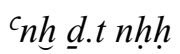

»Er war wohlbehalten, als er südwärts zog, um die junge Mannschaft in Ta-wer im Kopf des Südens auszuwählen im Jahr 25 unter der Majestät des Königs Amenemhet (III.), der ewig lebt.«

475 HELCK (1939: ebenda).

476 Für das Mittlere Reich: GoEdicke (1971: 68 ff.) und auch QUIRKE (1990: 170) (Arbeitseinheit); für das Neue Reich vgl. oben S. 131 Anm. 464; zur 10 als kleinste Truppeneinheit seit dem Alten Reich auch EICHLER (1993: 205) sowie HELCK (1975b: 129).

477 ERMAN und SCHÄFER (1900); Äg. Museum Berlin, Inv. Nr. 1198.

478 Zur Diskussion von tp-rsj t3-wr als eine Ortsangabe vgl. auch PARDEY (2003: 324 Anm. 14). 
Aus den Angaben des Sesostris-Kiosks, der für den Nomos eine Länge von 6 jtrw und 6 h ? $(67,138 \mathrm{~km})$ angibt, können die Grenzen des abydenischen Nomos (Ta-wer) im Süden am Gebel eț-Ṭârif und im Norden nördlich von el-Menschîja bestimmt werden. ${ }^{479}$ Nach Farouk GoMAÀ sind im Mittleren Reich für den abydenischen Nomos 30 Orte aus schriftlichen Quellen namentlich bekannt. ${ }^{480}$ Es steht außer Zweifel, daß mit diesen 30 bekannten Orten nicht der Gesamtbestand an Siedlungsplätzen in diesem Nomos erfaßt ist. Domänen und kleine Gehöftgruppen werden in dieser Zahl nicht enthalten sein. Im Gegensatz zur Zeit des Alten Reiches, da die Zahl der Domänen und kleinen Ansiedlungen die der regionalen Zentren überstiegen, dürfte eine Rekrutierung im Mittleren Reich bereits in den wenigen größeren Siedlungen die gewünschte Zahl an Personen zusammengebracht haben.

Wenn man GomaÀs Zahl von 30 Siedlungen sowie die Ausdehnung des Nomos nach dem Sesostris-Kiosk als Arbeitsgrundlage nimmt, läßt sich eine Verteilung von einer Siedlung auf zwei Kilometer errechnen. Dieses grobe Modell läßt sich mangels Informationen über die Rangfolge der einzelnen Orte nicht weiter verfeinern, obgleich GoMAÀ die besondere Stellung der Orte Thinis ( $\underline{T} n j)$ als Nomoshauptstadt sowie Abydos ( $3 b \underline{d} w$ ) als Kultzentrum herausgestellt hat. Auch der Versuch, mit Hilfe von Karl BuTZERs Hypothese ein Zahlenverhältnis einzelner Siedlungskategorien zu errechnen, führt zu keinerlei verwertbaren Ergebnissen. Bei einem Mindestverhältnis von 1:2 aufeinanderfolgender Siedlungskategorien und den wichtigsten Orten Thinis und Abydos als oberster Kategorie kann eine Relation von 2:4:8:16 ermittelt werden, was einerseits zufällig eine Summe von 30 ergibt und sich mit der Anzahl Orte in GoMAÀs Liste deckt, andererseits mangels jeglichen weiterführenden Datenmaterials keinen nennenswerten Informationsgewinn bietet. ${ }^{481}$

Die nächste und entscheidende Frage betrifft nun die Zahl der rekrutierten Männer. Auch hier müssen mangels konkreter Informationen plausible Vergleiche herangezogen werden. Die rekrutierte Truppe wurde im Text als militärische Einheit ausgewiesen. Jedoch scheint es fraglich, ob damit tatsächlich Leute für eine Kampfsituation ausgehoben werden mußten. Eher ist hierbei an die Zusammenstellung einer Expeditionstruppe oder dergleichen für zivile Aufga-

479 HELCK (1974: 93).

480 GomaÀ (1987: 187 ff.). Die Belegsituation fällt laut GomaÀ für das Mittlere Reich günstig aus, da die Namen der Ortschaften aufgrund ihrer bedeutenderen Rolle mehrfach und auch in späterer Zeit noch erwähnt worden sind. Damit steht die Belegsituation im Kontrast zu den verstreuten kleinen Domänen des Alten Reiches, die sich vielfach überhaupt nicht mehr inschriftlich oder archäologisch nachweisen lassen (GOMAÀ, S. 2). Dagegen meldet QUIRKE (1991a: 65 f.) grundsätzliche Bedenken an, ob jede Siedlung Altägyptens einen Namen besaß, wie es nach moderner Auffassung notwendig erscheint.

481 Zur Zentralort-Theorie und den zugrundegelegten Relationen vgl. BUTZER (1976: 72) sowie oben Kap. 1.4.1.1. 


\begin{tabular}{|c|c|c|c|}
\hline König & Datierung & Sinai & Wadi Hammamat \\
\hline Mentuhotep III. & Jahr 8 & & 3000 \\
\hline Mentuhotep IV. & Jahr 2 & & 10000 \\
\hline \multirow[t]{2}{*}{ Sesostris I. } & Jahr 16 & & 5000 \\
\hline & Jahr 38 & & 17000 \\
\hline \multirow[t]{7}{*}{ Amenemhet III. } & Jahr 2 & 734 & \\
\hline & Jahr 4 & $268+x$ & \\
\hline & Jahr 19 & & 2000 \\
\hline & Jahr 40 & $359+x$ & \\
\hline & Jahr 45 & $168+x$ & \\
\hline & $?$ & $209(213+x)$ & \\
\hline & $?$ & $214+x$ & \\
\hline Amenemhet IV. & Jahr 6 & 297 & \\
\hline
\end{tabular}

Tabelle 5.1: Zusammenstellung der Expeditionsgrößen des Mittleren Reiches

ben zu denken. Angaben zu Größe und Zusammensetzung derartiger Expeditionen sind aus den sogenannten Expeditionsinschriften des Mittleren Reiches bekannt (Tab. 5.1), ${ }^{482}$ deren Auswertung sich für diesen Fall jedoch als schwierig erweist. Während die Inschriften aus dem Rekrutierungsort näherliegenden Wadi Hammamat nur grob zwischen 2000 und 17000 Mann beziffert werden, sind die von Karl-Joachim SEYFRIED aus den Personallisten der Sinai-Expeditionen errechneten Truppengrößen zwischen 168 und 734 Personen vergleichsweise niedrig, wobei SEYFRIED zwar eine kleine Fehlermarge einräumt, aber insgesamt von realistischen Größenordnungen ausgeht, die nicht extrem nach oben korrigiert werden müssen. ${ }^{483}$ Die Differenz erklärt sich natürlich aus der Zielsetzung der Expeditionen. Das Wadi Hammamat war Steinbruchgebiet und entsprechend mußte eine große Anzahl an Personal zur Verfügung stehen, um die schweren Steinblöcke zurück in Richtung Niltal zu transportieren im Gegensatz zu den Türkis-Expeditionen in den Sinai. Die deutlichen Unterschiede bezüglich der Dimension der Expeditionen in den Sinai einerseits und in das Wadi Hammamat andererseits erschweren eine Interpretation dahingehend, ob möglicherweise auch kleine Expeditionen mit einer Personenzahl von weniger als 1000 Mann in das Wadi Hammamat aufbrachen. Da allerdings mehr als die Hälfte der bekannten Wadi Hammamat-Inschriften überhaupt keine Angaben dazu machen, kann es auch nicht völlig ausgeschlossen werden.

482 Belege zusammengestellt bei SEYFRIED (1981: 219 f., 269). In Klammern steht die aus den Einzelangaben errechnete Gesamtzahl, da die auf der Stele angegebene Summe falsch ist.

483 SEYFRIED (1981: 219 Anm. 84) geht für seine Berechnungen davon aus, daß in allen Fällen die wesentlichen Bestandteile einer Expeditionstruppe erwähnt wurden und die Größenordnungen damit ungefähr stimmen. 
Denkbar wäre zum Beispiel auch die Aushebung von Truppenteilen, die gar nicht die volle Mannschaftsstärke aufweisen. So berichtet der Nomarch Jmn-m-ḥ̉.t über zwei Expeditionen, bei denen er für den König Gold beschaffte: ${ }^{484}$

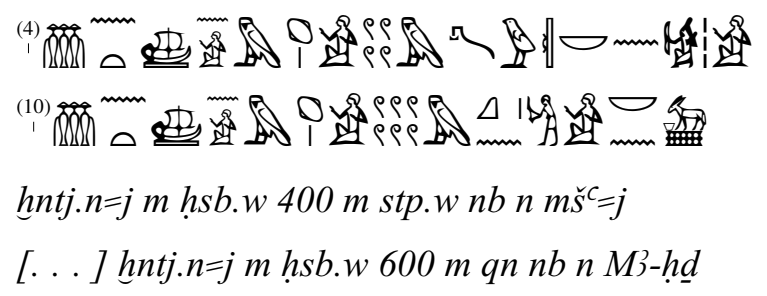

Die Erwähnung von zwei Einheiten verschiedener Größe sowie die explizite Angabe der Herkunft eines Truppenteils könnten Indiz für die Aushebung der Teilnehmer an verschiedenen Orten sein.

Eine Stützung dieser These liefert eine Stele aus dem Wadi el-Hudi, datiert in die Zeit Sesostris' I. ${ }^{485}$ Ashraf SADEK kann die eher zurückhaltenden Lesungen SEYFRIEDs vervollständigen und ermittelt aus dem Text neben einer Gesamtzahl von rund 1500 Teilnehmern auch die einzelnen Aufschlüsselungen der beteiligten Einheiten und Spezialisten. Wichtig ist auch die Differenzierung der Herkunft einzelner Teile der Truppe aus verschiedenen Orten, was sich bereits der Textbearbeitung SEYFRIEDs entnehmen läßt.

\begin{tabular}{|c|c|}
\hline Bezeichnung & Anzahl \\
\hline dim.w $n$ nfr.w & 1000 \\
\hline$c_{h} \zeta w . t j$ aus Elephantine & 200 \\
\hline$c h ̧ 3 w . t j$ aus Kōm Ombo & 100 \\
\hline$j m j-r 3 m^{\prime}-{ }^{C} 3 . w t$ & 41 \\
\hline Gruppe des Beamten Htp & 56 \\
\hline$j m j-r{ }^{C}{ }_{w}$ & 50 \\
\hline
\end{tabular}

Tabelle 5.2: Mannschaft einer Expedition in das Wadi el-Hudi

Neben der relativ hohen Zahl von 1000 Teilnehmern aus Theben, die sich vielleicht mit der Bedeutung Thebens als Metropole des Nomos erklären läßt, finden noch zwei weitere Zentren explizite Erwähnung. Die Aushebung von Truppenteilen in verschiedenen Orten oder

484 Biographie des Jmn-m-ḥ̉.t, genannt Jmnjj, Beni Hassan Grab Nr. 2, NEwBERRY (1893: Taf. 8); vgl. auch HELCK (1975b: 192).

485 WH Nr. 6; SEYFRIED (1981: 11 ff.); SADEK (1980: 16 ff.). 
Regionen wäre eine mögliche Erklärung für den Vorgang, der auf der Stele aus Abydos beschrieben worden ist. Es kam demnach nicht darauf an, die Gesamtstärke der Expeditionstruppe durch eine einzige Aushebung zu erreichen, sondern den jeweiligen >Pflichtteil $<$ des Ortes oder der Region abzurufen. Daß die auf der Kairener Stele erwähnten Rekruten dem $>$ Herrn der Kraft< $(n b-h p \breve{s})$ zugeführt werden sollen, deckt sich dabei hervorragend mit der Bezeichnung ${ }^{c} h 3 w . t j .{ }^{486}$

Aus den zuvor gezeigten Einzelbeobachtungen läßt sich nun folgende Situation konstruieren. Ausgehend von den niedrigen Teilnehmerzahlen der Expeditionen in den Sinai oder den Truppenteilen aus Elephantine und Kōm Ombo auf der Stele WH 6 sei zunächst als Grundgröße eine Truppe von 100 bis 200 Mann angenommen. Wenn diese auf den Gesamtbestand der wehrfähigen Männer hochgerechnet werden, den wir nach Auskunft der Stele CG 20732 mit $1 \%$ ansetzen, dann liegt die Zahl der wehrfähigen Männer im abydenischen Nomos zwischen 10000 und 20000 Mann. Bereits hier läßt sich erkennen, daß die hochgerechneten Zahlen plötzlich einer Schwankung unterliegen, die ihnen einen großen Teil ihrer Aussagekraft raubt. In einem letzten Schritt ließen sich die Gesamtzahlen auf die 30 Orte umlegen, so daß man am Schluß rein statistisch mit einer Zahl von ca. 333, beziehungsweise 666 wehrfähigen Männern pro Ort kalkulieren kann. Da in dieser Berechnung jedoch die einzelnen Siedlungsgrößen sowie ihre regionale Bedeutung als Metropolen nicht mit einfließen, sind diese Durchschnittsangaben für den Einzelfall recht grob. Wenn man etwa für Metropolen eine entsprechend größere Zahl an Wehrfähigen ansetzt, kommt man in Anbetracht der auf der Stele aus Wadi el-Hudi verzeichneten 1000 Mann aus Theben dennoch rasch in Erklärungsnot. Denn dies würde bedeuten, daß nahezu jeder Wehrfähige aus Theben für diesen Einsatz verpflichtet worden wäre.

Für ein besseres Verständnis derartiger Rekrutierungsmaßnahmen hilft ein Blick auf die Situation im Europa des Mittelalters. Aus Norditalien sind einige Akten des 12. Jahrhunderts überliefert, denen eine zusätzliche Einschätzung für den Umfang der Rekrutierung entnommen werden kann. Aus den Dokumenten jener Zeit geht hervor, daß die Bereitstellung von Soldaten eine Form der Steuerabgabe war, die im Bedarfsfall vom Grundherrn eingefordert werden konnte. ${ }^{487}$ Die zur Verfügung gestellten Männer waren entweder ausgebildete Soldaten, insbesondere Spezialisten für bestimmte Waffengattungen wie zum Beispiel Bogen, oder

$486 \mathrm{Zu}$ dieser Bezeichnung für eine Truppe Bewaffneter vgl. ausführlich SIMPSON (1959: 31).

487 LAne (1999: 130 f.). Eine ägyptische Parallele aus dem Alten Reich findet sich im sogenannten >KoptosDekret C<, das im Rahmen einer Liste der verschiedenen Steuerleistungen auch ausdrücklich Einberufungen für das »Ausschicken zu Lande und zu Wasser« einfordert (Urk. I 286,7-15); vgl. dazu auch HELCK (1975b: 112). 
sie dienten als Arbeitskräfte, etwa den modernen Pionieren vergleichbar. ${ }^{488}$ Die Anzahl dieser Rekruten, die ein Ort zu stellen hatte, war relativ gering. Für das Jahr 1162 verzeichnen die Dokumente, daß die Stadt Parpanese für die Belagerung Mailands zwei Bogenschützen und fünf >Pioniere< entsandte. Natürlich standen die Größenordnungen in direktem Verhältnis zu Größe und Reichtum des Ortes, doch darf man die Einbeziehung solcher Relationen als Voraussetzung auch für das Alte Ägypten annehmen. Es steht außer Frage, daß die ägyptischen Ortschaften üblicherweise im Rahmen ihrer Möglichkeiten einen Beitrag zur Aufstellung einer Truppe leisteten. Die geringe Anzahl mag zunächst kaum glaubhaft sein, doch muß dabei bedacht werden, daß diese Rekrutierungen zusätzlich zu einem stehenden Heer erfolgten, also lediglich eine Verstärkung der Kampfkraft bedeuteten. Wenn diese sieben Kämpfer auch in Italien unter jedem 100. der wehrfähigen Männer einer Stadt ausgewählt waren, kommt man auf eine Gesamtzahl von 700 Männern pro Stadt, was den zuvor dargelegten Überlegungen zum Alten Ägypten recht nahe kommt. Die tatsächliche Anzahl an Rekrutierungen bleibt jedoch auch im mittelalterlichen Kriegswesen mangels Belegen eine Sache von Schätzungen und Vermutungen, die nicht minder heftig umstritten sind. ${ }^{489}$

Ein letzter Aspekt ist die Bezeichnung der Rekruten. Die Stele Berlin 1198 bezeichnet sie als ḩwn(.w) $n f r(. w)$, die Stele Kairo CG 20732 dagegen als s3 ḩ3.t $m \check{s}^{c}$, also eine Vorhut. Aus den Expeditionsberichten sind diese Bezeichnungen nicht bekannt. Stattdessen findet man die üblichen Bezeichnungen wie hsb.w oder $\underline{d} 3 m . w$. Diese Diskrepanz in der Belegsituation hinterläßt zunächst Zweifel an der Aushebung zu Expeditionszwecken. Als Erklärung ließe sich anführen, daß es durchaus Belege für $n f r . w$-Einheiten bei Expeditionen gibt und eine Vorhut - hier vielleicht besser als > Vorauskommando< zu übersetzen - nicht unbedingt eine schriftliche Verewigung in dem von ihr erkundeten Gebiet hinterlassen muß.

\subsubsection{Steuer- und Abgabenerfassung}

Die Beurteilung altägyptischer Quellen in Bezug auf die Organisation der Abgaben- und Steuerverwaltung stellt sich als weitaus schwieriger dar als dies zuvor für den militärischen Bereich der Fall war. Dies hat seine Ursache besonders aufgrund der Tatsache, daß - anders als in griechisch-römischer Zeit - keine eindeutige Trennung von Voraussetzung (Zensus) und Folge (Besteuerung) vorgenommen werden kann. Anders gesagt: Eine Erfassung aller Landesbewohner durch einen Zensus stellte im altägyptischen Staat keine zwingende Voraus-

488 LANE (1999: ebenda).

489 Vgl. BACHRACH (1999) zu den Arbeiten des bekannten Militärhistorikers Hans DELBRÜCK. 
setzung für einen funktionierenden Steuerapparat dar. Das Verfahren, das dem ägyptischen Steuerwesen zugrundelag, verhielt sich grundsätzlich anders als die Steuersysteme moderner Gesellschaften. Dies hängt auch zu einem nicht unerheblichen Teil damit zusammen, daß Ägypten ein prä-monetäres System darstellt, dessen Abgabenleistungen in Naturalien festgelegt wurden. ${ }^{490}$ Darüber hinaus bildete die Redistribution als Wirtschaftssystem die Grundlage für die staatliche Einforderung von Abgaben. ${ }^{491}$ Dies war sicherlich nicht Verpflichtung des Einzelnen, sondern es hatten ganze Dorfgemeinschaften eine bestimmte Abgabenleistung zu erbringen, die nach Vermessung der Felder und Güte des Ackerbodens jährlich neu festgesetzt wurde oder bei Rindern und Kleinvieh durch Zählen des Bestands eine anteilige Abgabe errechnet werden konnte. ${ }^{492}$ Lediglich bei Besitz von Privatvermögen war der Verfügungsberechtigte verpflichtet, einen gewissen Anteil seines Surplus an den Staat abzuführen. Mit diesem Vorgang wurde in der Vergangenheit der Begriff $s ̌ 3 y t$ in Verbindung gebracht, der von HELCK als >personenbezogene Steuer< oder - im weiteren Sinne - als >Kopfsteuer < bezeichnet wurde. ${ }^{493}$ Da die $\check{s} 3 y t$ nicht als Steuer von weiten Teilen der Bevölkerung eingefordert wurde, sondern nur für bestimmte Personengruppen eine spezielle Verpflichtung bedeutete, ist HELCKs Bezeichnung etwas irreführend und sollte besser als >individuelle Besteuerung aufgefaßt werden. Empfänger waren in der Regel die Tempel als zuständige lokale Instanz. ${ }^{494}$

Das ganze wirtschaftliche Konzept der ägyptischen Administration baute also stärker auf den Erträgen selbst auf, die als lebensnotwendige Grundlage des Staates galten. Eine dezidierte Erfassung aller >Steuerpflichtigen < erschien unnötig, da bis auf die privilegierte Schicht der Inhaber von Privatvermögen alle Menschen im Rahmen ihrer handwerklichen oder agrarischen Tätigkeiten automatisch in das Abgabensystem mit eingebunden waren. Es scheint daher ausgeschlossen, aus diesem Themenkomplex verwertbare Akten zu ziehen, die Licht auf die dürftigen Kenntnisse über die demographische Situation jener Zeit werfen. Häufiger verzeichnen die Papyri nur die Ausnahmen von der Regel, etwa wenn bestimmte Berufsgruppen ausdrücklich von einer bestimmten Abgabeverpflichtung ausgenommen sind. ${ }^{495}$ Das System der Pharaonenzeit steht damit auch im Kontrast zu den fiskalischen Bedürfnissen der griechisch-römischen Epoche Ägyptens, deren Zielsetzung für Zensusmaßnahmen sich primär an der Erfassung steuerpflichtiger Bürger orientierte.

490 KeMP (1991: $248 \mathrm{ff}$.).

$491 \operatorname{KEMP}(1991: 232 \mathrm{ff}$.$) .$

492 Boochs (1988: 11 f., 16); siehe auch im folgenden die Ausführungen zu Papyrus Wilbour.

493 HELCK (1975b: 247 f.); skeptisch dagegen WARBURTON (1997: 278 ff.).

494 Warburton (1997: 323 ff.); Kemp (1991: 236); ein Beispiel für den Tempel als Empfänger von Abgabenleistungen gibt beispielsweise Papyrus Harris I (zuletzt ausführlich bearbeitet von GRANDET 1994), der die >Einzahlungen $<$ am Totentempel Ramses' III. in Medinet Habu auflistet.

495 Bekanntestes Beispiel dafür ist das Dekret Haremhabs (Urk. IV 2155,9 ff.), vgl. dazu auch ALLAM (2000). 
Am wahrscheinlichsten ließen sich noch aus den sogenannten >Katasterlisten<, wie sie uns etwa durch Papyrus Reinhardt ${ }^{496}$ oder besonders Papyrus Wilbour ${ }^{497}$ bekannt sind, als Quelle demographischer Einzelbetrachtungen heranziehen, obwohl sie nur einen indirekten Blick auf die Bevölkerungssituation erlauben. Papyrus Wilbour stammt aus der Regierungszeit Ramses' V. und verzeichnet auf der beeindruckenden Länge von über $10 \mathrm{~m}$ die genaue Lage und Größe tausender Parzellen an Ackerland zusammen mit den dazugehörigen Verwaltungsinstitutionen. Die Verwaltung oblag zumeist den staatlichen oder regionalen Tempeln, doch gab es auch Flächen, die direkt dem König unterstellt waren. Säkulare Einrichtungen spielten dagegen eine untergeordnete Rolle. ${ }^{498}$ Mit der Bewirtschaftung der Flächen wurden die Bewohner von Siedlungen betraut, deren Arbeitsverpflichtung sich anhand der Aufzeichnungen des Papyrus bis auf die Person genau feststellen ließ, der eine bestimmte Parzelle zugewiesen worden war, nebst der dazugehörigen Abgabenfestsetzung. Neben dem Namen der betreffenden Person ist gelegentlich deren Berufsbezeichnung und eine Filiationsangabe vermerkt.

Bereits David O'CONNOR hat aus den tausenden an Einzelinformationen erfolgreich eine Siedlungsstruktur herausarbeiten können. ${ }^{499}$ Das vom Papyrus Wilbour erfaßte Gebiet war faktisch von Norden nach Süden in vier Zonen eingeteilt, die mit den chronologisch fortlaufenden Aufzeichnungen der Feldvermesser korrelieren. Zwei große Zentren namens Hardai $(H r-d j)$ im Süden zwischen Zone III-IV und Neni-nesu (Nnj-nsw) im Norden zwischen Zone I-II, das mit dem späteren Herakleopolis gleichgesetzt wurde und heute bei Ihnāsīya al-Madīna liegt, stellen die regionalen Metropolen dar und liegen nur ungefähr $67 \mathrm{~km}$ auseinander. O'CONNOR konnte anhand der Häufigkeit von Ortsnamenerwähnungen im Text feststellen, daß die Zonen I und IV tendenziell dichter besiedelt waren und daher möglicherweise eine - relativ zu den Zonen II und III - höhere Population aufwiesen. ${ }^{500}$ Gleichzeitig konnte nachgewiesen werden, daß die Kontrolle über die Bodenfläche überwiegend in den Händen der Zonen I und IV lag. ${ }^{501}$

\section{Zentralort-Theorie und Rang-Größen-Regel}

Aufgrund seines zeitlich und geographisch eng gefaßten Datenmaterials erlaubt der Papyrus Wilbour in weitaus stärkerem Maße die Auswertung nach Mustern in den Siedlungsstrukturen

496 VLEEMiNG (1993).

497 Siehe dazu die aufschlußreiche Studie unter dem Aspekt der Besiedlungsstruktur von ADAMS (1997).

498 AdAMs (1997: 95).

499 O'CONNOR (1972a: 690 ff.).

500 O'CONNOR (1972a: 692).

501 ADAMs (1997: 97); er weist allerdings auch auf die Unschärfe der Daten hin, da es auch Siedlungen der untersten Ränge gegeben haben dürfte, die nicht für die institutionalisierten staatlichen Parzellen zuständig waren und daher im Papyrus Wilbour keine Erwähnung fanden. 
als es bisherigen Anwendern dieses Verfahrens geglückt war. ${ }^{502}$ Neben der bereits von BUTZER im Rahmen seiner Zentralort-Theorie etablierten Bifurkationsrate, die das zahlenmäßige Verhältnis von Siedlungen unterschiedlichen Ranges zueinander definiert, beschreibt Matthew ADAMS zusätzlich ein Populationsmodell, die sogenannte >Rang-Größen-Regel<. ${ }^{503}$ Der Unterschied beider Theorien liegt in der Definition der Ortsgrößen. Die Zentralort-Theorie arbeitet mit funktionalen Parametern, ${ }^{504}$ während die Rang-Größen-Regel auf demographischen Daten aufbaut und innerhalb einer Region die Bevölkerungsgröße einer Siedlung des Ranges $r$ als $\frac{1}{r}$ der größten Siedlung (Rang 1) in der Region definiert. ${ }^{505}$ Rangstufe und Größe sind in der graphischen Wiedergabe logarithmisch skaliert (Abb. 5.1). Die definitorische Idealverteilung beschreibt dabei eine Gerade. Dieser Fall ist jedoch eher theoretischer Natur.

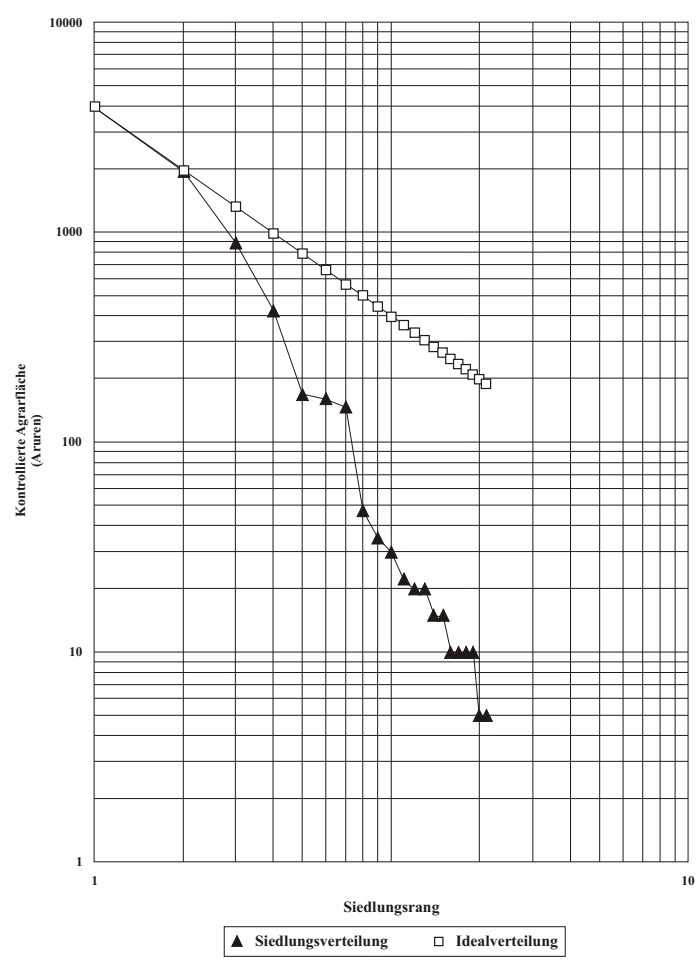

(a) Zone I-II

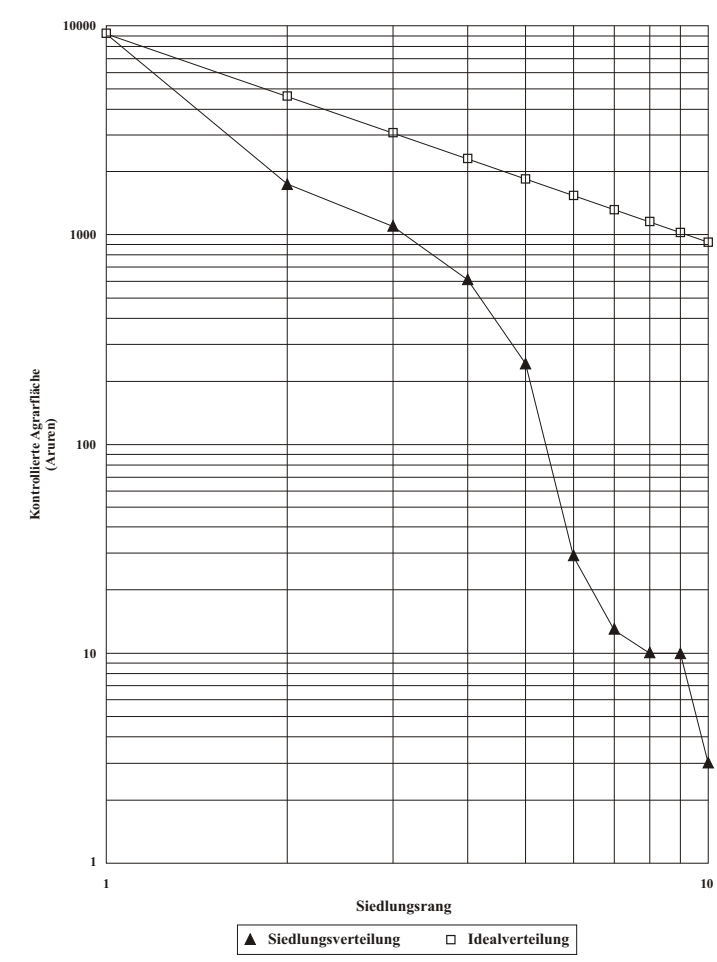

(b) Zone III-IV

Abbildung 5.1: Rang-Größen-Verteilung der Agrarflächen im Papyrus Wilbour

502 Vgl. Kap. 1.4.1.1 zu Karl BUTZERs chronologisch wie topographisch disparater Materialsammlung. 503 ADAMS (1997: 99).

504 Vgl. oben S. 19 mit Tab. 1.1.

505 Allgemein dazu: BERNBECK (1997: 175 ff.). 
Eine Schwäche in ADAMs' Ausführungen liegt in der Wahl seiner Datenbasis. Anstelle der üblicherweise anhand der Besiedlungsfläche zugrundegelegten Bevölkerungsgröße verwendet er die für die einzelnen Orte im Papyrus verzeichneten Agrarflächen. Dabei betont ADAMS allerdings, daß kein direkter Zusammenhang zwischen der verwalteten Landfläche einer Siedlung und deren Population hergestellt werden soll - quasi als Gleichung Mensch pro Quadratmeter, sondern die Argumentation eher indirekt funktioniert, indem Ortschaften mit viel Agrarfläche auch eine gewisse regionale Bedeutung besaßen, die sich nicht zuletzt in ihrer Größe und damit auch der Bevölkerungszahl niederschlug. ${ }^{506}$ Inwieweit sich dieses Modell künftig auch für konkrete Populationsgrößen heranziehen läßt, kann derzeit noch nicht abgeschätzt werden. Notwendig wären detaillierte Studien über einzelne Siedlungsplätze, die Datenmaterial für eine Gesamtbevölkerung pro Besiedlungsfläche liefern. ${ }^{507}$ Selbst bei stichprobenhafter Datensammlung könnte dann anhand von ADAMs' Diagrammen auf die Größe der Siedlungen anderer Ränge geschlossen werden.

Aus den Fragmenten eines Grundbuches des Neuen Reiches, die sich im Besitz des Berliner Ägyptischen Museums befinden, konnte Annie GASSE unter anderem eine Personenliste rekonstruieren. ${ }^{508}$ Auch in diesem Fall sind die Individuen anhand von Namen, Titeln und Filiation identifiziert. Neben einer Vielzahl von Soldaten, sind auch $w^{c} b$-Priester und sogar Steinbrucharbeiter (?) verzeichnet, denen eine bestimmte Fläche Boden zugewiesen worden war. Thebanische Namen bilden dabei auf einigen Fragmenten eine deutliche Mehrheit. Aus den einzelnen Summen von Personen läßt sich indes keine weitere Information ablesen. Weder ist die Gesamtfläche des Areals bekannt, auf das sich die Katasteraufstellung bezieht, noch können aus den Personenlisten seriöse Rückschlüsse auf Haushalte oder Familiengruppen gezogen werden. Zusammenfassend bleibt nur ein Erkenntnisniveau, wie es bereits der Papyrus BM 10068 offenbarte: eine Gruppe isoliert stehender Personen, die ein regional abgegrenztes Gebiet unbekannter Größe bewohnen. ${ }^{509}$

Ein interessanter Detailaspekt aus dem Berliner Grundbuch ist die auffällig hohe Zahl an Soldaten, denen ein Grundstück zugewiesen worden war. Mit dieser Beobachtung besteht die Möglichkeit, HELCKs Thesen mit den Belegen in Übereinstimmung zu bringen. ${ }^{510}$ So könnte

506 ADAMs (1997: 100).

507 Vgl. etwa die Ansätze von O'CONNOR (1972b), mit Hilfe von Nekropolenstatistiken und Versorgungsraten einen Näherungswert für ein fest umrissenes Gebiet zu ermitteln.

508 pBerlin 23251 ff.; GASSE (1988: 89 ff., bes. 93 f.).

509 Vgl. oben Kap. 3.4.

510 HelcK (1939: 20) bemerkt dazu: »Auch für diese Milizaushebungen waren genaue Listen über die ganze Bevölkerung und ihre wirtschaftlichen Verhältnisse nötig.« 
der Aktentypus des Berliner Grundbuchs indirekt auch anderen Verwaltungszwecken gedient haben, indem sich aus diesen Listen bestimmte Personenkreise und deren Grundstücksgrößen extrahieren ließen. Nach HELCKs Überlegungen konnten diese Listen dann im zivilen Bereich zur Aushebung von Arbeitergruppen für bestimmte Bauvorhaben dienen. Als Beispiel nennt er eine Stele Echnatons aus Gebel es-Silsile. ${ }^{511}$

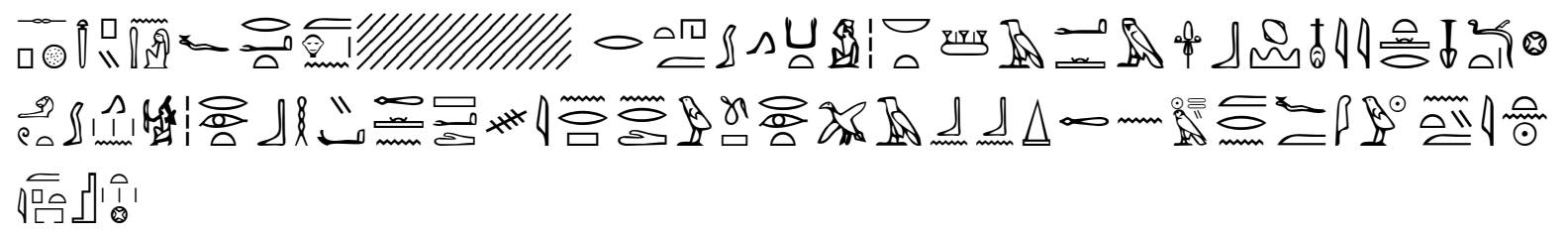

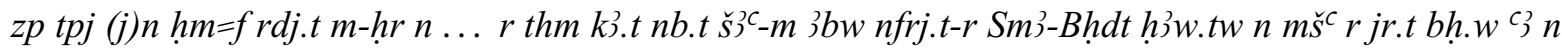
$\check{s}^{c} d j n r-n-r w \underline{d} p 3$ bnbn ${ }^{3} n R^{c}-H r-3 h t j m r n=f m \check{S} w n t j m$ Jtn $m$ Jp.t-sw.t

»Das erste Mal seiner Majestät, es wurde den ... befohlen, alle Arbeiten auszuführen von Elephantine bis Sema-Behedet. Die Befehlshaber der Truppe sollen eine große Arbeitsleistung erbringen für das Schneiden des harten Steins, um den großen Benben-Stein für Re-Harachte in seinem Namen als >Licht, das im Sonnenball ist $<$ in Karnak herzustellen.«

Bereits aus den in Kapitel 5.2.2.1 vorgeführten Textbelegen der Militärverwaltung wurde ersichtlich, daß die Militärschreiber nicht nur Rekrutierungsmaßnahmen in den Ortschaften durchführten, sondern auch in bestimmten Fällen für Besteuerungen zuständig waren, die sich vermutlich als Dienstverpflichtung verstehen lassen.

Einem Papyrusfragment des Mittleren Reiches kann man entnehmen, daß im Zusammenhang mit der Besteuerung die betroffenen Personengruppen explizit erfaßt werden konnten. Der Papyrus stammt aus Harageh am Ausgang des Fajjum und überliefert ein Journal von Arbeitseinsätzen eines Steuerbeamten zusammen mit Feldvermessern. ${ }^{512}$ Da auch der Aufbau des Papyrus Wilbour sich an der >Reiseroute< der Beamten und Landvermesser orientiert und deren einzelne Einsätze mit Tagesdatum vermerkt, stehen sich beide Papyri typologisch recht nahe. In Zeile 15 des Papyrus hat der Beamte vermerkt:

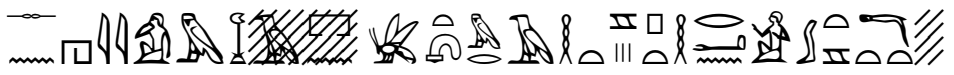

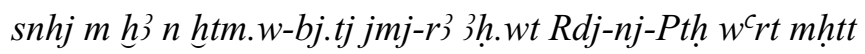

»Registrierung im Büro des Kronsiegelbewahrers und Vorstehers der Felder im nördlichen Verwaltungsbereich (namens) Rdj-nj-Ptḥ.«

511 LEGRAIN (1902: 263).

512 SMITHER (1941). 
Da der Terminus snhj überwiegend bei Personenzählungen Verwendung fand, ist auch in diesem Fall die Wahrscheinlichkeit groß, daß der auf solche Weise bezeichnete Registriervorgang sich auf die abgabepflichtigen Personen bezog. Zweifelsfrei belegen läßt sich diese These aus dem vorliegenden Material jedoch nicht.

\subsubsection{Subjekt und Objekt, oder: Was ist ein wpw.t?}

Der Terminus wpw.t tritt zuerst in Verwaltungsakten des Mittleren Reiches auf. Das Spektrum, das - mit unterschiedlichen Determinativen versehen - von diesem Begriff abgedeckt wird, reicht von >Haushaltsliste < bis zu >Liste, Inventar $<$ (Tab. 5.3). Die Streuung der Übersetzungen ist zugleich Zeugnis für ein gewisses Defizit an Quellen, das häufig zu ad hoc Interpretationen geführt hat. Im Einzelfall plausibel und nicht wirklich falsch, versperren die strikt getrennten Lemmata den Blick auf überschneidende Bedeutungsnuancen, die sich erst bei näherer Betrachtung erschließen. Im Neuen Reich sind sowohl der Begriff als auch der damit verbundene administrative Akt anscheinend wieder verschwunden. Lediglich im Kontext von Rinderlisten gibt es Belege, die dann allerdings mit zusätzlichem geschrieben werden.

Die Übereinstimmungen, die sich auf den ersten Blick zwischen den Aktenfragmenten des Mittleren Reiches und entsprechenden Beispielen für Zensuspapyri aus der griechischrömischen Zeit offenbaren, verführten bereits recht früh zu Spekulationen darüber, ob das Verfahren unverändert eineinhalb Jahrtausende überdauert hatte. Bereits wenige Jahre nach Francis Llewellyn GRIFFITHs Publikation stellte Ludwig BORCHARDT auf dem XIII. Orientalistenkongreß Überlegungen in dieser Richtung an, die allerdings nicht unwidersprochen blieben: ${ }^{513}$

»Besonders wichtig für die Chronologie könnten die Daten der Zählungen werden. Aus den citierten Beispielen scheint nämlich an einer Stelle mit Sicherheit, an zwei anderen mit Wahrscheinlichkeit hervorzugehen, dass diese Volkszählungen in Intervallen von 14 Jahren stattgehabt haben. Dies wäre umso wahrscheinlicher, als WILCKEN aus den griechischen Ostraka nachgewiesen hat, dass in römischer Kaiserzeit die Volkszählungen in Aegypten auch in 14-jährigen Abständen auf einander folgten. Es wäre also dies eine der vielen Sitten des kaiserlichen Aegyptens, die sich bis in die altägyptische Zeit zurückverfolgen liessen.«

Und noch in jüngerer Zeit stellte der Althistoriker Keith HOPKINS beim Vergleich der beiden Quellen fest: ${ }^{514}$

»The few surviving household lists from the twelfth Dynasty (ca. 1850 BC) and from the third century BC are remarkably similar to later Roman census returns.«

513 BORCHARDT (1904: 329).

514 HOPKINS (1980: 313 Anm. 34). 
Im folgenden soll zunächst eine knappe Darstellung der griechisch-römischen Belege erfolgen. Darauf folgt dann der Versuch eines Vergleichs und damit verbunden eine inhaltliche Neuinterpretation des Terminus wpw.t.

Das Steuerwesen der ptolemäischen wie auch der römischen Zeit baute auf der Erfassung und Auswertung zweier verschiedener Auskunftsbereiche auf: die Feststellung der Steuersubjekte (Personen) und -objekte (Besitz). ${ }^{515}$ Als Subjekte galten alle Bewohner eines Haushalts, sowohl die Familie des Haushaltsvorstands, als auch gegebenenfalls Mieter oder Bedienstete, ${ }^{516}$ die Objekte waren - neben Landbesitz, der gesondert mit den Katastern abgeglichen werden mußte - auch Getreidevorräte, Viehbestand und diverse Utensilien. ${ }^{517}$ Es war hierbei gesetzlich festgelegt, daß die Eigentümer den genauen Bestand an Besitz, und hierbei auch anteiligen Besitz an Objekten, sowie die Personen des Haushaltes regelmäßig den zuständigen Behörden zu melden hatten. Das Verfahren einer jährlichen Selbstdeklaration aller Hausbe-

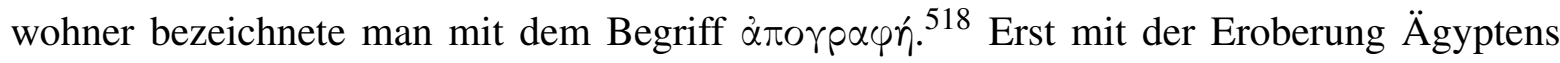
durch die Römer ergaben sich hier weitere Änderungen. Es wurde ein zusätzliches Zensusverfahren eingeführt, das nur noch alle 14 Jahre zur Deklaration verpflichtete und gleichzeitig in seinem Informatiosgehalt über die bisher durchgeführte $\alpha \pi \sigma \gamma \rho \alpha \varphi \hat{\eta}$ hinausging. Um zu betonen, daß diese Deklaration nun auch ausführliche Aufstellungen zu den Wohnhäusern enthielt,

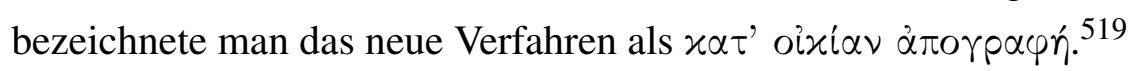

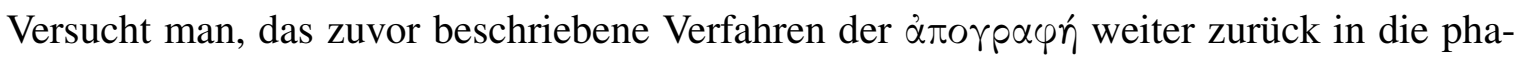
raonische Zeit zu schieben, stößt man recht schnell an die durch Quellenmangel gesteckten Grenzen. Aus dem Neuen Reich liegen keine vergleichbaren Beispiele vor, die Ähnlichkeit mit den griechischen Steuererhebungen aufweisen. Erst aus dem Übergang zwischen dem Mittleren Reich und der Zweiten Zwischenzeit liegen Hinweise auf Kombination von Subjekt und Objekt in den Verwaltungsakten ägyptischer Siedlungen vor, die mit dem Terminus wpw.t bezeichnet werden. Dieser Begriff wird laut $\mathrm{Wb}$ (I,303) zunächst mit »Leute jemds.« übersetzt, im weiteren Sinne aber dann auch als »Hausstand einer Person« sowie »Hausstandsliste (zur Steuer u. ä.)« aufgefaßt. Die Hauptquelle für die Kenntnis über die genaue Form dieser Erhebung liefern die Kahun-Papyri, die 1898 von GRIFFITH publiziert wurden. ${ }^{520}$ Die KahunPapyri bieten in verschiedenen Bereichen Einblicke in die Akten einer Arbeitersiedlung, die im Mittleren Reich auf Befehl Sesostris' I. angelegt worden war. Aus diesem umfangreichen

515 BAGNALL und FRIER (1994: 12 f.); WILCKEN (1912: 173 ff.).

516 BAGNALL und FRIER (1994: ebenda).

517 WILCKEN (1912: 175).

518 WiLCKEN (1912: 173).

$519 \mathrm{Zu}$ den Zensusverfahren in der griechisch-römischen Zeit siehe auch ausführlich Kap. 2.4.

520 Siehe dazu auch Kap. 3.3. 
Material stammen auch die Beispiele für die Erfassung aller Mitglieder eines Haushalts, die einem festen Formular folgen.

Neben den Aktenfunden aus Kahun gibt es in der Erzählung des Bauern ein weiteres Beispiel für die schriftliche Erfassung eines Haushalts und - als zusätzliche Besonderheit - des beweglichen Besitzes der Hausinhaber. ${ }^{521}$

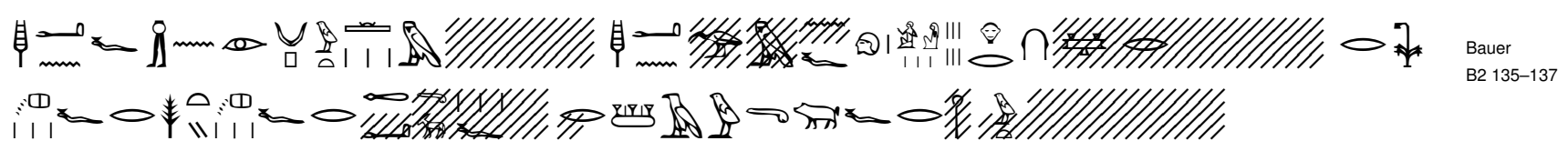

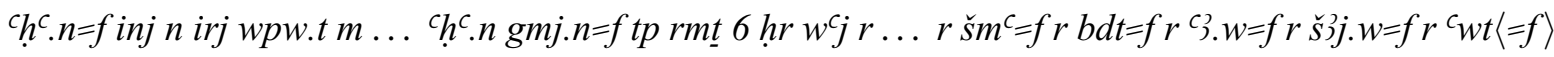
»Dann wurde er herbeigebracht und man erstellte eine Liste über [seinen Haushalt]. Er fand sechs Personen, dazu seine Gerste, seinen Emmer, seine Esel, seine Schweine, [sein] Kleinvieh ... «

Den in diesem Textbeispiel beschriebenen Vorgang mit den Verwaltungsakten zu vergleichen, scheint zunächst unmöglich. Die sogenannten >literarischen< Texte gelten in der neueren Forschung zunehmend als fiktionale Erzählungen, deren Wahrheitsgehalt des Beschreibungsapparates sich am ehesten noch in Form von >Realitätsfragmenten< definieren läßt. ${ }^{522}$ Häufig genug bestehen die Vorbehalte jedoch lediglich deshalb, weil es bisher nicht gelungen ist, schriftliche Beschreibung und archäologische Quelle zur Deckung zu bringen. In diese Richtung gehen auch die Einwände Friedrich VogELSANGs, der die Lesung von wpw.t als $>$ Haushaltsliste< in der Bauernerzählung schon früh angezweifelt hatte. Bereits 1913 schrieb er gegenteilig zu Alan GARDINER: ${ }^{523}$

»wpwt ist hier wohl nicht „Liste der Hausbewohner, Zensusliste“, wie ich früher angenommen hatte. Die in Pap. Kah. 9,2ff. mitgeteilten Listen haben ein ganz anderes Aussehen, als das Verzeichnis an unserer Stelle. Auf jenen sind nur Personen notiert, hier ist außer der Kopfzahl der Angehörigen auch sämtliches lebendes und totes Besitztum angegeben.«

VOGELSANGs Vorschläge haben sich jedoch nicht durchgesetzt. In der letzten Bearbeitung der Bauernerzählung von Richard PARKINSON verwirft dieser die Rekonstruktion VOGELSANGs mit Hinweis darauf, daß der Leerraum eben doch zu klein ist, um die fragliche Ergänzung einzufügen. ${ }^{524}$

521 PARKINSON (1991: 48).

522 Vgl. dazu auch Junge (2000: 157 f.) und speziell MoERs (2001: 55 f.): »Aus diesem Grund ist er [scil. der Verfasser] gezwungen, in einem ersten >Akt des Fingierens< verschiedene Selektionsprozesse vorzunehmen, um aus der Totalität der Welt die im Sinne seiner Intention relevanten Teile auszugrenzen. Diese Auswahlentscheidungen stehen ihrerseits wiederum sowohl in Abhängigkeit von je historischen Gegebenheiten als auch in Abhängigkeit von der intendierten Funktion des Textes.«

523 VOGELSANG (1913: $231 \mathrm{f}$.).

524 PARKINSON (1991: 48a (8.a)). 
Auf das zweite Argument - nämlich dem Unterschied zwischen der Beschreibung aus der Bauernerzählung und den Kahun-Akten - muß etwas ausführlicher eingegangen werden. VoGELSANG revidiert seine Übersetzung für wpw.t aufgrund des formalen Vergleichs mit den Beispielen aus den Kahun-Papyri. Unabhängig davon, daß seine Beobachtung natürlich richtig ist, verbietet sich jedoch der Vergleich zweier verschiedener Textgattungen. Die Erzählung des Bauern ist mit Sicherheit kein exakter Reflex einer formal korrekten Haushaltsliste. Für den erzählenden Charakter dieses Textes ist dies auch völlig unnötig. Neben der Aufzählung der Besitztümer des Hauses müßte dann auch die Form der Auflistung aller Personen des Haushalts unterschieden werden. Während die Kahun-Papyri jedes Mitglied des Haushalts beim Namen nennen, beschreibt die Bauern-Erzählung nur die Gesamtzahl der Personen ohne nähere Ausdifferenzierung. Die Unterschiede machen deutlich, daß in der Bauern-Erzählung keine Bestätigung für die Kahun-Papyri zu finden ist. Da aber einerseits das Prinzip dieser Form der Erfassung grundsätzlich aus späteren Epochen bekannt ist, andererseits auch in späterer Zeit verschiedene Typen von Deklarationen zeitgleich existieren, gibt es keinen Grund für die Annahme, daß in diesem Fall keine realistische Situation beschrieben wird. Es ist im Mittleren Reich durchaus im Rahmen des Möglichen, daß es neben der Subjektsdeklaration auch Fälle von Objektsdeklarationen gab. Dem trägt auch Richard PARKINSON in seiner Übersetzung der Bauern-Erzählung Rechnung, indem er an die ursprüngliche GARDINERÜbersetzung anknüpft und die Bedenken VoGELSANGs verwirft. 525

Die Unklarheit hinsichtlich einer adäquaten Übersetzung entstand aufgrund der Tatsache, daß für den Terminus wpw.t letztlich nur ein knappes Dutzend Belege vorliegen, von denen zwei laut Wb I,303 dem Bereich von Gefolgsleuten oder Untertanen zuzuordnen sind und daher hier entfallen können. Die restlichen Belege stammen hauptsächlich aus den Kahun-Papyri und - das ist der wesentliche Aspekt - in differierender Schreibung aus eher >literarischen< Texten. ${ }^{526}$ Während die Begriffe in den Schreibungen der Kahun-Papyri recht einheitlich mit den Zeichen für Mann/Frau determiniert werden, weisen die Schreibungen in der Bauernerzählung aber auch in den Admonitions (s. u.) eine dazu unterschiedliche Variante auf, die eine inhaltliche Zuordnung der Termini erschwert. Das Wb trennt deshalb die Bedeutungsnuancen und überträgt die Kahun-Termini mit »Hausstand einer Person«, die Begriffe aus der Bauernerzählung und den Admonitions dagegen - kaum verschieden - als »Hausstandsliste (zur Steuer u. ä.)«. Auszuschließen sein dürfte dagegen der zusätzliche Beleg von Ricardo CAMINOs, der

525 PARKINSON (1997: 75).

526 An dieser Stelle soll nicht das Problem der Textgattungen angeschnitten werden. Vielmehr dient die Bezeichnung der Texte als >literarisch< dazu, sie von den reinen Verwaltungsdokumenten abzugrenzen. 
- unter dem Stichwort wpwt von Raymond FAULKNER im >Concise Dictionary< zitiert - als Yho i wpyt verzeichnet wird, allerdings in unklarem Zusammenhang. ${ }^{527}$ Die Übereinstimmung des Determinativs $\propto$ mit der ebenfalls singulären Schreibvariante aus Admonitions 6,7 scheint als Erklärung unbefriedigend, zumal der Kontext von CAMINOS' Fragment keine weiteren Schlußfolgerungen erlaubt. Bis auf eine einzige (gänzlich undeterminierte) Ausnahme sind alle Schreibungen von wpw.t in den Kahun-Papyri mit dem Determinativ

\begin{tabular}{|c|c|c|c|c|}
\hline Quelle & Textstelle & Interpretation/Lesung & & Schreibweise \\
\hline \multirow[t]{3}{*}{ pKahun } & 9,$2 ; 9,8 ; 9,16$ & Hausstand einer Person & wpw.t & पP口过 \\
\hline & 9,27 & & & $Y_{0}$ \\
\hline & 10,$2 ; 10,24 \mathrm{a}$ & & & $Y_{0} 0 \frac{p_{1}}{11}$ \\
\hline Bauer & B2 135 & Hausstandsliste (zur Steuer u. ä.) & wpw.t & $\begin{array}{l}y=\infty \\
0=111\end{array}$ \\
\hline Admonitions & 6,7 & & & 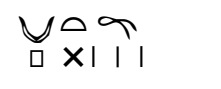 \\
\hline pBerlin P 10067 (Kahun) & & & & 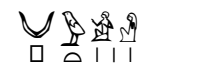 \\
\hline pSallier & IV vs. 4,1 & & & 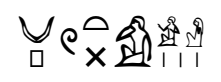 \\
\hline Beni Hassan & I Taf. 9b & Leute/Untergebene jmds. & wpw.t & You \\
\hline Kairo & 1609 & & & \\
\hline pKahun & 16,$13 ; 17,1$ & Viehliste/Viehzählung & $w p . t$ & $U_{0} x$ \\
\hline Kairo 21367 & & & & $\begin{array}{l}y 0 \\
0 x\end{array}$ \\
\hline pBoulaq 18 & 15,$14 ; 30,11$ & & & $y_{0 x}$ \\
\hline Urk. IV & 1115 & & & $\mathcal{U}_{\square} \mathbb{P}\left[\begin{array}{l}0 \\
1 \\
1\end{array}\right]$ \\
\hline
\end{tabular}

Tabelle 5.3: Vergleich der Schreibweisen für wpw.t/wp.t

In eine andere Richtung geht dagegen die Überlegung, ob die bisher im $\mathrm{Wb}$ vollzogene Trennung zwischen $w p . t$ »Einzelangabe/Viehzählung « einerseits und wpw.t »Haushaltsliste« andererseits in jedem Falle strikt durchgehalten werden kann. Für wp.t vermerkt das Wb eine Schreibung in Papyri der 19. und 20. Dynastie, die identisch zu der wpw.t-Schreibung in den

527 CAminos (1956: Taf. 22, Fragm. 13).

528 Vgl. ausführlich Tab. 5.3. 
Admonitions ist. ${ }^{529}$ Es eröffnen sich hier ernste Möglichkeiten, über einen Zusammenhang zwischen wp.t und wpw.t nachzudenken und damit einen Bezug zwischen Subjekts- und Objektslisten herzustellen. Trotz unterschiedlicher Determinierung zeigen die Kontexte deutlich, daß ein grundsätzlicher Zusammenhang zwischen beiden Termini gegeben ist, der sich allgemein mit >Listenartiger Erfassung < bezeichnen läßt. Das Beispiel des Neuen Reiches aus Urk. IV 1115 zeigt dabei, daß zumindest von einer Überschneidung der Begriffe auszugehen ist. Aus dem ursprünglichen wp.t ist ein wpw.t geworden. Da es sich jedoch um einen singulären Beleg handelt, sind Schlußfolgerungen nur mit Vorsicht zu ziehen. Als mögliche Szenarien ließen sich sowohl ein Irrtum des Schreibers als auch eine Verschiebung der Bedeutung zum ursprünglichen Begriff wpw.t anführen.

Einen noch deutlicheren Hinweis darauf, daß zwischen der Objektszählung von Rindern und der Subjektszählung ein engerer Bezug besteht als bisher vermutet wurde, liefert das PapyrusFragment Kahun I.3. ${ }^{530}$

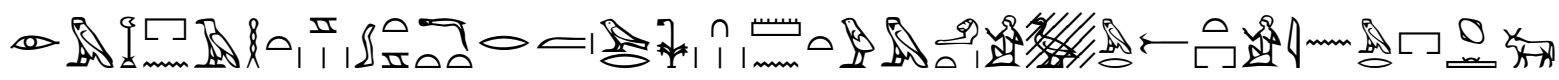

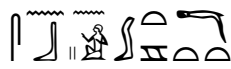

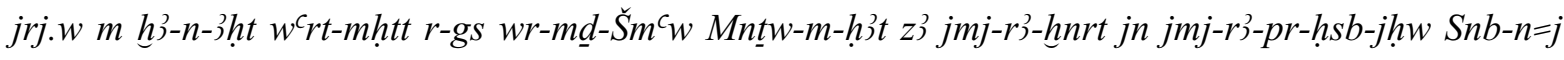
$w^{c} r t-m h t t$

»Erstellt im Büro der Felderverwaltung des nördlichen Bezirks - in Gegenwart eines Großen der Zehn von Oberägypten (namens) Mntw-m-ḥ̉t, Sohn des Aufsehers des Arbeitshofs ${ }^{531}$ - durch den Vorsteher des Hauses der Rinderzählung des nördlichen Bezirks (namens) Snb-n=j. «532

Die entscheidende Aussage des Zitats liegt in der Information, wer den vorliegenden Zensus erstellt hat. Obwohl es auch im Mittleren Reich ein eigenes Personenzählbüro gab, ${ }^{53}$ erfolgte die Aufzeichnung der vorliegenden Akten durch einen jmj-r3 pr-hsb-jhww (also

529 Leider liefert auch das Berliner Zettelarchiv keinen Hinweis darauf, woher diese Information stammt, da zu dieser Schreibung von wp.t kein Beispiel aufgenommen ist, sondern nur summarisch in der Liste der Schreibvarianten auftaucht. Daher muß man zwei Möglichkeiten in Erwägung ziehen: Entweder ist die Belegstelle nicht verzettelt worden oder es liegt eine Verwechslung mit dem Lemma wpw.t (Wb I,303) vor.

530 GRIFFITH (1898: Taf. 9).

531 Zur Problematik des Begriffs hnrt vgl. ausführlich QUIRKE (1988), der in Verbindung mit der Corvée eine Übersetzung als >umzäunter Hof< (enclosure, compound) vorschlägt.

532 Offenbar ist dem Schreiber des Dokuments ein Fehler unterlaufen. In Zeile 12 schreibt er Namen und Titel des Zeugen, der beim Aufsetzen des Schriftstücks zugegen war. Bei der Filiation dieses Zeugen >vergißt< er jedoch den Namen des Vaters und vermerkt lediglich dessen Titel jmj-r3-hnrt, der einen Vorsteher des Sperrbezirks oder Harems des Palastes bezeichnet (WARD 1982: Nr. 302); GRIFFITH (1898: 20) übersah den Fehler und übersetzte ohne Zögern den Titel als Namen >Mer·khent<. Zum Typus der Filiationsangabe des Mittleren Reiches vgl. auch GARDINER, EG $\S 85$.

533 Vgl. HelcK (1958: 153). 
einem für die Viehzählung zuständigen Beamten. ${ }^{534}$ Wenn in diesem Bürogebäude also eine Personenliste verfaßt wurde, läßt sich die Nähe der Begriffe wp.t und wpw.t kaum von der Hand weisen. Als Konsequenz daraus muß davon ausgegangen werden, daß eine - für die Zeit des Mittleren Reiches nicht näher bestimmbare - Konnexion zwischen diesen beiden Termini existierte. Auch der in den Admonitions erwähnte wpw.t paßt dabei nahtlos in die hier zu besprechende Gruppe von Ereignissen, wie bereits die Beobachtungen GARDINERs zeigen. Die entsprechende Passage in den Admonitions 6,7 lautet: ${ }^{535}$

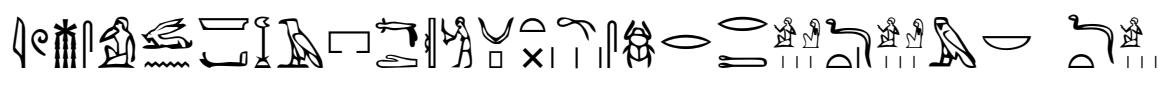

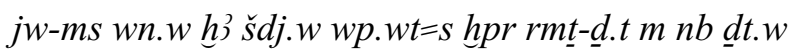

»Es ist doch so: Das Büro wurde geöffnet und seine Zensus-Akten entwendet. Ein Mitglied der Dienerschaft wurde zum Herrn über Diener.«

In den Kahun-Papyri sind neben den Hausbewohnern auch die Besitzverhältnisse über Diener des Haushalts vermerkt. Sowohl die Kahun-Papyri als auch die Admonitions bezeichnen diese Diener als $\underline{d} t .^{536}$ Unter der Prämisse, daß die $w p w . t$-Listen den einzigen Nachweis darüber darstellen, würde der Verlust dieser Listen zur Folge haben, daß die Besitzer keinerlei Beleg über ihre Diener vorweisen könnten. Die Diener könnten in einem solchen Fall also Freiheit für sich reklamieren. Ob dies auch in der Realität so geschehen ist, oder ob diese pessimistischen Beschreibungen als reiner literarischer Topos gelten müssen, bleibt offen. Doch läßt sich die Passage aus den Admonitions inhaltlich gut mit den bisher gewonnenen Erkenntnissen in Einklang bringen.

$\mathrm{Da}$ es sich bei den wpw.t definitiv nicht um einen Zensus im engeren Sinne gehandelt haben dürfte, war sicher schon GARDINER bewußt, da er seine Übersetzung der Textpassage dahingehend kommentiert hat. ${ }^{537}$ Vielmehr bietet sich der Vergleich mit den griechischen $\alpha \dot{\pi} 0-$

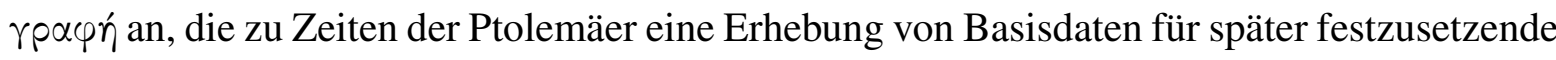
Steuern darstellte. Es ist jedoch nicht grundsätzlich auszuschließen, daß die Auflistungen der Haushalte, ihrer Bewohner und (in manchen Fällen) ihres zu versteuernden Besitzes Grundlage für eine Kompilation der Einwohnerzahlen einer Ansiedlung gewesen sein könnten, was bereits von Dominique VALBELLE vermutet wurde. ${ }^{538}$

534 Vgl. auch HelcK (1958: 171); zum Titel siehe auch WARD (1982: Nr. 160).

535 GARDINER (1909: 48); seine Pluralergänzungen scheinen allerdings unnötig.

$536 \mathrm{Zu}$ den Dienerlisten in den Kahun-Papyri vgl. oben Kap. 3.3.4.

537 GARDINER (1909: ebenda); Kritik an GARDINERs Übersetzung von FAULKNER (1964: 30): » 4 × $\times$ । is 'schedules' rather than 'census-lists', cf. Peas. B2,135, where, although the context is damaged, a 'census' of population can hardly be meant; in P.Kah. 17,1 the meaning 'inventory' seems clear, while ibid. 10,1 the word, determined with $\frac{2}{111}$, is a list of priestly personnel.«

538 VAlbelle (1987: 45). 
Im Zusammenhang mit den Kahun-Papyri muß an dieser Stelle auch darauf eingegangen werden, daß zwischen den einzelnen, aus den erhaltenen Texten bekannten Zählungen angeblich ein Intervall von vierzehn Jahren liegt. Es war BORCHARDT, der, wie oben zitiert und gegen den Einwand ERMANs, die Behauptung aufgestellt hatte, daß diese aus den Zensuserhebungen der römischen Zeit bekannte Zeitspanne in Zusammenhang mit den Erhebungen aus Kahun zu bringen ist, und damit ein weiteres Indiz für den direkten Bezug der römischen Haushaltserfassungen auf ihre altägyptischen Vorläufer gegeben ist, gleichsam eine unveränderte Übernahme pharaonischer Verwaltungsmaßnahmen durch die Römer. Diese These fand besonders in der älteren Forschung Widerhall und wurde relativ einhellig ablehnend zitiert. ${ }^{539}$

Tatsächlich stehen der Einschätzung BORCHARDTs mehrere schwerwiegende Argumente entgegen, während er selbst seine These nie mit Argumenten stützte. Einzig das Protokoll seines Vortrags auf dem Orientalisten-Kongreß beschreibt die Idee von den 14-Jahres-Abständen, ergänzt um ein paar weitere, vage Hinweise bei SCHÄFER, die darauf hindeuten, daß BORCHARDT nicht nur die datierten Personenzählungen aus den Kahun-Papyri in seine Überlegungen mit einbezogen hat, sondern zusätzlich auch die Katasterlisten und Viehzählungen. 540 Bereits GRIFFITH konnte lediglich anhand der seinerzeit bekannten Königschronologien vermuten, daß der Turnus der Haushaltserfassung in einer Zeitspanne von fünfzehn oder zwanzig Jahren erfolgte. ${ }^{541}$ Eine exakte Datierung der Papyri gestaltet sich ohnehin als äußerst schwierig, da es für den Übergang zwischen dem Mittleren Reich und der Zweiten Zwischenzeit nur unzureichende Daten gibt. ${ }^{542}$ Daher kann eine verläßliche Aussage über die Länge des Turnus anhand der vorliegenden Kahun-Papyri nicht getroffen werden.

Darüber hinaus muß eine mögliche Intention für den Zeitrahmen von vierzehn Jahren geklärt werden. Aus römischer Zeit wissen wir, daß den Jungen mit vierzehn Jahren, den Mädchen bereits mit zwölf Jahren ein >Erwachsenenstatus< zukam. ${ }^{543}$ Hier stand also möglicher-

539 Zum Beispiel Wilcken (1912: 173), WALlaCE (1938: 418), HoMBERT und PrÉAUX (1952: 43); Zustimmung lediglich bei SCHÄFER (1902: 9 Anm. 1).

540 SCHÄFER (1902: ebenda).

541 GRIFFITH (1898: 27).

542 Siehe dazu ausführlicher Kap. 3.3.

543 Die Quellen dazu sind recht uneinheitlich. Zuverlässige Zahlen finden sich erst im Codex Iustinianus 5/4/24 aus dem frühen 6. Jh. n. Chr., der mit den Angaben von 12, bzw. 14 Jahren allerdings das Alter der Heiratsfähigkeit beschreibt. WILCKEN (1899: 242) zitiert die gleichen Zahlen, behauptet jedoch, daß ab diesem Alter die Personen kopfsteuerpflichtig werden. Verbunden damit war bei den Römern eine religiöse Zeremonie am Tag der Liberalia (17. März), die im Ablegen der insigniae pueritiae gipfelte. Hinweise auf die Altersgrenze von 14 gibt es auch im 1. Jh. n. Chr. bei der Rechtsschule der >Proculejaner , die sich auf den Juristen Proculus beriefen (SOHM 1949: 238 Anm. 3). Weniger zuverlässig ist die Quelle des Aulus Gellius (X, 28.1-2), der Servius Tullius, den legendären sechsten König Roms, als Reformer der Altersklasseneinteilung bezeichnete, der den Übergang von der Kindheit zum Erwachsenenalter auf 17 Jahre festlegte. 
weise der pragmatische Zweck einer 14-jährlichen Erhebung im Vordergrund, der zugleich dem Staat unnötige Kosten sparte, die eine derartige Maßnahme zweifellos mit sich brachte. ${ }^{544}$ Ein Zusammenhang zwischen dem Turnus eines Zensus und der Adoleszenz kann für die pharaonische Zeit überhaupt nicht nachgewiesen werden. Wie bereits Rosalind und Jac JANSSEN aufzeigen konnten, gibt es kein festgelegtes Alter, in dem ein ägyptischer Jugendlicher offiziell in die Gemeinschaft der Erwachsenen aufgenommen wurde. Weder die Beschneidung, das >Umbinden des Gürtels< oder die Eheschließung, die noch am wahrscheinlichsten einen tieferen Einschnitt in das Leben eines jungen Mannes gebildet haben dürfte, können verbindlich einem entsprechenden Alter zugewiesen werden. Der Einstieg in das Berufsleben und die Welt der Erwachsenen erfolgte, wenn der Jüngling offenkundig reif dafür war. ${ }^{545}$ Zuletzt sei zu dieser Problematik nochmals auf die entsprechende Stelle bei Herodot (II 177,2) verwiesen, die ausdrücklich von einer jährlichen Deklaration spricht. Ein Verfahrenswechsel von vierzehn Jahren im Mittleren Reich herunter auf ein Jahr zur Zeit Herodots und dann wieder auf vierzehn Jahre während der römischen Herrschaft über Ägypten scheint wenig plausibel.

Obwohl es unwahrscheinlich ist, daß man das hier beschriebene Verfahren des wpw.t mit den aus der Ptolemäerzeit und Römischen Kaiserzeit bekannten Methoden in direkten Bezug setzen kann, die altägyptischen wpw.t gleichsam die direkten Vorläufer der griechischen Zensuserhebungen sind, lassen sich dennoch grundlegende Übereinstimmungen hinsichtlich der angestrebten Ergebnisse feststellen. Der Unterschied liegt eher darin, daß wir in keinem Fall eine Objektsdeklaration aus griechisch-römischer Zeit besitzen, die alle möglichen Elemente einer Zählung in sich vereint. Lediglich einzelne Beispiele für den Nachweis über Getreide oder Vieh liegen vor. Demgegenüber bietet die Bauernerzählung ansatzweise Evidenz dafür, daß tatsächlich der gesamte Haushalt in den altägyptischen Listen erfaßt werden konnte. $\mathrm{Ob}$ dies in allen Fällen das übliche Vorgehen war, läßt sich aus diesem singulären Hinweis leider nicht entnehmen. Der Sachverhalt zieht jedoch die prinzipiellen Ähnlichkeiten beider Verfahren nicht in Zweifel. Unterschiede sind eher im Bereich der nicht zur Familie gehörigen Personen festzustellen, wie etwa die aus den griechisch-römischen Akten bekannte Rubrik der >Sklaven<, die sich nicht direkt in den Kahun-Papyri wiederfinden läßt, da die bereits in Kap. 3.3.4 besprochenen $\underline{d} t$ aufgrund des anderen Status terminologisch keinesfalls mit dem griechischen $\delta$ oṽ $\lambda$ os gleichgesetzt werden dürfen.

544 HOFFMANN (2000: 54); WiLCKEN (1912: 193).

545 JANSSEN und JANSSEN (1990: 107 ff.), auch FEUCht (1995: 238 f.). 


\subsubsection{Arbeitsverpflichtung und Frondienst}

Bereits in Kapitel 3 konnte anhand der Gebelein-Papyri die früheste Erfassung von Personen für Arbeitsdienste in Form von Listen vorgeführt werden, für die sich auch bereits seit GRIFFITH die dem Französischen entnommende Bezeichnung >Corvée< etabliert hat. ${ }^{546}$ Diese offenbar rein zivile Liste steht neben den modern als >Rekrutenaushebungen< bezeichneten und militärisch konnotierten Listen. Häufig genug ist die Erwähnung von Arbeitsgruppen untrennbar verbunden mit der Existenz militärischer Einheiten, obwohl diese Gruppen rein zivile Aufgaben erfüllten. Dennoch dürfte der Vorgang der Rekrutierung von Arbeitskräften in der Praxis kaum anders vonstatten gegangen sein als der für militärische Truppen. Ein interessantes Detail offenbart die Zusammenstellung der Termini bei VALBELLE. ${ }^{547}$ Alle >geläufigen< Begriffe für Arbeitskräfte kommen erst ab dem Mittleren Reich auf und sind auch nur für diese Zeit gesichert, da neben dem bereits genannten Beispiel aus dem Alten Reich besonders aus dem Mittleren Reich zahlreiche Belege für solche Listen vorliegen. ${ }^{548}$ Aussagen wie die folgenden im Papyrus Reisner I aus der 12. Dynastie weisen deutlich auf die Existenz von Personallisten hin, denen eine Rekrutierung und damit einhergehend eine systematische Erfassung vorausgegangen sein kann.

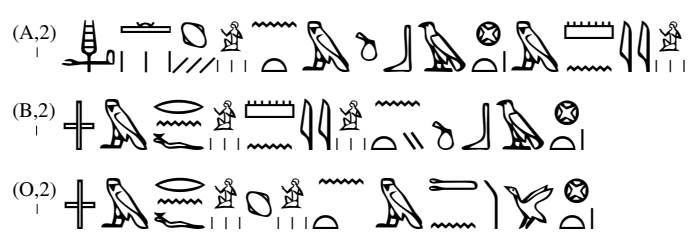

${ }^{c} h^{c}-h$ sh.w ntj $m$ Gbtw m mny.w

$j m j-r n=f m n y . w n t j$ Gbtw

jmj-rn=f ḩsb.w ntj $m \underline{T n j}$

»Dienstplan der Arbeitsdienstler in Koptos

Namensliste der Arbeitsdienstler von Koptos

Namensliste der Dienstleute von Thinis«

Aufgrund seiner häufigen Erwähnung in den Akten kommt dem Terminus hsb.w (o 通 eine hervorgehobene Stellung zu, ${ }^{549}$ der bereits etymologisch auf das >Zählen< $(h s b)$ bestimmter Personenkreise hinweist. Die Angaben in Papyrus Reisner I zeigen eine gewisse funktionale Nähe zwischen den $h s b . w$ und mny.w, so daß William SimPSON die Vermutung äußerte, der

546 GRIFFITH (1898: 43).

547 VALBelLE (1987: 47).

548 Mehrere Listen zum Beispiel aus dem Kahun-Archiv: QUIRKE (1991b: 143 f.); QUIRKE (1990: 164 f.).

549 HELCK (1975b: 148 ff.); BERLEV (1965: 266 ff.). 
Terminus $h s b . w$ könnte als allgemeiner Oberbegriff Verwendung gefunden haben. ${ }^{550} \mathrm{Zu}$ einem gegenteiligen Ergebnis kam dagegen Stephen QUIRKE anhand seiner Analysen der KahunPapyri. Die dort vorgefundene unterschiedliche Terminologie für Arbeitskräfte (mny.w, hsb.w, jth.w-jnr.w) stand jeweils unter verschiedenen funktionalen Gesichtspunkten. ${ }^{551}$ Nach QUIRKE war dabei mny.w die allgemeine Bezeichnung für ungelernte Kräfte mit niedrigem Status, während die hsb.w bereits zuvor für staatliche Projekte registriert worden waren und jth.w$j n r . w$ die konkrete Tätigkeit als Arbeiter bei Bauprojekten bezeichnet. ${ }^{552}$ Diese Interpretation ist allerdings nicht unproblematisch. Insbesondere die Trennung zwischen den Termini hssb.w und jth.w jnr.w läßt sich mit den Belegen aus den Kahun-Papyri nicht hinreichend erklären. QUIRKE bezieht sich offenbar auf die Textstellen 15,14 und 26a,22, die in beiden Fällen von hsb.w jth.w jnr.w sprechen und nicht als zwei getrennte Personengruppen aufzufassen sind, sondern als $\gg h s b . w$, die Steine schleppen «. ${ }^{553}$ Sollte QUIRKE dagegen mit seiner Unterscheidung zwischen $h s b . w$ und mny.w Recht behalten, dann muß von einer zentralen und dauerhaften Erfassung der arbeitstauglichen Kräfte ausgegangen werden. Der Pool der Arbeitskräfe stammte dabei nicht ausschließlich aus den kleinen Dörfern und Dömänen, die weit verstreut um die Metropolen lagen, sondern offenbar auch aus Zentren selbst, wie die Beispiele für Arbeitslisten aus Kahun zeigen. ${ }^{554}$

Oleg Berlev kam in seiner Rezension zu Simpsons Publikation von Papyrus Reisner I zu weitergehenden Schlußfolgerungen. ${ }^{555}$ Er analysierte nochmals die Dauer der Arbeitseinsätze und konnte aufzeigen, daß manche Arbeitskräfte fast das ganze Jahr über eingesetzt wurden, was durch den Abruf von großen Bevölkerungsteilen aus ihren Dörfern ökonomisch nicht zu leisten ist. ${ }^{556}$ Der Terminus $h s b . w$ bezeichnete nach seinem Urteil keine fest umrissene Gruppe von Leuten mit festen Aufgaben, sondern konnte fallweise für staatliche Arbeiter oder eigens rekrutierte Bevölkerung stehen. Irritierend ist dabei BERLEVs weitgefaßte Interpretation. Während die Expeditionsteilnehmer in das Wadi Hammamat offenbar aus der lokalen Bevölkerung stammten, ${ }^{557}$ sind die $h s b . w$ in anderen Fällen zusätzlich zu den >Einheimischen<

\footnotetext{
550 SIMPSON (1963: $34 \mathrm{f}$.).

551 QUIRKE (1990: 169 f.).

552 QUIRKE (1990: 170).

553 So auch HELCK (1975b: 149).

$554 \mathrm{Zu}$ den sog. >townsmen< (s-n-niwt-tn) und deren Funktion als Arbeitskräfte vgl. QUIRKE (1991b).

555 BERLEV (1965: $266 \mathrm{ff}$.).

556 HELCK (1975b: 150) dagegen vergleicht diese Dienstzeit mit dem modernen Militärdienst für junge Männer. Über den tatsächlichen personellen Umfang solcher Langzeit-Einsätze ist nichts bekannt, so daß BERLEVs Bedenken zu den wirtschaftlichen Problemen in den Orten nicht überprüfbar sind.

557 BERLEV (1965: 266).
} 
herangezogen worden. ${ }^{558}$ Der von BERLEV hergestellte Zusammenhang zwischen $h s b . w$ und Königssklaven ( $h m w$ - $n z w)$ wurde allerdings von HELCK zurückgewiesen. 559

Mit einem ganz anderen Erklärungsansatz versuchte EICHLER den außerordentlich hohen Zahlenangaben von $h s b . w$ in den Expeditionsinschriften beizukommen. ${ }^{560}$ Als Beispiel diente ihm ein Wadi Hammamat-Text aus dem 37. Jahr Sesostris' I., der von 17000 Teilnehmern berichtet (Tab. 5.1). EICHLER interpretierte diese Aussage nicht als Personenzahl, sondern als Angabe der Arbeitsleistung. Ein $h s b$ stellte demnach die Tagesleistung von 10 Personen oder 10 Tage Arbeit eines Mannes dar, wodurch sich die reale Anzahl der Expeditionsteilnehmer deutlich reduzieren ließe. Dieses Maß für 1/10 der Arbeitsleistung ist in der Tat bereits aus Papyrus Reisner I bekannt, ${ }^{561}$ doch ist gerade das von EICHLER gewählte Beispiel Hammamat 61 in seiner Angabe realer Personen (>Männer<) eindeutig: hsb.w n m̌́s jrj k’.wt zj 17000 , wie bereits Dieter MÜLLER anmerkte. ${ }^{562}$ In einzelnen Fällen scheint es hingegen tatsächlich möglich, den Terminus hasb.w sowohl als Person wie auch als Arbeitsleistung aufzufassen, weil im letzteren Fall auch Bruchzahlen angegeben sein können. 563

Da die ḩsb.w auch in Verbindung mit privaten Haushalten hochrangiger Persönlichkeiten erwähnt wurden, stellten sie nach Meinung HELCKs eine staatliche Zuweisung dar, die auch wieder abberufen werden konnte. ${ }^{564}$ Dabei ist jedoch gerade bei den $h s b . w$ der Übergang zwischen zivilen und militärischen Aspekten fließend. Bereits SIMPSON wies auf die immer wiederkehrenden Zusammenhänge zwischen $h s b . w$ und $m \check{s}^{c}$ hin, wie etwa die $h s b . w n m \check{s}^{c}$ in der zuvor genannten Inschrift Hammamat 61 oder aber auch den Truppenteilen in der Inschrift des Jmn-m-ḩ.t, genannt Jmnjj. ${ }^{565}$ Besonders letztere Inschrift dient HELCK als Beweis für die Verquickung von Soldaten und Arbeitern. ${ }^{566}$ Die eine Truppe bezeichnet Amenemhet als $h s b . w n m \check{s}^{c}$, die andere dagegen nur als $h s b . w$ mit dem Ort ihrer Herkunft, was nach HELCK auf eine frisch rekrutierte Truppe aus Zivilpersonen hindeutet. In Anbetracht des Missionsziels, nämlich die Beschaffung von Gold, ist eine militärische Konnotation auszuschließen.

Zusammenfassend betrachtet sind die Möglichkeiten einer Auswertung der Arbeiterlisten für demographische Zwecke wenig aussichtsreich. Selten genug sind diese Listen auf be-

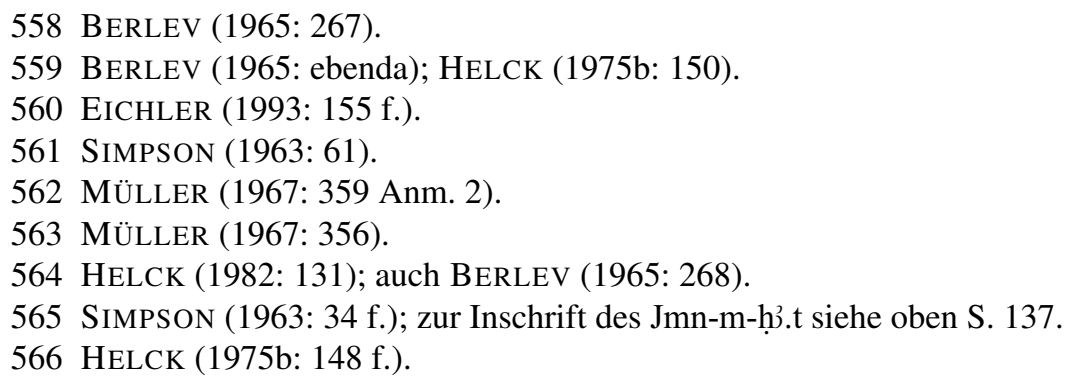


stimmte Einzelorte beschränkt oder stellen einen geschlossenen Personenkreis dar, von dem nicht einmal das Alter bestimmbar ist. Daher scheint diese Textgruppe bestenfalls geeignet, im Einzelfall die Plausibilität anderer Rekonstruktionen zu stützen. Mit der einzelnen Summe an Personen ist aus diesen Listen dagegen nichts gewonnen.

\subsubsection{Der demographische Wert der ältesten Belege}

Im allgemeinen ist sich die ägyptologische Forschung darin einig, daß seit den frühesten Anfängen die Zählung und - im weiteren Sinne - die Erfassung von Mensch und Material ein Grundpfeiler für die Entwicklung des ägyptischen Staates gewesen ist. ${ }^{567}$

Die frühesten Zeugnisse für Zählungen liegen aus dem Bereich des Kriegswesens vor. Gefallene Feinde wurden akribisch notiert oder Kriegsgefangene vor den siegreichen König geführt. In Zeiten langsamer demographischer Entwicklung stellten die Gefangenen eine wertvolle Ressource an Arbeitskraft dar, ${ }^{568}$ die in Form von Zwangsumsiedlung nach Ägypten verbracht wurden, was auch in späterer Zeit übliche Praxis blieb. Ob und inwieweit die auf diesen Denkmälern überlieferten Zahlenangaben realistisch oder reine Fiktion sind, bleibt im folgenden zu zeigen.

\subsubsection{Der Keulenkopf des Narmer}

Als früheste Inschrift gezählter Subjekte und Objekte und zugleich Beleg der höchsten von den alten Ägyptern je aufgeschriebenen Zahl gilt die Inschrift auf dem Keulenkopf des Narmer aus der 0. Dynastie, der gerade in den letzten Jahren wieder in das Zentrum des Interesses gerückt ist. ${ }^{569}$ Übereinstimmung herrscht bei allen Bearbeitern des Objekts über die Art der Einträge und die genaue Größe der Zahlenangaben. Es sind 400000 Rinder, 1422000 Stück Kleinvieh (Schafe und Ziegen?) und 120000 Gefangene verzeichnet. Differenzen bestehen hinsichtlich der Bewertung des Realitätsgehalts dieser Angaben, da vielen Bearbeitern die Zahlen viel zu hoch erscheinen. ${ }^{570}$ Jedoch weist Wafik GHONEIM in seiner Dissertation über

567 EICHLER (1992: 27), seine Aussage bezieht sich vermutlich auf HELCK (1975b: 104); des weiteren auch REINEKE (1978: 70) und ENDESFELDER (1991: 30 f.); dagegen weist WARBURTON (1995: 109) diese Hypothesen zurück, scheint sich allerdings nur gegen eine generelle Registratur aller Ägypter zu stellen.

568 BUTZER (1976: 86) schätzt die Wachstumsrate im Alten Reich auf höchstens 0,08 \%; zur Heranziehung von Kriegsgefangenen als Arbeitskräfte vgl. GUNDLACH (1994).

569 Zuletzt YurCo (1995); Millet (1990).

570 Millet (1990: 58 Anm. 16) mit Beispielen moderner Viehwirtschaft im Irak oder in Libyen. Diese Vergleiche sind allerdings nicht unproblematisch, da etliche Faktoren dabei außer acht gelassen werden: Umweltbedingungen für Viehhaltung, Ausschließlichkeit von Viehwirtschaft, andere (moderne) Möglichkeiten der 
das Rind im Alten Ägypten darauf hin, daß »die klimatischen Verhältnisse im Alten Reich noch günstiger waren und als Folge davon das Weideland noch größeres Ausmaß in diesem Gebiet besessen hat als zur Zeit Ramses III. im Neuen Reich. « ${ }^{571}$ Doch sprechen auch die Texte des Neuen Reiches davon, daß das Delta bevorzugter Ort der Viehhaltung war. ${ }^{572}$ Dies bedeutet aber auch, daß eine Konzentration von Vieh aller Art und damit entsprechend hohe Stückzahlen im Delta kein Zufall sind, sondern explizit auf die Qualität und den Stellenwert des dort vorhandenen Weidelandes hinweisen.

Im Grab des Jmn-m-ḩ.t in Beni Hassan ist ein Beispiel für Viehverwaltung in Mittelägypten erhalten geblieben. ${ }^{573}$ Auf der Abbildung eines Abrechnungspapyrus sind die Ergebnisse der Zählung des Viehbestandes aus dem Jahr 42 (Sesostris I.) im >Haus der Ewigkeit< und des gesamten 16. oberägyptischen Nomos zu lesen: 3000 Rinder und 30000 Esel. ${ }^{574}$ Im Vergleich zu den Größenordnungen, die aus dem Alten Reich berichtet werden, mutet der von Amenemhet geschilderte Umfang der Tierherden seines Nomos geradezu bescheiden an.

自公

$\left.{ }^{c} \underline{h}^{c} . n \operatorname{rdj} n=j j m j-r\right\} \underline{t z} . w t$ n gs.w-pr.w nw mnjw nw M3-ḥd k’3 3000 m nḥb.w=sn

»Es brachten mir die Vorsteher der Herden in der Verwaltung der Hirten von Ma-hedj 3000 Stiere von ihren Tributherden.«

Bei einer Jahresabgabe von allein 3000 Stieren als Steuerleistung an die königliche Residenz kann ein Gesamtbestand von 3000 Tieren nicht stimmen. Möglich wäre eine Interpretation dahingehend, daß die >Zählung « sich auf die Zusammenstellung einer Jahreslieferung an die Residenz bezieht und nicht den Gesamtbestand an Tieren verzeichnet.

Obwohl hier nicht die Zahlenangaben zum Vieh, sondern zu den Menschen im Mittelpunkt der Betrachtungen stehen sollen, kann die Zahl der gefangenen Menschen nicht unabhängig von der Zahl der Tiere betrachtet werden. Die Größenordnung der Angaben läßt nur den Schluß zu, daß alle Informationen entweder richtig oder falsch sein müssen. Für die

Ernährungssicherung, etc.; offizielle Statistiken nach Zählungen des Jahres 1983 aus Ägypten (IBRAHIM 1996: 111, Tab. 16) zeigen die modernen Dimensionen: 2,87 Mio. Rinder und 5,99 Mio. Stück Kleinvieh, was einem Verhältnis von rund 1:2 entspricht; BOESSNECK (1988: 66) befindet dagegen den Anteil an Eseln im Beispiel des Sahure-Tempels - verglichen zu den archäologischen Befunden - zu hoch; auch GoDRON (1951: 99 Anm. 3) zitiert eine Aussage von DRIOTON und VANDIER: „Comme toujours les nombres sont purement fantaisistes.«, befindet aber selbst: »Il n’y pas davantage impossibilité à ce que le roi ait capturé 120.000 prisonniers«.

571 GHONEIM (1977: 29).

572 Urk. IV 72,15-16:

573 Beni Hassan, Grab Nr. 2.

574 Urk. VII 23; NEWBERRY (1893: Taf. 21). 


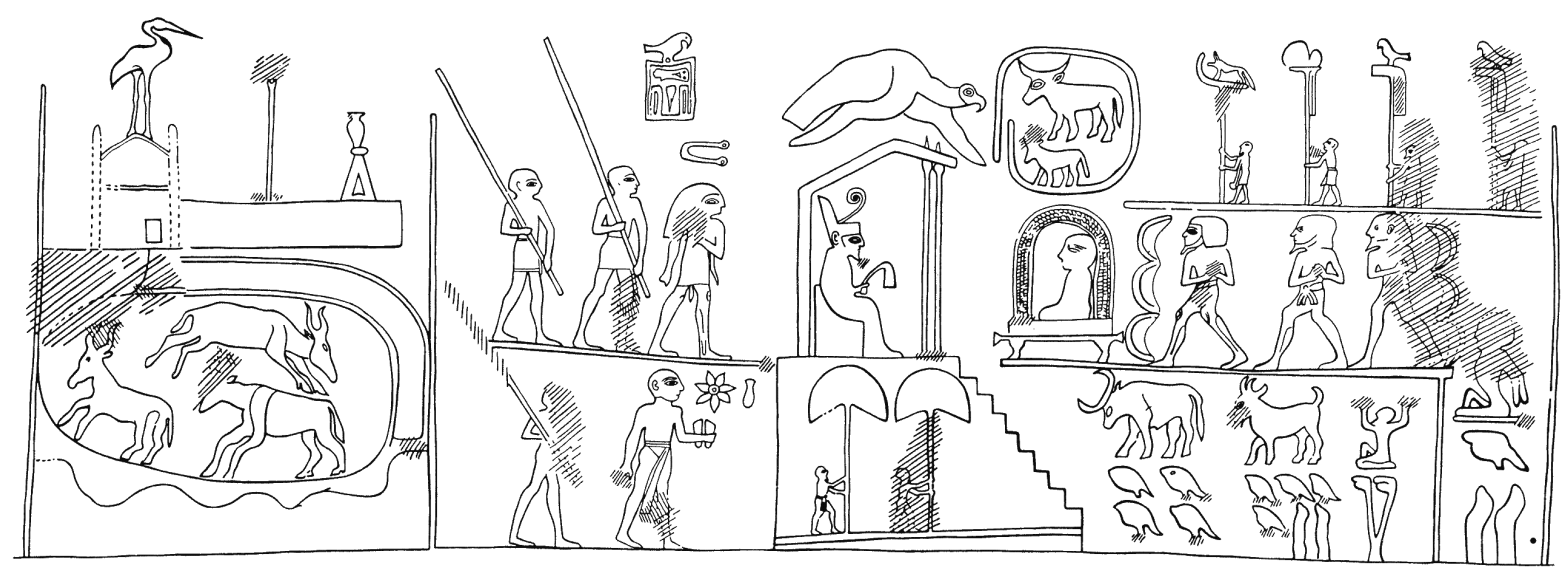

Abbildung 5.2: Der Keulenkopf des Narmer

120000 Gefangenen fehlt jedoch ein geeignetes Bezugssystem, das eine Plausibilitätsprüfung ermöglicht, so daß zunächst gezwungenermaßen die Viehherden in das Zentrum des Interesses rücken müssen. Im folgenden sollen zwei zentrale Fragen näher beleuchtet werden, nämlich einerseits das Verhältnis der Zahlenangaben von Rindern und Kleinvieh zueinander und andererseits die äußeren Bedingungen für die (Un-) Möglichkeit der überlieferten Zahlen. Wenn hierfür akzeptable Antworten gegeben werden können, darf man davon ausgehen, daß auch die Angaben zu den Gefangenen stimmen. Andernfalls müßte man auch diese Zahl zurückweisen, hätte dann aber der Narmerkeule keinerlei Informationen abgerungen.

\section{Literarische Quellen}

In einem ersten Schritt sollen nun parallele Angaben zur Narmerkeule aus der schriftlichen Quellen gegenübergestellt werden. Als Meßsystem dient dazu das zahlenmäßige Verhältnis von Rindern zum sogenannten >Kleinvieh<. Um einen besseren Überblick über die Größenordnung zueinander zu gewinnen, wenn in ägyptischen Texten gleichzeitig Rinder und Kleinvieh aufgelistet werden, sind eine Reihe von Belegen aus verschiedenen Dynastien in Tabelle 5.4 zusammengestellt. Unter der Rubrik >Kleinvieh< wurden nur Schafe und Ziegen gezählt sowie Aufsplittungen in einzelne Rinderarten zu einer einzigen Zahl zusammengefaßt. ${ }^{575}$ Esel und Schweine fanden in den Zusammenstellungen keine Berücksichtigung. Dies war notwendig, da diese beiden Tierarten nicht in allen Beispielen aufgelistet sind und grundsätzlich nicht einmal gesichert ist, ob Esel überhaupt zum Kleinvieh gezählt wurden. Die häufige Existenz einer eigenen Rubrik in den Texten läßt eher darauf schließen, daß eine Trennung von an-

575 Vgl. dazu auch HelcK (1975b: 161). 
deren Tierarten üblich war. ${ }^{576}$ Für Schweine ist die Situation nicht so eindeutig. Ausgehend von ihrer archäologisch nachgewiesenen wirtschaftlichen Bedeutung ${ }^{577}$ ist die seltene explizite Auflistung in den Inschriften ungewöhnlich und kann an dieser Stelle nicht befriedigend erklärt werden. Da die Bezeichnungen bzw. Abbildungen und Determinative in den Listen in der Regel jedoch in ihrer Darstellung von Schaf und Ziege als >Kleinvieh< eindeutig sind, wurde daher die Zusammenstellung auf Rind, Ziege und Schaf reduziert.

Das Verhältnis von Rindern zu Kleinvieh, wie es als Frühzeitbeleg auf der Narmerkeule verzeichnet ist, sticht im Vergleich zu den Quellen des Alten Reiches nicht besonders auffällig heraus und kann zur Stützung der Plausibilität dieser Angaben herangezogen werden. An den Belegen läßt sich ablesen, daß das Verhältnis im Alten Reich ungefähr im Bereich von $1: 3$ lag, während es im Neuen Reich auf etwa 1:10 anstieg, dabei jedoch eine signifikante Streuung aufweist. Sowohl das höhere Verhältnis als auch die Streuung könnten aber darauf zurückzuführen sein, daß der Ursprung der Tiere im Neuen Reich außerhalb des ägyptischen Territoriums lag, da hauptsächlich Auflistungen von Tributleistungen unterworfener Gebiete oder Beutegut eroberter Territorien außerhalb Ägyptens als Quellen vorliegen. Als weitere Gründe für die Streuung ließen sich etwa eine andere Zweckgebundenheit der Tiere anführen, die mehr auf Last und Transport ausgerichtet war. Auch eine Verlagerung der Ernährungsgrundlagen könnte der Grund für die Verschiebungen gewesen sein, die möglicherweise damit einherging, daß keine explizite $>$ Zucht $<$ bestimmter Arten mehr stattfand.

\begin{tabular}{|c|c|c|c|c|c|}
\hline Quelle & Beschreibung & Dyn. & Rinder & Kleinvieh & Ratio \\
\hline QuiBELl (1900: Taf. 26b) & Narmerkeule & 0 & 400000 & 1422000 & $1: 3,5$ \\
\hline \multirow[t]{2}{*}{ LD II,9 } & Gîza, Mastaba des $\mathrm{H}^{\mathrm{c} j}=\mathrm{f}-\mathrm{r}^{\mathrm{c}}-{ }^{\mathrm{C}} \mathrm{nh}$ & 4 & 1055 & 3208 & $1: 3$ \\
\hline & Totentempel Sahure & 5 & 123440 & 476101 & $1: 3,8$ \\
\hline PETRIE (1900: Taf. XI) & Inschrift des Nht & 10 & 33 & 100 & $1: 3$ \\
\hline Urk. IV 75 & Grab des Rnj & 18 & 122 & 1300 & $1: 10,6$ \\
\hline Urk. IV 664 & Eroberung Megiddos & 18 & 1929 & 22500 & $1: 11,7$ \\
\hline Urk. IV 669 & Tribut von Retjenu & 18 & 794 & 5703 & $1: 7,2$ \\
\hline Urk. IV 688 & Feldzug in Djahi, Tribute & 18 & 618 & 3636 & $1: 5,9$ \\
\hline Urk. IV 692 & Tribut von Retjenu & 18 & 276 & 4622 & $1: 16,7$ \\
\hline Urk. IV 699 & Tribut von Retjenu & 18 & 592 & 5323 & $1: 9$ \\
\hline KRI V 54 & $>$ Libysche Beute< Ramses' III. & 20 & 3609 & 28926 & $1: 10,5$ \\
\hline
\end{tabular}

Tabelle 5.4: Vergleich von Listen mit Angaben zu Rindern und Kleinvieh

576 Vgl. etwa die explizite Trennung der königlichen Weiden im Koptos-Dekret C (Urk. I 284,9): Rinderweiden, Eselweiden und Kleinviehweiden.

577 BOESSNECK (1988: 76 ff.). 
Obwohl die Zahlen der Narmerkeule gerundet sind, weisen die präziser gefaßten Angaben aus dem Totentempel des Sahure darauf hin, daß die verzeichneten Zahlen tatsächlich >gezählt< waren und es keinerlei Hinweise darauf gibt, daß die Angaben gezählte Zahlen nur imitieren. Dazu müßte zunächst bewiesen werden, daß das altägyptische Verständnis von Zahlen ähnlich unserem heutigen ist und im Alten Ägypten eine runde Zahl als weniger zuverlässig gegenüber einer $>$ krummen $<$ Zahl galt. Wichtiger als die präzise Information war in vielen Fällen eher die Vermittlung einer Größenordnung. Als weiteren Einwand könnte man auch das Zahlenverhältnis von 1:3 als einen Topos bezeichnen, den geübte Schreiber in Kenntnis der Versorgungsmöglichkeiten von Tieren zu jener Zeit in ihre Texte einbauten und damit frei erfundene Zahlen zusätzlich durch korrekte Proportionen aufwerteten. Die Konsequenz daraus wäre allerdings, bereits die Zahlen der Narmerkeule - zusammen mit allen späteren Angaben - als Topos zu charakterisieren. Mit anderen Worten: Der früheste schriftliche Beleg für das Verhältnis 1:3 müßte zugleich für alle späteren Belege des Alten Reiches der formal maßgebliche sein. Darüber hinaus sind die Verhältnisse trotz ihrer relativen Nähe zueinander immer noch einer gewissen Streuung unterworfen. Und nicht zuletzt gibt es durchaus einzelne Gegenbeispiele, die gar nicht in das hier zugrundegelegte Schema passen wollen. ${ }^{578}$

Zum Schluß soll noch auf die Frage des Kontextes eingegangen werden, die ebenfalls ein wichtiger Beitrag zum Verständnis der Texte ist. Das Ausgangsproblem, das sich dem Bearbeiter der Texte stellt, ist die große Differenz zwischen den Zahlenangaben bei Narmer und Snofru sowie den vergleichsweise geringen Zahlen sowohl der privaten Inschriften als auch der Berichte aus späterer Zeit, vorwiegend des Neuen Reiches. Das Gefälle zwischen über einer Million und einigen tausend Tieren scheint unüberwindbar, und allein diese Tatsache galt häufig genug als Argument für den Vorwurf der Übertreibung. Bei genauerer Betrachtung der kritisierten Quellen stellt man jedoch fest, daß hier im Gegensatz zu den anderen Belegen keine > Vorführung < der Herden stattfand, sondern lediglich eine Liste über die Tiere verfaßt wurde. Schon Rolf GUNDLACH sprach sich dafür aus, diese Zahlen nicht als Kriegsbeute zu betrachten, die erst nach Ägypten überführt werden mußte, sondern als Bestandsaufnahme der Ressourcen des besiegten Landes. ${ }^{579}$

\section{Der archäologische Befund}

Gegenüber den Informationen, die sich den schriftlichen Quellen entnehmen lassen, sind die archäologischen Befunde deutlich problematischer und weniger eindeutig. Die von Joachim

578 Die Angaben für Rinder, Esel und Kleinvieh in der Mastaba des Snb (Gîza, neuerdings in die 4. Dynastie datiert) stehen zum Beispiel durchweg im Verhältnis 1:1; vgl. dazu auch JUNKER (1940: 76).

579 GUNDLACH (1994: 95 ff.). 
BOESSNECK vorgeführten Ergebnisse aus vorgeschichtlicher Zeit ergeben ein uneinheitliches Bild, das zum Teil im diametralen Gegensatz zu den schriftlichen Quellen steht. ${ }^{580}$ Es ist allerdings kaum möglich, aus den archäologischen Befunden exakte Mengenangaben der Tiere herauszulesen. Meist bleiben die Auswertungen auf die >Fundzahlen< (die Anzahl aller Knochenteile einer bestimmten Gattung) und das >Knochengewicht $<$ beschränkt. Nicht immer erlauben es die Funde, auch die >Mindestzahl an Individuen< anzugeben, die für Vergleichszwecke am besten geeignet wäre. Immerhin besteht die Möglichkeit, anhand der Fundzahlen die Zahlenverhältnisse wenigstens in groben Dimensionen zu bestimmen. Den Anforderungen für präzisere Beurteilungen oder gar exakte Verhältnisangaben genügen die Daten jedoch nicht.

\begin{tabular}{|c|c|c|c|}
\hline Quelle & Ort & Rind & Schaf/Ziege \\
\hline VON DER WAY u. a. (1997: 207, Tab. 1) & Buto & ++ & -- \\
\hline WENKE u.a. (1988: 18 f.) & Kōm el-Ḥisn & -- & ++ \\
\hline BOESSNECK und VON DEN DRIESCH (1988: 118, Tab. 1) & Tell Ibrahim Awad & + & - \\
\hline BOESSNECK und VON DEN DRIESCH (1992: 49, Diag. 1) & Tell el-Dab ${ }^{c} a$ & $+/++$ & $+/++$ \\
\hline
\end{tabular}

++: sehr stark vertreten; +: stark vertreten; -: weniger stark vertreten; --: kaum vertreten

Tabelle 5.5: Osteoarchäologische Befunde aus dem Nildelta

Die Widersprüche der archäologischen Befunde zu den schriftlichen Überlieferungen können folgendermaßen zusammengefaßt werden. Zum einen lassen sich die zum Teil doch recht hohen Zahlen an Schafen und Ziegen archäologisch im Delta nicht fassen, zum anderen findet das Schwein, das offenbar als Fleischlieferant in der frühdynastischen Ernährung eine wesentliche Rolle gespielt hat, fast keinen Niederschlag in den Inschriften. Spekulationen darüber, ob dies möglicherweise mit dem sozialen Rang einer Person zusammenhängt, haben dazu keine schlüssige Lösung bieten können. ${ }^{581}$ Für Kōm el-Ḥisn im Westdelta dagegen beschreibt Robert WenKE, daß anhand der Knochenfunde domestizierter Tiere neben den Schweinen auch in annähernd gleichem Verhältnis Schafe und Ziegen gehalten wurden. ${ }^{582}$ Rinderknochen dagegen waren nur in geringer Zahl vorhanden. ${ }^{583}$

580 BOESSNECK (1988: 13 ff.).

581 In einer Diskussionsaufzeichnung von VAN DEN BRINK (1992: 481) wird von Karla KROEPER vermerkt, daß in Minshat Abu Omar Schweineknochen in ärmeren Gräbern gefunden wurden, in reicheren Gräbern dagegen nicht. Dies setzt KROEPER damit in Verbindung, daß in den Gräbern des Alten Reiches auch keine Abbildungen von Schweinen gefunden wurden und vermutet - unausgesprochen - Prestigegründe hinter den Befunden. Ihr Ansatz wurde jedoch vom Plenum sofort zurückgewiesen.

582 WENKE u.a. (1988: 18); siehe auch ausführlicher unten S. 173.

583 Zuletzt nochmals bestätigt von CAGLE (2003: 130 ff.). 
Die von BOESSNECK und VON DEN DRIESCH publizierten Ergebnisse aus den Siedlungsabfällen der vordynastischen Zeit bis in die 1. Dynastie vom Tell Ibrahim Awad im Ostdelta deuten darauf hin, daß dort Ziegen und Schafe zu jener Zeit praktisch keine Rolle gespielt haben. ${ }^{584}$ In ihrer Zusammenfassung geben sie zwar an, daß am Deltarand die Schafhaltung eine große Rolle spielte, im Delta selbst aber erst mit der Kultivierung des Bodens im Verlauf des Alten Reiches auch die notwendigen Bedingungen für eine erfolgreiche Schafhaltung erfüllt waren.

Wenn man die archäologischen Befunde der Frühzeit und des Alten Reiches zusammenfassend betrachtet, muß man feststellen, daß Befundkontexte und Ergebnisse zu disparat sind, als daß man eindeutige Schlußfolgerungen daraus ziehen könnte. Für die bearbeiteten Siedlungen ist in der Regel überhaupt nicht gesichert, welchen Zweck und Umfang die Viehhaltung hatte. Selbst die von WENKE für Kōm el-Ḥisn beschriebenen zwei Modelle der Viehwirtschaft (lokales Zentrum versus Zulieferer der Metropole), die als Arbeitshypothese vor Beginn des Surveys formuliert wurden, konnten nicht zweifelsfrei aus den Funden zugunsten des einen oder des anderen Modells entschieden werden. Es ist demnach gut möglich, daß das Überwiegen einer bestimmten Tierart in den Relikten sich aus dem Export einer anderen Tierart ergibt, die folglich archäologisch nicht greifbar ist. Erschwerend kommt hier die Stichprobenhaftigkeit der Funde hinzu, die üblicherweise auf wenige Schnitte verteilt sind und stratigraphisch getrennt ausgezählt werden. Es bleibt die Frage offen, inwieweit diese begrenzten Datenerhebungen repräsentativ für ein gesamtes Areal sind. Ein weiterer Aspekt ist die Frage nach dem Ursprung der vorgefundenen Knochenreste. Oftmals handelt es sich um Streufunde oder der Kontext der Knochenfunde ist archäologisch nicht sicher geklärt. ${ }^{585}$ Selbst der Hinweis auf eine Abfallgrube läßt immer noch die Frage offen, ob es sich beim Deponieren der Tierabfälle um eine einmalige Situation wie zum Beispiel ein Festmahl oder eine Opferhandlung handelt, oder ob sämtliche in einem bestimmten Kontext gefundenen Tierarten für den täglichen Bedarf der Ernährung zur Verfügung standen.

Bei abschließender Betrachtung wird deutlich, daß sich die bisher aufgearbeiteten einzelnen archäologischen Befunde aus der Frühzeit und dem Alten Reich nicht korrelieren lassen, sondern für jeden Standort isoliert zu betrachten sind. Trotz der Skepsis gegenüber den literarischen Quellen liefern die Tierknochenfunde bisher kein tragfähiges Gegenargument, das der Haltung einer großen Zahl von Kleinvieh zu jener Zeit widerspricht.

584 BOESSNECK und VON DEN DRIESCH (1988).

585 Die Unklarheit der Befunde wird auch von BOESSNECK und VON DEN DRIESCH (1992: 7) eingeräumt: »Ob Versorgungsmitgaben aus Gräbern, Opfer- oder Siedlungsabfälle vorliegen, ist von Fall zu Fall schwer oder auch gar nicht zu entscheiden.« 


\section{Folgerungen für die Zahl der Menschen}

Ausgehend von den Betrachtungen zu den literarischen und archäologischen Befunden ergibt sich für die Frage nach der Authentizität der Angaben von der Narmerkeule ein uneinheitliches Bild. Die zuvor aus den Vergleichen unterschiedlicher literarischer Quellen gewonnenen positiven Ergebnisse zur Größe der Tierherden ermöglichen es jedoch, die Zahl von 120000 Menschen als >wahrscheinlich $<$ zu klassifizieren. Es bleibt nun zu zeigen, wer die gezählten Personen waren. In der Vergangenheit wurde die Darstellung mehrfach als Wiedergabe einer Art von Zensus beschrieben. ${ }^{586}$ Die Art der Darstellung als Gefangene ist in diesem Fall aufgrund der Wahl des Determinativs eindeutig und vordergründig scheint die Interpretation als > Vorführung von Gefangenen $<$ zu überwiegen. ${ }^{587}$ Für eine weitergehende Beurteilung bleibt zu klären, wie mit diesen Gefangenen verfahren wurde, oder ob es sich überhaupt um (Kriegs-) Gefangene im modernen Sinne handelt. Die Szenarien, die in jüngerer Zeit zur sogenannten >Reichseinigung < beschrieben wurden, weisen nicht mehr auf militärische Maßnahmen im großen Umfang zwecks Eroberung Unterägyptens hin. ${ }^{588}$ Es muß deshalb davon ausgegangen werden, daß die Interpretation der 120000 Personen als eine militärische Größe nicht zutrifft. Dies schließt im Einzelfall kriegerische Auseinandersetzungen nicht aus, die Tod und Zerstörung über bestimmte Gebiete brachten. Die Reihen der toten Feinde mit abgeschlagenen Köpfen, die auf der Narmerpalette abgebildet sind, sprechen eine eindeutige Sprache. Als Kontrast lassen sich die Expeditionen des Alten Reiches anführen, die ausdrücklich eine militärische Streitmacht gegen Rebellen aufstellen. In der Biographie des Wnj ist von > vielen Zehntausenden< die Rede, doch wird die Zahl der Narmerkeule von Wnj's Heer längst nicht erreicht. Ein >Heer < in der Größe von über Hundertausend steht im Widerspruch zu den bisherigen Kenntnissen über die militärische Organisation des Alten Reiches. Wahrscheinlicher ist die Erfassung aller Bewohner der Region, die mittels des Determinativs als sbj.w (Rebellen) bezeichnet werden, wobei keine Unterscheidung zwischen Männern und Frauen getroffen wird. Die Narmerkeule beschreibt also die Räumung einer ganzen Region an Menschen und Tieren. Dies könnte als Endpunkt einer Zeit der allmählichen Einnahme oder Assimilation der Bevölkerung aufgefaßt werden. Insofern ließe sich tatsächlich von einer Form des Zensus sprechen, da hier versucht wurde, eine vollständige Bestandsaufnahme durchzuführen.

Was darüber hinaus mit den Menschen geschah, läßt sich indes nur schwer einschätzen. Wie bereits GUNDLACH richtig erkannte, dürfte eine Form der Zwangsumsiedlung in andere

586 MiLLET (1990); SCHOTT (1950: 24).

587 YuRCo (1995); GundlaCh (1994: 33); Husson und VAlbelle (1992: 109); HelCK (1987: 99).

588 KÖHLER (1995: 86 f.); VON DER WAY (1991: 423). 
Gebiete Ägyptens allein aus technischen Gründen entfallen. ${ }^{589}$ Der logistische Aufwand für Bewachung und Versorgung eines derart großen Trosses von Menschen wäre unverhältnismäBig hoch. ${ }^{590}$ Über Verbleib und Schicksal der 120000 Unterlegenen können keine verläßlichen Aussagen getroffen werden, jedoch erscheint eine Verschleppung der besiegten Bevölkerung an einen anderen Ort kaum sinnvoll, da gerade die Besiedlung und Bewirtschaftung dieser Region später wieder in das Zentrum des Interesses ägyptischer Könige rückte.

\subsubsection{Die Inschrift des Dewen auf dem Annalenstein}

Als frühester Beleg für eine Zählung von Menschen wird immer wieder ein Eintrag auf dem sogenannten Annalenstein zitiert, einem Fragment einer Dioritplatte, auf dem in einer gleichmäßigen Rastereinteilung die Könige der 1. bis 5. Dynastie das wichtigste Ereignis in jedem ihrer Regierungsjahre sowie die Höhe der jährlichen Nilüberschwemmung verzeichnet waren. ${ }^{591}$

Für das Jahr $\mathrm{x}+4$ des Königs Dewen, ${ }^{592}$ des fünften Herrschers der 1. Dynastie, ist auf diesem Stein ein Ereignis vermerkt (Abb. 5.3), das trotz grammatikalischer und inhaltlicher Probleme in der Vergangenheit als Textquelle für eine Zählung zitiert wurde. Als einem der frühesten Bearbeiter des Anna-

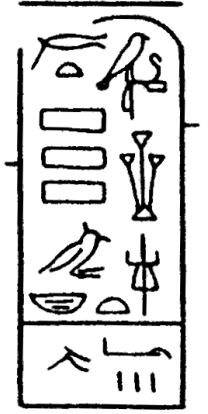

Abb. 5.3 lensteins blieb SCHÄFER der Sinn der Stelle noch völlig unklar. Zu einer Ergänzung im Sinne von >Zählung< konnte er sich noch nicht durchringen: »Die Übersetzung ist ganz fraglich. Es fehlt ein Verbum, oder steckt das in $\mathbb{P} ?$ ? 593 James BREASTED war in seiner Analyse mutiger und übersetzte die Textpassage als »(Numbering of) all people of the nomes of the west, north, and east « und erhob damit den Annaleneintrag in den Rang einer Zählung von Menschen. ${ }^{594}$ Die Vermutung liegt nahe, daß erst BREASTEDs Lesart in der Folgezeit für das häufige Zitieren des Annalensteins - besonders außerhalb der ägyptologischen Fachliteratur - als Quelle der ersten nachweisbaren >Volkszählung< verantwortlich war. ${ }^{595}$ Es bleibt allerdings unklar,

589 GUNDLACH (1994: 33).

590 Obgleich anzumerken ist, daß der logistische Kraftakt einer reibungslosen Versorgung bei den Expeditionen des Mittleren Reiches offenbar gelang - falls die in den Expeditionsinschriften berichteten hohen Teilnehmerzahlen (vgl. Tab. 5.1) realistisch sein sollten. Der Unterschied lag eher in der fehlenden Notwendigkeit einer militärischen Bewachung der Menschen.

591 Das größte Fragment dieser Platte befindet sich heute im Museum von Palermo, daher findet man auch stellenweise die Bezeichnung >Palermostein<; zuletzt ausführlich bearbeitet von WILKINSON (2000).

592 Zur Datierung vgl. WiLKInSON (2000: 103 ff.); HELCK (1987: 156 f.).

593 SCHÄFER (1902: 19).

594 BREASTED (1906: §106).

595 Hombert und PrÉaux (1952: 41 f.); Hopkins (1980: 313); dagegen VAlbelle (1987: 36) mit Vorbehalten. Beispiele für fachfremde Rezeption finden sich z. B. bei DUPÂQUIER und DUPÂQUIER (1985: 28) 
womit BREASTED seine Ergänzung begründet. Seine Anmerkung zur Textklammer läßt zwar vermuten, daß er sich bei der Zählung auf das Pflanzenzeichen bezog, doch liefert dieser Umstand keine Erklärung für die Klammerung, da das Zeichen auf dem Annalenstein klar erkennbar ist. Sollte er tatsächlich von der Lesung der Pflanze als ḩ ausgegangen sein und daraus das Wort $h 3 w »$ Zuwachs, Vermehrung «, aber auch »Überschuß, Reichtum« sowie schließlich mit Genitiv »Zuwachs, Überschuß an Ernteerträgen, Steuern, etc. « gelesen haben, ${ }^{596}$ wäre seine Klammerung überflüssig. Noch weniger plausibel scheint BREASTEDs Vermutung einer irrtümlichen >Fehlschreibung<der Pflanze anstelle des Zeichens für Süden ( $\Psi^{\mathbb{P}}>\frac{7}{\ddagger}$ ). Damit hätten zwar in der Inschrift alle vier Himmelsrichtungen Erwähnung gefunden, jedoch ohne Angabe eines Ereignisses.

Ganz anders deutet HELCK das besagte Jahresereignis. ${ }^{597}$ Ohne auf die bisherige Interpretation als >Zensus< näher einzugehen, liest er den Jahreseintrag als »Planen(?) und Graben(!) der westlichen und östlichen Kanäle (durch) alle rhjt«. Obwohl diese Übersetzung recht gut in den Kontext paßt, da das Anlegen eines Kanals im Sinne des Annalensteins durchaus ein Ereignis ist, das schriftlich festgehalten zu werden verdient, kommt auch HELCK nicht ohne einige Kunstgriffe bei der Interpretation von Zeichen und Zeichenfolge aus. So muß er gleich zweimal Zeichen umdeuten und damit andere Lesungen voraussetzen, nämlich $\bar{\gamma}(m h)>$ $(\check{s} d j)$ und $\square(\breve{s})>\Longleftarrow(m r)$. Erst diese Anpassungen erlauben es ihm, vom >Graben $<$ eines Kanals zu sprechen und eine Parallele aus dem Alten Reich im Grab des Nhb heranzuziehen, der in seiner Biographie den Bau eines Kanals beschreibt, den er für seine Majestät vorbereitet hat. ${ }^{598}$ Seine Übersetzung setzt zudem inhaltlich voraus, daß die rh.yt befriedet oder unterworfen sind, was allerdings für die Regierungszeit des Königs Dewen in der 1. Dynastie vermutet werden darf, da er - wie ebenfalls dem Annalenstein zu entnehmen ist - zahlreiche erfolgreiche Feldzüge durchführte ${ }^{599}$ und allem Anschein nach als Erster den neuen Königstitel $n z w$-bj.tj >König von Ober- und Unterägypten< trug. ${ }^{600}$

Auch inhaltlich kann ein starkes Argument für den Übersetzungsvorschlag von HELCK angeführt werden. Die Darstellungen auf der Prunkkeule des vordynastischen Königs Skorpion zeigen das Anlegen von Bewässerungssystemen oder Kanälen durch den König und andere

und KELLEY u.a. (1982: 34), die beide von ägyptischen Volkszählungen zu Beginn des 3. Jahrtausends v. Chr. ausgehen.

596 Wb III,16; vgl. zu der Kombination ḩ3t-jht als Steuerbegriff auch KAPLONY (1963: 1002 f.).

597 HELCK (1987: 157); akzeptiert von GUNDLACH (1994: 50 f.).

598 DUNHAM (1938: 2 Anm. 13).

599 HELCK (1987: 156 ff.).

600 GOdRON (1990: 179 ff.). 
Personen. ${ }^{601}$ Bereits in der Vergangenheit wurde immer wieder betont, daß die Fortschritte in der Bewässerungstechnik ein entscheidender Faktor für die Ägypter auf dem Weg zur hydraulischen Gesellschaft waren. ${ }^{602}$ Obgleich Wolfgang SCHENKEL unschlüssig über den genauen Zweck der auf der Keule des König Skorpion dargestellten Szene ist und neben einem Bewässerungskanal auch einen Schiffahrtskanal oder einen Schutzbau wie zum Beispiel einen Deich oder eine Drainage in Erwägung zieht, bleibt im Vergleich zu HELCKs Interpretation des Annalentextes die Übereinstimmung im Hinblick auf das Anlegen eines Kanals, welchen Zweck dieser Kanal auch immer erfüllt haben mag.

Der jüngste Versuch einer Übersetzung wurde kürzlich von Toby WILKINSON vorgelegt, der trotz teilweise anderer Auffassungen eine zu HELCK recht ähnliche Übersetzung anbot: »organising? the agricultural holdings? of the north-west(ern Delta) and all the people of the east(ern Delta) « ${ }^{603}$ WILKINSONs Argumente gegen die bisherigen Übersetzungsvorschläge beruhten im wesentlichen auf der Problematik der Reihenfolge der Zeichenlesungen. Um zu vermeintlich besseren Lesungen zu gelangen, ließen frühere Bearbeiter in der Regel Sprünge in der Zeichenabfolge zu, was WILKINSON ausdrücklich zurückwies. Seiner Meinung nach muß - wie bei den anderen Feldern des Annalensteins auch - von einer strikten Lesung in Zeilen jeweils von rechts nach links ausgegangen werden. Auch wenn WILKINSON selbst einräumt, daß die Textstelle schwierig bleibt und sein Übersetzungsvorschlag noch immer keine befriedigende Lösung darstellt, dürfte spätestens mit den Vorschlägen von HELCK und WILKINSON die Hypothese eines Zensus philologisch nicht mehr zu halten sein.

\section{Weitere Gegenargumente}

Der Annaleneintrag erwähnt am unteren Rand die rh.yt nb.t, also eine besiegte Volksgruppe - oder Teile davon - aus dem Westdelta. ${ }^{604}$ Aus einer deskriptiven Sicht von Süden wäre die Nennung dieser vierten Himmelsrichtung tatsächlich unnötig. Darüber hinaus bleibt in diesem Fall das Problem des fehlenden Verbums. Eine Ereignisangabe >Das rh.yt-Volk der Gaue aller vier Himmelsrichtungen < klingt wenig plausibel. Auch fehlt jeglicher Anhaltspunkt, daß sich dieses Ereignis mit dem sonst vom Annalenstein bekannten x.ten Mal der Zählung in

601 Quibell (1900: Taf. 26c).

602 SCHENKEL (1978: 28); BUTZER (1976); vgl. auch oben Kap. 1.4.1.1.

603 WILKINSON (2000: $108 \mathrm{ff}$.).

604 Die Interpretation der $r h . y t$ folgt hier der Argumentation von Claude VANDERSLEYEN (1989), der die umstrittene alte Bezeichnung >Bevölkerung Unterägyptens< geographisch präzisierte. Zur älteren Diskussion vgl. HELCK (1959) und GARDINER (1947: 104 f.). Zur Lokalisierung im Westdelta könnte auch eine Beobachtung von KAPLONY (1968: 41) passen, der vermutet, daß König Dewen besiegte Asiaten in das Westdelta umgesiedelt hat. 
Verbindung bringen läßt. Es kann vielmehr gut begründet werden, daß die rh.yt nicht im Zusammenhang mit den anderweitig bekannten gezählten Objekten stehen. Indiz dafür ist ein weiteres Fragment des Annalensteins, das sich heute im Petrie-Museum befindet. ${ }^{605}$ HELCK liest den teilweise stark zerstörten Bereich: ${ }^{606}$

Jahr 4: . . ; 1. Mal Zählung des Goldes

Jahr 5: Horusgeleit; 2. Mal der Zählung

Jahr 6: ...; .. der rhjt

Jahr 7: [Horusgeleit]; 3. Mal der Zählung

Die Angaben >x. Mal der Zählung< sind in diesem Fall lückenlos dokumentiert. Die rh.yt erscheinen jedoch nicht in Verbindung mit einer Zählung, so daß es mit der Ereignisangabe eine andere Bewandtnis haben muß. Die Zurückhaltung Harry STEWARTs bezüglich der Lesung als dritte Zählung scheint in Anbetracht seiner eigenen Umzeichnung unangebracht. ${ }^{607}$ Auch sind die Zeichen für die rh.yt relativ klar zu identifizieren, wenngleich sie in diesem Fall dreifach gesetzt sind und die normale Form anstelle der >klassischen $<$ geduckten

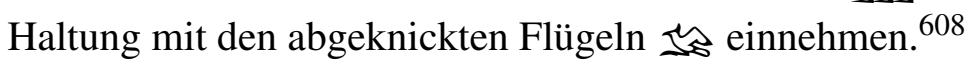

Es bleibt zum Schluß nur die Feststellung, daß der hier besprochene Eintrag des Annalensteins nicht mehr weiter als Beleg für die früheste Zählung von Menschen dienen kann. Weder lassen sich die rh.yt mit den sonst erwähnten Zählungen in irgendeinen Zusammenhang bringen, noch liefert die fragliche Textstelle selbst Evidenz für eine Zählung. Eine großangelegte unterägyptische Baumaßnahme, durchgeführt von >Einheimischen<, wie HELCK und WILKINSON als Übersetzung vorschlagen, scheint derzeit immer noch die plausibelste Erklärung für das beschriebene Ereignis zu sein. Aufgrund der hier vorgeführten starken Gegenargumente und der Tatsache, daß ein singulärer Übersetzungsvorschlag es zu einer gewissen Prominenz in der Forschung zur ägyptischen Demographie gebracht hat, der allerdings weder sprachlich noch inhaltlich abgesichert werden kann, dürfte der Hinweis auf den ältesten Nachweis eines Zensus damit hinfällig sein. Der Eintrag auf dem Annalenstein läßt sich dahingehend keinesfalls interpretieren.

605 Wilkinson (2000: 248 ff.); Helck (1987: 166); STEWART (1979: 6, Taf. 3,1).

606 Die Lesung stimmt im wesentlichen mit der von WILKINSON (2000: ebenda) überein.

607 STEWART (1979: 6).

608 Mit diesem Vergleich dürfte auch die Erklärung von Jacques PIRENNE verworfen sein (zitiert bei HOMBERT und PRÉAUX 1952: 41 Anm. 10), der eine lautliche Nähe zu rh.t $>$ Liste, Inventar< erkennen will, dabei allerdings das Aufzulistende wegfallen läßt. Ein Wortspiel annehmen zu dürfen, ist in diesem Fall wenig wahrscheinlich. 


\subsubsection{Die toten Feinde des Chasechemui}

Als es gegen Ende der 2. Dynastie (ca. 2700 v. Chr.) erneut zu Auseinandersetzungen zwischen Ober- und Unterägypten kam, trat ein oberägyptischer König namens Chasechemui an, die Einheit des Staates erneut herzustellen. ${ }^{609}$ Im Norden Ägyptens hatten sich offenbar eine Reihe von Herrschern an die Macht bringen können, die aus späten Königslisten in verderbter Namensform überliefert sind. ${ }^{610}$ Diese Aufstände galt es für Chasechemui in einem Feldzug zu beseitigen. Vom Sieg gegen den Norden, der allem Anschein nach blutig errungen wurde, zeugen neben Inschriften auf Steingefäßen ${ }^{611}(h\langle w j\rangle m h . w$, »Schlagen des Nordlandes «) zwei Statuetten, die den König die weiße Krone tragend und auf einem Thron sitzend darstellen. Auch der spätere Namenszusatz im Horusnamen $h t p-n b . w j-j m=f>$ Die beiden Herren (Horus und Seth) sind in ihm befriedet< zeugt vom Erfolg des Feldzuges. ${ }^{612}$

Beide Statuetten sind von James QUIBELL in Hierakonpolis gefunden und publiziert worden. ${ }^{613}$ Die erste Statuette besteht aus grünem Schiefer, hat eine Höhe von $56,5 \mathrm{~cm}$ und ist weitestgehend vollständig erhalten. Allerdings ist die rechte Hälfte des Kopfes weggebrochen, dazu weist die Figur etliche Bestoßungen und abgebrochene Kanten auf. ${ }^{614}$ Sie befindet sich heute im Ägyptischen Museum in Kairo. ${ }^{615}$ Die zweite Statuette wurde aus Kalkstein gefertigt und weist starke Beschädigungen auf. Das Oberteil einschließlich Brust ist vollständig weggebrochen, dazu Teile des rechten Beins sowie beide Hände. Dieses Objekt wird heute im Ashmolean Museum in Oxford aufbewahrt. ${ }^{616}$ Der Kopf der Statuette konnte separat geborgen werden.

Die Statuetten weisen jeweils auf der Sockelbasis eine im Wesentlichen übereinstimmende Dekoration auf. Auf allen vier Seiten sind tote Rebellen abgebildet, deren Identifikation als solche durch den jeweils auf der Frontseite angebrachten Vermerk sbj zusammen mit einer Zahlenangabe als gesichert gelten darf. ${ }^{617}$ Die Vermutung SCHNEIDERs, daß die Zahl einen Bevölkerungszensus wiedergibt, ${ }^{618}$ ist in Anbetracht der eindeutigen Bildmotive Gefallener

\footnotetext{
609 Allgemein dazu: HofFMAN (1979: 348 ff.); HelCK (1987: 106 f.).

610 VON BECKERATH (1997: 171 f.).

611 QuiBELl (1900: Taf. 36-38).

612 VON BECKERATH (1997: 171 f.).

613 QuiBell (1900: 11, Taf. 39-41).

614 Ein Eckstück der Basis konnte erst kürzlich aus der Sammlung Liverpool zugeordnet werden; siehe dazu ADAMS (1990).

615 Inv. Nr. JE 32161.

616 Inv. Nr. E 517.

$617 \mathrm{Zu}$ sbj vgl. KAHL (1994: 859).

618 SCHNEIDER (1997: 99).
} 
und der dazugehörigen Bezeichnung auszuschließen. Parallelen in der Art der Darstellung finden sich - mit eindeutigem Kontext - in allen ägyptischen Epochen, angefangen von den Gefallenen auf der Narmer-Palette bis hin zum meroitischen >Siegesdenkmal< des Sorakarora. Obwohl sich beide Statuetten in der Dekoration der Sockelbasis recht ähnlich sind, weisen beide eine deutlich unterschiedliche Qualität auf, die sich insbesondere an den Sockelritzungen bemerkbar macht. Auf dem Oxforder Exemplar sind die Konturen der menschlichen Körper prägnanter und die Zahlen auf der Frontseite besser lesbar als beim Kairener Gegenstück. Die Hieroglyphe $\int$ ist bei der Oxforder Statuette oberflächlich abgeplatzt, kann aber aus der anderen Statuette erschlossen werden. Die Qualität der Ausführung könnte zum Teil auch mit der Beschaffenheit des Werkmaterials zusammenhängen.

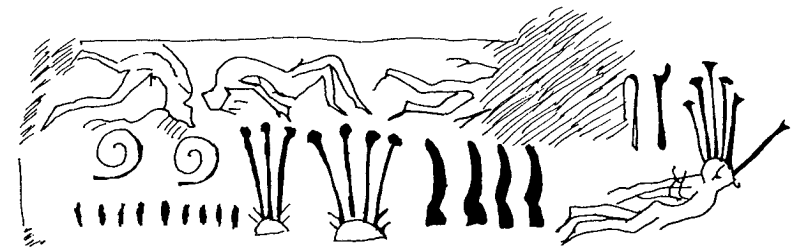

(a) Sockel der Statuette Oxford

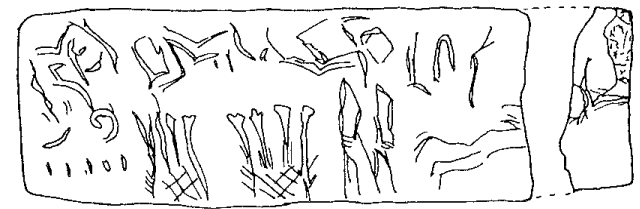

(b) Sockel der Statuette Kairo

Abbildung 5.4: Abbildungen auf den Sockeln der Chasechemui-Statuetten

Nach übereinstimmender Aussage bisheriger Betrachter ist die Zahlenangabe der (getöteten) Rebellen auf beiden Statuetten übereinstimmend $47209 .{ }^{619}$ Die Faszination dieser Zahl liegt in ihrer vermeintlichen oder tatsächlichen Genauigkeit. Während die übrigen bekannten Überlieferungen der Frühzeit eher unserer Vorstellung nach gerundeten Zahlenwerten entsprechen, findet sich hier eine bis auf die letzte Ziffernstelle präzise Information. Dennoch hat man

619 Die Eindeutigkeit dieser Übereinstimmung läßt sich allerdings weder anhand der von JUNKER (1955: Taf. 12) publizierten Fotos der Sockelbasen noch anhand der Umzeichnungen (zuletzt ADAMS 1990), die notwendigerweise gleichzeitig Interpretationen darstellen, nachvollziehen. Während die gängige Lesung 47209 auf der Statuette Oxford recht eindeutig bestätigt werden kann, weist die eingeritzte Zahl auf der Statuette Kairo weitaus mehr Unklarheiten auf. Von den zu erwartenden neun >Einer<-Strichen sind dort nur fünf zu erkennen und erscheinen demzufolge auch nicht auf den Umzeichnungen. Die >Tausender $<$ sind durch Kratzer und andere Einwirkungen undeutlich. Neben der bisher vertretenen $>$ sieben $<$ könnte dort auch eine $>$ acht $<$ stehen. Der zweite >Hunderter $<$ ist kaum erkennbar, läßt sich aber durch Reste einer Schlinge (९) rekonstruieren. Alle Einzelbeobachtungen zusammengenommen ließe sich die Zahl auf der Statuette Kairo auch als 48205 lesen. Gründe dafür könnten unterschiedliche Vorlagen, fehlerhafte Übermittlung an die Handwerker oder schlicht ein >Schreibfehler $<$ sein. Auch ein Abrieb der Statuenoberfläche ist nicht auszuschließen. Bei einer Differenz von nur knapp 1000 zwischen beiden Zahlenangaben kann zwar noch nicht von willkürlichen Zahlen gesprochen werden, doch es bleiben Zweifel. Da der Sachverhalt nicht am Original überprüft werden konnte, steht eine endgültige Klärung noch aus. 
wiederholt die Zahl als >übertrieben< bezeichnet. ${ }^{620}$ Tatsächlich ist die Beurteilung des Informationsgehaltes recht schwierig, da man einerseits keine genaue Kenntnis über die Gesamtgröße der Bevölkerung jener Zeit hat und damit die Dramatik der genannten Größenordnung kaum zu beurteilen ist, ${ }^{621}$ andererseits nicht einmal gesichert ist, ob die erschlagenen Feinde nur bewaffnete Rebellen im engeneren Sinne sind oder sich Männer, Frauen und Kinder gleichermaßen unter den Opfern befanden. Eine geschlossene Rebellenarmee allein scheint in Anbetracht der recht hohen Zahl unwahrscheinlich, jedoch nicht unmöglich. ${ }^{622}$

\subsubsection{Die Kriegsgefangenen des Snofru}

Konkreter als der Beleg für die früheste Menschenzählung scheint eine andere Stelle des Annalensteins zu sein, die einen Feldzug des Königs Snofru (4. Dynastie) beschreibt und die Beute dieses Unternehmens auflistet. Für die Regierungsjahre 14 und 15 ist dort vermerkt: ${ }^{623}$

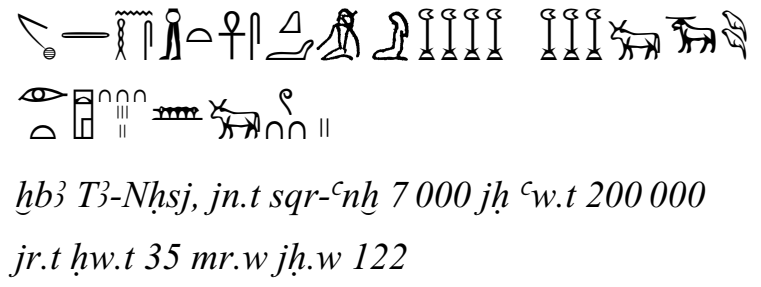

»(Jahr 14) Zerhacken des Nubier-Landes; Herbeibringen von 7000 Gefangenen und 200000 Stück Rindern \& Kleinvieh ${ }^{624}$

(Jahr 15) Gründung von 35 Domänen und 122 Weiden«

GUNDLACH mag bei der unmittelbaren Abfolge von Kriegszug und Siedlungsgründungen nicht an einen Zufall glauben und zieht beide Ereignisse zu einem einzigen zusammen: Die durch den Feldzug >erbeuteten< Menschen und Tiere werden auf ägyptischem Territorium in eigens eingerichteten Dörfern wieder angesiedelt. Wenn man die Personen und Tiere statistisch gleichmäßig auf die Siedlungen und Weiden aufteilt, so ergibt dies eine durchschnittliche Bewohnerzahl von 200 Menschen und rund 1600 Tieren pro Siedlung. ${ }^{625}$

620 OTTO (1953: 51); SCHNEIDER (1997: 99).

621 Die von GUNDLACH (1994: 64) getroffene Aussage einer »teilweise[n] Entvölkerung « ist nicht belegbar.

622 So wird zwar in der Biographie des Wnj (siehe S. 129) ebenfalls von einem Heer gesprochen, das aus > vielen Zehntausenden< bestand, doch muß man sich vergegenwärtigen, daß das dort aufgestellte Heer aus unterschiedlichen Gruppen verschiedenster regionaler Herkunft zusammengezogen wurde.

623 Urk. I 236; WiLKINSON (2000: 141 ff.); GUNDLACH (1994: 71 f.).

624 WiLKINSON (2000: ebenda) liest aus unbekannten Gründen trotz eindeutigen Determinativs »200,000 sheep and goats «.

625 Gundlach (1994: 89). 


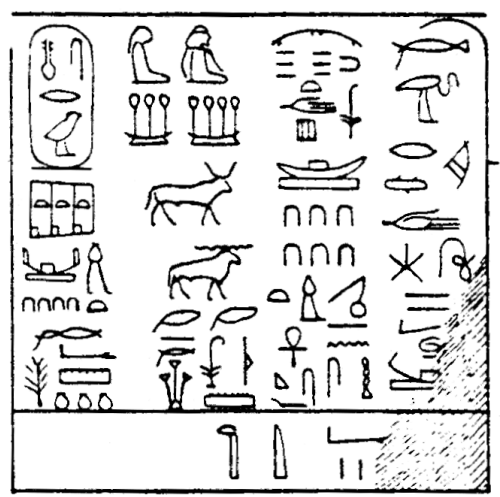

(a) Der Feldzug

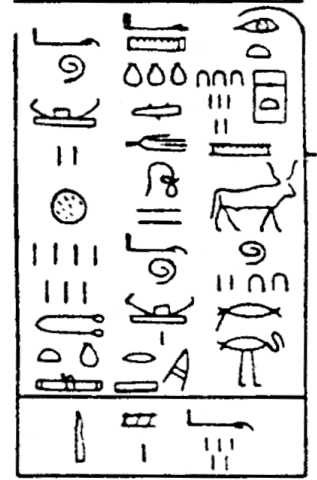

(b) Die Gründungen

Abbildung 5.5: Die Jahre 14 und 15 des Königs Snofru auf dem Annalenstein

Es bleibt allerdings die Frage offen, warum für eine Umsiedlungsmaßnahme ein derartiger Aufwand getrieben wurde und wie die Durchführung eines solchen Unternehmens technisch und logistisch geleistet wurde. Wenn man der Argumentation GUNDLACHs folgt, lag das Ziel der Umsiedlung im 13. und 14. unterägyptischen Nomos, also am Ostrand des Deltas. ${ }^{626} \mathrm{Da}$ die in den Annalen erwähnten Gefangenen aus den Gebieten südlich Ägyptens stammten, ${ }^{627}$ mußte folglich der gesamte Troß entlang des Nils durch Ägypten geführt werden, bis sie im Delta angesiedelt werden konnten. Diese Strapaze dürfte weder ohne logistische Kraftakte für Bewachung und Versorgung noch ohne Verluste an Mensch und Tier geblieben sein, so daß insgesamt der Sinn dieser Aktion fragwürdig bleibt. Der Hinweis GundLACHs, daß die Niederwerfung der Rebellen unter Chasechemui und die hohe Zahl von Toten in Unterägypten keine hundert Jahre zurücklag, weshalb bestimmte Regionen des Deltas quasi entvölkert waren und neu besiedelt werden sollten, ${ }^{628}$ klingt zwar plausibel, läßt sich jedoch mit den historischen Quellen nicht genügend in Einklang bringen. ${ }^{629}$ Da einerseits die Annaleninschrift selbst keine Auskunft über den Ort der Domänengründungen gibt und auch GUNDLACH aus den Inschriften im Taltempel des Snofru in Dahschur Güter sowohl in Ober- als auch in Un-

626 GUNDLACH (1994: 83, 87); in ähnlicher Weise äußert sich LEHNER (1997: 228).

627 Nach Zibelius (1972: 142) war das Toponym T3-Nhsj nur ein vager Ausdruck für ein Land südlich von Ägypten.

628 GUNDLACH (1994: 87) sowie oben Kap. 5.2.3.3.

629 Vgl. etwa die Bemerkungen von EYRE (1987: 37), der zwar die im großen Stil durchgeführten Zwangsumsiedlungen des Alten Reiches in das Delta bestätigt, als Begründung jedoch anmerkt, daß es einen erhöhten Versorgungsbedarf für eine ständig wachsende Klasse an Beamten und Angehörigen höherer Schichten gab. 
terägypten zitiert, ${ }^{630}$ ist die Wahrscheinlichkeit einer kürzeren Wegstrecke von Unternubien nach Oberägypten höher, wofür auch die Siedlungspolitik des Alten Reiches mit ihrem Schwerpunkt auf Oberägypten spricht. ${ }^{631}$

Die sich rein rechnerisch ergebende Größe für die Siedlungsgründung macht durchaus Sinn: Von der Siedlungsstruktur in viele kleine Ortschaften zergliedert, entspricht eine Ortschaft von 200 Personen und 1600 Tieren den ökonomischen Möglichkeiten. Die Spannweite der Größenverhältnisse solcher Ansiedlungen ist zwar weit gestreut, doch finden sich genügend Hinweise auf die Existenz von Kleinstgehöften. Ausgehend von der Prämisse, daß das prinzipielle Vorhandensein von Siedlungen aller Größen, von einer Gehöftgruppe bis zur Metropole, in pharaonischer wie in griechisch-römischer Zeit identisch ist (wobei die anteiligen Zahlenverhältnisse zur Gesamtzahl aller Ansiedlungen hier außer Acht gelassen werden), kann man hierfür Vergleiche aus den römischen Zensusauswertungen ziehen. ${ }^{632}$ Auch der Zweck dieser Maßnahme paßt in den historischen und ökonomischen Kontext. GUNDLACH hebt hervor, daß die Zwangsumsiedlungen im gleichen Zeitraum lagen, in dem die Pyramiden mit ihren Pyramidenbezirken errichtet wurden. ${ }^{633}$ Für letztere war unbedingt eine wirtschaftliche Basis in unmittelbarer räumlicher Nähe vonnöten, um entsprechende Zulieferungen landwirtschaftlicher Güter für den Kultbetrieb zu ermöglichen. Die Ansiedlungen entsprechen dann den sogenannten >Domänen<, also Gütern mit einem bestimmten gebundenen ökonomischen Zweck. Für das Delta gibt es bereits vereinzelt tiefere Kenntnisse über solche Siedlungen, zum Beispiel Kōm el-Ḥisn, das Mitte der 80er Jahre von WEnKE mittels Surveys und Sondagen untersucht wurde. ${ }^{634}$ Zwei Modelle waren zuvor entwickelt worden, um die Funktion einer solchen Siedlung zu beschreiben. Entweder fungierte Kōm el-Ḥisn als Zuchtstation für die Zulieferung von Vieh als Opfertiere an die Tempel von Memphis, oder es handelte sich um eine eher autarke Siedlung, die alle notwendigen Güter des täglichen Bedarfs entweder selbst produzierte oder durch regen Kontakt mit anderen Teilen Ägyptens erwarb. ${ }^{635}$ Obwohl WENKE die Ergebnisse seiner Untersuchungen zunächst offenlassen mußte, da er sich anhand

630 GundLACH (1994: 74); das Verhältnis von ober- zu unterägyptischen Gütern von 54:70 liegt zu dicht beieinander und kann daher nicht als Begründung für einen Schwerpunkt der Siedlungspolitik im Delta dienen.

631 Vgl. dazu oben Kap. 3.2.

632 ALSTON (2001: 162) vermutet für die kleinsten Siedlungen eine Bevölkerung von »under fifty«; BAGNALL und FRIER (1994: 55 f.) setzen als Untergrenze eine Zahl von 100 Personen an; für das moderne Ägypten zitiert HASSAN (1981: 52) Vergleichszahlen und schätzt kleine Dörfer auf eine Größe von wenigen Hundert bis 1000 Menschen.

633 Gundlach (1994: 88 f.).

634 WENKE (1986); WENKE u. a. (1988).

635 WeNkE u. a. (1988: 11). 
seiner Befunde nicht in der Lage sah, eine Entscheidung zugunsten eines der beiden Modelle zu fällen, gab es dennoch interessante Aspekte hinsichtlich der Struktur und Ökonomie einer Siedlung im Delta, auch wenn Kōm el-Hisn aufgrund der Vorläufigkeit von WENKEs Untersuchungsergebnissen noch nicht als Paradigma einer altägyptischen Siedlung gelten darf. ${ }^{636}$

Der Unsicherheitsfaktor hinsichtlich tiefergehender Aussagen zu den erwähnten Gefangenen wird hier besonders deutlich. Es gibt keinerlei Vorstellung davon, wer genau unter die Gefangenen gerechnet wurde, ob etwa Frauen, Kinder, Greise mitgezählt wurden oder nicht oder ob die Zahl von 7000 Gefangenen sich nur auf >Arbeitsfähige< oder auf ganze Familien bezieht. Wenn man mit GUNDLACH davon ausgeht, daß diese Zwangsumsiedlungen einem zentralen staatlichen Programm folgten, darf jedoch mit einiger Wahrscheinlichkeit davon ausgegangen werden, daß ganze Familien umgesiedelt wurden. Schließlich sollte über die Familiengründung und Kinderzeugung ein Beitrag zum ägyptischen Bevölkerungswachstum geleistet werden. Auch das Determinativ (je ein Mann und eine Frau) als Vorläufer des späteren $r m \underline{t}$ weist darauf hin, daß Männer und Frauen gleichermaßen erfaßt wurden.

Über die Praxis der Umsiedlung ganzer Sippen aus den besiegten Ländern auf das ägyptische Territorium berichten am deutlichsten die Quellen des Neuen Reiches, wie etwa eine Stele Amenophis' II. aus Memphis, auf der berichtet wird:

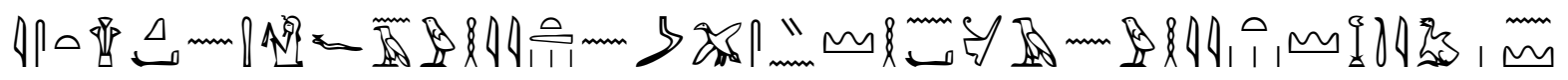

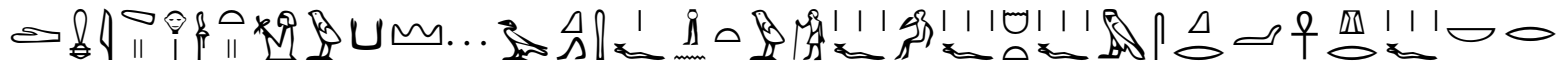
品1 II P)

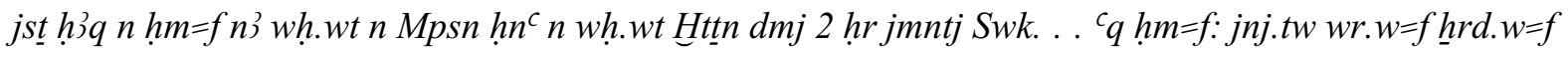

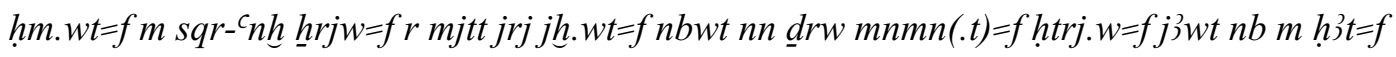

»Seine Majestät plünderte die Dörfer von Mapasin und die Dörfer von Chatsin, zweier Siedlungen westlich von Sukeh. ... Seine Majestät drang ein: Man erbeutete seine Großen, seine Kinder, seine Frauen als Gefangene, genauso wie alle seine Verwandten, all' seine Habe ohne Ende, sein Vieh, seine Gespanne, wobei alles Vieh vor ihm war.«

Im Gegensatz dazu wird auf einer Stele Amenophis' III. ausdrücklich von 30000 Männern (zj) gesprochen, so daß man in diesem Fall annehmen muß, daß sich die Angaben tatsächlich allein auf einen militärischen Gegner beziehen. Der Kontext der Schlacht und die Erwähnung der abgeschlagenen Hände als Siegeszeichen sprechen ebenfalls dafür. ${ }^{637}$

636 WENKE u. a. (1988: 34).

637 Vgl. dazu auch HelcK (1975a). 


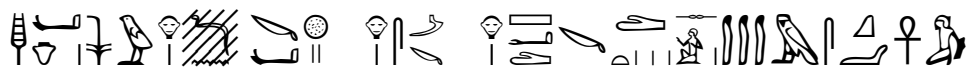

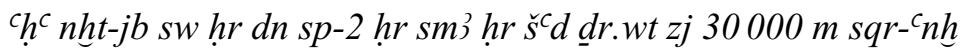

»Kühn war er beim Töten, beim Abschlachten und beim Abschneiden der Hände. 30000 Mann waren Gefangene.«

Ein indirekter Beleg für eine Zwangsumsiedlung liegt uns aus dem Neuen Reich vor. Es handelt sich dabei um eine teilweise zerstörte Grenzstele, die im Totentempel Thutmosis' IV. gefunden wurde und sich heute im Oriental Institute in Chicago befindet. ${ }^{638}$

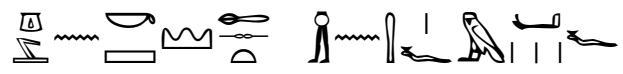

$\operatorname{grg} n K 3 \breve{s} \underline{h} z \cdot \operatorname{tjn}(j)(. w) \cdot n h m=f m n h t(. w)=f$

»Siedlung (der Leute aus dem) elenden Kusch, die Seine Majestät bei seinem Sieg herbeigebracht hat.«

Weitere Informationen über Umfang und Ort der Ansiedlung lassen sich dem Text leider nicht mehr entnehmen. Gelegentlich sind die Kriegsgefangenen auf Land angesiedelt worden, das der Verwaltung durch thebanische Tempel oblag. ${ }^{639} \mathrm{Daß}$ es in solchen Fällen häufig um eine Zwangsansiedlung ganzer Familien ging, die auf solche Art in ägyptisches Territorum >immigriert wurden<, zeigt das Beispiel aus den Siegesberichten von Ramses III. in Medinet Habu. ${ }^{640}$ Neben der separaten Erwähnung der Anführer werden sowohl Männer als auch Frauen in jeweils drei Altersklassen unterteilt und separat aufgelistet. Die Gesamtzahl der Gefangenen wird mit 2052 angegeben, das Verhältnis von Männern zu Frauen beträgt dabei ungefähr 3 : 1. Zusammen mit der Zahl der Getöteten, die 2175 beträgt, ergibt sich eine Summe von 4227 Personen, die auf der Seite des militärischen Gegners als Verlust zu beklagen waren. Die Tatsache, daß sowohl Männer als auch Frauen jeglichen Alters unter den Gefangenen waren, läßt darauf schließen, daß es Personengruppen wie diese waren, die als geschlossene Gruppe auf ägyptischem Boden angesiedelt wurden. Bemerkenswert ist auch die relativ niedrige Gesamtzahl, die nicht einmal 1/10 der Größenangaben erreicht, die in der Frühzeit und im Alten Reich hinsichtlich Gefangener und Gefallener getroffen wurden.

Wie sich eine solche - erzwungene oder freiwillige - >Einwanderung< dargestellt hat, kann ansatzweise aus einer Darstellung im Grab des ㅂnnmw-ḥtp II. in Beni Hassan erschlossen werden (Abb. 5.6). ${ }^{641}$ In Wort und Bild berichtet der Grabinhaber, ein Beamter der 12. Dynastie unter den Regenten Amenemhet II. und Sesostris II., an der Nordwand des Grabes davon, daß 37 >Asiaten $<\left({ }^{C} 3 \mathrm{mw}\right)$ angekommen sind, die wertvolle Augenschminke mitgebracht haben.

638 Chicago, OI 1363.

639 BORCHARDT (1898).

640 KRI V 53,1-2; zur Differenzierung der Altersgruppen vgl. bereits BRUGSCH (1876).

641 Beni Hassan Grab Nr. 3; NewBerRy (1893: Taf. 30). 


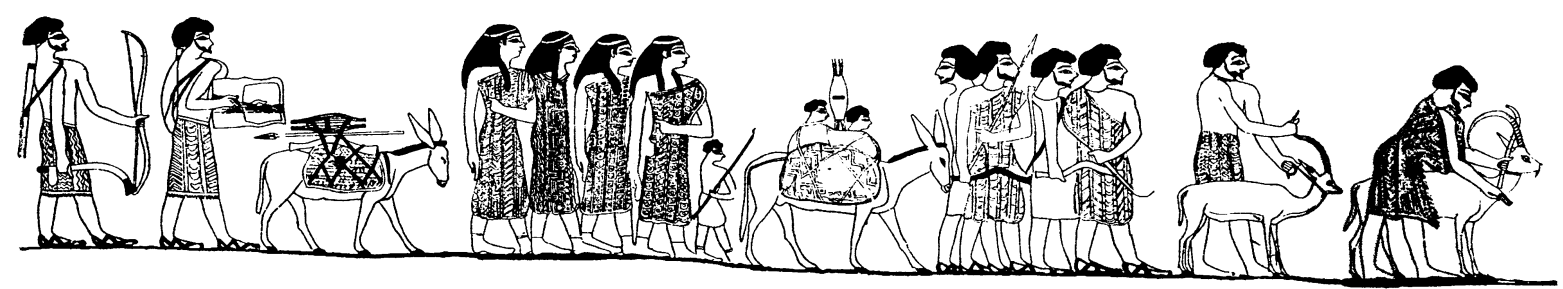

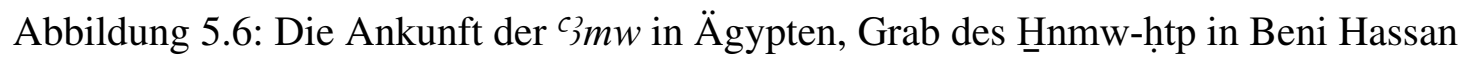

Mehrere Aspekte dieser Szene sind dabei interessant. Von den erwähnten 37 Personen sind an der Grabwand nur 15 Personen abgebildet: acht Männer, vier Frauen und drei Kinder. Zwei der Kinder sind dabei auf dem Tragegestell eines mitgeführten Esels verschnürt, was möglicherweise auf ihr recht junges Alter hindeutet, so daß sie noch nicht laufen können. Zwei ägyptische Beamten gehen dem Zug voran, der erste - tituliert als >Schreiber des Königs< -

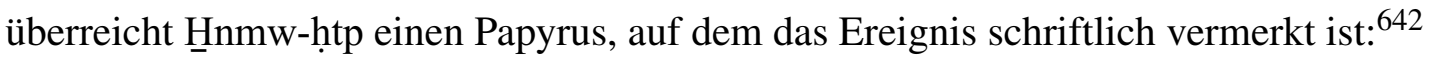

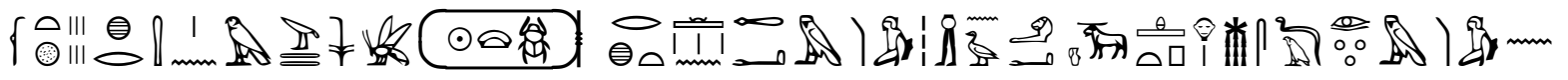

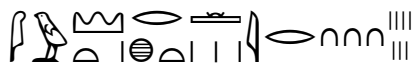

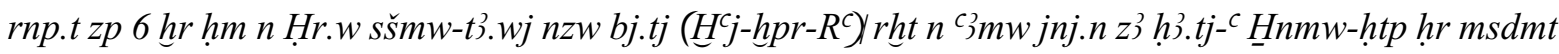
çmw $n$ Šwtw rh.t jrj 37

»Jahr 6 unter der Majestät des Horus, Führer der beiden Länder, König von Ober- und Unterägypten, Sesostris (II.); die Anzahl der Asiaten, gebracht vom Sohn des Nomarchen Hnmw-ḥtp wegen der Augenschminke, Asiaten des Landes Schutu, deren Anzahl 37 beträgt.«

An der Spitze der Asiatengruppe steht der >Herrscher des Fremdlandes` namens Ibscha, der eine Antilope mit sich führt. Dahinter steht ein anderer Mann mit einer zweiten Antilope. Insgesamt zwei Esel vervollständigen die Szene. Da sowohl der $>$ Papyrustext $<$ als auch die Bildbeischrift ausdrücklich von Augenschminke sprechen, die von den Fremden als Gabe überreicht werden, können die Tiere für diesen Zweck ausgeschlossen werden. Da auch nicht alle 37 Personen abgebildet sind, ergibt sich bei der Betrachtung des Ereignisses folgende Interpretation: Eine Gruppe Asiaten, die aus Männern, Frauen und Kindern besteht, ist zusammen mit ihrem Kleinvieh nach Ägypten gekommen. Das Determinativ des Ethnonyms ${ }^{3} m w$, das einen Gefangenen zeigt, ist deutliches Zeugnis der politischen Situation. ${ }^{643}$ Es stellt sich hier möglicherweise die Anwesendheit der Asiaten auf ägyptischem Boden weitaus weniger freiwillig dar als anfäglich vermutet. Die Szene im Grab des $\underline{H} n m w-h \underline{t p}$ könnte also

642 NEWBERRY (1893: Taf. 38,2).

643 Vgl. etwa die Schreibung in der Biographie des Wnj, Urk. I 101,9 das Eindringen der ${ }^{3} \mathrm{mw}$ auf ägyptisches Gebiet militärisch vorgehen mußte. 
tatsächlich eine Darstellung von Kleingruppen der Bevölkerung eines besiegten Landes handeln, die unter dem Eindruck ägyptischer Machtpräsenz auf ägyptisches Territorium übersiedelt. Die Herkunft der ${ }^{3} 3 m w$ ist kaum gesichert. Es könnte sich in diesem Fall um Angehörige

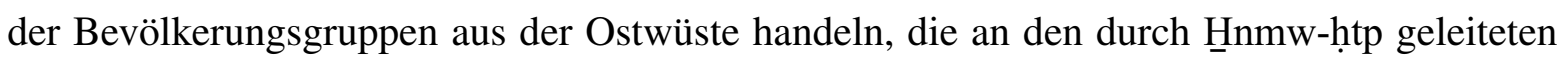
Nomos grenzt.

\subsection{Zusammenfassung}

Die Betrachtung der zweckgebundenen Zensustypen wie auch der Belege aus der Frühzeit und dem Alten Reich zeigen ein disparates Bild. Die einzelnen Textstellen sind in ihren Aussagen nicht immer zur Deckung zu bringen und stehen im Einzelfall sogar im diametralen Gegensatz zueinander. Dennoch ergaben sich durch neue Verknüpfungen mit themenfremdem Textmaterial andere Sichtweisen auf die altägyptische Form der Erfassung von Menschen. Insbesondere die Aussage des Hofbeamten Sbk-Nht, der jeden Hundertsten >Wehrfähigen< aus dem abydenischen Nomos rekrutierte, stellte sich in den Auswertungsmöglichkeiten ergiebiger dar als bisherige Bearbeiter ihr zugestanden hatten. Die Neubetrachtung der altägyptischen Steuerlisten brachte auch weitere Erkenntnisse zu dem bekannten Terminus wpw.t, dessen Reflexe in den >literarischen< Texten weitaus dichter an der Realität lagen als ihm bisher zugestanden wurde. Damit liegt jetzt neue Evidenz für eine Mischform von Subjekts- und Objektsdeklaration während des Mittleren Reiches vor. Erwartungsgemäß weniger aussagekräftig zeigen sich dagegen die altägyptischen Hinweise zur Arbeitsorganisation, die selten Möglichkeiten zu demographischen Hochrechnungen bieten und die Zwangsumsiedlungen, die zwar eine gewisse Relevanz für die Populationsbewegung des Alten Ägypten besitzen, jedoch in der Regel nicht quantifizierbar sind.

Dagegen konnte neues Licht in die frühesten Belege gebracht werden. Während einerseits lange tradierte Textstellen für demographische Angaben verworfen werden mußten, haben andere Quellen, obwohl sie bisher aufgrund ihrer unglaublichen Größenangaben zurückgewiesen wurden, durch den Aufbau indirekter Indizienketten neue und starke Fürsprache erfahren. Dabei stellen diese Quellen allerdings lediglich punktuelle Angaben konkreter Größenordnungen dar, die weder in einen Kontext zur demographischen Gesamtsituation eingebettet werden können, noch eine absolute Angabe der Bevölkerung zur jeweiligen Zeit erlauben. Insofern bleiben die Daten in ihrer Aussage isoliert und ungeeignet für darauf aufbauende Erkenntnisse. Doch auch in der Demonstration der beschränkten Möglichkeiten haben die in diesem Kapitel vorgeführten Beispiele ihren Zweck erfüllt. 


\section{Exogene demographische Faktoren}

Das Leben ist eine zähe Katze, und eine solche ist die Menschheit.

Thomas Mann, Nietzsches Philosophie im Lichte unserer Erfahrung

\subsection{Definition}

$\mathrm{D}$ IE Faktoren, die im folgenden einer näheren Betrachtung unterzogen werden sollen, können einerseits einen nachhaltigen Einfluß auf die demographische Entwicklung einer Gesellschaft bedingen, entziehen sich andererseits aber der Einflußnahme durch menschliches Handeln und sind daher als >exogen $<$ zu klassifizieren. Im weitesten Sinne handelt es sich bei diesen Faktoren um >Naturphänomene $<,{ }^{644}$ die sich prinzipiell in drei Kategorien unterteilen lassen. Erstens die klimatisch oder wetterbedingten Naturkatastrophen wie Dürre und Überschwemmung, zweitens die aus der ersten Kategorie häufig bedingten Hungersnöte und drittens der Bereich von Gesundheit und Hygiene.

\subsection{Naturkatastrophen: Überschwemmungen und Dürre}

Wie auch schon bei den Quellen zu den Hungersnöten ist es auch bei den altägyptischen Berichten über Naturkatastrophen sehr schwierig, aus den von Ideologie durchwirkten Texten den historischen Kern herauszuschälen. Dabei spielt die Frage, ob es sich in allen Fällen um einen Bericht eines historischen Ereignisses oder einer topischen Chaosbeschreibung handelt, für die Analyse der meteorologischen Hintergründe nur eine nachrangige Rolle. Die sogenannte >Unwetterstele< des Königs Ahmose I. (18. Dynastie) darf als das ausführlichste Beispiel für ein solches Ereignis gelten. Obwohl der teilweise stark zerstörte Text noch manche Verständnisprobleme birgt, ist der Abschnitt, der sich auf den Regensturm bezieht, mittlerweile 644 ADAMSON (1982: 7). 
recht gut erarbeitet. ${ }^{645}$ Zusammengefaßt nach der Übersetzung Peter BEYLAGEs berichtet die Stele folgendes: ${ }^{646}$ Ein Regensturm zieht auf, der Himmel verdunkelt sich durch eine dichte Wolkendecke. Das Unwetter tobt mit lautem Tosen über der Wüste. Nachdem der Sturm sich gelegt hat, sieht sich der König unmittelbar mit den zerstörerischen Folgen konfrontiert: überall eingestürzte Häuser und Lager, Tote unter den Trümmern begraben oder im Nil treibend und von dessen Strömung mitgerissen.

Die drastische Schilderung dieser Begebenheit und ihrer destruktiven Auswirkungen sind trotz des knappen Berichts recht eindringlich geraten. Auch wenn BEYLAGE in seiner Untersuchung zu Recht schreibt, daß »die Sprache dieser topischen Texte [...] bildreich und überzeichnet « ist, ${ }^{647}$ so müssen die auf solche Weise geschilderten Ereignisse zumindest als lebendiger Eindruck mit allen negativen Folgen den Ägyptern bekannt gewesen sein. Oder anders gesagt: Die zerstörerische Gewalt eines Regensturms kann nicht allein aus der Phantasie geboren sein. Aufgrund der klimatischen Gegebenheiten Ägyptens sind größere Unwetter eher als seltenes, wenngleich nicht unmögliches Ereignis einzustufen. ${ }^{648}$ Tatsächlich können sowohl aus archäologischer Evidenz wie auch aus der Beobachtung rezenter Regenschauer in Ägypten im Jahre 1994 übereinstimmende Auswirkungen auf den Lebensraum festgestellt werden. ${ }^{649}$

Weitere Indizien, die allerdings eher indirekte Aussagen zu Flut oder Niedrigwasser geben, hat Wolfhart WESTENDORF aus diversen medizinischen wie auch religiösen Texten zusammengetragen und stellt daraus einen Zusammenhang zu der >jährlichen Seuche< $(j 3 d t$ rnp.t) als Folge der Nilüberschwemmung her. ${ }^{650}$ Doch könnte dies als Kausalzusammenhang allein durch oberflächliche Beobachtungen genährt worden sein, wie auch die Aussagen europäischer Beobachter aus dem 19. Jahrhundert nahelegen. ${ }^{651}$ Die Folgen einer katastrophalen Überflutung dürften dabei die gleichen gewesen sein, die auch heute noch einen Teil des Gefahrenpotentials ausmachen und als Keimzelle für Seuchen gelten müssen. ${ }^{652}$ Direkte Konsequenzen nach einer Flut sind Verunreinigung mit Fäkalien und Tierkadavern sowie nach Ablauf des Wassers das Zurückbleiben von kontaminiertem Schlamm. Indirekte Auswirkun-

\footnotetext{
645 Zuletzt ausführlich BeYLAGE (2002: 77 ff.) sowie Allen und WiEnER (1998).

646 BeYLAGE (2002: 83).

647 BEYLAGE (2002: 609).

648 IBRAHIM (1996: 36).

649 ALLEN und WIENER (1998: 18 Anm. 16).

650 WESTENDORF (1981: 78).

651 KUHNKE (1990: 70) erwähnt Berichte, in denen europäische Reisende aufgrund der Tatsache, daß Epidemien beinahe so regelmäßig wie die jährliche Nilflut auftraten, einen kausalen Zusammenhang zwischen Pest und Nilüberschwemmung herstellten. Ein noch älteres Beispiel aus dem 16. Jh. zitiert LEITZ (1994: 206).

652 So auch WeStENDORF (1981: 80) für das Alte Ägypten.
} 
gen sind unter anderem die Flucht von Ratten und anderen Schädlingen vor dem ansteigenden Wasser auf höherliegende Landflächen und verstärktes Eindringen in die Gebiete der Menschen. ${ }^{653}$ Aus dem vielfältigen Vokabular der ägyptische Sprache für die Bezeichnung von Unwettern erhält der Terminus >jährlichen Seuche< eine besondere Bedeutung, ${ }^{654}$ da sich daraus ein zusammengesetzer Begriff snm rnp.t ableitet, der sich möglicherweise als Parallelbildung an $j 3 d t$ rnp.t anlehnt und dessen Etymologie nicht restlos geklärt ist. ${ }^{655}$ Als Bezugselement zwischen beiden Begriffen könnte in diesem Fall ein Unwetter als Ursache von Epidemien gemeint sein.

Die Auswirkungen einer > Dürre< sind im pharaonischen wie auch im modernen Ägypten etwas anders gelagert. Dies ist bedingt durch das einzigartige System Ägyptens, die Felder im wesentlichen durch den Nil zu bewässern, während etwa die Gebiete Zentralafrikas seit jeher auf günstige klimatische Verhältnisse mit regelmäßigen Regenperioden für das Einbringen einer guten Ernte angewiesen sind. Demnach müßte als äquivalentes Ereignis für Ägyten ein niedriger Nilpegel und damit verbunden die mangelhafte Nutzbarkeit des Ackerbodens angenommen werden. ${ }^{656}$ In beiden Fällen resultiert dann der Ernteausfall in einer Unterversorgung der Bevölkerung und somit in Hungersnöten. Die Erwähnung einer Hungersnot in den ägyptischen Quellen allein ist jedoch noch kein Indiz für eine zu niedrig ausgefallene Nilflut, oder wie Wolfgang SCHENKEL es prägnant formuliert: »Hungersnot und niedriger Nil sind nicht synonym «. ${ }^{657}$ Auf die Thematik der Hungersnöte wird an späterer Stelle in Kap. 6.4 separat eingegangen. Einstweilen sollen hier zunächst Ursachen und deren Ursprung interessieren. Die natürliche Bewässerung der Felder schwemmte auch in großer Menge Sedimente auf die Ackerböden. Inwieweit die im Nilwasser enthaltenen Schwebstoffe tatsächlich für die Bodenfruchtbarkeit von Bedeutung sind, ist in jüngerer Zeit wieder Gegenstand der Diskussion. ${ }^{658}$ Da jedoch auch die ägyptischen Texte selbst wiederholt einen Bezug zwischen niedrigem Nilstand und Hungersnöten herstellen, scheint das Ausbleiben der jährlichen Flut ein gravierendes Problem zu sein, das entsprechende Gegenmaßnahmen erfordert. ${ }^{659}$ Für das Ausbleiben der Nilflut sind verschiedene Hypothesen formuliert worden. Zum einen könnten Änderungen der klimatischen Bedingungen zu einer grundsätzlichen Abnahme der Nilflut geführt haben, zum anderen lassen sich Jahre mit besonders niedrigem Pegel in einen Zyklus von

653 Vgl. LeITZ (1994: 207).

654 Zur negativen Konnotation durch die Determinierung mit dem Seth-Tier Syl. auch VerhoEven (1986).

655 Zum Erklärungsversuch vgl. WESTENDORF (1981: 81 f.).

656 SCHENKEL (1978: 50).

657 SCHENKEL (1978: 52).

658 IBRAHIM (1996: $63 \mathrm{f}$.).

659 Siehe dazu unten den Exkurs auf S. 200. 
regelmäßig schwankenden Nilfluten einbetten. ${ }^{660}$ Wie auch bei den Hungersnöten fehlt es für die Nilstände an zuverlässigen Quellen, die eine sichere und mit archäologischen Befunden korrelierbare Datenbasis liefern. ${ }^{661}$

\subsection{Krankheiten}

Neben den alltäglichen Erkrankungen der altägyptischen Bevölkerung, die vielfach an $\mathrm{Mu}-$ mien nachgewiesen sind und an deren Symptomen die Betroffenen zwar litten aber nur unter bestimmten Bedingungen verstarben, soll es in diesem Kapitel im besonderen um diejenigen Infektionskrankheiten gehen, die unter den allgemeinen Begriff >Seuche< fallen. ${ }^{662}$ Als Seuche bezeichnet man eine plötzliche Erkrankung vieler Menschen durch eine Infektion, wobei drei Typen unterschieden werden. Die Epidemie stellt ein plötzliches, örtlich und zeitlich jedoch begrenztes Auftreten einer (Infektions-)Krankheit dar. Je nach Stärke des Anstiegs und Abfalls der Erkrankungsfälle unterscheidet man weiter zwischen Explosiv- und Tardivepidemien. Als Pandemie bezeichnet man die Ausbreitung einer Infektionskrankheit über Länder oder sogar Kontinente hinweg. Als jüngeres Beispiel für eine Pandemie können die aus Ostasien stammenden Fälle von Influenza oder die im Jahr 2003 ausgebrochene Lungenerkrankung SARS genannt werden. Der dritte Typus ist die Endemie, mit der man ein regelmäßig wiederkehrendes Vorkommen einer Erkrankung in einer begrenzten Region beschreibt. Das Auftreten einer Seuche oder eine hohe Infektionsrate sagt jedoch noch nichts über die Letalität der Krankheit aus. ${ }^{663}$ Dennoch darf auch bereits für den Mittelmeerraum der Bronzezeit von Massensterben durch Epidemien ausgegangen werden, wie Beschreibungen aus dem Hethitischen zeigen. ${ }^{664}$

\subsubsection{Die ’Pest‘}

\subsubsection{Definition}

Bei genauerer Betrachtung der Kontexte, in denen das Wort >Pest< als Beschreibung für eine Seuche in der Antike Verwendung findet, gelangt man schnell zu der Feststellung, daß häufig

660 SCHENKEL (1978: 52 ff.); zur Klimahypothese auch Henfling und Pflaumbaum (1991).

661 Morenz (1998: 97).

$662 \mathrm{Zu}$ Seuchen im Alten Ägypten zuletzt zusammenfassend WESTENDORF (2000).

663 Andererseits betont SCHEIDEL (2001b: 25), daß Krankheiten die häufigste Todesursache in Gesellschaften mit hoher Sterblichkeitsrate sind.

664 Vgl. SOUČEK (1963: 164) zu den sogenannten >Pestritualen< und dem hethitischen Wort hinkan für eine tödliche Epidemie. 
genug dieser Ausdruck ganz allgemein für Epidemien aller Art verwendet wird. Nicht immer ist die Pestkrankheit selbst gemeint und es bedarf einiger Aufmerksamkeit, die Verwendungsebenen der Begriffe auseinanderzuhalten. So finden sich immer wieder Übersetzungen von Texten, die mit dem Begriff >Pest< operieren, obwohl keine Klarheit darüber besteht, welches medizinische Phänomen tatsächlich in den alten Überlieferungen beschrieben wird. Deshalb hat die alltagshermeneutische Verwendung des Wortes >Pest< bisher mehr zur Verschleierung als zur Klärung der historischen Sachverhalte beigetragen, wozu sicher auch die englischen Bezeichnungen >pest, plague< beitrugen, deren Wortfeld im Sinne von >Seuche< deutlich weiter gefaßt war als durch die deutsche Übersetzung transportiert wurde.

Zunächst sei eine kurze Beschreibung der Pest den weiteren Betrachtungen vorangestellt. Sie ist ursprünglich eine Tierkrankheit und befällt hauptsächlich kleine Säugetiere. Neben wild lebenden Nagetieren können auch domestische Tiere (Ratten) Träger der Infektion sein. Der Mensch steht eigentlich gar nicht im Kreislauf der Krankheit. Der Rattenfloh als Überträger bevorzugt bei einem Wirtswechsel in der Regel wieder einen Nager und wechselt nur in besonderen Situationen auf den Menschen über, zum Beispiel, wenn die Wirtsratte stirbt und keine andere Ratte als Wirt in der Nähe ist. ${ }^{665}$

Die Medizin kennt drei Formen der Pest, alle drei werden jedoch durch den gleichen Erreger hervorgerufen: Beulenpest, Pestsepsis und Lungenpest, wovon die Beulenpest die am weitesten verbreitete Form dieser Krankheit darstellt. ${ }^{666}$ Auch der Krankheitsverlauf ist klar umrissen: Nach einer Inkubationszeit von zwei bis acht Tagen tritt zunächst hohes Fieber auf. Dazu kommen in vielen Fällen Krämpfe, Erbrechen, Schwindelgefühl, Lichtempfindlichkeit sowie Gliederschmerzen. Zwei bis drei Tage nach dem Ausbruch des Fiebers treten dann die charakteristischen Beulen auf, die der Krankheit ihren Namen gegeben haben. Es können auch sogenannte Petechien, rote oder bläuliche Punkte auf der Haut auftreten. Der Tod tritt aufgrund von Erschöpfung, Herzversagen oder inneren Blutungen ein. Wenn der Patient den Krankheitsschub für einen Zeitraum von acht bis zehn Tagen übersteht, kann er mit Genesung rechnen. Die Pestsepsis dringt direkt in die Blutbahn des Menschen ein und verursacht einen raschen Tod innerhalb von 24 bis 36 Stunden - noch bevor die Beulen auftreten. Die Lungenpest ist eine Form der Lungenentzündung, hervorgerufen durch den Pesterreger und kann als einzige aller drei Varianten durch Tröpfcheninfektion von Mensch zu Mensch übertragen werden. ${ }^{667}$

665 Herlihy (2000: 12 f.). Dies ist besonders der Fall bei Rattenpopulationen, die nicht immun gegen den Erreger sind und bei einer überspringenden Infektion ein Massensterben der Ratten einsetzt.

666 HeRLiHy (2000: ebenda) verweist auf die Daten aus China und Indien des vergangenen Jahrhunderts und gibt den Anteil der Beulenpest an der Gesamtzahl aller Erkrankungen mit ungefähr $75 \%$ an.

667 HERLiHy (2000: 13 f.). 
Als Indiz für eine >echte< Pestepidemie galt bisher die sogenannte Epizootie, das Massensterben von Ratten oder anderen Wirten, das dem Wechsel der Flöhe auf den Menschen vorangeht. Im Mittelalter ist dieses >Omen $<$ jedoch weder nachgewiesen, noch von zeitgenössischen Chronisten beschrieben worden. ${ }^{668}$

\subsubsection{Ratten und Flöhe als Überträger}

Ausschlaggebend für die Frage nach der Existenz von Pest im pharaonischen Ägypten scheint nun zu sein, ob es ein Kleinsäugetier gab, das als Überträger der Krankheit fungieren konnte. Die heute bekannte Hausratte (lat. Rattus rattus alexandrinus), die als >klassischer< Überträger des Erregers gilt, wanderte erst spät aus Asien nach Ägypten ein. Die bisher als älteste Belege vermuteten Skelettteile konnten bei erneuter Untersuchung der in Ägypten seit jeher heimischen Nilgrasratte (lat. Arvicanthis niloticus niloticus) zugewiesen werden. ${ }^{669} \mathrm{Ob}$ der Rattenfloh als Überträger bereits zuvor andere Kleinsäuger wie die Nilgrasratte als Wirte benutzte, bleibt ein Feld für Spekulationen. ${ }^{670}$ Auch in der jüngst erschienenen Arbeit von John ABERTH spricht dieser die Vermutung aus, daß die pesttragenden Rattenflöhe im Europa des Mittelalters von der Hausratte eingeschleppt wurden. ${ }^{671}$ Der älteste sichere Beleg für die Hausratte in Ägypten bleibt damit in der römischen Zeit des ersten und zweiten nachchristlichen Jahrhunderts angesiedelt. Dies wäre immerhin eine Parallelerscheinung zu den bekannten Epidemien der ägyptischen Spätantike.

Ein wichtiges Argument in der Indizienkette zur Existenz der Pest ist das Auftreten von Ratten als Überträgern. Wie bereits für die Ausbreitung der Pest in Mitteleuropa beschrieben, stellte eine teilweise Koinzidenz von Rattenplagen und dem Ausbruch der Seuche ein signifikantes Merkmal dar. Obwohl der Nachweis von Ratten oder anderen Nagern als Schädlingen nicht allein als Beleg für die Pest gelten darf, ist zumindest die Rückverfolgung von Indizien bis in die pharaonische Zeit ein notwendiger Teil der Beweisführung. Walter SCHEIDEL führt seine Belege bis in die Zeit des römischen Ägypten zurück und hält die Existenz von Ratten und Flöhen für mindestens seit dieser Zeit als gesichert. ${ }^{672}$ Die schwarze domestizerte Ratte kann nach SCHEIDEL sogar bis zurück in die hellenistische Zeit nachgewiesen wer-

668 HERLIHY (2000: 19 f.).

669 BOESSNECK (1988: 62 f.).

670 SCHEIDEL (2001a: 100) weist auf ein instabiles Verhalten der Pesterreger hin, die auch auf andere Nagetiere überspringen können.

671 ABERTH (2001: 112 f.).

672 SCHEIDEL (2001a: 99). Erwähnenswert auch die Schilderung Diodors (I 10,2), der über eine gelegentliche explosionsartige Vermehrung von Mäusen $(\mu \tilde{\cup} \varsigma)$ in der Thebais berichtet. 
den. Darüber hinaus gibt es Indizien für ein Einwandern der Ratten aus Indien in den Nahen Osten bereits im 2. Jahrtausend v. Chr. ${ }^{673}$ Der älteste aus Ägypten stammende Beleg dürfte aus Kahun stammen, wie William Matthew Flinders PETRIE in seinem Grabungsbericht vermerkt. Dort beschreibt er zahlreiche Tiergänge in den Ecken von Gebäuden, die zudem noch mit Steinen oder anderem Füllmaterial verstopft worden waren, um der Plage Herr zu werden. ${ }^{674}$ Dies erfährt durch die Neubesprechung des Fundes einer Lebendfalle für Nagetiere aus Kahun zusätzliche Unterstützung. ${ }^{675}$ Die Beschreibung PETRIEs wurde in jüngerer Zeit jedoch dahingehend relativiert, daß die Befunde in Kahun eher als >außergewöhnlich < gelten müssen, und auch in der Gegenwart Tiergänge nicht in einem derartigen Umfang zu finden sind. ${ }^{676}$ Eine Warnung davor, den Ratten zu nahe zu kommen, spricht auch ein Eintrag in einem sogenannten Tagewählkalender des Neuen Reiches aus, der unten auf S. 189 ausführlich besprochen wird.

Auch Flöhe waren den Ägyptern bekannt und wurden als >Lästlinge< bekämpft. So liefert beispielsweise der Papyrus Ebers in seiner Rubrik der >Hausmittel< ein Rezept gegen Flöhe im Haus. ${ }^{677}$ Im Übrigen ist der Floh - anders als von SCHEIDEL beschrieben - eher Überträger als Träger des Erregers Yersinia pestis, der als Verursacher aller drei Formen der Pest verantwortlich ist.

\subsubsection{Historische Evidenz}

Die gezielte Diagnose einer epidemischen Krankheit anhand historischer Überlieferungen ist generell ein unsicheres Unterfangen. Symptome können von den zeitgenössischen Chronisten falsch erkannt oder mangels Kenntnis um die medizinischen Zusammenhänge schlicht ignoriert werden. Daher ist es nötig, mehrere signifikante Indizien gleichzeitig beschrieben vorzufinden, um ein Minimum an Sicherheit hinsichtlich einer Beurteilung zu erlangen.

Unter diesen strikten Voraussetzungen wird bisher die Pandemie von $541 \mathrm{n}$. Chr. als frühester gesicherter Ausbruch der Pest angesehen, die in mehreren schweren Schüben in Zyklen zwischen neun und zwölf Jahren fast zweihundert Jahre lang den Nahen und Mittleren Osten im Griff hielt. ${ }^{678}$ Benannt wurde diese Pest nach Kaiser Justinian, der 542 in Konstantinopel herrschte, als die Pest die Stadt erreichte. Der genaue Ursprung des Ausbruchs ist unbekannt,

673 SCHEIDEL (2001a: ebenda Anm. 290).

674 PETRIE (1891: 8).

675 DRUMMOND u. a. (1990).

676 DRUMMOND u. a. (1990: 98).

677 WESTENDORF (1999: 458).

678 SCHEIDEL (2001a: 100); NorRis (1977: 2); ALSTON (2001: 182 ff.); Dols (1974: 372). 
doch geben zwei Zeitgenossen Hinweise auf den ungefähren geographischen Verlauf, den die Krankheit nahm. Ausführlich berichtet Prokop vom Ausbruch der Pest in Pelusium, einem ägyptischen Hafen, im Jahr 541. Von dort aus bewegte sich die Krankheit zum einen in Richtung Alexandria und weiter nach Ägypten, zum anderen strebte sie auf Palästina zu. ${ }^{679}$ Fast zeitgleich bezeugte Euagrius Scholasticus im Jahr 542 eine Pestepidemie in seiner Heimat Antiochia und behauptete, diese habe ihren Ursprung in Äthiopien genommen. ${ }^{680} \mathrm{Um}$ das Jahr 750 schien die Pest ihre Kraft verloren zu haben, da keine weiteren Vorkommen aus dem Mittleren Osten berichtet wurden. ${ }^{681}$ Spätere Berichte arabischer Autoren scheinen die These ihrer Herkunft ansatzweise zu bestätigen. Das medizinische Kompendium des ${ }^{\mathrm{C} A l i ̄ ~ i b n ~ R a b-~}$ ban at-Ṭabarī aus dem Jahr 850 gibt den Sudan als Ursprung an. Aus dem 16. Jahrhundert ist bekannt, daß die Pest aus Ostafrika ihren Weg über die Karawanenrouten nach Norden nahm. Andere medizinische Texte aus dem 13. und 14. Jahrhundert bringen die Pest ebenfalls mit Äthiopien in Verbindung, so daß der Verdacht naheliegt, die Pest könnte im Mittelalter endemisch in Äthiopien beheimatet gewesen sein und sich über Handelsverbindungen in den Mittelmeerraum ausgebreitet haben. ${ }^{682}$

Weitaus unklarer ist die Situation bei dem Ereignis, das als >Antoninische Pest< Einzug in die Geschichtsschreibung gehalten hat. Für die Datierung gibt es mehrere Ansätze. Die römischen Historiker berichten davon, daß römische Soldaten, die vom Partherkrieg unter Lucius Verus zurückkehrten, die Seuche zwischen den Jahren 166 und 167 n. Chr. nach Ägypten einschleppten. ${ }^{683}$ Die älteren Arbeiten bezogen sich in ihrer Argumentation hauptsächlich auf Auswertungen der Zensuslisten aus der Stadt Karanis zwischen den Jahren 165 und 171174 n. Chr., die einen Bevölkerungsrückgang um etwa $1 / 3$ zu belegen schienen. ${ }^{684}$ Die jüngere Forschung sieht dagegen eine Überlagerung mehrerer struktureller und sozio-kultureller Faktoren, die zur Depopulation ganzer Regionen führte. So betont Dominic RATHBone einerseits die regionalen Auswirkungen der Seuche auf die Population besonders von Unter- und Mittelägypten ab 166 bis in die späten Jahre des darauf folgenden Jahrzehnts, andererseits zeichneten sich etliche Gründe für regionalen Bevölkerungsrückgang bereits vor $165 \mathrm{n}$. Chr.

679 Prokop, Bella 2,22; Dols (1974: ebenda); Prokop war es auch, der durch eine recht genaue Beschreibung der Symptome eine Identifizierung der Seuche erst ermöglichte.

680 Euagrius, Historia ecclesiastica 4,29; Dols (1974: ebenda) vermutet dahinter jedoch eine Bezugnahme auf Thukydides und dessen Mutmaßung zum Ursprung der Seuche von Athen.

681 SCHEIDEL (2001a: 100).

682 DoLs (1974: 372 f.).

683 Eine andere Variante, die Äthiopien als Ursprung nennt, wird von RATHBone (1990: 114 Anm. 29) als weitere Kopie des Thukydides-Berichts bezeichnet.

684 BOAK (1959). 
ab, wie Überfälle oder Landflucht (Anachorese) wegen Nahrungs- und Wassermangel sowie möglicherweise Steuerbelastung oder Verfall der Administration. ${ }^{685}$ In den vergangenen Jahren wurde die >Antoninische Pest< zunehmend als Pockenepidemie identifiziert, so daß ein Bezug zur echten Pest ohnehin nicht mehr gegeben scheint. ${ }^{686}$ Dennoch wird - trotz der Einflüsse aller anderen Faktoren jener Zeit - der Epidemie ein tiefgreifender Einschnitt in die Populationskurve von 20-30\% attestiert, der jedoch bereits im frühen 3. Jahrhundert wieder aufgefangen werden konnte. ${ }^{687}$

\section{Die Biblische Überlieferung}

Auf Basis dieser Vorbedingungen gilt eine Beschreibung im 1. Buch Samuel des Alten Testaments als bisher ältester wahrscheinlicher Beleg für einen >echten< Pestausbruch. ${ }^{68}$ Dort heißt es unter anderem:

»(5,9) Als sie aber die Lade dahin getragen hatten, entstand in der Stadt ein sehr großer Schrecken durch die Hand des HERRN; denn er schlug die Leute in der Stadt, klein und groß, so daß an ihnen Beulen ausbrachen.

$(6,5)$ So macht nun Abbilder eurer Beulen und eurer Mäuse, die euer Land zugrunde gerichtet haben, daß ihr dem Gott Israels die Ehre gebt.«

Die Beschreibungen in der Bibel weisen zwei wesentliche Elemente auf, die auch später während der großen Pestepidemien im europäischen Mittelalter wieder in den Berichten der Chronisten anzutreffen sind: die Beulen am Körper der Erkrankten und die Mäuse- oder Rattenplagen, die als Überträger und damit auch Auslöser der Krankheit dem Aufkommen der Pest vorangehen. ${ }^{689}$

Das Problem bei der Heranziehung biblischer Texte für historische Zusammenhänge ist deren schlechte Datierbarkeit. Für die meisten Bücher des Alten Testaments können allenfalls Zeiträume und Eckdaten genannt werden, zwischen denen die geschilderte Handlung aufgrund bestimmter darin enthaltener Aussagen und Voraussetzungen angesiedelt sein muß. Dies gilt uneingeschränkt auch für die Samuelbücher, für die Reinhard KRATZ kürzlich zumindest ein historisches Gerüst formulierte. ${ }^{690}$ Demnach liegen die Ereignisse zwischen 720 und 597 v. Chr., der ersten Unterwerfung Jerusalems durch Nebukadnezar II., wobei eine zeitliche

685 Rathbone (1990: 114 ff.), akzeptiert von BAgnAlL und Frier (1994: 174); zu den Gründen des Strukturwandels auch ALSTON (2001: $165 \mathrm{f}$.).

686 SCHEIDEL (2001b: 31 Anm. 121), BAGNALL und FRIER (1994: 173 Anm. 17).

687 RATHBONE (1990: 119).

688 BLONDHEIM (1955).

689 ABERTh (2001: 112 f.); Herlihy (2000: 19 f.); das in der Bibel verwendete aramäische Wort bezeichnet gleichermaßen Mäuse und Ratten, vgl. dazu auch BLONDHEIM (1955: 337).

690 KRATZ (2000: 188). 
Nähe eher zum jüngeren Fixpunkt gegeben ist und damit zeitgleich zur ägyptischen Saïtenzeit (26. Dynastie) liegt. ${ }^{691}$ Ein Rückgriff der Bibelerzählungen auf ältere Überlieferungen aus dem 11. oder 10. Jahrhundert v. Chr. ist möglich, läßt sich aber nicht mehr nachweisen, so daß sich anhand dieser Quelle eine weitere Verschiebung in die Vergangenheit verbietet.

\section{Hinweise aus den medizinischen Papyri}

Auch aus Ägypten liegen Hinweise vor, die vereinzelt als Beschreibung von Pest-Symptomen interpretiert wurden. ${ }^{692}$ Insbesondere die Aussagen in einer Beschwörung des Medizinischen Papyrus Hearst, der in den Zeitraum um 1550 v. Chr. datiert, hat Hans GoEDICKE dazu bewegt, den dort als >Asiatische Krankheit< bezeichneten Fall als Beulenpest zu deuten. ${ }^{693}$

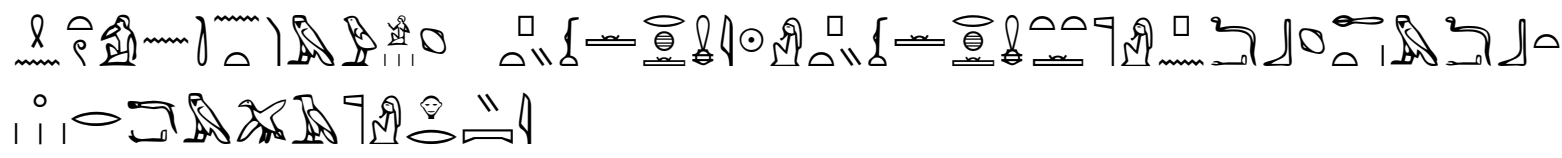

»Beschwörung der Asiaten-Krankheit: Wer ist allwissend wie Re? Wer ist ebenso allwissend? (Etwa) dieser Gott, der den Leib mit Holzkohle ankohlt? Ergriffen werde dieser >Oberste Gott< (d. h. unschädlich gemacht).«

Ansatzpunkt ist für GoEDICKE die Beschreibung des Körpers eines Erkrankten, der aufgrund des Vergleichs mit Holzkohle offenbar eine deutliche schwarze Verfärbung aufweist. ${ }^{694}$ Über die Identifizierung der Krankheit anhand des beschriebenen Symptoms herrscht jedoch keine Einigkeit. Während GOEDICKE aufgrund der Beschreibungen in Papyrus Hearst von der korrekten Deutung als Beulenpest überzeugt ist, vertritt WESTENDORF unter Berufung auf die Untersuchung von Thierry BARDINET die Identifikation der Asiaten-Krankheit mit Lepra. ${ }^{695}$ BARDINET analysiert zunächst die ägyptische Bezeichnung. Die Kreation eines eigenen Begriffs für die Krankheit wird von ihm in Verbindung gebracht mit dem Wissen um eine endemische Krankheit, die im syrisch-palästinensischen Gebiet grassierte und deren Kenntnis über Handelskontakte oder andere Wege zu den Ägyptern gelangte. Endemisch deshalb, weil nach BARDINETs Ansicht für ein singuläres Ereignis kaum ein neues Wort geschaffen worden

691 Kratz (2000: 331); Diese Angaben entstammen der theologischen Betrachtung der Texte und geben keinerlei Auskunft über den historischen Realitätsgehalt der Erzählung. Auf die Möglichkeit der Auswertung von einzelnen, dem Text entnommenen Beobachtungen hat dies jedoch keine Auswirkung.

692 Vgl. ausführlich GOEDICKE (1984a).

693 Hieroglyphentext nach GRAPOW (1958: 440); Erwähnung der >Asiatischen Krankheit< auch im Med. Pap. London (pBM 10059) 11,4-6; vgl. dazu GoEDICKE (1984a: 101 ff.) sowie WeSTENDORF (2000: 63).

694 GOEDICKE (1984a: 95).

695 WESTENDORF (2000: 62); WESTENDORF (1999: 311); BARDINET (1988); die Übersetzung folgt hier dem Vorschlag WESTENDORFs. 
wäre. ${ }^{696}$ Ein weiterer Grund für seine Identifikation könnten die beschriebenen Indizien für Hautverfärbungen sein, die im Fall von Lepra allerdings eher in Form von Depigmentierungen und dunklen Flecken auftreten. ${ }^{697}$

Darüber hinaus besteht auch Unklarheit über die Letalität, die von dieser Krankheit ausging. Es scheint kaum möglich, anhand der vagen Beschreibungen ein klares Bild darüber zu bekommen, wie sich die Verbreitung der Krankheit auf die demographische Entwicklung auswirkte, zumal keinerlei Kenntnisse über die Ansteckungskraft der Erreger (Kontagiosität) vorliegen. Sollte die >Asiatische Krankheit< tatsächlich mit Lepra gleichgesetzt werden können, so muß die Ansteckungsmöglichkeit wie auch die Letalität als gering eingeschätzt werden, gleichwohl könnte eine weitläufige Verbreitung vorgelegen haben. GOEDICKE spricht im Zusammenhang mit Indizien für Massenbestattungen gegen Ende der Hyksoszeit von der Erkrankung des ägyptischen Heeres, das die Seuche in das hethitische Reich brachte. ${ }^{698}$

\section{Was steht in den Amarnabriefen?}

Einen weiteren Hinweis auf den Ausbruch von Seuchen zur Zeit des Neuen Reiches - jedoch nicht unbedingt in Ägypten - hat man bisher aus einzelnen Berichten in den sogenannten >Amarnabriefen< gelesen. Die Amarnabriefe bezeichnen die Textgruppe diplomatischer Korrespondenz zwischen dem ägyptischen König Echnaton und verschiedenen Vasallenkönigen. Sie sind auf Tontafeln geschrieben und bedienen sich der akkadischen Keilschrift, die gleichsam die Diplomatensprache jener Zeit darstellte, der auch die ägyptischen Schreiber unterworfen waren. Die Liste der bekannten und bearbeiteten Texte umfaßt mittlerweile mehrere Hundert Tafeln.

Das wichtigeste Dokument aus den Armanabriefen stellt der Text EA 35 dar, in dem der König von Zypern über die verheerenden tödlichen Auswirkungen einer Seuche in seinem Land berichtet, daß »Nergal die Menschen getötet habe«. Auch der Sohn des Königs ist unter den Opfern. ${ }^{699}$ William MORAN übersetzt die fragliche Textpassage wie folgt: ${ }^{700} \gg$ Behold,

696 Eine andere, von BARDINET nicht berücksichtigte Voraussetzung für die Schaffung eines eigenen Begriffs könnte die verheerende Auswirkung einer solchen Krankheitswelle sein, die beispielsweise eine hohe Letalität mit sich bringt. Auch in solchen Fällen, die bereits als Nachricht in nicht betroffenen Regionen Schock und Bestürzung auslösen können, wäre die Neuschöpfung eines Wortes möglich.

697 Pschyrembel, s. v. Lepra.

698 Goedicke (1984a: 92); GoEdiCKE (1984b); zum Vorwurf der Hethiter gegen die Ägypter auch HELCK (1971: 183). Siehe auch dazu oben S. 181 zum hethitischen >Pestritual<.

699 HelcK (1971: 183); dagegen identifiziert MorAn (1992: 109 Anm. 9) die betroffene Person als junge Gemahlin des Herrschers.

700 MORAN (1992: 107 ff.). Es bleibt laut MORAN unklar, ob ${ }^{d}$ MAŠ.MAŠ tatsächlich Nergal bezeichnet, oder den westsemitischen Gott Reschef oder gar einen zyprischen >Pestgott<; für HELLBING (1979: 21 ff.) ist die Identifikation mit Reschef aufgrund der geographischen Nähe von Herkunft des Textes und Verehrungsort 
the hand of Nergal is now in my country; he has slain all the men of my country, and there is not a (single) copper-worker. « Die Betonung auf den Verlust der Kupferarbeiter liegt im eigentlichen Zweck des Briefs begründet, da der zyprische König sich nicht in der Lage sah, eine größere Menge Kupfer zu liefern und als Entschuldigungsgrund dafür die in seinem Land grassierende Seuche angab.

Auch ein Fürst in Megiddo befürchtet, daß seine belagerte Stadt erobert wird, falls die Bewohner an der Seuche zugrunde gehen (EA 244,31). ${ }^{701}$ Ob dies nur als Schwächung aufgefaßt werden kann, oder tatsächlich der Tod aller Stadtbewohner gemeint ist, läßt sich nicht sicher entscheiden. Andere Auszüge aus der Amarnakorrespondenz haben sich hinsichtlich ihrer Übersetzungen mit Pestbezug mittlerweile als nicht mehr haltbar erwiesen. Dazu gehören EA $16,43^{702}$ und EA $11,14^{703}$, die beide inhaltlich keinerlei Bezug auf Seuchen oder daruf zurückzuführende Todesfälle nehmen. $\mathrm{Ob}$ die in den Amarnabriefen erwähnten Epidemien tatsächlich auch ihren Weg nach Ägypten fanden, ist nicht eindeutig festzustellen. Sowohl Wolfgang HELCK als auch Donald REDFORD spekulieren dahingehend aufgrund der gehäuften Todesfälle in Echnatons Familie zum Ende seiner Regierungszeit. ${ }^{704}$ Doch fehlt ein letzter sicherer Beweis für diese Hypothese.

Es bleiben zusammengefaßt nur das Indiz aus EA 35 und der Hinweis GoEDICKEs, daß die Epidemien auch in der Levante im großen Umfang Todesfälle verursachten. Dies scheint jedoch zu wenig, um eine sichere Identifikation der Krankheit zu ermöglichen oder gar Analogien zu den Sterberaten im Mittelalter zu ziehen, da selbst letztere immer noch Basis heftiger Diskussionen sind. ${ }^{705}$

\section{Die Einträge in den Tagewählkalendern}

Eine weitere vermeintliche Erwähnung der Pest in einem altägyptischen Text hat zuletzt Christian LEITZ aus den Tagewählkalendern herausgearbeitet. Dabei handelt es sich um kommen-

der Gottheit eindeutig. Darin steckt auch eine gewisse Ambivalenz, da Reschef an seinem Ursprungsort in Ugarit sowohl Gott der Seuchen als auch der Fruchtbarkeit und der Heilung war. Für den Zusammenhang zwischen den Göttern Nergal und Reschef sind versuchsweise weitere ägyptische Textquellen herangezogen worden. HELCK setzt Reschef auch in Ägypten mit der >Pest< in Beziehung und verweist dabei auf den magischen Papyrus Leiden XI, in dem vom >Gift des Reschef< gesprochen wird, was HELCK (1971: 450 Anm. 34) als singuläre Anspielung auf dessen Eigenschaft als Pestgott auffaßt, weitergehende Evidenz dafür aber schuldig bleiben muß.

701 MORAN (1992: 298 f.): »Look, the city is consumed by pestilence«; zur gleichen Textstelle auch HELCK (1971: 183).

702 HelcK (1971: ebenda); jetzt: MoRAn (1992: 41 Anm. 16).

703 HELCK (1971: ebenda); jetzt: MORAN (1992: 21 ff.).

704 HELCK (1971: ebenda); REDFORD (1984: 187).

705 BENEDICTOW (1987). 
tierte Kalender, die gute Tage und schlechte Tage benennen und darüber hinaus Maßnahmen zu vorsichtigem Handeln beschreiben. Der Beleg, der zur Frage der Pest im Zentrum des Interesses steht, stammt vom Recto des Papyrus Sallier 4 und datiert in den Beginn der 19. Dynastie ${ }^{706}$ womit eine zeitliche Nähe zu den in den Amarnabriefen beschriebenen Ereignissen gegeben ist. Für den 12. Tag des 1. prt wird folgende Warnung ausgesprochen: ${ }^{707}$

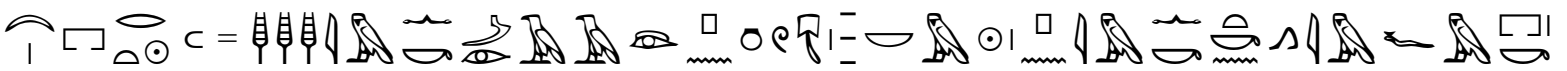

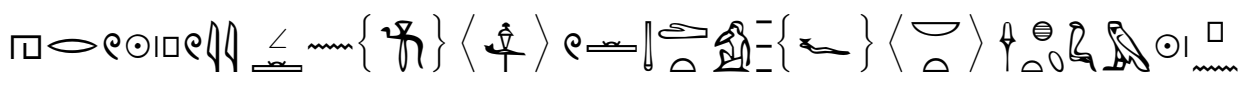

1. prt sw $12{ }^{c} h^{c} h^{c} h^{c} j m=k m 33$ pnw.w $n b m$ hrw pn jm=k tkn jm=fm pr.w=k hrw pwy $n$ hsf $m d w t$ nb.t Shm.t m hrw pn

»1. Monat der Aussaat, Tag 12: Ungünstig! Ungünstig! Ungünstig! Du sollst keine Ratten sehen an diesem Tag. Du sollst dich ihr nicht nähern in deinem Haus. Jener Tag, an dem man alle Angelegenheiten der Sachmet abwehrt an diesem Tag.«

Die ausdrücklichen Warnung vor den Ratten und die gleichzeitigen Erwähnung von Sachmet als Göttin der Seuchen stellen nach LEITZ Indizien für eine Bezugnahme auf die Beulenpest dar, die er mit der $j 3 d t$ rnp.t identifiziert. Als weitere Gründe nennt er: ${ }^{708}$

1. Die Verbreitung der Seuche durch Sachmet oder ihre Boten.

Sachmet kann als >Herrin der Krankheit< generell mit dem Ausbruch von Seuchen in Verbindung gebracht werden. Mehrfach wird sie im medizinischen Papyrus Edwin Smith im Zusammenhang mit der j3dt rnp.t erwähnt. ${ }^{709}$ Auch in der Erzählung des Sinuhe wird sie mit dem Grassieren einer Seuche in Verbindung gebracht:

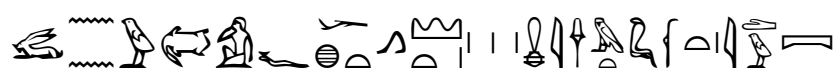

wnn.w snd $=$ f ht h his.wt mj Shm.t rnp.t j3dw

»Seine Furcht ist in allen Fremdländern, wie Sachmets jährliche Seuche.«

Zusammen mit dem Süd- und Westwind bringt Sachmet die jährliche Seuche über das Land. ${ }^{710}$ Im Falle einer Identifikation des Südwindes mit dem jährlichen Chamsin könnte diese Angabe auch auf eine Verbindung mit Tuberkulose hindeuten, wie Vergleiche

706 LEITZ (1994: 6).

707 Text nach der Bearbeitung von LEITZ (1994: 205) mit den durch pKairo JE 86637 verbesserten Lesungen.

708 LEITZ (1994: 207 f.).

709 Westendorf (1999: 742 ff.); zu den medizinischen Aufgaben der Sachmetpriester vgl. auch ausführlich ENGELMANN und HALLOF (1996).

$710 \operatorname{KEES}(1977: 13)$. 
mit zeitgenössischen Berichten des 19. Jahrhunderts zeigen. ${ }^{711}$ Die Beschwerden der Betroffenen wurden stärker und resultierten in der Folge in einer höheren Mortalität. ${ }^{712}$

2. Die Daten der Seuche im jahreszeitlichen Verlauf der Nilschwemme.

Zur saisonalen Sterblichkeit siehe unten ausführlich Kap. 6.3.1.4

3. Die Übertragung der Seuche durch die Pfeile der Sachmet.

Obgleich die subjektive Wahrnehmung der Schmerzsymptome durch die Betroffenen, die sich wie durch einen Pfeil getroffen fühlen, gut mit der Beschreibung des Handelns der Sachmet in Einklang zu bringen ist, so scheinen die Pfeile schießende und Feuer speiende Göttin doch nur aus dem kriegerischen Aspekt der Sachmet übernommene Metaphern. ${ }^{713}$

Darüber hinaus bestehen auch allgemeinere Probleme einer genauen Identifikation der hinter der Bezeichnung j3dt-rnp.t stehenden Krankheit. Das Medizinische Wörterbuch übersetzt $j 3 d t$ (rnp.t) allgemein als >Plage, Seuche< und erklärt den Zusatz rnp.t dahingehend, daß jede Seuche im Laufe eines Jahres damit gemeint ist. ${ }^{714}$ Dies ist jedoch kaum wahrscheinlich, da hier durch ein zusätzliches spezifizierendes Wort eine Unterspezifizierung der Aussage entsteht. Es wird kaum derart viele verschiedene in jährlicher Regelmäßigkeit wiederkehrende Epidemien gegeben haben, daß ein allgemeiner Oberbegriff für alle Epidemien benötigt wurde. In Zusammenhang mit den anderen zuvor dargelegten Vorbehalten sollte die Gleichsetzung von j3dt rnp.t mit der Pest noch nicht als bewiesen akzeptiert werden.

\subsubsection{Saisonale Sterblichkeitsraten}

Aufgrund der Erkenntnisse zum Verlauf von Infektionskrankheiten innerhalb eines Jahreszyklus' aus der jüngeren Medizingeschichte wurde immer wieder der Versuch einer Korrelation zwischen den altägyptischen Hinweisen auf Epidemien und den modernen Daten unternommen. SCHEIDEL schreibt zu den saisonalen Schwankungen: ${ }^{715}$

$»[\ldots]$ because infectious diseases are responsible for the majority of deaths in high-mortality populations, and the incidence of many major infections varies considerably depending on the season, seasonality patterns may reflect the underlying disease environment.«

711 SCHEIDEL (2001a: 92 Anm. 259).

712 SCHEIDEL (2001a: ebenda Anm. 260).

713 Sternberg (1984: 325 f.); vgl. zum Bild der Pfeile Gottes auch die Beschreibung in Homers Ilias I,49-52, in der Apollon erst die Tiere, dann auch die Achäer selbst mit todbringenden Pfeilen trifft.

714 MedWb 21.

715 SCHEIDEL (2001b: 25). 
Auch wenn die Sterblichkeitsraten großer Epidemien der jüngeren Zeitgeschichte besser überschaubar sind, so bleiben auch diese eine schwankende und keinesfalls eindeutige Datenbasis. Darüber hinaus kann etwa die Pest nicht als >jährliche Seuche< bezeichnet werden, da sie eher in unregelmäßigen Schüben auftritt und dann regelmäßige Perioden von etwa fünf bis zehn Jahren durchläuft. ${ }^{716}$ Richtig ist jedoch die Beobachtung einer gewissen regelmäßigen Wiederkehr in darauf folgenden Jahren, sobald die Seuche einmal ausgebrochen ist. ${ }^{717}$ Auch innerhalb des Jahreslaufes lassen sich für die Pest regelmäßige Verlaufsmuster nachweisen. Nach David HERLIHY weisen die mittelalterlichen Epidemien einen deutlichen Jahreszyklus auf. Die Epidemie gewann an Stärke bei zunehmend warmen Wetter mit einem Höhepunkt im Spätsommer oder Frühherbst. Im kalten Wetter des Spätherbstes und Winters gingen die Epidemien dagegen deutlich zurück. ${ }^{718}$ Ähnliches ist für die Epidemien Ägyptens im 19. Jahrhundert beobachtet worden. Ausgehend von den optimalen klimatischen Bedingungen für Rattenflöhe gibt Laverne KUHNKE eine Tabelle (6.1) für einen jahreszeitlich versetzten Verlauf der Pest in verschiedenen Regionen Ägyptens an die Hand. ${ }^{719}$ Dabei spielt auch die Erkenntnis eine Rolle, daß sowohl starke Hitze im Sommer genauso wie die Kühle der Wintermonate für die Ausbreitung der Pest ein Hemmnis bedeutet.

\begin{tabular}{|c|c|c|c|}
\hline Region & Anstieg & Höhepunkt & Abflauen \\
\hline Oberägypten & März & April & Mai \\
\hline Mittelägypten & April & Mai & Juni \\
\hline Delta und Suez & April & Juni & Juli \\
\hline Mittelmeerhäfen & Mai & Juli & Oktober \\
\hline
\end{tabular}

Tabelle 6.1: Die saisonale Verbreitung der Pest im modernen Ägypten

Zeitgenössische Berichte über das Ergreifen von Quarantänemaßnahmen verraten, daß die Epidemie typischerweise im Januar in einer der ägyptischen Hafenstädte ausbrach, ${ }^{720}$ was europäische Beobachter vor Ort vermutlich zu der falschen Annahme verleitet hat, die Pest würde ihren Ursprung in der Türkei, Kleinasien, Syrien oder Kreta nehmen und durch Handelsschiffe in die ägyptischen Häfen eingeschleppt werden. ${ }^{721}$

716 ECKERT (2000: 4).

717 Vgl. zu den in kurzer Folge über den Nahen Osten hereingebrochenen Wellen DoLS (1974) und KUHNKE (1990: $69 \mathrm{ff}$.$) .$

718 HERLIHY (2000: 20).

719 KuhnKe (1990: 73 Anm. 18); auch Scheidel (2001a: 97, Tab. 1.12).

720 KUHNKE (1990: 77).

721 KUHNKE (1990: 73). 
Bereits aus den Jahren 1798/99 und 1801 liegen uns Berichte über die Auswirkungen der Epidemie in Ägypten vor. Der Chronist Al-Jabarti hat Beschreibungen über die Ereignisse dieser Zeit zusammengestellt. Während 1798/99 Alexandria von der Pest schwer getroffen wurde, wütete die Epidemie zwischen 1800 und 1801 besonders in Kairo. Es wird berichtet, daß im April täglich durchschnittlich 100 Menschen starben, 2937 Menschen im gesamten Monat. ${ }^{722}$ Auch Oberägypten blieb nicht verschont und Augenzeugen berichten aus der Stadt Assiut, in der sogar mehr als 600 Personen täglich starben. Al-Jabarti fügt seinem Bericht den Brief eines Freundes aus Oberägypten bei, der vermutet, daß die Pest etwa 2/3 der Bevölkerung getötet hat. Auch im November des Jahres 1834 brach die Pest in Alexandria aus. ${ }^{723}$ Den Höhepunkt erreichte sie Ende März 1835, als etwa 180 bis 200 Tote täglich zu beklagen waren. Dabei fand die Epidemie in diesem Jahr ihren Weg auch in Regionen, die bisher nur selten von Seuchen heimgesucht worden waren. Sie reichte nach Oberägypten bis Theben und im Westen bis in das Fajjum. ${ }^{724}$ Mitte April nahm die Zahl der Todesfälle in Alexandria wieder rapide ab und sank unter ein Niveau von 100 Toten am Tag. Zur gleichen Zeit jedoch steuerte die Epidemie in Kairo auf ihren Zenith zu und die Bewohner der Stadt mußten täglich offenbar zwischen 600 und 1000 Tote beklagen. KUHNKE zitiert dazu Augenzeugenberichte eines Europäers namens Arthur Kinglake, der allem Anschein nach während des Höhepunkts der Epidemie in Kairo weilte. Er berichtet davon, daß während der 19 Tage seiner Anwesenheit die Sterblichkeitsrate von 400 auf 1200 Menschen täglich anstieg. ${ }^{725}$ Wenn man diese beiden genannten Werte als Minimum und Maximum nimmt und einen (hypothetischen) täglichen linearen Anstieg errechnet, ergibt das für den genannten Zeitraum eine Summe von 14800 Toten. Offiziell wurde das Ende der Epidemie am 30. Oktober 1837 verkündet - 40 Monate nach ihrem Ausbruch im Juli $1834 .{ }^{726}$

Berichte wie die zuvor für die Epidemien von 1800/01 und 1834/35 genannten, dienen LEITZ als weiteres Indiz für die Identifikation der altägyptischen $j 3$ dt rnp.t mit der Pest. Kern der Argumentation sind parallele Kurvenverläufe der Sterbeziffern mit übereinstimmenden Spitzenwerten zwischen den Monaten März bis Mai. Abgeglichen werden diese Daten von LEITZ mit den Sterbedaten von Mumienetiketten, die ebenfalls auf saisonale Unterschiede der Sterblichkeitshäufigkeit hinweisen und einen deutlichen Anstieg im Frühjahr in den Mo-

722 KuhnKe (1990: 76); SCHEIDEL (2001a: 98 Anm. 285).

723 KuhnKe (1990: 79); genaue Zahlen zu den monatlichen Todesfällen bei LEITZ (1994: 207 Anm. 20).

724 KUHNKE (1990: 83).

725 KUHNKE (1990: 84).

726 KUHNKE (1990: 86). 


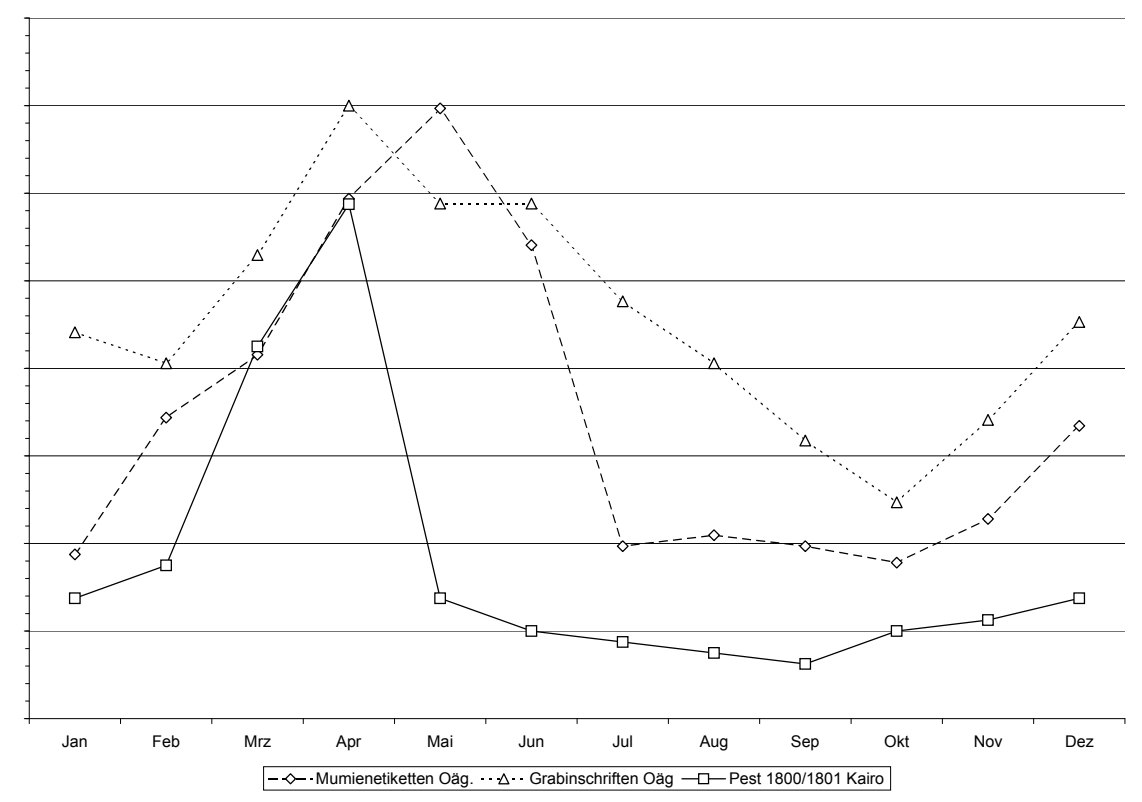

Abbildung 6.1: Vergleichende Verlaufskurve zur Mortalität

naten фармоүөı und пахџн verzeichnen. ${ }^{727}$ Erst kürzlich hat sich auch SCHEIDEL erneut der Auswertung der Mumienetiketten angenommen und aus dem vorliegenden Material saisonale Schwankungen der Mortalität herausgearbeitet. Dabei hat er auch Korrekturen an den bisherigen Interpretationen zur Auswertung der Datumsangaben auf den Etiketten vornehmen können, was zu chronologischen Verschiebungen um mehr als zwei Monate (70 Tage) führt. ${ }^{728}$ Die von SCHEIDEL präsentierten Daten weisen eine markante Übereinstimmung zwischen den Kurvenverläufen aus Grabinschriften und Mumienetiketten einerseits und dem Bericht über die Sterbefälle der großen Pestepidemie der Jahre 1800 und 1801 andererseits (Abb. 6.1) auf. $^{729}$

Geschmälert wird die Aussagekraft dieses Vergleichs durch die unterschiedliche Herkunft der Daten. Während die Quellen des frühen nachchristlichen Ägypten im wesentlichen aus Oberägypten vorliegen, stammen die Angaben zur Pest aus den Jahren 1800/01 aus Kairo.

727 LEITZ (1995: 15 Anm. 57).

728 SCHEIDEL (1998).

729 Für das Diagramm wurden griechische und koptische Quellen zusammengefaßt. SCHEIDEL (2001a: 5) gibt als Datierung für die griechischen Grabstelen das 4.-7. Jh. n. Chr. und für die koptischen das 8.-10. Jh. n. Chr. an. Mumienetiketten sind nur selten absolut datierbar, so daß im wesentlichen die darauf enthaltenen Tages- und Monatsangaben für eine relative Chronologie herangezogen wurden. Unberücksichtigt bleibt im Diagramm die Verschiebung der koptischen Monate gegen den modernen Kalender um etwa 8 bis 10 Tage, was für die Argumentation vernachlässigbar ist. 
Die frühchristlichen Grabinschriften aus Unterägypten weisen dagegen eine starke Streuung auf und können mit den anderen Kurven nicht in Deckung gebracht werden. ${ }^{730}$ Auch kann die unterschiedliche Datierung des Datenmaterials den Wert der Argumentation weiter verringern. Während die Erwähnung der j3dt rnp.t aus den Tagewählkalendern aus der 19. Dynastie stammen, sind die Grabinschriften und Mumienetiketten bereits ungefähr 1600 Jahre jünger. Weitere 1000 Jahre später sind die Daten der ägyptischen Pestepidemien gewonnen worden. Der Verlauf von Epidemien einer bestimmten Krankheit zwischen erstem Auftreten und Abflauen im Zeitraum eines Jahres ist - soweit vergleichbare Hinweise dafür vorliegen - zwischen Altertum und Moderne in Ägypten nicht zwangsläufig identisch und kann daher nicht generell als Identifikationskriterium herangezogen werden. ${ }^{731}$ Keinesfalls kann die absolute Datierung des Tagewählkalenders in den Zeitraum zwischen dem 24.9. und dem 22.11. mit den modernen Daten in Einklang gebracht werden. ${ }^{732}$

Inwieweit die überlieferten Zahlenangaben zur Mortalität während der Pestschübe der Wahrheit entsprechen, läßt sich nur schwer einschätzen. Allein die häufig widersprüchlichen Zahlen zwischen offiziellen Verlautbarungen und den Berichten unabhängiger Beobachter zeigen das Dilemma bei der Suche nach verläßlichen Quellen. ${ }^{733}$ Bereits die Überlieferungen des islamischen Mittelalters zur Zahl der Pesttoten wurden von zeitgenössischen Chronisten einer kritischen Prüfung unterzogen und gelegentlich als >bloße Vermutung $<$ und >Spekulation $<$ bezeichnet. ${ }^{734}$ Diese Situation hatte sich offenbar bis in das 19. Jahrhundert nicht verbessert. Neben den unregelmäßigen Ausbrüchen der Pest blieben auch andere Todesursachen als ständige Bedrohung der Bevölkerung unvermindert bestehen. Die frühen Statistiken sind daher das Abbild mangelhafter Differenzierungsmöglichkeiten oder auch schlicht falscher Diagnosen, so daß auf die detaillierten Einzeldaten in der Regel kein Verlaß ist. ${ }^{735}$

Als nahezu völlig unmöglich gestaltet sich schließlich das Unterfangen, Sterblichkeitsraten für das Alte Ägypten zu formulieren. Da im Einzelfall nicht einmal die Krankheit selbst zuverlässig identifizierbar ist, können auch keine Rückschlüsse auf die demographischen Einschnitte solcher Epidemien gezogen werden - sofern sie sich überhaupt als solche ansprechen lassen. Immerhin liegt für die Krankheitswellen der zweiten Hälfte des 2. Jahrhunderts n. Chr.

730 SCHEIDEL (2001a: $20 \mathrm{f}$.$) .$

731 SCHEIDEL (2001a: 113).

732 Zur Umrechnung der Datierung in heutige Datumsangaben zuletzt WESTENDORF (2000: 60); m. E. favorisiert LEITZ die Peulenpest-Hypothese doch stärker, als ihm WESTENDORF zubilligt.

733 KuHnKe (1990: 85 f.).

734 AYALON (1985: 10) zitiert den Kommentar des Historikers Ibn Taghrībirdī zur Epidemie des Jahres 1459/60.

735 SCHEIDEL (2001a: $105 \mathrm{ff}$.). 
indirekte Evidenz dafür vor, daß die Epidemie tiefgreifende Auswirkungen auf Wirtschaft und Gesellschaft jener Zeit hatte. Davon zeugen unter anderem Produktionslisten von Gütern des täglichen Bedarfs oder Münzemissionen, die eine deutliche Verringerung zeigen. ${ }^{736}$ Unter der Voraussetzung einer regelmäßigen Wiederkehr derartiger Epidemien darf daher auch in Unkenntnis ihrer tatsächlichen Ursache von einem spürbaren Druck auf die Bevölkerungsentwicklung ausgegangen werden - ganz analog zu den Beobachtungen, die aus anderen Epochen vorliegen. ${ }^{737}$

\subsubsection{Andere Infektionskrankheiten}

Trotz der eher vagen Angaben dürften die antiken Hinweise auf die Pest noch zu den besseren Indizien zählen. Weitaus schlechter ist die Überlieferungssituation für andere endemische und epidemische Krankheiten, deren Letalität zum Teil noch heute medizinisch nicht gebannt ist. Ein dabei nicht zu unterschätzender Faktor ist die Urbanität und damit verbunden eine hohe Bevölkerungsdichte in bestimmten begrenzten Gebieten, die eine sich rasch ausbreitende Ansteckung und damit explosionsartige Vermehrung eines Krankheitsausbruchs begünstigen. Insbesondere Kairo und Alexandria dürfen als Keimzellen der Epidemien des 19. Jahrhunderts gelten, denen jedoch keine vergleichbaren Strukturen des pharaonischen Ägypten gegenüberstellbar sind. ${ }^{738}$ Damit ist die Inzidenzrate (Anzahl der Neuerkrankungen innerhalb eines bestimmten Zeitraumes bezogen auf eine Gesamtzahl möglicher betroffener Personen) für Epidemien im Alten Ägypten praktisch nicht nachvollziehbar. Im folgenden soll die Evidenz der wichtigsten Krankheitsformen für das Alte Ägypten kurz skizziert werden.

Pocken: Obwohl die Existenz von Pocken (Variola) für die Antike nachgewiesen ist, bleibt die Situation für das Alte Ägypten weitgehend ungeklärt. Der einzige ernstzunehmende Hinweis scheint an der Mumie Ramses' V. vorzuliegen, an der Hautvernarbungen festgestellt worden sind. Doch auch diese Indizien sind wissenschaftlich noch umstritten. Alle anderen Befunde, die angeblich an Mumien nachgewiesen wurden, konnten keiner kritischen Überprüfung standhalten. Auch aus den medizinischen Papyri läßt sich kein Fall mit der Existenz von Pocken in Verbindung bringen. ${ }^{739}$ Der Nachweis von Pocken in der Antike läßt sich möglicherweise durch die vom Historiker Thukydides

736 SCHEIDEL (2001b: 30 f. Anm. 122).

737 Dols (1974: 381 f.).

738 Zusammenfassend auch SCHEIDEL (2001a: 114 ff.).

739 NUNN (1996: 77); ADAMSON (1982: 10). 
beschriebene große Seuche in Athen im Jahre 430 v. Chr. weiter in die Vergangenheit schieben, deren Identifikation mit Pocken mittlerweile breite Zustimmung findet. ${ }^{740}$ Es fällt jedoch schwer, daraus eine regelmäßige Wiederkehr der Erkrankungswelle zu ermitteln, da auch nach 430 v. Chr. die Pocken sich im Mittelmeerraum offenbar nicht etablierten. Aufgrund der Vergleiche mit dem neuzeitlichen Ägypten, in dem Pockenwellen hauptsächlich die Kinder der ersten Lebensjahre betrafen, insbesondere bei ansatzweiser Unterernährung, müßten auch die Endemien der Antike eine signifikante und damit beobachtbare Auswirkung gehabt haben. Die wenigen Indizien für jahreszeitlich abhängige Sterberaten lassen sich hingegen mit den Erkenntnissen über die Pockenausbreitung nicht in Einklang bringen und sind darüber hinaus im wesentlichen an Erwachsenen beobachtet worden. ${ }^{741}$

Tuberkulose: Auch diese durch Bakterien verursachte Infektionskrankheit, die vor allem in den Atemorganen lokalisiert ist, wurde in Einzelfällen an Mumien und Skelettfunden des pharaonischen Ägypten nachgewiesen. ${ }^{742}$ Die Evidenz bezieht sich dabei auf Körperveränderungen durch verschiedene Stadien von Immunität, die nur dadurch ausgelöst werden kann, wenn der Körper der Krankheit unmittelbar ausgesetzt war. ${ }^{743}$ Auch den medizinischen Papyri der koptischen Zeit können Hinweise auf das Spucken von Blut (Hämoptyse) als ein mit Tuberkulose in Verbindung zu bringendes Symptom entnommen werden, jedoch ohne Information über dessen Ursache. ${ }^{744}$

Typhus: Neben dem durch Salmonellen verursachten und schon seit der Antike bekannten Typhus abdominalis ${ }^{745}$ wird in der modernen Forschung in der Regel auf die durch Läuse übertragene epidemische Form Typhus exanthematicus, auch Fleckfieber gennant, Bezug genommen. Im 2. Jahrtausend v. Chr. sollen syrische Kriegsgefangene die Seuche bei den Hethitern eingeschleppt haben, ${ }^{746}$ was jedoch keinesfalls allgemein akzeptiert ist. ${ }^{747}$ Die nachgewiesene Existenz von Läusen reicht - wie auch bei den Ratten für

740 SCHEIDEL (2001a: 95); ganz anders dagegen LEVEN (2000), der den literarischen Aspekt von Thukydides' Schilderungen betont und kein Urteil über die medizinischen Hintergründe der Epidemie fällen mag.

741 SCHEIDEL (2001a: 96 f.).

742 SCHEIDEl (2001a: 91 f.); der direkte Nachweis des Bazillus ist erst kürzlich an einer Mumie des Neuen Reiches gelungen; vgl. WESTENDORF (1999: 460 f.).

743 AdAMSON (1982: $8 \mathrm{f}$.)

744 TILl (1951: 22); Hinweise aus medizinischen Papyri der pharaonischen Zeit fehlen gänzlich; vgl. NUNN (1996: 73 f.).

745 SCHEIDEL (2001a: 70 Anm. 168).

746 ADAMSON (1982: 9 f.).

747 SCHEIDEL (2001a: 68) weist darauf hin, daß nicht einmal grundsätzlich die Existenz von Typhus exanthe- 
die Pest - als Argument nicht aus. ${ }^{748}$ Die von P. B. ADAMSON aufgeführten Quellen beziehen sich ohnehin ausschließlich auf den assyrischen Raum, während für das Alte Ägypten vergleichbare Überlieferungen fehlen. Der früheste Nachweis eines Ausbruchs der Krankheit in Ägypten stammt erst aus dem 16. Jahrhundert. ${ }^{749}$ Deshalb gehen neuere Arbeiten zur altägyptischen Medizin wie NunN (1996) oder WESTENDORF (1999) mit gutem Grund überhaupt nicht auf diese Krankheit ein.

Lepra: Diese Hautkrankheit wird durch Bakterien verursacht und verursacht Störungen des Stoffwechsels, Lähmungen und Verstümmelungen. Der Beweis ihrer Existenz aus biblischen ${ }^{750}$ wie auch aus pharaonischen Quellen ${ }^{751}$ bleibt umstritten. Für Mumien aus pharaonischer Zeit gelang kein Nachweis, lediglich eine koptische Bestattung des 6. Jahrhunderts n. Chr. in El-Bigha wird mit Lepra in Verbindung gebracht, obwohl auch in diesem Fall der Erreger nicht nachgewiesen werden konnte. Die medizinischen Texte sind im Fall der Lepra ebenfalls nicht eindeutig. Die Beschreibungen des Papyrus Hearst sind ebenso mehrdeutig wie die zwei Fälle des Papyrus Ebers, die von den >Geschwüren des Chons < sprechen. ${ }^{752}$ In beiden Fällen kommen verschiedene Ursachen für die Symptome in Frage, wie etwa Krebsgeschwüre oder auch Pestbeulen.

\subsection{Hungersnot}

\subsubsection{Nahrungsmangel als demographischer Faktor?}

Das Thema Hungersnot hat seit langem die ägyptologische Forschung beschäftigt. Dies lag zu einem nicht unerheblichen Teil auch daran, daß für alle Epochen altägyptischer Geschichte Belege existieren, die auf einen - wie auch immer gearteten - Nahrungsmangel hinweisen. ${ }^{753}$ Auch die Historiker bedienten sich der Hungersnöte und formulierten eine Theorie, nach der die Hungersnöte als Folge zu niedriger Nilstände am Ende der 6. Dynastie zu einem Zusammenbruch des Staatssystems führten und den Übergang in die Erste Zwischenzeit markierten.

maticus für die Antike gesichert ist.

748 AdAmson (1982: 10 Anm. 24).

749 SCHEIDEL (2001a: 67).

750 AdAMSON (1982: 10 Anm. 29).

751 NUNN (1996: 74 f.).

752 Zur Identifikation der Beschreibungen in pHearst mit Lepra vgl. auch oben S. 187 Anm. 695; pEbers 874 und 877.

753 Ausführliche Zusammenstellung der Belege bei VANDIER (1936), neuerdings ergänzt von MoRENZ (1998). Zur Problematik der Datierbarkeit einzelner Ereignisse vgl. SCHENKEL (1978: 39). 
Hauptvertreter dieser Theorie war neben Karl BUTZER ${ }^{754}$ besonders Barbara BELL, ${ }^{755}$ deren Thesen jedoch keiner kritischen Prüfung standhielten. Die Argumente, die im einzelnen gegen die Ausführungen BELLs stehen, sind bereits ausführlich dargelegt worden und brauchen an dieser Stelle nicht nochmals wiederholt zu werden. ${ }^{756}$ Der Kernpunkt der Kritik lag in BELLs fehlgeschlagenem Versuch, ein naturwissenschaftliches Argument mangels geologischer Fakten mit Textbelegen aus dem Alten Reich und der Ersten Zwischenzeit beweisen zu wollen. Ohne notwendige Erfahrung im Umgang mit altägyptischen Texten verstrickte sie sich dabei jedoch in der vordergründigen Interpretation der dort vorgefundenen Aussagen. Die Problematik der ideologisch kontaminierten Überlieferungen hat zuletzt Ludwig MoRENZ deutlich angesprochen: ${ }^{757}$

»Mit einer idealen Fülle an Informationen ausgestattet, könnte ein Historiker versuchen, lokale, regionale und überregionale Hungersnöte sowie deren anthropogene und/oder klimatische Gründe zu bestimmen versuchen, doch genau dies lassen die insgesamt sehr wenigen und außerdem >ideologisch so stark geformten sprachlichen Daten aus der Ersten Zwischenzeit eben nicht zu.«

Zumindest kann aus verschiedenen Abbildungen des Alten und Mittleren Reiches auf eine prinzipielle Kenntnis der Ägypter geschlossen werden, welche physischen Folgen Mangelernährung mit sich brachte. ${ }^{758}$ Die abgemagerten Körper, deren Rippen sich am Brustkorb abzeichnen sowie die schlaff herabhängenden Brüste der Frauen sind typische Indizien für schwere Unterernährung, wie sie auch heute noch in Weltregionen mit Hungersnöten zu beobachten sind. Die Ägypter mußten also mit einer solchen Situation und ihren Konsequenzen für die Menschen vertraut gewesen sein. Daran ändert auch nichts die Interpretation dieser Darstellungen als Topos, der den Kontrast zwischen den notleidenen Fremdländern und dem reichen Ägypten beschreibt, wie überhaupt niemals Ägypter selbst in dieser Form dargestellt worden sind. ${ }^{759}$

Grundlage aller Betrachtungen zur Ernährung ist die prinzipielle Frage, wieviel Nährstoffe (Kalorien) ein menschlicher Körper täglich benötigt, um zu überleben. Für die heutige Zeit sind dazu verschiedene Durchschnittswerte errechnet worden. Es gibt eigene Tabellen für die USA, Großbritannien und eine Standard-Tabelle der >Food and Agriculture Organization<, wobei letztere die höchsten Werte vorgibt. Die Bevölkerung wird in vier Gruppen eingeteilt:

754 BUTZER (1976: 27 ff.).

755 BELL (1971).

756 Zur Kritik an den Thesen BelLs zuletzt ausführlich MüLleR-WoLleRMANN (1986: 106 ff.).

757 MORENZ (1998: 95).

758 SCHOTT (1965); BLACKMAN (1915: Taf. 3).

759 Guglielmi (1980: 83). 
Kinder (1-7 Jahre), Jugendliche, Erwachsene und Senioren, diese Gruppen wiederum werden getrennt nach Männern und Frauen gelistet. Die Spannweiten der Altersangaben differieren zwischen den drei Tabellen, genauso wie das durchschnittliche Gewicht eines erwachsenen Mannes und einer Frau. Dazu kommen noch unterschiedliche Umweltbedingungen, wie etwa die Schwere der täglichen Arbeit, die ein signifikantes Ansteigen der Ration erfordert, oder auch die Umgebungstemperaturen von unter $14{ }^{\circ} \mathrm{C}$ oder über $37^{\circ} \mathrm{C} .{ }^{760}$ Auf einer speziellen Tabelle des Kalorienbedarfs im Nahen Osten basiert die Untersuchung R. L. MiLleRs, der sich der Frage nach der Versorgungssituation im Alten Ägypten über Textquellen zu nähern versucht. ${ }^{761}$ Auch David WARBURTON stellt einige Berechnungen zur Versorgung an. Ausgangspunkt seiner Überlegungen sind die Angaben aus Papyrus Bologna 1086, aus denen eine Ertragsvorgabe pro Arbeiter von 200 Sack Getreide im Jahr ermittelt werden kann, die nach Meinung WARBURTONS den jährlichen Bedarf von 15 Personen decken. ${ }^{762}$

\section{Exkurs: Niedrige Nilfluten und Hungersnot}

Wie bereits zuvor im Kapitel 6.2 über Hochwasser und Dürre angedeutet, bedeutet ein niedriger Nilpegel allein noch keine Hungersnot für die betroffenen Menschen. Das drohende Ausbleiben der jährlichen Nilflut zwang die Administration jedoch bereits früh zu entsprechenden Vorsorgemaßnahmen, wie zum Beispiel einer verstärkten Vorratswirtschaft, die ebenso wie die Nilflut selbst ein Kriterium für die Fähigkeit zur Versorgung der Bevölkerung war und dafür ausreichend bemessen sein mußte. ${ }^{763}$ Ein Versagen der Getreideverwaltung bedeutete in letzter Konsequenz also ebenfalls Hungersnot. ${ }^{764}$ Diese letztere anthropogene Ursache von Hungersnöten scheint jedoch ein lokal bregrenztes Phänomen gewesen zu sein, wie die Aussagen in den Biographien einzelner Lokalfürsten trotz ihrer starken Toposhaftigkeit vermuten lassen. Wenn Anchtifi etwa davon berichtet, wie er nicht nur die eigenen Siedlungen sondern auch andere Orte mit Korn belieferte, dann entspricht dies dem typischen Schema: >Ich konnte meine Leute versorgen, während man anderswo hungern mußte. ${ }^{765}$

760 LIVI-BACCI (1991: 24).

761 MiLler (1991).

762 Warburton (1991: 80 f. Anm. 19); dabei wird von ihm eine Rate von 3000 kcal pro Person und Tag zugrunde gelegt, was allerdings nach den Kalkulationen von MILLER (1991: 258 f.), der aus verschiedenen altägyptischen Quellen zur Versorgung Angaben zwischen ca. 1700 und $3200 \mathrm{kcal}$ extrahiert hat, bereits an der oberen Grenze liegt; BAER (1963: 14 Anm. 89) kalkuliert 5000 kcal für ein Ehepaar mit einem Kleinkind.

763 Reflexe dieser unbedingten Notwendigkeit finden sich auch außerhalb der ägyptischen Überlieferung wieder, etwa in der biblischen Josephs-Erzählung, Gen. 41-47.

764 SCHENKEL (1978: $52 \mathrm{f}$.).

765 Morenz (1998: 87); zur Anchtifi-Textstelle vgl. SCHENKEL (1965: 54). 
Die Evidenz für einen direkten kausalen Zusammenhang zwischen niedriger Nilflut und Mangelversorgung ist nur selten aus den altägyptischen Quellen gegeben. Meist verschleiern die Berichte ihre Aussagen über einen geringen Nilstand hinter Metaphern wie >Sandbank $(\underline{t z}),{ }^{766}$ oder werfen dem Nil vor, er sei $>$ faul< $(w z f)$ oder $>$ geizig $<$ mit seinem Wasser $\left({ }^{c} w n\right.$ jb). ${ }^{767}$ Erst kürzlich hat MORENZ zu diesem Themenkomplex ein Fragment aus Dendera ins Zentrum des Interesses gerückt, das offenbar eine unverhüllte Aussage zu den Ereignissen während eines mageren Jahres trifft. ${ }^{768}$

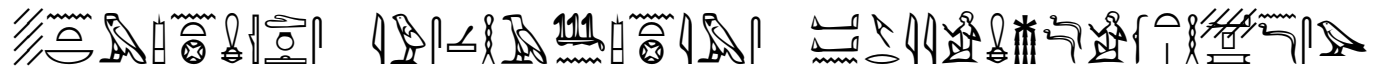

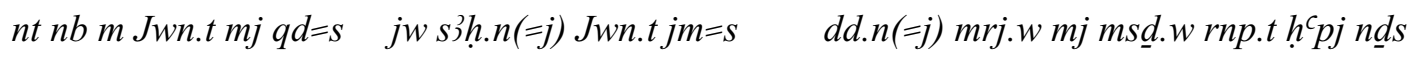

$\gg .$. alles in Dendera in seiner Gesamtheit. Ich beschenkte Dendera mit ihm. Dem Geliebten wie auch dem Verhaßten gab ich während des Jahres des geringen Nils.«

Während im Gegensatz zu anderen Genre-typischen Texten in diesem Fall eindeutig vom niedrigen Nil ( $\left.h^{c} p j n \underline{d} s\right)$ gesprochen wird, ist die Dendera zuteil gewordene Maßnahme noch interpretationsbedürftig, zumal die Schenkung nicht bezeichnet wird, sondern nur als $j m=s$ Erwähnung findet. In Analogie zu anderen Quellen vermutet MoREnZ dahinter Darlehen von Getreide, wie sie beispielsweise auch Anchtifi von sich berichtet. ${ }^{769}$

\subsubsection{Entkoppelung von Hungersnot und Populationsrückgang}

Die Auswirkungen, die eine Hungersnot auf die Bevölkerungsentwicklung haben konnte, sind in der Forschung immer wieder Gegenstand kontroverser Diskussion gewesen. Insbesondere die Vertreter der Thesen von Thomas MALTHUS sahen einen zwingenden Zusammenhang zwischen Bevölkerungswachstum und unbeschränkten Zugriff auf Ressourcen - und auch dessen Umkehrung.

Dieser Argumentation folgt auch ABERTH, der sich ausführlich mit einschneidenden Faktoren des Mittelalters - Hunger, Krieg und Krankheiten - auseinandergesetzt hat. ${ }^{770}$ Er geht davon aus, daß während der Zeiten von Hungersnöten nur ein kleiner Teil der Todesfälle direkt auf Verhungern zurückzuführen ist. Der überwiegende Teil der Menschen verstarb da-

766 SCHENKEL (1978: $50 \mathrm{f}$.$) .$

767 WESTENDORF (1981: $79 \mathrm{f}$.).

768 MORENZ (1998: $84 \mathrm{f}$.).

769 Guglielmi (1980: 82).

770 ABERTH (2001). 
gegen an epidemischen Krankheiten. ${ }^{771}$ Der kritische Aspekt an dieser Argumentation ist, daß aus medizinischer Sicht keine Koppelung von Hunger und Krankheit vorausgesetzt werden kann. Dafür gibt es - historisch betrachtet - zwei wesentliche Gründe. Der erste Grund liegt darin, daß im Falle eines Zusammenhanges zwischen Unterernährung und Krankheit die Angehörigen höherer gesellschaftlicher Schichten aufgrund besserer Versorgung tendenziell einer geringeren Bedrohung durch schwere Krankheiten ausgesetzt gewesen sein müssen, was jedoch nicht der Fall war. Als Beispiel führt ABERTH eine Abtei im englischen Canterbury des 15. Jahrhunderts an, in der die Mönche sich gesund und abwechslungsreich mit Brot, Milch, Käse, Obst und Gemüse sowie Fisch und Fleisch ernähren konnten. Dennoch verzeichnet die Abtei im Verlauf des 15. Jahrhunderts unter den Mönchen den Ausbruch von mindestens 27 größeren Krankheitsfällen und eine durchschnittliche Lebenserwartung von gerade einmal 23 Jahren - laut ABERTH eine der niedrigsten jemals verzeichneten Lebenserwartungen. ${ }^{772}$

Der zweite Grund bezieht sich darauf, daß die großen Epidemien des Mittelalters zu Zeiten von Hungersnöten ausgebrochen sein müssen. Auch dies war jedoch nicht der Fall. Im Jahr 1348, zu Beginn der Pestepoche in Europa, lag die Getreideernte in England leicht über dem Durchschnitt, und auch in den folgenden Jahren blieben die Getreidepreise niedrig - ein Indiz für ausreichende Versorgung. ${ }^{773}$ Dies hing sicher auch damit zusammen, daß trotz eines signifikanten Ausfalls des Erntebetriebs die Bevölkerung bereits soweit dezimiert war, daß die verbleibenden Vorräte ausreichten. Auch die Kindersterblichkeit verhielt sich keineswegs proportional zu den allgemeinen Entwicklungen, weshalb ABERTH die Schlußfolgerung zog, eine leichte chronische Unterernährung hätte einen positiven Effekt auf die Abwehr von Krankheitserregern. ${ }^{774}$ Die Kindersterblichkeit in europäischen Gesellschaften des 18. Jahrhunderts stand auch im Zentrum der Betrachtungen von Massimo LIVI-BACCI. In seiner Zusammenfassung der Ergebnisse betont er einerseits den Zusammenhang zwischen Kindersterblichkeit und der durchschnittlichen Lebenserwartung in der Bevölkerung. Unterschiedlich hohe Kindersterblichkeit kann demzufolge Schwankungen in der statistischen Gesamtlebenserwartung bis zu mehreren Jahren verursachen. Andererseits ist durch Stillen der Kleinstkinder in den ersten Monaten ihres Lebens kein direkter Zusammenhang zur allgemeinen Versorgungslage herstellbar. Auch die Ernährung der stillenden Mütter stellt nur einen untergeordneten Fak-

771 Aberth (2001: 15 ff.); auch Herlihy (2000: 31) weist auf den fehlenden Zusammenhang zwischen den Hungersnöten in den Jahren vor der Pest und einer Verringerung der Bevölkerungszahlen hin.

$772 \mathrm{Zu}$ ähnlichen Schlußfolgerungen für die privilegierten Schichten des 16. und 17. Jahrhunderts gelangt auch LIVI-BACCI (1991: $66 \mathrm{f}$.).

773 Ganz anders HeRLiHY (2000: 29, 52 f.).

774 ABERTH (2001: 17); in gleicher Weise auch LIVI-BACCI (1991: 36). 
tor dar. Daher liegen die Gründe für die deutlichen Schwankungen der Kindersterblichkeit in den verschiedenen Ländern Europas eher in der Kombination von Versorgungsnotlage und Umwelt-, Klima- oder kulturellen Faktoren. ${ }^{775}$ Allgemein kann folgendes Szenario für Versorgungsmangel formuliert werden: Eine Unterernährung bis an die Grenzen der körperlichen Auszehrung führt zu stark erhöhter Mortalität. Eine graduelle Unterernährung führt dagegen nicht zu einer graduell höheren Mortalität, da in diesem Stadium der Effekt einer verstärkten Übertragbarkeit von Infektionen durch Zerwürfnisse im sozialen Gefüge, Hygienedefiziten oder sonstigen Umweltfaktoren die Mangelversorgung überlagert. ${ }^{776}$ In eine ähnliche Richtung geht eine Anmerkung SCHEIDELs, der darauf hinweist, daß gut versorgte römische Eliten nicht notwendigerweise eine höhere Lebenserwartung vorweisen konnten als Angehörige der armen Bauernschichten. ${ }^{777}$ Selbst wenn eine Hungersnot über eine Gesellschaft oder bestimmten gesellschaftlichen Gruppen hereinbricht, ist der Regenerationsgrad der Bevölkerung extrem schnell. ${ }^{778}$

\subsection{Geburtsraten und Kindersterblichkeit}

Bereits Edmé JOMARD fügte seiner Abhandlung in der Description de l'Égypte eine kurze Betrachtung zur Fruchtbarkeit ägyptischer Frauen bei und stellte Vergleiche zwischen Antike und Moderne an. ${ }^{779}$ Dabei zog er als Vergleichsmaterial auch zeitgenössische Daten aus europäischen Metropolen wie Paris oder London heran. Zunächst verglich JOMARD die Geschlechtsgliederung zwischen Europa und Ägypten und stellte für Europa ein Gleichgewicht bei den Geburten fest, während in Ägypten offenkundig ein Frauenüberschuß vorlag. Nach den Sterbetafeln aus Kairo zu urteilen, starben jedoch auch deutlich mehr Frauen als Männer. Eine für JOMARD verblüffende Beobachtung war auch der frühe Zeitpunkt der Geschlechtsreife bei ägyptischen Mädchen, die bereits mit 12 Jahren Kinder zur Welt brachten und deren Zeit optimaler Gebärfähigkeit sich über die ersten sechs Jahre nach der Heirat erstreckte. Der frühe Eintritt in die Geschlechtsreife bedingte aber auch eine Verschiebung des gesamten Lebenszyklus'. Schon mit 30 Jahren gebaren die Frauen keine Kinder mehr, waren diversen

775 LiVI-BACCI (1991: 78).

776 LIVI-BACCI (1991: 47).

777 SCHEIDEL (2001b: 29).

778 SCHEIDEL (2001b: 69 Anm. 283).

779 JOMARD (1818: 100 ff.). 
Erkrankungen ausgesetzt und galten nach Meinung JOMARDs bereits als >Alte< (vieilles). ${ }^{780}$ Seine Informationen über die Antike holte sich JOMARD einmal mehr bei den klassischen Autoren. Insbesondere befaßte er sich mit Mehrlingsgeburten, für die es auch mehrfach antike Hinweise gibt. ${ }^{781}$ Der letzte von JOMARD betrachtete Aspekt ist die Kindersterblichkeit, die er als Augenzeuge in Kairo als recht hoch einschätzt und dies unter anderem Krankheiten wie den Pocken zuschreibt. Ohne die überdurchschnittliche Fruchtbarkeit ägyptischer Frauen würde nach seiner Meinung die Population zurückgehen oder zumindest stagnieren. So sah er als Hemmnisse der Fertilität das frühe Ende der Gebärfähigkeit sowie die hohe Kindersterblichkeit.

\subsubsection{Konzeption und Kontrazeption}

Obwohl es bereits verschiedene Mittel zur Schwangerschaftsverhütung gab, dürfte dies kein wirklich effektives Element zur Geburtenkontrolle gewesen sein. ${ }^{782}$ Mehr noch als die Verhütung dürfte den Frauen des Alten Ägypten eine Schwangerschaft ohne Zwischenfälle und die Geburt eines gesunden Kindes am Herzen gelegen haben. Die letale Gefahr war für die werdende Mutter durch auftretende Komplikationen während der Zeit ihrer Schwangerschaft und bei der Niederkunft relativ hoch. ${ }^{783}$ Dieser zunächst aus den medizinischen Papyri gewonnene Eindruck findet seine Entsprechung in den Untersuchungen von Nekropolen. Der anthropologische Befund der Nekropole von Tell el-Dab ${ }^{c}$ a ergab eine außergewöhnlich hohe Kindersterblichkeit. Die Mütter hingegen starben in frühadultem Alter an Kindbettfieber. Sämtliche Personen aus den insgesamt 140 untersuchten Bestattungen wiesen ein Alter von unter 60 Jahren auf. ${ }^{784}$ Auch Grabungsergebnisse aus den Siedlungen von Deir el-Medineh und Amarna geben indirekte Hinweise. So wurden in Deir el-Medineh Wandbilder mit Darstellungen einer >Wochenlaube< in den Räumen mit Hausaltären gefunden und in der Main City Amarnas la-

780 JOMARD (1818: 101). Möglicherweise entspringt die Einschätzung JOMARDs auch den Malthusianischen Konzepten, die als Geburtenkontrolle eine möglichst späte Heirat empfahlen. Verdächtig auch sein Fazit: »On n'en peut donc tirer aucune induction pour conclure le fait ou la possibilité d'une population excédant toute mesure, telle que plusieurs savans l'ont admise.« Vergleichszahlen für das 19. Jahrhundert, die ungefähr zwischen 28 und 30 Jahren liegen, bei MARSCHALCK (1984: 39 f.).

781 Strabon, Geographica XV 1,22 unter Berufung auf Aristoteles; Plinius, Naturalis historiae VII 3,33. Alle Autoren geben übereinstimmend den fruchtbarkeitsfördernden Nil (vgl. auch oben Kap. 2.3) als Ursache dafür an. Eine interessante Ausnahme davon bildet eine zusätzliche Angabe Strabons, der von einer Frau z. Zt. des Augustus berichtet, die zweimal hintereinander Zwillinge bekam, und dies mit der zu jener Zeit drohenden Hungersnot (!) begründet.

$782 \mathrm{Zu}$ den Verhütungsmitteln zusammenfassend NUNN (1996: 196); WESTENDORF (1999: 426).

783 WESTENDORF (1999: 448).

784 BIETAK (1974/77: 316). 
gen am Hausaltar eines Gebäudes zwei Modellbetten, ein sogenanntes Konkubinenfigürchen sowie eine Stele, die Mutter und Kind vor der Göttin Thoëris zeigt. Übereinstimmend werden solche Funde als an die Gottheiten gerichteter Wunsch nach komplikationsfreier Empfängnis und Schwangerschaft gedeutet. ${ }^{785}$ Auch Amulette wie das $t j t$ - oder Isisblut-Symbol (书) werden als Schutz gegen Fehlgeburten interpretiert. ${ }^{786}$

Im Spiegel der Textquellen scheint dagegen die Problematik der Fehlgeburten fast gänzlich ausgespart. Selbst in den medizinischen Papyri finden sich nur Umschreibungen oder Andeutungen. Fast scheint es, als sei der Komplex von Schwangerschaftskomplikationen, Fehl- und Totgeburten aufgrund seines Auftretens als Schicksalsschlag tabuisert gewesen. So gibt es im Ägyptischen und Koptischen nur wenige Termini, die sich mit der Bezeichnung einer Fehlgeburt in Verbindung bringen lassen. Da ist zunächst einmal das koptische Wort 20Y2€ $(<w h j-$ h.t), das von Wilhelm SPIEGELBERG als >Fehlgeburt $<$ identifizert wurde, in seiner Deutung allerdings singulär bleibt. ${ }^{787}$ Die etymologische Herleitung aus dem Ägyptischen ist als eigener zusammengesetzter Begriff nicht belegt und sowohl Jaroslav ČERNÝ als auch WESTENDORF beziehen sich in ihren Lexika einzig auf den Vorschlag SPIEGELBERGs. ${ }^{788}$

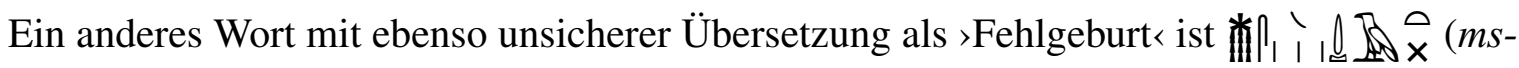
d).t), für das es aufgrund seiner singulären Verwendung keine Bestätigung aus anderen Texten gibt. ${ }^{789}$ Ein drittes Wort, das im Zusammenhang mit Fehlgeburten stehen soll, bietet das Medizinische Wörterbuch unter $\int_{\Omega}^{m m} \sim(b n d / b n \underline{d}) .{ }^{790}$ Da dieser Terminus allerdings nur im Gegensatz zu einer >glücklichen Geburt< verwendet wurde und man daher auf eine Erklärung als $>$ schwierige Geburt< oder sogar >Fehlgeburt< verfiel, ist auch hier nur eine geringe Evidenz für das Wort gegeben. Alternativ erklärt das MedWb bnd auch als >Koitus ohne Befruchtung $<$. Da sämtliche Autoren für die Frage der Übersetzung von bnd nur den Fall 196 des medizinischen Papyrus Berlin als einzige Belegstelle heranziehen können, erhärtet sich der Verdacht, daß hier ein Hapax vorliegt. ${ }^{791}$ Das gebotene Material läßt keinerlei Schluß zu, ob sich der Terminus tatsächlich auf eine Fehlgeburt bezieht, weshalb bereits Walter WRESZINSKI Bedenken hegte und allgemeiner mit >schwierige Geburt< übersetzte. ${ }^{792}$

785 PINCH (1983).

786 WESTENDORF (1966).

787 SPIEGELBERG (1923).

788 ČERnÝ, CED, 305; WeSTENDORF, KoptHWB, 406.

789 pTurin 1984, R114-115; die Bearbeitung stammt von EDWARDS (1960: 66 Anm. 68), dessen Interpretation allein darauf beruht, daß $\underline{d} 3 y . t$ allgemein $>$ Unheil< beschreibt und negativ konnotiert ist.

$790 \mathrm{MedWb} 251$.

791 Med. pBerlin 3038, vs. 1,10.

792 WESTENDORF (1999: 436 Anm. 776) bleibt bei der Übersetzung >Fehlgeburt<, weist aber auf die eigentliche 


\subsubsection{Sterbeziffern, Konstitution und Fertilität}

Ein einzelner Artikel aus dem Jahr 1972 hat die Meinung der ägyptologischen Fachwelt zur Fertilität und Gebärfähigkeit der Frau im Alten Ägypten wie kein zweiter geprägt. Es handelt sich um einen Beitrag der italienischen Anthropologen Melchiorre MASALI und Brunetto Chiarelli, die in der Sammlung des Ägyptischen Museums in Turin Hunderte von Skeletten aus den Nekropolen der Orte Gebelein und Assiut untersucht hatten. ${ }^{793}$ Aus Gebelein lagen dabei sowohl Funde aus prädynastischer als aus der dynastischen Zeit vor, so daß auch ansatzweise diachrone Vergleiche möglich waren.

Die Analyse der Skelette erbrachte im wesentlichen zwei Ergebnisse. Zum einen konnten Tendenzen in der Bestimmung der Sterblichkeitsraten festgestellt werden. Die statistische Auswertung ergab eine Reduktion der erwachsenen Bevölkerung um die Hälfte nach dem 25. Lebensjahr in der prädynastischen und nach dem 30. Lebensjahr in der dynastischen Zeit. Bis zu diesem Alter gab es auch eine deutliche weibliche Übersterblichkeit, das heißt es starben im gleichen Zeitraum mehr Frauen als Männer. In den höheren Altersgruppen glich sich die Sterblichkeit von Männern und Frauen wieder einander an. ${ }^{794}$ Der identische Zeitraum weiblicher Übersterblichkeit und der Phase höchster Fertilität scheint dabei kein Zufall zu sein. Die Indizien deuten darauf hin, daß Komplikationen während der Schwangerschaft und Geburt einen deutlichen Einfluß auf die Mortalität der Frauen haben mußten. In der Tat fand man bei näherer Untersuchung der Konstitution weiblicher Skelette auffällige Übereinstimmungen der Statur des Beckenbereiches. Der Körperbau der Frauen war sehr schmal und auch die Beckenbreite zeigte zu Vergleichszahlen anderer Ethnien unterdurchschnittliche Werte. ${ }^{795}$ Auch die aus adulten Bestattungen bekannten Totgeburten oder Säuglinge weisen auf häufige tokologische Probleme hin. ${ }^{796}$

In ihrer Zusammenfassung der Ergebnisse weisen MASALI und CHIARELLI auf die Umweltfaktoren im ägyptischen Niltal hin. Obgleich die Sterblichkeit recht hoch war, wäre nach Meinung der Verfasser aufgrund der hohen Fruchtbarkeit des Bodens, der großen Zahl an jungen und aktiven Menschen und die geringe Lebenserwartung für Alte und Behinderte ein starker Bevölkerungsdruck zu erwarten gewesen. Diese Situation läßt sich aber zu keinem Zeitpunkt für das Alte Ägypten nachweisen. Auch verstärke Mortalität durch Einwirkung äu-

Bedeutung des Wortes als >Ausscheidung $<$ hin; WrESZINSKI $(1909: 46,107)$.

793 Masali und ChiARELli (1972).

794 MASALI Und CHIARELLI (1972: 165).

795 Detaillierte Angaben bei MASALI (1972: 194 f. Tab. 3).

796 Masali und Chiarelli (1972: 165 f.); MASAli (1972: 196 Abb. 1). 
ßerer Gewalt wurde anhand der niedrigen Sterberate junger Männer widerlegt, obwohl diese Gruppe am stärksten den Gefahren durch Jagd oder Kriege ausgesetzt gewesen sein müßte. ${ }^{797}$ So blieb als einziges plausibles Erklärungsmodell die Hypothese einer allgemein niedrigen Geburtenziffer im pharaonischen Ägypten als Grundlage demographischer Stabilität. Dabei scheint es jedoch offenbar einen Wandel im Übergang von der prädynastischen zur pharaonischen Zeit gegeben zu haben. Die ursprünglich kräftige Konstitution der prädynastischen Bewohner Ägyptens wich einer zunehmenden >Grazilität<, die vielleicht mit geänderten und insgesamt leichteren Lebensumständen zusammenhing. ${ }^{798}$

Bereits wenige Jahre nach ihrer Veröffentlichung verwendete Jac JANSSEN die Turiner Ergebnisse als Grundlage für seine >Prolegomena< zur ägyptischen Wirtschafts- und Sozialgeschichte und stellte basierend auf der Stagnation der Bevölkerungsentwicklung ein Gleichgewicht zwischen Ressourcen und Population fest. ${ }^{799}$ Auch Michael ATZLER befaßte sich im Rahmen seiner Studie zur Genese des ägyptischen Staates mit demographischen Aspekten und griff die Zusammenhänge von Beckenbreite und Geburtsschwierigkeiten erneut auf. Er differenzierte dabei jedoch zwischen prädynastischer und pharaonischer Zeit und wies nachdrücklich auf die bessere Konstitution der prädynastischen Frauen hin. ${ }^{800}$

Auch andere Ägyptologen zogen die Aussagen MASALIs und CHIARELLIs für ihre Untersuchungen heran, gelegentlich jedoch mit eigenwilligen Interpretationen. So begründete HELCK das Phänomen der schlankwüchsigen Ägypterinnen mit einer »Auslese aus Schönheitsgründen «. ${ }^{801}$ Zuletzt erwähnte auch NUNN das schmale Becken in seiner Monographie über altägyptische Medizin, zog daraus allerdings keine tokologischen Konsequenzen. Er wies jedoch erneut darauf hin, daß die lange und schmale Darstellung von Frauen in den Abbildungen des Neuen Reiches möglicherweise keine idealisierte Darstellung bedeuten, sondern anthropologisch begründet sind. ${ }^{802}$ Die Feststellung über die Körperstatur der Ägypterinnen scheint bereits in der Antike bekannt gewesen zu sein, wie das Bibelzitat Exodus 1,19 nahelegt, das den anthropologischen Befund stützt:

\footnotetext{
»Die Hebammen antworteten dem Pharao: Die hebräischen Frauen sind nicht wie die ägyptischen, denn sie sind kräftige Frauen. Ehe die Hebamme zu ihnen kommt, haben sie geboren.«
}

797 MASAli und CHIARELli (1972: 168).

798 MASALI (1972: 196).

799 JANSSEN (1975b: 135).

800 ATZLER (1981: $78 \mathrm{f}$.).

801 HELCK (1977b: 307 Anm. 13).

802 NUNN (1996: 20); so bereits auch von MASALI (1972: 196) beschrieben. 
Auch wenn die darauf basierende Interpretation Gerhard PFEIFERs, die Frauen seien »weniger lebenskräftig (sic!) und gebärtüchtig als die Hebräerinnen «, ${ }^{803}$ in ihrer Absolutheit fehlgeht, so stellt diese biblische Beobachtung doch eine bemerkenswerte Parallele zu den Ergebnissen MASALIS und CHIARELLIS dar.

Die offenbar schlechtere Gebärfähigkeit ägyptischer Frauen läßt sich möglicherweise auch mit den Befunden aus den Zensuspapyri (Kap. 3) in Einklang bringen, die sämtlich - entgegen allen Erwartungen für vorindustrielle Gesellschaften - eine geringe Anzahl Kinder verzeichnen. Dabei muß allerdings berücksichtigt werden, daß sämtliche Belege, Zensuspapyri und Bestattungen gleichermaßen aus dem Lebensumfeld einer privilegierten Oberschicht stammen. Weil über die weitaus größere Zahl an Bauern und Angehörigen der Unterschicht keine verläßlichen Daten vorliegen, muß die Diskrepanz zwischen dem aus demographischen Modellen prognostizierten Kinderreichtum und den Einzelbeobachtungen einstweilen offen bleiben. Zumindest kann aus den von MASALI und CHIARELli beschriebenen Befunden keine Verallgemeinerung für alle gesellschaftlichen Schichten und $\mathrm{zu}$ allen Zeiten deduziert werden. Die Modelle von Roger BAGNALl und Bruce FRIER für das römische Ägypten stehen im deutlichen Gegensatz zu den anthropologischen Befunden an den pharaonischen Gebeinen. In dieser späten Epoche war die Geburt von sechs lebenden Kindern pro Mutter notwendig, um die Population stabil zu halten. ${ }^{804}$

\subsection{Soziale Auswirkungen demographischer Einbrüche}

Obgleich die Kenntnisse über die realen und täglichen Auswirkungen exogener Faktoren auf das soziale Gefüge der altägyptischen Gesellschaft praktisch nicht vorhanden sind, so stellt sich dennoch die Frage, welche Folgen derartige Faktoren prinzipiell auf eine Gesellschaft haben können und möglicherweise auch für das Alte Ägypten Gültigkeit besaßen. Als Vergleichsobjekt und zugleich Kontrastfolie bieten sich sowohl das europäische Mittelalter in der Zeit nach den großen Pestepidemien, als auch das Europa nach den Weltkriegen des 20. Jahrhunderts an. Beide Ereignisse dürfen als gravierender Einschnitt in das demographische Gefüge der betroffenen Gesellschaften gelten und haben deshalb auch in der Folgezeit tiefgreifende Konsequenzen für die sozialen Entwicklungen, die in diesem Kapitel kurz skizziert werden sollen.

803 PFEIFER (1995: 32).

804 Vgl. Bagnall und Frier (1994: 139 Anm. 16) zur Angabe der sogenannten Gesamtfruchtbarkeitsziffer von 5,979 . 


\section{Die Konsequenzen in der Arbeitswelt}

Die Auswirkungen von demographischen Einbrüchen auf die Arbeits- und Berufswelt des Mittelalters und der Moderne ist nicht direkt mit dem Alten Ägypten vergleichbar. Während hier die gesellschaftliche Struktur auf ein Heer von ungelernten Bauern und Tagelöhnern zurückgreifen kann, wovon die Administration zu allen Zeiten der ägyptischen Geschichte regen Gebrauch machte, ${ }^{805}$ weisen das Mittelalter mit seinem verbreiteten Zunftwesen und dem ausgeprägt spezialisierten Handwerk wie auch die Moderne nach den Umwälzungen der industriellen Revolution völlig andere Strukturen auf. Dennoch gewähren beide Epochen interessante Einblicke in das menschliche Handeln während Krisenzeiten, das in modifizierter Form möglicherweise auch auf die ägyptische Gesellschaft Einfluß hatte.

Zunächst soll die Situation während der Höhepunkte der Pestepidemien des Mittelalters näher beleuchtet werden. Die ökonomischen Strukturen jener Zeit standen unter einem schweren Druck, weil die benötigten Arbeitskräfte entweder starben oder die Flucht aus den betroffenen Gebieten ergriffen. ${ }^{806}$ Die dadurch gegebene Diskontinuität schlug sich in mehreren Bereichen nieder. Zum einen griff man verstärkt auf jüngere Arbeitskräfte zurück, die jedoch entsprechend weniger Berufserfahrung mitbrachten und aufgrund der reduzierten durchschnittlichen Lebensdauer möglicherweise nur kurz produktiv für die Gesellschaft arbeiten konnten. ${ }^{807}$ Dies wiederum, gepaart mit einem Ausgreifen auf Personen außerhalb der unmittelbaren Familienangehörigen bei der Anwerbung von Lehrlingen, resultierte in einer tendenziell geringeren Qualität der hergestellten Produkte und Dienstleistungen. ${ }^{808}$ Doch da die Gesellschaft verschiedenste Dienstleistungen benötigte, mußten bestimmte Bereiche gezwungenermaßen gewisse >Privilegien< gewähren. So konnten Frauen als Zeuge vor Gericht auftreten oder Personen ohne Notarstatus rechtsgültige Verträge aufsetzen. ${ }^{809}$ Berufsgruppen wie Totengräber, Ärzte und Priester sahen sich einer verstärkten Nachfrage ausgesetzt - eine makabere Hausse in den Zeiten von Leiden und Tod. ${ }^{810}$ Insgesamt blieb die Zahl der Zunftzulassungen nach Auskunft der Zunftbücher offenbar konstant, obgleich die Zahl der Bevölkerung um etwa $1 / 3$ zurückgegangen war. Daraus kann gefolgert werden, daß die Gesellschaft sich bemühte, den Anteil an Berufstätigen weitestgehend konstant zu halten, auch um den Preis von

805 Vgl. dazu auch oben Kap. 5.2.2.5 zu >Arbeitsverpflichtung und Frondienst<.

806 HERLIHY (2000: 41).

807 HERLIHY (2000: 45).

808 HERLIHY (2000: 50 f.).

809 HERLIHY (2000: 45).

810 HERLIHY (2000: 42 f.). 
qualitativen Zugeständnissen. ${ }^{811}$ Aber auch die Löhne stiegen. Die verzweifelte Nachfrage nach Arbeitskräften ermöglichte selbst Angehörigen des niedrigen und ungelernten Personals die Einforderung dreister Lohnvorstellungen. ${ }^{812}$

Auch die Situation vor und während des Ersten Weltkrieges war von sozialen Umbrüchen und Veränderungen geprägt, die in ihren prinzipiellen Konsequenzen verblüffende Parallelen zu den Phänomenen während der Pestzeit aufweisen. Der durch die allgemeine Mobilmachung verursachte Mangel an Arbeitskräften führte in der Folge zu der paradoxen Situation stärkerer Lohnerhöhungen für Frauen als für Männer, blieb aber im Verhältnis immer noch hinter den Männern zurïck. ${ }^{813}$ Auch hier wirkte sich die verstärkte Nachfrage nach der Arbeitskraft auf die Lohnentwicklung aus, die ein rasches Auffüllen der entstandenen Lücken zum Ziel hatte. Eine weitere Folge dieses Verfahrens war das schnelle Anlernen weiblicher Arbeitskräfte an den verlassenen Arbeitsplätzen. Den staatlichen Forderungen nach gründlicher und adäquater Ausbildung weiblicher Lehrlinge kamen die Unternehmen nicht nach, sondern sie kompensierten ihren Facharbeitermangel durch »Schmalspurqualifikationen«.814

\section{Die soziale und ökonomische Dimension}

Neben den gravierenden Verschiebungen in der Arbeitswelt des Mittelalters standen auch praktische Probleme der Versorgung und des Warenaustauschs im Vordergrund. Aus Frankreich berichtet der Dichter Guillaume de Machaut vom Zusammenbruch der Landwirtschaft aufgrund des Personalmangels. Bauern vernachlässigten ihre Höfe, Tiere wurden nicht mehr versorgt, Getreide verblieb ungeerntet auf den Feldern. ${ }^{815}$ Die Produktion von Nahrungsmitteln war unter dem Eindruck der Pest dramatisch gesunken und resultierte in einer Inflation der Preise. ${ }^{816}$ Während der beiden Weltkriege war die Versorgungslage unterschiedlich und zumeist gegen Ende des Krieges kam es immer wieder zu Hungersnöten. ${ }^{817}$ Die Verknappung von Nahrungsmitteln bedeutete immer einen Anstieg der Preise, und diese Steigerung der Lebenshaltungskosten bewirkte Veränderungen des Speiseplans. Während der Konsum von Brot, Fleisch und Fett zurückging, stieg der Verzehr von Kartoffeln gegenüber der Vorkriegszeit um das Dreifache an. ${ }^{818}$ Insgesamt überwog die einseitige Ernährung mit Kartoffeln, Steckrüben

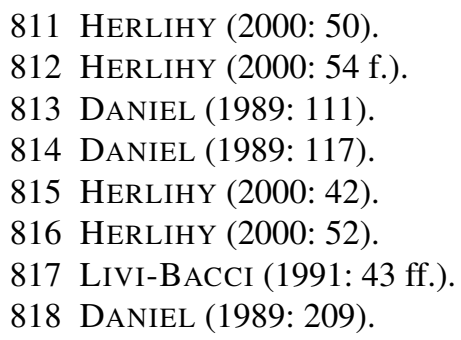


sowie >Ersatzlebensmitteln < wie Milchpulver oder Kunsthonig, die den Bedarf des Körpers an Nährstoffen nur unzureichend deckten. ${ }^{819}$

Aufgrund der immer noch umstrittenen demographischen Auswirkungen der Pest auf die Bevölkerungszahl Europas können repräsentative Erhebungen von Haushalten (Mikrozensus) für diese Zeit nicht gegeben werden. ${ }^{820}$ Bessere Beobachtungen demographischer Folgen lassen sich dagegen nach den Weltkriegen der jüngsten Geschichte tätigen. ${ }^{821}$ So beschreibt Doris SCHUBERT etwa das soziale Umfeld der Frauen nach dem Zweiten Weltkrieg. ${ }^{822}$ Die hohen militärischen Verlustzahlen unter jungen Männern bedeuteten deutliche Verschiebungen in der Alterspyramide wie auch der Sexualproportionen. ${ }^{823}$ Der Überschuß an Frauen bedingte zunächst weniger Eheschließungen, wodurch sich auch das Verhältnis von verheirateten zu unverheirateten Frauen verschob. Dazu kam ein spürbarer Geburtenrückgang zwischen 1914 und 1918 und die Haushaltsgrößen verkleinerten sich proportional. Während ein Rückgang von Haushalten mit sechs oder mehr Personen zu verzeichnen war, nahmen Kleinhaushalte zwischen zwei und fünf Personen zu. ${ }^{824}$ Auch während des Zweiten Weltkrieges nahm der Anteil Frauen bis zum Jahr 1946 um 7,3 \% zu, während im gleichen Zeitraum die Männerquote um $2 \%$ sank. Nach Ende des Krieges bestand für viele Frauen, die die Position ihres Ehemannes als Versorger der Familie einnehmen mußten, die Notwendigkeit, den Lebensunterhalt durch Aufnahme einer Berufstätigkeit zu bestreiten. Allerdings stieg mit deren erhöhter Nachfrage nach Arbeitsplätzen nicht der Anteil an Stellen in frauenspezifischen Berufen, so daß als zwangsläufige Folge die Frauen in vormals typische Männerberufe einstiegen. Dies stellte jedoch keine Erweiterung weiblicher Rechte dar, sondern lag allein im Mangel an männlichen Arbeitskräften begründet. ${ }^{825}$

\section{Stabile Veränderungen oder vorübergehende Kompromisse?}

Es bleibt abschließend zu beurteilen, inwieweit die unter äußerem Druck zustande gekommenen gesellschaftlichen Veränderungen eine langfristige Perspektive besaßen oder nur so lange Akzeptanz fanden, bis sich die sozialen Bedingungen wieder verbessert hatten. Die Ergebnisse für Mittelalter und Moderne sind übereinstimmend pessimistisch. Insbesondere die Männerwelt zeigte sich nicht gewillt, den status quo als fortan gültiges Modell zu akzeptieren.

819 DANIEL (1989: 210).

820 Schätzungen für den Bevölkerungsrückgang im 14. Jahrhundert: ECKERT (2000: 2): 8-15\%; HERLIHY (2000: 7): um 1420 ca. $66 \%$; zur stark schwankenden Morbidität während der Pestepidemien seit dem 16. Jahrhundert vgl. BENEDICTOW (1987).

821 DANIEL (1989: 127 ff.); MARSCHALCK (1984: 69).

822 SCHUBERT (1984: 235 f.).

823 Vgl. dazu die detaillierte Studie für Großbritannien von WINTER (1976).

824 DANIEL (1989: 137 m. Tab. 28).

825 SCHUBERT (1984: $250 \mathrm{f}$.). 
Der durch die Pestepidemien seit 1348 verursachte Rückgang der Bevölkerungszahlen und damit auch Mangel an Arbeitern konnte zwar erfolgreich durch Frauen aufgefüllt wurde. Doch betrachtete man dies als vorübergehendes Phänomen und keinen grundsätzlichen gesellschaftlichen Umschwung. Spätestens Ende des 15. Jahrhunderts wurde durch die Zunftmeister vieles wieder rückgängig gemacht. Der Mangel an Arbeit wurde zugunsten der Männer entschieden und der Wettbewerb erneut eingeschränkt. ${ }^{826}$ Ganz ähnlich erging es den Kriegsfrauen nach den beiden Weltkriegen. Geschürt durch ein unterschwelliges Konkurrenzdenken seitens der Männer und der Furcht der Gewerkschaften vor steigenden Lohndruck, gab es nach Kriegsende starke Bestrebungen, die Situation wieder zugunsten der Zustände rückgängig zu machen, die vor dem Krieg herrschten. ${ }^{827}$ Die Bewertung der Arbeitssituation zwischen 1914 und 1918 als > vorläufig < spielte dabei bereits in den Diskussionen während des Krieges eine entscheidende Rolle.

\subsection{Zusammenfassung}

Obwohl alle in diesem Kapitel beschriebenen Naturphänomene auf die eine oder andere Art auch über die Menschen des Alten Ägypten hereingebrochen sind, können die tatsächlichen demographischen Auswirkungen nicht präzise benannt werden. Es ist nicht einmal sicher, ob diese Faktoren für die ägyptische Gesellschaft als >katastrophal< bezeichnet werden können. Für eine Katastrophe wurden in der Vergangenheit verschiedene Definitionen formuliert, die von Robin TORRENCE und John GRATTAN zusammenfassend wie folgt beschrieben wurden. ${ }^{828}$ Im einfachsten Fall hat man einen Schwellwert an Todesopfern angesetzt. Komplexere Definitionen beziehen sich auf den Zusammenbruch des sozialen und ökonomischen Gefüges, das ohne externe Hilfe nicht wiederaufbaubar war. Alles in allem gilt ein Schaden an Individuen und deren Besitz als Grundlage aller Definitionsversuche, so daß die Kernaussage »Katastrophen sind soziale Phänomene« unbedingte Gültigkeit hat.

Entscheidend sind in allen von TORRENCE und GRATTAN vorgestellten Definitionen die gesellschaftlichen Folgen, die - wie in diesem Kapitel gezeigt werden konnte - auch den demographischen Aspekt mit einschließen. Eine länger anhaltende hohe Mortalität unter dem Eindruck von Hunger und Krankheit hat dabei tiefergreifende Auswirkungen auf die Populationskurve als kurze Unwetterkatastrophen. Auch drücken die vermutlich recht häufigen Todes-

826 HERLIHY (1990: 180).

827 DANIEL (1989: 117 ff.).

828 TORRENCE und GRATTAN (2002: 5 f.). 
fälle von Müttern im Kindbett und die hohe Kindersterblichkeit während der ersten Lebensjahre die durchschnittliche Lebenserwartung. Ähnlich, wie es bereits BAGNALL und FRIER bei der Frage nach einer >stabilen Bevölkerung< der römischen Zeit beschrieben haben, kann auch für die älteren Epochen der ägyptischen Geschichte eine gewisse Instabilität vorausgesetzt werden, die sich jedoch nicht konkret fassen läßt. ${ }^{829}$ Beide Autoren betonen jedoch den hohen demographischen Druck auf die Bevölkerung, der typischerweise gleichermaßen zu hoher Fertilität wie auch hoher Mortalität führte. Der von BAGNALL und FRIER als »>Chinese< situation « bezeichnete Zustand bedeutete einerseits niedrige Letalität von Epidemien, zwang aber aufgrund sozialer Umstände andererseits zu früher Heirat. Die Folge waren hohe Fertilität und - als Hemmnis des rapiden Wachstums - eine entsprechend hohe Mortalität. ${ }^{830}$ Wichtig ist im Zusammenhang mit dem Auftreten zeitlich begrenzter Ereignisse die aus den Vergleichen gewonnene Erkenntnis einer schnellen Regeneration der Bevölkerungsentwicklung, sobald das Hemmnis überwunden ist. Beispiele dafür können auch aus dem Wachstum der Bevölkerung des vormodernen Ägypten abgelesen werden, die im Blickfeld der Betrachtungen des folgenden Kapitels steht.

829 BAGNALL und FRIER (1994: 173 ff., bes. 177 f.); als stabile Bevölkerung bezeichnet man eine geschlossene Bevölkerung (ohne Zu- und Abwanderung), die über einen längeren Zeitraum hinweg eine konstante Wachstumsrate aufweist.

830 BAgNALl und FRIER (1994: 177 Anm. 28); unsicher bleibt, ob für das historische China tatsächlich ein paradigmatischer Verlauf nach dem Modell von MALTHUS angenommen werden darf. 


\section{Die ägyptische Population im 19. Jahrhundert}

Seit Tausenden von Jahren bewohnt in physisch unveränderter Gestalt das Volk der Ägypter die Ufer des Nils.

Baedeker, Ägypten und der Sûdan, 1928

\subsection{Moderne Bevölkerungsstatistiken}

$\mathrm{D}$

A das erhaltene altägyptische Datenmaterial offenkundig zu gering ist, um Entwicklungstrends der Populationskurve verläßlich beschreiben zu können, gab es in der Vergangenheit immer wieder Versuche, stattdessen die leichter zugänglichen Daten des modernen Ägypten heranzuziehen, um daraus Rückschlüsse auf die pharaonische Zeit zu ziehen oder zumindest anhand des Zahlenmaterials die für das Alte Ägypten vermuteten Phänomene als plausibel zu deklarieren. ${ }^{831}$ Dabei bedarf es zweifellos einer gewissen Vorsicht, da Vergleiche dieser Art nur dann zulässig sind, wenn die grundsätzlichen Parameter der beiden zu vergleichenden Epochen eine gewisse Übereinstimmung aufweisen. Und auch das Zahlenmaterial selbst, das aus dem 19. und 20. Jahrhundert vorliegt, kann nicht als unbelastet von tendenziösen Einflüssen gelten, die aus den häufig unzureichenden Umständen ihrer Erhebung resultieren. Um aus den für das Ägypten des 19. Jahrhundert gewonnenen Kenntnissen über demographische Zusammenhänge parallele Entwicklungen für das pharaonische Ägypten ableiten zu können, müßte zunächst der Beweis erbracht werden, daß sich beide Gesellschaften deterministisch, also in gleichen Situationen identisch, verhalten. Das heißt, daß die Effekte von ökonomischen Entwicklungen, Innovationen und exogenen Faktoren bei gleichen Ausgangsdaten (Entwicklungszeitraum und Bevölkerungsgröße) zu übereinstimmenden Resultaten hinsichtlich der Bevölkerungsentwicklungen führen. Diesen Beweis ist man bisher 831 Rathbone (1990: 107 f.); Bowman (1986: 17); siehe zum Versuch Bowmans unten S. 220. 
schuldig geblieben, stattdessen erfolgt a priori eine simplifizierende Gleichsetzung des alten und vormodernen Ägypten als Prämisse. Die Folge der auf solchen Grundlagen entwickelten universellen Modelle ist ihr geringer Informationsgehalt, der dem Anspruch antiker Gesellschaften auf soziokulturelle Eigenständigkeit nicht gerecht wird. ${ }^{832}$

Wenn in diesem Kapitel also ein ausführlicherer Blick auf die Bevölkerungsentwicklung Ägyptens im 19. und 20. Jahrhundert geworfen wird, dann mit dem primären Zweck einer Demonstration von demographischen Mechanismen und deren Wirkungen, besonders im Ägypten vor der Industrialisierung. Die quantitative Verwertbarkeit ist dabei ausgeschlossen, das dahinterstehende Modell kann jedoch mögliche Hinweise auf die gesellschaftliche Entwicklung des Alten Ägypten liefern.

\subsubsection{Volkszählungen und Modellrechnungen als Datenbasis}

Generell spielt die Art und Weise einer Datenerhebung eine wichtige Rolle. Aus verschiedenen Gründen kann das Ergebnis einer Zählung nach oben oder unten verfälscht sein. Als Beispiel eines zu hohen Ergebnisses gilt die Volkszählung in Nigeria von 1963, in der die regionalen Clanführer die Macht ihres Königs zu stärken suchten, indem sie eine größere Zahl von Personen meldeten als tatsächlich existierten. ${ }^{833}$ Doch allgemein wird von dem Grundsatz ausgegangen, daß Menschen bei einem Zensus eher gar nicht als mehrfach erfaßt werden, so daß die tatsächliche Bevölkerungszahl eines Landes in der Regel unterschätzt wird. Dies gilt um so mehr in den weniger entwickelten Ländern, in denen die Daten über die Bevölkerung ohnehin häufig nur einen fragmentarischen Charakter haben. ${ }^{834}$

Diese Defizite in der Datenqualität und der Vollständigkeit ihrer Erhebung sind auch für das moderne Ägypten zu beobachten. Insbesondere die Bevölkerungszahlen vor dem Jahr 1882 basieren häufig auf Schätzungen, die sich auf andere Verwaltungslisten wie zum Beispiel Kataster stützen. Aus diesem Grund ist es notwendig, in der nachfolgenden Analyse der Zensusergebnisse zwischen den >offiziellen< Zahlen und deren Entstehungsgeschichte einerseits sowie den von Zensuszahlen unabhängig entworfenen Rechenmodellen andererseits zu differenzieren. Eine ausführliche Betrachtung der Schätzungen und Zählungen der ägyptischen Bevölkerung vom Ende des 18. bis in das frühe 20. Jahrhundert hat kürzlich Walter

832 Der Politikwissenschaftler Friedrich Heckmann (1992: 194) hat es in seiner Studie über inner-ethnische

Beziehungen prägnant wie drastisch formuliert: »Wer [...] Kimbern und Teutonen und Gastarbeiter in einer Wanderungstheorie unter einen Hut bringen will, muß mit >dünnen Abstrakta< enden.«

833 HOLLINGSWORTH (1969: 29 f.).

834 Hollingsworth $(1969: 28,33)$. 
SCHEIDEL vorgelegt. ${ }^{835}$ SCHEIDEL beschreibt die Bevölkerungssituation des 19. Jahrhunderts zunächst auf der Grundlage zweier jüngerer Aufsätze zu dieser Thematik, die von ihm miteinander verglichen und auf ihre Tragfähigkeit hin analysiert werden. ${ }^{836}$ Beiden Autoren gemeinsam ist der Untersuchungszeitraum. Während jedoch Justin MCCARTHY die einzelnen Zensus-Ergebnisse einer Einzelkritik unterzieht und daher zwangsläufig von Fall zu Fall einen > Wahrheitsgehalt < abschätzen muß, verwirft Daniel PANZAC alle Daten vor dem Zensus aus dem Jahr 1897 und schließt von dessen Ergebnissen durch Extrapolation auf die vorangegangenen Jahrzehnte. ${ }^{837}$ Daß dieses Verfahren trotz aller statistischen Schwächen vermutlich bessere Ergebnisse liefert, sei im folgenden anhand einer kurzen Einzelkritik der Zensusdaten von 1800 bis 1882 erläutert.

\section{Offizielle und inoffizielle Zensuszahlen}

Wenn man die groben Schätzungen früher Ägyptenreisender aus der Zeit vor der Napoleonischen Expedition 1798 außer acht läßt, dann gilt die Abhandlung von Edmé JOMARD als die erste $>$ wissenschaftliche $<$ Bearbeitung einer Bevölkerungsanalyse des antiken wie des modernen Ägypten. ${ }^{838}$ Die Arbeit darf zwar als verdienstvoll gewürdigt werden, bleibt jedoch aufgrund methodischer Mängel in ihrem Ergebnis praktisch nicht verwertbar. ${ }^{839}$ Die Kritik setzt im wesentlichen an der Datenbasis an, da JOMARD für seine Berechnungen von einer Reihe Prämissen ausging, die weder empirisch gesichert, noch prinzipiell wahrscheinlich waren. Zum einen untersuchte er die Bevölkerungssituation in der Metropole Kairo, für die er die zwei Prämissen ansetzte, daß Kairo mit Paris vergleichbar sei und es in Kairo durchschnittlich pro Haus zehn Bewohner gebe. Beide Annahmen waren falsch. ${ }^{840}$ Für die flächendeckende Untersuchung suchte er sich die Provinz Minia als repräsentative Region aus und extrapolierte das Datenmaterial auf das ganze Land. Das Ergebnis von 2488950 Einwohnern $^{841}$ ohne die nomadische Bevölkerung gilt heute allgemein als deutlich zu niedrig angesetzt. Als Ausgangsbasis der darauf folgenden Zählungen und demographischen Studien wird sie dennoch in die Liste der Bevölkerungszahlen mit aufgenommen.

835 SCHEIDEL (2001a: $201 \mathrm{ff}$.).

836 SCheidels Daten basieren auf den Arbeiten von PANZAC (1987) und MCCARTHY (1976).

837 SCHEIDEL (2001a: 207).

838 Siehe dazu ausführlich auch oben Kap. 1.3.3.1.

839 Der von SCHEIDEL als Kronzeuge zitierte MCCARTHY (1976) war nicht der erste Kritiker. Beinahe ein halbes Jahrhundert früher veröffentlichte EL-DARWISH (1929) eine ausführliche Rezension von JOMARDs Abhandlung.

840 MCCARTHY (1976: 2).

841 JOMARD (1818: 100). 


\begin{tabular}{rrrl}
\hline Jahr & Bevölkerung & & Quelle \\
\cline { 1 - 1 } 1800 & 2488950 & & Schätzung von Edmé JOMARD/Napoleonische Expedition \\
1821 & 2536400 & & Schätzung durch Mohammed Ali anhand der Hauslisten \\
1846 & 4476439 & & 1. Katasteraufnahme durch Mohammed Ali \\
1873 & 5250000 & & $>1$. Volkszählung \\
1882 & 6817262 & & Volkszählung \\
1897 & 9734405 & & Volkszählung \\
1907 & 11287359 & Volkszählung \\
\hline
\end{tabular}

Tabelle 7.1: Schätzungen und Zensusdaten aus dem Ägypten des 19. Jahrhunderts

Dies gilt ebenfalls für die kaum höhere Zahl aus dem Jahr 1821, die von ScHEIDEL als Zensus deklariert, jedoch nicht näher erläutert wird. ${ }^{842}$ Die zugrundegelegte Datenbasis war die Anzahl der Wohnhäuser des Landes, aus der man die Bevölkerung zu errechnen versuchte, um für den Rekrutierungsbedarf des Militärs sowie für die geplante >Haussteuer $<$ geeignetes Zahlenmaterial zu besitzen. ${ }^{843}$ Demnach handelt es sich nicht um einen echten Zensus, sondern eher um eine von Mohammed Ali veranlaßte Schätzung, die eine Zahl von 2536400 ergab. ${ }^{844}$ Da das Ergebnis der Erhebung von 1821 sogar den Weg in eine spätere Auflage von JOMARDs Abhandlung aus dem Jahr 1829 fand, ${ }^{845}$ besteht der Verdacht, daß in zeitlicher Nähe eine Konfirmation der Ergebnisse JOMARDs aus dem Jahr 1800 erbracht werden sollte. Inoffizielle Bestandsaufnahmen der steuerpflichtigen Haushalte wurden 1827 und 1833 unternommen. Eine ausführliche Aufnahme der Landbesitzverhältnisse für Steuerzwecke fand noch unter Mohammed Ali zwischen den Jahren 1846 und 1848 statt. ${ }^{846}$ Ein Nebeneffekt war dabei die detaillierte Erfassung der Haushalte und ihrer Angehörigen, die ähnlich zu den aus pharaonischer und griechisch-römischer Zeit bekannten Listen nicht nur akribisch die Familienmitglieder - Männer und Frauen gleichermaßen - auflisteten, sondern oft auch den Beruf des männlichen Familienvorstands - ganz analog zu den aus dem Alten Ägypten bekannten Akten. ${ }^{847}$ Der Zensus des Jahres 1873 wird fast nie in neueren Tabellen vermerkt. Es scheint

842 SCHEIDEL (2001a: 203), der die Angabe als »defective beyond redemption« bewertet und darin mit ELDARWISH und AZMI (1934: 43) übereinstimmt, die vorschlugen »we ought to omit altogether the figure of 1821 in trying to calculate the rate of growth of the population of Egypt«.

843 CUNO und REIMER (1997: 197).

844 SCHEIDEL (2001a: 204 f.) vermutet hinter dem Ergebnis gar politische Motivation eines »massive underestimate $\ll$.

845 Russell (1966: 70 Anm. 10).

846 CUNO und REIMER (1997: 199 f.).

847 Cuno (1999: 309 ff.); CUNO und REIMER (1997: 204); zu den altägyptischen Listen vgl. oben Kap. 2.4, 3.3 und 3.4 . 
sich dabei um eine Art > Vorlauf< der ersten offiziellen Volkszählung im Jahr 1882 gehandelt zu haben, die nach modernen Gesichtspunkten durchgeführt worden war. Da die Generalprobe jedoch mit etlichen Mängeln behaftet war, findet sie in der neueren Forschung in der Regel keine Berücksichtigung. ${ }^{848}$ Der Zählung von 1873 kann jedoch kaum eine größere Unzuverlässigkeit als den vorangegangenen Erhebungen zugemessen werden, weshalb sie als historisches Dokument im Diagramm der ägyptischen Zensusdaten (Abb. 7.1 a) berücksichtigt wird. Die Diagramme in diesem Kapitel sind dabei alle nach dem gleichen Schema aufgebaut: Die zu einer Linie verbundenen Punkte geben die absoluten Bevölkerungszahlen des zugrundeliegenden Jahres wieder, während die Balkendiagramme die mittlere jährliche Wachstumsrate zwischen zwei Erhebungen darstellen.

\section{Bevölkerungsmodelle}

Weil die Zensusdaten sich als nicht genügend zuverlässig für weiterführende Analysen erwiesen, mußten auch für das vormoderne Ägypten Modelle entwickelt werden, die eine verläßlichere Aussage über das Bevölkerungswachstum treffen. SCHEIDEL versucht zwei unterschiedliche Ansätze: Das erste Szenario setzt eine ursprünglich niedrige Population als Ausgangsbasis und erlaubt eine hohe Wachstumsrate, das zweite Szenario startet dagegen umgekehrt mit einer relativen hohen Population, unterstellt aber ein niedriges, jedoch stetig ansteigendes Wachstum. ${ }^{849}$ Der dieser Betrachtung zugrundeliegende Zeitraum ist zwischen den Jahren 1800 und 1907 angesiedelt, letzteres als Datum des ersten relativ verläßlichen Zensus. Dabei arbeitet SCHEIDEL leider bereits mit den überarbeiteten Zensusergebnissen von MCCARTHY und PANZAC. Obwohl es nach den Umständen ihrer Herkunft prinzipiell begründet scheint, für die Wachstumsraten nicht von den ursprünglichen Zensusdaten auszugehen, so muß doch konstatiert werden, daß auch die Korrekturen von MCCARTHY und PANZAC keinen unbedingten Anspruch auf höheren Wahrheitsgehalt haben, zumal SCHEIDEL auf den Mittelwert aus den unterschiedlichen Ergebnissen beider Autoren zurückgreift. ${ }^{850}$

Aufgrund von Unstimmigkeiten, die sich aus dem ersten Szenario unter Berücksichtigung der Epidemien im Verlauf des 19. Jahrhunderts ergaben, favorisiert SCHEIDEL seine zweite Variante. MCCARTHYs und PANZACs Zahlenbasis korrigiert SCHEIDEL nochmals in Hinblick auf die großen Cholera-Epidemien, die seit 1831 wiederholt ausbrachen und erst ab dem Jahr 1865 wirkungsvoll bekämpft werden konnten. ${ }^{851}$ Mit der tendenziell hohen Population

848 Erwähnung und Kommentar bei Schamp (1977: 409 f., Tab. IV,3).

849 SCHEIDEL (2001a: 208 ff.).

850 Vgl. bei ScheIDEL (2001a: 205 f.) z. B. Tabelle 3.1.

851 Vgl. dazu ausführlich KUHNKE (1990: 49 ff.). 


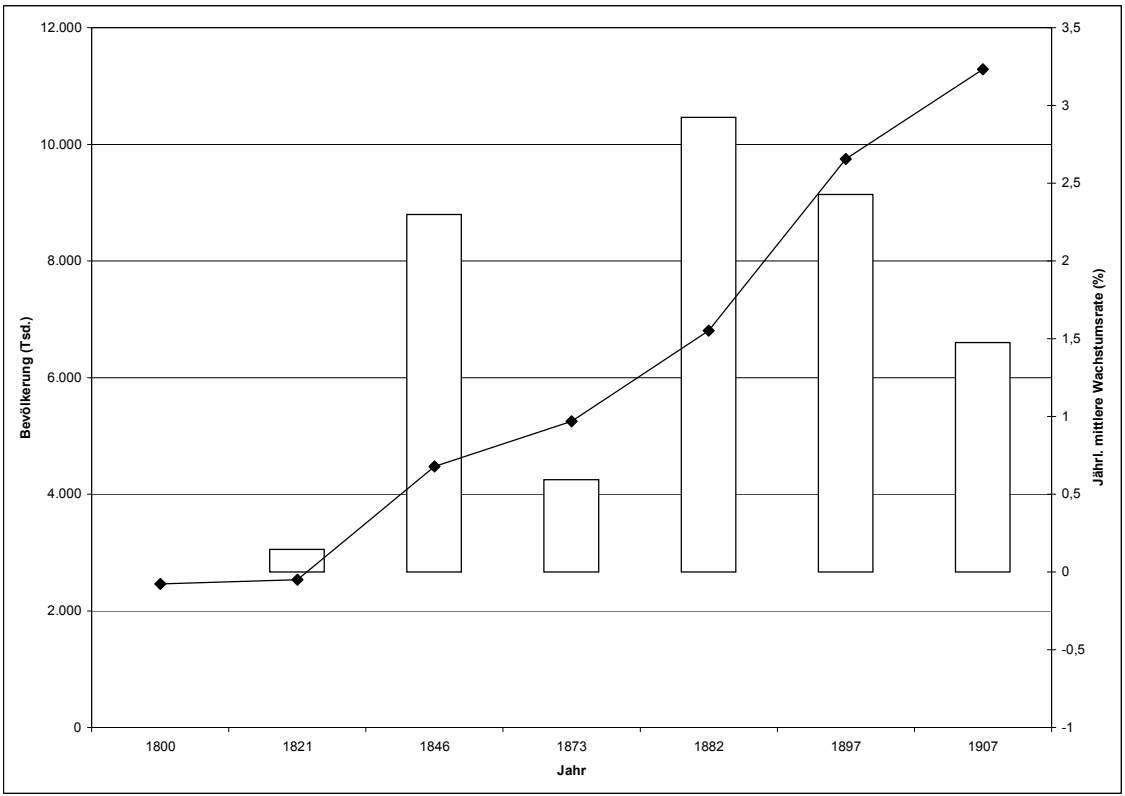

(a) Bevölkerungsentwicklung und jährliche Wachstumsraten nach den offiziellen Zensusdaten

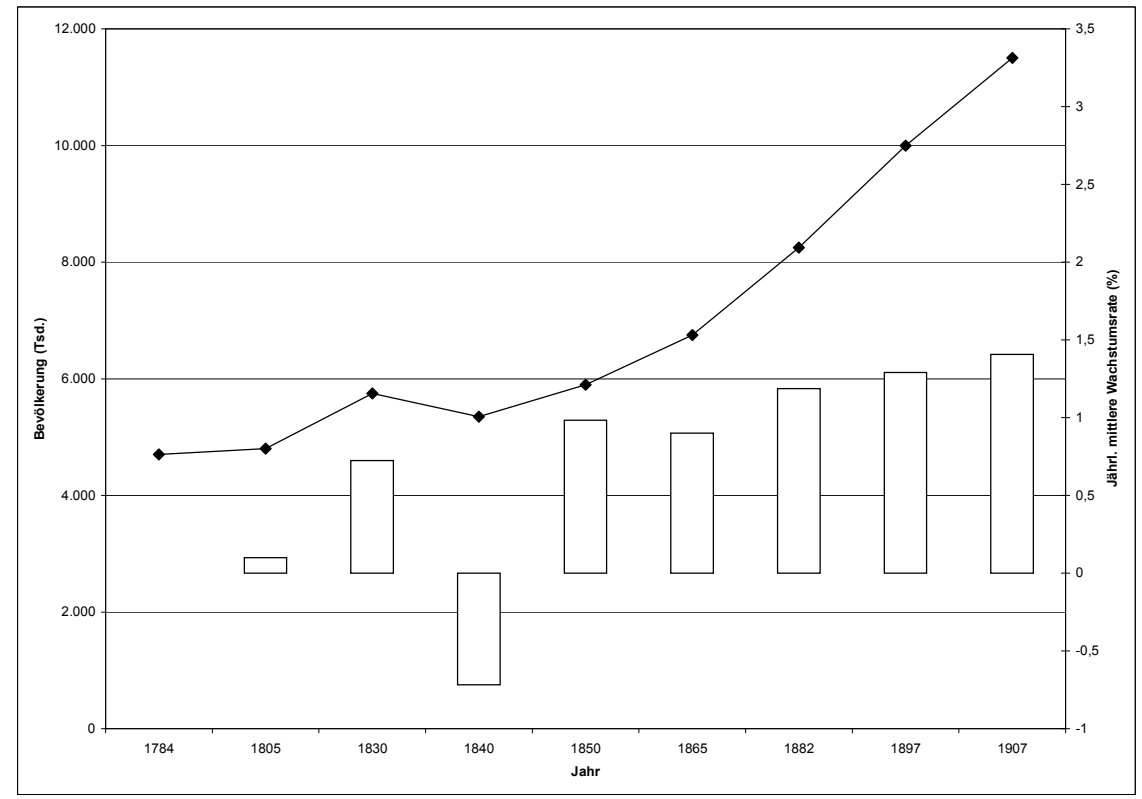

(b) Bevölkerungsentwicklung und jährliche Wachstumsraten nach SCHEIDEL (2001a: 212) Szenario II

Abbildung 7.1: Bevölkerungsentwicklung in Ägypten seit 1800 


\begin{tabular}{|c|c|c|c|}
\hline Jahr & Bevölkerung & $\varnothing$ Wachstum & Epidemien \\
\hline 1784 & 4700000 & & -400000 Tote \\
\hline 1805 & 4800000 & $0,10 \%$ & \\
\hline 1830 & 5750000 & $0,72 \%$ & -700000 Tote \\
\hline 1840 & 5350000 & $-0,72 \%$ & \\
\hline 1850 & 5900000 & $0,98 \%$ & \\
\hline 1865 & 6750000 & $0,90 \%$ & -300000 Tote \\
\hline 1882 & 8250000 & $1,19 \%$ & \\
\hline 1897 & 10000000 & $1,29 \%$ & \\
\hline 1907 & 11500000 & $1,41 \%$ & \\
\hline
\end{tabular}

Tabelle 7.2: Bevölkerungsentwicklung im Ägypten des 19. Jhs. nach SCHEIDEL

von etwa 4,4 Millionen Menschen um das Jahr 1800 als Ausgangsbasis verbindet SCHEIDEL zunächst die niedrige Wachstumsrate von 0,75\% zwischen 1805 und 1830, die bis 1907 auf 1,4\% anstieg und das verstärkte Wachstum der ägyptischen Bevölkerung einleitete (Tab. 7.2 und Abb. 7.1 b).

\subsubsection{Beobachtungen und Ergebnisse}

Die jährlichen Wachstumsraten lagen bei der Auswertung der Bevölkerungsentwicklung im neuzeitlichen Ägypten von jeher im Zentrum des Interesses. Dies hatte seine Ursache in den Hypothesen zum Bevölkerungswachstum im Alten Ägypten, das generell als sehr niedrig eingestuft wurde. ${ }^{852}$ Diese Erkenntnis stand in einem scheinbaren Widerspruch zu den Ergebnissen der Volkszählungen des 19. Jahrhunderts, die ein weitaus schnelleres Wachstum der ägyptischen Population suggerierten. Seitens der demographischen Forschung galten selbst mittlere Wachstumsraten von knapp unter $1 \%$ schon als >rapide< und bereits $3 \%$ wurden als nur über kurze Zeiträume mögliches Phänomen erklärt, das zudem durch starke Immigration begünstigt wurde. ${ }^{853}$ Diese Obergrenze beeinflußte im weiteren Verlauf immer wieder die wissenschaftliche Diskussion, wie beispielsweise die Vergleiche Alan BOWMANs zeigen, der zwischen dem antiken und modernen Ägypten Analogieschlüsse zu ziehen versuchte: ${ }^{854}$

»The increase from a Late Pharaonic population estimated at 3 million to one of 7.5 million would in fact take only about fifty years at an average annual increase of 20/1000 or 2 per cent, completely discounting any effects of immigration. As an useful analogy, an increase of this order can be documented for the years

$852 \mathrm{Vgl}$. dazu auch oben Kap. 2.6.2.

853 HOLLINGSWORTH (1969: 97).

854 BOWMAN (1986: 17). 
1821-46, under the influence of political and economic improvements in the country brought by Mohammed Ali and although the rate of increase slowed thereafter, the census figures of 1882 record a population of 6.8 million.«

Dabei weisen weder das korrigierte Datenmaterial von SCHEIDEL, noch die überlieferten Rohdaten der Bevölkerungszählungen des 19. und 20. Jahrhunderts eine mittlere Wachstumsrate von über drei Prozent auf. Gleichwohl ist bei der Schätzung von Wachstumsraten Vorsicht geboten. Bereits kleine Schwankungen der Ausgangsdaten können dramatische Änderungen der berechneten Ergebnisse hinsichtlich der Bevölkerungsentwicklung bewirken, wie bereits John DURAND im caveat seiner Einleitung festgestellt hat. ${ }^{855}$ DURAND wies darauf hin, daß bereits geringe Veränderungen in der Wachstumsrate $(0,1 \%)$ über einen längeren Zeitraum betrachtet zu starken Schwankungen der Gesamtbevölkerung führen. Bei der Ausgangsbasis von 4,8 Millionen Menschen im Jahr 1805 nach SCHEIDELs Szenario II würde bis zum Jahr 1907 mit konstanter Wachstumsrate $(0,72 \%)$ bereits eine Schwankung von 0,05\% zu einer Differenz von einer halben Million Menschen führen. Dies führt drastisch die Schwierigkeiten vor Augen, ein verläßliches Modell zu formulieren. Sofern man SCHEIDELs Szenario der ägyptischen Populationsentwicklung akzeptiert, können weitere überraschende Auffälligkeiten an seinem Datenmaterial festgestellt werden. Während die Epidemien zwischen 1784 und 1805 sowie 1831 deutliche Auswirkungen auf die durchschnittliche jährliche Wachstumsrate haben, scheint der Trend - sobald der >Schwellenwert< von $1 \%$ überschritten ist - nicht mehr umkehrbar, wie an ein paar Beispielen gezeigt werden kann. Für das Jahr 1840 vermutet SCHEIDEL aufgrund der hohen Zahl zu beklagender Opfer sogar einen Bevölkerungsrückgang. Und auch 1805 hätte die Wachstumsrate ohne die zusätzlichen Todesfälle immerhin noch bei 0,48 \% gelegen. Doch bereits 1882 differierte die Wachstumsrate ohne die Cholera-Opfer nur um etwa $0,2 \%$ und stieg danach immer rascher an, was nach Meinung SCHEIDELs das Ergebnis der verbesserten hygienischen und gesundheitlichen Bedingungen sowie des Wirtschaftswachstums war. ${ }^{856}$

\subsubsection{Lineare Wachstumsberechnungen}

Das Verfahren, aufgrund der unausgewogenen Beschaffenheit des Datenmaterials ein lineares Wachstum zwischen zwei überlieferten Zensusjahren anzusetzen, dient einerseits der Vereinfachung und ist andererseits methodisch nicht unüblich, da es bereits in der Vergangenheit für

855 DURAND (1972: 370).

856 SCHEIDEL (2001a: 208). 
die Berechnungen eingesetzt wurde. ${ }^{857}$ Aus diesem Grund können für die folgenden Berechnungen bekannte Formeln abgewandelt werden. Zunächst das lineare Wachstum einer Bevölkerung $P_{0}$ innerhalb von $n$ Jahren, wenn eine jährliche Wachstumsrate von $r$ Prozent angesetzt wird:

$$
P_{n}=P_{0} \times\left(1+\frac{r}{100}\right)^{n}
$$

Des weiteren wird als Umkehrung der Formel noch eine Möglichkeit benötigt, die lineare Wachstumsrate $r$ zu bestimmen, wenn Anfangs- $\left(P_{0}\right)$ und Endgröße $\left(P_{n}\right)$ einer Bevölkerung sowie der Entwicklungszeitraum von $n$ Jahren bekannt sind:

$$
r=\left(\sqrt[n]{\frac{P_{n}}{P_{0}}}-1\right) \times 100
$$

oder bei praktischer Anwendung besser nachvollziehbar:

$$
r=\left(\left(\frac{P_{n}}{P_{0}}\right)^{\frac{1}{n}}-1\right) \times 100
$$

Dieses Verfahren kann allerdings der tatsächlichen demographischen Situation im Einzelfall nicht gerecht werden, da Einbrüche durch Epidemien etc. nicht präzise erfaßt werden, sondern nur im tendenziell niedrigeren statistischen Mittel ihren Niederschlag finden. ${ }^{858}$ Doch selbst dies kann durch einen raschen regenerativen Ausgleich kompensiert werden, so daß die Wachstumsraten keinen Blick auf die dahinterliegenden Ereignisse erlauben. Daher gilt die Faustregel, daß der Einblick in den Verlauf der Populationskurve umso präziser ist, je dichter die Intervalle der einzelnen Erhebungen liegen. Die linearen Wachstumsberechnungen können und wollen den nichtlinearen Modellen nicht ebenbürtig sein, haben sich aber für rasche Trendbestimmungen von Durchschnittswerten in der Praxis gut bewährt.

857 MCCARThy (1976: 34 Anm. 1).

858 Genaugenommen bleiben auch eine Reihe von andere Faktoren bei der linearen Berechnung unberücksichtigt, wie etwa die Sexualproportion (Zahlenverhältnis zwischen Männern und Frauen), Altersspezifische Bevölkerungsentwicklungen sowie Schwankungen im Verhältnis von Geburten- zu Sterbeziffern. Um diese Einflüsse zu berücksichtigen, wurden in den vergangenen Jahren spezielle nichtlineare mathematische Modelle entwickelt, die in ihren Ergebnissen spezifische demographische Variablen mit einbeziehen. Zu den komplexen Rechenmodellen, die an dieser Stelle nicht weiter verfolgt werden können, vgl. ausführlich ISLAM (2002) und BAZYKIN (1998). 


\subsection{Grenzen der Vergleichbarkeit}

Im Jahr 1941 verblüffte der polnische Historiker Tadeusz WAŁEK-CZERNECKI die Fachwelt mit einem Aufsatz, in dem er für die Saïtenzeit eine Population von etwa 20 bis 21 Millionen Menschen in Ägypten feststellte. ${ }^{859}$ Kernthese seiner Untersuchung war das Herausarbeiten von Parallelen zwischen den gesellschaftlichen Entwicklungen des pharaonischen und dem modernen Ägypten des 19. und 20. Jahrhunderts: ${ }^{860}$

»Or nous verrons dans la suite qu'une analogie poussée très loin existe dans ce domaine entre l'Égypte aux $\mathrm{XIX}^{\mathrm{e}}$ et $\mathrm{XX}^{\mathrm{e}}$ siècles et certaines époques pharaoniques. Dans la mesure où cette analogie est valable, une évaluation du chiffre total des habitants aux époques respectives de l'antiquité est possible.«

WAŁEK-CZERNECKIs Grundthese für alle seine weiteren Betrachtungen ist die prinzipielle Vergleichbarkeit des ägyptischen 19. Jahrhunderts unter der Regierung Mohammed Alis und seiner Nachfolger einerseits sowie der altägyptischen 26. Dynastie (664-525 v. Chr.), die sogenannte Saïtenzeit, andererseits. Die Population zu Beginn der Regierungszeit Psammetichs I. setzte er als Prämisse relativ niedrig an, unterhalb der Zahlen Diodors und näher an den Schätzungen für das Jahr 1800 n. Chr. Die Wahl der 26. Dynastie als idealer Untersuchungszeitraum hatte für ihn drei Gründe. Da der Zeitraum der Saïtenzeit (140 Jahre) und der Zeit zwischen 1805 und 1937 - dem Jahr, in dem die Untersuchung verfaßt wurde - nahezu identisch sind, entfiel eine unterschiedliche Gewichtung des Zeitfaktors für beide Epochen. Zweitens stellte die 26. Dynastie diejenige pharaonische Epoche dar, die den ältesten schriftlichen Überlieferungen zur Gesamtbevölkerung zeitlich am nächsten lag. Und schließlich waren nach Meinung WAŁEK-CZERNECKIs die allgemeinen Bedingungen der Saïtenzeit hinreichend aus griechischen und ägyptischen Quellen bekannt. Bei gleicher Zeitdauer und prinzipiell gleichen äußeren Bedingungen erschien ihm also eine Bevölkerungsentwicklung, die im Jahr 525 v. Chr. als Minimum den Stand von 16,5 Millionen Menschen des Jahres 1937 erreichte, als zwingend. ${ }^{861}$ Um einen Eindruck von WAŁEK-CZERnECKIs Argumentationswegen zu erhalten und gleichzeitig die Fehler eines solchen Verfahrens aufzuzeigen, sollen einige seiner Thesen im folgenden etwas ausführlicher diskutiert werden.

859 WAŁEK-CZERnECKI (1941); spätere Zitate dieser Zahl bei MeulenAere (1951: 123 Anm. 221) und HoLLINGSWORTH (1969: 307 f.); kritische Beurteilung bei BRAUNERT (1964: 20 Anm. 20), der sich allerdings eher am Vergleich zwischen dem antiken und modernen Ägypten stört, als an der hohen Zahl selbst. Zuletzt bezeichnete HUSS (2001: 23 Anm. 32) die Berechnung als »nicht geglückt «.

860 WAŁEK-CZERNECKI (1941: 40).

861 WAŁEK-CZERNECKI (1941: 45, 59). 
Ausgehend vom rapiden Wachstum, das die ägyptische Bevölkerung im 19. Jahrhundert nach Auswertung der Volkszählungen nahm, suchte WAŁEK-CZERNECKI nach einzelnen Faktoren, die für diese Entwicklung verantwortlich waren und sich in Form und Auswirkung auch im Alte Ägypten wiederzufinden sind. Entscheidend für den Aufschwung der ägyptischen Wirtschaft war seiner Meinung nach die Regierungszeit von Mohammed Ali (»l'homme de génie«) in den Jahren von 1805 bis 1848 und dessen völlige Neuorganisation des ägyptischen Staates, die eine allgemeine Verbesserung der Lebensbedingungen bewirkte. Tatsächlich wurden die Technologien der Industriestaaten Europas in Ägypten unter Mohammed Ali und seinen Nachfolgern schneller und in größerem Umfang eingeführt, als in anderen nichteuropäischen Ländern. Dazu gehörten die zwischen 1852 und 1854 erbaute Eisenbahnstrecke, die Telegraphenlinien sowie ein landesweiter Postdienst. ${ }^{862}$ Auch die mechanische Industrialisierung der Landwirtschaft wurde vorangetrieben. ${ }^{863}$ Eine ausschließlich positive Beurteilung der Leistungen Mohammed Alis wird allerdings nicht einhellig geteilt. So schreibt etwa Fouad IBRAHIM über die Anwendung von zweifelhaften Methoden, mit denen Mohammed Ali die Industrialisierung vorantrieb und die gerade gegen Ende seiner Regierungszeit negative Auswirkungen hatten. Zwischen 1838 und 1882 ließ sich ein stetiger Rückgang des ägyptischen Industrialisierungsprozesses beobachten. Europa zwang Mohammed Ali zur Verringerung seiner Streitkräfte, was in der Folge zu einem Schrumpfungsprozeß in der Rüstungsindustrie führte, deren Auswirkung auch andere Wirtschaftszweige zu spüren bekamen. Angeschlagen durch Mißmanagement, der Beschäftigung von Zwangsarbeitern und hohen Produktionskosten gerieten weitere Unternehmen in den Abwärtssog und mußten schließen. ${ }^{864}$ Zudem führten die Kredite, die Ägypten zum Bau des Suezkanals aufnahm, einerseits zu einer politischen und wirtschaftlichen Abhängigkeit von Europa und brachten andererseits den ägyptischen Staat in die Nähe eines Staatsbankrotts, so daß ab 1876 eine britisch-französische Kommission die Verwaltung der ägyptischen Staatsfinanzen zur Wahrung der eigenen Interessen übernahm. Schließlich waren es die englischen Besatzer, die ab 1882 aufgrund ihres Verlangens nach ägyptischer Baumwolle die Bewässerungstechnik vorantrieben und zugleich auch die Erntefläche Ägyptens innerhalb von 35 Jahren bis 1917 nochmals um 68,4\% vergrößerten. ${ }^{865}$ Die Steigerung der Agrarfläche führte dabei nicht zwingend zu einem parallelen Wachstum der Population. Die von WAŁEK-CZERnECKI als bemerkenswert eingeschätzte

862 TOLEDANO (1998: 261).

863 Alleaume (1999: $338 \mathrm{f}$.).

864 IBRAHIM (1996: 115), übereinstimmend auch TOLEDANO (1998: 273).

865 IBRAнIм (1996: 115); zur Verdoppelung der ägyptischen Agrarfläche bereits zwischen 1820 und 1840 und nochmals zwischen 1840 und 1880 vgl. AlLEAUME (1999: 336). 
Wachstumsrate zwischen den Jahren 1821 und 1846 erweist sich in der neueren Forschung mit einem Wert von 0,3-0,5\% als eher niedrig. ${ }^{866}$ Erst zwischen 1840 und 1860 stieg die jährliche Rate auf über $1 \%$ und nahm in der Folgezeit weiter zu.

Ein eklatanter Fehler unterlief WAŁEK-CZERNECKI bei der Untersuchung der agrarfähigen Fläche Ägyptens, für die er sich auf die Angaben des Textes auf der Umfassungsmauer des Edfu-Tempels stützte. ${ }^{867}$ Obgleich diese Quelle von ihm als >unzuverlässig< und >schwierig zu interpretieren< eingestuft wird, deutet er die dort überlieferte Gesamtfläche Ägyptens von 27000000 Aruren in die Angabe der besiedelten Fläche Kulturlands um und ging für seine weiteren Überlegungen von einer Fruchtlandfläche von über 74000 qkm - statt der tatsächlichen 24800 qkm - aus. ${ }^{868}$ In zwei abschließenden Schritten sollte die Beweisführung für die Richtigkeit der zugrundeliegenden Thesen erbracht sein. Aufgrund der tendenziell besseren Lebensbedingungen in der Saïtenzeit erachtete er die Bevölkerungsgröße von 1937 (16,5 Millionen) als Untergrenze der ausgehenden 26. Dynastie und die doppelte Zahl (33 Millionen) als hypothetische Obergrenze. ${ }^{869}$ Für realistisch hielt er einen Mittelwert von 20-25 Millionen Menschen, den er auch durch eine Gegenrechnung mit einer durchschnittlichen Besiedlungsdichte von 400 Menschen/qkm zu stützen suchte und als Berechnungsgrundlage die von ihm ermittelte Flächenangabe des Edfu-Tempels nahm. ${ }^{870}$ Auch die ökonomischen Rahmenbedingungen der Getreideexporte Ägyptens in der griechisch-römischen und der Saïtenzeit wurden von WAŁEK-CZERNECKI falsch eingeschätzt. Er betonte die wichtige Rolle, die der Export in griechisch-römischer Zeit spielte und verwies darauf, daß es keine vergleichbaren Exporte während des 6. Jahrhunderts v. Chr. gab, was seiner Meinung nach auch von Ägypten selbst nicht >gewollt< war. Er erklärte sich dieses Verhalten mit dem erhöhten lokalen Bedarf Ägyptens, das aufgrund seiner großen Population keinen Ernteanteil für Exporte entbehren konnte. ${ }^{871}$ Diese Begründung kann nur teilweise aufrecht erhalten werden, da spätestens seit der

866 WAŁEK-CZERNECKI (1941: 43); neuere Zahlen bei SCHEIDEL (2001a: 213) und TOLEDANO (1998: 254). 867 WAŁEK-CZERNECKI (1941: 47 f.).

868 Korrekturen bei SchlOTT-SCHWAB (1981: 137 f. Anm. 80), dort auch die exakten Umrechnungen und Vergleiche mit statistischen Daten aus den Jahren 1949/50 (nach KEES 1977: 1): die Gesamtfläche beträgt nach Angaben der Edfu-Inschrift 74425,5 qkm, davon 24 808,5 qkm Fruchtland; im Jahr 1950 betrug die Fläche des genutzten Fruchtlandes 24982 qkm. Die Umrechnung erfolgt von SCHLOTT-SCHWAB auf Basis

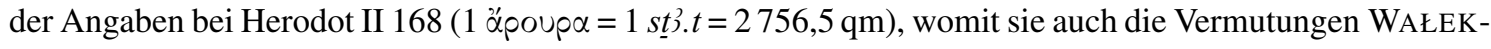
CZERNECKIs zu wechselnden Maßsystemen für die Arure verwirft. Zum Fruchtland vgl. auch SCHEIDEL (2001a: $220 \mathrm{ff}$.).

869 WAŁEK-CZERNECKI (1941: 59).

870 WAŁEK-CZERNECKI (1941: 60); hier ist ihm offenbar auch ein Rechenfehler unterlaufen, da sich aus den zugrundegelegten 400 Personen/qkm und 74000 qkm Fläche eine Gesamtpopulation von 29,6 Millionen und nicht - wie von ihm angegeben - 20-21 Millionen Menschen errechnet.

871 WAŁEK-CZERNECKI (1941: 58 f.). 
Machtergreifung durch die Römer der Getreideexport im großen Stil aus der >Kornkammer Roms < kaum eine freiwillige Leistung darstellte. Der fehlende Nachweis für Getreidehandel während der pharaonischen Zeit ist allerdings auch nicht monokausal mit >Eigenbedarf $<\mathrm{zu}$ erklären, da es durchaus Beispiele für Getreidelieferungen an Nachbarländer gibt, die unter Nahrungsmangel litten und auf Importe aus Ägypten angewiesen waren. Bekanntestes Beispiel dafür dürften die Lieferungen an die Hethiter unter Ramses II. und Merenptah während der 19. Dynastie sein, die zum Teil auch politisch motiviert waren. ${ }^{872}$ Der während der Ptolemäerzeit erwirtschaftete Überschuß an landwirtschaftlichen Gütern wurde dagegen - wie von WAŁEK-CZERNECKI richtig erkannt - gewinnbringend über den Handelsknoten Alexandria weiterverkauft. ${ }^{873}$ Dies mag auch mit der langen Tradition des Handelswesens zusammenhängen, die von den Griechen nach Ägypten mitgebracht wurde. ${ }^{874}$ Nicht vergessen sollte man in diesem Zusammenhang die Preisinflationen für Getreide, die aufgrund von Aufständen, verstärktem innenpolitischen Druck und außenpolitischen Engagements seit der Regierungszeit Ptolemaios' VI. hervorgerufen wurden. ${ }^{875}$ Schließlich bliebe zu erklären, warum es einen dramatischen Bevölkerungsrückgang zwischen der 26. Dynastie und der griechisch-römischen Zeit gab. Die Daten der antiken Historiker lagen - wie bereits in Kap. 2 ausführlich dargelegt - deutlich näher an der Wahrheit, als WAŁEK-CZERNECKI ihnen zugestehen will. Dem von ihm geschmähten Herrscher Ptolemaios XII. (Auletes) weist er eine Teilschuld am Niedergang des ägyptischen Reiches zu, doch liegt dazwischen auch die Blütezeit unter den ersten drei Ptolemäern und ein signifikanter demographischer Einbruch in der Zeit bis zur Übernahme der Herrschaft durch die Römer im Jahr 44 v. Chr. ist nicht nachweisbar.

Neben der problematischen Bewertung und Gewichtung von historischen Einzelaspekten können in der Argumentationskette WAŁEK-CZERnECKIs drei Schwachpunkte festgestellt werden, die das Ergebnis seiner Untersuchung völlig in Frage stellen. Den geringsten Anteil hat dabei noch sein blindes Vertrauen in die Korrektheit der Zensusdaten des 19. und 20. Jahrhunderts, die sich jedoch - wie bereits gezeigt werden konnte - durch moderne Analysen als recht unzuverlässig erwiesen haben. Schwerwiegender sind seine Gleichsetzungen der allgemeinen Bedingungen von 26. Dynastie und dem modernen 19. Jahrhundert. Sie beruhen im wesentlichen auf vordergründigen Gleichsetzungen von ökonomischer Situation, politischen >absolutistischen< Verhältnissen unter der Regierung Mohammed Alis sowie anderen sozialen

872 HELCK (1971: 371).

873 Bagnall (1993: 314); BowMAN (1986: 220).

874 BOWMAN (1986: $106 \mathrm{f}$.$) .$

875 HuSs (2001: 593). 
Rahmenbedingungen wie beispielsweise der Sterblichkeitsraten, die von ihm für die Saïtenzeit sogar als geringer eingeschätzt wurde. ${ }^{876}$ Insgesamt wird hierbei ein Bild der 26. Dynastie gezeichnet, das weder den historischen Bedingungen noch den komparatistischen Notwendigkeiten gerecht wird. ${ }^{877}$ Die Entwicklungen des 19. Jahrhunderts stellen sich in vielen Punkten durchaus anders dar, als von WAŁEK-CZERNECKI beschrieben wurde. Obgleich er Unterschiede der Ökonomie wahrnimmt, wie etwa die Einbindung des neuzeitlichen Ägypten in die Weltwirtschaft mit allen sich daraus ergebenden Konsequenzen, zieht er keinerlei Schlüsse für seine eigenen Betrachtungen. ${ }^{878}$ Auch die Ausblendung des Einflusses technischer Innovationen ist problematisch, da die industrielle Revolution auch und besonders in die Landwirtschaft und das Leben der Landbevölkerung Ägyptens eingriff. ${ }^{879}$ Am schwersten wiegt jedoch WAŁEK-CZERnECKIs Wahl eines deterministischen Modells, mit dem er die Meinung vertritt, daß gleiche Ausgangsbedingungen über einen fest definierten Zeitraum zu identischen Entwicklungen führen. Das Prinzip des Determinismus, das bereits ausführlich in Kap. 1.4.2 besprochen wurde, muß für die Beschreibung gesellschaftlicher Prozesse in jeder Hinsicht als diskreditiert gelten.

\subsection{Faktoren der Bevölkerungsentwicklung Deutschlands}

Um über die Einzelphänomene, die als Verursacher von Aufwärts- oder Abwärtstendenzen der Bevölkerungsentwicklung gelten müssen, ein besseres Bild zu gewinnen, bietet sich der Vergleich mit dem Verlauf anderer zeitgleicher Populationen an. Voraussetzung dafür ist eine bessere und dichtere Erfassung der demographischen Daten sowie genaue Kenntnis über die sozialen, wirtschaftlichen und politischen Hintergründe, die diese Entwicklung begleiteten. Die Wahl fiel dabei auf Deutschland, dessen Datenmaterial gut aufbereitet ist und für das trotz seiner wechselhaften Geschichte - und den damit verbundenen Schwankungen der >Deutschland < zuzurechnenden Landesfläche - Zahlen vorhanden sind, die einen diatopischen

876 WAŁEK-CZERNECKI (1941: 59).

877 SCHEIDEL (2001a: 216) wies zuletzt auf die Unmöglichkeit eines komparatistischen Vergleiches zwischen römischer und vormoderner Zeit hin, da über beide Epochen nicht genügend grundlegende Kenntnis besteht.

878 WAŁEK-CZERNECKI (1941: 53); dagegen beschreibt TOLEDANo (1998: 270 ff.) die tiefgreifenden Konsequenzen des Welthandels für die ägyptische Wirtschaft, die - eingebettet in die allgemeinen Umwälzungen und gefördert durch den Einfluß der europäischen Okkupatoren - Wachstum und ansatzweise auch verbesserte Lebensbedingungen schufen.

879 WAŁEK-CZERNECKI (1941: 50); zum Einfluß der industriellen Revolution in der Landwirtschaft vgl. ausführlich AllEAUME (1999). 


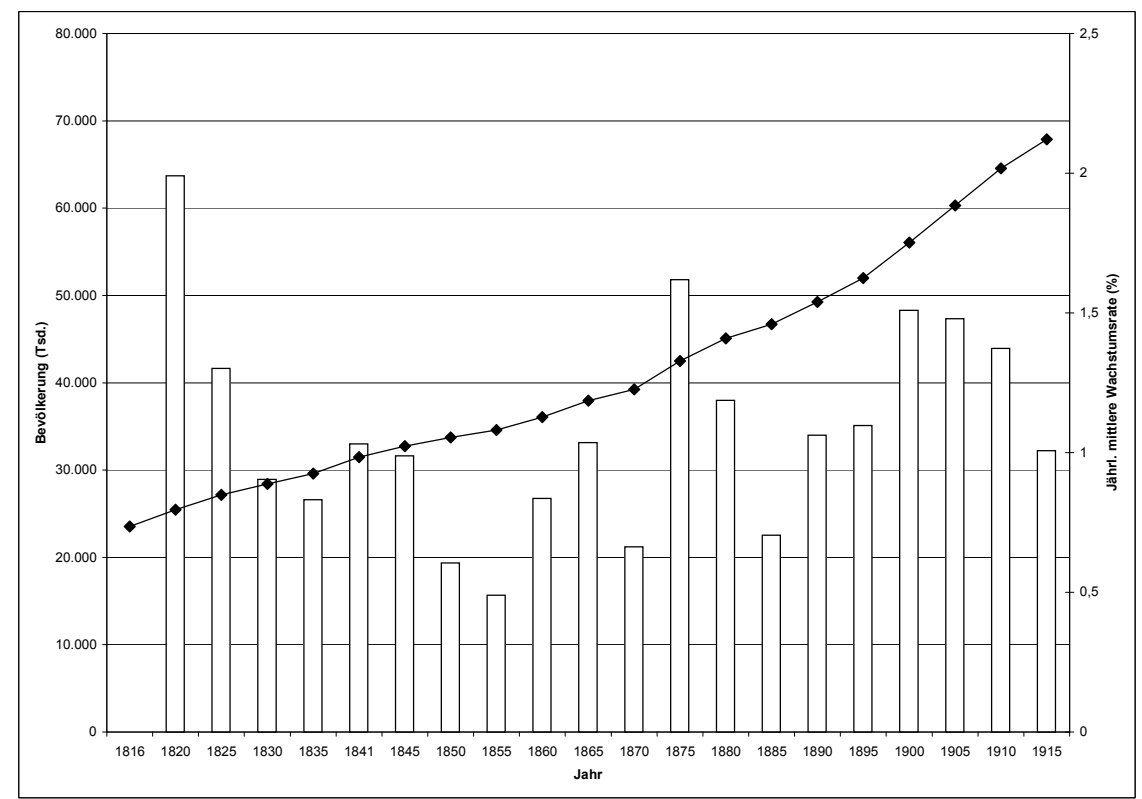

Abbildung 7.2: Bevölkerungsentwicklung in Deutschland zwischen 1816 und 1915

Vergleich erlauben. ${ }^{880}$ Mit der Gründung statistischer Ämter in allen Regionen Deutschlands, beginnend mit Preußen im Jahre 1805, beginnt die Ära regelmäßiger staatlicher Volkszählungen, die 1833 länderübergreifend gestaltet und 1872 durch die Reichsstatistik ersetzt wurden. ${ }^{881}$ Die zeitliche Obergrenze des für unsere Zwecke ausgewählten Datenmaterials wurde auf 1915 festgesetzt, weil es bereits nach dem Ersten Weltkrieg signifikante Gebietsabtretungen und damit neben den Kriegsverlusten unter der männlichen Bevölkerung auch eine rein rechnerische Umschichtung der Bevölkerung gab. Diese Ereignisse setzten sich als Faktoren auch während des Dritten Reiches bis 1945 fort und mündeten schließlich in der Gründung der Bundesrepublik Deutschland.

In dem Diagramm (Abb. 7.2) lassen sich zwei besonders signifikante Zeiträume gut ablesen. Zum einen sind dies die hohen mittleren Wachstumsraten von knapp $2 \%$ zwischen den Jahren 1816 und 1820 sowie immerhin noch 1,62 \% zwischen den Jahren 1870 und 1875. Dagegen offenbart sich ein deutlicher Tiefpunkt des Wachstums zwischen 1845 und 1855, der im Mittel nur etwa 0,55 \% betrug. Diese Einzelbeobachtungen der Entwicklung beziehen sich dabei nicht auf das gesamte Staatsgebiet, sondern die überdurchschnittlichen Wachstumsraten und Geburtenüberschüsse blieben auf einzelne Regionen beschränkt, während die Menschen

880 Die im folgenden verwendeten Daten zum >Deutschen Reich < basieren ausschließlich auf der handlichen Zusammenstellung von MARSCHALCK (1984).

881 MARSCHALCK (1984: 10 f.). 
aus verarmten Gebieten auswanderten und in die >reichen< Regionen drängten, was von den statistischen Mittelwerten nicht abgebildet werden kann. Insgesamt stellten der Zuwachs an Erwerbsstellen, der Wegfall von Ehebeschränkungen und die Veränderungen in der Arbeitswelt die stärksten Faktoren für Wachstum dar, während die Fruchtbarkeits- und Sterbeziffern relativ konstant blieben. ${ }^{882}$ Wenn dieser Zustand über einen längeren Zeitraum hinweg erhalten werden kann, spricht man demographisch von einer $>$ stabilen Bevölkerung $<.{ }^{883}$ Doch auch Deutschland blieb im 19. Jahrhundert nicht verschont von Krisensituationen, die sich auf die demographische Entwicklung auswirkten. Mißernten lösten in den Jahren 1816 und 1817 eine große Hungersnot aus und trieben die Sterbeziffern in die Höhe. ${ }^{884}$ Darüber hinaus brach zwischen 1831 und 1855 mehrfach die Cholera in Deutschland aus und ließ die Sterbeziffern auf 3-3,5\% ansteigen. Diese Einschnitte wurden jedoch mit nur geringer Verzögerung wieder ausgeglichen, da sehr bald die Eheschließungen und kurz darauf die Geburtenziffern überdurchschnittlich stiegen, so daß durch diese Kompensation in der Summe immer noch ein durchschnittliches Wachstum von ca. $1,1 \%$ zu verzeichnen war. ${ }^{885}$

\section{Ein deutsches Problem: Die Auswanderungswelle nach Amerika}

Weitaus stärker als die möglichen Einbrüche durch die Cholera-Epidemien wirkte sich die Auswanderungswelle der Deutschen auf die Wachstumsraten der Population aus. Seit 1840 beschleunigte sich der Prozeß durch die regionalen wirtschaftlichen Probleme. Ursachen gab es viele: Das starke Bevölkerungswachstum zu Beginn des 19. Jahrhunderts führte zu einer Massenverelendung ( $>$ Pauperismus $<$ ), dem die Obrigkeiten durch rigide Beschränkungen der Eheschließungen und Geburten entgegenzutreten versuchten oder gar Auswanderungswillige förderten. ${ }^{886}$ Die zuvor bereits genannten Cholera-Epidemien taten ein Übriges, so daß zwischen 1845 und 1858 etwa 1,3 Millionen Auswanderer ihr Glück in Amerika und zu einem geringen Teil auch anderswo suchten. ${ }^{887}$ Für das bis dahin hohe Wachstum von 1,25\% bedeutete dies einen massiven Einbruch, der sich auch deutlich in der Populationskurve niederschlägt.

882 MARSCHALCK (1984: 27). Die ehebeschränkenden Maßnahmen sind entweder von Behördenseite angeordnet worden und setzten ein materielles Existenzminimum der Heiratswilligen für die Eheschließung und Fortpflanzung voraus. Oder aber sie werden als freiwillige präventive (Geburten-)Kontrolle im Sinne von Thomas Malthus verstanden, der das Aufschieben der Eheschließung bei gleichzeitiger sexueller Enthaltsamkeit (>moral restraint<) vor der Ehe propagierte. Zu MALTHUS' Ansichten vor dem politischen Hintergrund seiner Zeit vgl. auch BIRG (1996: 34 f.) und APPLEMAN (1976: xviii, 130 ff.).

883 Vgl. zur Definition auch HöHN u. a. (1987: § 703).

884 MARSCHALCK (1984: 34).

885 MARSCHALCK (1984: ebenda).

886 MARSCHALCK (1984: $30 \mathrm{f}$.).

887 MARSCHALCK (1984: 32). 
Der Wegfall der Geborenenüberschüsse und die Verlangsamung des Bevölkerungswachstums haben ihre Ursache in der Alterszusammensetzung der Auswanderer, die zwischen 15 und 40 Jahren die höchste Mobilität aufwies. ${ }^{888}$ Diese Altersgruppe ist es auch, die Familien gründet und Kinder großzieht. Ein Rückgang dieser Gruppe in der Alterspyramide hatte daher eine signifikante Auswirkung auf den gesamten Entwicklungsprozeß.

\section{Urbanisierung und der Beginn der Industrialisierung}

Die moderne Demographie begründet das europäische Bevölkerungswachstum nicht mehr durch einen Anstieg der Geburtenziffer, sondern durch das Sinken der Sterbeziffer. ${ }^{889}$ Dieser Sachverhalt wurde in der sogenannten Theorie der demographischen Transformation formuliert, die den Übergang in die Industriegesellschaft beschreibt. Die sozio-ökonomische Entwicklung der Gesellschaft vollzog sich demnach von der > vorindustriellen Bevölkerungsweise $<$ mit hohen Geburten- und Sterbeziffern zur >industriellen Bevölkerungsweise< mit niedrigen Geburten- und Sterbeziffern, wobei der Geburtenüberschuß (das natürliche Wachstum) in beiden Fällen mit ca. $1 \%$ relativ niedrig blieb. ${ }^{890}$ Verstärktes Wachstum ergab sich in der Übergangsphase selbst, in der die Sterberate früher als die Geburtenrate sank und die daraus resultierende Differenz zugunsten höheren Wachstums Wirkung zeigte, bevor sich beide Raten wieder einander angeglichen haben. ${ }^{891}$

Die höhere Lebenserwartung war also der entscheidende Faktor für den demographischen Wandel, der in Deutschland seit 1880 beschleunigt stattfand. Die altersspezifischen Sterbeziffern waren dabei recht unterschiedlich, paradoxerweise nahm die Säuglingssterblichkeit insbesondere in den Städten sogar noch zu. ${ }^{892}$ Auch war die Fertilität in den Städten trotz ihrer relativ jungen Bevölkerung geringer als auf dem Lande, was jedoch zunächst kaum Auswirkungen hatte, da um 1870 lediglich $5 \%$ der Gesamtbevölkerung in Großstädten lebten. Doch durch die aus der Binnenwanderung resultierende zunehmende Verstädterung wohnten um 1900 bereits $16 \%$ der Menschen in Städten, weshalb negative Folgen für das mittlere

888 MARSCHALCK (1984: 30).

889 BiRG (1996: 50 f.); MARSCHALCK (1984: 41 ff.).

890 Die Wachstumsrate weist dabei Analogien zur Entwicklung in Ägypten während des gleichen Zeitraums auf; vgl. dazu Abb. $7.1 \mathrm{~b}$

891 BIRG (1996: 57); dies ist nur die erste von zwei Kernthesen der Transformationstheorie. Die zweite These, die sich als völlig falsch erwies, soll an dieser Stelle jedoch nicht verschwiegen werden. Sie besagt, daß nach Abschluß der Transformation die Geburtenüberschußziffer größer Null bleibt, es also weiterhin eine positive Wachstumsrate gibt, die allein ausreicht, um die Bevölkerungsgröße ohne zusätzliche Einwanderung zu halten.

892 MARSCHALCK (1984: 41 f.). 
Wachstum nicht ausblieben. ${ }^{893}$ Ein langfristiger Effekt lag auch in der >Aufwertung< der Geburt. Während für die Bestandserhaltung der Bevölkerung in der Mitte des 19. Jahrhunderts noch vier Geburten je Frau notwendig waren, lag diese Rate um das Jahr 1900 nur noch bei drei. ${ }^{894}$ Der allgemeine Rückgang der Kinderzahlen betraf dabei eher die Geburt des zweiten oder dritten Kindes, während die Zahl der Erstgeburten im gleichen Zeitraum nur um $10 \%$ sank. ${ }^{895}$

\subsection{Zusammenfassung}

Wie bereits zu Beginn dieses Kapitels erwähnt, ist die Suche nach absoluten Wachstumsraten zum Vergleich mit dem Alten Ägypten verlockend. Zumindest läßt sich am Datenmaterial des 19. Jahrhunderts ablesen, daß die schlechten allgemeinen Lebensbedingungen das jährliche mittlere Wachstum lange Zeit auf unter $1 \%$ hielten und erst mit dem langsamen Beginn der Mechanisierung und Industrialisierung ein stärkerer Anstieg einherging, der bis heute anhält. Noch interessanter sind die Folgen nach Krisenfaktoren, die einen kurzfristigen zusätzlichen Druck erzeugen und die Bevölkerungsentwicklung einknicken lassen. Hier können zwischen Ägypten und Deutschland am Vorabend zur Industriellen Revolution interessante Ähnlichkeiten festgestellt werden. Falls man den regenerativen Mechanismen eine universelle Gültigkeit unterstellt, dürften in sämtlichen Fällen die Auswirkungen von Hunger und Epidemien in kurzer Zeit wieder aufgefangen worden sein. Selbst die Massenauswanderung in Deutschland scheint den Trend nur kurzfristig verlangsamt, nicht aber gestoppt zu haben. Daher muß insgesamt von einer stärkeren regenerativen Kraft, bzw. Resistenz einer Population ausgegangen werden.

Der Nutzen eines Vergleichs zwischen antiken und modernen Gesellschaften bleibt letztendlich problematisch. Die Tabellen für die wahrscheinliche Lebensdauer eines Menschen sind sämtlich von modernen Gesellschaften abgeleitet und deren Anwendung auf die Antike bleibt ein nach heutigem Forschungsstand unbeweisbares Modell, das sich in die vagen historischen Überlieferungen einpassen muß. Die einzige Möglichkeit eines Vergleichs besteht hinsichtlich der ökonomischen Variablen. Wie zuvor gezeigt werden konnte, muß dabei jedoch eine gewisse Vorsicht walten, welche Zeiträume des vormodernen Ägypten für eine

893 MARSCHALCK (1984: 44 f., 50).

894 MARSCHALCK (1984: 42).

895 MARSCHALCK (1984: 54 f.); die Angabe betrifft den Zeitraum zwischen den Eheschließungsjahrgängen 1900 und 1922/25. 
Gegenüberstellung geeignet sind. Während WAŁEK-CZERNECKI in seiner Beurteilung der Vergleichbarkeit fehlging, zog SCHEIDEL eine strikte Grenze an Ägyptens Übergang zur Industrialisierung im letzten Viertel des 19. Jahrhunderts und beschränkte sich im wesentlichen auf die ökonomischen Parameter. Die von Kleinbauerntum geprägte ägyptische Gesellschaft wies auf diese Weise genügend Übereinstimmung auf, um Parallelen zum römischen Ägypten zu ziehen. Dies läßt sich einerseits nicht in absoluten Zahlen ausdrücken, da weder die modernen Zensusergebnisse, noch die Überlieferungen römischer Historiker anhand verläßlicher Daten auf ihre Korrektheit geprüft werden können. ${ }^{896}$ Andererseits ist dieses Modell der einzige Weg, sich der historischen Situation im römischen Ägypten zu nähern. Wenn SCHEIDEL in seinem Fazit dafür wirbt, daß die pessimistischen Urteile über die Entwicklung Ägyptens in römischer Zeit ein wenig abgemildert werden und eine größere Prosperität und Population denkbar ist, dann trägt dies auch einen kleinen Teil zur Rehabilitation der historischen Quellen bei.

896 SCHEIDEL (2001a: 246 f.). 


\section{Zusammenfassung der Ergebnisse}

Great difference of opinion exists as to the population of Egypt, both in past and present times.

John Bowring, Report on Egypt

$\mathrm{E}$ IN Blick auf die einzelnen Untersuchungsergebnisse dieser Arbeit zeigt, daß es jenseits rein statistischer Arbeit immer noch möglich ist, aus den wenigen überlieferten Textquellen sinnvolle demographische Aussagen herauszuarbeiten. Für die historischen Quellen, die nicht selten als > unwahrscheinlich< oder >übertrieben< charakterisiert werden, muß stets eine sorgfältige Argumentation bezüglich ihrer Plausibilität geführt werden, die das erste subjektive Urteil stützt oder widerlegt. Dieser Weg nötigt in vielen Fällen aufgrund des Mangels an überlieferten Informationen indirekte Beweisketten ab, die im folgenden einer abschließenden Betrachtung unterzogen werden sollen.

Die Texte der antiken Autoren Diodor und Josephus stellen - als schriftliche Quelle - den einzigen Zugang zu einer Schätzung der Gesamtbevölkerung Ägyptens dar, obgleich sie auf den griechisch-römischen Zeitraum festgelegt sind. Der Widerspruch, der zwischen den relativ niedrigen Bevölkerungszahlen nach Karl BuTZERs Berechnungen für das pharaonische Ägypten einerseits und den recht hohen Angaben der römischen Autoren andererseits vordergründig herrscht, ist tatsächlich weitaus geringer. Selbst wenn man mit einer Untergrenze von knapp 5 Millionen Menschen zu Beginn der Perserzeit (525 v. Chr.) rechnet und als Ausgangspunkt des Verlaufs Butzers Schätzung von 2,9 Millionen Menschen für das Neue Reich (1250 v. Chr.) nimmt, ergibt dies eine moderate lineare Wachstumsrate von 0,075\% in einem Zeitraum von 725 Jahren. Dieser Wert ist bereits für das Alte Reich angenommen worden, ${ }^{897}$ so daß man für das Neue Reich und die Spätzeit sogar von einer höheren mittleren Wachstumsrate ausgehen darf, die eine entsprechend höhere Population bedingt. Diese Vermutung wird gestützt durch die von Roger BAGNALL und Bruce FRIER erarbeitete Wachstumsrate von $0,2 \%$ für die römische Zeit. Spätestens an dieser Stelle gerät die Forschung jedoch in ein an-

897 BUTZER (1976: 86). 
deres Dilemma, da bei einer höheren mittleren Wachstumsrate entweder der Anfangswert des Neuen Reiches immer noch zu hoch ist, oder die bisher geäußerten Bevölkerungsgrößen der griechisch-römischen Zeit zu konservativ sind. An der Grenze zur > historischen < Zeit der Griechen, frühestens aber seit der Zeit der Perserherrschaft klafft eine zusätzliche Erklärungslücke. Sollte die in Kap. 2 vorgeschlagene Frühdatierung zutreffen, muß zumindest über die Auswirkungen der Ereignisse in der Perserzeit auf die demographische Entwicklung Ägyptens neu nachgedacht werden. Die Skepsis gegenüber einer relativ hohen Population der griechischrömischen Zeit scheint daher mehr davon genährt, den Übergang in die pharaonischen Epochen zu glätten. Auf der anderen Seite stehen die Erklärungsversuche für das Alte Ägypten, die fast ausschließlich auf Ernährungsmodellen, der carrying capacity sowie Hochrechnungen für die ägyptische Wirtschaft basieren. Doch sollte man bei derartigen Argumenten eher davon ausgehen, daß der ägyptische Boden zu allen Zeiten seine Bevölkerung ernährt hat. Eine rechnerische Obergrenze festzusetzen heißt auch, daß der Bevölkerung Ägyptens unterstellt wird, sie habe dauerhaft am Limit ihrer Versorgungsgrundlagen gelebt. Die Frage, in welcher der beiden Epochen die Angaben zur Bevölkerungsgröße korrigiert werden sollten, muß vorerst unbeantwortet bleiben. Schließlich sollte auch nicht unterschätzt werden, welche Konsequenzen die Kalkulation einer Gesamtbevölkerung auf die zugrunde liegenden Haushaltsgrößen hat. Eine generelle Anhebung der Gesamtzahl bedeutet zwangsläufig auch eine Strukturveränderung der zugrunde gelegten Siedlungen. Der Mikro-Level darf daher bei einer Beurteilung des Makro-Levels nicht außer Acht gelassen werden. ${ }^{898}$

Als mindestens ebenso schwierig hat sich die Bewertung einzelner Zensusdaten herausgestellt, die aus dem Alten Ägypten erhalten geblieben sind. Obwohl sie in der Aussagekraft ihren römischen Epigonen nicht nachstehen, kann aufgrund ihrer geringen Anzahl keine Auswertung mit statistischer Signifikanz erstellt werden. Diese Textzeugnisse bleiben Einzelbeobachtungen, die jedoch zumindest einen Eindruck davon vermitteln, mit welchen sozialen Strukturen in einem altägyptischen Haushalt zu rechnen ist. Dies ist als Grundlage für Berechnungen auf Basis von Familiengrößen zumindest realitätsnäher, als grobe Peilungen zur Personenzahl aufgrund (vor-)moderner Vergleiche.

Die wenigen schriftlichen Quellen aus der pharaonischen Zeit können ebensowenig die Problematik erhellen, ob manche Bevölkerungsgruppen nur marginal oder gar nicht durch die Administration erfaßt wurden. Die Arbeitslisten bezogen sich ausschließlich auf die staatlichen Projekte, die detaillierten Zensusakten aus Kahun stammen von Priestern, Soldaten oder

898 SCHEIDEL (2001b: 59 f.). 
zumindest Angehörigen einer im weitesten Sinne als >privilegiert< zu bezeichnenden Schicht. Lediglich Papyrus Wilbour liefert Indizien für eine flächendeckende Erfassung steuerpflichtiger Bauern, die den staatlichen Institutionen unterstellt waren. Eine detaillierte Einzeldeklaration seiner Haushaltssituation dürfte jedoch keiner dieser Bauern jemals abgegeben haben. Die Arbeitslisten können aber als grober Anhaltspunkt dafür dienen, von welcher Größenordnung kleiner Dörfer wir auszugehen haben, sofern sich die Listen ausdrücklich auf einzelne Orte beziehen. Unter der Prämisse, daß alle arbeitsfähigen Frauen und Männer in diesen Listen erfaßt worden waren, kann - unter Vorbehalt - auf die ungefähre Gesamtgröße der Bevölkerung dieser Siedlung geschlossen werden, obwohl die dabei mögliche Fehlerrate hinsichtlich der nicht erfaßten Personen beträchtlich ist: Ein alternatives Szenario wäre beispielsweise eine Siedlung, in der ausschließlich die Werktätigen zusammengefaßt sind, also weder Kinder noch Alte einen Bevölkerungsteil der Siedlung bilden.

Ein weiterer Anhaltspunkt für Siedlungsgrößen ergibt sich aus den Quellen, die auf Umsiedlungsmaßnahmen hindeuten. Hier ergaben sich aus der zusammenhängenden Betrachtung einzelner Ereignisse Anhaltspunkte, die recht genau die Größenbestimmung der neu gegründeten Siedlungen ermöglichten. Dagegen kann das Auffüllen eines Gebietes mit Menschen - auch als Peuplierung bezeichnet - wie auch die teilweise Entvölkerung ganzer Regionen als demographisches Phänomen der altägyptischen Geschichte zwar erfaßt, in ihren quantitativen Konsequenzen auf regionaler Ebene jedoch nicht beurteilt werden. Starke Schwankungen dürften diese Maßnahmen allerdings nicht ausgelöst haben, da noch im Deutschland des 18. Jahrhunderts die Migration auf die Gesamtbevölkerung gerechnet im Promillebereich lag. 899

Nicht bewährt haben sich als Berechnungsgrundlage von Siedlungsgrößen die Konzepte von Versorgungsraten und der carrying capacity aufgrund der recht schwachen Datengrundlage und der daraus resultierenden Unsicherheitsfaktoren. Noch risikoreicher ist die Heranziehung von archäologisch bezeugten Getreidespeichern und Silos, aus denen sich lediglich ableiten läßt, für wieviele Menschen eben jene Vorratsbehältnisse konzipiert waren. Ob dies auch der Gesamtzahl der angesiedelten Menschen entspricht, kann daraus nicht geschlossen werden. Diese Verfahren sind momentan nicht geeignet, um verläßliche Aussagen über Siedlungsgrößen zu treffen oder diese auch nur zu bestätigen. Vielversprechender sind hingegen die Arbeiten zu den Wirtschaftsakten des Neuen Reiches. Die Anwendung der Rang-Größen-Regel auf den Papyrus Wilbour läßt hoffen, anhand künftig hinzugewonnenen Datenmaterials Licht

899 MARSCHALCK (1984: 21). 
in Größe und Relation einzelner Siedlungen innerhalb der altägyptischen agrarischen Wirtschaftsstruktur zu bringen und hierbei auch mit konkreten Zahlen zu operieren. Die Einzelergebnisse können jedoch für keine andere Region repräsentativ sein als für diejenige, auf die sie sich beziehen. Dies stellt auch gleichzeitig das Grundproblem der auf historischen Quellen und Aussagen basierenden demographischen Forschung dar: Die im konkreten Einzelfall für einen bestimmten Zeitpunkt oder geographischen Raum gewonnenen Erkenntnisse können keineswegs leichthin als universell gültig definiert werden. Die Modellhaftigkeit, mit der die Daten einzelner Epochen auf die Analyse anderer Epochen angewendet werden müssen, bleibt auch weiterhin die Schwachstelle für die aus historischem Zahlenmaterial errechneten Ergebnisse.

Eine weitere Unüberbrückbarkeit zweier unterschiedlicher historischer Aussagen liegt in den Überlieferungen zur Fruchtbarkeit. Während die Autoren der klassischen Antike übereinstimmend vom >fruchtbaren $<$ und > volkreichen $<$ Ägypten berichten und immer wieder den Kinderreichtum betonen, weisen die anthropologische Befunde, wie auch das antike Wissen um die Risikogeburten der Ägypterinnen andererseits auf einen gegenteiligen Effekt hin. Auch dieser Widerspruch ist nach Lage der zur Verfügung stehenden Quellen nicht lösbar. Hypothetisch ließe sich vermuten, daß sich im Zeitraum zwischen der pharaonischen Zeit und der griechisch-römischen Epoche ein geringfügiger anatomischer Wandel vollzogen hat, der das zunehmende Bevölkerungswachstum wie auch dessen Beobachtung durch die antiken Historiker erklärt, etwa durch Einfluß der aus dem griechischen Raum eingewanderten Bevölkerungsgruppen. Doch ohne eine grundlegende anthropologische Untersuchung bleibt eine solche These reine Spekulation.

Die rechnerischen durchschnittlichen Lebenserwartungen von unter 30 Jahren verzerren das Bild, das gemeinhin über die altägyptische Bevölkerung gewonnen wird. Die hohe Sterblichkeit von Müttern im Kindbett und Kindern in den ersten Lebensjahren drücken den statistischen Mittelwert nach unten. Dabei konnten bereits BAGNALL und FRIER für das römische Ägypten zeigen, wie mit steigendem Alter auch die Lebenserwartung weiter wächst. Alte Menschen waren keine Seltenheit, doch war die Wahrscheinlichkeit, ein solches Alter zu erreichen, deutlich niedriger als heute.

In der vorliegenden Arbeit wurde versucht, die düstere Situation bezüglich der demographischen Forschung zum Alten Ägypten etwas aufzuhellen. Es bleibt am Schluß die Feststellung, daß der oft genug vorgebrachte Pessimismus zur Quellenlage nicht immer gerechtfertigt ist. In einigen Fällen, wenngleich in einem zeitlich oder räumlich eng begrenzten Rahmen, konnten Aussagen formuliert werden, die über vage Vermutungen hinausgehen und dennoch nicht 
letzte Gewißheit für sich beanspruchen dürfen. Da im Fall des pharaonischen Ägypten die quantitativ-statistisch operierende Demographie mangels Daten kaum eine höhere Qualität gegenüber der traditionellen Forschung für sich beanspruchen kann, bleibt nur der Versuch, durch Erfolge im kleinen Maßstab das Wissen um einzelne Aspekte der altägpytischen Bevölkerung zu vermehren. David HENIGE hat das pessimistische Credo historischer Demographie prägnant auf den Punkt gebracht: ${ }^{900}$

»Ordinarily, or so it might seem, the existence of independent testimony is a boon to the historian intent on fixing with a degree of certainity some aspect of the past. Paradoxically, searching for multiplicity in numbers entails finding variation, difference, contradiction, and dissappointment.«

Es bleibt zu hoffen, daß diese Arbeit und ihre darin aufgezeigten Möglichkeiten und Grenzen dazu beitragen, daß der künftigen Forschung wenigstens hin und wieder die von HENIGE formulierten Enttäuschungen erspart bleiben.

900 HENIGE (1998: 100). 


\section{Siglen und Abkürzungen}

Die Zitate der Papyri folgen im allgemeinen den Konventionen des Lexikons der Ägyptologie (LÄ). Für weiterführende Angaben sei an dieser Stelle auf die ausführliche Bibliographie von Madeleine BELLION (1987) verwiesen.

BGU Aegyptische Urkunden aus den Königlichen (Staatlichen) Museen zu Berlin, Griechische Urkunden, Berlin $1892 \mathrm{ff}$.

CED Jaroslav ČERnÝ, Coptic Etymological Dictionary, Cambridge 1976.

EG Alan H. GARdiner, Egyptian Grammar, 3. rev. Aufl., London 1969.

KoptHWB Wolfhart WESTENDORF, Koptisches Handwörterbuch, Heidelberg 1965-77.

KRI Kenneth A. Kitchen, Ramesside Inscriptions, 7 Bde., Oxford 1968 ff.

LD Karl Richard LePsius, Denkmaeler aus Aegypten und Aethiopien, 12 Bde. und Erg.bd., Berlin 1849-58, Leipzig 1913.

MedWB Hildegard von DEINES, Wolfhart WESTENDORF, Wörterbuch der Medizinischen Texte, Berlin 1961 (Grundriß der Medizin der alten Ägypter VII,1.2).

Pschyrembel Pschyrembel Klinisches Wörterbuch, 258. Aufl., Berlin 1998.

Urk. I Kurt SETHE, Urkunden des Alten Reiches, Leipzig ${ }^{2} 1933$.

Urk. IV Kurt SETHE, Urkunden der 18. Dynastie, Parts 1-16, Urkunden des Ägyptischen Altertums IV, Leipzig 1906-1909; Wolfgang HELCK, Urkunden der 18. Dynastie, Parts 17-22, Urkunden des Ägyptischen Altertums IV, Berlin 1955-1958.

Urk. VII Kurt SETHE, Historisch-biographische Urkunden des Mittleren Reiches, Leipzig 1935.

Wb Wörterbuch der ägyptischen Sprache, hg. von Adolf ERMAN und Hermann GRAPOW, 6 Bde., Berlin und Leipzig ${ }^{2} 1957$. 


\section{Literaturverzeichnis}

Dieses Literaturverzeichnis folgt formal den Richtlinien der Norm DIN 1505 Teil 3 für die Publikation wissenschaftlicher Arbeiten.

ABERTH 2001 ABERTH, John: From the brink of the apocalypse: confronting famine, war, plague, and death in the later middle ages. New York [u. a.] : Routledge, 2001

Adams 1990 ADAms, Barbara: A fragment from the Cairo statue of Khasekhemwy. In: Journal of Egyptian Archaeology 76 (1990), S. 161-163

Adams 1997 Adams, Matthew D.: A Textual Window on the Settlement System in Ancient Egypt. In: LuSTIG, Judith (Hrsg.): Anthropology and Egyptology. A Developing Dialogue. Sheffield : Academic Press, 1997 (Monographs in Mediterranean Archaeology 8), S. 90-105

Adamson 1982 Adamson, P. B.: Human Diseases and Deaths in the Ancient Near East. In: Die Welt des Orients 13 (1982), S. 5-14

Allam 2000 Allam, Schafik: Der Steuer-Erlaß des Königs Haremhab. In: Zeitschrift für Ägyptische Sprache und Altertumskunde 127 (2000), S. 103-111

Alleaume 1999 Alleaume, Ghislaine: An Industrial Revolution in Agriculture? Some Observations on the Evolution of Rural Egypt in the Nineteenth Century. In: BowmAN, Alan K. (Hrsg.) ; RogAn, Eugene (Hrsg.): Agriculture in Egypt. Oxford : Oxford University Press, 1999 (Proceedings of the British Academy 96), S. 331-345

Allen und Wiener 1998 Allen, James P. ; Wiener, Malcolm H.: Separate Lives: The Ahmose Tempest Stela and the Theran Eruption. In: Journal of Near Eastern Studies 57 (1998), S. 1-28

Alston 2001 Alston, Richard: Urban population in Late Roman Egypt and the end of the ancient world. In: SCHEIDEL, Walter (Hrsg.): Debating Roman demography. Leiden [u. a.] : E. J. Brill, 2001 (Mnemosyne: Supplementum 211), S. 161-204

Anthes 1964 Anthes, Rudolf: Die Felsinschriften von Hatnub. Hildesheim : Georg Olms, 1964 (Untersuchungen zur Geschichte und Altertumskunde Ägyptens 9)

Appleman 1976 Appleman, Philip (Hrsg.): Thomas Robert Malthus: An Essay on the Principle of Population. New York [u. a.] : W. W. Norton \& Co., 1976 (Norton Critical Editions) 
ATZler 1981 AtZler, Michael: Untersuchungen zur Herausbildung von Herrschaftsformen in Ägypten. Hildesheim : Gerstenberg, 1981 (Hildesheimer Ägyptologische Beiträge 16)

Ayalon 1985 Ayalon, David: Regarding Population Estimates in the Country of Medieval Islam. In: Journal of the Economic and Social History of the Orient 28 (1985), S. 1-19

BACHRACH 1999 BACHRACH, Bernard S.: Early Medieval Military Demography: Some Observations on the Methods of Hans Delbrück. In: KaGAY, Donald J. (Hrsg.) ; Villalon, L. J. Andrew (Hrsg.): The circle of war in the Middle Ages. Woodbridge : The Boydell Press, 1999 (Warfare in history), S. 3-20

BADAWY 1966 BADAWY, Alexander: A History of Egyptian Architecture. The First Intermediate Period, the Middle Kingdom, and the Second Intermediate Period. Berkeley [u. a.] : University of California Press, 1966

BADAWY 1968 BADAWY, Alexander: A History of Egyptian Architecture. The Empire (the New Kingdom). Berkeley [u. a.] : University of California Press, 1968

BAER 1962 BAer, Klaus: The Low Price Of Land in Ancient Egypt. In: Journal of the American Research Center in Egypt 1 (1962), S. 25-45

BAER 1963 BAER, Klaus: An Eleventh Dynasty Farmer's Letters to his Family. In: Journal of the American Oriental Society 83 (1963), S. 1-19

Bagnall 1993 Bagnall, Roger S.: Egypt in Late Antiquity. Princeton : Princeton University Press, 1993

BAgNall und Frier 1994 Bagnall, Roger S. ; Frier, Bruce W.: The demography of Roman Egypt. Cambridge : Cambridge University Press, 1994 (Cambridge Studies in Population, Economy and Society in Past Time 23)

BAINES und EyRE 1983 BAINES, John ; EyRe, Christopher J.: Four notes on literacy. In: Göttinger Miszellen 61 (1983), S. 65-96

BAKIR 1952 BAKIR, Abd el-Mohsen: Slavery in Pharaonic Egypt. Kairo : Institut Français d'Archéologie Orientale, 1952 (Supplément aux Annales du Service des Antiquités de l'Égypte 18)

BARDinet 1988 BARDinet, Thierry: Remarques sur les maladies de la peau, la lèpre, et le châtiment divin. In: Revue d'Égyptologie 39 (1988), S. 3-36

BAZYKIN 1998 BAZYKIN, Alexander D.: Nonlinear Dynamics of Interacting Populations. Singapur [u. a.] : World Scientific Publishing, 1998 (World Scientific Series on Nonlinear Science, Series A 11)

VON BECKERATH 1997 BECKERATH, Jürgen von: Chronologie des pharaonischen Ägypten. Mainz : Philipp von Zabern, 1997 (Münchner Ägyptologische Studien 46)

VON BECKERATH 1999 BECKERATH, Jürgen von: Handbuch der ägyptischen Königsnamen. Mainz : Philipp von Zabern, 1999 (Münchner Ägyptologische Studien 49) 
Behlmer 1998 Behlmer, Heike: Das Alter im christlichen Ägypten. In: Hallesche Beiträge zur Orientwissenschaft 26 (1998), S. 5-25

BELl 1971 Bell, Barbara: The Dark Ages in Ancient History I. The First Dark Age in Egypt. In: American Journal of Archaeology 75 (1971), S. 1-26

BELlion 1987 Bellion, Madeleine: Catalogue des Manuscrits hiéroglyphiques et hiératiques et des Dessins, sur Papyrus, Cuir ou Tissu, Publies ou Signales (Égypte Ancienne). Paris, 1987

BELOCH 1886 Beloch, Julius: Die Bevölkerung der griechisch-römischen Welt. Leipzig : Von Duncker \& Humblot, 1886

Benedictow 1987 Benedictow, Ole J.: Morbidity in Historical Plague Epidemics. In: Population Studies 41 (1987), S. 401-431

BERLEV 1965 Berlev, Oleg D.: [Rezensionsartikel zu] William K. Simpson, Papyrus Reisner I. The Records of a Building Project in the Reign of Sesostris I. (Boston 1963). In: Bibliotheca Orientalis 22 (1965), S. 263-268

BERNBECK 1997 BernBeCK, Reinhard: Theorien in der Archäologie. Tübingen [u. a.] : Francke, 1997 (Uni-Taschenbücher 1964)

Beylage 2002 Beylage, Peter: Aufbau der königlichen Stelentexte vom Beginn der 18. Dynastie bis zur Amarnazeit. Wiesbaden : Otto Harrassowitz, 2002 (Ägypten und Altes Testament 54)

BIETAK 1974/77 BIETAK, Manfred: [Ausgrabungen in] Ägypten. In: Archiv für Orientforschung 25 (1974/77), S. 310-336

BIETAK 1975 BIETAK, Manfred: Tell el-Dabca II. Der Fundort im Rahmen einer archäologischgeographischen Untersuchung über das ägyptische Ostdelta. Mit einem geodätischen Beitrag von Josef Dorner und Heinz König. Wien : Verlag der Österreichischen Akademie der Wissenschaften, 1975 (Denkschriften der Gesamtakademie 4)

BILlER 2000 BILler, Peter: The measure of multitude: population in medieval thought. Oxford : Oxford University Press, 2000

BIRG 1996 BIRG, Herwig: Die Weltbevölkerung. Dynamik und Gefahren. München : C. H. Beck, 1996 (Beck'sche Reihe 2050)

Blackman 1915 Blackman, Aylward M.: The Rock Tombs of Meir II. The Tomb-Chapel of Senbi's Son Ukh-Hotp (B, No. 2). London : Egypt Exploration Fund, 1915 (Archeological Survey of Egypt 23)

BLondheim 1955 Blondheim, S. Hillel: The First Recorded Epidemic of Pneumonic Plague: The Bible, I Sam. VI. In: Bulletin of the History of Medicine 29 (1955), S. 337-345

BOAK 1959 BOAK, Arthur E.: Egypt and the Plague of Marcus Aurelius. In: Historia 8 (1959), S. $248-250$ 
BoesSneck 1988 BoesSneck, Joachim: Die Tierwelt des Alten Ägypten. München : C. H. Beck, 1988

BOESSNECK und VON DEN DRIESCH 1988 BOESSNECK, Joachim ; DRIESCH, Angela von den: Tierknochenfunde vom Tell Ibrahim Awad im östlichen Delta. In: BRINK, Edwin C. M. van den (Hrsg.): The Archaeology of the Nile Delta, Egypt: Problems and Priorities. Amsterdam : Netherlands Foundation for Archaeological Research in Egypt, 1988, S. 117-122. - (Proceedings of the Seminar held in Cairo, 19-22 October 1986, on the occasion of the fifteenth anniversary of the Netherlands Institute of Archaeology and Arabic Studies in Cairo)

BOESSNECK und VON DEN DRIESCH 1992 BOESSNECK, Joachim ; DRIESCH, Angela von den: Tell el-Dabca VII. Tiere und historische Umwelt im Nordost-Delta im 2. Jahrtausend v. Chr. anhand der Knochenfunde der Ausgrabungen 1975-1986. Wien : Verlag der Österreichischen Akademie der Wissenschaften, 1992 (Denkschriften der Gesamtakademie 11)

BontTy 1997 BontTy, Mónica: Concerning hp. In: The Journal of the Society for the Study of Egyptian Antiquities 27 (1997), S. 1-8

Boochs 1988 Boochs, Wolfgang: Fiskaldelikte. In: Discussions in Egyptology 10 (1988), S. 9-19

BORCHARDT 1898 BORCHARDT, Ludwig: Ansiedelung Kriegsgefangener in Tempeln. In: Zeitschrift für Ägyptische Sprache und Altertumskunde 36 (1898), S. 84

BORCHARDT 1899 BORCHARDT, Ludwig: Der zweite Papyrusfund von Kahun und die zeitliche Festlegung des mittleren Reiches der ägyptischen Geschichte. In: Zeitschrift für Ägyptische Sprache und Altertumskunde 37 (1899), S. 89-103

BORCHARDT 1904 BORCHARDT, Ludwig: Zählkarten von Volkszählungen aus der Zeit des Mittleren Reiches. In: Verhandlungen des XIII. internationalen Orientalisten-Kongresses, Hamburg, September 1902. Leiden [u. a.] : E. J. Brill, 1904, S. 329

BOSERUP 1970 BOSERUP, Ester: The Conditions of Agricultural Growth. The Economics of Agrarian Change under Population Pressure. 4. Aufl. London : Allen \& Unwin, 1970

BOSERUP 1981 Boserup, Ester: Population and Technological Change. A Study of Long-Term Trends. Chicago : The University of Chicago Press, 1981

Bотті 1922 Bотті, Giuseppe: Frammenti di Registri di Stato Civile della XX ${ }^{\mathrm{a}}$ Dinastia. In: Rendiconti della R. Accademia dei Lincei. Classe di Scienze morali, storiche e filologiche 31 (1922), S. 391-394

Bowman 1986 Bowman, Alan K.: Egypt after the Pharaohs. London : British Museum Publications, 1986

BOWRING 1998 Bowring, John: Report on Egypt 1823-1838 under the reign of Mohamed Ali. London : Triade Exploration, 1998 
BraCK 1977 Brack, Annelies und Artur: Das Grab des Tjanuni. Theben Nr. 74. Mainz : Philipp von Zabern, 1977 (Archäologische Veröffentlichungen. Deutsches Archäologisches Institut Abteilung Kairo 19)

BRAUnERT 1964 BRAUnERT, Horst: Die Binnenwanderung. Studien zur Sozialgeschichte Ägyptens in der Ptolemäer- und Kaiserzeit. Bonn : Ludwig Röhrscheid, 1964 (Bonner Historische Forschungen 26)

BREASTED 1906 Breasted, James H.: Ancient Records of Egypt. Bd. 1-5. Chicago : The University of Chicago Press, 1906

VAN DEN BRINK 1992 BRINK, Edwin C. M. van den (Hrsg.): The Nile Delta in Transition; 4th.3rd. Millenium B. C. Tel Aviv : Israel Exploration Society, 1992. - (Proceedings of the Seminar held in Cairo, 21-24 October 1990, at the Netherlands Institute of Archaeology and Arabic Studies)

BRUGSCH 1876 BRUGSCH, Heinrich: Altertumskunde 14 (1876), S. 71-77

BRUNNER 1977 Brunner, Hellmut: s. v. >Fruchtbarkeit<. In: HelcK, Wolfgang (Hrsg.) ; WESTENDORF, Wolfhart (Hrsg.): Lexikon der Ägyptologie Bd. 2: Erntefest-Hordjedef. Wiesbaden : Otto Harrassowitz, 1977, S. 336-344

BRUYÈRE 1939 BRUYÈRE, Bernard: Rapport sur les fouilles de Deir el Médineh (1934-1935). Kairo : Imprimerie de l'Institut Français d'Archéologie Orientale, 1939 (Fouilles de l'Institut Français du Caire 16)

DE BUCK 1954 Buck, Adriaan de: The Egyptian Coffin Texts V. Texts of Spells 355-471. Chicago : The University of Chicago Press, 1954 (The University of Chicago Oriental Institute Publications 73)

BURTON 1972 Burton, Anne: Diodorus Siculus Book I. A Commentary. Leiden [u. a.] : E. J. Brill, 1972 (Études préliminaires aux Religions Orientales dans l’Empire Romain 29)

Butzer 1976 ButZer, Karl W.: Early Hydraulic Civilization in Egypt. A Study in Cultural Ecology. Chicago [u. a.] : The University of Chicago Press, 1976 (Prehistoric Archaeology and Ecology 3)

BUTZER 1984 BUtZer, Karl W.: s. v. >Siedlungsgeographie<. In: HelCK, Wolfgang (Hrsg.) ; WeStENDORF, Wolfhart (Hrsg.): Lexikon der Ägyptologie Bd. 5: Pyramidenbau-Steingefäße. Wiesbaden : Otto Harrassowitz, 1984, S. 924-933

Cagle 2003 Cagle, Anthony: The Spatial Structure of Kom el-Hisn: An Old Kingdom Town in the Western Nile Delta, Egypt. Oxford : Archaeopress, 2003 (British Archaeological Reports 1099)

Caminos 1956 Caminos, Ricardo A.: Literary Fragments in the Hieratic Script. Oxford : Griffith Institute, 1956

ČERNÝ 1973 ČERnÝ, Jaroslav: A Community of Workmen at Thebes in the Ramesside Period. Kairo : Institut Français d'Archéologie Orientale, 1973 (Bibliothèque d'Étude 50) 
Chevereau 1987 Chevereau, Pierre-Marie: Contribution à la prosopographie des cadres militaires de l'Ancien Empire et de la Première Période Intermédiaire. In: Revue d'Égyptologie 38 (1987), S. 13-48

Crawford 1971 Crawford, Dorothy J.: Kerkeosiris. An Egyptian village in the Ptolemaic period. Cambridge : Cambridge University Press, 1971 (Cambridge Classical Studies)

Cuno 1999 Cuno, Kenneth M.: A Tale of Two Villages: Family, Property, and Economic Activity in Rural Egypt in the 1840s. In: Bowman, Alan K. (Hrsg.) ; RogAN, Eugene (Hrsg.): Agriculture in Egypt. Oxford : Oxford University Press, 1999 (Proceedings of the British Academy 96), S. 301-329

Cuno und Reimer 1997 Cuno, Kenneth M. ; Reimer, Michael J.: The Census Registers of Nineteenth-century Egypt: a New Source for Social Historians. In: British Journal of Middle Eastern Studies 24 (1997), S. 193-216

Czerny 1999 Czerny, Ernst: Tell el-Dabca IX. Eine Plansiedlung des frühen Mittleren Reiches. Wien : Verlag der Österreichischen Akademie der Wissenschaften, 1999 (Denkschriften der Gesamtakademie 16)

DANIEL 1989 DANIEL, Ute: Arbeiterfrauen in der Kriegsgesellschaft. Göttingen : Vandenhoeck \& Ruprecht, 1989 (Kritische Studien zur Geschichtswissenschaft 84)

DAVID 1986 DAVID, A. Rosalie: The Pyramid Builders of Ancient Egypt. London [u. a.] : Routledge \& Kegan Paul, 1986

DaVIES 1902 DaVIES, Norman de Garis: The Rock Tombs of Deir el Gebrawi I. London : Egypt Exploration Fund, 1902 (Archeological Survey of Egypt 11)

DAVIES 1923 DAVIES, Norman de Garis: The tombs of two officials of Thutmosis the Fourth (nos. 75 and 90). London : Egypt Exploration Society, 1923 (The Theban Tombs Series 3)

DemaréE 1993 Demarée, Robert J.: Recent work on the administrative papyri in the Museo Egizio : Torino, 1-8 settembre 1991. In: CURTO, Silvio (Hrsg.) u. a.: Sesto Congresso Internazionale di Egittologia, Torino Bd. 2. Turin, 1993, S. 101-105

Dols 1974 Dols, Michael W.: Plague in Early Islamic History. In: Journal of the American Oriental Society 94 (1974), S. 371-383

Donadoni 2000 Donadoni, Sergio: Theben: Heilige Stadt der Pharaonen. München : Hirmer, 2000

Drummond u. a. 1990 Drummond, David C. ; JANSSEN, Rosalind M. ; J., Janssen J.: An Ancient Egyptian Rat Trap. In: Mitteilungen des Deutschen Archäologischen Instituts in Kairo 46 (1990), S. 91-98

Dunham 1938 Dunham, Dows: The Biographical Inscriptions of Nekhebu in Boston and Cairo. In: Journal of Egyptian Archaeology 24 (1938), S. 1-8 
DUPÂQUIER und DUPÂQUIER 1985 DUPÂQUIER, Jacques ; DUPÂQUIER, Michel: Histoire de la Démographie. Paris : Librairie Académique Perrin, 1985

DURAND 1972 Durand, John D.: The Viewpoint of Historical Demography. In: Spooner, Brian (Hrsg.): Population Growth: Anthropological Implications. Cambridge/Mass. [u. a.] : MIT Press, 1972, S. 370-374

ECKERT 2000 ECKeRT, Edward A.: The Retreat of Plague from Central Europe, 1640-1720: A Geomedical Approach. In: Bulletin of the History of Medicine 74 (2000), S. 1-28

EDWARDS 1960 EdWARDS, I. E. S.: Oracular Amuletic Decrees of the Late New Kingdom. London : Trustees of the British Museum, 1960 (Hieratic Papyri in the British Museum, Fourth Series)

EICHLER 1992 EICHLER, Eckhard: Polanyi - Keynes - Warburton: Zur Rekonstruktion des altägyptischen Wirtschaftssystems. In: Göttinger Miszellen 131 (1992), S. 25-31

EICHLER 1993 EICHLER, Eckhard: Untersuchungen zum Expeditionswesen des ägyptischen Alten Reiches. Wiesbaden : Otto Harrassowitz, 1993 (Göttinger Orientforschungen, IV. Reihe: Ägypten 26)

EL-DARWISH 1929 El-Darwish, Mahmoud: Analysis of some estimates of the population of Egypt before the XIX ${ }^{\text {th }}$ Century. In: L'Égypte contemporaine 20 (1929), S. 273-286

El-Darwish und Azmi 1934 El-Darwish, Mahmoud ; Azmi, H. El-S.: A Note on the Population of Egypt. In: Population. Journal of the International Union for the scientific investigation of population problems 1 (1934), February, Nr. 2, S. 43-56

ENDESFELDER 1991 ENDESFELDER, Erika: Die Formierung der altägyptischen Klassengesellschaft. Probleme und Beobachtungen. In: ENDESFELDER, Erika (Hrsg.): Probleme der frühen Gesellschaftsentwicklung im Alten Ägypten. Berlin : Humboldt-Universität, 1991, S. 5-61

ENDRUWEIT 1994 ENDRUWEIT, Albrecht: Städtischer Wohnbau in Ägypten. Klimagerechte Lehmarchitektur in Amarna. Berlin : Gebr. Mann, 1994

Engelmann und Hallof 1996 Engelmann, Heinz; Hallof, Jochen: Der Sachmetpriester, ein früher Repräsentant der Hygiene und des Seuchenschutzes. In: Studien zur Altägyptischen Kultur 23 (1996), S. 103-146

Erman 1903 ERman, Adolf: Zur Erklärung des Papyrus Harris. Berlin : Verlag der Königlichen Akademie der Wissenschaften, 1903 (Sitzungsberichte der Königlich Preußischen Akademie der Wissenschaften, Philosophisch-Historische Klasse 21)

ERMAN 1917 ERman, Adolf: Römische Obelisken. Berlin : Verlag der Königlichen Akademie der Wissenschaften, 1917 (Abhandlungen der Königlich Preußischen Akademie der Wissenschaften, Philosophisch-Historische Klasse 4)

ERMAN und SCHÄFER 1900 ERMAN, Adolf ; SCHÄFER, Heinrich: Zwei Rekrutenaushebungen in Abydos aus dem mittleren Reich. In: Zeitschrift für Ägyptische Sprache und Altertumskunde 38 (1900), S. 42-45 
EYRE 1987 EYRE, Christopher J.: Work and the Organisation of Work in the Old Kingdom. In: Powell, Marvin A. (Hrsg.): Labor in the Ancient Near East. New Haven : American Oriental Society, 1987 (American Oriental Series 68), S. 5-47

FAUlKner 1953 FaUlKner, Raymond O.: Ancient Egyptian Military Organization. In: Journal of Egyptian Archaeology 39 (1953), S. 32-47

FAULKNER 1964 FAULKNER, Raymond O.: Notes on 'The Admonitions of an Egyptian Sage'. In: Journal of Egyptian Archaeology 50 (1964), S. 24-36

FeCHT 1956 FeCHT, Gerhard: Die $H 3 t j w{ }^{-}{ }^{c}$ in $T h n w$, eine ägyptische Völkerschaft in der Westwüste. In: Zeitschrift der Deutschen Morgenländischen Gesellschaft 106 (1956), S. 37-60

FEIX 1963 FeIX, Josef (Hrsg.): Herodot: Historien. München : E. Heimeran, 1963 (Serie Tusculum)

Feucht 1995 Feucht, Erika: Das Kind im alten Ägypten. Die Stellung des Kindes in Familie und Gesellschaft nach altägyptischen Texten und Darstellungen. Frankfurt/Main [u. a.] : Campus, 1995

Franke 1998 Franke, Detlev: Kleiner Mann (nd $d s)$ - was bist Du? In: Göttinger Miszellen 167 (1998), S. 33-48

FrIER 2001 FrIER, Bruce W.: More is Worse: Some Observations on the Population of the Roman Empire. In: ScheIDEL, Walter (Hrsg.): Debating Roman demography. Leiden [u. a.] : E. J. Brill, 2001 (Mnemosyne: Supplementum 211), S. 139-159

FritZ 1970 Fritz, F. P.: Theokrit: Gedichte. München : E. Heimeran, 1970 (Serie Tusculum)

GARDINER 1909 Gardiner, Alan H.: The Admonitions of an Egyptian Sage. Leipzig : J. C. Hinrichs, 1909

GARDINER 1932 Gardiner, Alan H.: The Theory of Speech and Language. Oxford : Clarendon Press, 1932

Gardiner 1937 Gardiner, Alan H.: Late-Egyptian Miscellanies. Brüssel : Fondation Égyptologique Reine Élisabeth, 1937 (Bibliotheca Aegyptiaca 7)

Gardiner 1947 Gardiner, Alan H.: Ancient Egyptian Onomastica. Bd. 1. Oxford : Oxford University Press, 1947

GARDINer 1964 Gardiner, Alan H.: The inscription of Mes: a contribution to the study of Egyptian juridical procedure. Hildesheim : Georg Olms, 1964 (Untersuchungen zur Geschichte und Altertumskunde Ägyptens 4). - [Reprint der Ausgabe Leipzig : J. C. Hinrichs, 1905]

GARR 1987 GARR, W. Randall: A Population Estimate of Ancient Ugarit. In: Bulletin of the American Society for Oriental Research 266 (1987), S. 31-43

GASSE 1988 GASSE, Annie: Données nouvelles administratives et sacerdotales sur l'organisation du domaine d'Amon, $X X^{e}-X X I^{e}$ dynasties. Kairo : Institut Français d'Archéologie Orientale, 1988 (Bibliothèque d'Étude 104) 
Ghoneim 1977 Ghoneim, Wafik: Die ökonomische Bedeutung des Rindes im Alten Ägypten. Bonn : Rudolf Habelt, 1977 (Habelts Dissertationsdrucke, Reihe Ägyptologie, Heft 3)

GNIRS 1996 GNIRS, Andrea: Militär und Gesellschaft. Ein Beitrag zur Sozialgeschichte des Neuen Reiches. Heidelberg : Heidelberger Orientverlag, 1996 (Studien zur Archäologie und Geschichte Altägyptens 17)

Godron 1951 Godron, Gérard: Deux notes d'épigraphie thinite. In: Revue d'Égyptologie 8 (1951), S. 91-100

GODRON 1990 Godron, Gérard: Études sur l'Horus Den et quelques problèmes de l'Égypte archaïque. Genf : Patrick Kramer Éditeur, 1990 (Cahiers d'Orientalisme 19)

GOEDECKEN 1976 GOEDECKEN, Karin: Eine Betrachtung der Inschriften des Meten im Rahmen der sozialen und rechtlichen Stellung von Privatleuten im ägyptischen Alten Reich. Wiesbaden : Otto Harrassowitz, 1976 (Ägyptologische Abhandlungen 29)

GOEDICKE 1971 Goedicke, Hans: Re-Used Blocks from the Pyramid of Amenemhet I at Lisht. New York : The Metropolitan Museum of Art, 1971 (The Metropolitan Museum of Art Egyptian Expedition 20)

GOEDICKE 1984a Goedicke, Hans: »The Canaanite Illness«. In: Studien zur Altägyptischen Kultur 11 (1984), S. 91-105

Goedicke 1984b Goedicke, Hans: s. v. >Seuche<. In: Helck, Wolfgang (Hrsg.) ; WestenDORF, Wolfhart (Hrsg.): Lexikon der Ägyptologie Bd. 5: Pyramidenbau-Steingefäße. Wiesbaden : Otto Harrassowitz, 1984, S. 918-919

GomaÀ 1987 GomaÀ, Farouk: Die Besiedlung Ägyptens während des Mittleren Reiches. Wiesbaden : Dr. Ludwig Reichert, 1987 (Beihefte zum Tübinger Atlas des Vorderen Orients, Reihe B 66)

Grandet 1994 Grandet, Pierre: Le Papyrus Harris I (BM 9999). Kairo : Institut Français d'Archéologie Orientale, 1994 (Bibliothèque d'Étude 109)

GrAPOW 1958 GRAPOW, Hermann: Die medizinischen Texte in hieroglyphischer Umschreibung autographiert. Berlin : Akademie-Verlag, 1958 (Grundriß der Medizin der Alten Ägypter V)

GrIFFITH 1898 Griffith, Francis L.: Hieratic Papyri from Kahun and Gurob. London : Bernard Quaritch, 1898 (The Petrie Papyri)

Grohmann 1999 Grohmann, Heinz: Zur Planung eines Zensus in Deutschland. In: GroHmanN, Heinz (Hrsg.) ; SAHner, Heinz (Hrsg.) ; WIEGERT, Rolf (Hrsg.): Volkszählung 2001. Von der traditionellen Volkszählung zum Registerzensus. Göttingen : Vandenhoeck \& Ruprecht, 1999 (Sonderhefte zum allgemeinen statistischen Archiv. Organ der Deutschen Statistischen Gesellschaft 33), S. 25-33

Guglielmi 1980 GugLielmi, Waltraud: s. v. >Hunger<. In: Helck, Wolfgang (Hrsg.) ; WeSteNDORF, Wolfhart (Hrsg.): Lexikon der Ägyptologie Bd. 3: Horhekenu-Megeb. Wiesbaden : Otto Harrassowitz, 1980, S. 82-84 
GundLACH 1994 GundLaCH, Rolf: Die Zwangsumsiedlung auswärtiger Bevölkerung als Mittel ägyptischer Politik bis zum Ende des Mittleren Reiches. Stuttgart : Franz Steiner, 1994 (Forschungen zur Antiken Sklaverei 26)

Gutgesell 1983 Gutgesell, Manfred: Die Datierung der Ostraka und Papyri aus Deir elMedineh und ihre ökonomische Interpretation. Hildesheim : Gerstenberg, 1983 (Hildesheimer Ägyptologische Beiträge 18/19)

Hammel und Laslett 1974 Hammel, Eugene A. ; Laslett, Peter: Comparing Household Structure Over Time and Between Cultures. In: Comparative Studies in Society and History 16 (1974), S. 73-109

Hassan 1973 Hassan, Fekri A.: On mechanisms of population growth during the Neolithic. In: Current Anthropology 14 (1973), S. 535-542

Hassan 1975 HASSAn, Fekri A.: Determination of the Size, Density, and Growth Rate of HuntingGathering Populations. In: Polgar, Steven (Hrsg.): Population, Ecology, and Social Evolution. Den Haag [u. a.] : Mouton, 1975 (World Anthropology), S. 27-52

Hassan 1981 Hassan, Fekri A.: Demographic Archaeology. London : Academic Press, 1981 (Studies in Archaeology)

HASSAN 1993 HASSAN, Fekri A.: Town and village in ancient Egypt: ecology, society and urbanization. In: SHAw, Thurstan (Hrsg.) ; Sinclair, Paul (Hrsg.) ; ANDAH, Bassey (Hrsg.) ; OKPOKO, Alex (Hrsg.): The archaeology of Africa. Food, metals and towns. London [u. a.] : Routledge, 1993 (One World Archaeology 20), S. 551-569

Hassan 1994 Hassan, Fekri A.: Population Ecology and Civilization in Ancient Egypt. In: CRUMley, Carole L. (Hrsg.): Historical Ecology: cultural knowledge and changing landscapes. Santa Fe, New Mexico : School of American Research Press, 1994, S. 155-181

Hayes 1955 Hayes, William C.: A Papyrus of The Late Middle Kingdom in the Brooklyn Museum (Papyrus Brooklyn 35.1446). New York : The Brooklyn Museum, 1955

HeCkmann 1992 Heckmann, Friedrich: Ethnische Minderheiten, Volk und Nation. Soziologie inner-ethnischer Beziehungen. Stuttgart : Ferdinand Enke, 1992

HelcK 1939 Helck, Wolfgang: Der Einfluß der Militärführer in der 18. ägyptischen Dynastie. Leipzig : J. C. Hinrichs, 1939 (Untersuchungen zur Geschichte und Altertumskunde Ägyptens 14)

Helck 1958 Helck, Wolfgang: Zur Verwaltung des Mittleren und Neuen Reichs. Leiden [u. a.] : E. J. Brill, 1958 (Probleme der Ägyptologie 3)

HelCK 1959 HelCK, Wolfgang: Die soziale Schichtung des ägyptischen Volkes im 3. und 2. Jahrtausend v. Chr. In: Journal of the Economic and Social History of the Orient 2 (1959), S. 1-36

Helck 1971 Helck, Wolfgang: Die Beziehungen Ägyptens zu Vorderasien im 3. und 2. Jahrtausend v. Chr. Wiesbaden : Otto Harrassowitz, 1971 (Ägyptologische Abhandlungen 5) 
Helck 1974 Helck, Wolfgang: Die altägyptischen Gaue. Wiesbaden : Dr. Ludwig Reichert, 1974 (Beihefte zum Tübinger Atlas des Vorderen Orients, Reihe B 5)

HelCK 1975a Helck, Wolfgang: Abgeschlagene Hände als Siegeszeichen. In: Göttinger Miszellen 18 (1975), S. 23-24

HelCK 1975b HelcK, Wolfgang: Wirtschaftsgeschichte des Alten Ägypten im 3. und 2. Jahrtausend vor Chr. Leiden [u. a.] : E. J. Brill, 1975 (Handbuch der Orientalistik I 1,5)

HeLCK 1977a HelcK, Wolfgang: Die Lehre für König Merikare. Wiesbaden : Otto Harrassowitz, 1977 (Kleine Ägyptische Texte)

HelCK 1977b HelcK, Wolfgang: s. v. >Fremde in Ägypten<. In: HelcK, Wolfgang (Hrsg.) ; WESTENDORF, Wolfhart (Hrsg.): Lexikon der Ägyptologie Bd. 2: Erntefest-Hordjedef. Wiesbaden : Otto Harrassowitz, 1977, S. 306-310

HeLCK 1982 Helck, Wolfgang: s. v. >Militär . In: Helck, Wolfgang (Hrsg.) ; Westendorf, Wolfhart (Hrsg.): Lexikon der Ägyptologie Bd. 4: Megiddo-Pyramiden. Wiesbaden : Otto Harrassowitz, 1982, S. 128-134

HeLCK 1987 HelCK, Wolfgang: Untersuchungen zur Thinitenzeit. Wiesbaden : Otto Harrassowitz, 1987 (Ägyptologische Abhandlungen 45)

Hellbing 1979 Hellbing, Lennard: Alasia Problems. Göteborg : Paul Åströms, 1979 (Studies in Mediterranean Archaeology 57)

Henfling und Pflaumbaum 1991 Henfling, Edwin ; Pflaumbaum, Holger: Neue Aspekte zur klimatischen Interpretation der hohen pharaonischen Nilflutmarken am 2. Katarakt aus ägyptologischer und geomorphologischer Sicht. In: Würzburger Geographische Arbeiten 80 (1991), S. $87-109$

HeNige 1998 Henige, David: Numbers from Nowhere. The American Indian Contact Population Debate. Norman : University of Oklahoma Press, 1998

Herlihy 1990 Herlihy, David: Opera Muliebria: Women and Work in Medieval Europe. Philadelphia : Temple University Press, 1990

HeRLIHY 2000 Herlihy, David: Der schwarze Tod und die Verwandlung Europas. Berlin : Klaus Wagenbach, 2000

Hoffman 1979 Hoffman, Michael A.: Egypt Before the Pharaohs. The Prehistoric Foundations of Egyptian Civilization. New York : Dorset Press, 1979

HofFmann 2000 Hoffmann, Friedhelm: Ägypten. Kultur und Lebenswelt in griechisch-römischer Zeit. Eine Darstellung nach den demotischen Quellen. Berlin : Akademie-Verlag, 2000 (Studienbücher Geschichte und Kultur der Alten Welt) 
HöHN u. a. 1987 HöHN, Charlotte (Hrsg.) u. a.: Mehrsprachiges Demographisches Wörterbuch. Deutschsprachige Fassung. Boppard am Rhein : Boldt, 1987 (Schriftenreihe des Bundesinstituts für Bevölkerungsforschung 16)

HöLbL 1994 HöLBL, Günther: Geschichte des Ptolemäerreiches. Darmstadt : Wissenschaftliche Buchgesellschaft, 1994

HollingSWORTh 1969 Hollingsworth, Thomas H.: Historical Demography. London : Hodder and Stoughton, 1969 (The Sources of History: Studies in the Uses of Historical Evidence)

Hombert und Préaux 1952 Hombert, Marcel ; PrÉaux, Claire: Recherches sur le recensement dans l'Égypte Romaine. Leiden [u. a.] : E. J. Brill, 1952 (Papyrologica Lugduno-Batava 5)

HOPKINS 1980 HopKIns, Keith: Brother-Sister Marriage in Roman Egypt. In: Comparative Studies in Society \& History 22 (1980), S. 303-354

Huss 2001 Huss, Werner: Ägypten in hellenistischer Zeit 332-30 v. Chr. München : C. H. Beck, 2001

Hume 1964 Hume, David: Of the Populousness of Ancient Nations. In: Green, Thomas H. (Hrsg.) ; Grose, Thomas H. (Hrsg.): Essays - Moral, Political, and Literary, by David Hume Bd. 1. Aalen : Scientia Verlag, 1964, S. 381-443. - [Reprint der Ausgabe London 1882]

Husson und Valbelle 1992 Husson, Geneviève ; Valbelle, Dominique: L'état et les institutions en Égypte des premiers pharaons aux empereurs romains. Paris : Armand Colin, 1992

IBRAHIM 1996 IBRAhIM, Fouad N.: Ägypten. Eine geographische Landeskunde. Darmstadt : Wissenschaftliche Buchgesellschaft, 1996 (Wissenschaftliche Länderkunden 42)

IMHAUSEN 1996 Imhausen, Annette: Das Zahlensystem der Ägypter - (k)ein Dezimalsystem? In: Discussions in Egyptology 36 (1996), S. 49-51

ISLAM 2002 Islam, Md. Shahidul: Non-linear Age-time Dependent Population Dynamics. Göttingen, Georg-August-Universität, Dissertation, 2002

JACOBY 1957 JACOB Y, Felix: Die Fragmente der griechischen Historiker ( F Gr H). Leiden [u. a.] : E. J. Brill, 1957

JACQUET-GORDON 1962 JACQUET-GORDON, Helen: Les noms des domaines funéraires sous l'Ancien Empire Égyptien. Kairo : Institut Français d'Archéologie Orientale, 1962 (Bibliothèque d'Étude 34)

JANSSEn 1975a Janssen, Jac J.: Commodity Prices from the Ramessid Period. Leiden : E. J. Brill, 1975

JANSSEN 1975b Janssen, Jac J.: Prolegomena to the Study of Egypt's Economic History during the New Kingdom. In: Studien zur Altägyptischen Kultur 3 (1975), S. 127-185 
JANSSEn 1979 Janssen, Jac J.: The Water Supply of a Desert Village. In: Medelhavsmuseet Bulletin (1979), Nr. 14, S. 9-15

JANSSEN 1983 JANSSEN, Jac J.: El-Amarna as a residential City. In: Bibliotheca Orientalis 40 (1983), S. 273-288

JansSen 1992 JAnSSEN, Jac J.: A New Kingdom Settlement. The Verso of Pap. BM. 10068. In: Altorientalische Forschungen 19 (1992), S. 8-23

JANSSEN 1997 JANSSEN, Jac J.: Village Varia. Ten Studies on the History and Administration of Deir el-Medina. Leiden : Nederlands Instituut vor het Nabije Oosten, 1997 (Egyptologische Uitgaven 11)

JANSSEN und JANSSEN 1990 JansSen, Rosalind M. ; JANSSEn, Jac J.: Growing up in Ancient Egypt. London : The Rubicon Press, 1990

JenNy 1962 Jenny, Hans: Model of a Rising Nitrogen Profile in Nile Valley Alluvium, and Its Agronomic and Pedogenic Implications. In: Proceedings of the Soil Science Society of America 26 (1962), S. 588-591

JOMARD 1818 JOMARD, Edmé F.: Mémoire sur la population comparée de l'Égypte ancienne et moderne. In: Description de l'Égypte. Paris : L'Imprimerie Royale, 1818 (Mémoires, Antiquités 2), S. $87-142$

JUNGE 2000 JungE, Friedrich: Die Rahmenerzählung des Beredten Bauern: Innenansichten einer Gesellschaft. In: Lingua Aegyptia 8 (2000), S. 157-181

Junker 1940 Junker, Hermann: Die Maștaba des Śnb (Seneb) und die umliegenden Gräber. Wien [u. a.] : Hölder-Pichler-Tempsky, 1940 (Bericht über die von der Akademie der Wissenschaften in Wien auf gemeinsame Kosten mit Dr. Wilhelm Pelizaeus unternommenen Grabungen auf dem Friedhof des Alten Reiches bei den Pyramiden von Gîza, Band 5)

JUNKer 1955 Junker, Hermann: Die Feinde auf dem Sockel der Chasechem-Statuen und die Darstellung von geopferten Tieren. In: FIRCHOw, Otto (Hrsg.): Ägyptologische Studien (Festschrift Hermann Grapow zum 70. Geburtstag). Berlin : Akademie-Verlag, 1955 (Deutsche Akademie der Wissenschaften zu Berlin, Institut für Orientforschung, Veröffentlichungen Nr. 29), S. 162-175

KADRY 1986 KADRY, Ahmed: The Social Status and Education of Military Scribes in Egypt during the 18th Dynasty. In: Oikumene 5 (1986), S. 155-162

KAHL 1994 KAHL, Jochem: Das System der ägyptischen Hieroglyphenschrift in der 0.-3. Dynastie. Wiesbaden : Otto Harrassowitz, 1994 (Göttinger Orientforschungen, IV. Reihe: Ägypten 29)

KAPLONY 1963 KAPLONY, Peter: Die Inschriften der ägyptischen Frühzeit. Wiesbaden : Otto Harrassowitz, 1963 (Ägyptologische Abhandlungen 8)

KAPLONY 1968 KAPLONy, Peter: Steingefäße mit Inschriften der Frühzeit und des Alten Reichs. Brüssel : Fondation Égyptologique Reine Élisabeth, 1968 (Monumenta Aegyptiaca 1) 
Kauffman 1981 Kauffman, Barbara E.: The Maximal Covering Location Problem as a Stimulation of Decision Making in Ramessid Egypt, The University of Chicago, Magisterarbeit, 1981

KEES 1962 KeES, Hermann: Der sogenannte oberste Vorlesepriester. In: Zeitschrift für Ägyptische Sprache und Altertumskunde 87 (1962), S. 119-139

KeES 1977 KeES, Hermann: Das alte Ägypten - Eine kleine Landeskunde. 3. Auflage. Berlin : Akademie-Verlag, 1977

Kelley u. a. 1982 Kelley, Allen C. ; Khalifa, Atef M. ; El-KhoraZaty, M. Nabil: Population and development in rural Egypt. Durham : Duke University Press, 1982 (Studies in social and economic demography)

KeMP 1984 KemP, Barry J.: Amarna Reports I. London : Egypt Exploration Society, 1984 (Occasional Publications 1)

KeMP 1987a Kemp, Barry J.: Amarna Reports IV. London : Egypt Exploration Society, 1987 (Occasional Publications 5)

KeMP 1987b KemP, Barry J.: The Amarna Workmen's Village in Retrospect. In: Journal of Egyptian Archaeology 73 (1987), S. 21-50

KeMP 1991 KemP, Barry J.: Ancient Egypt. Anatomy of a Civilization. London : Routledge, 1991

KнатTAв 1982 KнаттAB, Aleya: Das Ägyptenbild in den deutschsprachigen Reisebeschreibungen der Zeit von 1285-1500. Frankfurt/Main : Peter Lang, 1982 (Europäische Hochschulschriften 517)

KöHLER 1995 KöHLER, E. Christiana: The State of Research on Late Predynastic Egypt: New Evidence for the Development of the Pharaonic State? In: Göttinger Miszellen 147 (1995), S. 79-92

KolB 1984 Kolb, Frank: Die Stadt im Altertum. München : C. H. Beck, 1984

Kramer 1980 Kramer, Carol: Estimating Prehistoric Populations: an Ethnoarchaeological Approach. In: L'archéologie de l'Iraq du début de l'époque néolithique à 333 avant notre ère. Perspectives et limites de l'interpretation anthropologique des documents. Paris : Éditions du centre national de la recherche scientifique, 1980 (Colloques internationaux du Centre national de la recherche scientifique 580), S. 315-334

Kratz 2000 Kratz, Reinhard G.: Die Komposition der erzählenden Bücher des Alten Testaments. Göttingen : Vandenhoeck \& Ruprecht, 2000 (Uni-Taschenbücher 2157)

Kóthay 2001 Kóthay, Katalin A.: Houses ans Households at Kahun: Bureaucratic and Domestic Aspects of Social Organization During the Middle Kingdom. In: GYôRY, Hedvig (Hrsg.): »le lotus qui sort de terre " - Melanges offerts à Edith Varga. Budapest, 2001 (Bulletin du Musée Hongrois des Beaux-Arts, Supplement 2001), S. 349-368

Kuhnke 1990 Kuhnke, Laverne: Lives at Risk: Public Health in Nineteenth-century Egypt. Kairo : The American University in Cairo Press, 1990 
LANE 1852 LANE, Edward W.: Sitten und Gebräuche der heutigen Egypter. Bd. 1-3. Leipzig : Dyk'sche Buchhandlung, 1852. - [Deutsche Ausgabe von Julius Theodor Zenker]

Lane 1999 Lane, Steven G.: Rural Population and the Experience of Warfare in Medieval Lombardy: The Case of Pavia. In: Kagay, Donald J. (Hrsg.) ; Villalon, L. J. Andrew (Hrsg.): The circle of war in the Middle Ages. Woodbridge : The Boydell Press, 1999 (Warfare in history), S. $127-134$

LEGRAIN 1902 Legrain, Georges: Notes d'Inspection: Les Stèles d'Aménôthès IV à Zernik et à Gebel Silsileh. In: Annales du Service des Antiquités de l'Égypte 3 (1902), S. 259-266

LEHNER 1997 LeHner, Mark: The Complete Pyramids. London : Thames \& Hudson, 1997

LEITZ 1994 LeITZ, Christian: Tagewählerei. Das Buch ḩ3t nḥh ph.wy dt und verwandte Texte. Wiesbaden : Otto Harrassowitz, 1994 (Ägyptologische Abhandlungen 5)

LEITZ 1995 LeITZ, Christian: Altägyptische Sternuhren. Löwen : Peeters, 1995 (Orientalia Lovaniensia Analecta 62)

LEVEN 2000 LEVEN, Karl-Heinz: »Das Einzige von allem, was wirklich jede Erwartung überstieg «: Thukydides, Perikles und die Pest in Athen. In: Karenberg, Axel (Hrsg.) ; Leitz, Christian (Hrsg.): Heilkunde und Hochkultur I. Geburt, Seuche und Traumdeutung in den antiken Zivilisationen des Mittelmeerraumes. Münster [u. a.] : Lit Verlag, 2000 (Naturwissenschaft - Philosophie Geschichte 14), S. 71-84

Livi-BaCCI 1991 LiVI-BACCI, Massimo: Population and nutrition. An essay on European demographic history. Cambridge : Cambridge University Press, 1991 (Cambridge Studies in Population, Economy and Society in Past Time 14)

LiVi-BACCI 1992 LiVi-BACCI, Massimo: A Concise History of World Population. Oxford : Blackwell, 1992

LlOYD 1988 Lloyd, Alan B.: Herodotus Book II. Commentary 99-182. Leiden [u. a.] : E. J. Brill, 1988 (Études préliminaires aux Religions Orientales dans l’Empire Romain 43)

LOPRIENO 1986 LOPRIENO, Antonio: s. v. >Zahlwort<. In: HELCK, Wolfgang (Hrsg.) ; WeSTENDORF, Wolfhart (Hrsg.): Lexikon der Ägyptologie Bd. 6: Stele-Zypresse. Wiesbaden : Otto Harrassowitz, 1986, S. 1306-1319

MARSChALCK 1984 MARSCHALCK, Peter: Bevölkerungsgeschichte Deutschlands im 19. und 20. Jahrhundert. Frankfurt/Main : Suhrkamp, 1984 (edition suhrkamp 1244)

MaSali 1972 MaSAli, Melchiorre: Body Size and Proportions as Revealed by Bone Measurements and their Meaning in Environmental Adaptation. In: Journal of Human Evolution 1 (1972), S. $187-197$

Masali und Chiarelli 1972 Masali, Melchiorre ; Chiarelli, Brunetto A.: Demographic Data on the Remains of Ancient Egyptians. In: Journal of Human Evolution 1 (1972), S. 161-169 
Mattha 1975 Mattha, Girgis: The Demotic Legal Code of Hermopolis West. Kairo : Institut Français d'Archéologie Orientale du Caire, 1975 (Bibliothèque d'Étude 45)

MCCARThy 1976 MCCARThy, Justin A.: Nineteenth-century Egyptian population. In: Middle Eastern Studies 12 (1976), Nr. 3, S. 1-39

MCDowell 1999 McDowell, Andrea G.: Village Life in Ancient Egypt. Oxford : Oxford University Press, 1999

Meulenaere 1951 Meulenaere, Herman de: Herodotos over de 26ste Dynastie. Leuven : Instituut voor Oriëntalisme, 1951 (Bibliothèque du Muséon 27)

Michel und BAUERnFEIND 1959 Michel, Otto (Hrsg.) ; BAuERnfeind, Otto (Hrsg.): Flavius Josephus: De Bello Judaico / Der Jüdische Krieg. Darmstadt : Wissenschaftliche Buchgesellschaft, 1959

Miller 1991 Miller, R. L.: Counting Calories in Egyptian Ration Texts. In: Journal of the Economic and Social History of the Orient 34 (1991), S. 257-269

Millet 1990 Millet, Nicolas B.: The Narmer Macehead and Related Objects. In: Journal of the American Research Center in Egypt 27 (1990), S. 53-60

Mitchell 1979 Mitchell, Bruce: Geography and resource analysis. London : Longman, 1979

MOERS 2001 MoERs, Gerald: Fingierte Welten in der ägyptischen Literatur des 2. Jahrtausends v. Chr. Leiden [u. a.] : E. J. Brill, 2001 (Probleme der Ägyptologie 19)

Moran 1992 Moran, William L.: The Amarna Letters. Baltimore [u. a.] : The Johns Hopkins University Press, 1992

Moreno Garcia 2000 Moreno Garcia, Juan Carlos: Acquisition de serfs durant la Première Période Intermédiaire: une étude d'histoire sociale dans l'Égypte du $\mathrm{III}^{\mathrm{e}}$ millénaire. In: Revue d'Égyptologie 51 (2000), S. 123-139

Morenz 1998 MorenZ, Ludwig D.: Hungersnöte in der Ersten Zwischenzeit zwischen Topos und Realität. In: Discussions in Egyptology 42 (1998), S. 83-97

MÜLLER 1967 MÜLLER, Dieter: Neue Urkunden zur Verwaltung im Mittleren Reich. In: Orientalia 36 (1967), S. 351-364

MÜLLER 1997 MÜLLER, Klaus E.: Geschichte der antiken Ethnologie. Reinbek : Rowohlt, 1997

Müller-Wollermann 1986 Müller-Wollermann, Renate: Krisenfaktoren im ägyptischen Staat des ausgehenden Alten Reichs. Tübingen, Eberhard Karls Universität, Dissertation, 1986

MÜLleR-WOLlermann 1987 Müller-Wollermann, Renate: sw.tjw-Bauern als Kolonisatoren. In: Varia Aegyptiaca 3 (1987), S. 263-267 
Müller-Wollermann 1991 Müller-Wollermann, Renate: Präliminierungen zur ägyptischen Stadt. In: Zeitschrift für Ägyptische Sprache und Altertumskunde 118 (1991), S. 48-54

NAROLl 1962 NAROLl, Raoul: Floor Area and Settlement Population. In: American Antiquity 27 (1962), S. 587-589

NAVILle 1886 NAVILle, Edouard: Das aegyptische Todtenbuch der XVIII. bis XX. Dynastie. Berlin : Asher \& Co., 1886

NewberRy 1893 Newberry, Percy E.: Beni Hassan I. London : Kegan Paul [u. a.], 1893

Newell 1988 Newell, Colin: Methods and Models in Demography. London : Belhaven Press, 1988

NORRIS 1977 NORRIS, John: East or West? The Geographic Origin of the Black Death. In: Bulletin of the History of Medicine 51 (1977), S. 1-24

NOTHERS 1992 NOTHERS, Thomas (Hrsg.): Diodors Griechische Weltgeschichte Buch I-X. Übersetzt von Gerhard Wirth (Buch I-III) und Otto Veh (Buch IV-X). Erster Teil. Stuttgart : Anton Hiersemann, 1992 (Bibliothek der Griechischen Literatur 34)

NunN 1996 NunN, John F.: Ancient Egyptian Medicine. London : British Museum Press, 1996

O'CONNOR 1972a O'CONNOR, David: The geography of settlement in ancient Egypt. In: UCKO, Peter J. (Hrsg.) ; Tringham, Ruth (Hrsg.) ; Dimbleby, Geoffrey W. (Hrsg.): Man, settlement and urbanism. London : Duckworth, 1972, S. 681-698. - (Proceedings of a meeting of the Research Seminar in Archaeology and Related Subjects held at the Institute of Archaeology, London University)

O'Connor 1972b O'Connor, David: A Regional Population in Egypt to circa 600 B. C. In: SPOONER, Brian (Hrsg.): Population Growth: Anthropological Implications. Cambridge/Mass. [u. a.] : MIT Press, 1972, S. 78-100

O’Connor 1997 O'Connor, David: The Elite Houses of Kahun. In: PHILliPS, Jacke (Hrsg.) u. a.: Ancient Egypt, the Aegean, and the Near East. Studies in Honour of Martha Rhoads Bell. Van Siclen Books, 1997, S. 389-400

Oldfather 1968 OldFather, Charles H. (Hrsg.): Diodorus of Sicily in twelve volumes, I: Books I and II, 1-34. London: William Heinemann, 1968 (The Loeb Classical Library 279)

Отто 1953 Отто, Eberhard: Ägypten. Der Weg des Pharaonenreiches. Stuttgart : W. Kohlhammer, 1953

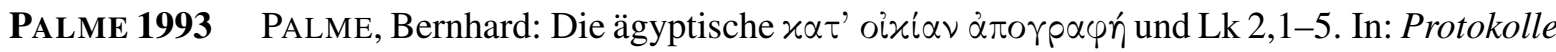
zur Bibel 2 (1993), S. 1-24

Pamminger 1991 Pamminger, Peter: Das Trinken von Überschwemmungswasser - eine Form der jährlichen Regeneration des Verstorbenen. In: Göttinger Miszellen 122 (1991), S. 71-76 
Panzac 1987 Panzac, Daniel: The population of Egypt in the nineteenth century. In: Asian and African Studies : Annual of the Israel Oriental Society 21 (1987), S. 11-32

Pardey 2003 Pardey, Eva: Die Datierung der »Dienstanweisung für den Wesir« und die Problematik von $T p$ rsj im Neuen Reich. In: KlOTh, Nicole (Hrsg.) ; PARDEY, Eva (Hrsg.): Es werde niedergelegt als Schriftstück. Festschrift für Hartwig Altenmüller zum 65. Geburtstag. Hamburg : Helmut Buske Verlag, 2003 (Studien zur Altägyptischen Kultur. Beihefte 9), S. 323-334

Parkinson 1991 Parkinson, Richard B.: The Tale of the Eloquent Peasant. Oxford : Griffith Institute, Ashmolean Museum, 1991

PARKINSON 1997 PARKInson, Richard B.: The Tale of Sinuhe and other Ancient Egyptian Poems 1940-1640 BC. Oxford : Clarendon Press, 1997

Peet 1930 Peet, Thomas E.: The Great Tomb-Robberies of the Twentieth Egyptian Dynasty. Oxford : Clarendon Press, 1930

Petrie 1891 Petrie, William Matthew F.: Illahun, Kahun and Gurob. Warminster : Aris \& Phillips, 1891

Petrie 1900 Petrie, William Matthew F.: Dendereh : 1898. London : Egypt Exploration Fund, 1900 (Memoirs of the Egypt Exploration Fund 17)

Petrie u. a. 1923 Petrie, William Matthew F. ; Brunton, Guy ; Murray, Margaret A.: Lahun II. London : Bernard Quaritch, 1923 (British School of Archaeology in Egypt and Egyptian Research Account [26. Year, 1920] 33)

Pfeifer 1995 PfeIfer, Gerhard: Ägypten im Alten Testament. München, 1995 (Biblische Notizen $\cdot$ Beihefte 8 )

PinCH 1983 PInCH, Geraldine: Childbirth and Female Figurines at Deir el-Medina and el- ${ }^{c}$ Amarna. In: Orientalia 52 (1983), S. 405-414

Posener-Kriéger 1975 Posener-Kriéger, Paule: Les papyrus de Gébélein. Remarques préliminaires. In: Revue d'Égyptologie 27 (1975), S. 211-221

Posener-Kriéger 1986 Posener-Kriéger, Paule: Old Kingdom papyrus: external features. In: BIER BRIER, Morris L. (Hrsg.): Papyrus: Structure and Usage. London : Trustees of the British Museum, 1986 (British Museum Occasional Papers 60), S. 25-41

QUibell 1900 Quibell, James E.: Hierakonpolis, Part I. London : Bernard Quaritch, 1900 (Egyptian Research Account 4)

QUIRKE 1988 QUIRKE, Stephen: State and Labour in the Middle Kingdom. A Reconsideration of the Term hnrt. In: Revue d'Égyptologie 39 (1988), S. 83-106

QUIRKE 1990 QUIRKE, Stephen: The Administration of Egypt in the Late Middle Kingdom. Whitstable : Sia Publishing, 1990 
Quirke 1991a Quirke, Stephen: The Egyptological Study of Placenames. In: Discussions in Egyptology 21 (1991), S. 59-71

QUIRKE 1991b QUIRKE, Stephen: "Townsmen" in the Middle Kingdom. On the Term s-n-niwt-tn. In: Zeitschrift für Ägyptische Sprache und Altertumskunde 118 (1991), S. 141-149

Rathbone 1990 Rathbone, Dominic: Villages, Land and Population in Graeco-Roman Egypt. In: Proceedings of the Cambridge Philological Society 216 (1990), S. 103-142

REDFORD 1984 REDFORD, Donald B.: Akhenaten, the Heretic King. Princeton : Princeton University Press, 1984

Reher und SChOFIEld 1993 Reher, David S. (Hrsg.) ; Schofield, Roger (Hrsg.): Old and New Methods in Historical Demography. Oxford : Clarendon Press, 1993

REINEKE 1978 REINEKE, Walter F.: Gedanken zum vermutlichen Alter der mathematischen Kenntnisse im Alten Ägypten. In: Zeitschrift für Ägyptische Sprache und Altertumskunde 105 (1978), S. $67-76$

RICKE 1932 RICKE, Herbert: Der Grundriß des Amarna-Wohnhauses. Leipzig : J. C. Hinrichs, 1932 (Ausgrabungen der Deutschen Orient-Gesellschaft in Tell el-Amarna 4)

ROIK 1988 RoIK, Elke: Das altägyptische Wohnhaus und seine Darstellung im Flachbild. Frankfurt/Main [u. a.] : Peter Lang, 1988 (Europäische Hochschulschriften, Reihe 38: Archäologie 15)

RostovtzeFF 1984 RostovtZeFF, Michael: Gesellschafts- und Wirtschaftsgeschichte der hellenistischen Welt. Darmstadt : Wissenschaftliche Buchgesellschaft, 1984. - [Übersetzung der Originalausgabe Oxford : Clarendon Press, 1941]

Russell 1966 Russell, Josiah C.: The Population of Medieval Egypt. In: Journal of the American Research Center in Egypt 5 (1966), S. 69-82

RyHOLt 1997 Ryнolt, Kim S. B.: The Political Situation in Egypt during the Second Intermediate Period c. 1800-1550 B. C. Kopenhagen : Museum Tusculanum Press, 1997 (Carsten Niebuhr Institute Publications 20)

SACKS 1990 SaCKS, Kenneth S.: Diodorus Siculus and the first century. Princeton : Princeton University Press, 1990

SADEK 1980 SADEK, Ashraf I.: The amethyst mining inscriptions of Wadi el-Hudi. Warminster : Aris \& Phillips, 1980

SANDERS 1984 SANDERS, Guy: Reassessing ancient populations. In: Annual of the British School at Athens 79 (1984), S. 251-262

SChAEDEl 1936 Schaedel, Herbert D.: Leipziger Ägyptologische Studien. Bd. 6: Die Listen des großen Papyrus Harris. Ihre wirtschaftliche und politische Ausdeutung. Glückstadt [u. a.] : J. J. Augustin, 1936 
SCHÄFER 1902 SCHÄFER, Heinrich: Ein Bruchstück altägyptischer Annalen. Berlin : Akademie der Wissenschaften, 1902 (Phil.-hist. Abh. nicht zur Akad. gehör. Gelehrter)

SCHÄFERS 2001 SCHÄFERS, Bernhard (Hrsg.): Grundbegriffe der Soziologie. Opladen : Leske + Budrich, 2001 (Uni-Taschenbücher 1416)

SChAMP 1977 Schamp, Heinz (Hrsg.): Ägypten. Tübingen [u. a.] : Horst Erdmann, 1977

SCHEIDEL 1998 SCHEIDEL, Walter: The meaning of dates on mummy labels: seasonal mortality and mortuary practice in Roman Egypt. In: Journal of Roman Archaeology 11 (1998), S. 285-292

SCHEIDEl 2001a SCHEIDEL, Walter: Death on the Nile. Disease \& the Demography of Roman Egypt. Leiden [u. a.] : E. J. Brill, 2001 (Mnemosyne: Supplementum 228)

SCHEIDEL 2001b SCHEIDEL, Walter: Progress and problems in Roman demography. In: SCHEIDEL, Walter (Hrsg.): Debating Roman demography. Leiden [u. a.] : E. J. Brill, 2001 (Mnemosyne: Supplementum 211), S. 1-81

SCHENKEL 1965 SCHENKel, Wolfgang: Memphis, Herakleopolis, Theben. Die epigraphischen Zeugnisse der 7.-11. Dynastie Ägyptens. Wiesbaden : Otto Harrassowitz, 1965 (Ägyptologische Abhandlungen 12)

SCHENKEL 1978 SCHENKEL, Wolfgang: Die Bewässerungsrevolution im Alten Ägypten. Mainz : Philipp von Zabern, 1978 (Sonderschrift des Deutschen Archäologischen Instituts, Abteilung Kairo 6)

SChlotT-SCHWAB 1981 SchlotT-Schwab, Adelheid: Die Ausmaße Ägyptens nach altägyptischen Texten. Wiesbaden : Otto Harrassowitz, 1981 (Ägypten und Altes Testament 3)

SCHNEIDER 1997 SChNeIder, Thomas: Lexikon der Pharaonen. Düsseldorf [u. a.] : Artemis \& Winkler, 1997

Sснотт 1950 Sснотт, Siegfried: Hieroglyphen. Untersuchungen zum Ursprung der Schrift. Mainz : Verlag der Akademie der Wissenschaften und der Literatur, 1950 (Akademie der Wissenschaften. Abh. der Geistes- und Sozialwissenschaftlichen Klasse 24)

Sснотт 1965 Schотт, Siegfried: Aufnahmen vom Hungersnotrelief aus dem Aufweg der Unaspyramide. In: Revue d'Égyptologie 17 (1965), S. 1-13

SChÜRmAnn 1991 SCHÜRMAnN, Astrid: Griechische Mechanik und antike Gesellschaft. Studien zur staatlichen Förderung einer technischen Wissenschaft. Stuttgart : Franz Steiner, 1991 (BOETHIUS - Texte und Abhandlungen zur Geschichte der Mathematik und der Naturwissenschaften 27)

SCHUBERT 1984 SCHUBERT, Doris: Frauen in der deutschen Nachkriegszeit, Band 1: Frauenarbeit 1945-1949: Quellen und Materialien. Düsseldorf : Schwann, 1984 (Geschichtsdidaktik: Studien, Materialien 21) 
SEIDL 1982 SEIDL, Erwin: Das Grundsteuerkataster in Ägypten vor der Ptolemäerzeit. In: ModrZeJews KI, Joseph (Hrsg.) ; LieBS, Detlev (Hrsg.): Symposion 1977. Vorträge zur griechischen und hellenistischen Rechtsgeschichte (Chantilly, 1.-4. Juni 1977). Köln [u. a.] : Böhlau, 1982 (Akten der Gesellschaft für griechische und hellenistische Rechtsgeschichte 3), S. 179-182

SeIPEL 1984 Seipel, Wilfried: Staat und Gesellschaft. In: EgGebrecht, Arne (Hrsg.): Das Alte Ägypten. München : C. Bertelsmann, 1984, S. 117-196

SEThE 1905 SETHE, Kurt: Die Entwicklung der Jahresdatierung bei den alten Ägyptern. In: Untersuchungen zur Geschichte und Altertumskunde Ägyptens 3 (1905), S. 60-100

SETHE 1916 Sethe, Kurt: Von Zahlen und Zahlworten bei den alten Ägyptern. Straßburg : Karl J. Trübner, 1916 (Schriften der Wissenschaftlichen Gesellschaft Straßburg 25)

SEYFRIED 1981 SEYFRIED, Karl-Joachim: Beiträge zu den Expeditionen des Mittleren Reiches in die Ostwüste. Hildesheim : Gerstenberg, 1981 (Hildesheimer Ägyptologische Beiträge 15)

Shaw 1981 Shaw, Thurstan: Towards a Prehistoric Demography of Africa. In: FyFE, Christopher (Hrsg.) ; MCMASTER, David (Hrsg.): African Historical Demography Bd. 2. Edinburgh : Centre of African Studies, 1981, S. 581-606

SiMPSON 1959 SiMPSON, William K.: Historical and lexical notes on the new series of Hammamat inscriptions. In: Journal of Near Eastern Studies 18 (1959), S. 20-37

SimPSON 1963 SimPSON, William K.: Papyrus Reisner I. The Records of a Building Project in the Reign of Sesostris I. Transcription and Commentary. Boston : Museum of Fine Arts, 1963

Smither 1941 Smither, Paul C.: A tax-assessor's journal of the Middle Kingdom. In: Journal of Egyptian Archaeology 27 (1941), S. 74-76

Sонм 1949 Sонм, Rudolph: Institutionen, Geschichte und System des Römischen Privatrechts. Berlin : Von Duncker \& Humblot, 1949

Sonntag 1987 Sonntag, Regine: Studien zur Bewertung von Zahlenangaben in der Geschichtsschreibung des früheren Mittelalters: Die Decem Libri Historiarum Gregors von Tours und die Chronica Reginos von Prüm. Kallmünz/Opf. : Michael Laßleben, 1987 (Münchner Historische Studien, Abteilung Mittelalterliche Geschichte 4)

SOUČEK 1963 SOUČEK, Vladimír: Ein neues hethitisches Ritual gegen die Pest. In: Mitteilungen des Instituts für Orientforschung 9 (1963), S. 164-174

SPIEGELBERG 1923 SPIEgelBerg, Wilhelm: Die Etymologie von 2OY2€ >Fehlgeburt<. In: Zeitschrift für Ägyptische Sprache und Altertumskunde 58 (1923), S. 56

SPOERRI 1991 SPOERRI, Walter: Diodorea. In: Museum Helveticum 48 (1991), S. 310-319

Sternberg 1984 Sternberg, Heike: s. v. >Sachmet<. In: Helck, Wolfgang (Hrsg.) ; WeStenDORF, Wolfhart (Hrsg.): Lexikon der Ägyptologie Bd. 5: Pyramidenbau-Steingefäße. Wiesbaden : Otto Harrassowitz, 1984, S. 323-333 
Sternberg-el Hotabi 2000 Sternberg-el Hotabi, Heike: Politische und sozio-ökonomische Strukturen im perserzeitlichen Ägypten. In: Zeitschrift für Ägyptische Sprache und Altertumskunde 127 (2000), S. 153-167

Stewart 1979 Stewart, Harry M.: Egyptian Stelae, Reliefs and Paintings from the Petrie Collection. Part Two: Archaic Period to Second Intermediate Period. Warminster : Aris \& Phillips, 1979

STRUdwick 1995 STRUdwick, Nigel: The population of Thebes in the New Kingdom. Some preliminary thoughts. In: Assman, Jan (Hrsg.) u. a.: Thebanische Beamtennekropolen. Neue Perspektiven archäologischer Forschung. Internationales Symposion Heidelberg 9.-13.6.1993. Heidelberg : Heidelberger Orientverlag, 1995 (Studien zur Archäologie und Geschichte Altägyptens 12), S. 97-105

ThOMPSON 1997 Thompson, Dorothy J.: The Infrastructure of Splendour: Census and Taxes in Ptolemaic Egypt. In: Cartledge, Paul (Hrsg.) ; Garnsey, Peter (Hrsg.) ; Gruen, Erich (Hrsg.): Hellenistic Constructs. Essays in Culture, History, and Historiography. Berkeley [u. a.] : University of California Press, 1997 (Hellenistic Culture 26), S. 242-257

Thompson 1999a Thompson, Dorothy J.: Irrigation and Drainage in the Early Ptolemaic Fayyum. In: Bowman, Alan K. (Hrsg.) ; RogAn, Eugene (Hrsg.): Agriculture in Egypt. From Pharaonic to Modern Times. Oxford : Oxford University Press, 1999 (Proceeding of the British Academy 96), S. 107-122

Thompson 1999b Thompson, Dorothy J.: New and Old in the Ptolemaic Fayyum. In: Bowman, Alan K. (Hrsg.) ; Rogan, Eugene (Hrsg.): Agriculture in Egypt. From Pharaonic to Modern Times. Oxford : Oxford University Press, 1999 (Proceeding of the British Academy 96), S. 123-138

Tietze 1985 Tietze, Christian: Amarna. Analyse der Wohnhäuser und soziale Struktur der Stadtbewohner. In: Zeitschrift für Ägyptische Sprache und Altertumskunde 112 (1985), S. 48-84

TILl 1951 Till, Walter C.: Die Arzneikunde der Kopten. Berlin : Akademie-Verlag, 1951

Toledano 1998 Toledano, Ehud R.: Social and economic change in the »long nineteenth century «. In: DALY, M. W. (Hrsg.): The Cambridge history of Egypt. Volume two: Modern Egypt from 1517 to the end of the twentieth century. Cambridge : Cambridge University Press, 1998, S. 252-284

TORREnCE und Grattan 2002 TORREnCE, Robin ; Grattan, John: The archaeology of disasters: past and future trends. In: Natural Disasters and Cultural Change. London [u. a.] : Routledge, 2002 (One World Archaeology 45), S. 1-18

Tosi und RocCATI 1972 Tosi, Mario ; Roccati, Alessandro: Stele e altre epigrafi di Deir el Medina n. $50001-n .50262$. Turin : Edizioni d'Arte Fratelli Pozzo, 1972 (Catalogo del Museo Egizio di Torino. Serie Seconda - Collezioni 1)

Trigger u. a. 1983 Trigger, Bruce G. (Hrsg.) u. a.: Ancient Egypt. A Social History. Cambridge : Cambridge University Press, 1983 
UNRUH 2001 UnRUh, Frank: »... Dass alle Welt geschätzt würde.«Volkszählung im Römischen Reich. Stuttgart : Theiss, 2001 (Schriften des Limesmuseums Aalen 54)

Valbelle 1985a Valbelle, Dominique: Eléments sur la démographie et le paysage urbains, d'après les papyrus documentaires d'époque pharaonique. In: Cahier de Recherches de l'Institut de Papyrologie et d'Égyptologie de Lille 7 (1985), S. 75-87

Valbelle 1985b Valbelle, Dominique: «Les ouvriers de la tombe». Deir el-Médineh à l'époque ramesside. Kairo : Institut Français d'Archéologie Orientale, 1985 (Bibliothèque d'Étude 96)

VALBELLE 1987 VAlbelle, Dominique: Les recensements dans l'Egypte pharaonique des troisième et deuxième millénaires. In: Cahier de Recherches de l'Institut de Papyrologie et d'Égyptologie de Lille 9 (1987), S. 33-49

VANDERSLEYeN 1989 VANDERSLEYen, Claude: The rekhyt and the Delta. In: NibBI, Alessandra (Hrsg.): Proceedings of Colloquium >The Archaeology, Geography and History of the Egyptian Delta in Pharaonic Times<, Wadham College 29-31 August 1988, Oxford. Oxford : DE Publications, 1989 (Discussions in Egyptology Special Number 1), S. 301-304

VANDIER 1936 VANDIER, Jacques: La famine dans l'Égypte ancienne. Kairo : Institut Français d'Archéologie Orientale, 1936 (Recherches d'archéologie, de philologie et d'histoire 7)

VARILLE 1968 VARILLE, Alexandre: Inscriptions concernant l'architecte Amenhotep fils de Hapou. Kairo : Institut Français d'Archéologie Orientale, 1968 (Bibliothèque d'Étude 44)

Verhoeven 1986 Verhoeven, Ursula: s. v. >Unwetter<. In: Helck, Wolfgang (Hrsg.) ; WeStendoRf, Wolfhart (Hrsg.): Lexikon der Ägyptologie Bd. 6: Stele-Zypresse. Wiesbaden : Otto Harrassowitz, 1986, S. 860-861

VERnUS 1986 Vernus, Pascal: Le surnom au Moyen Empire. Rom : Biblical Institute Press, 1986 (Studia Pohl 13)

Vleeming 1993 Vleeming, Sven P.: Papyrus Reinhardt. An Egyptian Land List from the Tenth Century B. C. Berlin : Akademie-Verlag, 1993 (Hieratische Papyri aus den staatlichen Museen zu Berlin)

Vogelsang 1913 Vogelsang, Friedrich: Kommentar zu den Klagen des Bauern. Leipzig : J. C. Hinrichs, 1913 (Untersuchungen zur Geschichte und Altertumskunde Ägyptens 6)

WACHSMUTH 1903 WACHSMUTH, Curt: Zwei Kapitel aus der Bevölkerungsstatistik der alten Welt. 1. Die Ziffer der Bevölkerung Ägyptens. In: Klio 3 (1903), S. 272-280

WAŁeK-CZernecki 1937 WaŁeK-CZerneCKI, Tadeusz: La population de l'Égypte ancienne. In: Congrès international de la population Bd. 2. Paris, 1937, S. 7-13

WAŁeK-Czernecki 1941 WaŁeK-Czernecki, Tadeusz: La population de l'Égypte à l'époque saïte. In: Bulletin de l'Institut d'Egypte 23 (1941), S. 37-62 
Wallace 1753 Wallace, Robert: A Dissertation on the Numbers of Mankind in Antient and Modern Times. Edinburgh : G. Hamilton \& J. Balfour, 1753

Wallace 1938 Wallace, Sherman L.: Census and Poll-Tax in Ptolemaic Egypt. In: American Journal of Philology 59 (1938), S. 418-442

WARBURTON 1991 WARBURTON, David: Keynes'sche Überlegungen zur altägyptischen Wirtschaft. In: Zeitschrift für Ägyptische Sprache und Altertumskunde 118 (1991), S. 76-85

WARbUrton 1995 Warburton, David: The Economy of Ancient Egypt Revisited Yet Again or Die wirtschaftliche Bedeutung der Erweiterung des Bestehenden. In: Göttinger Miszellen 146 (1995), S. 103-111

WARBURTON 1997 WARBURTON, David: State and Economy in Ancient Egypt. Fribourg : University Press, 1997 (Orbis Biblicus et Orientalis 151)

WARD 1982 WARD, William A.: Index of Egyptian Administrative and Religious Titles of the Middle Kingdom. Beirut : American University of Beirut, 1982

VON DER WAY 1991 WAY, Thomas von der: Die Grabungen in Buto und die Reichseinigung. In: Mitteilungen des Deutschen Archäologischen Instituts in Kairo 47 (1991), S. 419-424

VON DER WAY u.a. 1997 WAY, Thomas von der u. a.: Tell el-Fara'în-Buto. Ergebnisse zum frühen Kontext. Kampagnen der Jahre 1983-1989. Mainz : Philipp von Zabern, 1997 (Archäologische Veröffentlichungen. Deutsches Archäologisches Institut Abteilung Kairo 83)

WeHR 2002 WeHr, Marco: Der Schmetterlingsdefekt. Turbulenzen in der Chaostheorie. Stuttgart : Klett-Cotta, 2002

Wenke 1986 Wenke, Robert J.: Old Kingdom Community Organization in the Western Egyptian Delta. In: Norwegian Archaeological Review 19 (1986), S. 15-33

WENKE u.a. 1988 WenKE, Robert J. u. a.: Kom el-Hisn: Excavation of an Old Kingdom Settlement in the Egyptian Delta. In: Journal of the American Research Center in Egypt 25 (1988), S. 5-34

WESTENDORF 1966 WESTENDORF, Wolfhart: Beiträge aus und zu den medizinischen Texten. In: Zeitschrift für Ägyptische Sprache und Altertumskunde 92 (1966), S. 128-154

WeSTENDORF 1981 WeStendorf, Wolfhart: Beiträge aus und zu den medizinischen Texten. 5. Der >lastende< Nil und >die Seuche des Jahres<. In: Göttinger Miszellen 49 (1981), S. 77-83

WESTENDORF 1999 WESTENDORF, Wolfhart: Handbuch der altägyptischen Medizin. Leiden [u. a.] : E. J. Brill, 1999 (Handbuch der Orientalistik 36)

WeSTENDORF 2000 Westendorf, Wolfhart: Seuchen im Alten Ägypten. In: KAREnBerg, Axel (Hrsg.) ; LEITZ, Christian (Hrsg.): Heilkunde und Hochkultur I. Geburt, Seuche und Traumdeutung in den antiken Zivilisationen des Mittelmeerraumes. Münster [u. a.] : Lit Verlag, 2000 (Naturwissenschaft - Philosophie - Geschichte 14), S. 55-69 
WIESEHÖFER und RENGER 1997 WIESEHÖFER, Josef ; RENGER, Johannes: s. v. >Bevölkerung<. In: CANCIK, Hubert (Hrsg.) ; SCHNEIDER, Helmuth (Hrsg.): Der Neue Pauly: Enzyklopädie der Antike Bd. 2. Stuttgart ; Weimar : J. B. Metzler, 1997, S. 599-606

WILCKEN 1899 WiLCKEN, Ulrich: Griechische Ostraka aus Aegypten und Nubien. Leipzig [u. a.] : Von Giesecke \& Devrient, 1899

WiLCKEN 1912 WiLCKEN, Ulrich: Grundzüge und Chrestomathie der Papyruskunde. Leipzig [u. a.] : B. G. Teubner, 1912

Wilfong 1994 Wilfong, Terry G.: The Egyptological Papers of Klaus Baer. In: Silverman, David P. (Hrsg.): For His Ka. Essays Offered in Memory of Klaus Baer. Chicago : The Oriental Institute of the University of Chicago, 1994 (Studies in Ancient Oriental Civilization 55), S. 285323

Wilkinson 2000 Wilkinson, Toby A.: Royal Annals of Ancient Egypt. The Palermo Stone and its associated fragments. London [u. a.] : Kegan Paul, 2000

WiNKLER 1934 WinKLER, Hans A.: Bauern zwischen Wasser und Wüste. Volkskundliches aus dem Dorfe Kimân in Oberägypten. Stuttgart : W. Kohlhammer, 1934

Winter 1976 WinTER, J. M.: Some Aspects of the Demographic Consequences of the First World War in Britain. In: Population Studies 30 (1976), S. 539-552

WIRTH 1993 WIRTH, Gerhard: Diodor und das Ende des Hellenismus. Mutmaßungen zu einem fast unbekannten Historiker. Wien : Verlag der Österreichischen Akademie der Wissenschaften, 1993 (Sitzungsberichte der österreichischen Akademie der Wissenschaften, Phil.-Hist. Klasse 600)

WitTfogel 1962 WitTfogel, Karl A.: Die orientalische Despotie. Eine vergleichende Untersuchung totaler Macht. Köln [u. a.] : Kiepenheuer \& Witsch, 1962

Wolf 1930 Wolf, Walther: Papyrus Bologna 1086: ein Beitrag zur Kulturgeschichte des Neuen Reiches. In: Zeitschrift für Ägyptische Sprache und Altertumskunde 65 (1930), S. 89-97

WreSZINSKI 1909 WresZINSKI, Walter: Der grosse medizinische Papyrus des Berliner Museums (Pap. Berl. 3038). Leipzig : J. C. Hinrichs, 1909 (Die Medizin der alten Ägypter 1)

YURCO 1995 YuRCO, Frank J.: Narmer: First King of Upper and Lower Egypt. A Reconsideration of His Palette and Macehead. In: The Journal of the Society for the Study of Egyptian Antiquities 25 (1995), S. 85-95

ZiBelius 1972 Zibelius, Karola: Afrikanische Orts- und Völkernamen in hieroglyphischen und hieratischen Texten. Wiesbaden : Dr. Ludwig Reichert, 1972 (Beihefte zum Tübinger Atlas des Vorderen Orients, Reihe B 1)

ZORN 1994 Zorn, Jeffrey R.: Estimating the Population Size of Ancient Settlements: Methods, Problems, Solutions, and a Case Study. In: Bulletin of the American Society for Oriental Research 295 (1994), S. 31-48 